\title{
STBiO
}

-1. $\bigcirc 1 \bigcirc \begin{aligned} & \text { Sistemática, Taxonomia anima } \\ & \text { e Biodiversidade - MZUS P }\end{aligned}$

Arthur de Lima Oliveira e Silva

\section{SKELETAL ANATOMICAL DESCRIPTION OF ISOGOMPHODON OXYRHYNCHUS (MÜLLER \& HENLE, 1839) AND DISCUSSION OF ITS PHYLOGENETIC POSITION WITHIN CARCHARHINIDAE (CHONDRICHTHYES, CARCHARHINIFORMES)}

DESCRIÇAO ANATÔMICA DO ESQUELETO DE ISOGOMPHODON OXYRHYNCHUS (MÜLLER \& HENLE, 1839) E DISCUSSÃO SOBRE SEU POSICIONAMENTO FILOGENÉTICO DENTRO DE CARCHARHINIDAE (CHONDRICHTHYES, CARCHARHINIFORMES) 


\title{
Skeletal anatomical description of
} Isogomphodon oxyrhynchus (Müller \& Henle, 1839) and discussion of its phylogenetic position within Carcharhinidae (Chondrichthyes, Carcharhiniformes)

\author{
DESCRIÇAO ANATÔMICA DO ESQUELETO DE ISOGOMPHODON \\ OXYRHYNCHUS (MÜLLER \& HENLE, 1839) E DISCUSSÃO SOBRE \\ SEU POSICIONAMENTO FILOGENÉTICO DENTRO DE \\ CARCHARHINIDAE (CHONDRICHTHYES, CARCHARHINIFORMES)
}

Corrected Version

Dissertation submitted to the Graduate Program of the Museu de Zoologia da Universidade de São Paulo in partial fulfillment of the requirements for the degree of Master of Science (Systematics, Animal Taxonomy and Biodiversity).

Advisor: Prof. Dr. Mario Cesar Cardoso de Pinna

Co-Advisor: Prof. Dr. Thiago Silva Loboda 
I authorize the reproduction and dissemination of this work in part or entirely by any electronic or conventional means, for study and research, provide the source is cited.

\section{Serviço de Biblioteca e Documentação}

\section{Museu de Zoologia da Universidade de São Paulo}

\section{Cataloging in Publication}

Silva, Arthur de Lima Oliveira e

Skeletal anatomical description of Isogomphodon oxyrhynchus (Müller \& Henle, 1839) and discussion of its phylogenetic position within Carcharhinidae (Chondrichthyes, Carcharhiniformes).= Descrição anatômica do esqueleto de Isogomphodon oxyrhynchus (Müller \& Henle, 1839) e discussão sobre seu posicionamento filogenético dentro de Carcharhinidae (Chondrichthyes, Carcharhiniformes). / Arthur de Lima Oliveira e Silva; orientador Mario Cesar Cardoso de Pinna. São Paulo, 2021.

$355 \mathrm{p}$.

Dissertação de Mestrado - Programa de Pós-Graduação em Sistemática, Taxonomia Animal e Biodiversidade, Museu de Zoologia, Universidade de São Paulo, 2021.

Versão corrigida

Volume único

1. Isogomphodon oxyrhynchus - anatomia. 2. Chondrichthyes, Carcharhiniformes - filogenética. I. Pinna, Mario Cesar Cardoso de, orient. II. Título. 
de Lima Oliveira e Silva, Arthur

Skeletal anatomical description of Isogomphodon oxyrhynchus (Müller \& Henle 1839) and discussion of its phylogenetic position within Carcharhinidae (Chondrichthyes, Carcharhiniformes

Dissertation submitted to the Graduate Program of the Museu de Zoologia da Universidade de São Paulo in partial fulfillment of the requirements for the degree of Master of Science (Systematics, Animal Taxonomy and Biodiversity).

Date approved:

\section{COMMITTEE MEMBERS}

Prof. Dr.

Institution:

Decision:

Signature:

Prof. Dr.

Institution:

Decision:

Signature:

Prof. Dr.

Institution:

Decision:

Signature: 
To the art of smilling everytime the world says 'no' 


\section{ACKNOWLEDGMENTS}

I know I may be exaggerated, but I learned to always remember the ones who somehow helped us along the way, and I try my best to never forget them. What I am about to register here goes beyond the two years I spent in MZUSP writing this dissertation, it talks about the biologist I am today and how grateful I am for all the contributions I received along the way. And during this dissertation, I got a lot of support from my friends and family, by which I would never forgive myself if I did not put all their names here. I would like to remember and thank each and every one of these people.

Firstly, an immense thank you to my supervisors Dr. Mario de Pinna and Dr. Thiago Loboda, who not only showed me the right path to be the scientist I intend to be someday but also were extremely dedicated during all the steps of this project.

To my family, who has always been my strongest base to allow me to continue on pursuing my dream and my career: Evandro, Ana Lucia, Lucas, Gustavo, Camila, Clarissa, Adriana, Karina, Rosilda, Juraci, Luíza, Ana Carla, Andréia, Bruno, Maurício, Wesley, Therezinha, Laura, Nilson, José Alves, José Neto, Adriana, Vitória, Ismael, Creuzi, Breno and Davi.

A huge thank you to lab mates from the Ichthyological Collection, and although some are already somewhere else, they will always be part of this lab as in the day I arrived: Manoela Marinho, Priscilla Camelier, Paulo Presti, Murilo Pastana, George Vita, Vinicius Carvalho, Vinícius Espíndola, Gustavo Ballen, Illana Fichberg, Marina Loeb, Roberto Camacho, Michel Donato, Osvaldo Oyakawa, Fábio Pupo, Vitor Abrahão, Verônica Slobodian, Péricles Gentile, Henrique Varela, Alessio Datovo, Heraldo Britski, Naércio Menezes, José Lima, Guilherme Dutra, Karolina Reis, Jaqueline Batilana, Luz Ochoa and João Pedro Moto. A special reminder to Luiz Antônio Wanderley Peixoto for the tremendous effort in bringing the adult specimen from Belem to the MZUSP collection and to Victor A. Tagliacollo for performing the molecular analysis.

I want to dedicate a special thanks to Alberto Carvalho and Vanessa Yamamoto from the CT-Scan lab of MZUSP, who put a tremendous effort in scanning and editing the images from my specimens to the best quality possible, therefore being crucial for the project, and to Lara Guimarães from the SEM lab, who spent hours alongside me collection the dermal denticles images which was extremely helpful. Also, I would like to leave a special note to Dione Seripierri for the enormous support with the literature and checking the format of this 
final version. A special reminder to the MZUSP staff Marta Grobel, Mariana Shinohara, Selma Midori and Sônia Favaro for all the support with documentations.

My museum mates and academic mates that also helped me along the way, directly or indirectly: Tamires Andrade, Juan Pablo, Thiago Polizei, Rafaela Lumi, Jose Eduardo Serrano, Gustavo Rizzi, Arthur Sena, Paulo Bernarde, Bruno Augusto, Afonso Santiago, Pedro Paulo, Flora Roncolatto, Daniela Genari, Lucas Pizentin, Rodolfo Santos, Letizia Migliore, Rafael Souza, Aline Benetti, Francisco Eriberto, Edgar Blois, Paulo Pachelle, Paola Ancajima, Jordan Goetze, Todd Bond, Matt Birt, Bernardo Ferraz, Paulo César Motta, Nathan Carvalho, Marcelo Melo and Amanda Alves. A special note to Caio Guilherme Pereira, who in that late night shifts cutting leafs taught me in my early academic years what I should do to be a good biologist, and to Daniela França and Kleber Mathuabara, who not only are great biologists in their areas but also very dear friends who became a part of my family.

To my friends who also work on elasmobranch research. Please know that I'm tremendously honoured to call you colleagues: Ana Clara Athayde, Aline Poscai, Bianca Rangel, Jade Medeiros, Gabriela Machado, Giovanni Torres, Karla Soares and Natascha Wosnick. A special note to Andre Casas who helped me a lot in my chapter 2, to João Paulo Capretz for his advice and counselling during pretty much the whole dissertation conduction, to Diego Vaz for all the chats and supports to become the researcher I intend to be and to Professor Otto Gadig for all the support with available material and for living up to my expectations as my childhood idol.

To the school friends who are still my dear mates and will for sure be as so for a lifetime: My brother Murillo Brederode Gonçalves, although born from another mother, it is my family as much as my blood relatives; My brother Cauê Chaves, who will always be my brother regardless of how long time passes; My friend Gabriel Neiva, who doesn't know that but he has been one of my great examples in life in many aspects; My friend Stefano Romani, who has an incredible potential to any of his endeavours; My friend Thiago Nicolaidis, the smartest man I know; My friend Matheus Santinello, who is an extraordinary basketball player and a true friend; My brothers Matheus and Rafael Martins, who basically grew up with me and taught me that true friendship never dies; My friend Guilherme Figueiredo, who I personally admire for his strength, both physically and mentally; My friend Thiago Gulmine, who is brilliant in any game he plays; My friend Vítor Bichara, who not only is a smart man but also gave me the coolest handshake ever; My friend Paulo Kimura, who recently became closer to me and truly has a great heart; My friend Gustavo Soato, who in the few moments we had he gave me great 
memories. And last but not least, my friend Camila Martinelli, who doesn't know that but taught me what true friendship is about, and I am extremely grateful to her.

To the Ratos de Praia, the guys were basically born in the sand and know how to play beach football: Victor Costa, one of my best friends and a tremendously kind person; Victor Chaves, a legendary player and dancer; Matheus Pimenta, the best futvolêi player the world is about to witness; Fabio Zago, the best truco partner I could have asked for; Daniel Ferrari, my school friend who became very special quite recently; Gianlucca Carpaneda, a recent but true friend; Hugo Costa, the best father I know; Hudson Monteiro, a very kind soul. To the legend Rodrigo Pxoto, who unfortunately passed away too soon but it will always be remembered.

To the women of my life: Clara Brito for being my partner in crime and in many other memories, Priscilla Magalhães Santos for being my life advisor on many occasions, and to Marina Coriolano, who has been more than one of my best friends but also my safehouse in every storm. Also, thanks to her husband Bruno who also became a very dear friend.

The friends from the BBBritz fish course, who will for sure be brilliant scientists: Lorena Agostinho, Nathalie Amorim, Emilia Wendt, Karla Soares, Ariane, Cintia Oliveira, Manuela Dopazo, Junior Chuctyaya, Carolina Vieira, Daniel Coutinho and Sérgio Santos.

The members of COSom: Ana Paula Cantamessa, André Vésper, Daniel Dovigo, Eduardo Carvalho, Eduardo Gonçalves, Gabriel Pacheco, Henrique Rivero, João Lucas Falcão, Madson Campos, Marla Calazans, Matheus Silva, Miguel Machnicki, Pedro Barros, Priscilla Magalhães Santos, Yasmin Maccari.

The friends that UnB gave me: William Januário, Bernardo de Paula, Erick Leal, Bernardo Kerr, Antônia Arlina César, Roberto Oliveira, Ravenna Horana, Dhara Reis, Bruna Lisboa, Arthur Rodrigues Garcia, Larissa Nunes, Marcella Campos, Leticia Mallmann, Bianca Moura, Bryam Amorim, Victor Marques, Mayumi, Nayara Rodrigues, Nathalia Miranda, Matheus Costa, Alex Resende, Fernando Pagels, João Fregonasse, Caroline Leppa, Ingrid Mutinelli, Mariana Bicalho, Mariana Mathias, Marina Minari, Marcos Simão, Matheus Simão, Tomás Maldonado, Lucas Viana, Bernardo Brixi, Lucas Xavier, Rodrigo Campelo, Marcelo Coelho, Paula Schettman, Victor Andrade, Felipe Maia, Guilherme Ferraz, Vinicius Ferraz, Guilherme Andrade, Rodrigo Born, Marina Moreira, Erica Marina, Erico Grassi, Rodolfo Patini, Yan Felipe, Lorena Neno, Mariana Zischegg, Beatriz Rocha, Samara Borges, Mariana Caldeira, Barbara Neumann, Beatriz Bogoni, Clara Vida, Rafaela Dandara, Daniella Vieira, Débora Resende, Diogo Telheiro, Diogo Lott, Elias Trotti, Flávia Santos, Filipe Sartório, Gabriel Drose, Gabriel Cubas, Gabriela Santos, Anderson de Lima, Ana Cecilia Holler, Igor 
Lima, João Martins, Karina Mendes, Karina Simon, Carolina Nappo, Lara Nóbrega, Liviane Claver, Lorrane Fortes, Luana Alves, Lucas Bohrer, Lucas Girotto, João Pedro Terra, Luiz Miguel Rottili, Marcos Medeiros, Luciana de Oliveira, Isabela Ornelas, Milla Gois, Murilo Durão, Nick Nunes, Pedro de Souza Marques, Radan Souza, Clara Siracusa, Matheus Mourão, Thiago Albuquerque. João Lucas Prado, Vitor Sena, Ricardo Cotts, Lucas Rodrigues, Matheus Rodrigues, Lucas Leon, Hérik Hédem, Matheus Gonzaga, Gabriel Caputo, Luiz Fernando Reis, Eduardo Rossi, Marcos Reis, Reynaldo Magalhães, Raquel Santos, Raffael Araújo, Sarah Dobkowski, Samuel Montenegro, Leticia Velasco, Gabriella Ferreira, Igor Cutrim, Jairo, Jesine Neto, Gabriel Ginani, Guilherme Hirata, Catarina Vargas, Stefano Aires, Thaís Carvalho, Victor Fernandes, Thais Damasceno, Texugo Matos, Takeshi Ohashy, Pedro Nogueira, Vinicius Trindade, Priscila Maia, Artur Morbeck, Harlen Ferreira, Igor Daniel, Juliana Gonçalves, Eliza Teles, Vanessa Carla, Lucas Ramalho, Arthur Ribeiro, Guilherme Andrade, Arthur Sandoval, Bárbara Nascimento, Laura Pertence, Victor Santos, Victor Mendes, Vitoria Vilarinho, Waira Saraiva, Wanderson Lacerda, Vinicius Paes, Vinicius Teixeira, Rayane Leal and Pedro Gomes.

My australian mates who gave me the best memories during my abroad time in Perth: Freya Crozier, Michael Taylor, Renee Cooper, Caeleb Simkin, Sinai-Jade Wright, Tahlia Gibson, Benjamin Plunkett, Jonathan Heir, Jeff Bennet, Imogene Weatherly, Rhisi Ray, Saddiq Kalam, Naya, Cassandra Greenwell, Kongo Kimani, Denis Dmitrievich, Alex King, Danni Fuller, Dillon Jevon, Jordyn Rose, Yasmin Coutinho, Jerrysson Yee, Joshua Keen, Laura, Joshua Wedd, Shae Layton, Erin Oliver, Terence Au, Quenton Cornell, Louie Go, Ellen D'Cruz, Nathan Beerkens, Julian Keller and Curtis Spencer. A special note to Alyssa Shapland who always kept me close and never let me forget that I am always welcome in Australia. A special reminder to Dr. Dave and Nurse Gail, who gave me a place to call home in Paynes Find.

My brazilian mates from UWA: Adam Sordi, Jonathan Marinho Felipe Sturaro, Guilherme Suzano, Igor Abreu, Thiago Haruki, Luiz Gustavo Pereira, Gustavo Lauton, Arthur Matos, Gabriel Sampaio, Ocimar Junior, Renato de Paula, Marcos Paulo Marshall, João Bruno, Felipe Avarena, Rafael Mol, Gustavo Terra, Vitor Santos Guimarães, Vitor Felício, Natasha Costa, Maitê Rodrigues, Natália Escaleira, Gabriela Sardinha, Fernanda Fukai, Maristela Melo, Fernando Pacheco, Ramon Zanow, Raphael Cordeiro, Nicolas Brugnolo, André Rati, Érika Rocha, Isabel Leoni, Leonardo Tavolaro, Andressa Knopp, Marcos Paulo, Luana Castelo, Lorena Monteiro, Ívenis Pita, Lorena Oliveira, Ana Elisa Ferreira, Narelly Almeida, Lígia 
Barbosa, Maria Augusta Starling, Daniele Cristine, Mariana Porto Lopes, Rafael Floriano, Vitor Hugo.

My friends and mentors from Primato: Matheus Primo and Victor Canato, who were responsible for many of the happiest moments and parties in my life, which I will never forget. To my party mates, Filipe Lins, Tainá Ivo, Bruno Santos, Felipe Redorat, Izabel Nunes, Walber Junior, Victor Cleto, Alessandra Maringolo, Natália Calhau, Rafaela Llorente, Renato Barbosa, Ian Souza, Adriano Henrique and Luiz Cláudio Rosas.

The bug players Cláudio Andrade, Júlia Andrade, Felipe Coutinho, Iain Hauber, Sophia, and Cesar Augusto. A special note to Adriana Zimbarg for giving me a place to call home in São Paulo. The friends from São Paulo and from the Biology courses around Brazil: Thyelle Cipriano, Paula Ramalho, Bruna Batista, Beca Moreno, Flavia Sant'anna, Suellen Paiva, Fernando Nodari, Gabrielle Rizzato, Luiz Renato, Raissa Rosa, Duda Romanini, Paloma Garcia, Pedro Yoshi, Isabel Oliveira, Yuli Novski, Eloísa Zanin, Ana Noronha, Caio Ribeiro, Guilherme Ambar, Igor Caetano, Otávio Reis, Paula Krusicki, Maurício Pavani, Igor Drummond, Leonardo Lessa, Roberta Figueiredo, Luiz Pol, Luiza Gondim, Elias Araújo, Matheus Tizo, Pedro Castro, Rafael Pereira da Silva, Stefanie Jordan, Rafael Pagipe, Ryan, Thiago Abreu, José Felici, Carolina Moraes, Camila Dourado, Gustavo Brito, Guilherme Toledo, Julia Pinna, Karla Cunha, Marcelo Kai, Marcos Vasconcelos, Arthur de Lima, Gabriel Sontachi, Thiago Zupelli, Ricardo Custodio, Lucas Silva, Scheila Araujo and Maryani.

The friends I randomly met and became very special: William Rodrigues, Felipe Ramos, Marcella Schmidt, Lucas Vascun, Júlia Haick, Isabela Simas, Gabriella Miranda, Bruno Abarno, Nathaly Plaza, Camilla Bandeira, Rafaela Braga and Fernando Pires.

Finally, I would also like to thank the institutions Zoology Museum of the University of São Paulo, for all the support in terms of structure, material and supervision during this project and Wolmar Wosiacki from Museu Paraense Emilio Goeldi for providing the adult specimen of Isogomphodon oxyrhynchus, which significantly enriched the present work.

This study was financed in part by the Coordenação de Aperfeiçoamento de Pessoal de Nível Superior - Brasil (CAPES) - Finance Code 001. 
"I can't tell where the journey will end...

But I know where to start." 


\begin{abstract}
The Daggernose shark Isogomphodon oxyrhynchus (Müller \& Henle, 1839), sole species in its genus, is endemic to northern South America, from Southern Maranhão State in Brazil to Trinidad and Tobago. Although the species is traditionally classified as estuarine, it occurs also in entirely marine environments. Such habitat shift is seasonal, related to the rain regimen. $I$. oxyrhynchus is easily recognized by the elongated snout, enlarged pectoral fins and reduced eyes, characteristics which account for its unique general aspect in the family Carcharhinidae. The anatomy of $I$. oxyrhynchus remains poorly known and its phylogenetic position is still controversial. In this study, we propose a contribution to the knowledge of I. oxyrhynchus, offering: 1. A description of the entire skeleton and dermal denticles, based on dissections, computerized tomography and Scanning Electron Microscopy; 2. A comparative anatomical study of $I$. oxyrhynchus with other representatives of Carcharhinidae, which is compared with results from a novel molecular analysis; 3. Review of fossil taxa assigned to Isogomphodon. The skeletal description revealed much new information for I. oxyrhynchus, including new information on complexes which were previously entirely unknown in the taxon, such as the appendicular skeleton, mandibular arch and clasper. The comparative and phylogenetic study is organized into 46 morphological characters, which qualitatively supports Carcharhinus porosus as sister-group of I. oxyrhynchus. Results also support the hypothesis of Isogomphodon as a modified member of the genus Carcharhinus. Lastly, a critical review of the fossil taxa assigned to Isogomphodon demonstrated that from the five species previously assigned to Isogomphodon, only two can be positively attributed to the genus.
\end{abstract}

Keywords: Carcharhinidae. Isogomphodon oxyrhynchus. Skeletal. Anatomy. CT-Scan. Comparative anatomy. Fossil. 


\section{RESUMO}

O cação-pato Isogomphodon oxyrhynchus (Müller \& Henle, 1839), única espécie em seu gênero, é endêmica da porção norte da América Sul, do Sul do Maranhão ao arquipélago de Trinidad e Tobago. Embora tradicionalmente seja classificada como estuarina, sua área de ocorrência inclui localidades de água inteiramente salgada, sendo tal mudança de habitat um fenômeno sazonal correlacionado com o regime de chuvas. I. oxyrhynchus é facilmente reconhecido pelo alongamento do focinho, nadadeiras peitorais largas e olhos diminutos, características que lhe outorgam um aspecto geral único na família Carcharhinidae. A anatomia e posicionamento filogenético de $I$. oxyrhynchus ainda permanecem pouco conhecidos ou controversos. No presente trabalho, propomos uma contribuição ao conhecimento da espécie, oferecendo: 1. Descrição completa do esqueleto e dentículos dérmicos, realizada através de dissecções, tomografia computadorizada e Microscopia Eletrônica de Varredura; 2. Estudo da anatomia comparada de I. oxyrhynchus com outros membros de Carcharhinidae, para obtenção de subsídios relevantes para o entendimento de seu posicionamento filogenético na família, sendo esta avaliada também de acordo com uma nova filogenia molecular; 3. Revisão crítica dos registros fósseis alocados em Isogomphodon. A descrição do esqueleto da espécie trouxe informações novas sobre todos os complexos esqueléticos, sendo que o esqueleto apendicular, arco mandibular e clásper são inteiramente inéditos. O estudo comparativo e filogenético propõe 46 caracteres morfológicos potencialmente informativos, indicando Carcharhinus porosus como o provável grupo irmão de I. oxyrhynchus. Os resultados também suportam a hipóteses de Isogomphodon como um membro modificado do gênero Carcharhinus. Por fim, a revisão dos registros fósseis demonstrou que das cinco espécies descritas como Isogomphodon, apenas duas podem positivamente ser atribuídas ao gênero.

Palavras-chave: Carcharhinidae. Isogomphodon oxyrhynchus. Esqueleto. Anatomia. CT-Scan. Anatomia comparada. Fóssil. 


\section{CONTENTS}

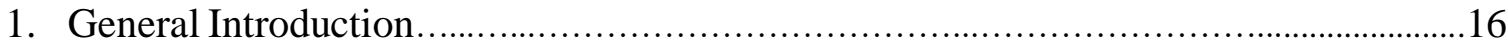

1.1 From Chondrichthyes to Carcharhinidae: major classifications ...................16

1.2 The Daggernose Shark Isogomphodon oxyrhynchus (Müller \& Henle

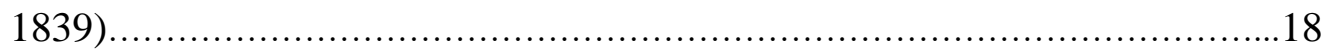

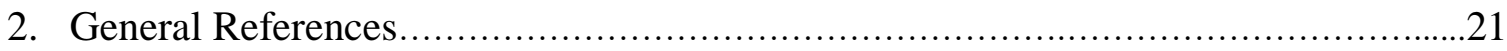

Chapter 1 - Description of the skeletal anatomy and dermal denticles of Isogomphodon

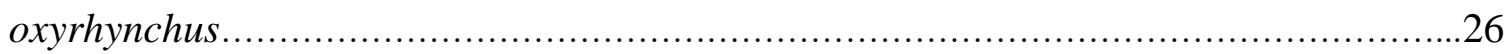

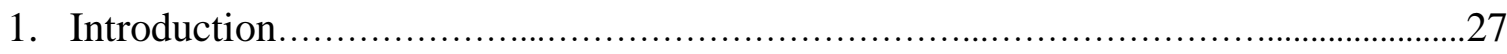

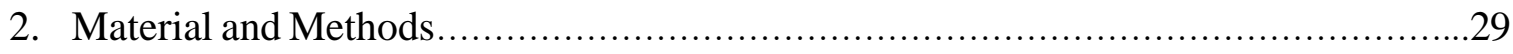

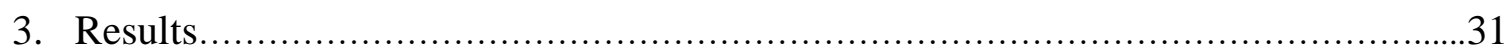

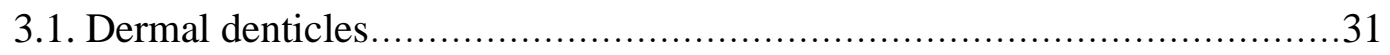

3.1.1. Head Section...................................................... 31

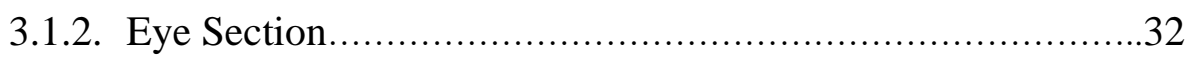

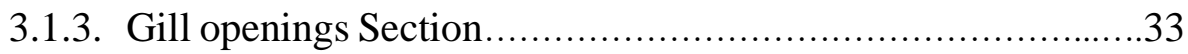

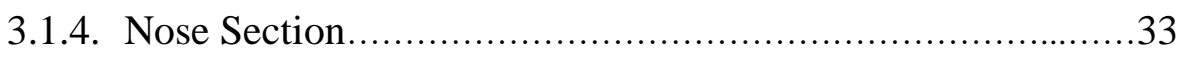

3.1.5. Body Section........................................................ 34

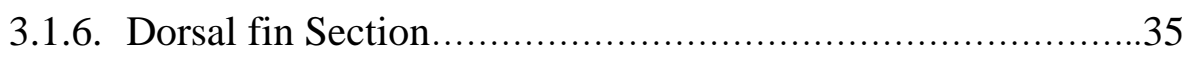

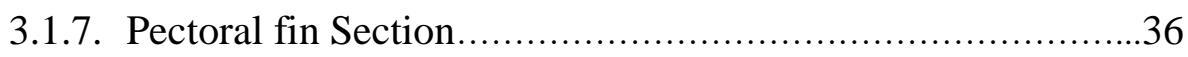

3.1.8. Caudal fin Section..................................................... 37

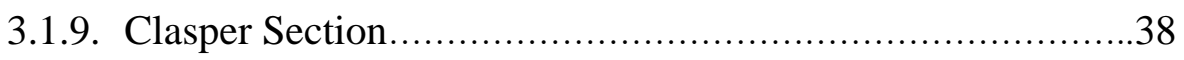

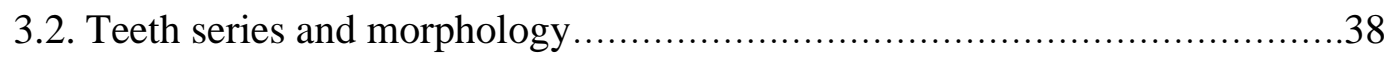

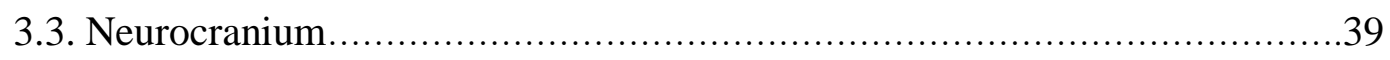

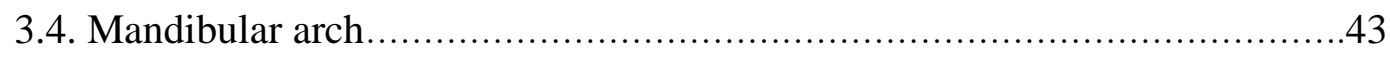

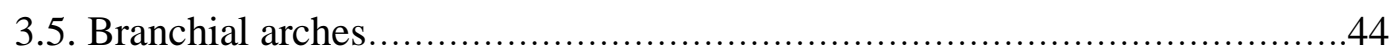

3.5.1. Pharyngobranchials ...............................................45

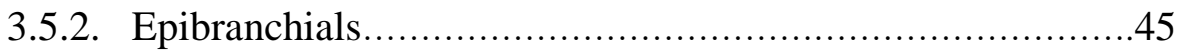

3.5.3. Ceratobranchials..............................................46

3.5.4. Hypobranchials...................................................46

3.5.5. Basibranchial/Basibranchial copula.............................46

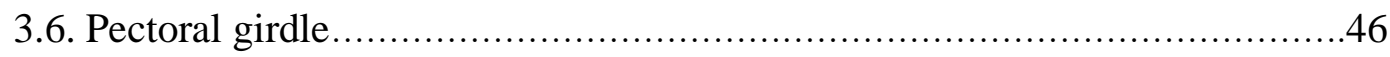

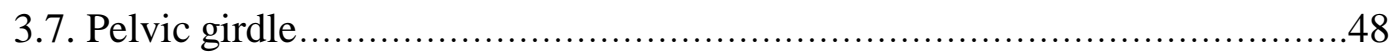

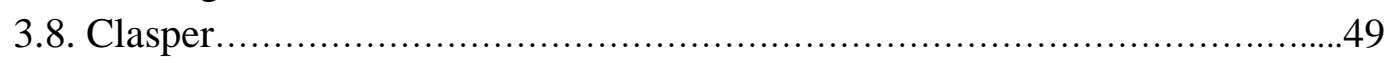

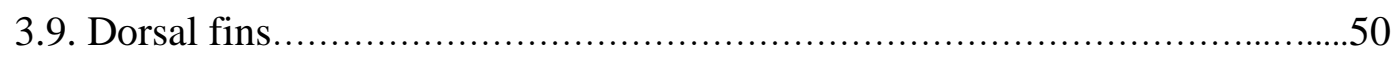

3.9.1. First dorsal fin.....................................................50

3.9.2. Second dorsal fin................................................51

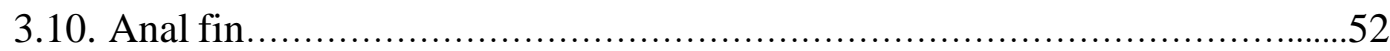

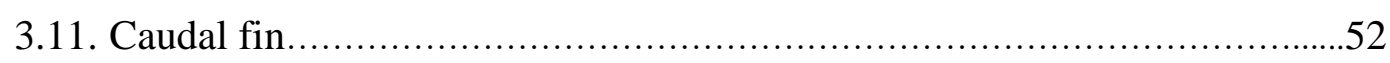

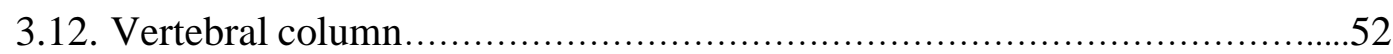

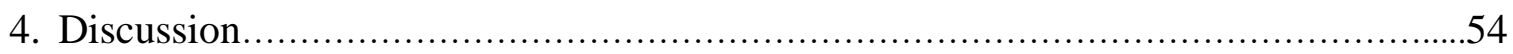




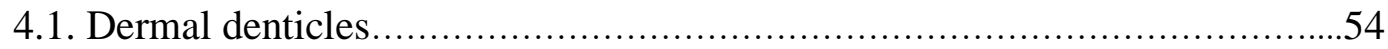

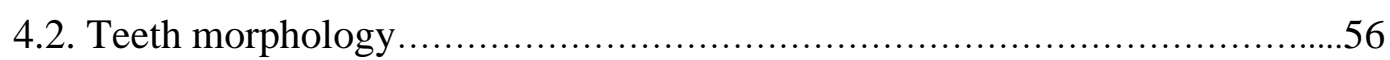

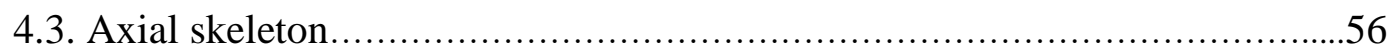

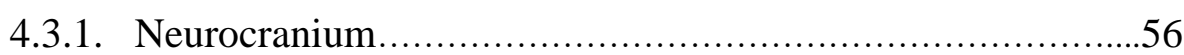

4.3.2. Splanchnocranium............................................57

4.3.2.1. Mandibular and Hyoid arches.........................57

4.3.2.2. Branchial arches.......................................58

4.3.3. Vertebral Column.....................................................59

4.3.4. Caudal fin................................................................6

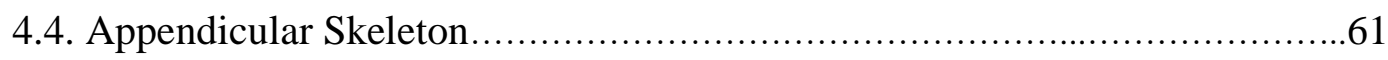

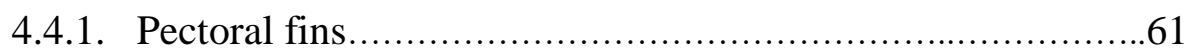

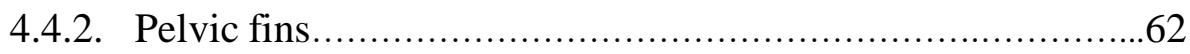

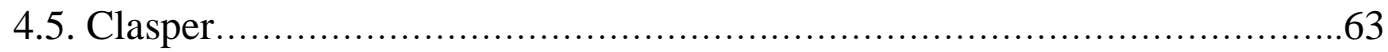

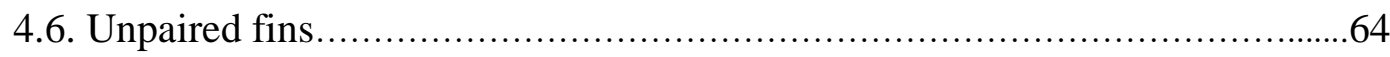

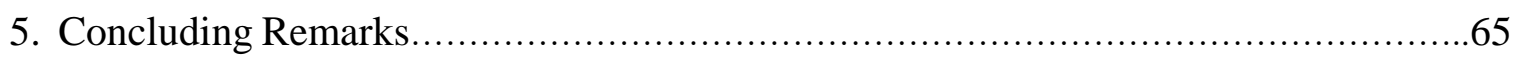

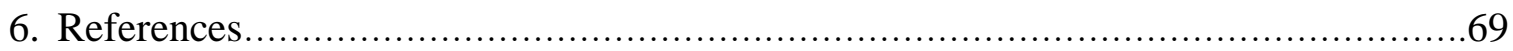

7. Appendix A - Analysed Material............................................................... 73

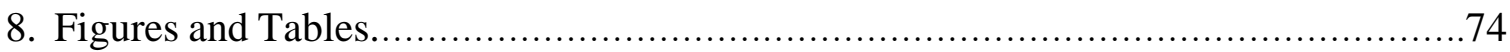

Chapter 2 - Isogomphodon oxyrhynchus within Carcharhinidae: Comparative anatomy and

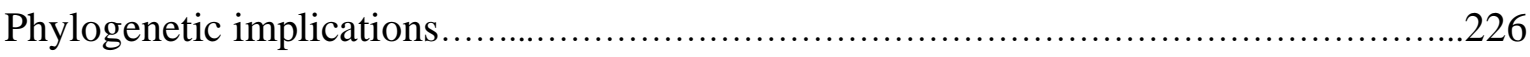

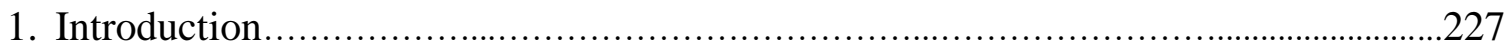

1.1.Morphological complexes and their significance in Selachii phylogeny.....227

1.2.Phylogenetic position of Isogomphodon within Carcharhinidae.................228

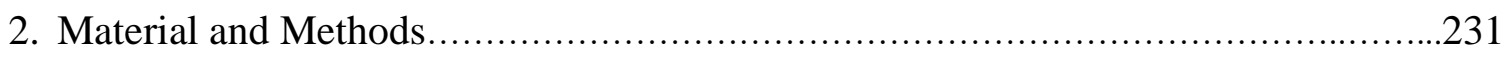

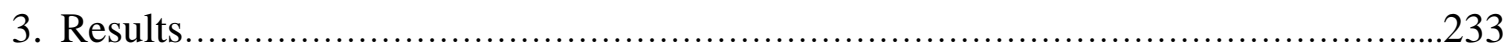

3.1.Comparative anatomy - Comparison of Isogomphodon oxyrhynchus within

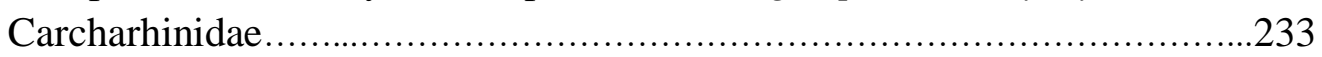

3.1.1. Isogomphodon oxyrhynchus and Carcharhinus porosus.....233

3.2.List of Morphological characters - Carcharhinidae............................234

3.2.1. Neurocranium.................................................234

3.2.2. Mandibular Arch....................................................243

3.2.3. Pectoral Girdle...................................................249

3.2.4. Pelvic Girdle......................................................252

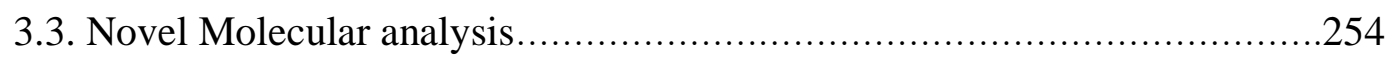

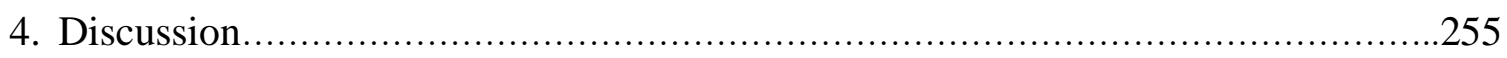

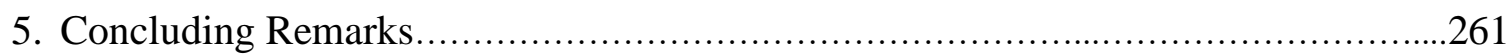

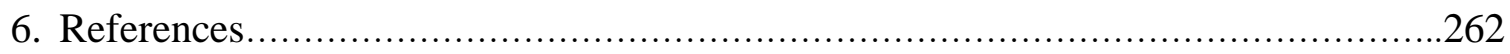

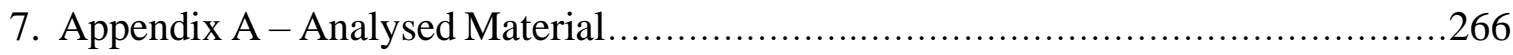

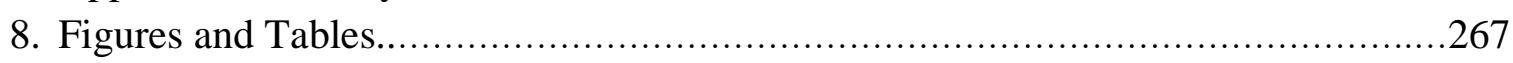


Chapter 3 - A critical overview of fossil taxa attributed to Isogomphodon (Gill, 1862) based on tooth specimens.

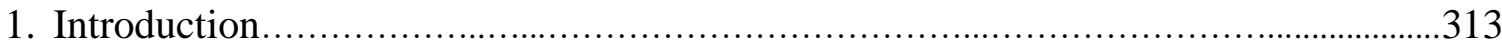

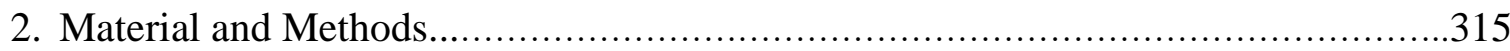

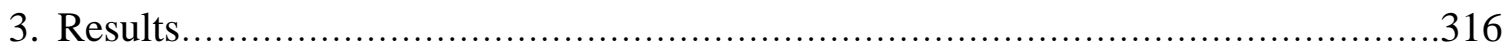

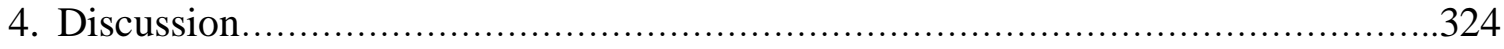

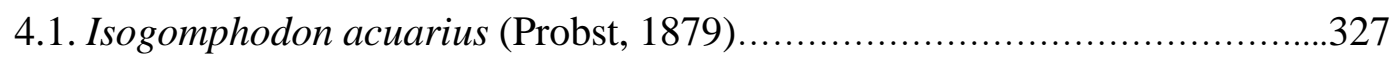

4.2. Isogomphodon lerichei (Datervelle \& Casier, 1943).............................329

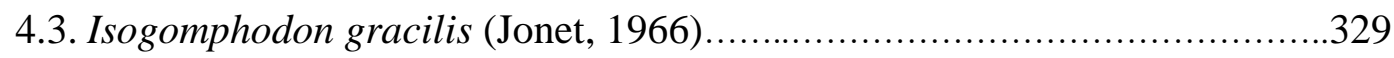

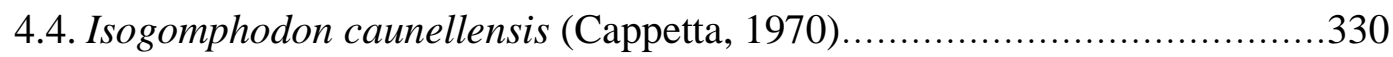

4.5. Isogomphodon aikenensis (Cicimurri \& Knight, 2019)........................331

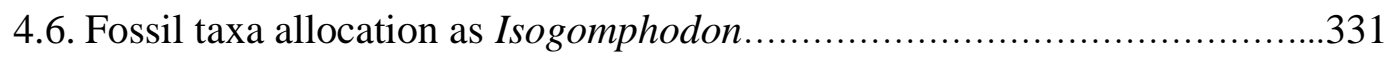

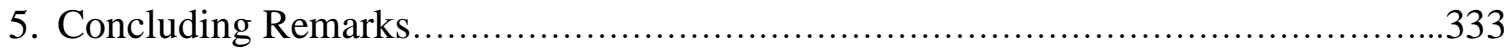

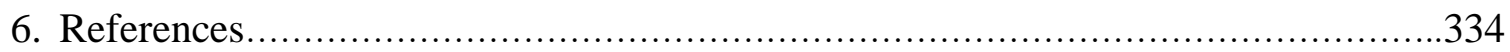

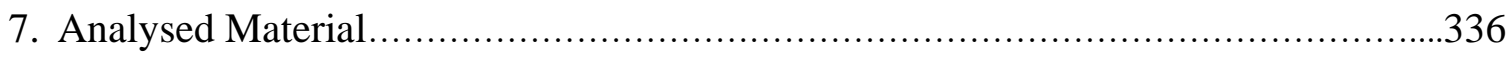

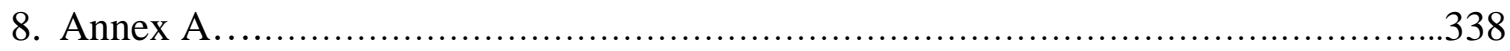

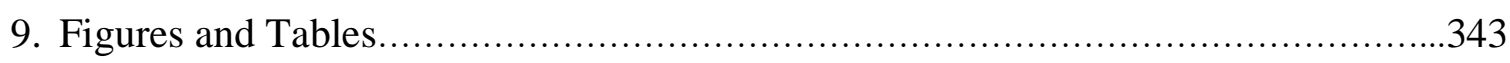




\section{General Introduction}

\subsection{From Chondrichthyes to Carcharhinidae: major classifications and history of art in a systematic perspective}

The Chondrichthyes are a monophyletic group of cartilaginous fish with approximately 1200 described species, present in every ocean of the planet. They are divided into two subclasses: Holocephali, which comprises the Chimeras, and Elasmobranchii, with sharks and rays (Compagno 1977, 1984, 1988; Hickman et al. 2001; Musick \& Ellis 2005; Weigmann 2016). The Elasmobranchii are split in two superorders: Selachii, with sharks, and Batoidea with skates and rays (Compagno 1984, 1988; Naylor et al. 2012;).

A number of authors support the Neoselachii as a valid taxonomic category comprising all living sharks and rays plus few Mesozoic shark species (Compagno 1977, Shaeffer 1981; Shirai 1992; de Carvalho 1996; Kriwet et al. 2008). Within Selachii there are two subdivisions proposed by Compagno (1977): Squalomorphii, with the orders Hexanchiformes, Pristiophoriformes, Echinorhiniformes, Squatiniformes and Squaliformes, and Galeomorphii, with Heterodontiformes, Orectolobiformes, Lamniformes and Carcharhiniformes (Compagno 1977; Douady et al. 2003; Naylor et al. 2005; Vélez-Zuazo \& Agnarsson 2011, Naylor et al. 2012). However, morphological and molecular phylogenies diverge regarding the recognized orders inside Selachii, with some authors proposing seven (Human et al. 2006) and others eight (Compagno 1984; Shirai 1996; Douady et al. 2003; Naylor et al. 2012) or nine orders (de Carvalho 1996).

It is important, first of all, to delimit what is an Elasmobranchii, a valid question even today. Generally, three main linages are recognized: Cladoselache, Euselachii, comprising fossil taxa such as Xenacanths and Hybodontiforms (De Carvalho 1996), and Neoselachii composed of modern sharks with few fossil taxa such as Paleospinax (Maisey 1984; Compagno 1999a). Cladoselache, which dates from the Devonian, Euselachii, ranging from the Perminan to the Triassic (Xenacanths and Hybodontiformes), and Neoselachii, from the Mesozoic to the present. The group Neoselachii has been employed as valid by a number of authors (Compagno 1977; Maisey 1984; Shirai 1996; De Carvalho 1996). There is general consensus that sharks (i.e., Neoselachii excluding fossil taxa such as Paleospinax) are monophyletic (Compagno 1988; Shirai 1996 Maisey 2012). Originally, it was believed that Chondrichthyans are dated to have risen during the Devonian (i.e., around 400 m.y.a), nonetheless scales that resemble a 
'Chondrichthyan-like' condition are also reported to the Middle Ordovician (Andreev et al., 2015). The ancestor clade of sharks (i.e., Cladoselache) is dated to the Late Devonian and has no evidence of significant decline for more than 60 million years (Compagno 1990; Maisey 2012). Phylogenetically, sharks are diagnosable by a number of synapomorphies, among which the most conspicuous is a polyhedral calcification pattern: The prismatic calcification (Applegate 1967 apud Compagno 1999b).

The order Carcharhiniformes, popularly known as the Ground Sharks, is the most diverse subgroup of Galeomorphii sharks, with nearly 300 described species and a rather conserved morphology when compared to remaining orders within Neoselachii (Compagno1988). Although accepted as monophyletic by some specialists (Compagno 1988; Shirai 1996), ongoing controversy feeds continued investigations, both in molecular and morphological phylogenies (Carvalho 1996; Vélez-Zuazo \& Agnarsson 2011; Naylor et al. 2012; Silva e Carvalho 2015). Compagno 1988 and Shirai 1996 consider Carcharhiniformes as monophyletic based on a number of morphological synapomorphies, for example the presence of a true nictitating membrane with associated musculature in the eye region. A paraphyletic Carcharhiniformes arose with phylogenies based on molecular markers (e.g., Vélez-Zuazo \& Agnarsson 2011; Naylor et al. 2012). Eight families are recognized within Carcharhiniformes: Proscyllidae, Leptochariidae, Scyliorhinidae, Triakidae, Pseudotriakidae, Hemigaleidae, Carcharhinidae and Sphyrnidae (Compagno 1988).

Popularly known as Réquiem-Sharks, the family Carcharhinidae includes 59 species in 12 genera, including famous sharks as the Blacktip Shark - Carcharhinus limbatus (Müller \& Henle, 1839) and the Tiger Shark - Galeocerdo cuvier (Péron \& Lesueur, 1822) (Compagno 1988; Fricke, Eschmeyer \& Van der Laan 2020). Although most taxa are exclusively marine, some species inhabit freshwater environment (Speartooth Shark - Glyphis gangeticus), estuaries (Daggernose Shark - Isogomphodon oxyrhynchus) or even both, such as the Bull Shark - Carcharhinus leucas (Müller \& Henle, 1839) which is capable of freely transit between marine and freshwater environments, having been reported from locations far from the ocean. Carcharhinidae is apomorphically characterized by a well-defined caudal sulcus, highly mobile and muscular nictitating eye membranes and a first dorsal fin strongly ahead of the pelvic girdle, among other features (Castro 2010; Gomes et al. 2010). Commercially and socially, carcharhinid sharks include species of great importance some of which responsible for attacks on humans, such as Galeocerdo cuvier and Carcharhinus leucas (Compagno 1988). There has been long-term consensus among previous authors that Carcharhinidae, excluding 
Sphyrnidae, is a monophyletic group (Compagno 1988). However, recent analyses have hypothesized that Galeocerdo is the sister group to Hemigaleidae and Carcharhinidae + Sphyrnidae (Compagno 1988, Naylor et al. 2012; 2021). If that is true, then Carcharhinidae, as traditionally composed, is paraphyletic. The first evidence for such hypothesis were raised by Compagno 1988 based in morphological autapomorphies.

\subsection{The Daggernose Shark - Isogomphodon oxyrhynchus (Müller \& Henle 1839)}

The Daggernose shark, also popularly called "cação-pato" or "cação-quati" by local fishermen, is an endemic Carcharhinidae from northern South America. Its habitat is restricted to the turbid waters of mangroves and estuaries, although it can also be found in coastal waters during the rainy season (Lessa et al. 1999; Lessa et al. 2000). The species has confirmed occurrence in Brazil (States of Maranhão, Pará and Amapá), Suriname, Guiana, French Guiana and northern Trinidad Tobago, which is possibly the most restricted geographical distribution of any marine elasmobranch (Lessa et al. 2000; Lessa et al. 2016).

In terms of ecology, I. oxyrhynchus drifts its habitat seasonally, occupying estuaries during the dry season and migrating to marine habitats during the wet season possibly following salinity thresholds (Lessa et al. 1999; Lessa et al. 2000 Lessa et al. 2016). It has been reported from entirely freshwater environments (Feitosa et al. 2019). Males reach maturity at $1.03 \mathrm{~m}$ and females approximately at $1.15 \mathrm{~m}$ in total length. The species has a fairly regular reproductive cycle and probably give birth during the first months of the year, with the incubation period between January and December, i.e., nearly a year long (Lessa et al. 1999).

Once ranked as the fourth most abundant species in demographic sampling studies, the Daggernose shark now faces population reduction rates that are likely driving the species to extinction (Lessa et al. 2016). Today, I. oxyrhynchus is listed as critically endangered by the IUCN. Its population is under sharp decline due to fishing pressure, making it the least resilient shark in the northern coast of Brazil (Lessa et al. 2006; Lessa et al. 2016). Additionally, the once abundant species (Lessa et al. 1986; Lessa et al. 1987), is now nearly absent in new surveys of the same areas (Rodrigues-filho et al. 2009). Some authors estimate a population reduction rate of $18 \%$ per year, with a total of $90 \%$ of reduction in the number of individuals in the past 14 years (Lessa et al. 2006). Low fecundity, long gestation period, late sexual maturity age and biennial reproduction contribute significantly to such numbers (Nachtigall et al. 2017).

Isogomphodon Gill, 1862 was described to allocate a single species, Carcharias (Prionodon) oxyrhynchus Müller \& Henle, 1839. It was later synonymized under Carcharhinus 
Blainville, 1816 by Bigelow \& Schroeder (1948), Garrick \& Schultz (1963) and Garrick (1967). The genus is today considered valid due to works by Compagno $(1970 ; 1979 ; 1988)$, Compagno \& Vergara (1978) and Garrick (1982). Five nominal species are included in Isogomphodon, four fossil and one living. The only extant species, I. oxyrhynchus, is easily recognizable by its long snout, relatively large pectoral fins and reduced eye size, the latter possibly directly associated with its estuarine habit (Müller \& Henle 1841; Compagno 1984; Lessa et al. 1999). On the basis of morphological divergence, Compagno (1988) proposed the tribe Isogomphodontini to separate the Daggernose shark from other tribes of Carcharhininae (Triaenodontini, Carcharhinini and Rhizoprionodontini). Isogomphodon oxyrhynchus indeed exhibits significant morphological differences when compared to all other members of Carcharhinidae. Although its biology and distribution are relatively well studied (Compagno 1984, Lessa et al. 1999; Lessa et al. 2016), anatomical descriptions are still scarce or superficial (Compagno 1984, 1988; Martins et al. 2015).

Five fossil species of Isogomphodon are described solely based on teeth specimens, being: Isogomphodon acuarius (Probst, 1879); Isogomphodon lerichei (Darterville \& Casier, 1943); Isogomphodon gracilis (Jonet, 1966); Isogomphodon caunellensis (Cappetta 1970); Isogomphodon aikenensis (Cicimurri \& Knight 2019). These taxa, with the exception of $I$. aikenensis that was already described as Isogomphodon, were initially described as Carcharias (Aprionon) and later included in Isogomphodon by Lauritto 1999 (I. acuarius, I. lerichei, I. gracilis) by Cappetta 1987 (I. caunellensis). Gill 1862 described the genus Isogomphodon as a group in which its members have a head with elongated and conical snout, with minute teeth or poorly teethed, the mandibular arch is nearly straight in a key-shape, nearly constricted in its base and with dorsal fins strongly or totally anterior to the pectoral fins. Therefore, dental characters were not considered in detail, even though the name Isogomphodon refers to dental features ('Iso' - from Greek means 'equal', 'gomphos', derives from 'nail-like' and 'Odon' refers to teeth). Consequently, fossil taxa were diagnosed and described as novel species of Isogomphodon based on the extant I. oxyrhynchus.

Phylogenetic hypothesis, based both on morphological and molecular data, disagree on the position of I. oxyrhynchus among Carcharhinidae (Compagno 1988; Rodrigues-Filho et al. 2009; Vélez-Zuazo \& Agnarsson 2011; Sorenson et al. 2014; Naylor et al. 2012a; 2012b, 2018;). Vélez-Zuazo \& Agnarsson (2011) propose that the species occupies a distal position within Carcharhinidae, while Compagno (1988) and Naylor (2012a) place it as sister group of Triaenodon obesus in a basal portion of the phylogeny. On the other hand, Sorenson et al. 
(2014) place I. oxyrhynchus as the sister group to Carcharhinus macloti, while Rodrigues-filho et al. (2009) and Naylor et al. (2012b) allocate the species as sister group to Carcharhinus porosus.

Even with important research been done on the biology and ecology of I. oxyrhynchus (e.g., Lessa et al. 1999; 2000; 2016; Feitosa et al. 2019), anatomical and systematic knowledge on the species remain precarious. The scarcity of specimens of I. oxyrhynchus in collection means that information of this sort is difficult to obtain. Herein, we present the first detailed description of the skeleton of $I$. oxyrhynchus. New data thus obtained allow a renewed view of the phylogenetic relationships of the species and of the affinities of its fossil presumably congeneric taxa. 


\section{General References}

Applegate, S. P. (1967). A survey of shark hard parts. In. Compagno, L. J. V. (1999b). Endoskeleton. Sharks, skates, and rays: The biology of elasmobranch fishes, 69-92.

Bigelow, H. B. \& Schroeder, W. C. (1948). Sharks. Mem.Sears Found.Mar.Res., (1):53-576

Blainville, H. M. (1816). Prodrome d'une nouvelle distribution systématique du regne animal. Bull. Des Scies de la Société. Philomatique Paris, 8: 105-24.

Castro, J. I. (2010). The sharks of North America. Oxford University Press.

Cappetta, H. (1970) Les Sélaciens du Miocène de la région de Montpellier. Palaeovertebrata, 3(extraordinaire):1-139 p., 22 fig., 27 pl.

Castro, J. I. (2010). The sharks of north America. Oxford University Press.

Cicimurri, D. J. \& Knight, J. L. (2019). Late Eocene (Priabonian) elasmobranchs from the Dry Branch Formation (Barnwell Group) of Aiken County, South Carolina, USA. Paleobios, 36:1-31.

Compagno, L. J. (1970). Systematics of the genus Hemitriakis (Selachii: Carcharhinidae), and related genera. Proceedings California Academy of Science, 33(4): 63-98.

Compagno, L. J. (1977). Phyletic relationships of living sharks and rays. American zoologist, 17(2): 303-322.

Compagno, L. J. \& Vergara R. R. (1978). Triakidae. In FAO species identification sheets for fishery purposes. Western Central Atlantic. Fishing” Area 31. Edited by W. Fischer. Rome, FAO. v. 5: pag. var.

Compagno, L. J. (1979). Carcharhinoid sharks: morphology, systematics and phylogeny. Unpublished Ph. D. Thesis, Stanford University, 932 p. Available from University Microfilms International, Ann. Arbor, Michigan.

Compagno, L. J. (1984). Sharks of the World: an annotatedand illustrated catalogue of the sharks know to date. Rome, FAO. v. 4, Part 2. (FAO Fisheries Synopsis, n. 125)

Compagno, L. J. (1988). Sharks of the order Carcharhiniformes. Princeton, Princeton University Press.

Compagno, L. J. (1990). Alternative life-history styles of cartilaginous fishes in time and space. Environmental Biology of Fishes, 28(1-4): 33-75.

Compagno, L. J. (1999a). Systematics and body form. In: Hamlet, W.C. Sharks, skates and rays: The biology of elasmobranch fishes. Baltimore, Johns Hopkins University Press. 42p. 
Compagno, L. J. V. (1999b). Endoskeleton. In: Hamlet, W.C. Sharks, skates and rays: The biology of elasmobranch fishes. Baltimore, Johns Hopkins University Press. p. 69-92.

De Carvalho, M. R. (1996). Higher-level elasmobranch phylogeny, basal squaleans, and paraphyly. In: Stiassny, M.1.J.; Parenti, L.R. \& Johnson, G.D.(Eds). Interrelationships of fishes. San Diego, Academic Press. p. 35-62.

Datervelle, E. \& Casier, E. (1943). Les poisons fossils du Bas-Congo et des régions voisines. Annales du Musée du Congo Belge, Sér. A (Minéralogie Géologie, Paleontogie), 3, 2 (1): 1200, fig. 1-60, pl. 1-16.

Douady, C. J.; Dosay, M.; Shivji, M. S. \& Stanhope, M. J. (2003). Molecular phylogenetic evidence refuting the hypothesis of Batoidea (rays and skates) as derived sharks. Molecular Phylogenetic Evolution, 26: 215-221.

Feitosa, L. M.; Martins, A. P. B.; Giarrizzo, T.; Macedo, W.; Monteiro, I. L.; Gemaque, R.; Nunes, J. L. S.; Gomes, F.; Scheinder, H.; Sampaio, I.; Souza, R.; Sales, J. B.; RodriguesFilho, L. F.; Tchaicka, L. \& Carvalho-Costa, L. F. (2018). DNA-based identification reveals illegal trade of threatened shark species in a global elasmobranch conservation hotspot. Scientific reports, 8(1): 3347.

Fricke, R., Eschmeyer, W. N. \& Van der Laan, R. (eds) 2020. Eschmeyer's Catalog of Fishes: Genera, Species, Available at: (http://researcharchive.calacademy.org/research/ichthyology/catalog/fishcatmain.asp)

Electronic version accessed: [22th March 2020].

Garrick, J. A. \& Schultz L. P. (1963) A guide to the kinds of potentially dangerous sharks. In: Gilbert, P. W.; Garrick, J. A. F. \& Schultz, L. P. (Eds). Sharks and survival. Boston, D. C. Heath and Company. p. 3-60.

Garrick, J. A. (1967). Revision of sharks of genus Isurus with description of a new species (Galeoidea, Lamnidae). Proceedings of theUnited States National Museum, 118(3337): 66390.

Garrick, J. A. (1982). Sharks of the genus Carcharhinus. NOAA Techniccal Report NMFS Circ., (445):1-194.

Gill, T. (1862). XXXII.-Analytical Synopsis of the Order of Squali; and Revision of the Nomenclature of the Genera. Annals of the New York Academy of Sciences, 7(1): 367-408.

Gomes, U. L.; Signori, C. N.; Gadig, O. B. F. \& Santos, H. R. S. (2010). Guia para identificação de tubarões e raias do Rio de Janeiro. Rio de Janeiro, Technical Books Editora. 
Hickman, C. P.; Roberts, L. S.; Larson, A.; Ober, W. C. \& Garrison, C. (2001). Integrated principles of zoology. 11 ed. New York, McGraw-Hill.

Human, B. A.; Owen, E. P.; Compagno, L. J. V.; Harley, E. H. (2006). Testing morphologically based phylogenetic theories within the cartilaginous fishes with molecular data, with special reference to the catshark family (Chondrichthyes; Scyliorhinidae) and the interrelationships within them. Molecular Phylogenetic Evolution, 39: 384-391.

Jonet, S. (1966). Notes d'ichthyologie miocène. II-Les Carcharhinidae. Boletim do Museu e Laboratorio Mineralógico e Geológico da Faculdade de Ciências, 10(2):65-88.

Kriwet, J.; Klug, S.; Canudo, J. I. \& Cuenca-Bescos, G. (2008). A new early Cretaceous lamniform shark (Chondrichthyes, Neoselachii). Zoological Journal of the Linnean Society, 154(2): 278-290.

Laurito, C. A. M. (1999). Los Seláceos fósiles de la localidade de Alto Guayacán (y otros ictiolitos associados): Mioceno-Superior-Plioceno Inferior de la formación Uscari. San Jose. 166p.

Lessa, R. P. (1986). Levantamento faunístico dos elasmobrânquios (Pisces, Chondrichthyes) do litoral ocidental do estado do Maranhão, Brasil. Boletim do Laboratório de Hidrobiologia, São Luiz, 7(1):27-41.

Lessa, R. P. T. (1987). Aspectos da biologia do cação-quati, Isogomphodon oxyrhynchus (Mueller \& Henle, 1839) (Chondrichthyes: Carcharhinidae), das reentrâncias maranhenses. Boletim de Ciencias do Mar, Fortaleza, 44:1-19.

Lessa, R.; Batista, V. \& Almeida, Z. (1999). Occurence and biology of the daggernose shark Isogomphodon oxyrhynchus (Chondrichthyes: Carcharhinidae) off the Maranhão coast (Brazil). Bulletin of Marine Science, 64(1): 115-128.

Lessa, R.; Santana, F. M.; Batista, V. \& Almeida, Z. (2000). Age and growth of the daggernose shark, Isogomphodon oxyrhynchus, from northern Brazil. Marine and freshwater research, 51(4): 339-347.

Lessa, R., Batista, V. S., \& Santana, F. M. (2016). Close to extinction? The collapse of the endemic daggernose shark (Isogomphodon oxyrhynchus) off Brazil. Global Ecology and Conservation, 7:70-81.

Martins, A. P. B.; Silva Filho, E.; Feitosa, L. M.; Silva, L. P. N.; Almeida, Z. S. \& Nunes, J. L. S. (2015). Sexual dimorphism of sharks from the amazonian equatorial coast. Universitas Scientiarum, 20(3): 297-304.

Maisey, J. G. (1984). Higher elasmobranch phylogeny and biostratigraphy. Zoological Journal of the Linnean Society, 82(1-2): 33-54. 
Maisey, J. G. (2012). What is an 'elasmobranch'? The impact of palaeontology in understanding elasmobranch phylogeny and evolution. Journal of Fish Biology, 80(5): 918951.

Moreira, R. A. \& De Carvalho, M. R. (2019). Clasper Morphology of the Japanese Sawshark, Pristiophorus japonicus Günther, 1870 (Chondrichthyes: Elasmobranchii). The Anatomical Record, 302(9), 1666-1670.

Musick, J.A \& Ellis J.K. 2005. In: Hamlett, W.C. Reproductive biology and physiology. Plimouth, Science Publishers. v.3, p. 45-79.

Naylor, G. J. P.; Ryburn, J. A.; Fedrigo, O.; López, J. A. (2005). Phylogenetic relationships among the major lineages of modern elasmobranchs. In: Hamlett, W.C.; Jamieson, B.G.M. (Eds.), Reproductive Biology and Phylogeny, vol. 3. Enfield, NH, Science Publishers, Inc. v.3, p. 1-25.

Naylor, G. J., Caira, J. N., Jensen, K., Rosana, K. A., Straube, N., \& Lakner, C. (2012a). Elasmobranch phylogeny: a mitochondrial estimate based on 595 species. The biology of sharks and their relatives, 31-56.

Naylor, G. J., Caira, J. N., Jensen, K., Rosana, K. A. M., White, W. T., \& Last, P. R. (2012b). A DNA sequence-based approach to the identification of shark and ray species and its implications for global elasmobranch diversity and parasitology. Bulletin of the American Museum of Natural History, 2012(367), 1-262.

Probst, J. (1879). Beiträge zur Kenntniss der fossilen Fische aus der Molasse von Baltringen. Hayfische. Jahreshefte des Vereins für vaterländische Naturkunde in Württemberg, 35:127191.

Rodrigues-Filho, L. F. D. S.; Rocha, T. C. D.; Rêgo, P. S. D.; Schneider, H.; Sampaio, I. \& Vallinoto, M. (2009). Identification and phylogenetic inferences on stocks of sharks affected by the fishing industry off the Northern coast of Brazil. Genetics and molecular biology, 32(2): 405-413.

Schaeffer, B. (1981). The xenacanth shark neurocranium, with comments on elasmobranch monophyly. Bulletin of the American Museum of Natural History, v. 169(1):1-66.

Shirai, S. (1992). Phylogenetic relationships of the angel sharks, with comments on elasmobranch phylogeny (Chondrichthyes, Squatinidae). Copeia, (1992): 505-518.

Silva, J. P. (2014). Filogenia dos principais grupos de Chondrichthyes baseada na anatomia comparada do esqueleto das nadadeiras pares e suas cinturas. (Doctoral dissertation, Universidade de São Paulo). 
Sorenson, L.; Santini, F. \& Alfaro, M. E. (2014). The effect of habitat on modern shark diversification. Journal of evolutionary biology, 27(8):1536-1548.

Vélez-Zuazo, X., \& Agnarsson, I. (2011). Shark tales: a molecular species-level phylogeny of sharks (Selachimorpha, Chondrichthyes). Molecular phylogenetics and evolution, 58(2): 207-217.

Weigmann, S. (2016). Annotated checklist of the living sharks, batoids and chimaeras (Chondrichthyes) of the world, with a focus on biogeographical diversity. Journal of Fish Biology, 88(3), 837-1037. 
Chapter 1

Description of the skeletal anatomy and dermal denticles of Isogomphodon oxyrhynchus 


\section{Introduction}

The skeleton of chondrichthyans, differently from that of the Osteichthyes, is rather simplified in terms of structural delimitation. Although it is popularly known that the skeleton on sharks is essentially composed of cartilage, it has nonetheless a calcification type that is a synapomorphy to the group: prismatic calcification (Moss 1977; Compagno 1977; 1999). The main sections are subdivided into three main regions according to the allocated structures: cranial skeleton, axial skeleton, and appendicular skeleton (Compagno 1999). Externally, elasmobranchs are known by a modified scale type called dermal denticles, which is homologous in its internal structure to oral teeth: an external dentine with an internal pulp nourished by blood vessels. Morphologically, these structures are not only taxonomically informative, but also variable within body sections on the same species (Dillon et al. 2017).

Unlike other carcharhinids, which have a rather conserved and stereotyped morphology, Isogomphodon oxyrhynchus exhibits features of external anatomy that are a direct reflection of its skeleton, such as an elongated rostrum, broad pectoral fins and reduced eyes. Available knowledge on the skeleton of Isogomphodon oxyrhynchus is severely limited and although this taxon is relatively well studied in ecology and reproductive biology (e.g., Lessa et al. 2000; Lessa et al. 2016) only few authors have looked into the species' anatomy. The latest anatomical description for I. oxyrhynchus focused on its caudal fin (Moreira et al. 2018). Compagno (1988) provides the most detailed description of general anatomical structures of $I$. oxyrhynchus, focusing on external morphology, neurocranium, dental series, vertebral counts and external clasper morphology.

Studies on Elasmobranch anatomy were classically based on preparations such as dissections, clearing and staining techniques and dry skeleton preparations. Recently, a protocol to skeletal preparation for fresh and frozen specimens has been proposed by Enault et al. (2016). The development of modern computerized tomography techniques allowed access to skeletal information without damage to specimens, which has been invaluable in studying rare species. This is exactly the case of Isogomphodon oxyrhynchus, a critically endangered species poorly represented in scientific collections.

Such factors have resulted in a large knowledge gap about Isogomphodon oxyrhynchus. Major features of its skeletal anatomy remain poorly explored. For example, the splanchnocranium and appendicular skeleton remain completely unknown. Information on the dermal denticles of this species is limited to Compagno (1988). Intraespecific variation of most 
anatomical structures have never been studied. Herein, we offer a description of the complete skeleton of I. oxyrhynchus based on classical and modern methodologies, including CT-Scan, Scanning Electronic Microscopy (SEM) and dissections. Our results for the first time permit a detailed comparison of the anatomy of I. oxyrhynchus with potentially related taxa. 


\section{Material and Methods}

Descriptions on the skeleton were performed based on two specimens: an adult male (MPEG 3455, $1130 \mathrm{~mm}$ TL) and a juvenile male specimen (MZUSP 101214, $604 \mathrm{~mm}$ TL). Therefore, general statements are valid for both specimens while specific traits of each life stage are detailed within the manuscript. Both specimens were scanned in a CT-Scan (Modelo: Phoenix v|tome $x$ m microfocus - General Electric). During the process, the individuals were covered in a plastic film and inserted in waterproof recipients to prevent alcohol leaking (juvenile in a plastic tube with a circular cover base and the adult on a traffic cone). The scanning time was approximately six hours for both specimens. The juvenile was scanned in 6 connected scans to be posteriorly merged in a single image, although it was not here included as the file size was significantly dense to the MZUSP computers. On the other hand, the adult was scanned 5 independently scans to obtains images from specific anatomic landmarks, once, as well as the juvenile individual, the computational capacity of the MZUSP machines were not able to render the full image of the adult. The employed software for editing were VGStudio and MyVGL for visualization, both developed by Volume Graphics. The equipment is MZUSP property, as well as the software licenses. Due to accidents in the preservations of the specimen, the tripodal rostral cartilages were damaged, however no further impact within the descriptions was reported. MZUSP 37289, a juvenile male specimen, was partially dissected to enhance the description of regions that presented flaws in their visualization on the CT-Scan images. The employed literature for structure identification and description is listed on Table 1. The abbreviations were inserted in the images using Paint 3D (Microsoft). The nomenclature followed for the description of each anatomical region is summarized on Table 1, the analysed specimen used for this description is available on Table 2 and the foramina numbering on Table 3.

Regarding the dermal denticles, the body sections where skin samples were collected from the specimens of I. oxyrhynchus followed Dillon et al. (2017) with adaptations (e.g., only the dermal denticles from the eye nictitating membrane were sampled and described) to better detect variations among the dermal denticles of this taxon (see Fig. 1). The nomenclature followed Compagno (1988) with adaptations (Table 1), as well as the descriptions of denticles from the neonate, juvenile and adult. Overall, 20 skin regions (see Fig 1. for abbreviations of body sections locations and Fig. 2 for used nomenclature) were selected for sample collection, each had approximately $0.5 \mathrm{~cm}^{2}$. Additionally, two sections of P3 (Fig. 3) were sampled in the 
juvenile specimen: one close to the fin margin and the other close to the fin insertion. Three specimens (MZUSP 101214 - Neonate male; MZUSP 37289 juvenile male; MPEG 3455 Adult male) had skin samples collected from to be analysed with Scanning Electronic Microscopy (SEM - Check MZUSP model). For preparations, these tissue samples were dried to the critical point, in which they were dehydrated with alcohol $100 \%$ prior to the liquid $\mathrm{CO}_{2}$ baths that precede the metallization. Once metalized in gold and positioned in the stubs, samples were inserted in the equipment.

Once the focus of the present work is to detect and describe morphological variations in each body section from I. oxyrhynchus, the functional morphologies described by Dillon et al. (2017) were not here considered. It is important to state that these body sections were never analysed individually in a species level for I. oxyrhynchus, therefore showing novel perspectives for future analysis. Previous authors, however, corroborated that morphological variations occur along different body regions (Rangel et al. 2019). The insertion base of each denticle was not included in the descriptions, once this region was not always visible in the images and, when so, was either damaged or not completely apparent. 


\section{Results}

Both MPEG 3455 and MZUSP 101214 were successfully scanned, although MPEG 3455 (adult specimen) was only analysed on the neurocranium, teeth, dermal denticles, anal fin, vertebral centra and branchial arches due to limitation on the access of the CT-Scan equipment with the COVID-19 pandemics. MZUSP 101214 was entirely analysed in its skeleton and dermal denticles.

\subsection{Dermal denticles}

The body sections where skin samples were collected from the specimens of $I$. oxyrhynchus followed the body sections compiled in Dillon et al. (2017) with adaptations to better detect variations among the dermal denticles of I. oxyrhynchus (Fig. 1). Overall, 20 skin samples from 3 individuals, being one neonate (MZUSP 101214), one juvenile (MZUSP 37289) and one adult (MPEG 3455), (see fig 1. for abbreviations of body section locations from where the samples were collected and fig. 3 for used nomenclature and abbreviations) were collected and analysed with Scanning Electronic Microscopy (SEM).

Overall, the general morphology of the dermal denticles in most regions has three (in most cases) to five (few cases, usually the external are discrete to nearly absent) crests, with mild variations along few of the body sections, although some regions are evidently different from the general morphology, which are detailed below.

\subsubsection{Head section (Figs. 4 - 43)}

The dermal denticles on the head sections are wide and characterized by two to three ridges with rounded to oval posterior edges and a rugged surface on the entire crown. On the insertion region, the medial portion is circular and curved, as well as the lateral edges.

\section{Neonate (H1: Figs. 4 - 7; H2: Figs. 17 - 20; H3: Figs. 32 - 35)}

Generally, the denticles are round to oval shapes, with two to three crests. H1 presented emerging scales, with rounded posterior edges and crests that do not reach the scale edge, i.e., disappear approximately on the medial portion of the scale surface, although on $\mathrm{H} 2$ some scales have crests reaching the end. $\mathrm{H} 3$ did not present emerging denticles, only a rugged surface of dermal tissue.

Juvenile (H1: Figs. 8 - 11; H2: Figs. 21 - 27; H3: Figs. 36 - 39) 
Similar to the neonate, the distal facets on $\mathrm{H} 1$ are rounded and the crests, which are now discrete within the surface and more prominent close to the scale insertion, do not reach the posterior edge. In an oblique view, it is possible to notice that the crests are irregularly originating from the base and have different heights. Regarding $\mathrm{H} 2$, the scales have three pointed edges with taller crests than $\mathrm{H} 1$ and with most scales having three defined crests (rare examples presented four). Additionally, some scales in this region the medial crest is shorter or even absent. The skin surface on this region also presented a lower number of denticles. H3, on the other hand, the pointed peaks are stronger and longer, with a more regular general shape: three well defined crests, nearly equal in height and dilated on lateral surface adjacent to the lateral crests.

\section{Adult (H1: Figs. Figs. 12 - 16; H2: Figs. 28 - 31; H3: Figs. 40 - 43)}

The head section for the adult presented rather different morphologies for each section. On H1, the number of crests on the adult increase in the head section, with three-crested scales becoming rare, most scales presenting four to five and some even reaching six crests. Additionally, the posterior edges are also oval-shaped, although some have linear facets. H2 has a different morphology, with three shallow and plain crests, with the medial being wider and slightly taller than the laterals. Similar to H1, H2 has three pointed scales. Nonetheless, the H3 sections showed a different general morphology. The crests are short and, in some cases, not visible. The lateral edges are rounded, with three pointed posterior edges and the medial crest being the most evident extremity. Additionally, the surface is smoother than the other regions, and the lateral crests transit towards the lateral edge with a curved line.

\subsubsection{Eye section (Figs. $44-51$ )}

The scales of nictitating lower eyelid are rugged in their entire surface, thinner and shorter in diameter, although similar in morphology to the observed on the clasper, nose and P3 scales. Discrete ridges occur in anterior insertion of few scales of the juvenile that become more apparent in the adult.

\section{Neonate (Figs. 44 - 45)}

The nictitating eye membrane in the neonate did not show emerging scales, instead presented dermal denticles covered in dermal tissue. Beneath this layer, the denticles have round to oval shape and with no apparent crests.

Juvenile (Figs. 46 - 47) 
Within the juvenile, the dermal denticles in this region are rounded and with discrete emerging crests or even absent in some denticles. The surface of the crown is relatively smoother when compared to other regions, although still presents the characteristic prismatic pattern.

\section{Adult (Figs. 48 - 51)}

In the adult, the scales are round to trapezoid shaped, the crests, which vary from three in most case to five in some denticles, are evident close to the anterior margin and do not reach the posterior edge. The overlap of denticles in this section is discrete to nearly absent.

\section{$\underline{\text { 3.1.3 Gill opening section (Figs. } 52-62 \text { ) }}$}

The dermal denticles on gill openings, in general, are thinner than on other corporal regions, with its prismatic pattern being more accentuated and with more spaced crests (Figs. 193 -203). Similar to other regions, the scales here are three-ridged and three-cusped, although the ridges are apparently equal in height and likely absent on the neonate.

\section{Neonate (Figs. 52 - 54)}

Although few emerging scales were visible, on the neonate the gill slits scales agree with the general stated morphology, although its prismatic patterns seem to have more space between the polygons. Additionally, the ridges are low to nearly absent.

\section{Juvenile (Figs. 55 - 58)}

The scales on the juvenile are wide and broad, with three crests and large lateral edges. In terms of conformations, the denticles are an apparently thin and agglomerated, showing no regions without scales coverage. The hexagonal pattern is highly evidenced in the scales from this section.

\section{Adult (Figs. 59 - 62)}

Differently from the observed in the juvenile, the adult has more spaced scales and with more accentuated cusps, nonetheless they are also three-crested, three cusped and with less angular transition between cusps. Figs. 200 - 203 also show that the denticles in this region are obliquely inclined, differently from the juvenile that showed parallelly positioned scales, parallel to the dermal surface.

\subsubsection{Nose section (Figs. $63-78$ )}

The dorsal nose section presents round, patterned dermal denticles, with signs of rugosity emerging in the lateral edges, close to the regions of insertion on the epidermis area, 
and the ridges are absent or discrete in the crown. On the other hand, the dermal denticles on the ventral sections showed a rather different morphology: these denticles are mildly crested on the crown, with three visible crests (some have four), rugged in their surface and present a round morphology, although some have slightly pointed edges.

\section{Neonate (Ndors: Figs. 62 - 64; NVent: Figs. 72 - 73)}

The Ndors in the neonate shows circular denticles with mild but visible emerging crests, although not enough to determine their exact quantity. Regarding the Nvent, the denticles have a similar morphology to the observed in Ndors, although the rough prismatic pattern is more evident in the ventral section.

\section{Juvenile (Ndors: Figs. 65 - 67; NVent: Figs. 74 - 75)}

Within the juvenile, the Ndors section has round shaped scales with stronger roughness in the lateral and anterior edges. The crests are absent and the anterior edge close to the scale insertion has a trapezoid-shape. Regarding the Nvent scales, the crests become more apparent, which are either three or four, and the posterior edge is mildly circular to plain in some denticles. The prismatic pattern on the crown is also more visible and has wider polygons, especially on the medial portion.

\section{Adult (Ndors: Figs. 68 - 71; NVent: Figs. 76 - 78)}

The Ndors and Nvent sections presented a rather similar general morphology: ovalshaped denticles with discrete ridges, usually more evident near the anterior edge, and with a weaker rugosity in the crown, although on the dorsal portion horizontal lines (parallel to the anterior edge insertion) are visible. Additionally, the ventral nostril scales on the adult are apparently more agglomerated with mild overlaps between individual denticles.

\section{$\underline{\text { 3.1.5 Body section (Figs. } 79-109 \text { ) }}$}

Generally, body scales are slightly narrower than those from the head sections, specially than on $\mathrm{H} 2$ from the adult. In terms of number of crests and peaks, all scales in this region are three ridged and three peaked. These three crests within the crown have rugged pattern surfaces that converge from the crests until the pointed ends. However, as the neonate presented few emerging scales in this region, the descriptions are limited. Nonetheless, mild variations occur among the analysed life stages.

\section{Neonate (B1: Figs. 79 - 80; B2: Figs. 89 - 91; B3: Figs. 99 - 100)}

The B1, B2 and B3 on neonate did not show many emerging scales, although some emerging dermal denticles suggest that, in these sections, the scales are also three-peaked in 
their posterior edge with discrete lateral edges. On B2, however, the emerging scales present a rounded base within the scale insertion.

\section{Juvenile (B1: Figs. 81 - 84; B2: Figs. 92 - 94; B3: Figs. 101 - 104)}

The general morphology for B1, B2 and B3 are similar on the juvenile: three well defined and equally high crests, three peaks and a rugged hexagonal pattern on the crown surface. On B3, however, the lateral edges are slightly more expanded.

Adult (B1: Figs. 85 - 88; B2: Figs. 95 - 98; 60 - 63; B3: Figs. 105 - 109)

Differently from the juvenile (which have curved crests), the crests on the adult are plain, especially the medial, and more spaced between each other. The lateral edges are more expanded on B1, which originate in the first third of the scale. On all sections within the adult, the scales are less rugged, with an aspect that resembles a screwed cover, and have broader lateral edges than those on the juvenile scales.

\section{$\underline{\text { 3.1.6 Dorsal fin section (Figs. } 110-138 \text { ) }}$}

Generally, the dermal denticles on first dorsal fin are more agglomerated than on other body regions. There are three well pronounced ridges, as well as in the other sections, with three cusps, being the medial slightly more prominent. The lateral edges, similar to the observed on B3, are more expanded and equally high or higher than the medial ridge in all three sampled areas. The medial ridge originates more anteriorly than the lateral ones and is also further extended, although on D1 from the juvenile the crest have nearly the same length.

\section{Neonate (D1: Figs. 110 - 111; D2: Figs. 119 - 121; D3: Figs. 128 - 131)}

The D1 scales have round shapes, with three crests and eventually one prominent medial projection that could eventually be classified as a cusp. These regions have more overlapped denticles and the medial crest appears to be slightly higher, although some scales have the lateral crests as the highest. On D2, no emerging scales were visible, nonetheless they are covered in skin tissue which reveals an oval-shaped crown. Regarding D3, the few emerging denticles showed an oval crown with three crests, three cuspids and the medial being the longest.

\section{Juvenile (D1: Figs. 112 - 114; D2: Figs. 122 - 124; D3: Figs. 132 - 134)}

The morphology on the scales from the juvenile showed little variation in the dorsal fin, as they are generally characterized by the characteristic rugged surface three crests, three cusps and an agglomerated pattern. However, D1 showed less pronounced cusps and narrower lateral edges than D2 and D3, with some scales presenting four ridges. 


\section{Adult (D1: Figs. 115 - 118; D2: Figs. 125 - 127; D3: Figs. 135 - 138)}

In the D1 section, the posterior edges are oval while the crests surfaces are straight and plain, with the medial one posteriorly projecting creating a cusp on the edge. Some denticles on this section are four-crested and all of them reach the posterior edge. The D2 the general morphology is rather similar to D1, although the lateral edges are more pronounced. On D3, however, some peculiarities were evidenced. The crests are slightly curved whereas the other crests are straight, and the cusps have close extremities, i.e., the concavity between them is less angular.

\subsubsection{Pectoral fin section (Figs. 139-178)}

Generally, the dermal denticles on the pectoral region present one of the strongest variations among homologous regions. The scales on P1 and P2 are mostly three-ridged, with a rugged surface pattern as most scales from other regions, and three-cusped, with the medial cusp being the more evident. In terms of surface, the hexagonal rugged pattern is also present in the scales from those regions. The P3 section on the juvenile and on the adult, nonetheless, presented a completely different morphology: smooth round-shaped scales, with no cusps and discrete crowns that become absent near the base or near the medial portion of the crown surface.

Neonate (P1: Figs. 139 - 140; 104 - 105; P2: Figs. 151 - 154; P3: Figs. 164 - 165)

The P1 on the neonate showed few emerging scales and, although still partially covered in skin tissue, they are oval-shaped crowns and have generally three crests. P2 and P3 on the other hand, all denticles were covered in skin tissue, which showed oval to circular denticles with mild peaks in the posterior facet. The number of crests within the crown is not well visible, although in the listed figures, the scale prints suggest three.

Juvenile (P1: Figs. 141 - 147; P2: Figs. 155 - 159; P3: Figs. 166 - 169; P3m: Figs. 174 - 178)

On P1 samples, three to four crests are present, in which the medial eventually originates as two crests that converge at the posterior region. P2 has three well defined equally high crests and mild but fairly visible lateral edges. On the other hand, P3 on the juvenile specimen was analysed in two different sections: P3, close to the posterior fin insertion, and P3m, which is close to the fin margin. There is an evident difference between those two samples, as P3 agrees with the morphology from the adult and P3m showed denticles without crests, with a hexagonal 
patterned surface, three-cusped (being the medial the most prominent) and with broad, anteriorly expanded bases.

\section{Adult (P1: Figs. 148 - 150; P2: Figs. 160 - 163; P3: Figs. 170 - 173)}

Within the P1 section from the adult, the scales have discrete cusps and broad lateral edges, with three crests presenting mild curvature. P2 presented a similar morphology, although with higher crests that reach the posterior end of the slightly stronger cusps. The P3 section, as postulated in the general description, are round shaped with smooth surfaces and agglomerated arrangements. However, differently from the juvenile, this region on the adult has crests with ridges that disappear near the scale anterior base or are even absent. Differently from the juvenile, the prismatic pattern on the adult is evidently desquamated.

\subsubsection{Caudal fin section (Figs. 179 - 208)}

Generally, the scales of caudal region are thin and have mildly less pronounced crests that, in some individual scales, do not reach the cusp ends. Posteriorly in the crown, the characteristic hexagonal pattern within the scale surface presented shorter polygons, especially on the $\mathrm{C} 1$ region. The crowns on the $\mathrm{C} 3$ region are less pronounced to nearly absent, with also an agglomerated arrangement. The $\mathrm{C} 1$ section scales have discrete cusps and nearly absent ridges. However, they are present on insertion region of some scales, with medial cusps being more pronounced.

\section{Neonate (C1: Figs. 179 - 181; C2: Figs. 189 - 191; C3: Figs. 200 - 202)}

Within the $\mathrm{C} 1$ of the neonate, the cusps are discrete, and, in some scales, they are resumed to a single posterior projection in an oval-shaped scale. Three ridges are present, whose position varies from scale to scale, being nearly absent in some cases or reaching the cusp ends. The C2 denticles showed a similar morphology, although in most scales the crests reach the cusp ends and some have four crests. Finally, within C3 scales, the crests are reduced to nearly absent, being closely positioned to each other and having three cusps.

\section{Juvenile (C1: Figs. 182 - 185; C2: Figs. 192 - 196; C3: Figs. 203 - 205)}

In $\mathrm{C} 1$, the scales morphology agrees with the general description, with no evident differences for this section within this life stage. The $\mathrm{C} 2$, however, the medial crest is doubled, converging medially in the crown on a single medial crest (see Figs. 158, 159 and 161). Additionally, Fig. 159 shows an emerging three cusped scale with an evident long medial ridge. The C3, on the other hand, has agglomerated scales without crests, three cusped and with thinner crowns. 


\section{Adult (C1: Figs. 186 - 188; C2: Figs. 197 - 199; C3: Figs. 206 - 208)}

The adult $\mathrm{C} 1$ section presents apparently thicker scales, with short ridges that do not reach the cusps ends and have smoother surfaces, although still with the pattern. $\mathrm{C} 2$, on the other hand, besides the three eventually four converging ridges, the scales are generally wider and with prominent lateral edges, although with short and narrow cusps. The scales within C3 are similar to the observed in $\mathrm{C} 1$, although the crown surface has a rougher surface pattern.

\subsubsection{Clasper section (Figs. 209-219)}

The samples of the dermal denticles from the clasper section present a round to oval shape, similar to the denticles observed on nose sections, although its entire surface has a rugged pattern and no ridges or cusps. Additionally, the scales are also slightly more aggregated. Hexagonal pattern on the external tissue is visible on the juvenile specimen.

Neonate (Figs. 209 - 210)

No emerging scales were seen in the clasper sample from the juvenile, showing only a smooth surface of dermal tissue.

\section{Juvenile (Figs. 211 - 212)}

The juvenile presented round to oval-shaped denticles, with no apparent ridges and with a surface pattern that is smooth posterior edges and rough anterior facets.

\section{Adult (Figs. 213 - 219)}

In contrast, the scales on the adult present anterior portion of the clasper present denticles in a very unique morphology, while posteriorly their morphology drifts to a similar form to the observed on the juvenile. As observed in figs. 178 to 184, the clasper dermal denticles on the adult are diamond-shaped, have strong, pointed medial cusps, with very deep transitions between cusps, and lack crests within the smooth, curved crown. The lateral edges are confluent with the lateral cusps and have pointed margins, which resembles a new cusp. The anterior insertions of the scale are narrow, and gradually transit to the lateral edges in curved margins. Additionally, the invaginations between the cusps are deepen and mildly angled (i.e., $>30^{\circ}$ ).

\subsection{Teeth series and morphology}

Compagno (1988) provided a detailed description of I. oxyrhynchus dentition, which is here updated and redescribed based on the CT-Scan imaging. The teeth rows present a morphological gradient, both on the upper and lower jaws. Generally, the teeth present narrow, 
high and smooth cusps, broad curved roots and transverse notches that are deeper on lower teeth. The anterior and lateral rows present higher crowns and roots than the posterior ones. Additionally, the teeth are symmetric in most regions, with the upper anterolateral teeth having narrow cuspids and strong curves on the edges of its base, while the medial lower teeth, which Compagno (1988) named 'alternates', are significantly broader and with higher cusps than the medial ones from upper rows. The cusps on anterior teeth, in both upper and lower rows, are higher and narrower than the posterolateral teeth ones. From anterior to posterior rows, the cusps gradually reduce in height, with most posterior ones without cusps, therefore creating a visible morphological gradient along the jaws (Figs. 221 and 222). The mesial edges of most teeth have a discrete ridge (which was detected with low resolution on the CT-images). A mild, although evident, dignathic heterodonty is present, in which the upper anterolateral teeth presenting broad roots and short cusps, while the lower rows in the same position have higher cusps and narrow roots (Figs. 221 and 222). In both jaws, the anterior teeth have slightly more curved lateral root edges than the adjacent ones. The posterior most rows lose their cusps from the 22 nd teeth onwards. Some ontogenetic variation on dentary morphology was detected for this species, and, although weak, it is more evident on the lower teeth rows, with the adult specimen presenting deeper transverse notches than the juvenile. No serrations were detected in any teeth with the CT-Scan. In terms of morphology, the adult and the juvenile do not present a significant difference on the teeth morphology, although in the adult the cusps are slightly bulkier. The tooth row formula from both the adult and juvenile is described below.

TOOTH ROW FORMULA (Juvenile - MZUSP 101214): Alternates: 1 /Medial: 3; Intermediates: L0 - R0; Symphysials - 1; Anterior: Upper L7 - R7/Lower L7 - R7; Laterals: Upper L10 - R12/Lower L10 - R10; Posteriors: Upper L8 - R8/ Lower L7 - R7.

TOOTH ROW FORMULA (Adult - MPEG 3455): Alternates: 1 /Medial: 5; Intermediates: L0 - R0; Symphysials - 1; Anterior: Upper L5 - R5/Lower L1 - R1; Laterals: Upper L18 - R17/Lower L17 - R16; Posteriors: Upper L7 - R8/ Lower L5 - R7.

\subsection{Neurocranium (Figs. 223 - 231)}

CT-Scan results indeed presented similar characters of those observed by Compagno (1988), although novel structures and interpretations were included in the present description. The 
neurocranium of $I$. oxyrhynchus is characterized by its long tripodal rostrum, a V-shaped fontanelle, round to oval nasal capsules and a reduced preorbital process (Figs. 223 - 231). Additionally, the orbits are large and wide when compared to the optic capsule and the occipital condyles have wing-shaped lateral processes that are confluent with the vagus nerve foramina aperture. Four views are here described, although both analysed specimens have their rostrum broken with their preservation and allocation in collection. The rostrum of I. oxyrhynchus is tripodal, consistent with other carcharhinids, therefore composed by three elements (2 laterals rods and one medial) that are connected anteriorly on the snout tip forming a rostral node.

Dorsal view: The rostrum is elongated, with medial and lateral rostral cartilages being nearly equal in size related to total length (Medial of juvenile and male specimens respectively 29\% and $31 \% \mathrm{TL}$, and lateral $32 \%$ and $34 \% \mathrm{TL}$ ). The length of medial rostral cartilage is approximately four times the distance between the lateral rostral cartilages (Figs. 225 and 226). A small elliptical rostral fenestra is visible in the CT-Scan images on ventral view of the juvenile neurocranium (Fig. 229), although in the adult this character clearly visible (Fig. 230). The medial rostral cartilage is dilated on its base, having discrete signs of hyper calcifications detected on the rostral node region on the CT-Scan images and not reported by Compagno (1988) (Figs. 225 and 226). The anterior fontanelle length fits approximately four times in nasobasal length, which corresponds to 2.4 times the distance from medial rostral cartilage base to dorsal edge of anterior fontanelle. The posterior base of lateral rostral cartilages gradually transits to lateral edges of anterior fontanelle (there is no evident division of those two regions, as described by Compagno (1988) which has an anterior fontanelle with evident V-shape that extends few posteriorly to the line of orbitonasal foramen. The cranial roof is narrow on the ectethmoid region, close to the fontanelle, while broad and flattened on the optic capsule. However, this region is apparently narrower on the adult (Figs. 225 and 226). The orbitonasal foramina presents wide apertures and crest-like processes are evident on its external lateral margins on the juvenile and absent on the adult (Figs. 225 and 226). The preorbital process is reduced in size, (i.e., truncated) and located just below the posterior margins of nasal capsule. On the other hand, the postorbital process is slender, cylindrical and originates parallelly to the line of the anterior edge of sphenopterotic ridge. In the adult, this structure is slightly more pronounced than on the juvenile. Curiously, the CT-Scan images reveal no calcified path connecting the postorbital process to the optic capsule, suggesting these structures are maybe separated. The suborbital shelves are visible in dorsal view and parallel to each other (Figs. 225 and 226). An oval-shaped parietal fossa is visible and deepened, with size approximately 1.3 
times the anterior fontanelle length in the juvenile and nearly equal in length on the adult, being located aligned with the sphenopterotic ridge. Undetermined hyper calcifications signs were visible dorsal to the parietal fossa in the CT-Scan images, with the perilymphatic foramen located laterally to those structures. A medial sulcus posteriorly to the parietal fossa is present (Figs. 225 and 226, posterior end of parietal fossa) and extends to the anteromedial part between the endolymphatic foramina, dorsal to the occipital condyle. The sphenopterotic ridge has a curvy-shaped edge and is located below the postorbital process insertion (Figs. 225 and 226). Ten foramina (five on each side) located in a semicircular arrangement are located dorsal to the occipital condyles and laterally to the magnum foramen, which are more accurately identified in the caudal view (Fig. 231). Discrete tubercles are laterally visible laterally to those foramina formation. The glossopharyngeal foramina (IX) are similar to the orbitonasal foramen of the juvenile: with wide openings and crest-like processes on one of their edges. The vagus nerve foramina $(\mathrm{X})$ are located anteriorly to the occipital condyle, hardly seen on dorsal view, and presenting wide apertures. The occipital condyle is dilated in posterior margin and has distinct wing-like projections, which are confluent with the opening of the vagus nerve foramina $(\mathrm{X})$.

Lateral view: The basal plate has a concave arrangement on ectethmoid region, and is flattened on the orbit region, with a medial keel visible posterior to ectethmoid condyles (Fig. 227 and 228). The optic nerve foramen (II) is large in its aperture, being in the posterior inferior region on the orbit wall, while the trochlear nerve foramen (IV) is divided in two foramina, being positioned on the line of supraorbital crest. The cranial roof is flattened, feature better observed in lateral view. The suborbital shelf is undulated in lateral view and extends from the orbitonasal foramen to hyomandibular facet, being confluent with lateral commissure (Fig. 227 and 228). A triangular conformation of three foramina is present posteriorly to the optic foramen (II), composed by: oculomotor foramen (III, dorsal), foramen for efferent spiracular artery (anteroventrally) and abducens nerve foramina (VI, posteroventrally). Posteriorly to this formation, an oval and wide orbital fissure is located anteriorly to stapedial foramen (Figs 227 and 228). The foramen for superficial ophthalmic nerve is dorsal to the triangular formation and anterior to the optic capsule. The lateral commissure extends to the line of hyomandibular facet and the sphenopterotic ridge has discrete crests and is oval-shaped (Fig. 227 and 228). The glossopharyngeal nerve foramen (IX) is posteriorly positioned and confluent with hyomandibular facet (Fig. 227 and 228). The vagus nerve foramen (X) is also visible on lateral view. The occipital condyle has wing-like posterior projections that are confluent with inferior part of vagus nerve foramen $(\mathrm{X})$. 
Ventral view: The lateral ectethmoid foramina are present between nasal capsules and ectethmoid condyles (Figs. 229 and 230). The subethmoid fossa has a Y-shape and the medial keel, with ectethmoid condyles, is located beneath nasal capsules and anteriorly to the basal plate, however not prominent (Figs. 229 and 230). Although both the scanned individual presented calcification failures, the nasal capsules are oval and present anteriorly positioned apertures. The width of nasal capsules is approximately $20 \%$ shorter than the distance between the nasal apertures (Figs. 229 and 230). The paired medial ectethmoid foramina have enlarged and oval apertures (when compared to other foramina), being located below the ectethmoid condyles. Lateral dilatations, or the 'crest-like' projections, of the orbitonasal foramen are visible on ventral view on the juvenile (Figs. 229), located in the line of the midpoint of the medial keel, while on the adult they become absent (Figs. 230). Both on the juvenile and on the adult, the width of basal plate at the orbital notches line corresponds to three times the nasobasal length and is slightly wider than the width between the suborbital shelves (corresponding to approximately 2.6 times the nasobasal length). The suborbital shelves are parallel to each other and extend from the line of orbitonasal foramen to nearly the internal carotid foramen, with a discrete curvature better observed on lateral view (Figs. 229 and 230). The basal plate is flattened on the orbit region and concave on ectethmoid region. The wide stapedial foramina are located completely separated from the internal carotid foramina as the distance from the internal carotid foramen is wider to each other than to a stapedial foramina. The hyomandibular facets are medially curved and located below stapedial foramina. The occipital condyle is elongated and present wing-like shaped with symmetric dilatations. On the other hand, the occipital centrum is straight-edged and located in between those wing-like projections, fused to basal plate. The vagus nerve foramina $(\mathrm{X})$ are confluent with the occipital condyle projections.

Caudal view: The magnum foramen aperture diameter is wider than occipital centrum diameter, with its size approximately $20 \%$ larger. The undetermined hypercalcified structures are apparent on the dorsal region of parietal fossa, medially to the perilymphatic fenestrae. Laterally to those, two parallel tubercles on external edges of the parietal fossa are present. A medial sulcus originating from the posterior part of parietal fossa and extending to slightly dorsal to the magnum foramen is visible (Fig. 231). Around the magnum foramen occurs a semicircular arrangement of foramina: endolymphatic foramen (most dorsal), perilymphatic foramen (second, beneath the endolymphatic foramen) and two lateral branches of vagus nerve (X, most ventral) (Fig. 231). The vagus nerve foramina (X) main openings are located beneath such arrangement and dorsal to the occipital condyle. A medial keel-like 
process occurs between vagus nerve foramen and its wider lateral branch (below perilymphatic foramen), originating from the posteroinferior portion of glossopharyngeal foramina (IX). The concavity of basal plate is apparent on ventral view and the posterior edge of occipital condyle is triangular-shaped and projecting itself posteriorly from neurocranium. The occipital centrum is fused with posterior basal plate, near the internal carotid foramina.

\subsection{Mandibular arch (Figs. 232 - 237)}

The splanchnocranium, or cartilaginous visceral arches, of sharks is composed by mandibular, hyoid, and branchial arches (Compagno 1988; 1999). Mandibular arch (or jaws) and hyoid arch are closely associated with the neurocranium, being composed by paired palatoquadrates, labial cartilages, Meckel's cartilages (mandibular arch), hyomandibula and ceratohyals (hyoid arch). Laterally, the labial cartilages are superficially located on the mandibular arch. Descriptions on this region are pioneer for Isogomphodon oxyrhynchus.

Palatoquadrate: In terms of morphology, the palatoquadrate of I. oxyrhynchus is semisphenoidal in ventral view and nearly equal in diameter with the ceratohyal. The ethmoid process of the palatoquadrate length is bigger than its height, while total length of palatoquadrate corresponds to approximately 2.2 times the hyomandibula length. The ethmoid process has a semicircular and not broadly prominent shape, originating and ending, approximately, between the 7th and 11th teeth, respectively (Figs. 233 and 234). The first dentary row represents the functional active teeth, while the remaining four to six rows are not functional, whose number gradually decreases posteriorly (i.e., less teeth closer to the articular process). The posterior surface is mildly curved near the articulation and mostly straight on the medial dorsal facet, with the only elevation been resumed to the ethmoid process of the palatoquadrate. Articular region with Meckel's cartilage presenting a discrete tubercle on the external facet and a mild curvature ventrally and posterior to the ending of teeth rows.

Labial cartilages: As most species in Carcharhinidae, I. oxyrhynchus has three labial cartilages located closer to the articulations of the mandibular arch than to the anterior part, with the posterodorsal labial cartilage being the biggest in size, the anterior superior the smallest and the ventral labial cartilage located more ventrally with a discrete curvature in a boomerang shape (Fig. 233). The junction of posterosuperior and inferior labial cartilages has an accentuated 
connection and nearly fused posteriorly, with contact area corresponding to approximately $2 / 3$ of total size of each section (Fig. 233). The anterior extremity of inferior labial cartilage is located on the medial line of the 'C-shaped' recesses of Meckel's cartilage.

Meckel's cartilage: Generally, they are narrowed and pointy in anteroventral regions, where the first teeth rows originate. In terms of size, the Meckel's cartilages are dilated and rounded on the posterior region, close to the articulations with the palatoquadrate. Regarding its proportions, the first two-thirds are similar in diameter. There are ' $\mathrm{C}$-shaped' concavity present on the medial ventral part of this structure, located approximately on the line of the anterior extremity of anterior superior cartilage (Fig. 234). The anterior origin of these 'C-Shaped' recesses have more prominent extension than those on the posteriors. The posterior inferior region of Meckel's cartilage is extended and circularly long, while the origin of articulation with palatoquadrate is mildly curved and appears on the line of anterior portion of ceratobranchial I. Additionally, discrete prominent tubercles are present on the external facet of articulation region, which also has a shallow concave sulcus where the posterior articulation of palatoquadrate inserts.

Hyoid Arch: Overall, the hyomandibula has a rectangular shape in lateral view, with its length corresponding to three times its width (Figs. 235 and 236). The ceratohyal is rather elongated and curved on the articulation region with hyomandibula, with its total size ranging from posterior region of Meckel's cartilage to the C-shaped recesses (Figs. 234 - 236). Posteriorly, the hyomandibula gradually increases in circumference diameter from anterior to posterior region. Dilatations (mild elevations) on the external anterior parts of ceratohyal are visible on dorsal view by the line of the C-Shaped recesses (Fig. 235 and 236). The anterior external facet of ceratohyal has discrete dorsal crests on the external edges and the ceratohyal has tuberclelike projections emerging from dorsal external facet. The basihyal has a 'Spade-shape', in which the posterior facet is enlarged on the lateral edges that narrows on the anterior margin and, in the posterior region, it bifurcates to the connection facets to the ceratohyal (Fig. 237)

\subsection{Branchial arches (Figs. 238 - 241).}

Overall, five pharyngobranchials, five epibranchials, five ceratobranchials and two hypobranchial are visible, while the unpaired structure is resumed in a single fused basibranchial copula. In terms of articulation of branchial elements, the following conformation was observed: The pharyngobranchial 1 is a minute structure with no apparent articulation, the 
pharyngobranchial 2 is articulated with epibranchial 1, the pharyngobranchial 3 with epibranchial 2, the pharyngobranchial 4 with the epibranchial 3 and the pharyngobranchial 5 with the epibranchial 4 (see Figs. 237 and 239). The gill rakers are poorly visible and associated with the epibranchials and ceratobranchials (Fig. 238 and 240). Descriptions of each branchial element are detailed below.

\subsubsection{Pharyngobranchials}

Although poorly calcified, especially in the posterior ones, five pharyngobranchial were visible and connected to an epibranchial, in an arrangement where the pharyngobranchial 1 has not an associated branchial element and the pharyngobranchial 2 articulates with the epibranchial 1, following this conformation onwards as seen in Figs 238 and 240. In terms of positioning, the pharyngobranchials $2,3,4$ and 5 originate dorsal to the anterior edge of epibranchial 1 and are diagonally arranged towards the posterior region of basibranchial. The pharyngobranchials 2 and 3, which are anteriorly bifurcated, present a Blade-like shape that gradually decreases in diameter posteriorly and are the biggest in size, while the pharyngobranchials one, four and five are minute. The first pharyngobranchial is resumed to a reduced structure closely located to the anterior edge of ceratobranchial 1 . In the adult specimen, the pharyngobranchial 5 is fused is the epibranchial 5 (Figs. 237 and 239). Both anterior and posterior edges of these branchial elements are rounded, which is only partially visible in Figs. 238 and 240.

\subsubsection{Epibranchials}

As observed in the pharyngobranchials, the calcification was poorly developed in the epibranchials and pharyngobranchials in both specimens, although fair descriptions are still plausible. Morphologically, the epibranchial 1 is more visible in detail, therefore its description shall be considered similar to the following epibranchials. However, the epibranchials 1 and 2 of the left side are relatively well evidenced in both specimens, thus facilitating their morphological description. Additionally, there is a decreasing gradient from the epibranchial one to five. Essentially, these structures are cylindrical with the external margin slightly wider. Origin of epibranchial 1 is dorsal to the ceratobranchial 1 and apertures in the posterior edge were detected with the CT-Scan imaging, which are possibly to facilitate the passage of branchial musculature. In the adult, the epibranchial 5 is fused with the pharyngobranchial 5. 
Round edges both in the external and in the internal facets are present, and articulations with their respective pharyngobranchials is described above.

\subsubsection{Ceratobranchials}

In morphological terms, the ceratobranchials are thicker in their anterior portion, having, in the ceratobranchial 1 , a tubercle-like projection towards the interior region of the branchial basket. Only the ceratobranchial 5 presents 'hook-like' concavities in the external posterior facet, resembling a u-shaped evagination in a hook-like form (Figs. 237 -240). Apart from these peculiarities, the other ceratobranchials have similar morphologies: cylindrical shapes with dilations on the internal margins.

\subsubsection{Hypobranchials}

The hypobranchials are essentially two paired structures connected with their respective ceratobranchials and to the basibranchial copular in a diagonal conformation (Figs. Figs. 238 and 240). In terms of size and proportion, they present a rectangular shape, with round edges, in which, in the hypobranchial 1, the length is approximately 4,6 times the width and the length corresponds 3,2 times the width in the hypobranchial 2.

\subsubsection{Basibranchial/Basibranchial copula}

A single fused structure originating immediately posterior to the ceratobranchial 3 . The basibranchial copula is rectangular with curved edges and gradually reducing in diameter in the anterior-posterior way. In morphological terms, the basibranchial copula originates in a rectangular shape with curved edges and gradually reduces in width until it turns in a conicalshaped posterior plate. Additionally, the basibranchial copula here observed is divided into two pieces in both analysed specimens (juvenile and adult).

\subsection{Pectoral girdle (Figs. 242 - 245).}

Overall, and based on available data on the literature, the pectoral girdle of $I$. oxyrhynchus resembles other carcharhinids, although it also presented differences that require a detailed description. Generally, there are three basal fin elements with modern sharks,

specially carcharhinids, being the metapterygium as the largest in size (Figs. 242 - 245). In Isogomphodon, the conformation agreed with the previously stated. An enlarged, oval diazonal 
foramen is positioned in the internal ventral side of the scapula-coracoid, between the insertion of propterygium and mesopterygium. As for the coracoid bar, it has a posteroinferior sulcus, which is nearly confluent with it. Additionally, no fusion of the coracoid bar in the ventral midline was detected in the scanned specimen. The posterodorsal triangular process of scapula is present and located slightly below the scapula superior edge (Figs. 244). The radials are long and numerous (MZUSP 101214 total radial counting: 25 proximal, 28 medial 30 distal), with the distal radials being the largest in size, a direct reflex from the external morphology of the pectoral fins of this species. The first proximal radial, directly associated with the propterygium, is thicker, which likely represents a fusion of the first proximal radial with its respective medial radial, while the last (i.e., the last associated with the metapterygium) is also elongated, with two radials associated to it. The tip from the scapula superior edge is pointed and has a discrete division originating from the posterodorsal triangular process (Figs. 242 - 244). In terms of condyles, three independent condyles for each basal element are present for I. oxyrhynchus. The posterodorsal foramen has a small aperture and is located medially within the coracoid bar in the junction point with the scapula (Fig. 242 and 243), with probably a foramen for the subclavian artery. The propterygium is rectangular shaped, with a unique fusioned and thicker radial associated with it, which has no accessory radials connected with it (other carcharhinids present such feature - see Silva \& Carvalho, 2015 p. 556). The mesopterygium is approximately quadrangular shaped and has six proximal radials, while the posteriorly elongated metapterygium has a short narrow facet with the coracoid bar, presenting 17 associated proximal radials being the last enlarged when compared to the others (Figs. 243). Among the three facets of radial elements, the facet for metapterygium is the narrowest, followed by the facet for the mesopterygium (Figs. 242 - 244). The diazonal foramen is confluent with the propterygium and the mesopterygium facets, which are also enlarged and located, in dorsal view, on the line of the mesopterygium. The posterodorsal foramen is located approximately in the midpoint of the coracoid bar (Figs. 242 and 243). The region between the coracoid bar inferior edge, close to the junction, and the scapular process shows a linear shaped conformation, with its total length corresponding to 1.4 times the length of the metapterygium internal margin. The posterodorsal triangular process of the scapula is mildly edged and basically confluent with the scapula itself, while the coracoid bar is more dilated in the inferior portion, with a pointed edge in the posteromedial region (Fig. 243). A medial posterior concavity is located on the ventral portion of the coracoid bar, which has no fusion of the two bars as illustrated on Fig. 245. The metapterygium has a crest-like projection in the internal 
facet, which is closely positioned to the facet for the coracoid bar. No other foramina are visible in ventral view besides the diazonal foramen.

\subsection{Pelvic girdle (Fig. 246 - 247).}

The puboischial bar is flattened in the upper edge and mildly concave in the lower edge, in a 'boomerang-like' shape (i.e., straight, narrow edged on the anterior margin and gradually increasing in width until the posterior edge, which is concave), also with two obturator foramina visible in both dorsal and ventral views and with basipterygium having multiple indentations, each associated with the proximal end of an individual proximal radial. Those indentations are more pronounced in the middle of the range, less so anteriorly and posteriorly (Figs. 246 and 247). Similarly, as observed by Silva (2014), carcharhinid sharks in general presented similarities to the observed here in I. oxyrhynchus, as described below. The first radial is enlarged and bifurcated on the external facet to be associated with two distal radials and to articulate directly with the puboischial bar, as observed in Figs. 246 and 247. The bifurcation begins closer to external margin than to the internal, with lower extension being larger than the upper. There are 15 proximal radials associated with each side of the basipterygium, being 14 within the basipterygium itself and one enlarged associated to the puboischiadic bar, as previously described. In morphological terms, the proximal and distal radials are simple and non-bifurcated (except the first enlarged radial), with proximal radial presenting a rectangular cylindrical shape and being larger than the distal ones, which are also rectangular although their length is nearly equal to their width on anterior ones. Additionally, the first distal radial immediately posterior to the first enlarged radial is shorter than the following ones. The anterior distal radials are slightly larger than the posterior ones, gradually reducing on length from the fifth distal radial and, in the first radials, the width increases significantly, especially in the first three radials. Besides the first enlarged radial, each proximal radial is associated with a single distal radial. In dorsal view, the are two tubercle-like crests located close to the facet for the basipterygium and immediately posterior to the obturator foramen. The basipterygium is discreetly curved on both edges, with the most prominent curvature on the caudal facet. The obturator foramina are located close to the facet for the basipterygium, which are oval-shaped and close to the facet for the first enlarged radial (Figs. 247). 


\subsection{Clasper (Figs. 248 - 252).}

Overall, the clasper of $I$. oxyrhynchus can be classified as a Carcharhinoid or Hemigaleoid type of clasper (see Compagno 1988, p. 43). This kind of clasper is characterized by its circular to oval cover rhipidion located in the rear end of the structure, by the presence of a curved to slightly curved clasper groove and by the presence of a mesorhipidion. As an adult male was analysed within the CT-Scan, not only descriptions of the external view but also of the internal cartilages from this species were here performed.

External view: The dorsal surface is rather smoother than the ventral, once the dermal denticles are only present in the ventral facet. The apopyle line is confluent with the clasper groove, which is mildly sinuous and ends in a circular line that corresponds to the region above the hypopyle. The cover rhipidion is circular, located slightly anterior to the posterior edge of the clasper (Fig. 248). The pseudopera and the pseudosiphon are located on the midline of mesorhipidion and cover rhipidion (Figs. 248 - 250). Within the rear end of the clasper itself, there is a cavity/sulcus that is confluent with the line from the clasper groove and cover rhipidion (Figs. 248 and 249). The rhipidion is similar in size with the cover rhipidion and bigger than the mesorhipidion, which is rugged in the ventral surface. Between the rhipidion and the mesorhipidion, a cavity/sulcus is present, while the pseudopera is positioned in the midline of the rhipidion. Dorsal to the hypopyle and in the terminal part of the clasper groove, a delimited oval to circular region is evident, although it is not clear what structure this corresponds. The 'terminal sulcus' has a rugged confluent margin with the mesorhipidion rear end and is slightly dark coloured in the margin connected to the rhipidion (Figs. 248 - 250).

Internal skeletal structures: The internal facet of the dorsal marginal cartilage is rectangular shaped (i.e., edges with vertices that resemble a rectangle corner) while the ventral marginal cartilage is linear anteriorly and discretely curved posteriorly (Fig. 251). The accessory dorsal marginal cartilage (RD2) is oval and, unlike the cover rhipidion, which is the correspondent structure on external view, extends until the posterior end of the clasper skeleton, suggesting that the 'terminal-sulcus' is not a calcified region but rather composed by dermal tissue (Fig. 250). Associated with the ventral terminal cartilage (TV) there is a discrete but thick cartilage, called end-style, that is connected to the ventral marginal cartilage (RV) and with the accessory terminal cartilage (TD3, which is inside the mesorhipidion), as shown on figure 251. The endstyle $(\mathrm{G})$ is rectangularly delimited, originating slightly anterior to the ventral terminal cartilage 
(TV) confluent with the axial cartilage (AX). Both claspers have a tubercle-like projection on the anterior edge, close to the insertion with the basipterygium. The anterior edge of the external calcified cover of the apopyle is strongly cornered (i.e., nearly perpendicular) while the internal coverages of the apopyle are straight edges in their anterior margins. Additionally, the region of insertion with the basipterygium has an accentuated calcification and is located close to the anterior tubercles on axial cartilage. Similar to the observed on the external view, the terminal cartilages (i.e., RD2, TV, TD2 and TD3) are equally the same in terms of size, although the accessory terminal cartilage is slightly larger than the others.

\subsection{Dorsal fins (Figs. 253 - 257).}

\subsubsection{First dorsal fin}

Although stated in Compagno (1999), the dorsal fins are composed, apart from the three kinds of radials, by basal elements, in the CT images those structures were not visible and are absent within I. oxyrhynchus unpaired fins anatomy. Generally, the first dorsal fin structure of this species is composed by three main radial components: proximal, medial, and distal. The total count is 18 slender, cylindrical proximal radials and one posterior expanded proximal radial, 26 medial radials, being the most posterior larger and bifurcated, and 26 distal radials, with the last two being connected to a single medial radial (Figs. 253 - 256). In terms of size, the proximal radial is nearly equal sized in length and width with each other, and the medial radials are shorter in the anterior and posterior region, as well as the distal radials (Figs. 253 256).

In more detailed descriptions, the anterior region is solely composed by two radials (proximal and medial), being curved in their connection. Therefore, the distal radials appear only from the second pterygiophore onwards. Externally, the first dorsal fin on I. oxyrhynchus has a free margin, and the last proximal radial (epr) before the beginning of this free margin is expanded and triangular, being connected to four medial radials (Fig. 253). Apart from such radial element, each proximal radial is connected to a single medial radial and are slightly curved in the anterior region (Fig. 253).

Regarding the medial radials, the anterior most is not connected to a distal radial and the posterior most is slightly bifurcated, connected to two distal radials (Figs. 253 and 254). These radials are narrower in the lower portion and dilated in the upper portion. The anterior distal radials, on the other hand, are pointed in the upper portion while the posterior ones are narrower and shorter, with the last three ones (except the last) being slightly curved. The 
transitional distal radials (i.e., whose shape is nearly triangular and represents the transition from the slender, pointed form to the short, narrow morphology) occur from the 15th to the 17th distal radial. Additionally, the last distal radial is also bifurcated.

\subsubsection{Second dorsal fin}

As well as the first dorsal fin, no basal elements were detected in the CT images of the second dorsal fin, therefore sustaining that those elements are absent in I. oxyrhynchus. Generally, the second dorsal fin is rather similar to the first dorsal, not only in terms of the morphology of the radial elements but also on the presence of fused proximal and medial radials. Overall, the distal radials originate only from the second radial element and begin to drift their morphology on the sixth pterygiophore (i.e., from a cylindrical shape to a spade-like, triangular shape). However, from the eleventh proximal radial onwards, the cylindrical shape appears again. The medial radials are also cylindrical, with the radials on the medial region being the biggest in size. Finally, the proximal radials 6, 7 and 8 are larger than 2, 3, 4 and 5, while the first is rather minute (Fig. 257). The total radial count is resumed: proximal radials = 11 , with four being fused in a single element; medial radials $=14$, with being two being fused in a single element; distal radials $=15$, with no fused elements.

\subsection{Anal fin (Fig. 258).}

Similar to the results on dorsal fins, the posterior radial elements are expanded and possibly fused. Three radial elements are present: proximal radials, medial radials and distal radials, with two fused posterior elements that are here described as 'expanded proximal radial' and 'expanded medial radial' (Fig. 258). In terms of morphology, the radials are shorter in the anterior portions, higher in the medial region and slightly shorter on the posterior region before the expanded elements. The distal radials are narrow in their connection region with the medial radials, with the three anterior ones with pointed tips and the adjacent presenting dilated ends. The expanded proximal radial is triangular and is possibly the result of a fusion between three proximal radials, while the expanded medial radial, which is also triangular, is likely a product of four medial radials fused. The two medial radials that precede the expanded medial radial are medially connected, with the anterior one having two distal radials associated to it. 


\subsection{Caudal fin (Fig. 259).}

The caudal fin skeleton of I. oxyrhynchus has been described and illustrated by Moreira et al. (2019). Here, the present descriptions shall not only follow the nomenclature used by these authors, but also contribute to the available descriptions with the CT-Scan imaging.

In the caudal fin of I. oxyrhynchus there are six to seven anterior vertebral centra, which are characterized by its diplospondylic profile (i.e., with both interdorsal and basidorsal dorsal to a single centrum). A total of 78 caudal vertebrae are present in MZUSP 101214 (juvenile specimen), considering the categorization method for the caudal fin employed in Moreira et al. (2018). Firstly, the epaxial elements are described by the supraneurals, basidorsals and interdorsals, by which the supraneurals are numerous and start from the 12th until the end of the caudal fin (Fig. 259). In terms of shape, these structures are cylindrical to conical, by which the first is reduced and the following ones gradually grows until they reach the maximum size around the 18th vertebral centrum of the caudal fin, when they start to decrease again in a slower rate. Additionally, bifurcations are present in some of them. The interdorsals and basidorsals have similar quadrangular shapes, although the basidorsals have dilatations in their ventral portion. On the other hand, in the hypaxial elements, described by the basiventrals and haemal spines, are present only in the seven anterior haemal spines, while from the eighth onwards these two structures are fused. The haemal spines are thicker in the anterior region and gradually become thinner in the anteroposterior way. Morphologically, these structures are cylindrical with dilated ventral tips and become thinner in the point when they fuse with the basiventrals. Regarding the vertebral centra, these are rectangular shaped with mild dorsal and ventral concavities in which the dorsal (basidorsals and interdorsals) and ventral (haemal spines) elements are attached to.

\subsection{Vertebral column (Figs. 260 - 266).}

In lateral view, the vertebral centra is nearly equal in height and length, although they vary slightly in width along the column axis (Figs. 260 to 263). The most prominent variation in these measurements occur after the first vertebral centra (from the $17^{\text {th }}$ centrum onwards, which marks the beginning of the trunk region. After the transition from monospondylic centrum to diplospondylic centra, all the dorsal vertebral elements were considered and named as neural arches. As viewed in layer images from the CT-Scan images, the morphology of the centrum has two narrow projections (dorsal and ventral) with 'V-shaped' areas and two lateral 
expansions in a fan-shaped conformation (Fig. 264). In terms of length and width, the transition between the monospondylic and diplospondylic vertebral centrum, using such measurements as a tool, is not detectable, although these slightly vary along the vertebral column axis. On the other hand, such transition, in terms of classification between centrum types, occurs abruptly, when the interdorsals and basidorsals are considered. This becomes visible between the $33^{\text {rd }}$ and $34^{\text {th }}$ centra on the juvenile (Fig. 262). There is no evident mark to identify, based on the anatomy of vertebral column, the beginning of the caudal fin (i.e., caudal vertebral centra). Within the monospondylic region, the differentiation between interdorsal and basidorsal elements is evident, although on the diplospondylic region, those elements were all classified as neural arches as they become morphologically similar (a square/rectangular shape).

The first rows of basidorsal arches are characterized by having a lateral foramen in both sides, and in MZUSP 101214 these foramina are completely closed in their circumference only in the first five arches, which begin to gradually disappear from the sixth until the nineth, when they become completely absent (Fig. 261). On the adult specimen, these foramina begin to fade away in the seventh basidorsal arch, when they merge with the lateral facet of the vertebral centrum. The haemal archers become completely absent on the $10^{\text {th }}$ vertebral centrum as observed with the CT-Scan images. A total of 78 caudal vertebrae are present in MZUSP 101214 (juvenile specimen), and the caudal fin starts on the $56^{\text {th }}$ vertebral centrum. Regarding the calcification pattern, the dorsal and ventral solid intermedialia is ' $\mathrm{V}$-shaped', with the dorsal having a U-shaped scallop in the dorsal facet, while the ventral has a round facet. The lamellae between these regions are also ' $\mathrm{V}$-shaped' in a ' $\mathrm{X}$ ' conformation. 


\section{Discussion}

\section{Dermal denticles}

In Isogomphodon oxyrhynchus, a high variation in the dermal denticles morphology occurs not only among the different body sections but also among life stages. Comparatively, within some sections from the adult (e.g., body and gill slits) the scales present a desquamated surface, in which the polygonal pattern was not as visible and well-delimited as the results from the juvenile. Additionally, the cusps of some scales in this same section of the adult presented damaged cusps and lateral edges, which might be a result of foraging behaviour or merely swimming in turbid waters. Consequently, these results also suggest that age is a factor that would influence the morphology of the dermal denticles, not only the surface coverage.

The dorsal fin sections seem to have more agglomerated denticles when compared to other sections (i.e., apparently more scales per sampled area), which probably enhances the hydrodynamic profile of the region. In the pectoral section, although most dermal denticles are rather similar to most of the other regions (except P3), few of them present four and five ridges. Curiously, the morphology of the scales on P3, on the adult and juvenile, is rather similar to the observed on the Ndors section: a round-shaped scale with rugged surface on the insertion region with no crests or ridges on the crown and smooth on the terminal endings. On the other hand, P3 from the neonate, cusps are visible in the covered denticles. The P3m sampled for the juvenile showed denticles with a hydrodynamic profile: rough crown surface with three cusps. It is important to state that the P3 samples were collected from the surface near the posterior insertion of the dorsal fin, a less hydrodynamic region than the pectoral edges (see Fig. 2), which probably explains the morphological difference.

Biomechanical in vitro tests on hydrodynamic propel capacity suggest that overlapped scales with crested and cusped morphologies have enhanced speed performances and, therefore, facilitate the passage of a water flux (Wen et al. 2015). Additionally, along the body axis of sharks, the water flow velocity during the swimming tends to be less accentuated on the posterior insertion of the pectoral fin and strong on the dorsal and caudal fins (Díez et al. 2015). These observations suggest that, on those regions with round-shaped scales (e.g., P3), the water flow is reduced compared to other body locations and, therefore, do not require an enhanced hydrodynamic format. The morphology observed on P3m also corroborates with the fact that in the pectoral margin the water flux is more enhanced (Díez et al. 2015), therefore requiring an equally hydrodynamic format for the dermal denticles. Interestingly, the scales in regions 
with lateral line canals (e.g., body sections) the scale overlap was not recurrent, which would probably allow an enhanced water flow beneath them. Additionally, in the adult, placoid scales have more spaced ridges, which could potentially be useful for a hydrodynamic profile on the scale.

Some scales on $\mathrm{P} 1$ and $\mathrm{C} 1$ are damaged, probably due to specimen preservation, sampling collecting process or a result of the species habits (i.e., the contact of the pectoral region with the substrate during hunt for benthonic prey). Across body sections, dermal denticles are nearly identical in general morphology. However, the lateral edges, or reticulated depressions, on B3 (i.e., ventral scales) are slightly more pronounced than on the B1 and B2. The caudal fin section showed some denticles with bifurcated medial ridges that medially converge to a single ridge in the crown, especially on $\mathrm{C} 2$. Nonetheless their morphology resembles more closely that observed in other sections: three ridges and three cusps, with the medial one most prominent. Scales in that region are often damaged in their cusps and sometimes entirely broken, which limits the description of a standard morphology for the $\mathrm{C} 1$ section. Additionally, a rather different scale was detected in an emerging position. This suggests that the scales within the caudal fin would have this morphology in a situation where damage, either post-mortem or caused by the environment, are minimal.

The main difference of the scales within gill openings is that their lateral edges and cusps are broader and flattened. Additionally, the distance between medial and lateral cusps and ridges is shorter than in scales of other regions. Scales in that region are more desquamated on the adult that on the juvenile. The adult shows spaced-out denticles whereas the juvenile has an overlapped arrangement. On the nostril regions, some scales in the ventral portion of the adult show horizontal 'scars', although the characteristic prismatic pattern is still visible. Considering that I. oxyrhynchus also preys on benthonic fauna (Lessa et al. 2016), this morphology on the crown of this denticles are likely a result of foraging behaviour. The adult scales were often desquamated, a common phenomenon as the shark ages.

The clasper sections lack scales in the neonate. The juvenile has round to oval-shaped scales whereas the adult has three-cusped scales, with deep grooves between each cusp and no ridges. The clasper of the juvenile is not entirely developed because its distal margin does not reach the posterior region of the pectoral fin. The adult clasper is elongated, with its distal portion being significantly posterior to the distal margin of the pelvic fin. The dermal denticles of the adult are from the anterior portion on the clasper, within the perimeter of the pectoral fins, while those of juveniles were taken from an immature, short clasper. Microscopic 
observations directly on the clasper show that, posteriorly, dermal denticles are also round, similarly to the condition in the juvenile.

\subsection{Tooth Morphology}

Apart from the symphyseal and intermediates, all tooth group rows described by Compagno (1988) are present. Muller \& Henle (1839) and Compagno (1988) report that delicate serrations are present. No serrations were observed herein, even in SEM images of a juvenile specimen. Compagno (1988) also reports on a mesial notch at the medial portion on the anterior teeth, disappearing in posterior rows, a feature confirmed here. The grooves, however, were not accurately visible in CT-images. Based on the present results, teeth morphology of sharks can only be described in its general outlines by CT-Scan. A more detailed discussion on the tooth morphology of I. oxyrhynchus is provided in Chapter 3.

\subsection{Axial Skeleton}

The axial skeleton in Neoselachii comprises the head region (neurocranium and splanchnocranium) which is associated with the vertebral column that sustains the post-cranial anatomy and the caudal fin (Compagno 1999). Anatomical descriptions on the axial skeleton of sharks are well documented from the late $19^{\text {th }}$ to early $20^{\text {th }}$ century (e.g., Gadow 1888; Goodey 1910; Alis 1923; Lightoller 1939, El-Toubi 1949), although some significant late $20^{\text {th }}$ century works exist (e.g., Compagno 1977; 1988; 1999; Maisey 1980; Stone \& Shimada 2019). Regarding Isogomphodon oxyrhynchus, Compagno (1988) and Moreira et al. (2019) described the neurocranium and caudal fin respectively but did not cover the vertebral column elements or the splanchnocranium. The present chapter fills such gaps, adding novel observations on those anatomical landmarks.

\subsubsection{Neurocranium}

In Chondrichthyes, the neurocranium is a compact structure which, despite its simplicity, is potentially informative for a species-level identification (Compagno 1999; Maisey and Springer 2013). Gegenbaur 1865 (apud Compagno 1999b) initially proposed four main regions for the neurocranium (ethmoidal region, orbital region, otic region and occipital region) while Compagno 1988 proposed a more elaborate division with seven major sections (rostrum, nasal capsules, cranial roof, basal plate, orbits, otic capsules and occiput). Among families, the 
neurocranium varies in terms of morphology and associated musculature. In Carcharhinidae, the chondrocranium is rather reduced, with robust muscles (Moss 1972).

The neurocranium of I. oxyrhynchus is possibly the structure where most morphological peculiarities are concentrated. Compagno (1988) was the first author to give detailed descriptions on the anatomy of the species, although restricted to neurocranium, teeth, externalmorphological features and dermal denticles from the trunk. Although Compagno (1988) describes the orbit as relatively elongate, the eyes are reduced compared to other carcharhinids. Compagno (1988) did not report a basirostral fenestra for the species, however two small apertures are present in the rostral base, raising the question whether they are late calcification points or actual basirostral fenestra. The cranial roof seems slightly narrower in the adult than in the juvenile. Additionally, the crests on the orbitonasal foramen are absent in the adult specimen, which raises the question whether they are lost with growth or not detected in the CT-Scan. Compagno (1988) reported that the anterior fontanelle is approximately 6.3 times the nasobasal length, while in the scanned individual herein that distance is about 4 times. Comparisons between the adult and the juvenile reveal few differences, with the preorbital process larger in the adult, the absence of crests in the orbitonasal foramen in the adult and the parietal fossa slightly wider in the juvenile. The rostral fenestrae are more well-delimited in the adult than in the juvenile, which had thicker calcification in the rostral node. These differences, however, were detected solely using CT-Scan, and may reflect some artefact of image software (although a similar potential effect was not seen in the absence of crests on the orbitonasal foramen, clearly observed in the juvenile.

\subsubsection{Splanchnocranium}

The Splanchnocranium is composed of three major components: mandibular arch, hyoid arch, and branchial basket. As postulated in Compagno 1999, these elements are paired structures that are generically named as visceral arches, which can vary from seven to nine depending on the major groups.

\subsubsection{Mandibular Arch}

The mandibular arch is the first and anterior-most visceral arch, which is homologous to that in other Gnathostomata (Compagno 1999; Hickman et al. 2004). Those arches comprise a pair of palatoquadrates, the upper jaws and a pair of Meckel's cartilages, the lower jaw (Compagno 1999). Associated with those components, close to the mouth corners, labial 
cartilages are present in shapes that differ from group to group (Allis 1923; Compagno 1999, Shimada et al. 2009; Stone \& Shimada 2019). Orbital processes of the palatoquadrate, or ethmoid process depending on the group (Maisey, 1980), are present posterior to the nasal capsule, connected with the neurocranium by ligaments (Compagno 1999). Sharks are capable of forward jaw protrusion, which involves morphological adaptations of both the mandibular and hyoid arches (Moss 1972; Wu 1994; Wilga et al. 2001; Motta \& Wilga 1995; Motta et al. 1997; Nakaya et al. 2016). The muscles associated with the hyoid arch forwardly protrudes the mandibular arches, which are not directly connected to the chondrocranium, but instead anchored by elastic ligaments and muscles associated with the retraction (Nakaya et al. 2016). The hyoid arch is composed of two paired structures: hyomandibula (dorsal) and ceratohyal (ventral). Those structures articulate posteriorly with the neurocranium in the optic capsule region (hyomandibular facet) and with the distal facets of the mandibular arch (Compagno 1999).

The general 'arrow-like' morphology of the mandibular arch might be a facilitator form to allow an efficient cruising on the turbid, murky waters that I. oxyrhynchus is known to occur (Lessa et al. 2000). Overall, the mandibular and hyoid arches of $I$. oxyrhynchus did not present major differences from those in other carcharhinids. However, the ' $\mathrm{C}$-shaped' entrances, observed in the ventral portion of Meckel's cartilages, are likely to be autapomorphic, as it is not present in any other carcharhinid. The extremities of this 'C-Shaped' entrance are connected by a tendon, which although also present in other carcharhinids, is more pronounced in $I$. oxyrhynchus. Although Compagno $(1988,1999)$ adopt the nomenclature 'orbital process of the palatoquadrate', Maisey (1980) stated: "Other sharks with a corresponding orbital process in this position include the hexanchoids, squaloids, Squatina and Pristiophorus. No other elasmobranchs, living or fossil, have a comparable process, and I regard this as a shared derived character defining a monophyletic group of neoselachians. There are no conflicting characters to suggest that this derived condition has evolved more than once." Considering that, the so called "orbital process of the palatoquadrate" is here called "ethmoid process of the palatoquadrate".

\subsubsection{Branchial arches}

The most distal region of the splanchnocranium, preceded by the hyoid arch, is the branchial basket. Its arches are composed by six elements: pharyngobranchials, epibranchials, ceratobranchials, hypobranchials, basibranchials and a basibranchial copula. The 
pharyngobranchials correspond to the dorsal portion of the pharynx, the epibranchials and ceratobranchials (located laterally on the basket) are equivalent to the hyomandibula and ceratohyal, respectively, the hypobranchials are the ventral elements, often reduced in size and shorter in number of pairs, the basibranchials correspond to the ventral minute elements that are connected to the hypobranchials (usually the hypobranchial one is not included) and the basibranchial copula in a well delimited plate located dorsal to the cardiac cavity (Compagno 1999). As previously stated, the number of branchial arches vary from five, on most shark species, to six or seven in members of Hexanchidae. Additional accessory circular elements (dorsal extrabranchial and ventral extrabranchial) give mechanical support and are occasionally present between the arches depending on the group (Compagno 1999).

Ceratobranchial 5, has hook-like projections in the distal margin. Within the basibranchial copula, the two separated regions were here observed as one single structure. The minute structures on the dorsal edge of ceratobranchial 1 are named here as pharyngobranchial 1. Minor differences were detected between the juvenile and the adult. The observation of the divided basibranchial on both analysed specimens either indicates they are either different pieces or a single structure broken in both specimens. On the other hand, the enlarged anterior structure, here called basibranchial, possibly represents a fusion of hypobranchials 3 and 4 and the minute posterior piece is the hypobranchial 5. The epibranchial 5 is detected as fused with the pharyngobranchial 5, which is also likely possible for epibranchial 4 with pharyngobranchial 4 . Nonetheless the calcification failures limit the reliability of such assumptions. In the epibranchials and pharyngobranchials 3, 2 and 1, no fusion was detected.

\subsubsection{Vertebral column}

Within Elasmobranchii, the vertebral column is composed by two types of centrum that are defined based on the allocation of ribs and on the number associated dorsal arches: Monospondylic, the anterior vertebral centrum directly connected with neurocranium, having one basidorsal and one interdorsal, and Diplospondylic, presenting more than two dorsal elements by centrum and appearing immediately posterior to the gastrointestinal cavity (Compagno 1999). The latter is subdivided into two categories: diplospondylic pre-caudal and diplospondylic caudal vertebrae based on their location relative to the caudal fin (Compagno 1970; 1988; 1999; Moreira et al. 2018). A single vertebra is composed of a vertebral centrum, a pair of dorsal arches denominated basidorsals (which are separated from each other along the column axis by interdorsals and pierced by a spinal nerve foramen) and paired basiventrals 
(Compagno 1999). The transition between monospondylic and diplospondylic centra occurs in the line dorsal to the digestive tract or at the end of the rib series attached to the basiventrals (Compagno 1999). Calcification patterns of the central regions of the vertebral centrum are taxonomically informative (White 1937; Compagno 1988).

The general structure of the vertebral column observed here is similar to that reported in Compagno (1999) for the vertebral column of elasmobranchs. Ribs were not detected in CTScan images, although that does not imply actual absence, as their calcification is likely weak.

The calcification pattern of the vertebral centrum is highly variable among sharks, and potentially informative about phylogenetic relationships (Compagno, 1988). The vertebral centra of I. oxyrhynchus externally resembles that in other carcharhinids. The central area of the vertebral centrum is where the peculiarities of the taxon reside, such as the mild scallop on the dorsal solid intermedialia. Monospondylic centra appear to be narrower than diplospondylic pre-caudal centra (Fig. 262).

\subsubsection{Caudal fin}

The caudal fin in Elasmobranchii is classically defined as Heterocercal (classification initially postulated by Agassiz 1833), meaning that the upper and lower lobes have different lengths, with the lower usually shorter, although on Lamniformes and Squatiniformes the fin lobes are relatively equal in size (Compagno 1984; Gomes et al. 2010). In terms of structural composition, the caudal fin of sharks is composed of vertebral centra (which can either be precaudal diplospondylic or caudal diplospondylic), supraneurals, basidorsals, interdorsals, basiventrals and haemal spines (Agassiz 1833; Compagno 1999; Moreira et al. 2018). The upper lobe of this fin allocates the terminal portions of the vertebral column whereas the lower lobe is composed of haemal spines (or hypochordal rays) and ceratotrichia (Compagno 1999; Moreira et al. 2018). The variation on this region within shark taxa is not only vast, as observed by Little \& Bemis 2004 but also phylogenetically informative within Carcharhiniformes (Moreira et al. 2018).

Most morphological data here provided agrees with Moreira et al. (2019), which report that the caudal fin of I. oxyrhynchus is characterized by having seven diplospondylic vertebral centra on the anterior region. Here, this number was seen to vary between six and seven, in which the juvenile has six and the adult, seven. Moreira et al. (2019) state that the basiventral elements are rectangular. 


\subsection{Appendicular skeleton}

The appendicular skeleton is defined by the paired pectoral and pelvic fins. Posterior to the branchial arches described the zone where the pectoral fin girdle is located, followed by the pelvic girdle. Both anatomical regions allocate important structures for the body locomotion, once these elements allow an efficient stability during their cruise swimming, which had deep importance in the survival of elasmobranchs throughout their evolution (Moss, 1982). Morphologically, Compagno 1999 provides significant descriptions on how these structures are disposed and structured in Elasmobranchii, although recent works have not only provided species level descriptions (Silva 2014) but also phylogenetic implications of coded characters based on these anatomical regions (Silva et al. 2015).

\subsubsection{Pectoral fins}

Primordially divided into basal proximal region and distal radial region (Zangerl, 1981), the pectoral girdle is composed by, in a general overview: (1) a fused u-shaped scapulocoracoid bar, comprising the scapula dorsolaterally and the coracoid bar ventrally, although some primitive groups as Hexanchidae, the ventral junction of the paired scapulocoracoid is joined by a sternal cartilage (Compagno 1999; Silva 2014); (2) three basal elements denominated as propterygium (posterior, reduced), mesopterygium (medial, large) and metaptertygium (distal, elongated), which the pro and mesopterygium have one facet for both and the metapterygium has it unique condyle that articulates with the lateral portion of the coracoid bar (3) radial elements (proximal, medial, distal), with the first proximal usually being a fusion of two radials or simply enlarged (Compagno 1999). Considering such composition, two kinds of pectoral girdles are described: aplesodic, present in sharks, described for fins which the radials do not reach the border, and plesodic, described for rays, which have the radials reaching the edge of the fins (Compagno 1999; Maia et al. 2012). Regarding the basal elements, each are directly attached to radial elements and, within sharks, they not only correspond to fused radials but also vary in their fusion patterns (Compagno 1999; Silva 2014). For example, some Squalomorphii sharks, such as Centroscymnys coelolepis, have the pro and mesopterygium fused (Silva et al. 2015; Vaz 2015), while in some Orectolobiformes, such as Hemiscyllium ocellatum, the propterygium is absent (Goto et al. 1997). Once the pectoral region is a highly active region in metabolic terms, they therefore require blood nutrition and strong nervous control, which is evidenced by nerve and blood vessel foramina, such as the diazonal foramen located within the scapulocoracoid (Compagno 1999; Silva 2014; Silva et al. 2015). 
For the pectoral girdles, nomenclatural terms and structures followed Silva (2014) and Silva et al. (2015), who described a vast number of pectoral girdles, some from carcharhinids, and unfortunately did not include $I$. oxyrhynchus in their analysed specimens. Consequently, the present description in this region for this species is pioneer.

The foramen located dorsally to the diazonal foramen was called posterodorsal foramen, based on the illustrations provided by Compagno (1999). However, its name is still not clear, once this would only be possible to confirm in dissected specimens. The general anatomy of pectoral girdle, in terms of structures and part positioning, is relatively conserved among Carcharhinidae, therefore the major variations of I. oxyrhynchus reside in few characteristics, such as the number of radials on the radial elements and the presence of some foramina. Regarding the radials size, it was observed that the distal radials presented the largest length amongst the remaining ones, which is a direct consequence in the external anatomy of $I$. oxyrhynchus, i.e., enlarged pectoral fins. Regarding the calcification failures, they did not significantly limit the present description, although it gave the impression that the scapulocoracoid bar in I. oxyrhynchus is composed by two separated pieces whereas this structure is described, as well as for other carcharhinids, as single jointed U-shaped girdle by Silva (2014). Additionally, the articulation facets are not easily visible in the CT-Scan pictures, although the condition observed here suggests that I. oxyrhynchus has a condition which the meso- and metapterygium have two separated articulation condyles. As observed here, the posterodorsal triangular process of scapula is present and located slightly below the scapula superior edge, as this structure is not always present within Carcharhinidae (Silva et al., 2015).

\subsubsection{Pelvic fins}

Also denominated as puboischiadic bar (Compagno 1999), the pelvic girdle is rather simplified in terms of composition when compared to the pectoral girdle. Generally, the pelvic girdle is composed by: (1) a single curvy piece identified as puboischiadic bar; (2) a basipterygium, similar to the basal elements on the pectoral girdle, in which the radials are inserted; (3) proximal radials, with the first usually being fused and having a condyle in the puboischiadic bar; (4) distal radials. As a whole, the pelvic girdle is completely separated from the vertebral column in terms of skeletal anatomy, and it is aplesoidic in most shark species (including I. oxyrhynchus) except alopiids (Compagno 1999). Laterally, this girdle is perforated by obturator foramina, whose quantity among groups is not a constant feature (Cappetta 1987). Overall, the general structure of the pelvic fin from I. oxyrhynchus is cohesive 
with the general description on this region provided by Compagno (1999), although with the exclusive features of the species here described. In terms of visualization on the CT-Scan images, discrete calcification failures were detected in the distal edges, but with no significant impact on the descriptions. Externally, the clasper is visible in both specimens, although the adult has a more elongated and rigid structure. The clasper was not significantly calcified due to the juvenile condition of one of the scanned specimens, and therefore, was not visible in CTscan images from MZUSP 101214, while on the adult this structure was clearly visible. The pelvic girdle of the adult was not described, once the images of this specimen were obtained aiming for the clasper.

\subsection{Clasper}

The pelvic girdle in Chondrichthyes allocates the clasper, a relatively well known male copulatory feature present in all extant sharks and rays (Compagno 1999). Those organs have complex, highly variable internal skeletal structures, and are hypothesized as modifications of the posterior-most radials of the pelvic basipterygium (Compagno 1999). Compagno 1988 described major clasper categories based on morphological features that are related at family level. The clasper is ventrally composed of a long axial cartilage that ends in the end-style and in the terminal ventral cartilages, and dorsally (from the anterior to the posterior region), by the apopyle, the clasper groove, the hypopyle and by the terminal dorsal cartilages (Compagno 1988; Vaz 2015). These last cartilages comprise the region where most of the clasper complexity resides, and they are generally subdivided in cover rhipidion, rhipidion, mesorhipion, pseudopera and pseudosiphon (Compagno 1988).

As observed on the juvenile images, all major skeletal structures were detected on the CT-Scan images, except the clasper. This indicates that the clasper is one of the last structures to receive calcium allocation, which comes with sexual maturation. The adult clasper, on the other hand, was perfectly detected.

Anterior cylindrical cartilages on the clasper, close to the connection with the basipterygium directed towards the internal margin, called intermediate segments (Compagno, 1988 - Fig. 5.1), are described to occur in the clasper of some Carcharhiniformes. In Isogomphodon oxyrhynchus, two other cartilages that are morphologically similar and closely positioned with an occasional intermediate segment (i.e., anterior to the apopyle and connected on the posterior region of the pelvic basipterygium) were visualized in the posterior-most proximal radials of the pelvic fins. These radials are thicker and have similar morphologies 
those previously mentioned elements, therefore giving a false impression they are intermediate segments, as described by Compagno (1988).

\subsection{Unpaired fins}

Overall, the unpaired fins are defined by the dorsal, either one or two, and the anal fins. According to major group classification, shark orders are also categorized based on the number of dorsal fins and by the presence of the anal fin (Compagno 1977; 1984; 1988; Gomes et al. 2010; Last et al. 2009; Naylor et al. 2012). Hexanchiformes, for example, are known by the occurrence of a single dorsal fin and by the presence of six or seven gill openings (Compagno 1984; 1988; Last et al. 2009). Structurally, the dorsal and anal fins are composed by series of radial elements (proximal, medial and distal) and by basal element that give support to the fin structure (Compagno 1999). Additionally, the first ray of both the first and second dorsal fin are modified into spines in Squaliformes, Heterodontiformes and vestigial in Pristiophoriformes, which are anchored in the basal cartilages (Compagno 1999). Regarding the anal fin, no basal elements are reported, therefore it is only composed by radial elements (Cappetta 1987 apud Silva 2014). These basal cartilages are structures that support the fin radials, and within Galeomorphii, this feature is only present in Heterodontiformes that have fin spines in both dorsal fins (Compagno 1999). However, the basal elements in Carcharhiniformes, Lamniformes and Orectolobiformes are reduced to absent (Compagno 1999), which means that these fins bases are supported by the proximal radials and associated musculature. Connected to the distal radials, there are thin, cylindrical filaments that give rigidity and extension to the fin: the Ceratotrichia.

Within Galeomorphii, only in Heterodontiformes the basal elements, which give support not only to the radials but for the spines, occur in the dorsal fins while the anal fin is known by the absence of such structures (Compagno, 1999). In I. oxyrhynchus, the two dorsal and anal fins are not significantly different from what is usually observed in other Carcharhiniformes, with only minor details such as the expanded radials, which are probably a radial fusion of the posterior proximal radials, detected on all images from these fins. In the first dorsal fin, the posterior-most proximal radial (epr), located before the beginning of the free margin is expanded and triangular, being connected to four medial radials (Fig. 253). Such radial morphology suggests a fusion of the posterior proximal radials (Fig. 253). Within the second dorsal fin, the expanded proximal radial possibly is a result of four radials being fused while 
the expanded medial radial possibly corresponds to two fused radials (Fig. 257), although only with a categorical ontogenetic study such assumption can be assured. 


\section{CONCLUDING REMARKS}

The Daggernose shark, Isogomphodon oxyrhynchus, is a morphologically modified Carcharhinidae. The elongated tripodal rostrum, the long distal radials on the pectoral fin, the large orbital region, reduced to nearly truncated preorbital process, the slender and cylindrical post-orbital process, the 'C-Shaped' grooves on Meckel's cartilages and the narrow cranial roof are examples of unique or unusual traits among sharks. A high variation on the morphology of the dermal denticles was observed not only among different life stages but also along the body sectors. The use of computerized tomography is useful for this kind of study, especially when it involves rare species. However, classic dissections and skeletal preparations are still necessary to ensure some foramina identifications and to detected poorly calcified regions that are bypassed in CT-Scan images. 


\section{References}

Agassiz, L. (1833). Recherches Sur Les Poissons Fossiles (Vol. 5). Imprimerie De Petitpierre, 160 p.

Allis Jr., E. P. (1923). The Cranial Anatomy of Chlamydoselachus anguinesus. Acta Zoologica, 4(2-3), 123-221.

Andreev, P. S., Coates, M. I., Shelton, R. M., Cooper, P. R., Smith, M. P., \& Sansom, I. J. (2015). U pper O rdovician chondrichthyan-like scales from $\mathrm{N}$ orth $\mathrm{A}$ merica. Palaeontology, 58(4), 691-704.

Cappetta, H. (1987). Mesozoic and Cenozoic elasmobranchii. Chondrichthyes II. Handbook of paleoichthyology, 3 .

Compagno, L. J. (1970). Systematics of the genus Hemitriakis (Selachii: Carcharhinidae), and related genera. Proc. Calif. Acad. Sci., 33(4): 63-98

Compagno, L. J. (1977). Phyletic relationships of living sharks and rays. American zoologist, 17(2), 303-322.

Compagno, L. J. (1984). Sharks Of The World Vol 4 Part 2.

Compagno, L. J. (1988). Sharks of the order Carcharhiniformes (Vol. 486). Princeton, New Jersey: Princeton University Press.

Compagno, L. J. V. (1999b). Endoskeleton. Sharks, skates, and rays: The biology of elasmobranch fishes, 69-92.

Díez, G., Soto, M., \& Blanco, J. M. (2015). Biological characterization of the skin of shortfin mako shark Isurus oxyrinchus and preliminary study of the hydrodynamic behaviour through computational fluid dynamics. Journal of fish biology, 87(1), 123-137.

Dillon, E. M., Norris, R. D., \& Dea, A. O. (2017). Dermal denticles as a tool to reconstruct shark communities. Marine Ecology Progress Series, 566, 117-134.

El-Toubi, M. R. (1949). The development of the chondrocranium of the spiny dogfish, Acanthias vulgaris (Squalus acanthias). Part I. Neurocranium, mandibular and hyoid arches. Journal of Morphology, 84(2), 227-279.

Enault, S., Auclair, C., Adnet, S., \& Debiais-Thibaud, M. (2016). A complete protocol for the preparation of chondrichthyan skeletal specimens. Journal of Applied Ichthyology, 32(3), 409-415. 
Gadow, H. F. (1888). XVII. On the the modifications of the first and second visceral arches, with especial reference to the homologies of the auditory ossicles. Philosophical Transactions of the Royal Society of London. (B.), (179), 451-485.

Gill, T. (1862). Analytical synopsis of the Order of Squali and revision of the nomenclature of the genera. Annals of the Lyceum of Natural History of New York, 7, 367-108.

Gomes, U. L., Signori, C. N., Gadig, O. B. F., \& Santos, H. R. S. (2010). Guia para identificação de tubarões e raias do Rio de Janeiro. Technical Books Editora.

Goodey, T. (1910, August). A Contribution to the Skeletal Anatomy of the Frilled Shark, Chlamgdoselachus anguineus Gar. In Proceedings of the Zoological Society of London (Vol. 80, No. 2, pp. 540-571). Oxford, UK: Blackwell Publishing Ltd.

Goto, T., Nishida, K., \& Nakaya, K. (1999). Internal morphology and function of paired fins in the epaulette shark, Hemiscyllium ocellatum. Ichthyological Research, 46(3), 281-287.

Hickman, C. P., Roberts, L. S., \& Larson, A. (2004). Principios Integrados de Zoologia. Ed.

Last, P. R., Stevens, J. D., Swainston, R., \& Davis, G. (2009). Sharks and rays of Australia.

Lessa, R., Santana, F. M., Batista, V., \& Almeida, Z. (2000). Age and growth of the daggernose shark, Isogomphodon oxyrhynchus, from northern Brazil. Marine and freshwater research, 51(4), 339-347.

Lessa, R., Batista, V. S., \& Santana, F. M. (2016). Close to extinction? The collapse of the endemic daggernose shark (Isogomphodon oxyrhynchus) off Brazil. Global Ecology and Conservation, 7, 70-81.

Lightoller, G. H. S. (1939). Probable Homologues. A study of the Comparative Anatomy of the Mandibular and Hyoid Arches and their Musculatures. - Part 111. The Columella and Ossicula Auditus. The Transactions of the Zoological Society of London, 24(5), 403-444.

Maia, A. M., Wilga, C. A., \& Lauder, G. V. (2012). Biomechanics of locomotion in sharks, rays, and chimaeras. Biology of sharks and their relatives, 1, 125-151.

Maisey, J. G. (1980). An evaluation of jaw suspension in sharks. American Museum novitates; no. 2706.

Maisey, J. G., \& Springer, V. G. (2013). Chondrocranial morphology of the Salmon Shark, Lamna ditropis, and the Porbeagle, L. nasus (Lamnidae). Copeia, 2013(3), 378-389.

Moreira, R. A., Gomes, U. L., \& de Carvalho, M. R. (2019). Systematic implications of the caudal fin skeletal anatomy in ground sharks, order Carcharhiniformes (Chondrichthyes: Elasmobranchii). Zoological Journal of the Linnean Society, 185(1), 193-211. 
Moss, S. A. (1972). The feeding mechanism of sharks of the family Carcharhinidae. Journal of Zoology, 167(4), 423-436.

Moss, M. L. (1977). Skeletal tissues in sharks. American Zoologist, 17(2), 335-342.

Motta, P. J., \& Wilga, C. A. (1995). Anatomy of the feeding apparatus of the lemon shark, Negaprion brevirostris. Journal of Morphology, 226(3), 309-329.

Motta, P., Tricas, T., \& Summers, R. (1997). Feeding mechanism and functional morphology of the jaws of the lemon shark Negaprion brevirostris (Chondrichthyes, Carcharhinidae). Journal of Experimental Biology, 200(21), 2765-2780.

Nakaya, K., Tomita, T., Suda, K., Sato, K., Ogimoto, K., Chappell, A., Sato, T., Takano, K. \& Yuki, Y. (2016). Slingshot feeding of the goblin shark Mitsukurina owstoni (Pisces: Lamniformes: Mitsukurinidae). Scientific reports, 6, 27786.

Naylor, G. J., Caira, J. N., Jensen, K., Rosana, K. A., Straube, N., \& Lakner, C. (2012). Elasmobranch phylogeny: a mitochondrial estimate based on 595 species. The biology of sharks and their relatives, 31-56.

Shimada, K., Rigsby, C. K., \& Kim, S. H. (2009). Labial cartilages in the smalltooth sandtiger shark, Odontaspis ferox (Lamniformes: Odontaspididae) and their significance to the phylogeny of lamniform sharks. The Anatomical Record: Advances in Integrative Anatomy and Evolutionary Biology: Advances in Integrative Anatomy and Evolutionary Biology, 292(6), 813-817.

Silva, J. P. C. B. D. (2014). Filogenia dos principais grupos de Chondrichthyes baseada na anatomia comparada do esqueleto das nadadeiras pares e suas cinturas. (Doctoral dissertation, Universidade de São Paulo).

Silva, J. P. C., \& De Carvalho, M. R. (2015). Morphology and phylogenetic significance of the pectoral articular region in elasmobranchs (Chondrichthyes). Zoological Journal of the Linnean Society, 175(3), 525-568.

Stone, N. R., \& Shimada, K. (2019). Skeletal Anatomy of the Bigeye Sand Tiger Shark, Odontaspis noronhai (Lamniformes: Odontaspididae), and Its Implications for Lamniform Phylogeny, Taxonomy, and Conservation Biology. Copeia, 107(4), 632-652.

Vaz, D. F. B. (2015). Revisão taxonômica e morfológica do gênero Centroscymnus Barboza du Bocage \& Britto-Capello, 1864, com comentários no arranjo genérico da família Somniosidae (Chondrichthyes: Squaliformes) (Doctoral dissertation, Universidade de São Paulo). 
Wen, L., Weaver, J. C., Thornycroft, P. J., \& Lauder, G. V. (2015). Hydrodynamic function of biomimetic shark skin: effect of denticle pattern and spacing. Bioinspiration \& biomimetics, 10(6), 066010.

White, E. G. (1937). Interrelationships of the elasmobranchs with a key to the order Galea. Bulletin of the AMNH; v. 74, article 2.

Wilga, C. D., Hueter, R. E., Wainwright, P. C., \& Motta, P. J. (2001). Evolution of upper jaw protrusion mechanisms in elasmobranchs. American Zoologist, 41(6), 1248-1257.

$\mathrm{Wu}$, E. H. (1994). Kinematic analysis of jaw protrusion in orectolobiform sharks: a new mechanism for jaw protrusion in elasmobranchs. Journal of Morphology, 222(2), 175-190.

Zangerl, R. (1981). Chondrichthyes I: Paleozoic Elasmobranchii (Vol. 1). Lubrecht \& Cramer Limited. 
7. Appendix A - Analysed Material

Institutional abbreviations:

MZUSP - Museu de Zoologia da Universidade de São Paulo;

\section{Carcharhinidae}

Isogomphodon oxyrhynchus - MZUSP 101214, 1 Newborn (alc); MZUSP 101214, 1 Juvenile Male (Alc); MZUSP 37289, 1 Juvenile Male (alc); MPEG 3455, 1 Adult male (alc). 


\section{Figures and Tables}

Table 1. Described anatomical regions of Isogomphodon oxyrhynchus skeleton and dermal denticles with the literature followed for nomenclatural indications.

Anatomical region

Neurocranium

Splanchnocranium

Branchial arches

Pectoral girdle

Pelvic girdle

Vertebral column

Unpaired fins

Caudal fin

Clasper

Dentition

Dermal denticles

\section{Cited literature}

Allis 1923; Gilbert 1973; Compagno 1988; 1999b; Vaz 2015

Allis 1923; Maisey 1980; Compagno 1988; 1999b

Compagno 1999b; Vaz 2015

Compagno 1988; Silva 2014; Silva et al. 2015

Compagno 1988; Silva 2014; Silva et al. 2015

Compagno 1988; Compagno 1999b; Moreira et al. 2018

Compagno 1988; Compagno 1999b;

Compagno 1999b; Moreira et al. 2018;

Compagno 1988; Moreira et al. 2019; Vaz 2015

Compagno 1988; Laurito 1999;

Compagno 1988; Dillon et al. 2017;

Table 1. Tooth row formula of the analysed juvenile (MZUSP 101214) and adult (MPEG3455).

\begin{tabular}{|lcc|}
\hline & Juvenile & Adult \\
Alternates & 1 & 1 \\
Medial & 3 & 5 \\
Intermediates & L0-R0 & L0-R0 \\
Symphyseal & 1 & 1 \\
Anterior Upper & L7-R7 & L5-R5 \\
Anterior Lower & L7-R7 & L1-R1 \\
Lateral Upper & L10-R12 & L18-R17 \\
Lateral Lower & L10-R10 & L17-R16 \\
\hline
\end{tabular}




\begin{tabular}{|lcc|}
\hline Posterior Upper & L8-R8 & L7-R8 \\
Posterior Lower & L7-R7 & L5-R7 \\
\hline
\end{tabular}

Table 2. List of specimens used in the anatomical description of each skeletal region.

\begin{tabular}{|cc|}
\hline Anatomical region & Specimen \\
& MZUSP 101214 (Neonate), MZUSP \\
Dermal denticles & 37289 (Juvenile) and MPEG 3455 \\
& (Adult) \\
Neurocranium & MZUSP 101214 (Juvenile) and MPEG \\
& 3455 (Adult) \\
Mandibular arch + Hyoid arch & MZUSP 101214 (Juvenile) \\
Branchial arches & MZUSP 101214 (Juvenile) and MPEG \\
Pectoral girdle & MZUSP 101214 (Juvenile) \\
Pelvic girdle & MZUSP 101214 (Juvenile) \\
Dorsal fins & MZUSP 101214 (Juvenile) \\
Anal fin & MPEG 3455 (Adult) \\
Clasper & MPEG 3455 (Adult) \\
Caudal fin & MZUSP 101214 (Juvenile)
\end{tabular}

Table 3. Nerve nomenclature associated with the numeration.

\begin{tabular}{|cc|}
\hline Foramen Numeration & Associated nerve \\
I & Olfactory nerve \\
II & Optic nerve \\
III & Oculomotor nerve \\
IV & Trochlear nerve \\
V & Trigeminus nerve \\
VI & Abducens nerve \\
VII & Facial nerve \\
VIII & Auditory nerve \\
IX & Glossopharyngeal nerve \\
X & Vagus nerve \\
\hline
\end{tabular}




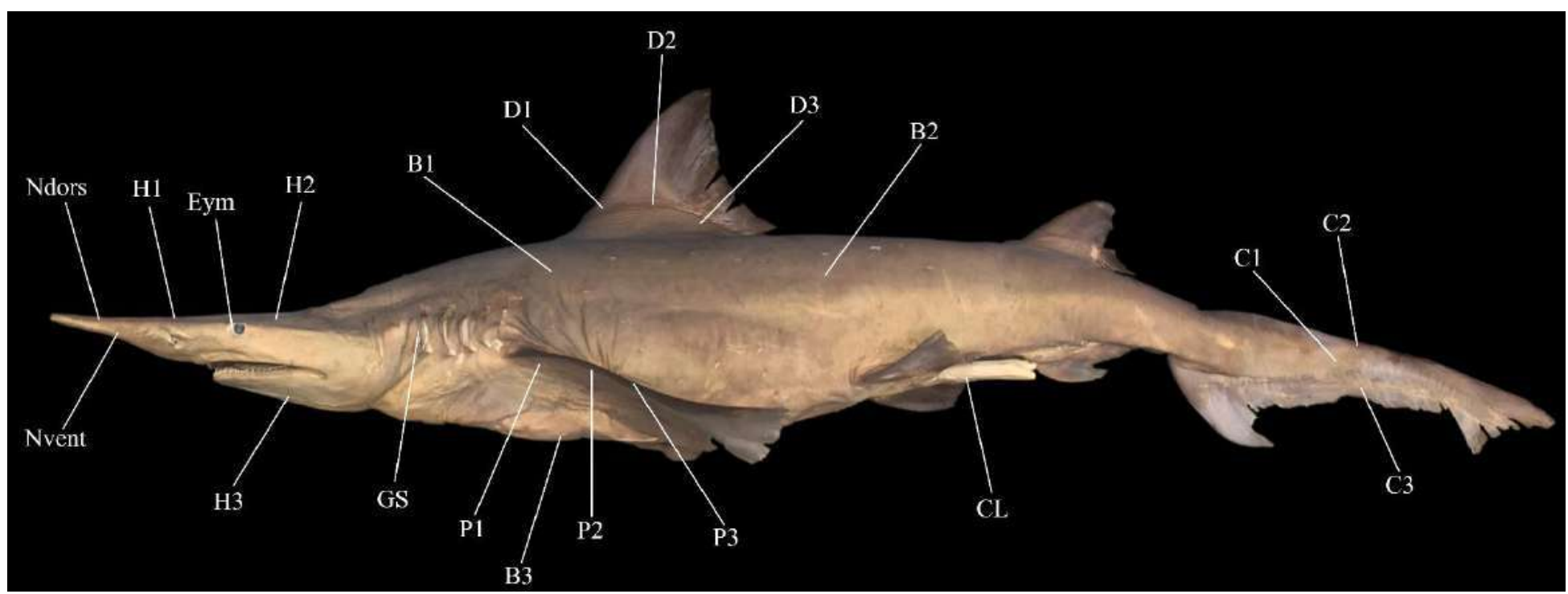

Figure 1. Schematic illustration of the Daggernose Shark Isogomphodon oxyrhynchus (Müller \& Henle 1839) showing the location from where skin samples were obtained, following Dillon et al. 2017 with adaptations. Images photoshop edited for esthetical purposes. Abbreviations: $\mathrm{H}$ - Head; B - Body; P - Pectoral; D - Dorsal fin; C - Caudal fin; N - Nostril; Eym - Eye membrane; GS - Gill Slits; CL - Clasper;

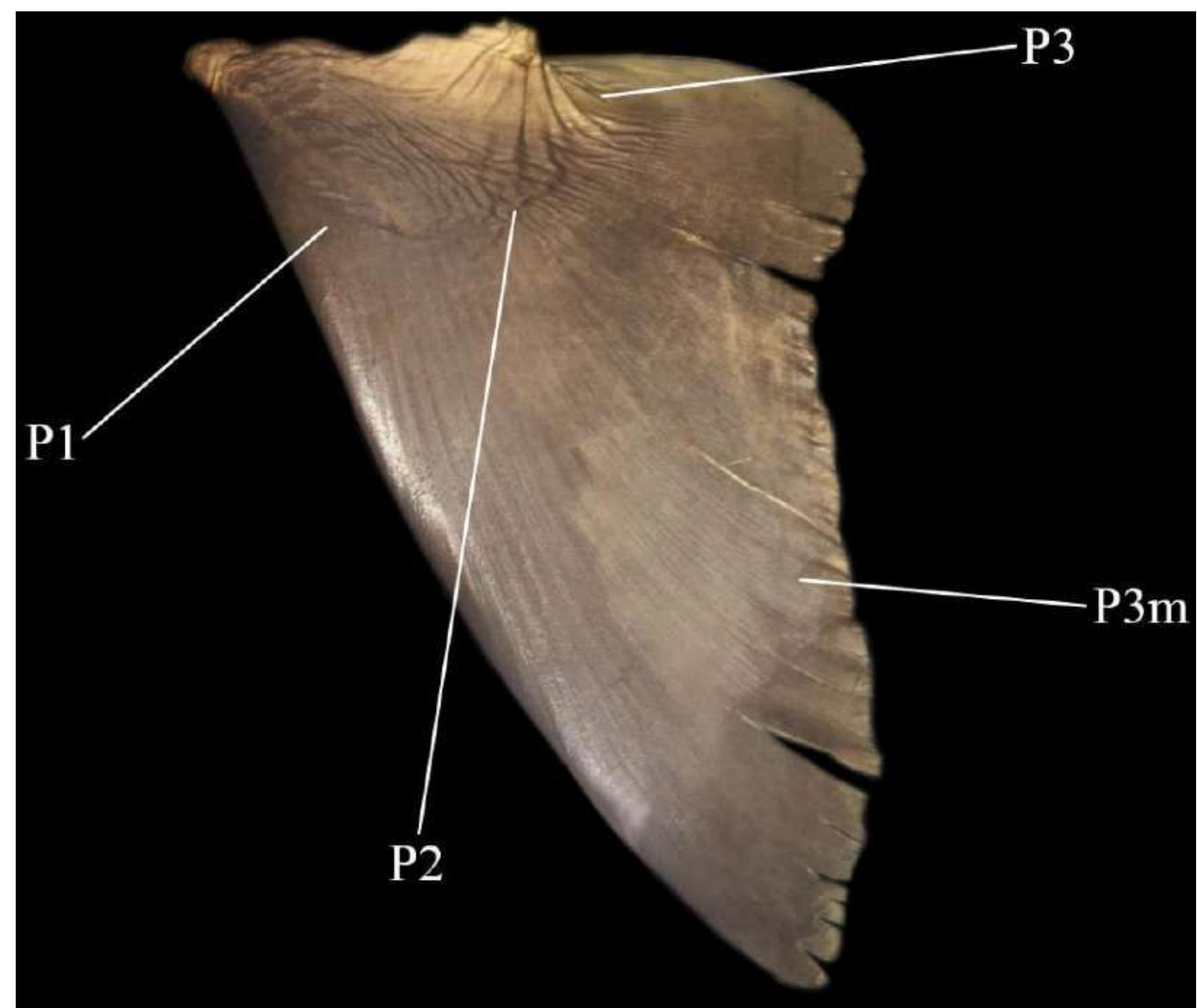

Figure 2. Schematic illustration of the right pectoral fin of Isogomphodon oxyrhynchus (Müller \& Henle 1839) showing the location from where skin samples were obtained, following Dillon et al. 2017 with adaptations. Image horizontally flipped for schematic purposes. Abbreviation: P - Pectoral; P3m - Sample P3 on marginal region; 


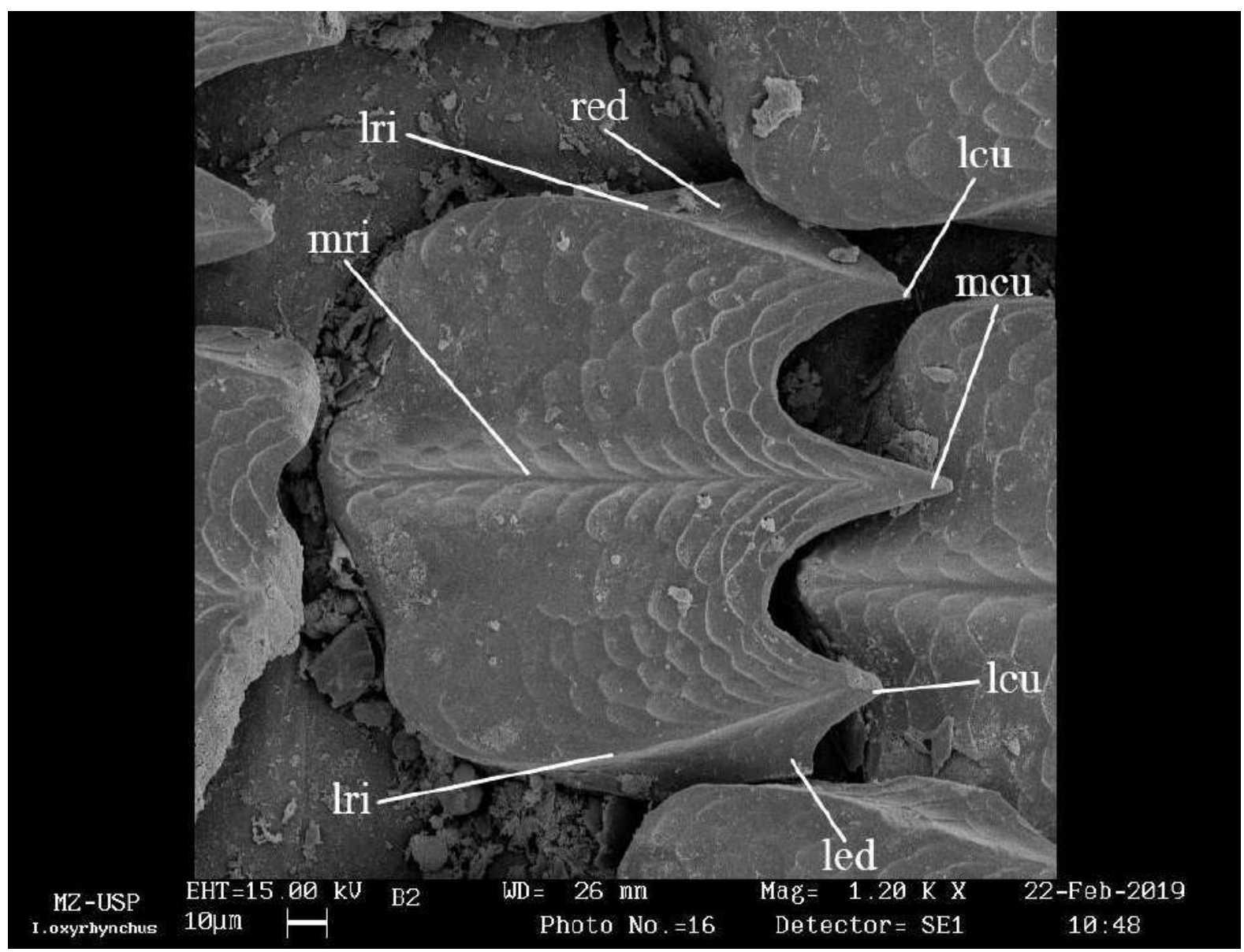

Figure 3. Schematic SEM image of a dermal denticles of Isogomphodon oxyrhynchus from section B2 (Fig. 1) showing the anatomical structures as stated by Compagno 1988. Anatomical abbreviations: lcu, lateral cusp; led, lateral edge; lri, lateral ridge; mcu, medial cusp; mri, medial ridge; red, reticulated depression. Additional information in the image footer. 


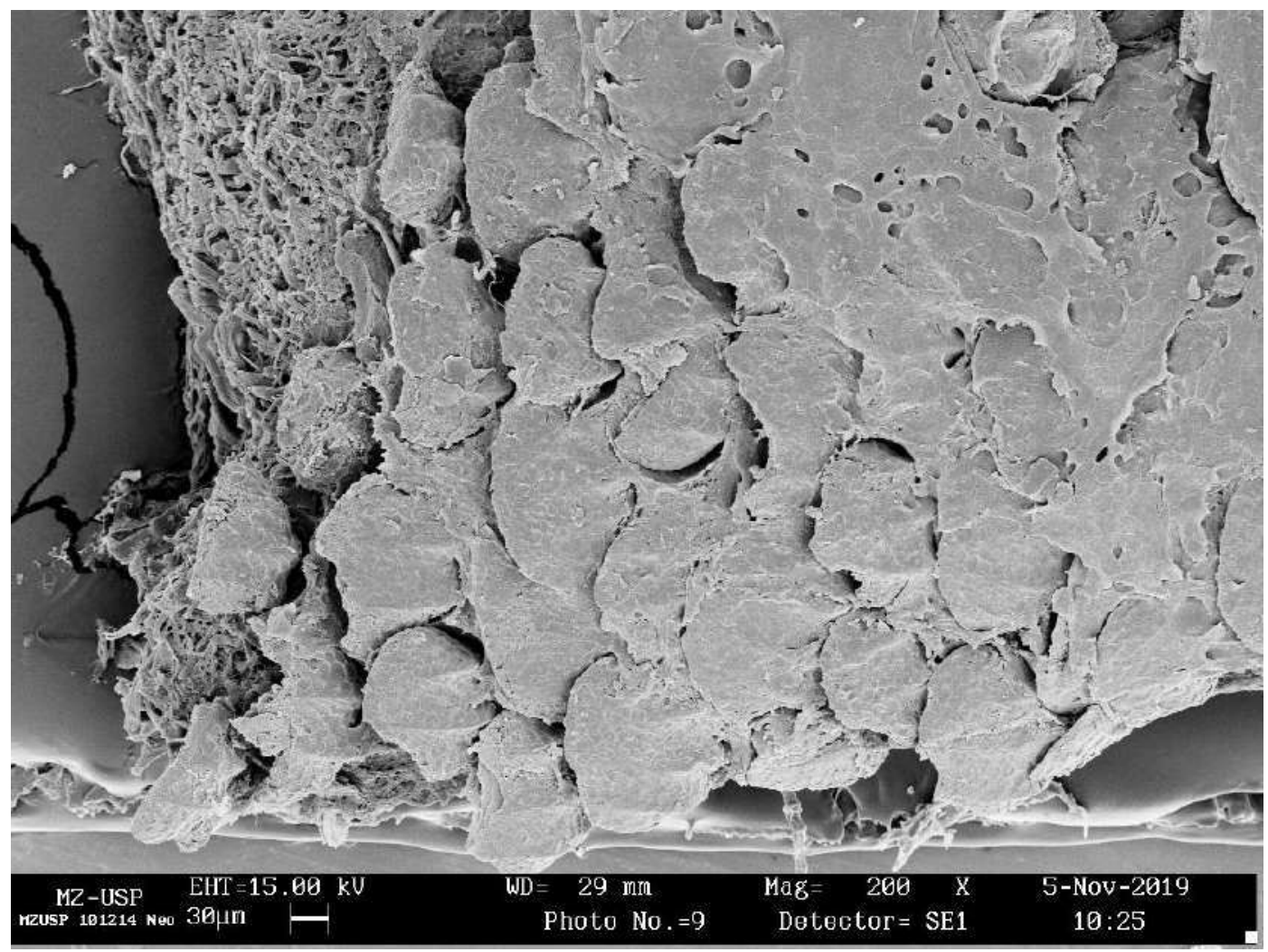

Figure 4. Dermal denticles collected from the section H1 (see fig. 1) of the neonate Isogomphodon oxyrhynchus (MZUSP 101214). Additional information in the image footer.

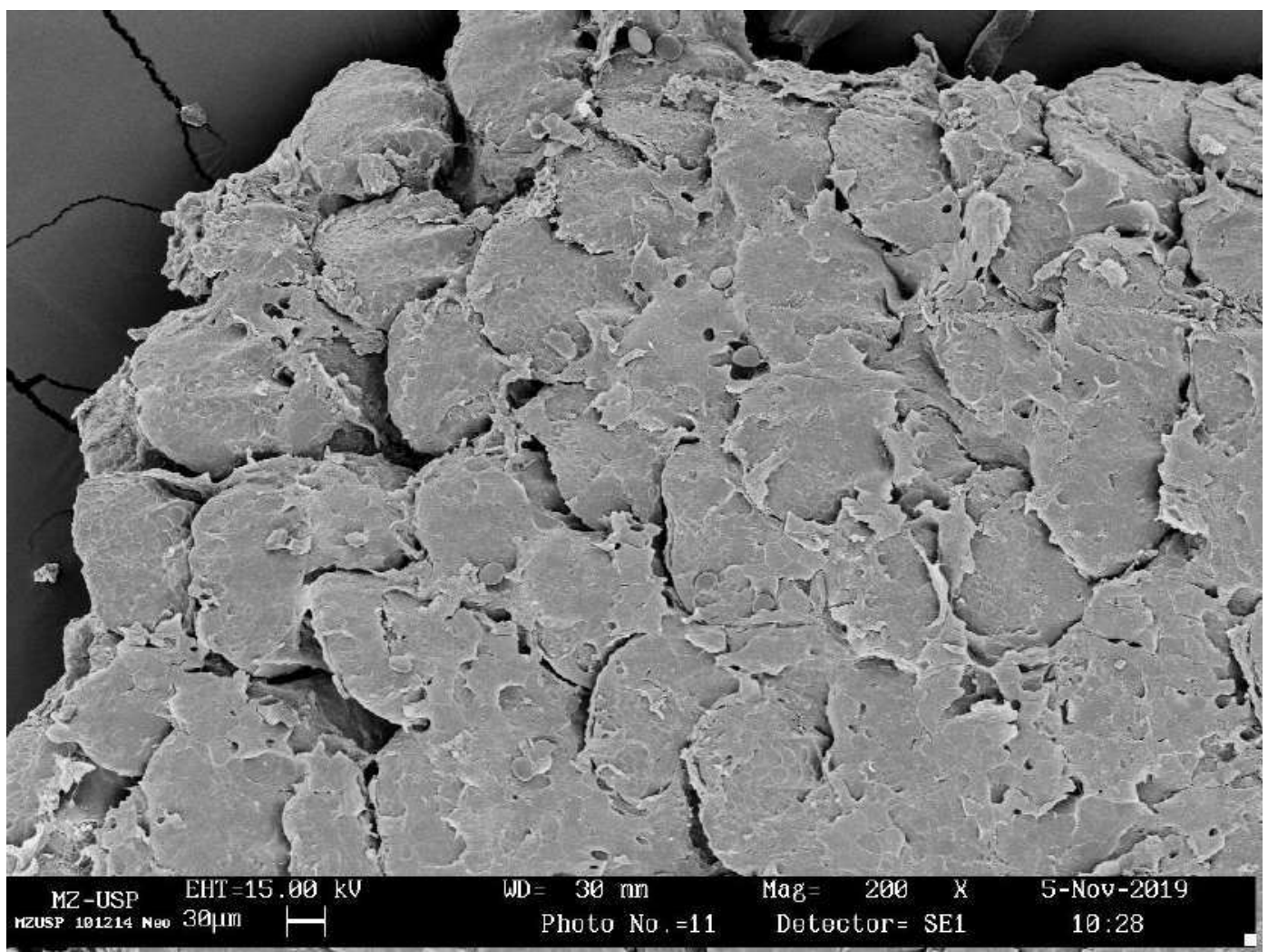

Figure 5. Dermal denticles collected from the section H1 (see fig. 1) of the neonate Isogomphodon oxyrhynchus (MZUSP 101214). Additional information in the image footer. 


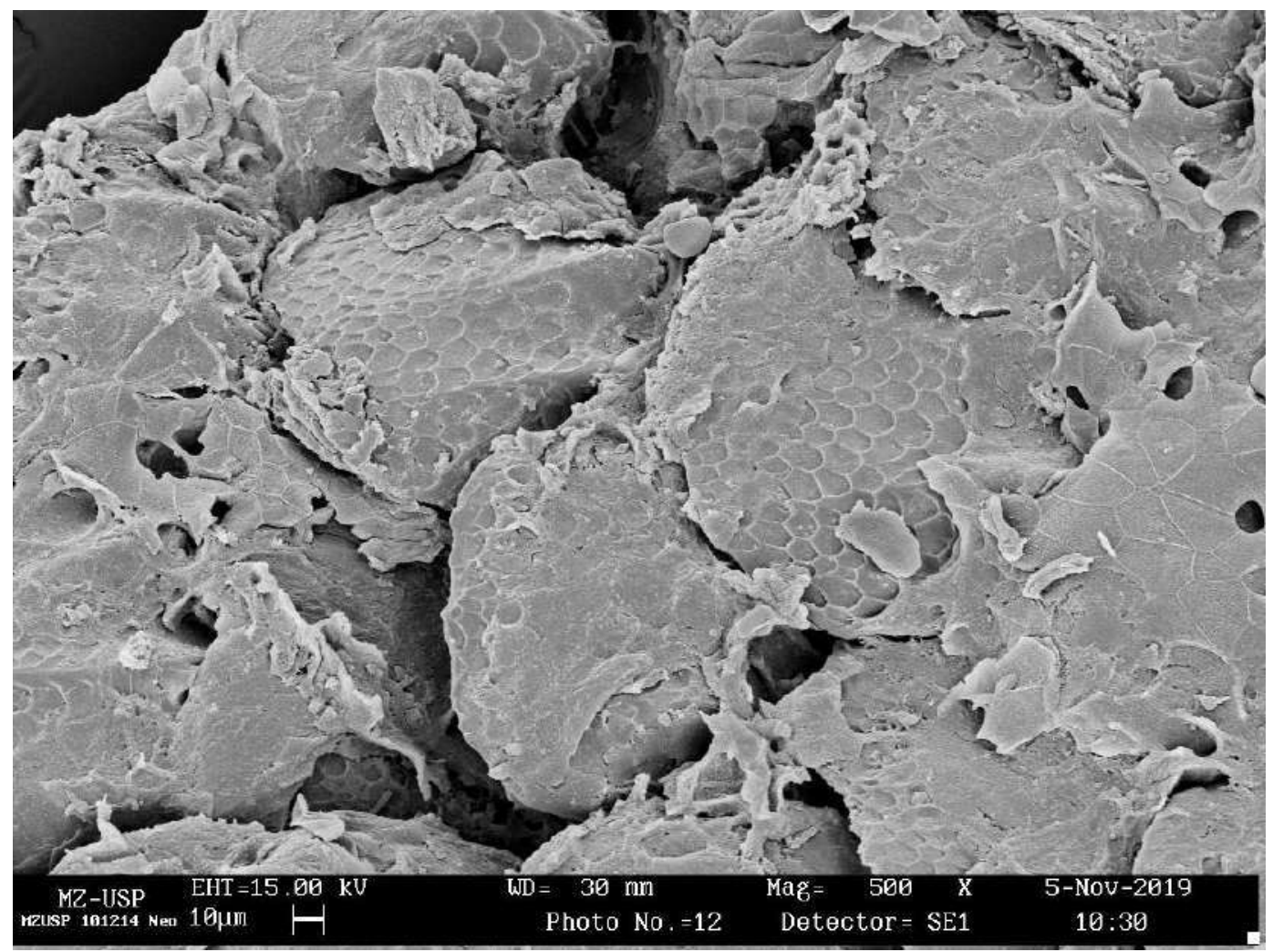

Figure 6. Dermal denticles collected from the section H1 (see fig. 1) of the neonate Isogomphodon oxyrhynchus (MZUSP 101214). Additional information in the image footer.

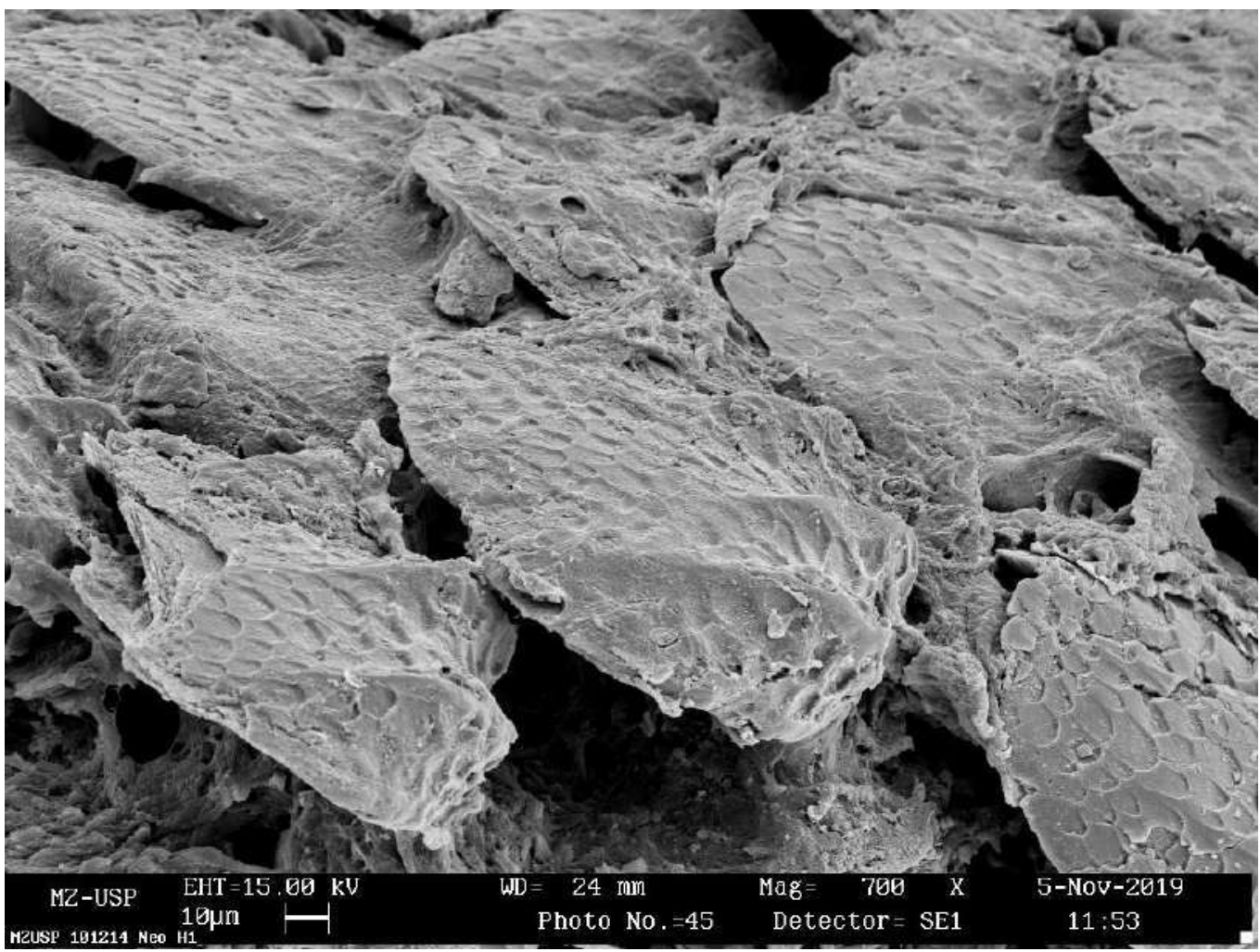

Figure 7. Dermal denticles collected from the section H1 (see fig. 1) of the neonate Isogomphodon oxyrhynchus (MZUSP 101214). Additional information in the image footer. 


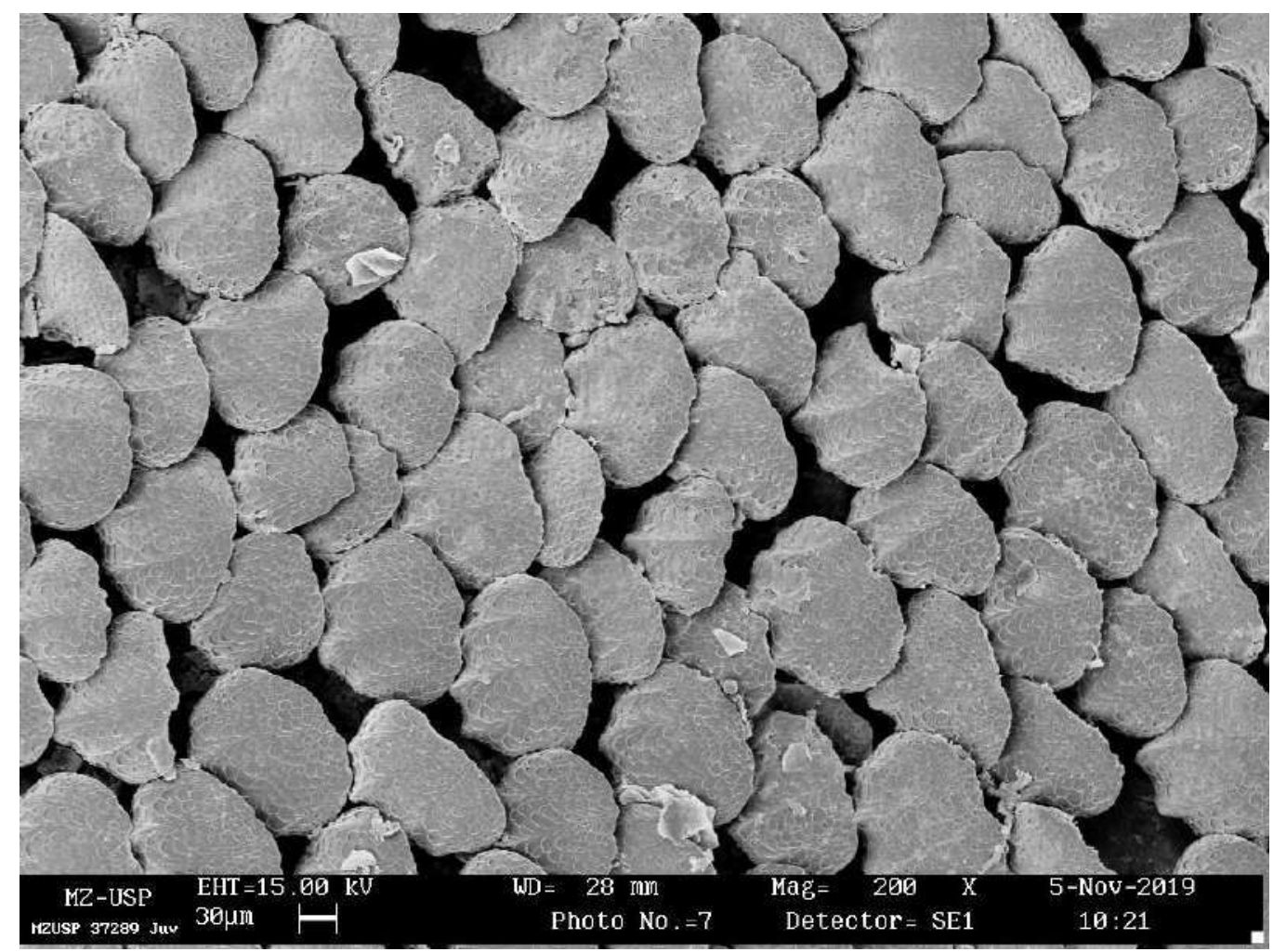

Figure 8. Dermal denticles collected from the section H1 (see fig. 1) of the juvenile Isogomphodon oxyrhynchus (MZUSP 37289). Additional information in the image footer.

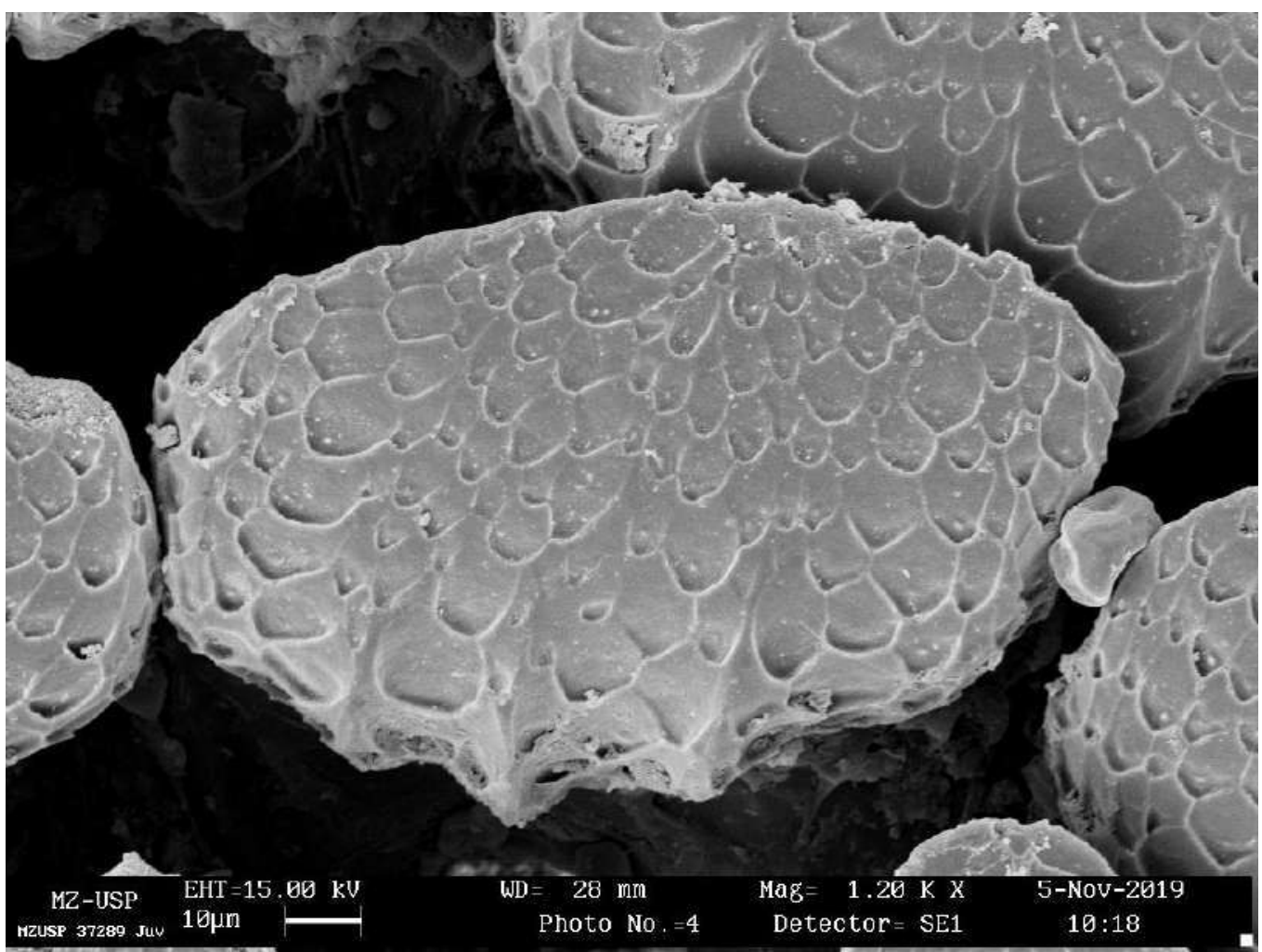

Figure 9. Dermal denticles collected from the section H1 (see fig. 1) of the juvenile Isogomphodon oxyrhynchus (MZUSP 37289). Additional information in the image footer. 


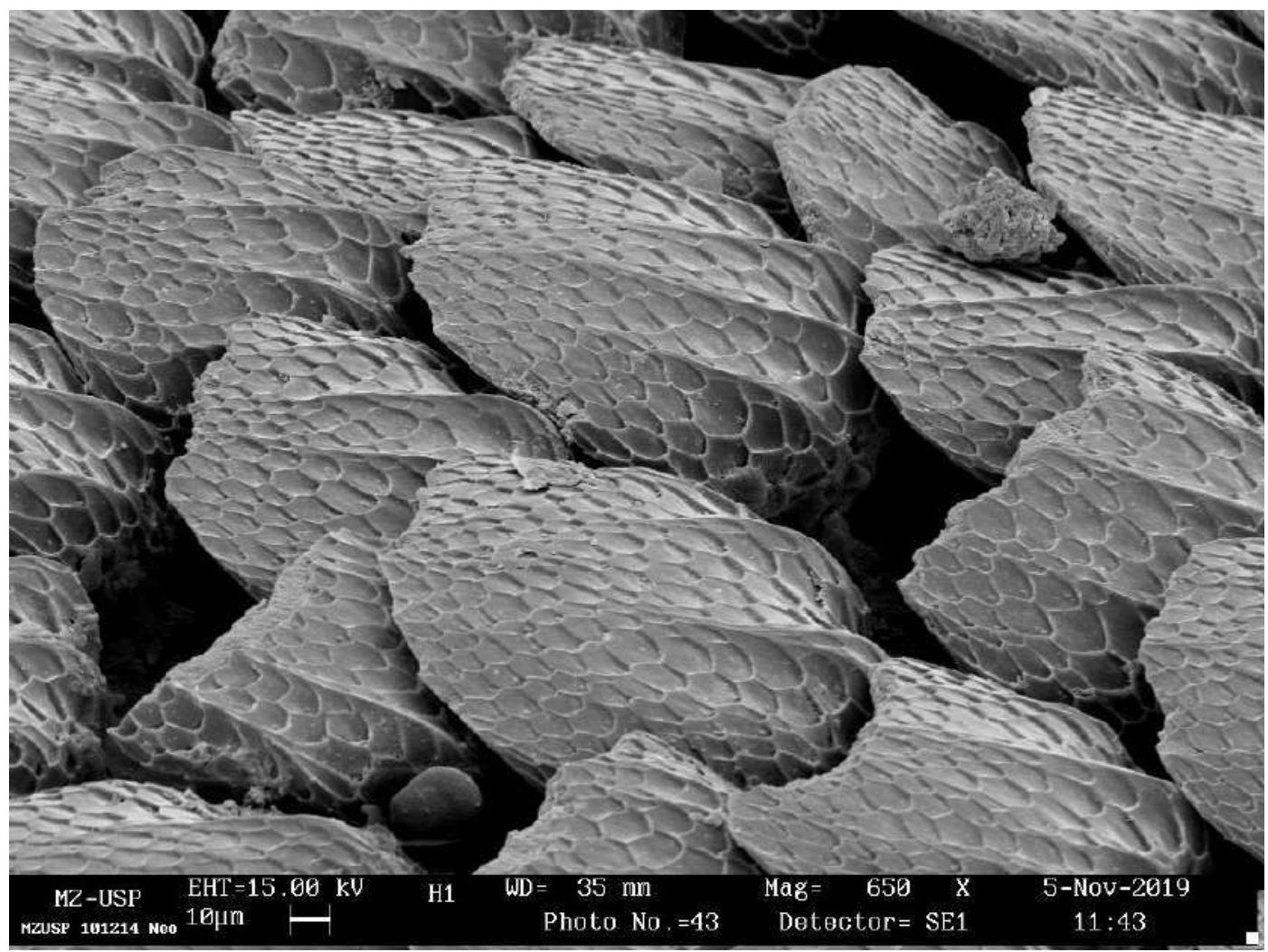

Figure 10. Dermal denticles collected from the section H1 (see fig. 1) of the juvenile Isogomphodon oxyrhynchus (MZUSP 37289). Additional information in the image footer.

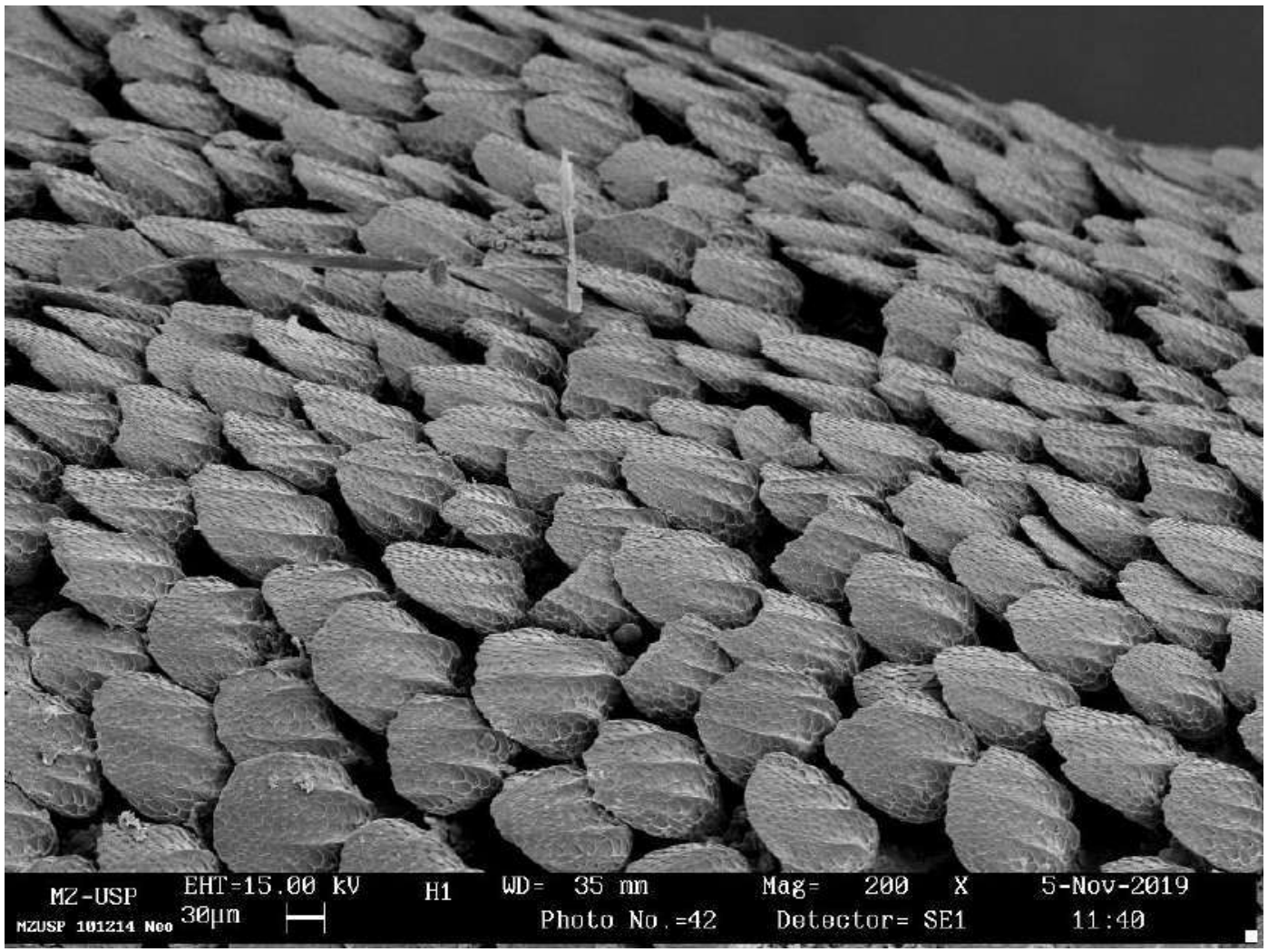

Figure 11. Dermal denticles collected from the section H1 (see fig. 1) of the juvenile Isogomphodon oxyrhynchus (MZUSP 37289). Additional information in the image footer. 


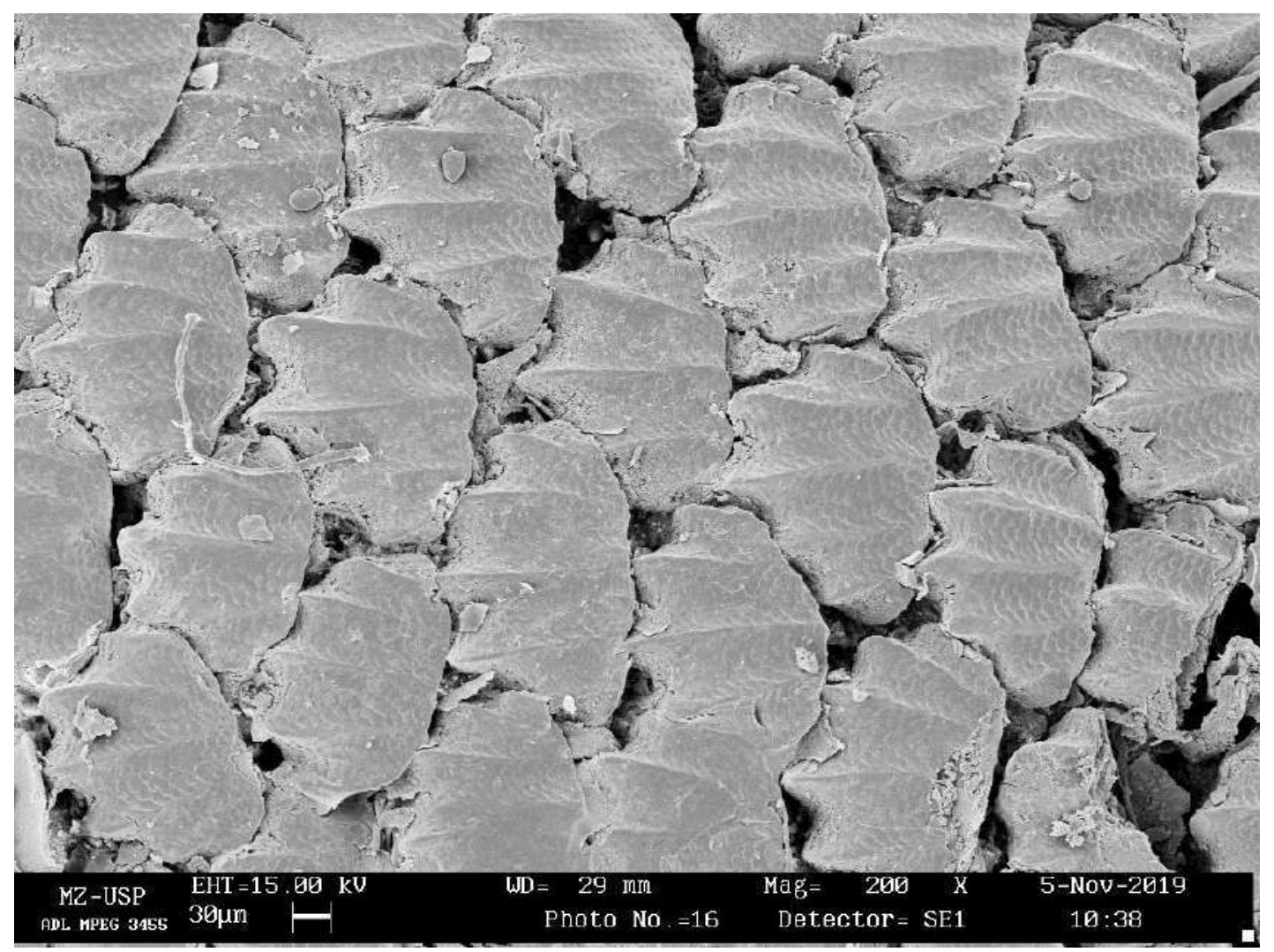

Figure 12. Dermal denticles collected from the section H1 (see fig. 1) of the adult Isogomphodon oxyrhynchus (MPEG 3455). Additional information in the image footer.

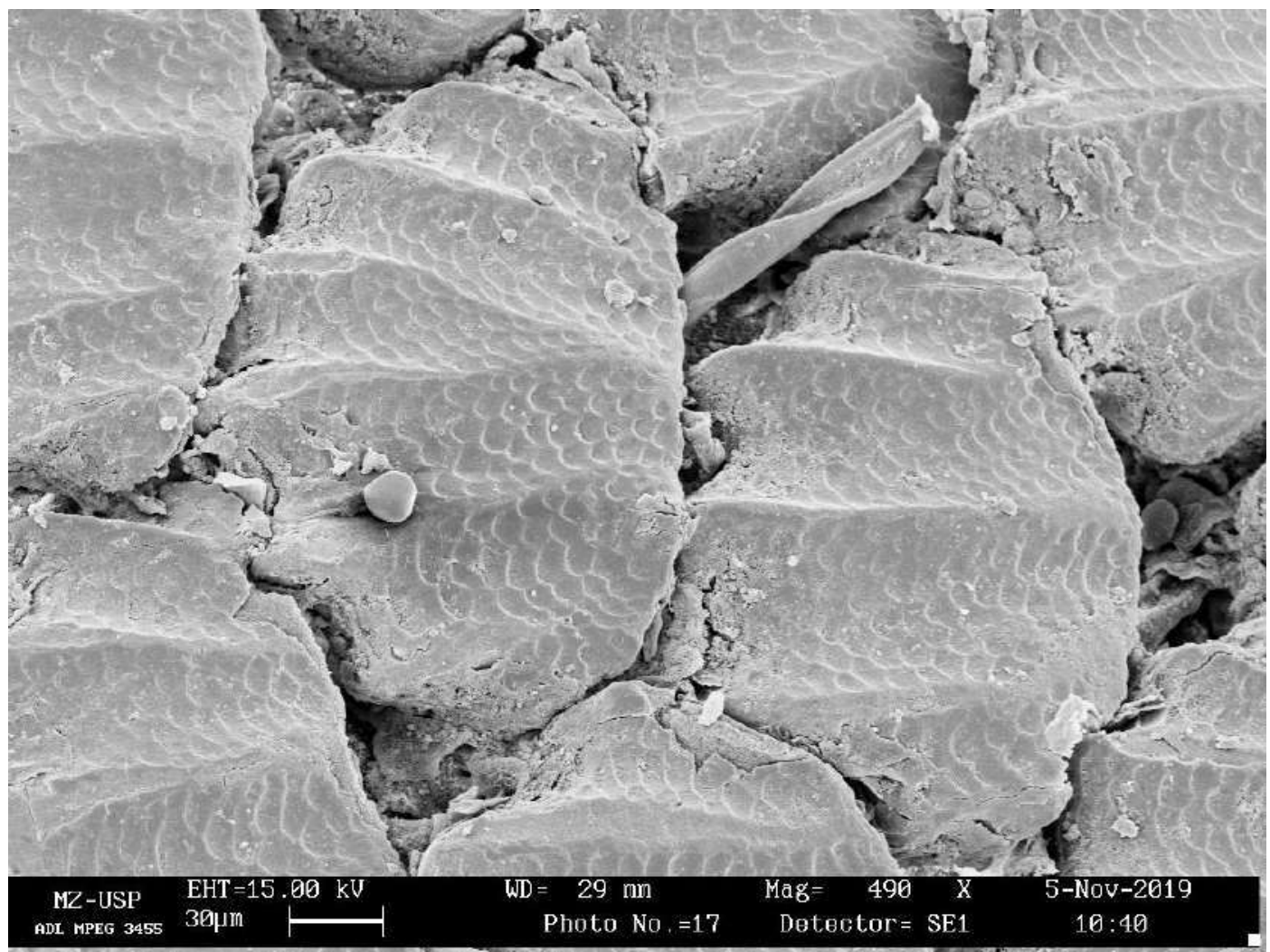

Figure 13. Dermal denticles collected from the section H1 (see fig. 1) of the adult Isogomphodon oxyrhynchus (MPEG 3455). Additional information in the image footer. 


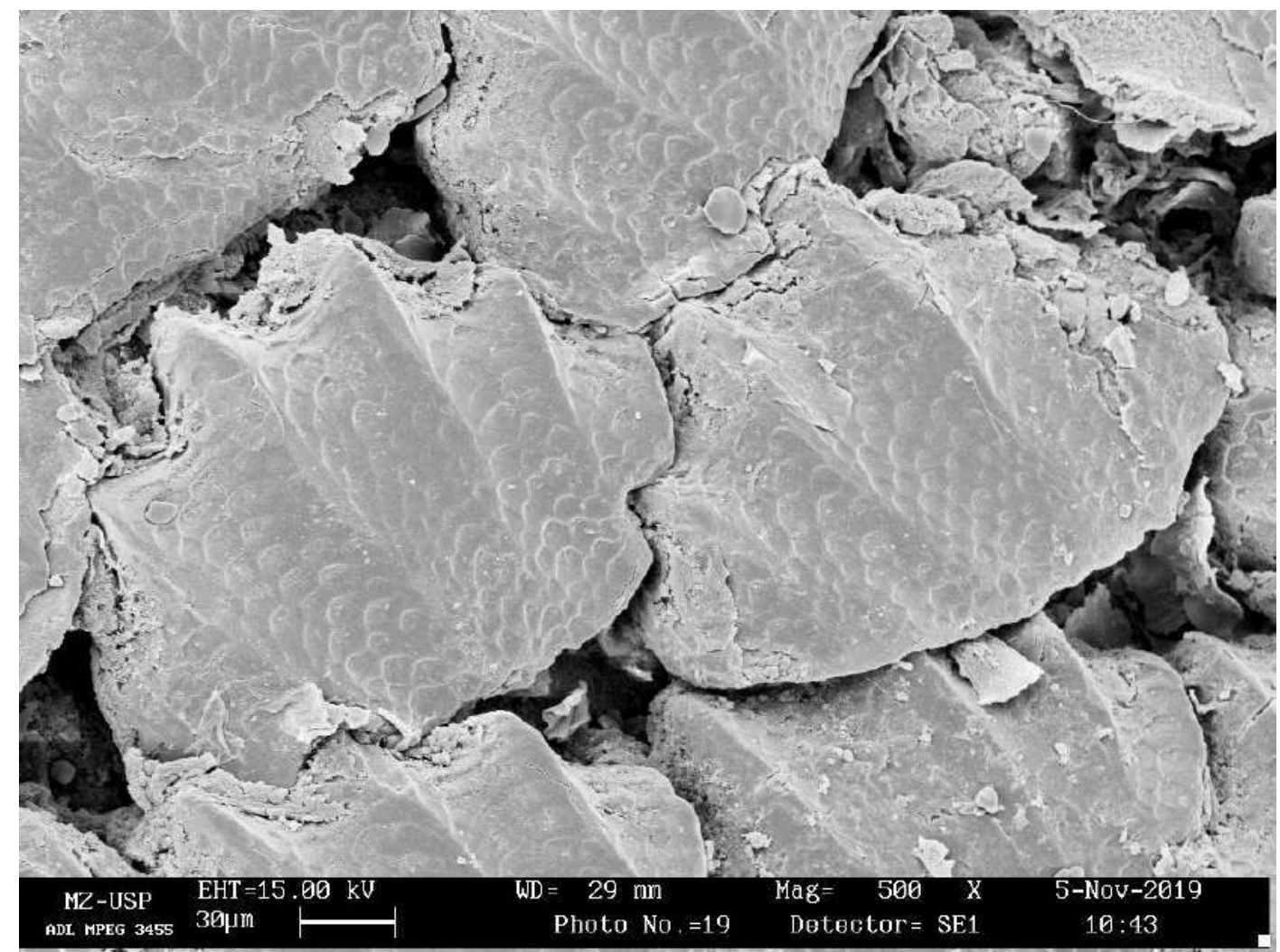

Figure 14. Dermal denticles collected from the section H1 (see fig. 1) of the adult Isogomphodon oxyrhynchus (MPEG 3455). Additional information in the image footer.

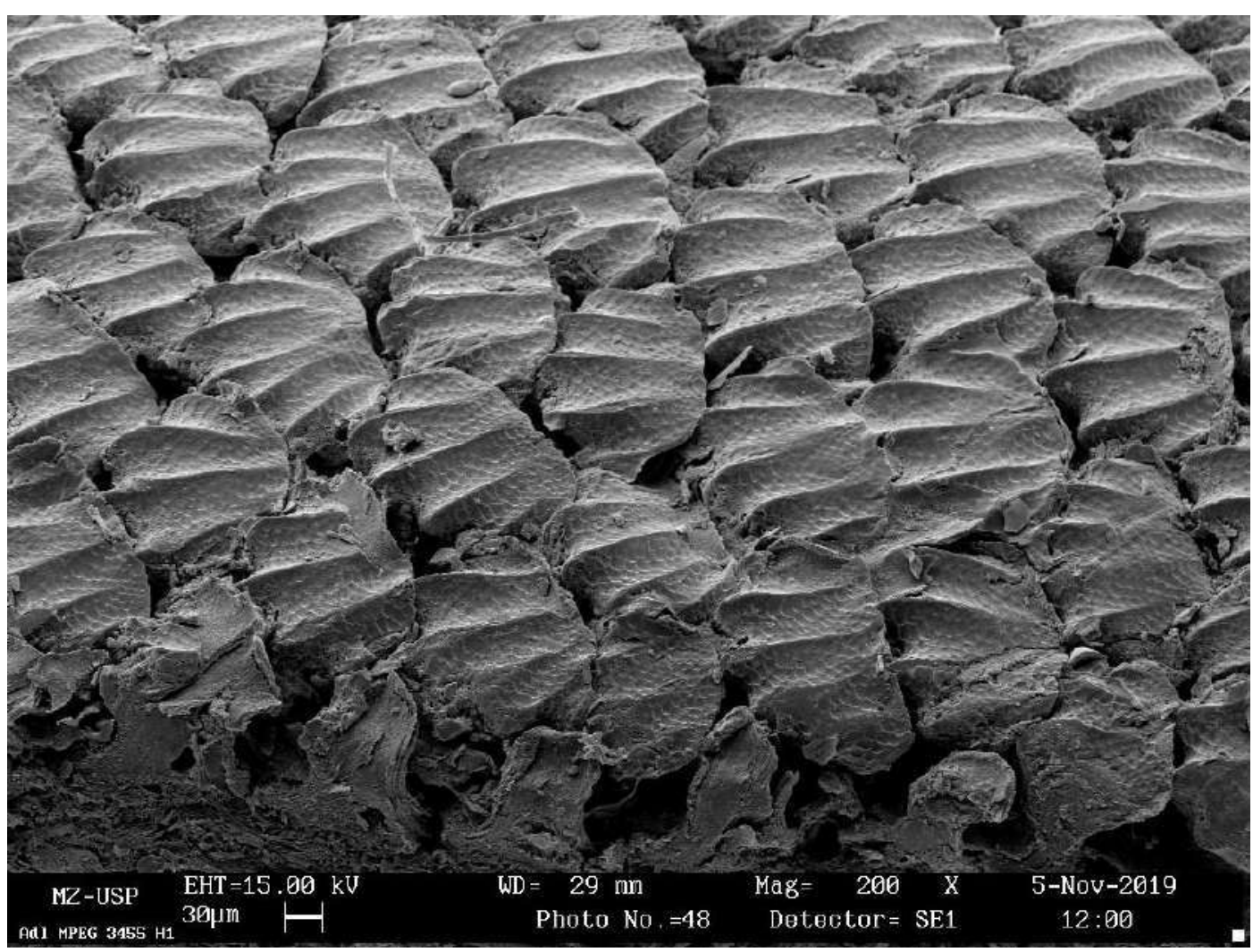

Figure 15. Dermal denticles collected from the section H1 (see fig. 1) of the adult Isogomphodon oxyrhynchus (MPEG 3455). Additional information in the image footer. 


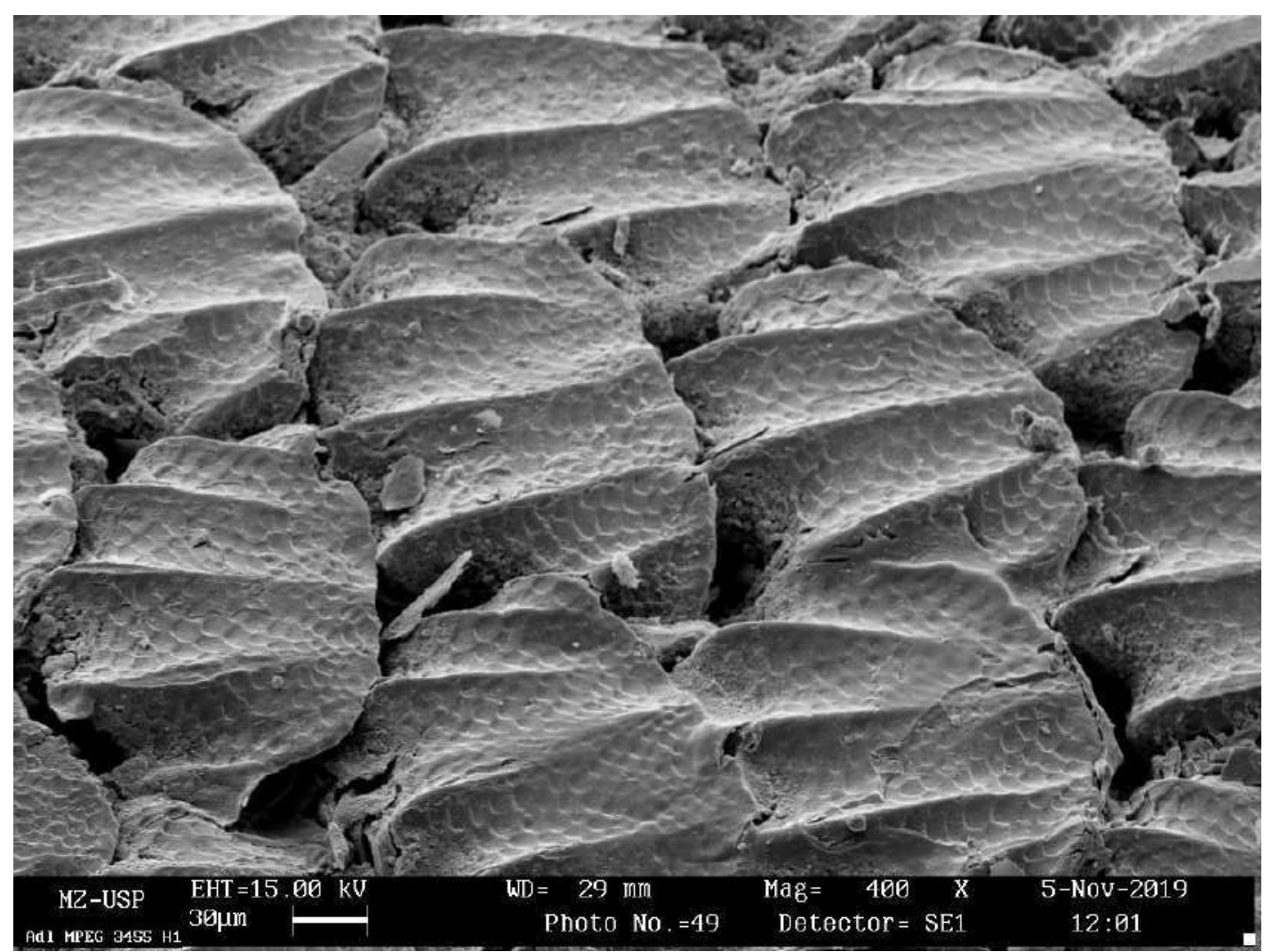

Figure 16. Dermal denticles collected from the section H1 (see fig. 1) of the adult Isogomphodon oxyrhynchus (MPEG 3455). Additional information in the image footer.

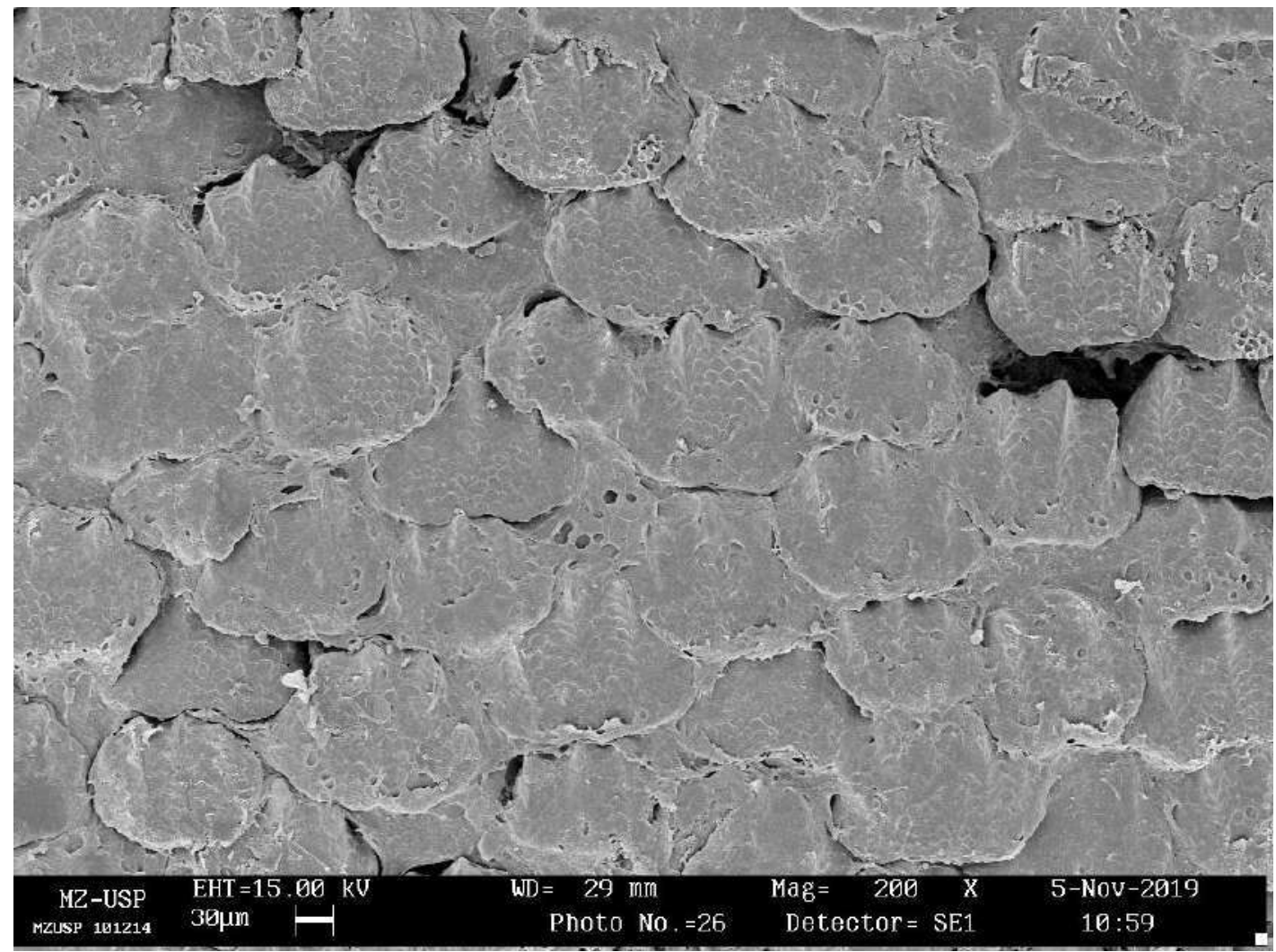

Figure 17. Dermal denticles collected from the section H2 (see fig. 1) of the neonate Isogomphodon oxyrhynchus (MZUSP 101214). Additional information in the image footer. 


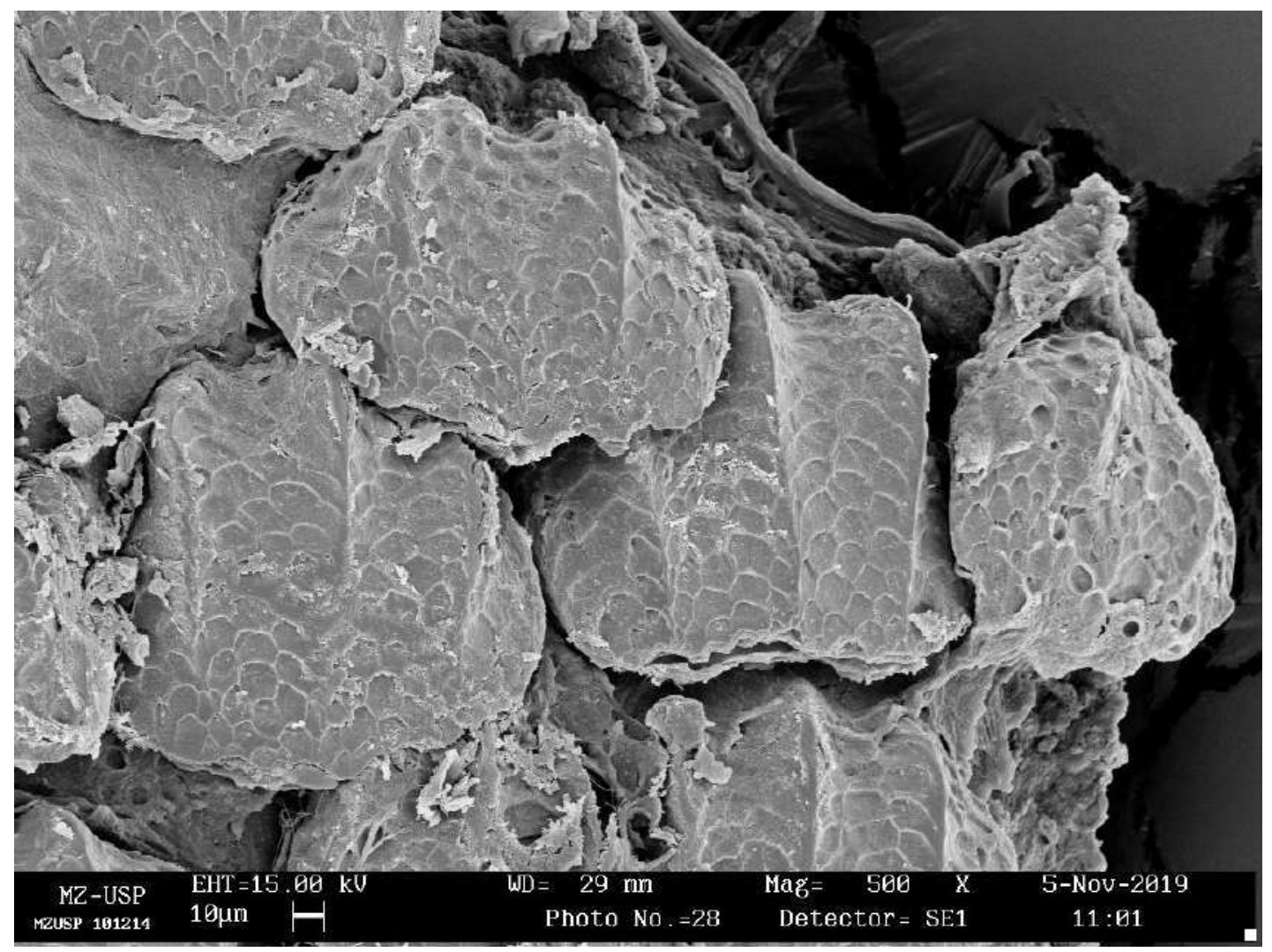

Figure 18. Dermal denticles collected from the section H2 (see fig. 1) of the neonate Isogomphodon oxyrhynchus (MZUSP 101214). Additional information in the image footer.

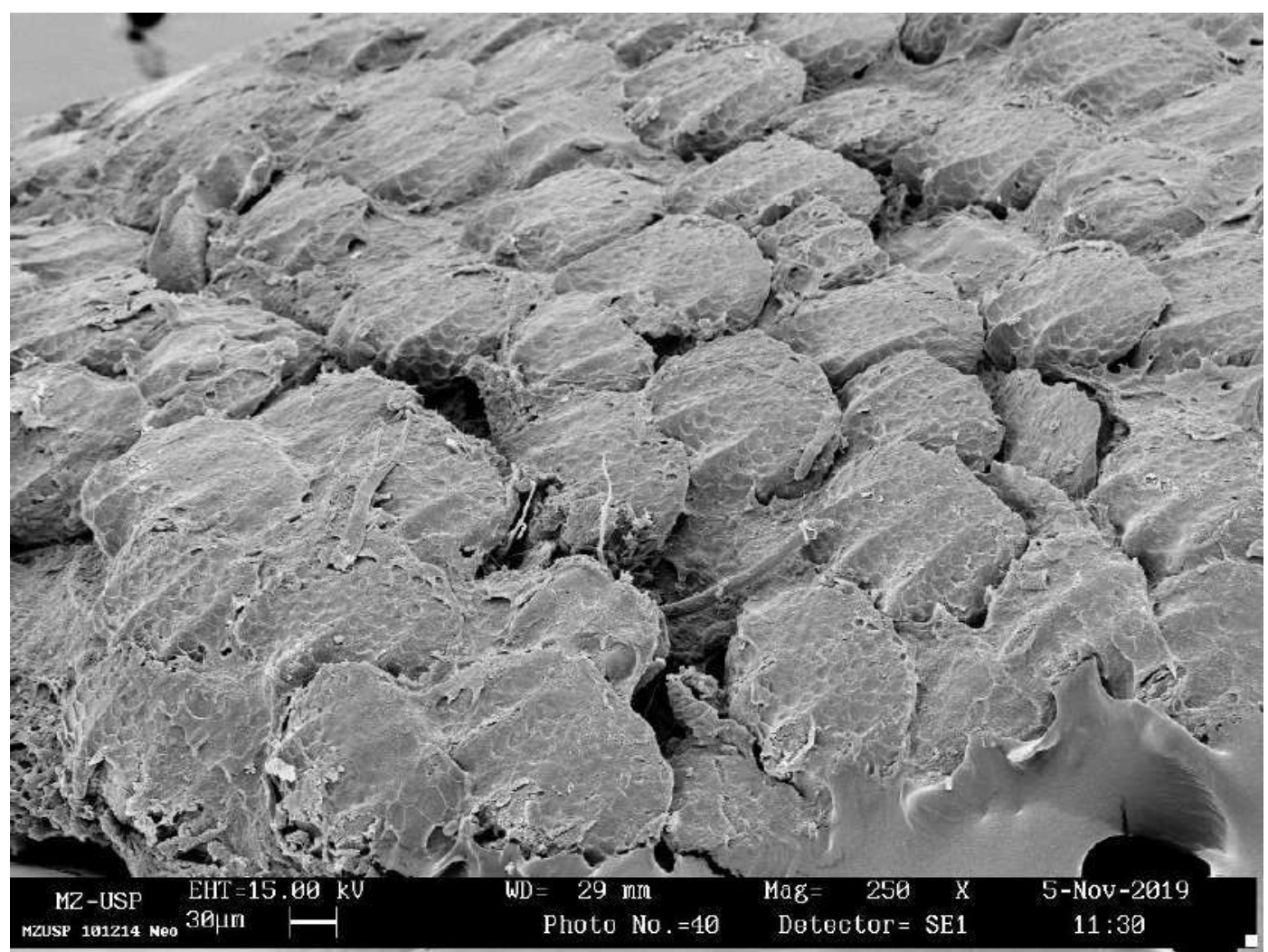

Figure 19. Dermal denticles collected from the section H2 (see fig. 1) of the neonate Isogomphodon oxyrhynchus (MZUSP 101214). Additional information in the image footer. 


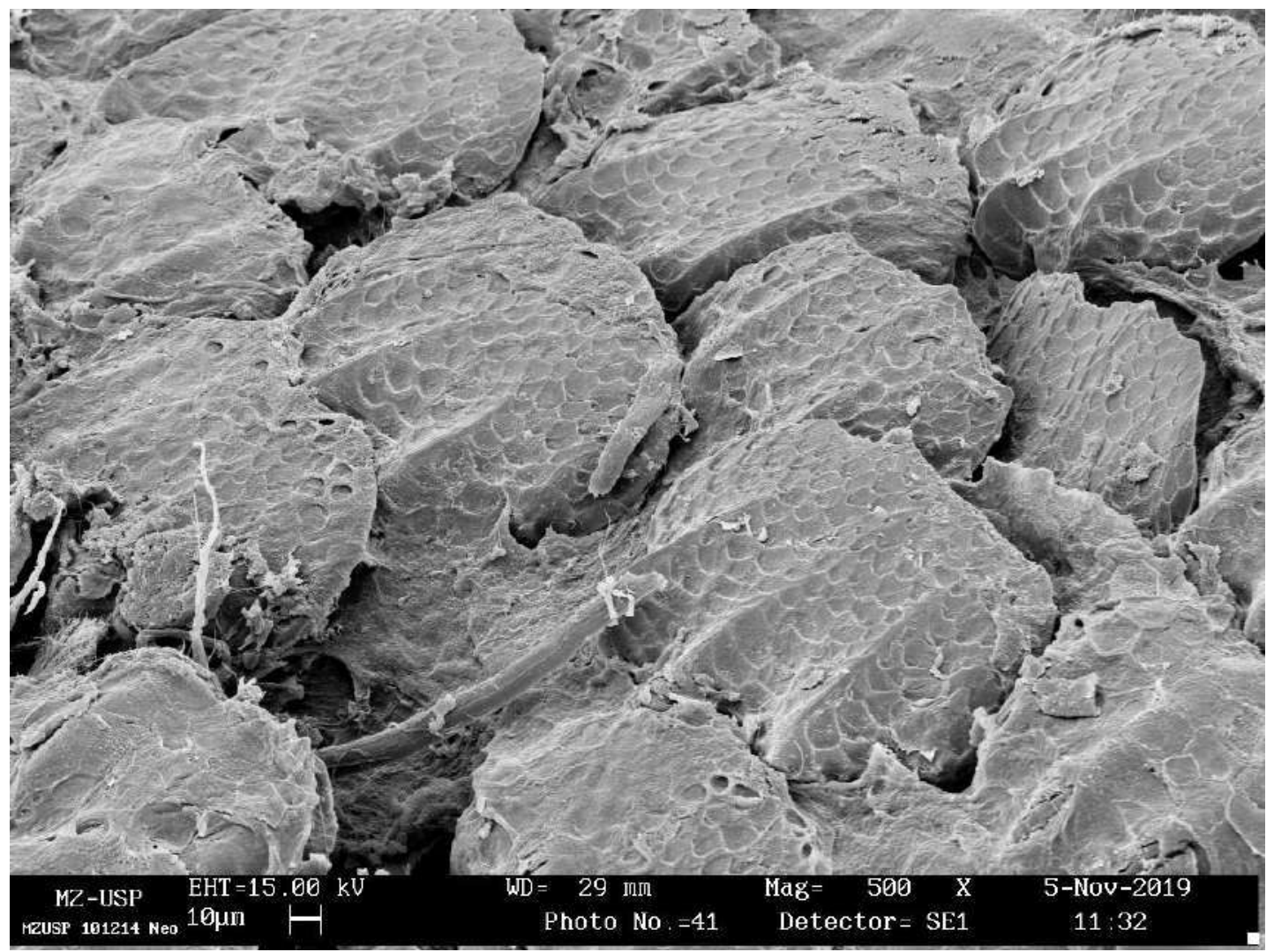

Figure 20. Dermal denticles collected from the section H2 (see fig. 1) of the neonate Isogomphodon oxyrhynchus (MZUSP 101214). Additional information in the image footer.

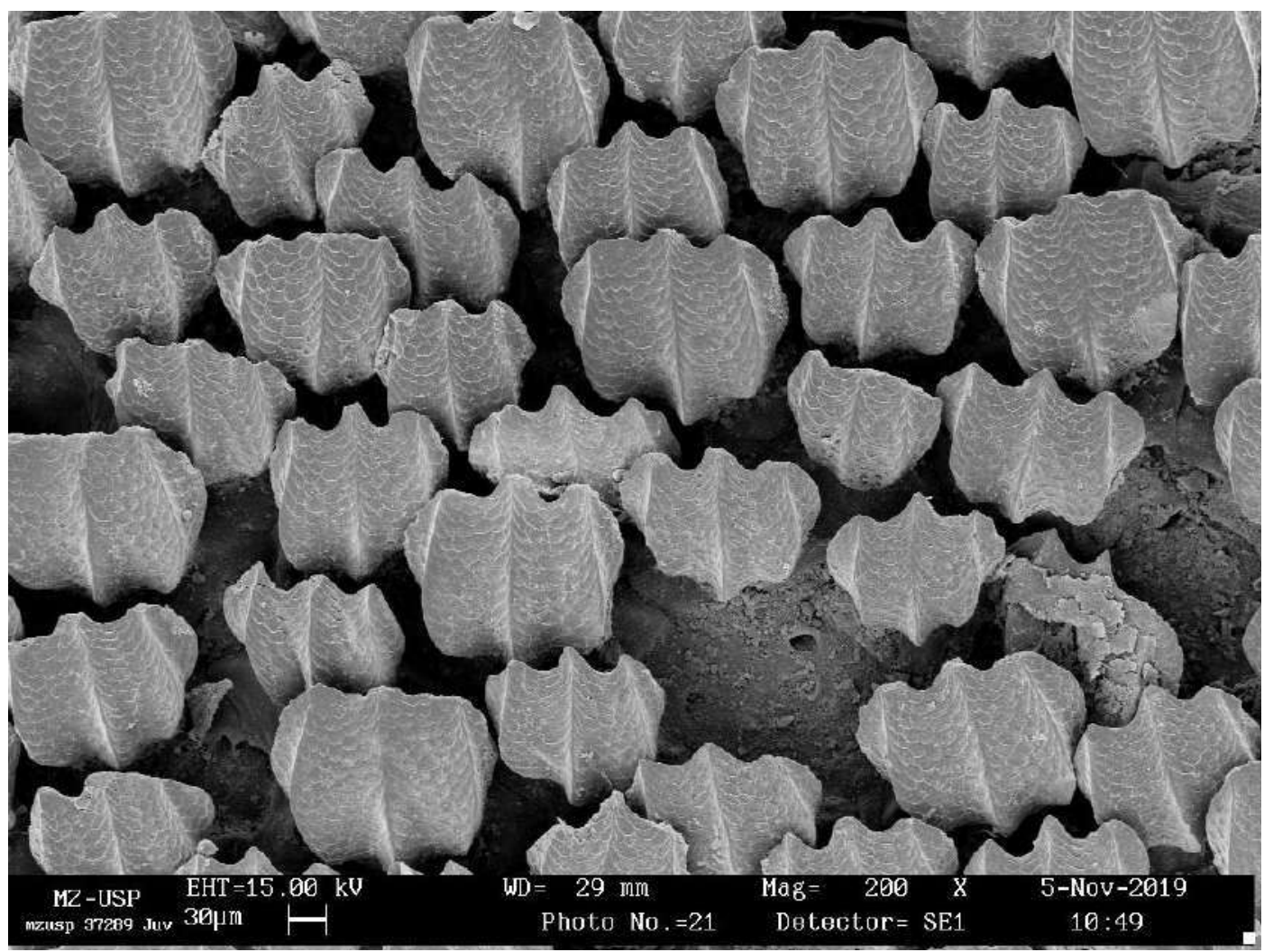

Figure 21. Dermal denticles collected from the section H2 (see fig. 1) of the juvenile Isogomphodon oxyrhynchus (MZUSP 37289). Additional information in the image footer. 


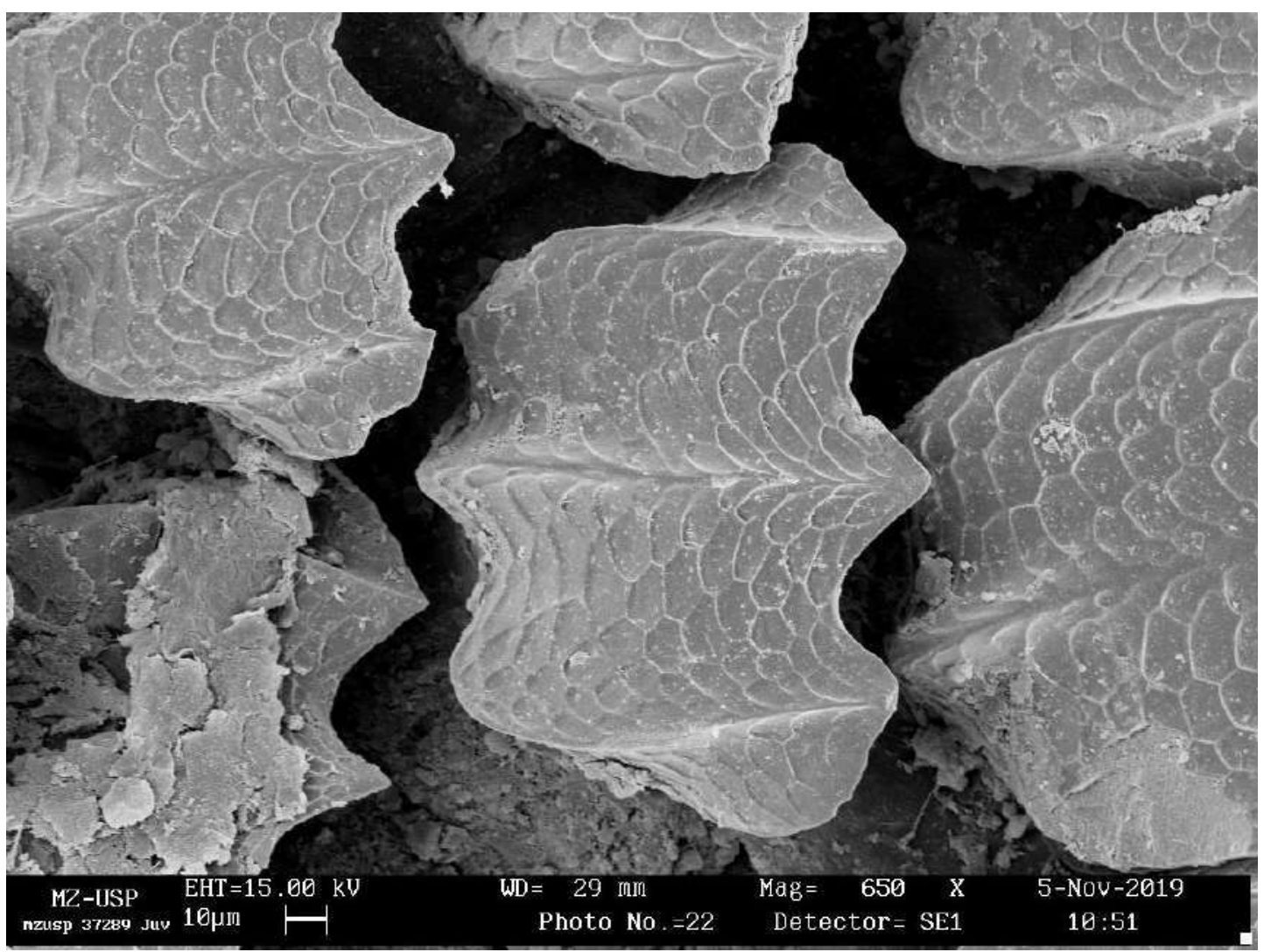

Figure 22. Dermal denticles collected from the section H2 (see fig. 1) of the juvenile Isogomphodon oxyrhynchus (MZUSP 37289). Additional information in the image footer.

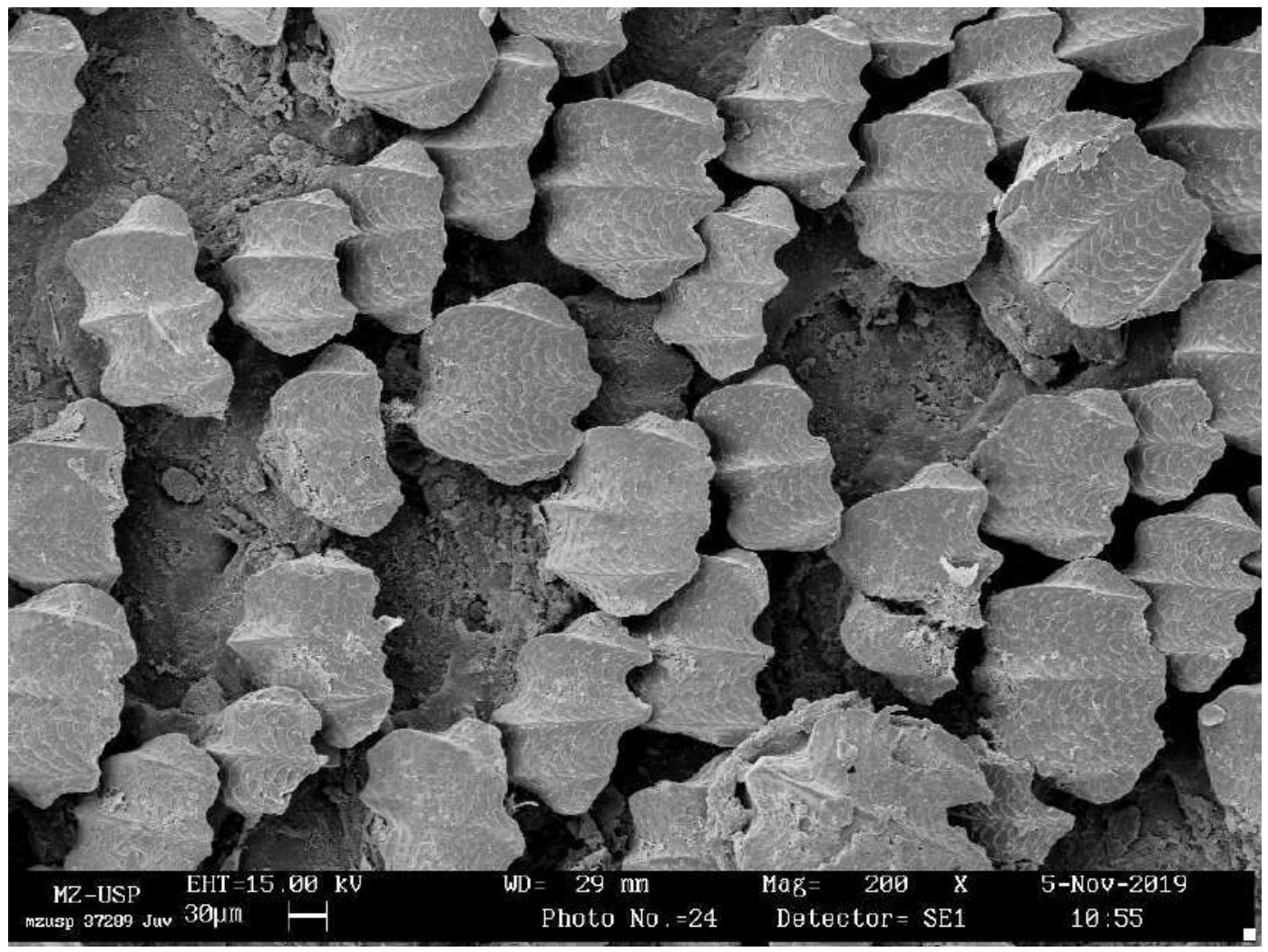

Figure 23. Dermal denticles collected from the section H2 (see fig. 1) of the juvenile Isogomphodon oxyrhynchus (MZUSP 37289). Additional information in the image footer. 


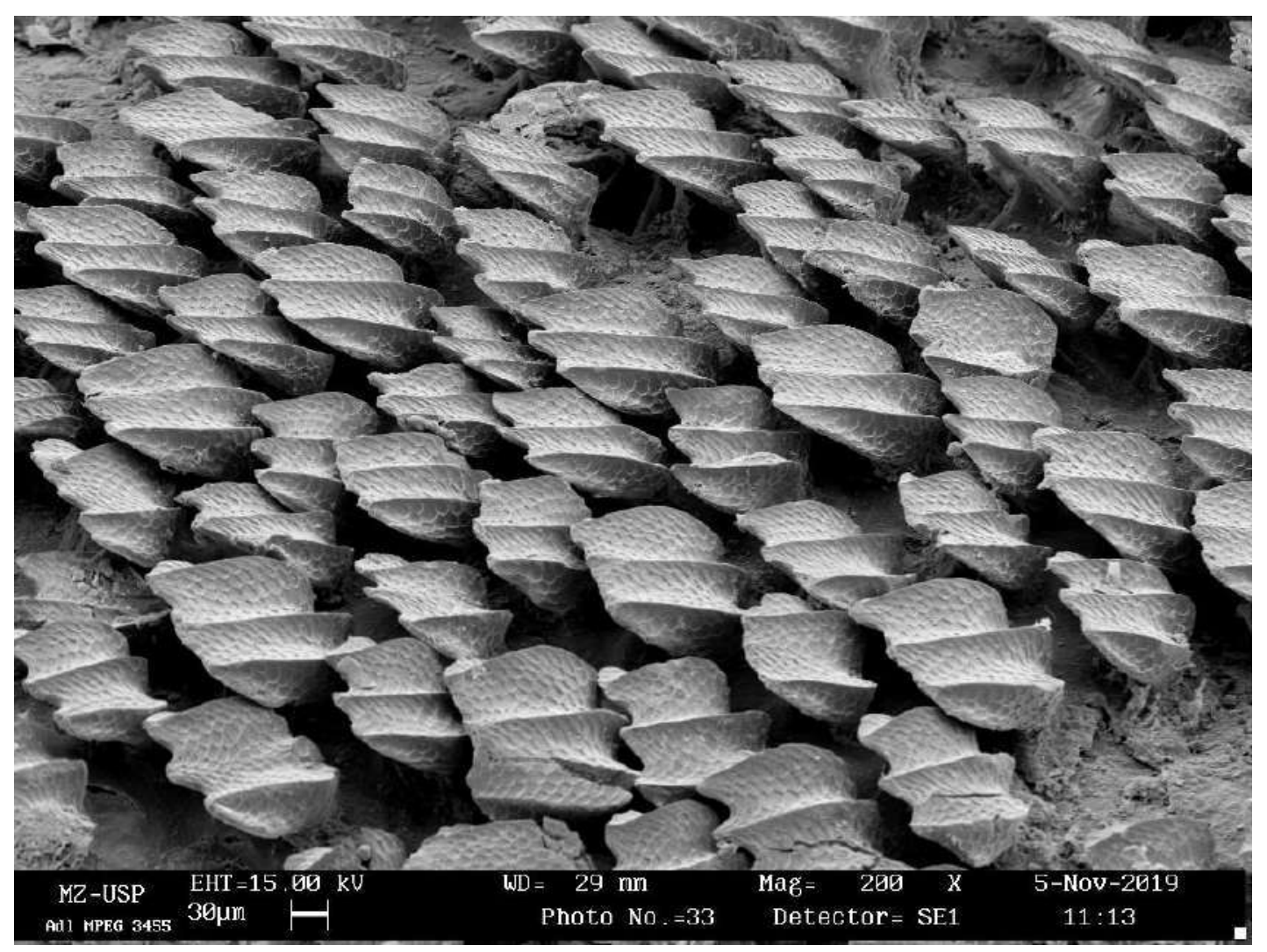

Figure 24. Dermal denticles collected from the section H2 (see fig. 1) of the juvenile Isogomphodon oxyrhynchus (MZUSP 37289). Additional information in the image footer.

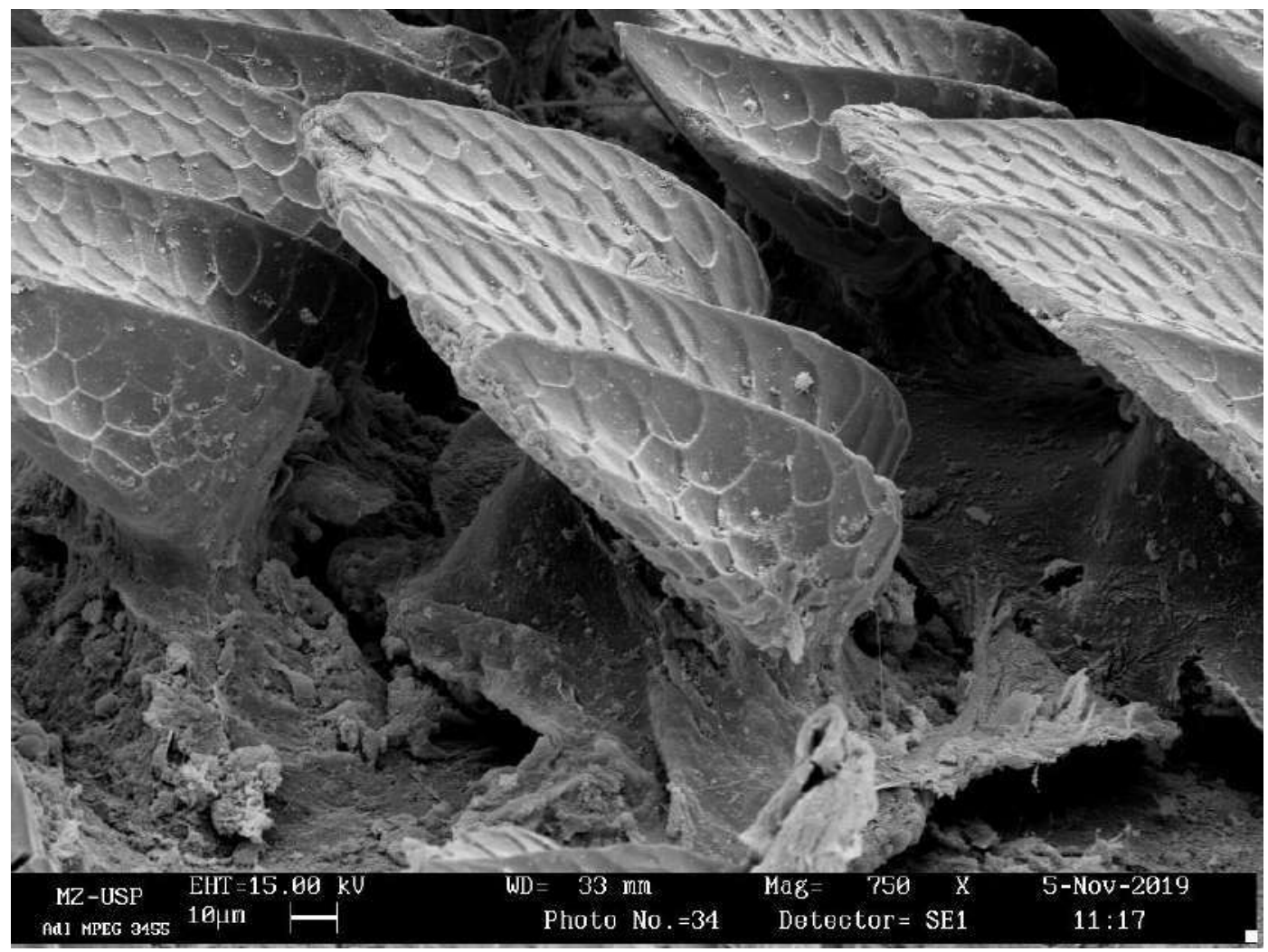

Figure 25. Dermal denticles collected from the section H2 (see fig. 1) of the juvenile Isogomphodon oxyrhynchus (MZUSP 37289). Additional information in the image footer. 


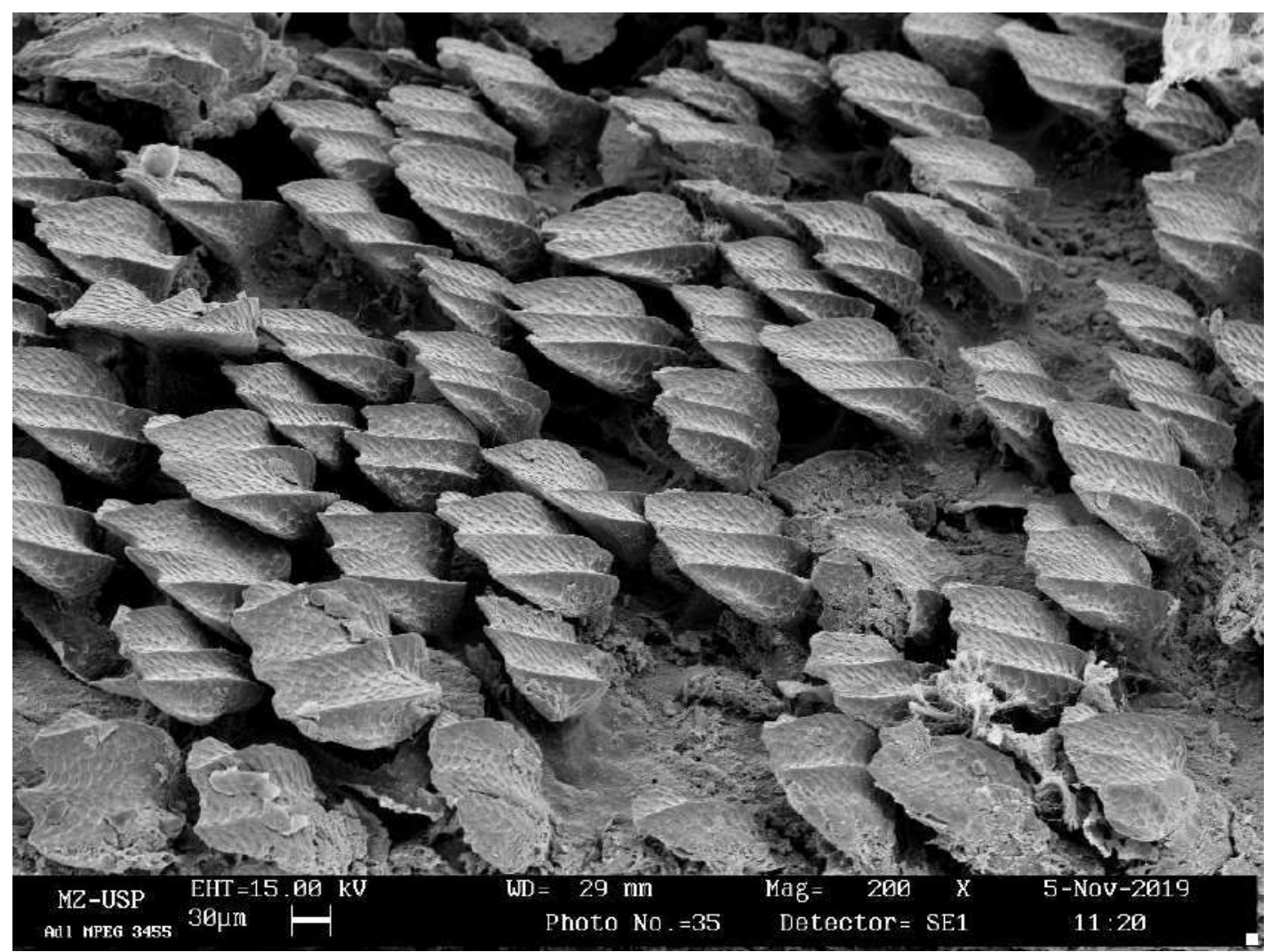

Figure 26. Dermal denticles collected from the section H2 (see fig. 1) of the juvenile Isogomphodon oxyrhynchus (MZUSP 37289). Additional information in the image footer.

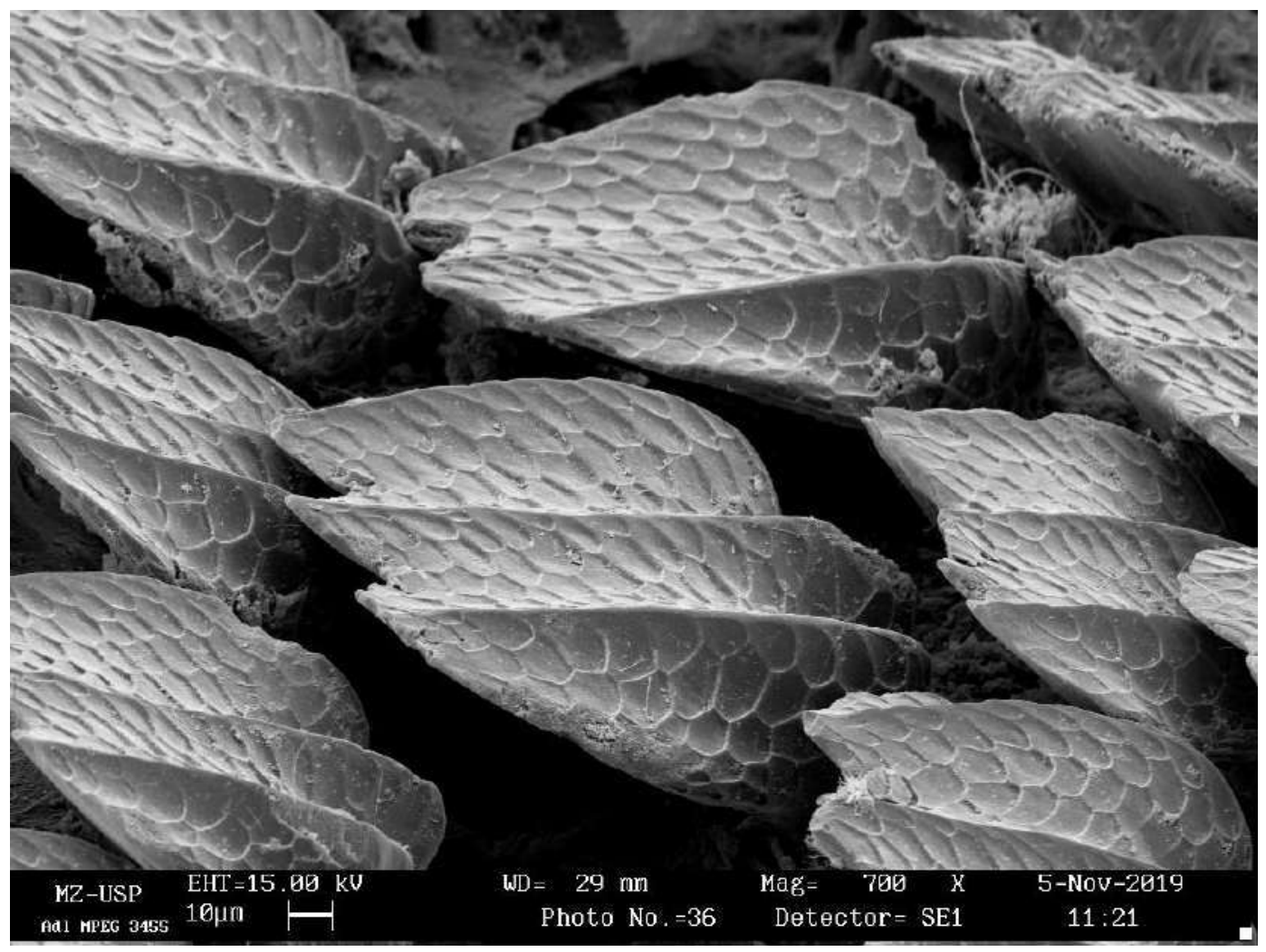

Figure 27. Dermal denticles collected from the section H2 (see fig. 1) of the juvenile Isogomphodon oxyrhynchus (MZUSP 37289). Additional information in the image footer. 


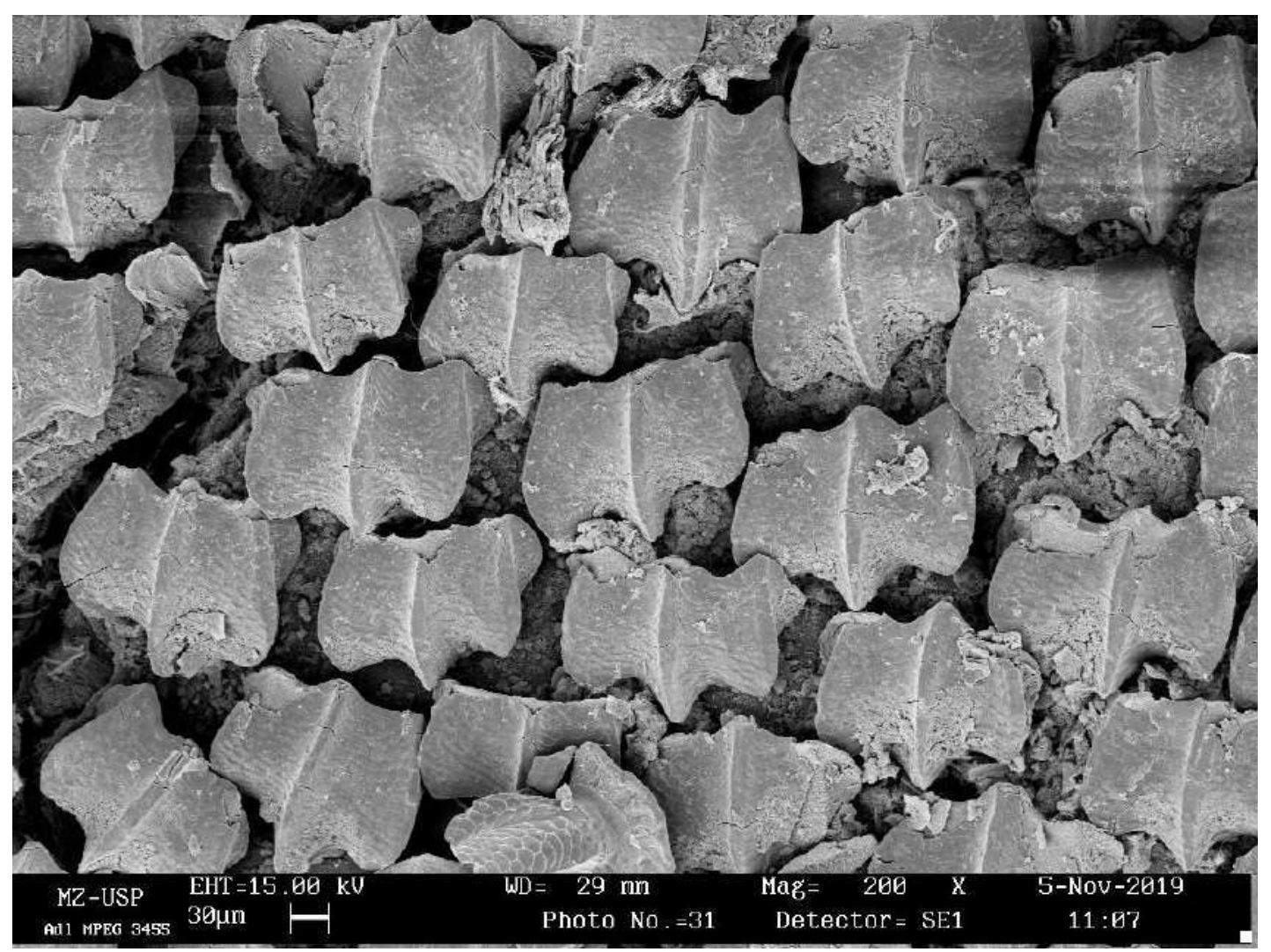

Figure 28. Dermal denticles collected from the section H2 (see fig. 1) of the adult Isogomphodon oxyrhynchus (MPEG 3455). Additional information in the image footer.

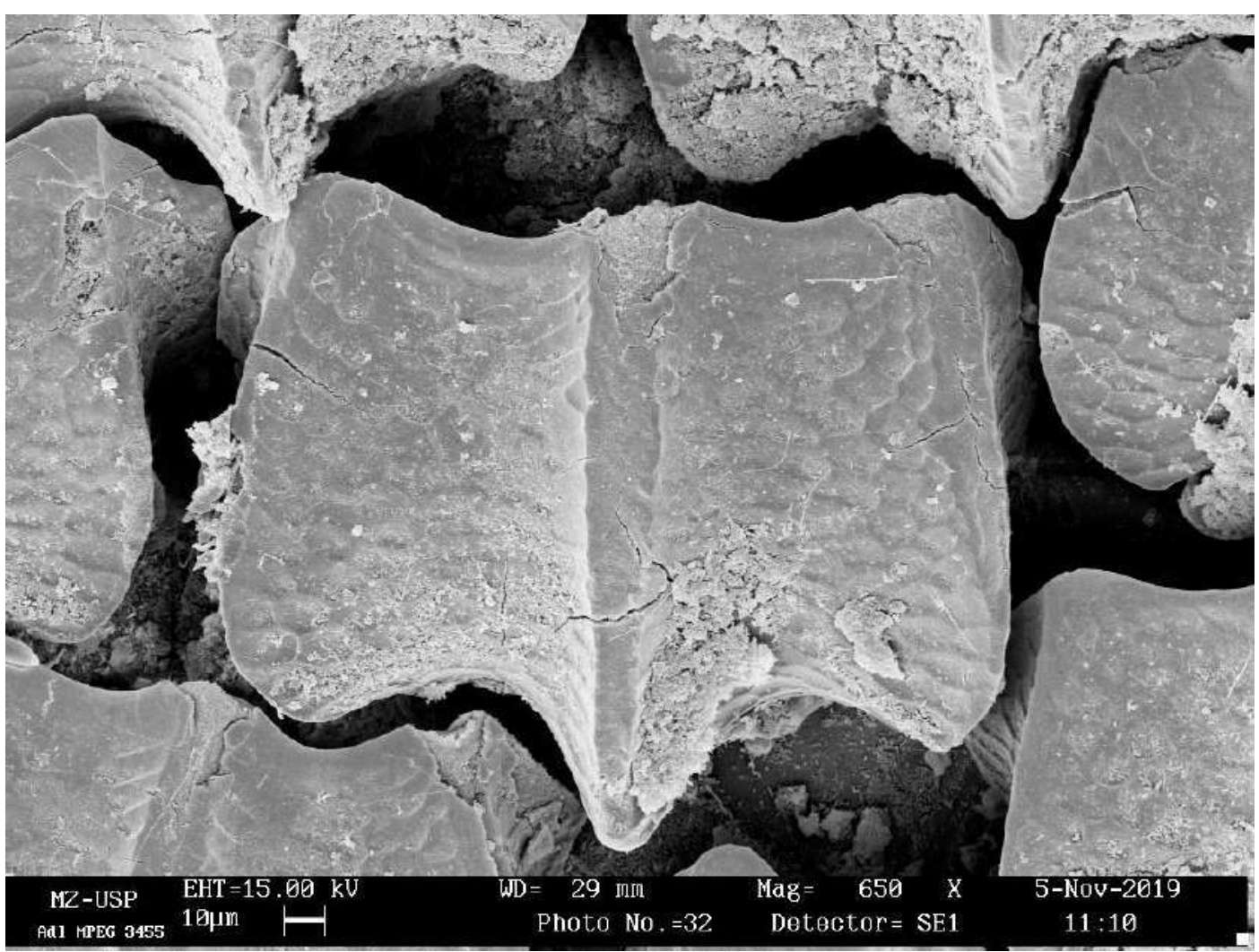

Figure 29. Dermal denticles collected from the section H2 (see fig. 1) of the adult Isogomphodon oxyrhynchus (MPEG 3455). Additional information in the image footer. 


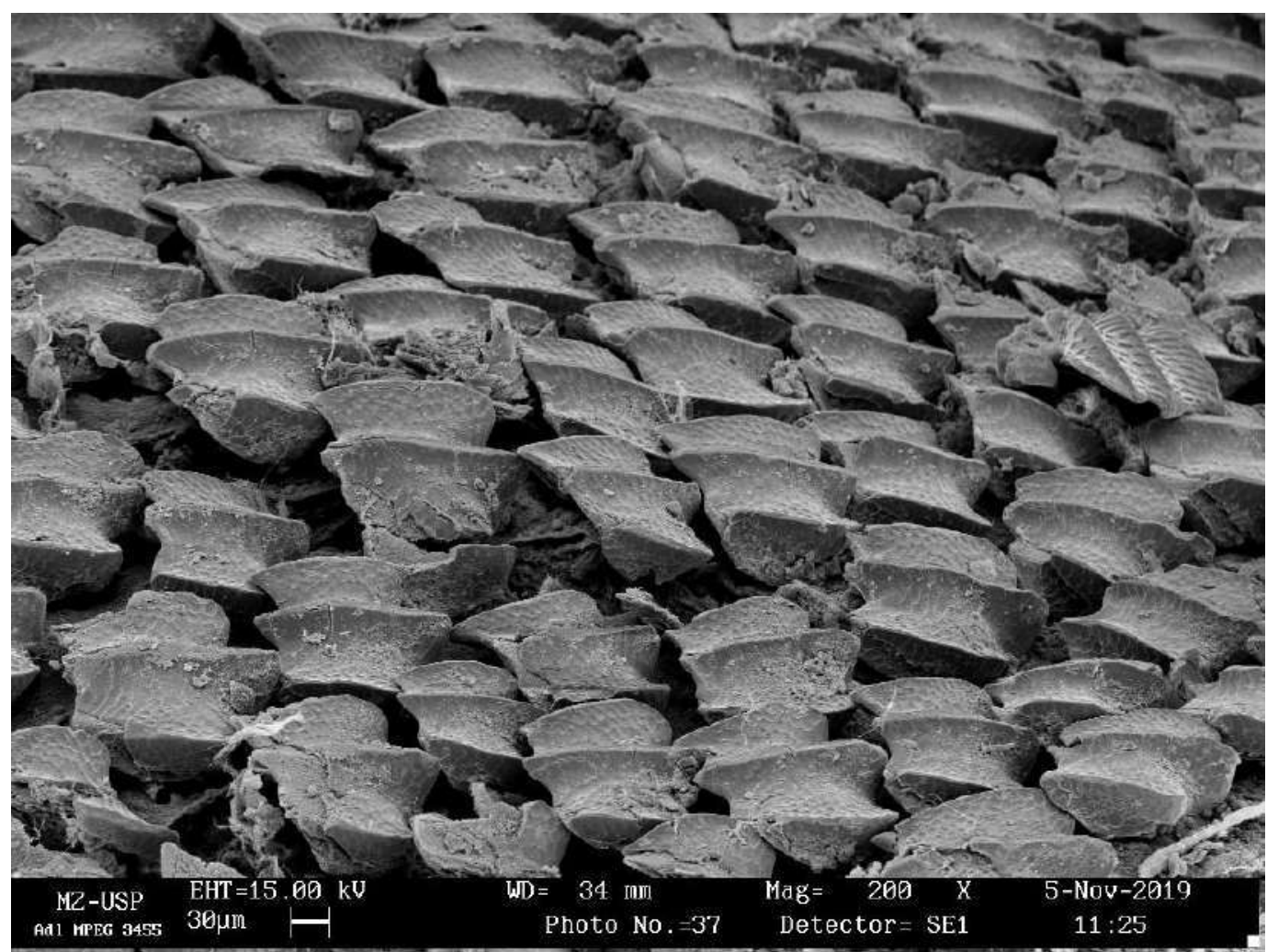

Figure 30. Dermal denticles collected from the section H2 (see fig. 1) of the adult Isogomphodon oxyrhynchus (MPEG 3455). Additional information in the image footer.

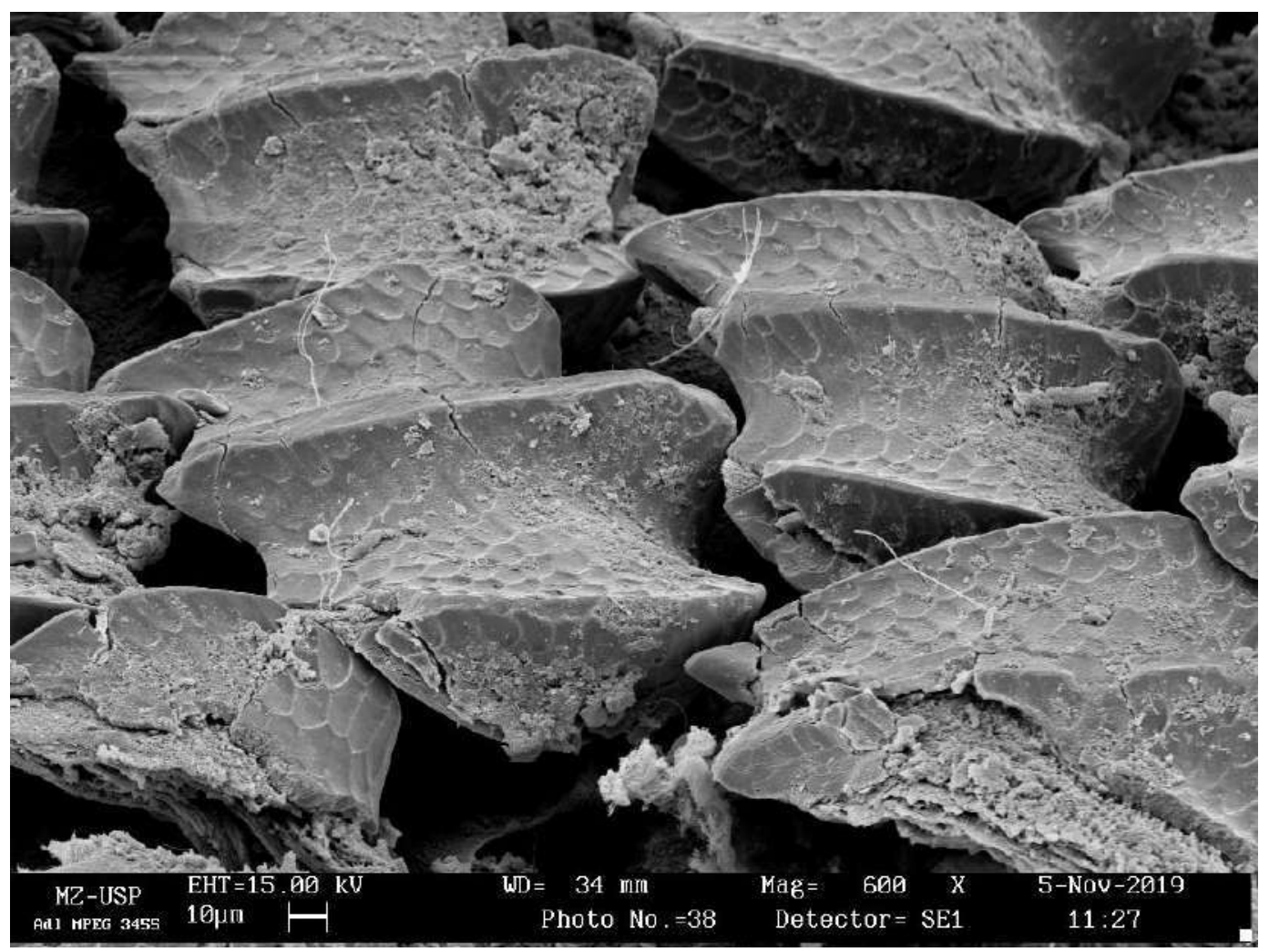

Figure 31. Dermal denticles collected from the section H2 (see fig. 1) of the adult Isogomphodon oxyrhynchus (MPEG 3455). Additional information in the image footer. 


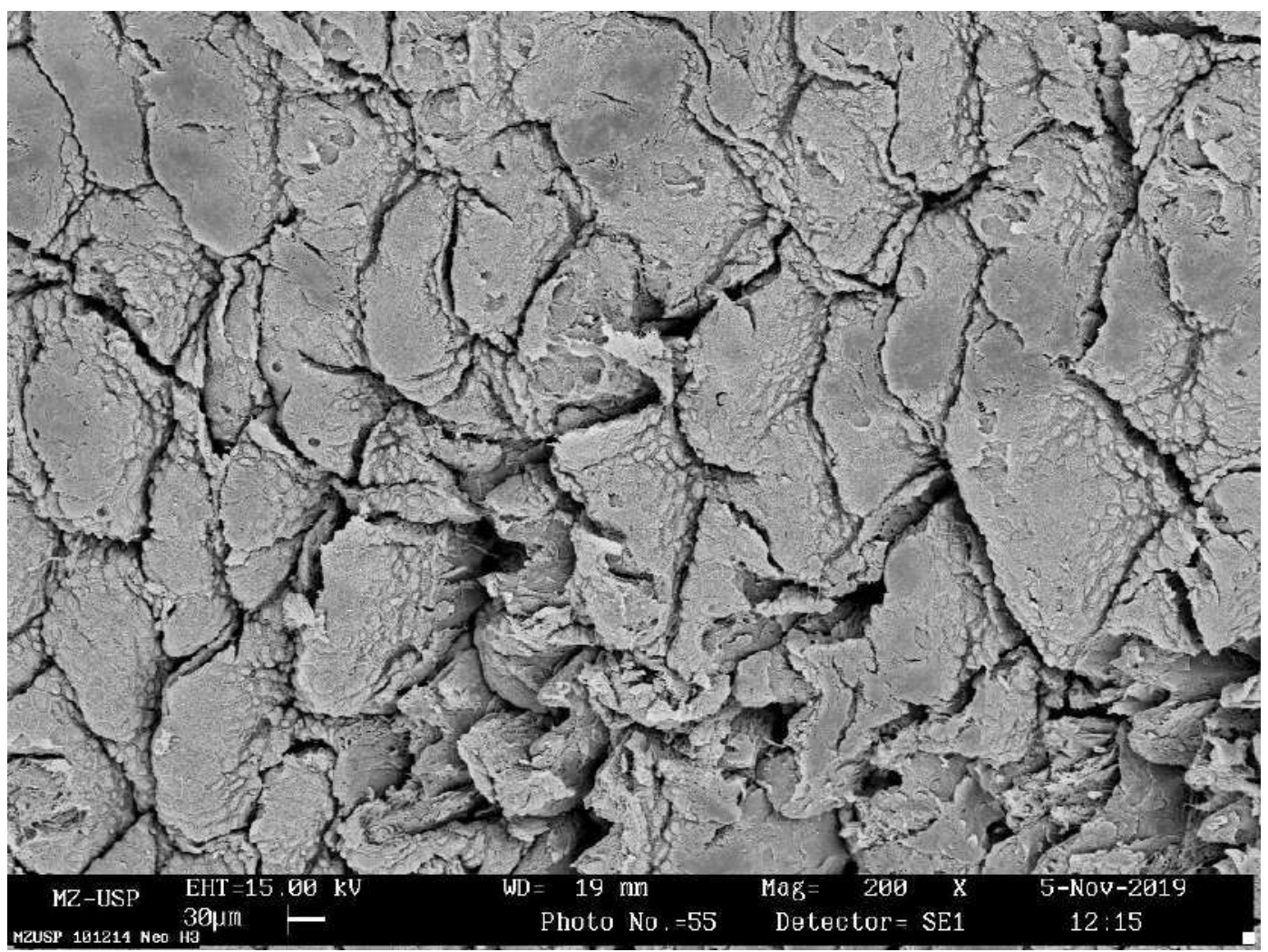

Figure 32. Dermal denticles collected from the section H3 (see fig. 1) of the neonate Isogomphodon oxyrhynchus (MZUSP 101214). Additional information in the image footer.

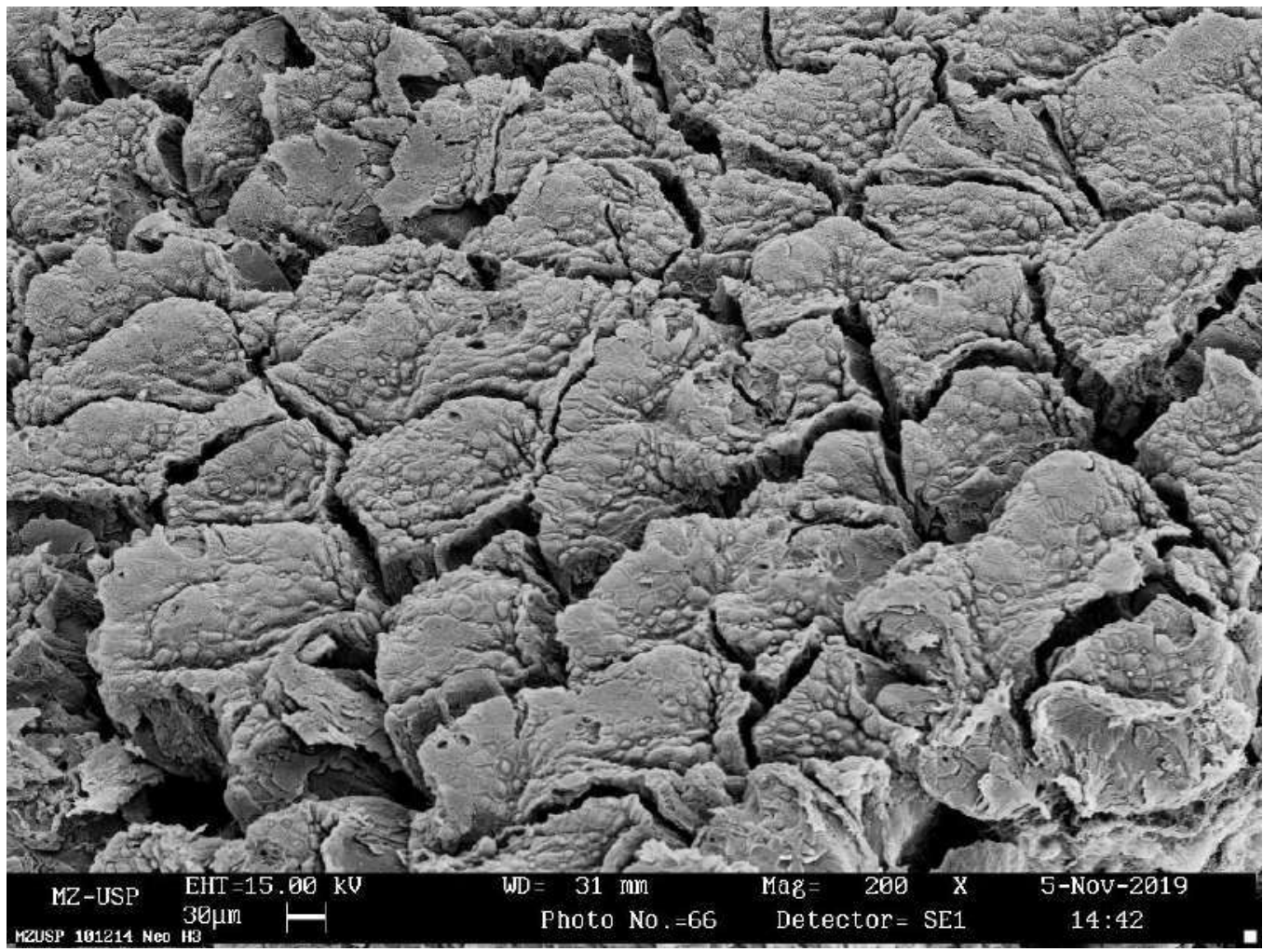

Figure 33. Dermal denticles collected from the section H3 (see fig. 1) of the neonate Isogomphodon oxyrhynchus (MZUSP 101214). Additional information in the image footer. 


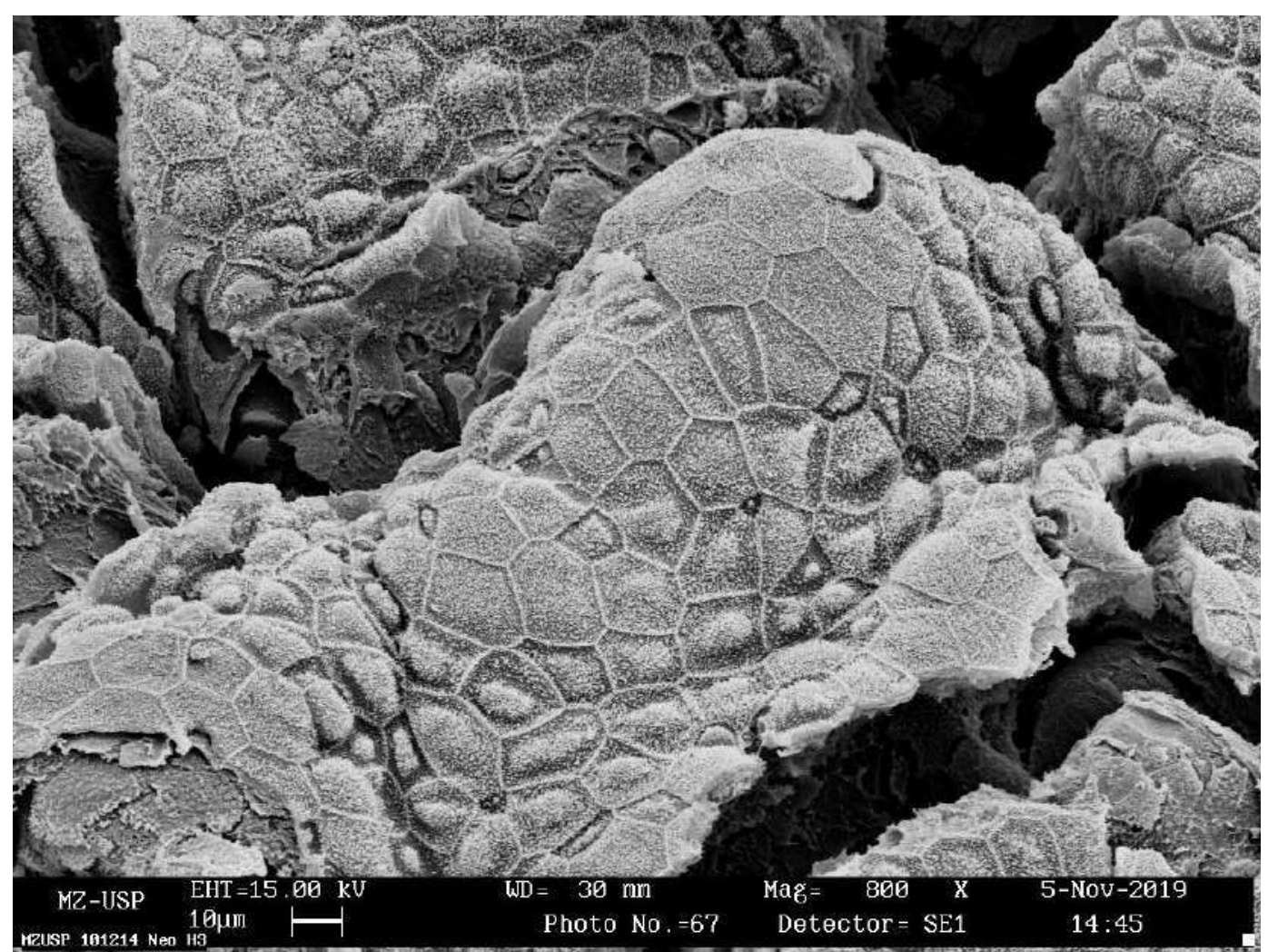

Figure 34. Dermal denticles collected from the section H3 (see fig. 1) of the neonate Isogomphodon oxyrhynchus (MZUSP 101214). Additional information in the image footer.

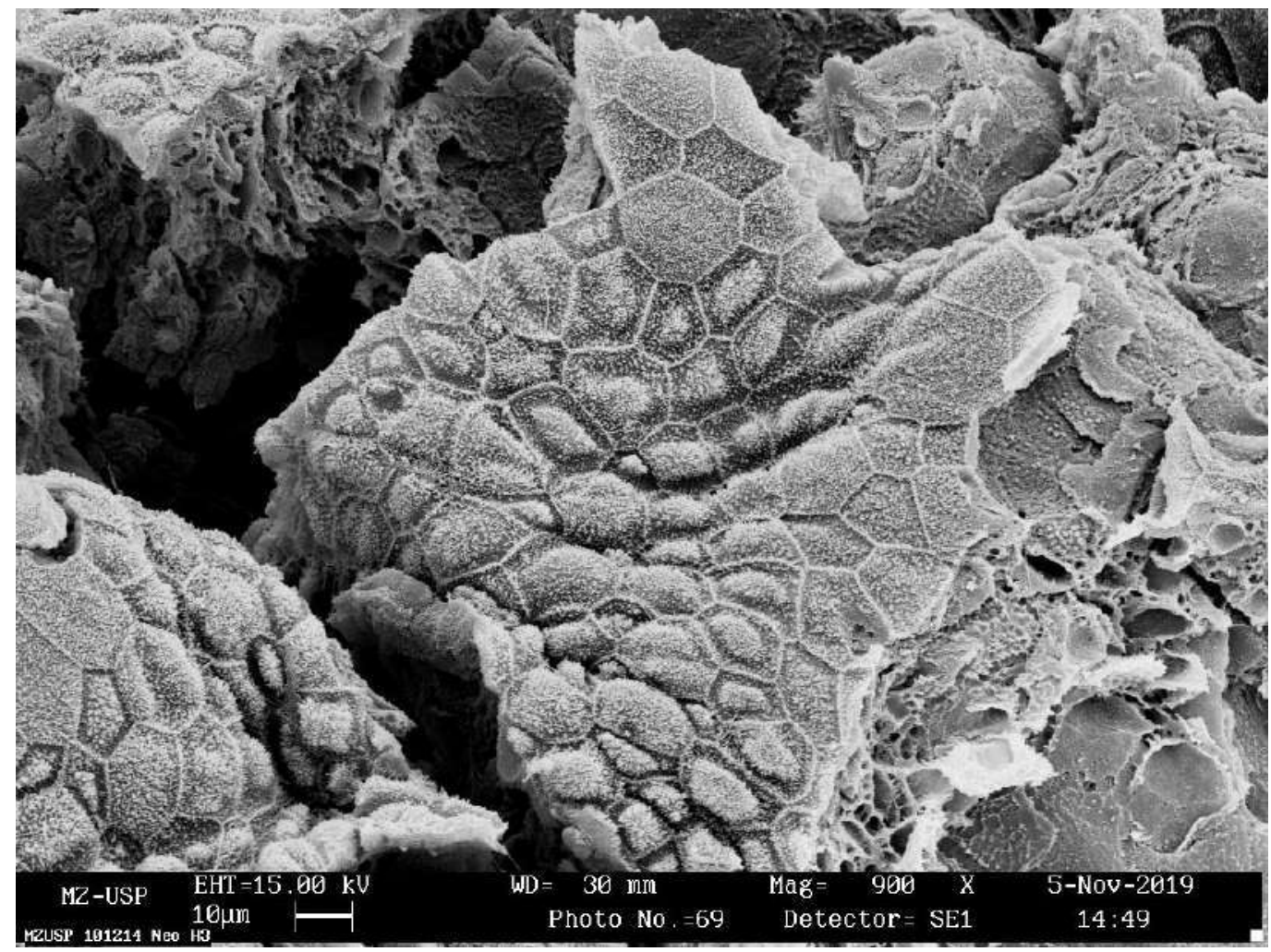

Figure 35. Dermal denticles collected from the section H3 (see fig. 1) of the neonate Isogomphodon oxyrhynchus (MZUSP 101214). Additional information in the image footer. 


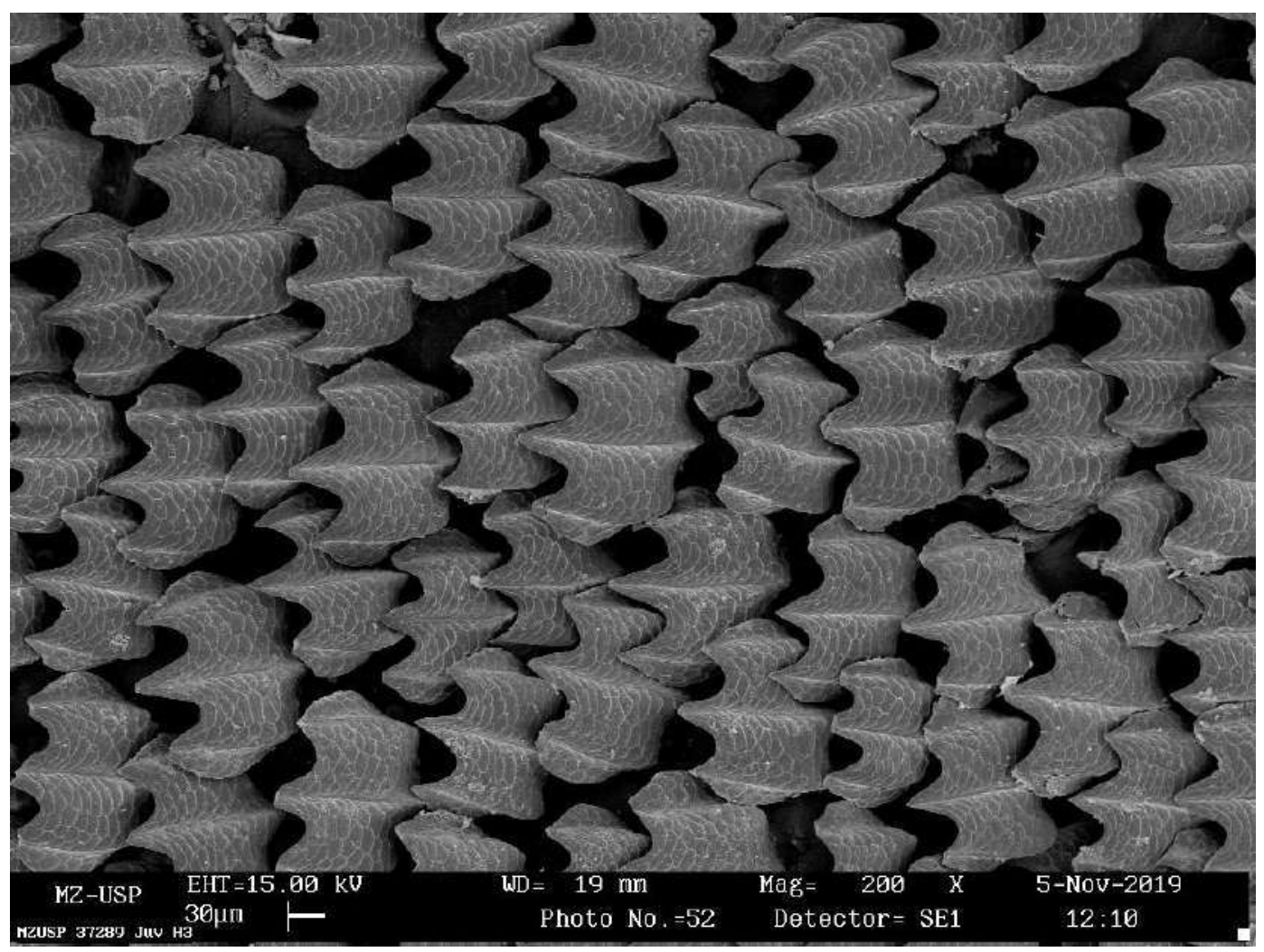

Figure 36. Dermal denticles collected from the section H3 (see fig. 1) of the juvenile Isogomphodon oxyrhynchus (MZUSP 37289). Additional information in the image footer.

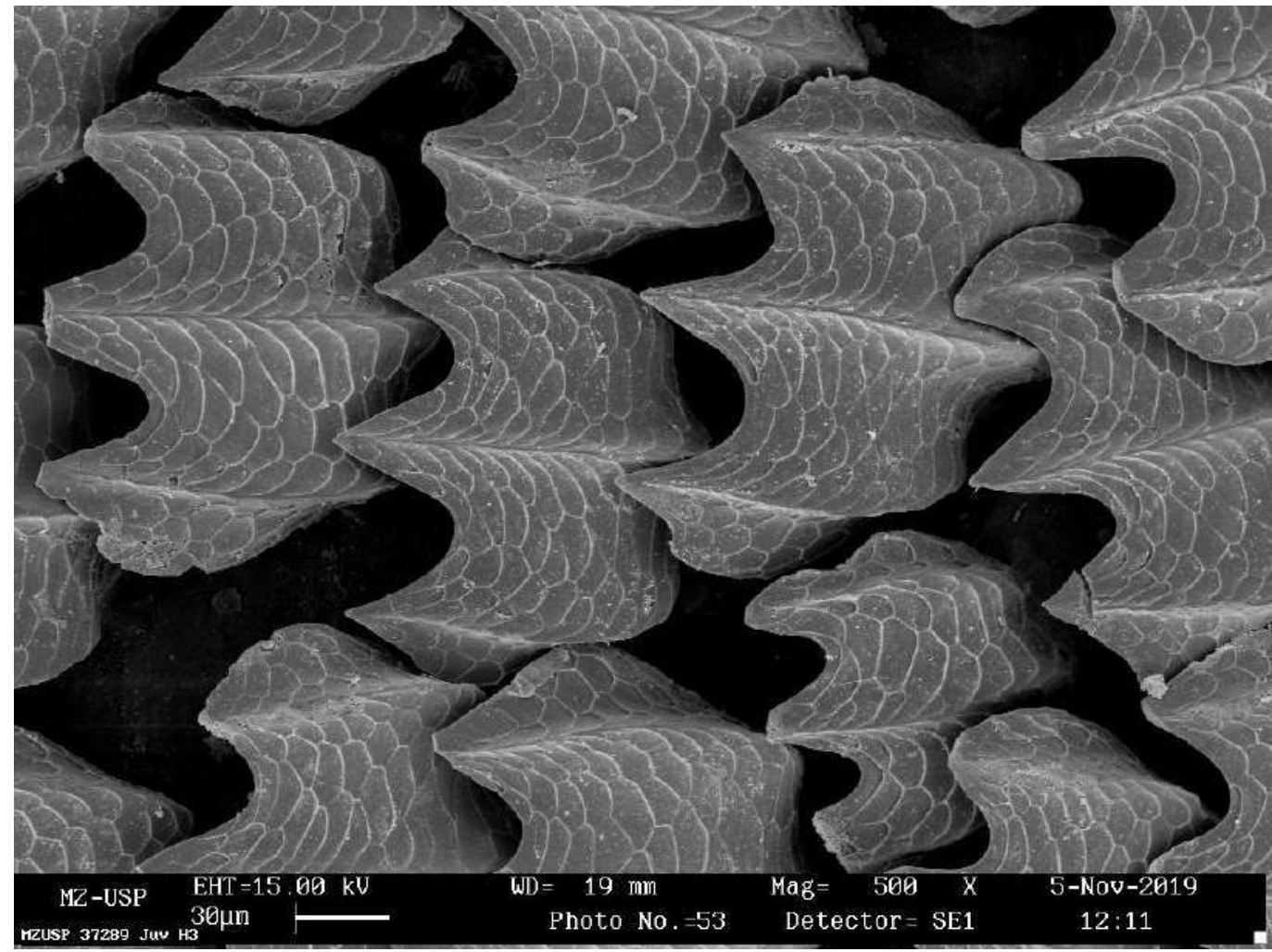

Figure 37. Dermal denticles collected from the section H3 (see fig. 1) of the juvenile Isogomphodon oxyrhynchus (MZUSP 37289). Additional information in the image footer. 


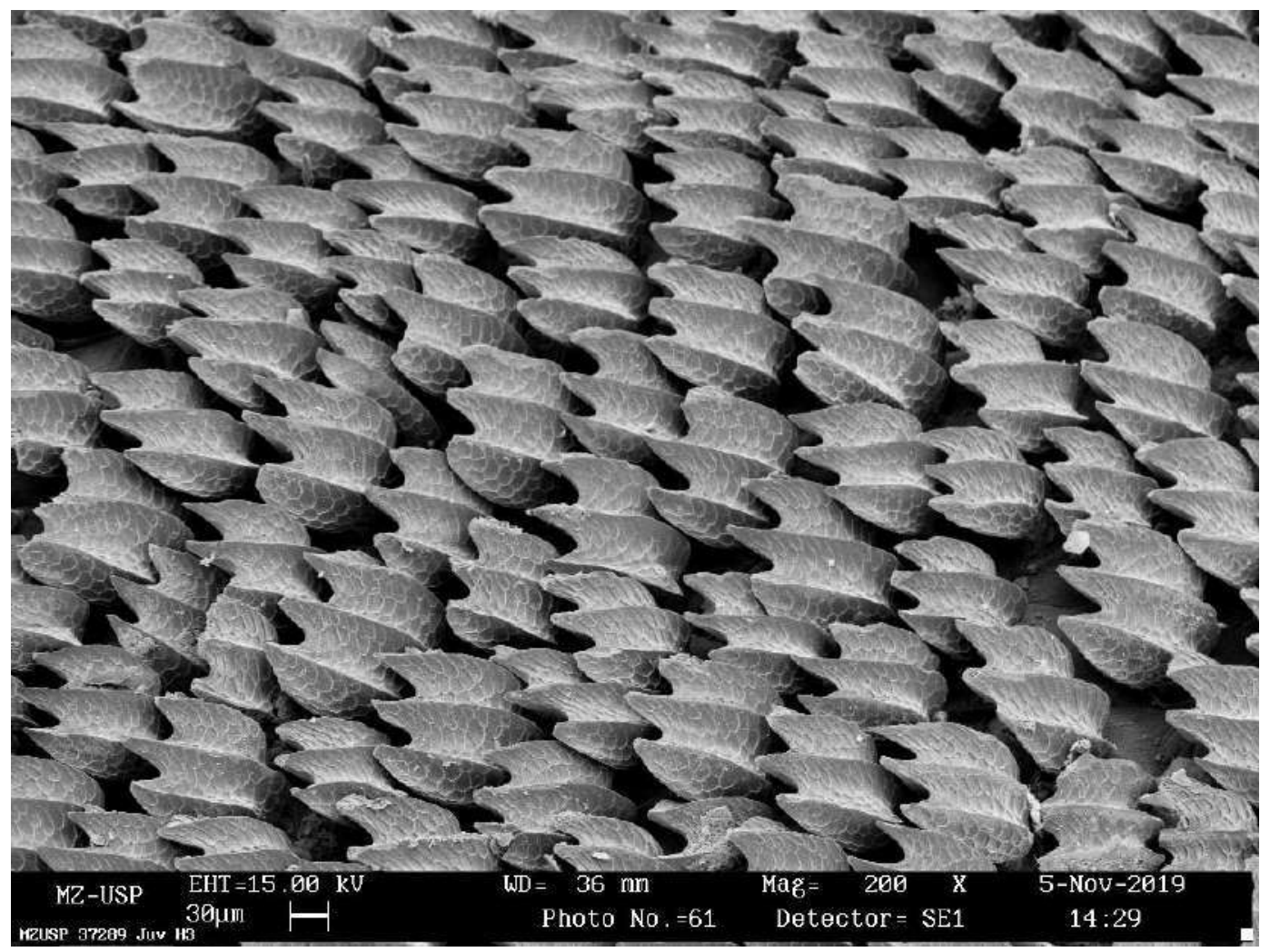

Figure 38. Dermal denticles collected from the section H3 (see fig. 1) of the juvenile Isogomphodon oxyrhynchus (MZUSP 37289). Additional information in the image footer.

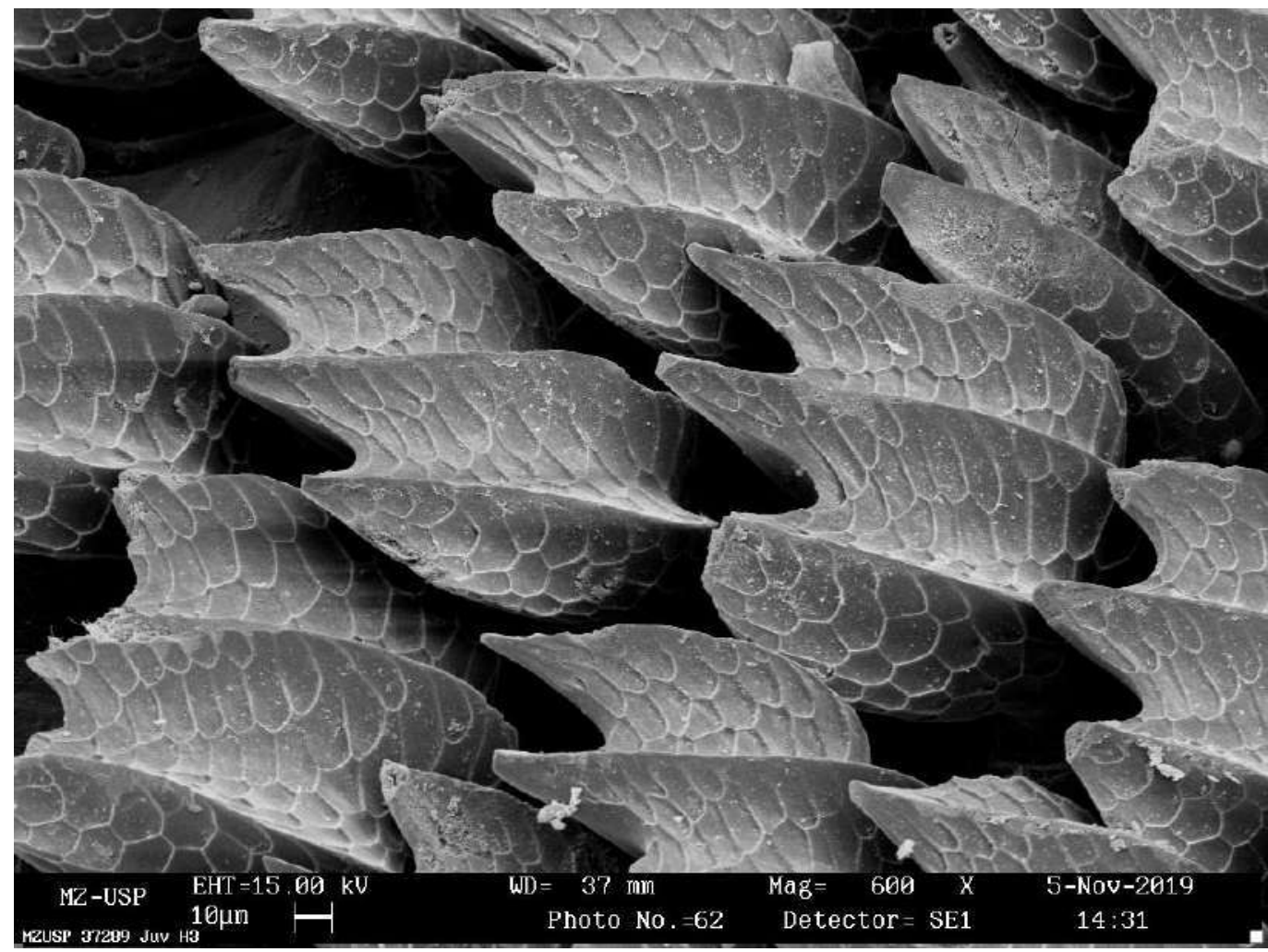

Figure 39. Dermal denticles collected from the section H3 (see fig. 1) of the juvenile Isogomphodon oxyrhynchus (MZUSP 37289). Additional information in the image footer. 


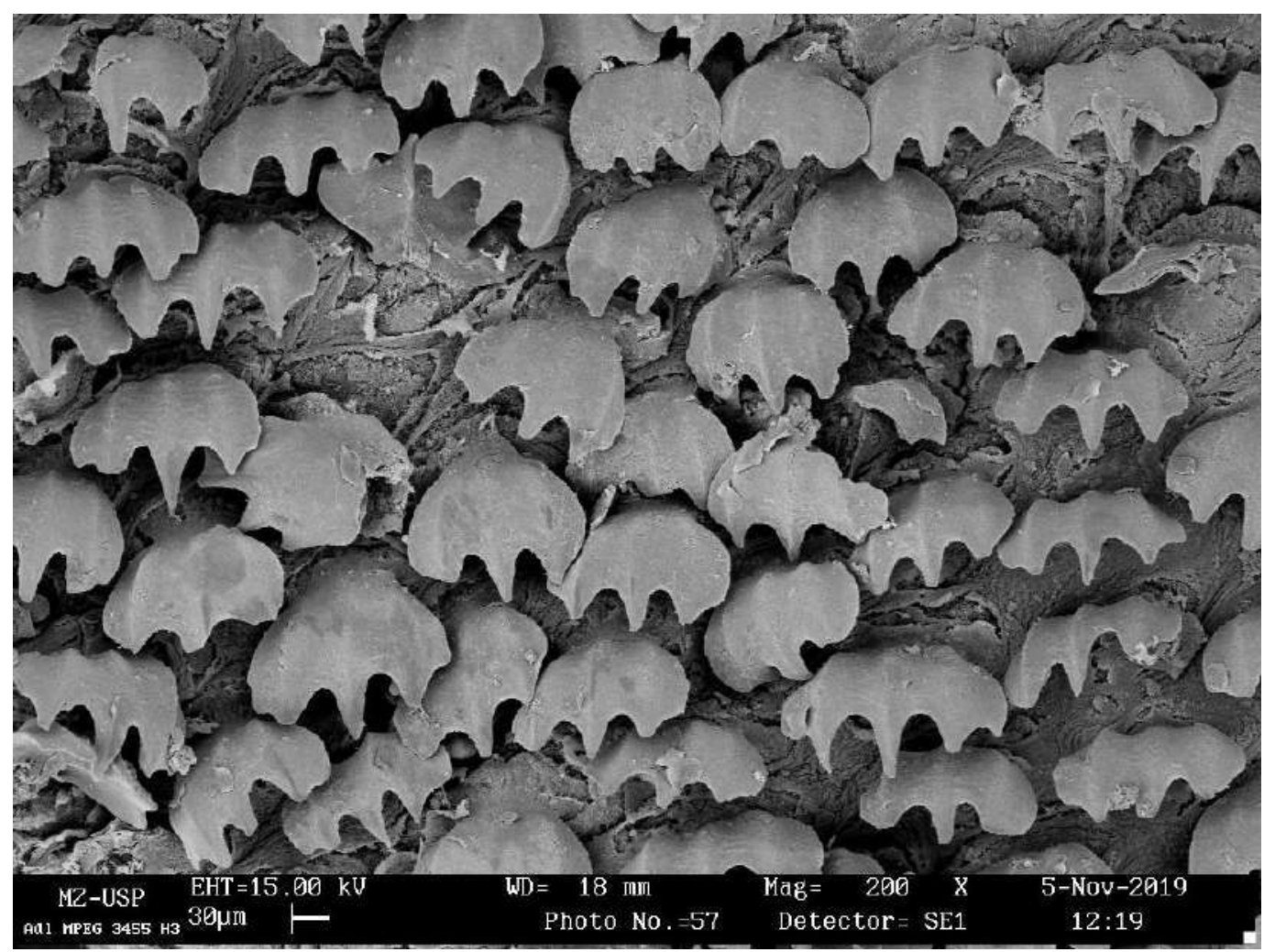

Figure 40. Dermal denticles collected from the section H3 (see fig. 1) of the adult Isogomphodon oxyrhynchus (MPEG 3455). Additional information in the image footer.

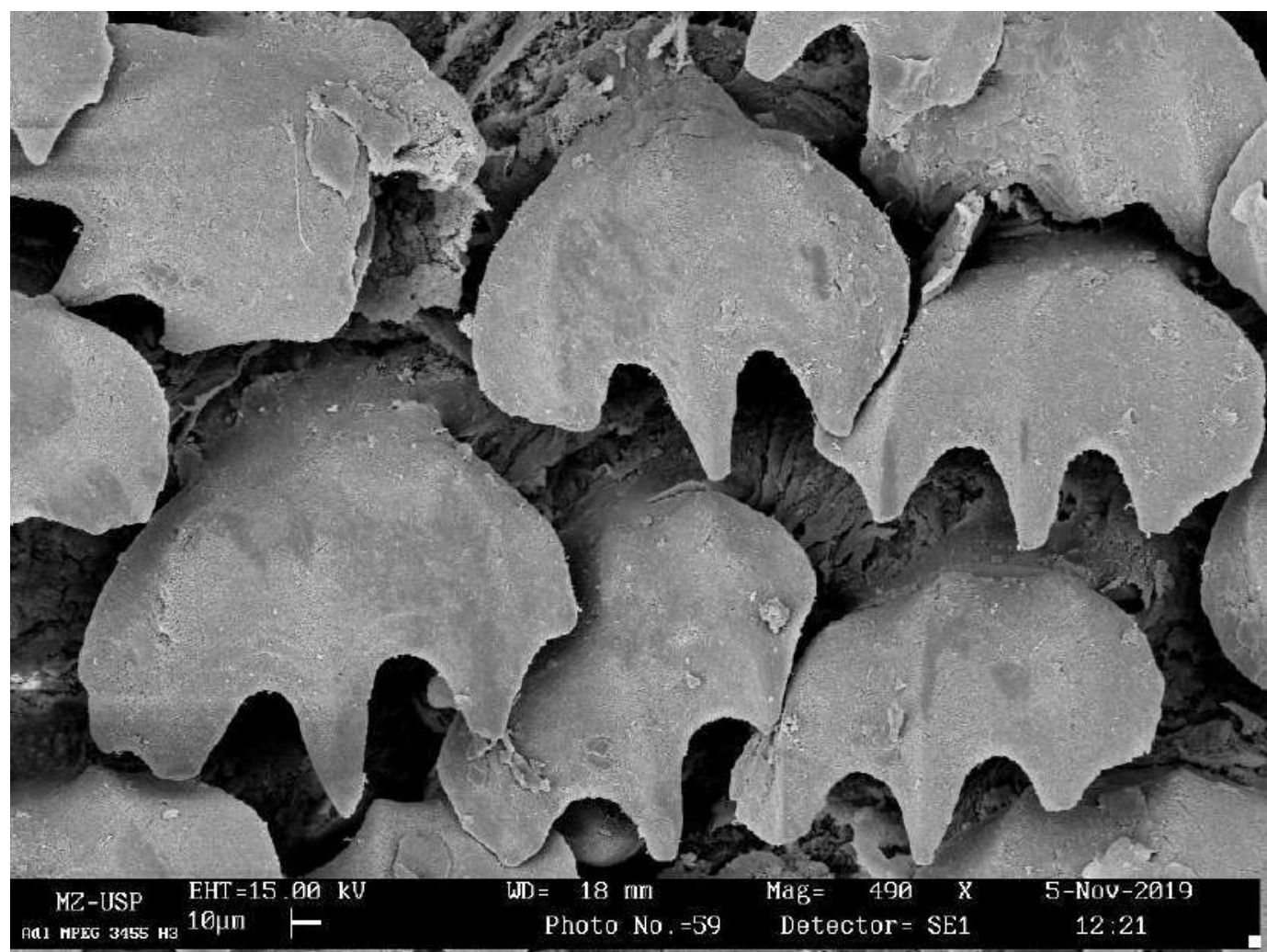

Figure 41. Dermal denticles collected from the section H3 (see fig. 1) of the adult Isogomphodon oxyrhynchus (MPEG 3455). Additional information in the image footer. 


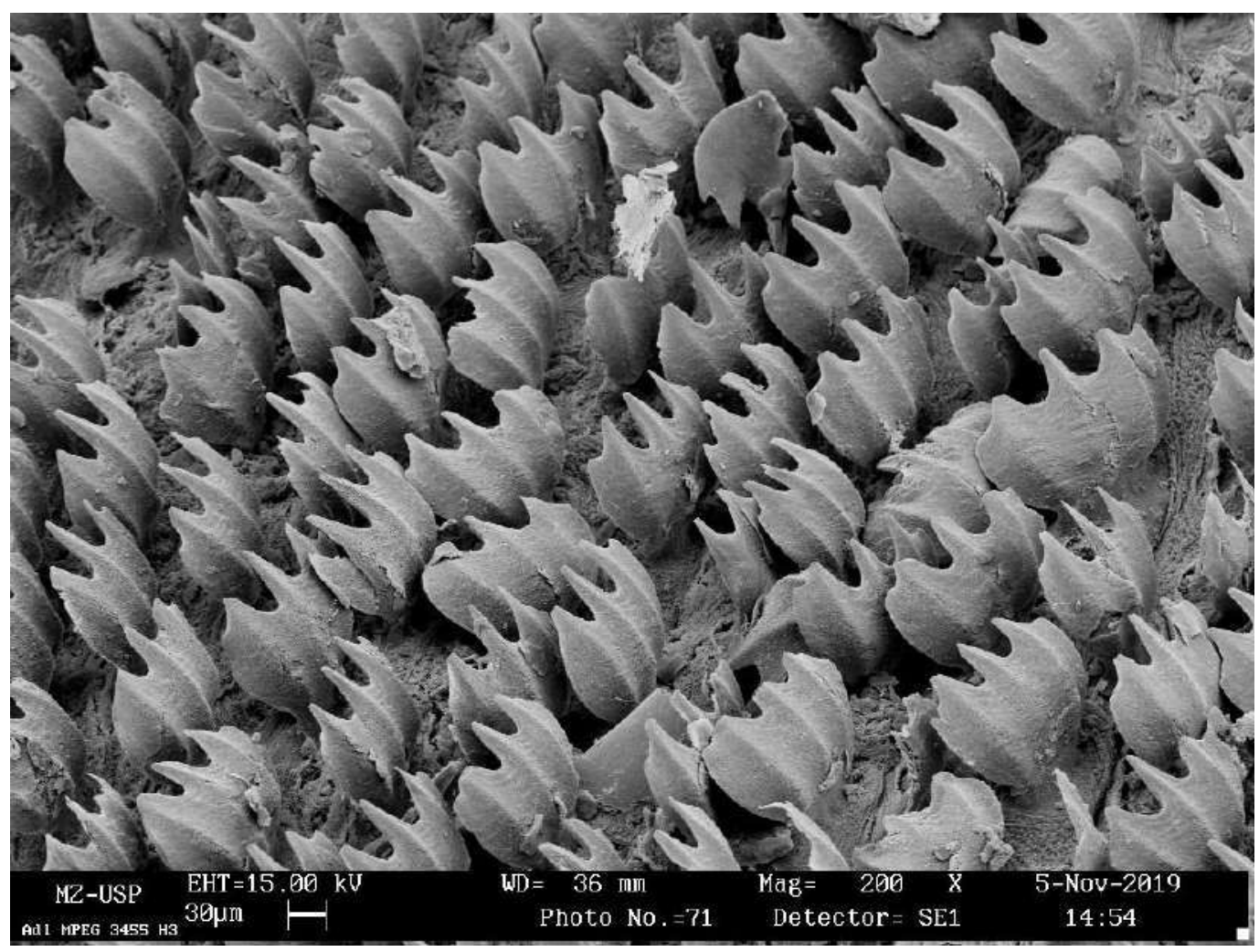

Figure 42. Dermal denticles collected from the section H3 (see fig. 1) of the adult Isogomphodon oxyrhynchus (MPEG 3455). Additional information in the image footer.

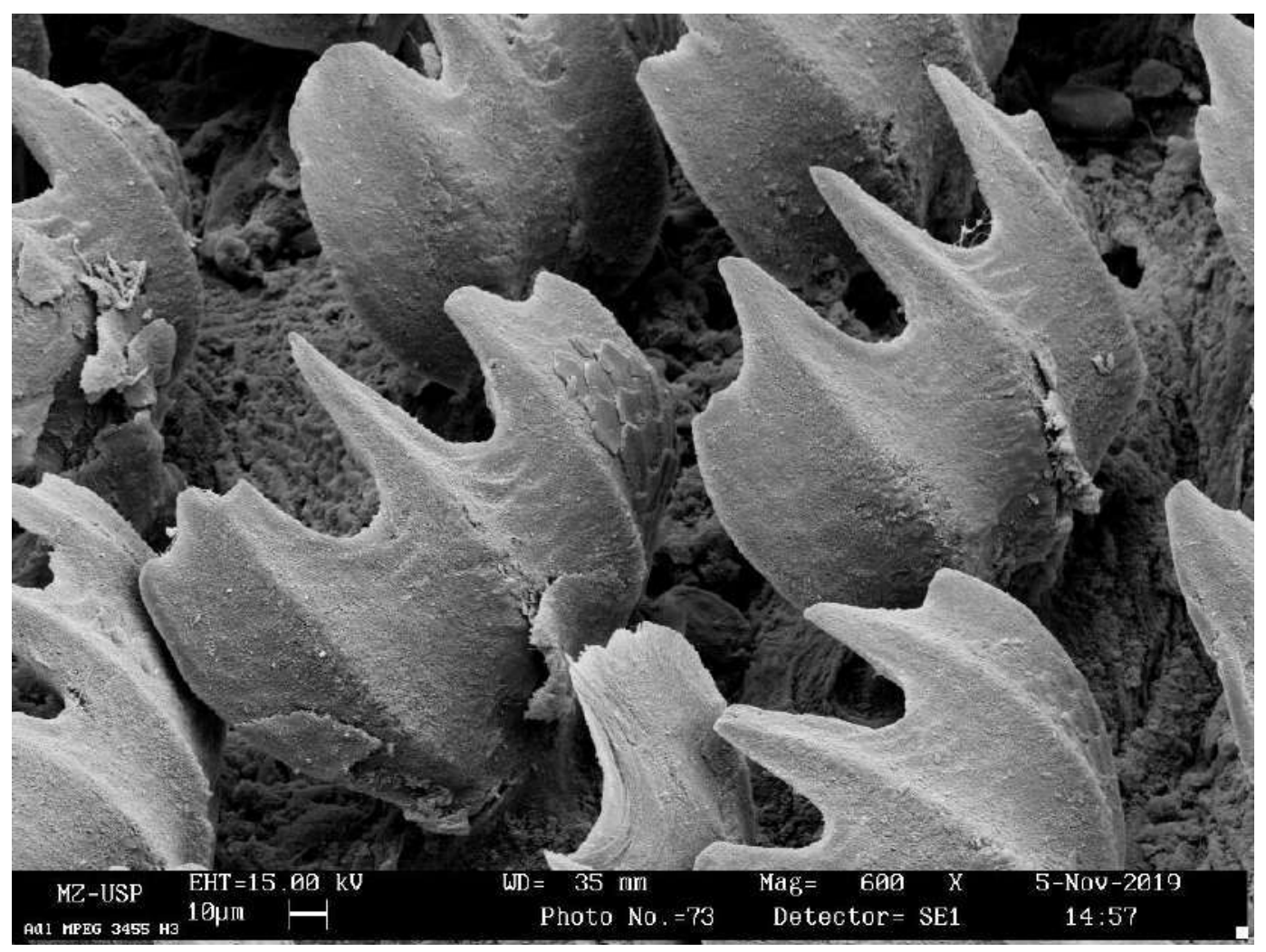

Figure 43. Dermal denticles collected from the section H3 (see fig. 1) of the adult Isogomphodon oxyrhynchus (MPEG 3455). Additional information in the image footer. 


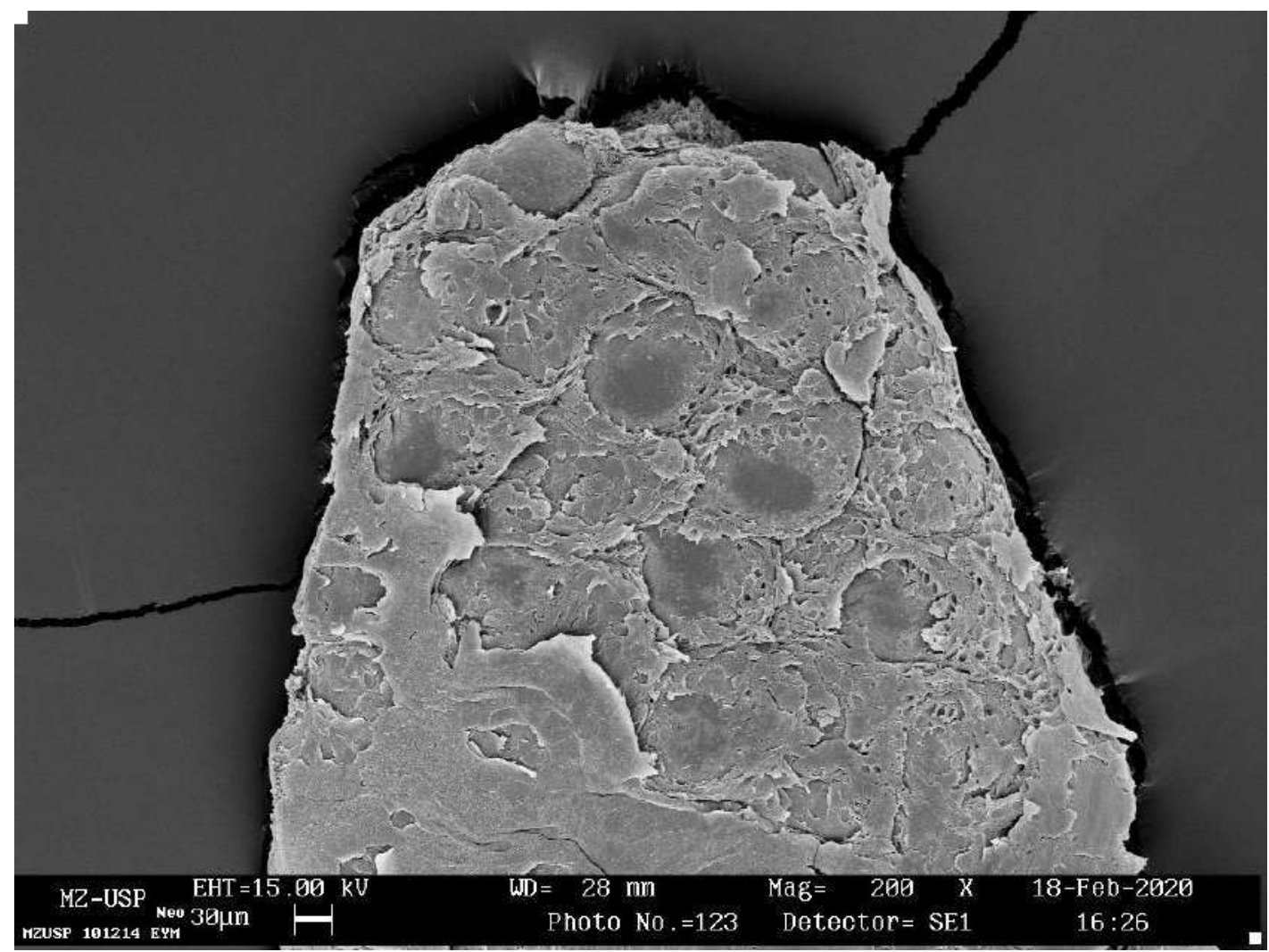

Figure 44. Dermal denticles collected from the section Eym (see fig. 1) of the neonate Isogomphodon oxyrhynchus (MZUSP 101214). Additional information in the image footer.

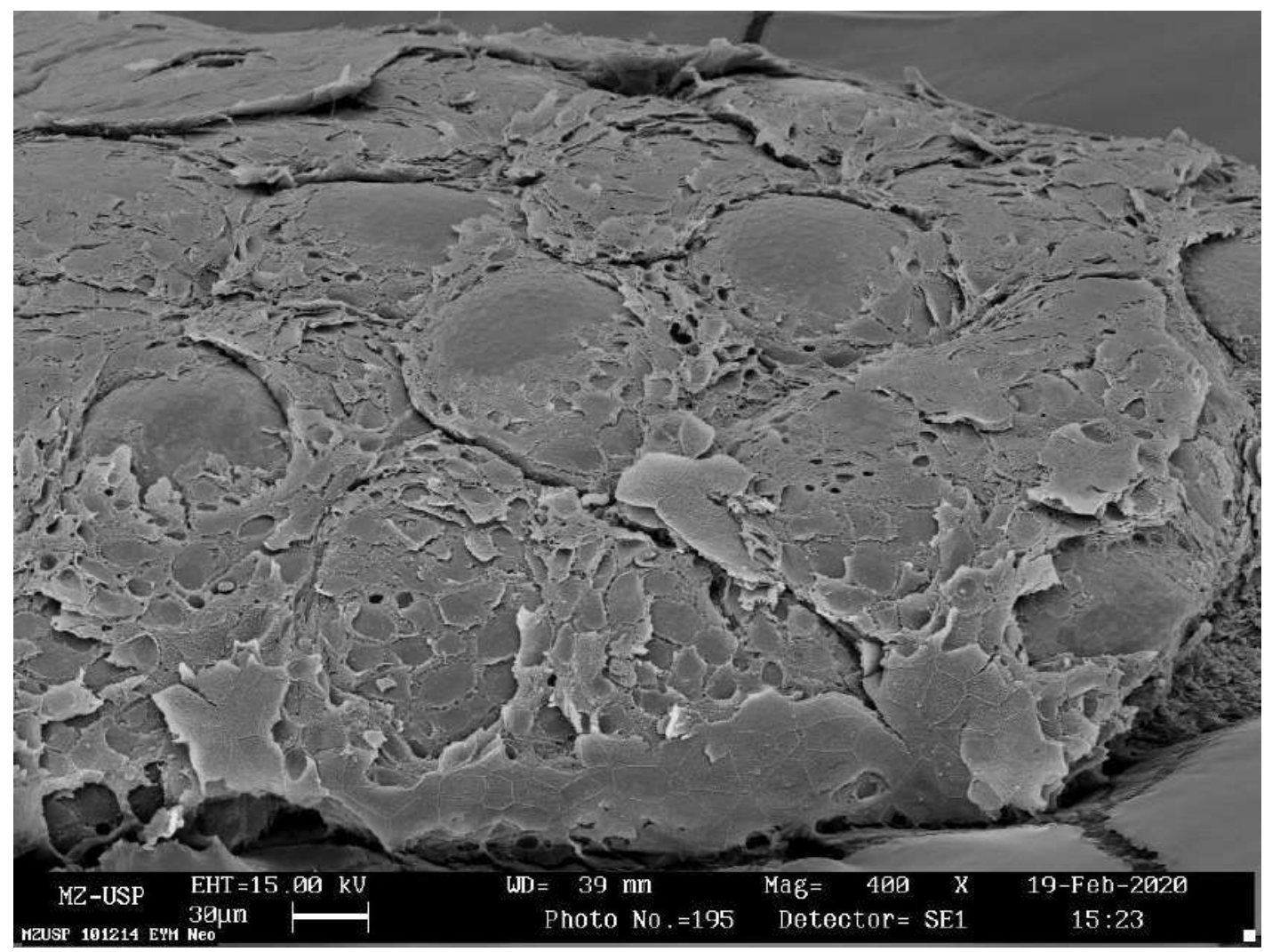

Figure 45. Dermal denticles collected from the section Eym (see fig. 1) of the neonate Isogomphodon oxyrhynchus (MZUSP 101214). Additional information in the image footer. 


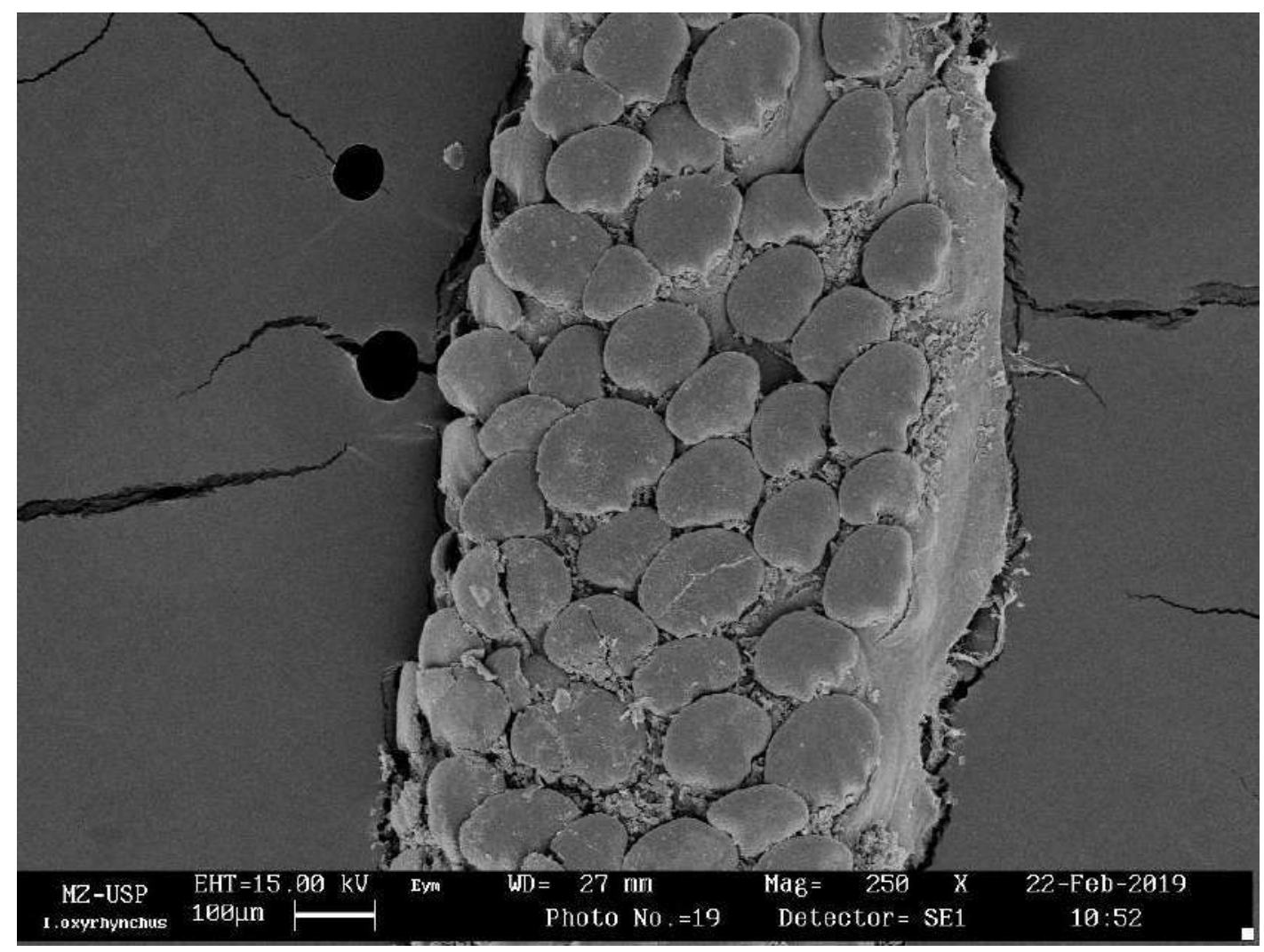

Figure 46. Dermal denticles collected from the section Eym (see fig. 1) of the juvenile Isogomphodon oxyrhynchus (MZUSP 37289). Additional information in the image footer.

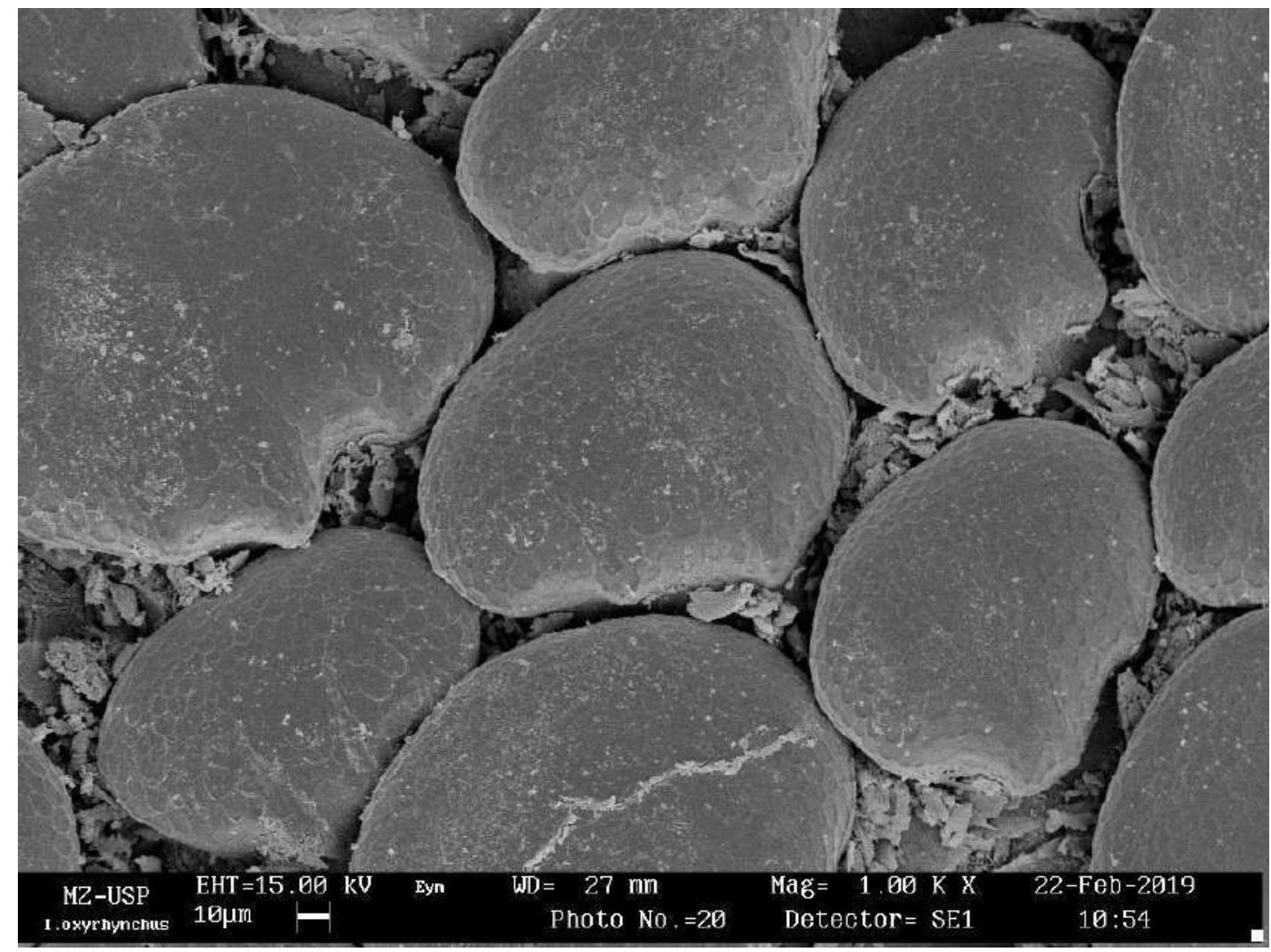

Figure 47. Dermal denticles collected from the section Eym (see fig. 1) of the juvenile Isogomphodon oxyrhynchus (MZUSP 37289). Additional information in the image footer. 


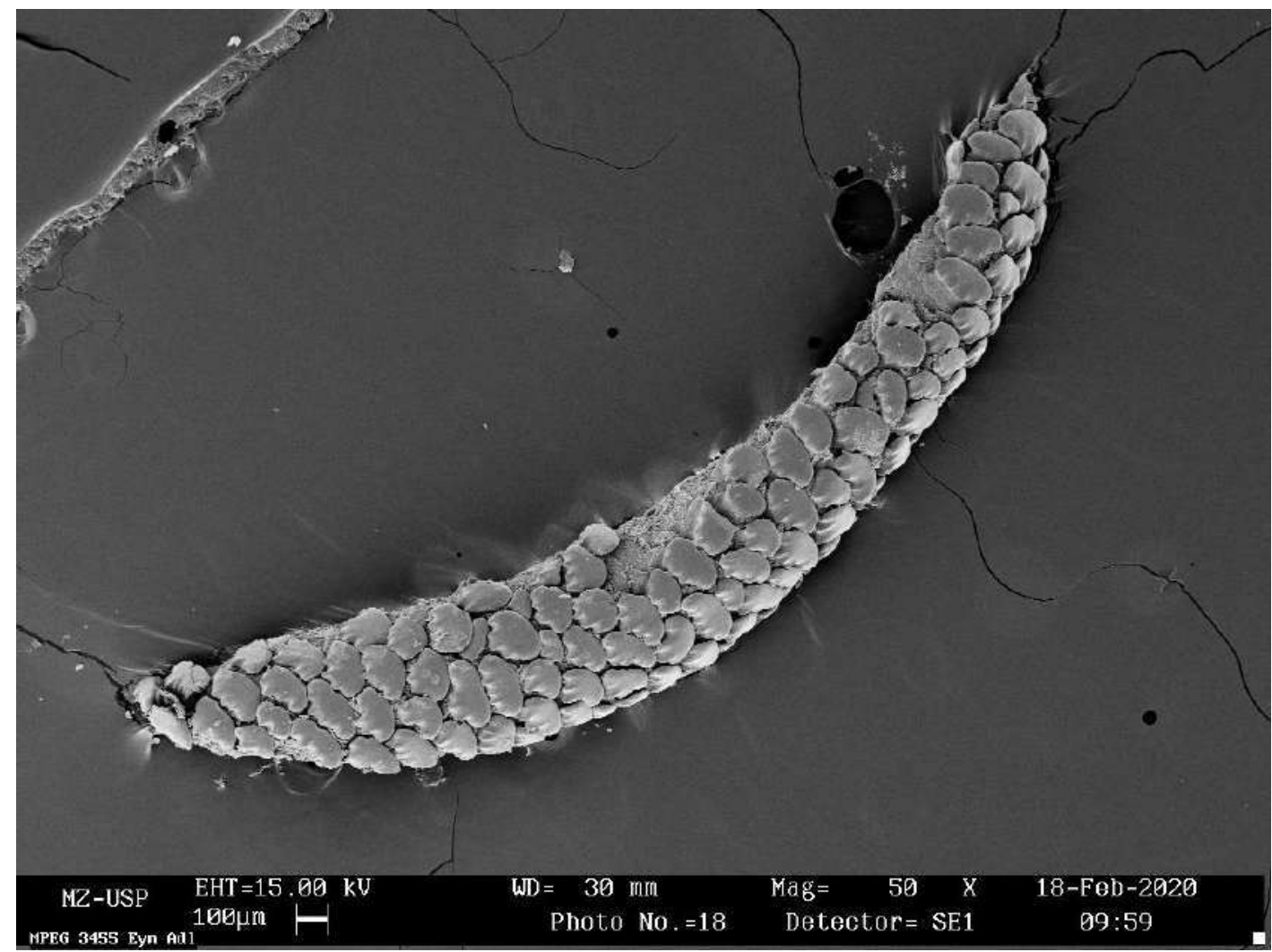

Figure 48. Dermal denticles collected from the section Eym (see fig. 1) of the adult Isogomphodon oxyrhynchus (MPEG 3455). Additional information in the image footer.

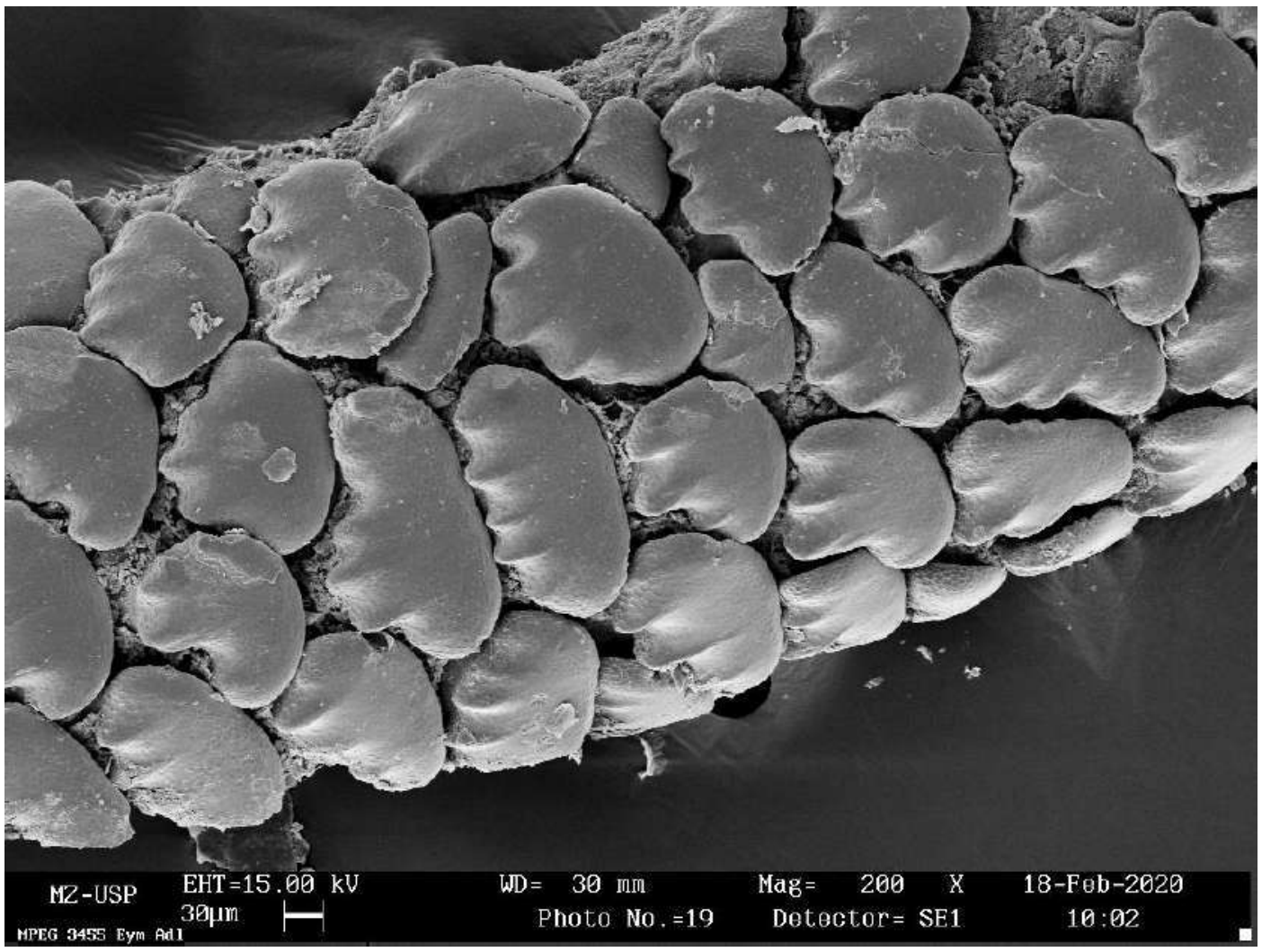

Figure 49. Dermal denticles collected from the section Eym (see fig. 1) of the adult Isogomphodon oxyrhynchus (MPEG 3455). Additional information in the image footer. 


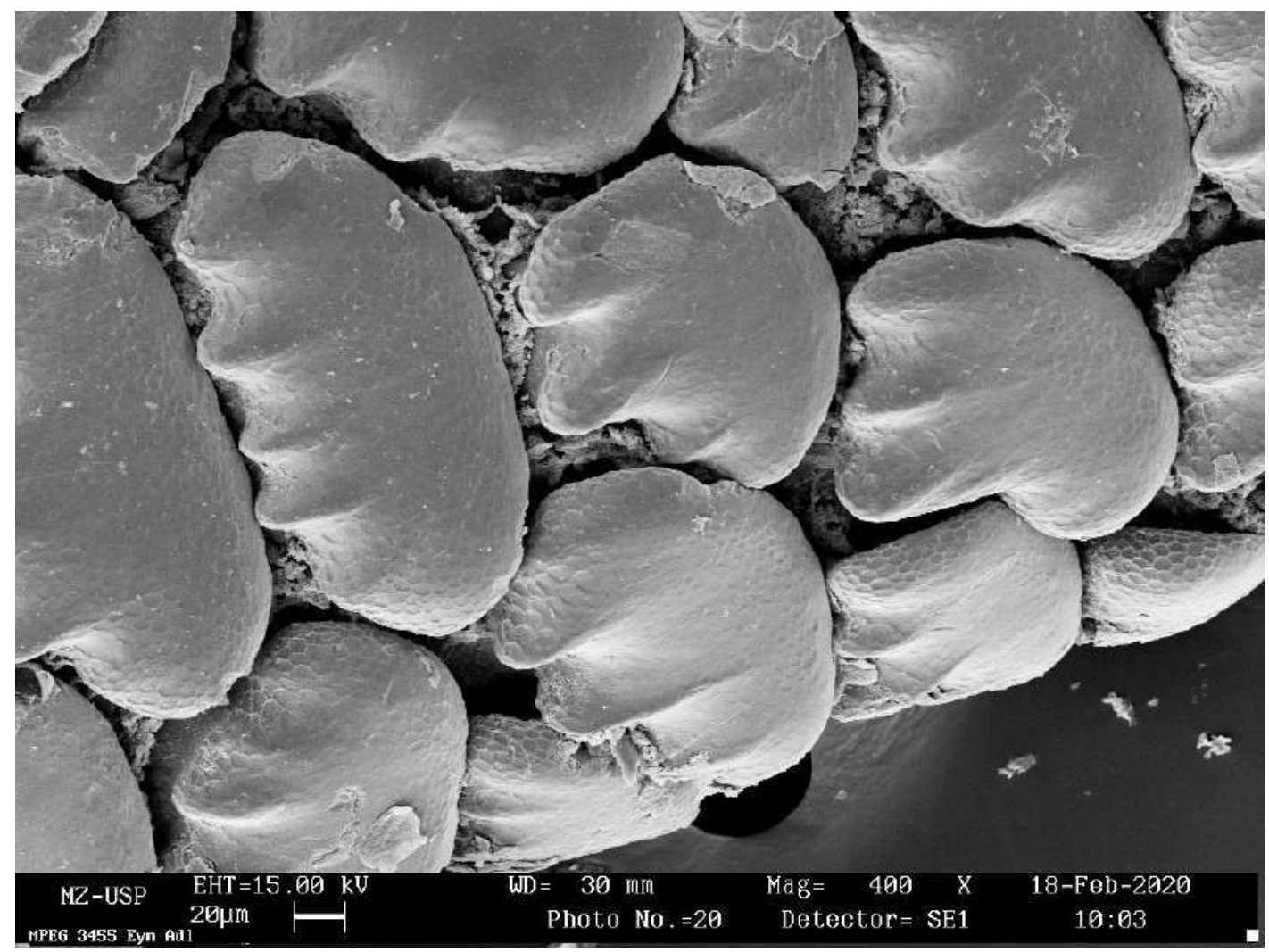

Figure 50. Dermal denticles collected from the section Eym (see fig. 1) of the adult Isogomphodon oxyrhynchus (MPEG 3455). Additional information in the image footer.

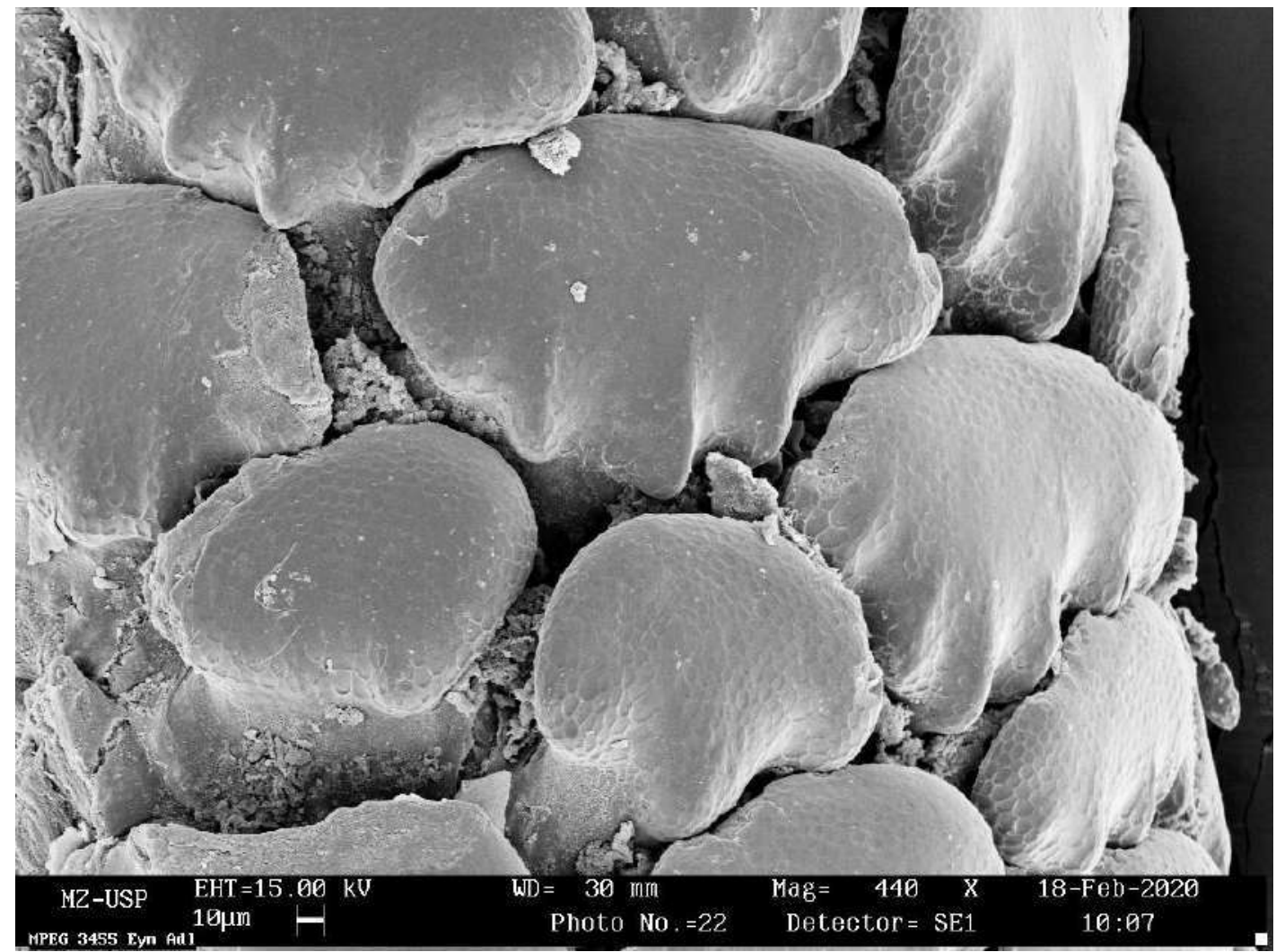

Figure 51. Dermal denticles collected from the section Eym (see fig. 1) of the adult Isogomphodon oxyrhynchus (MPEG 3455). Additional information in the image footer. 


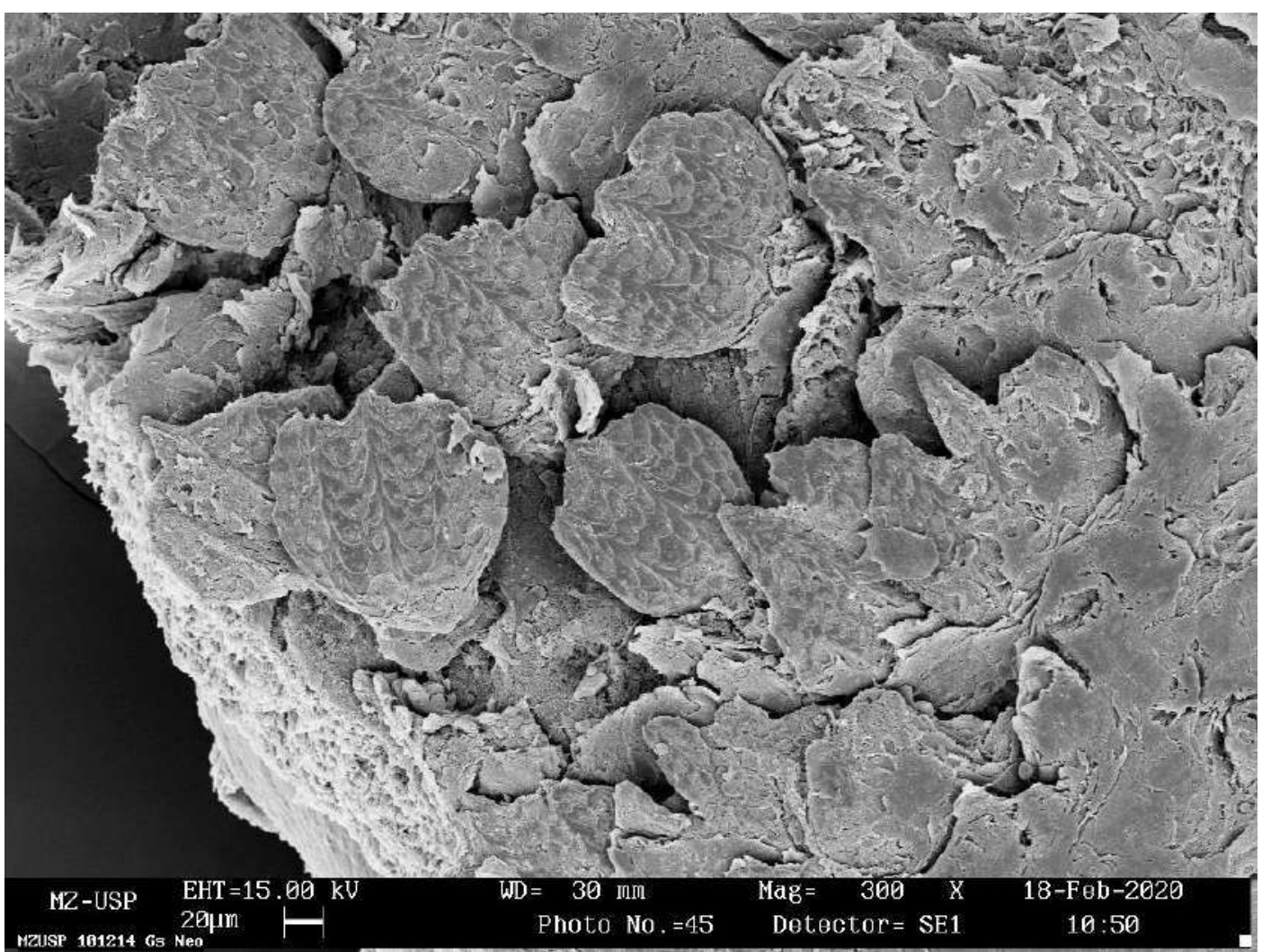

Figure 52. Dermal denticles collected from the section GS (see fig. 1) of the neonate Isogomphodon oxyrhynchus (MZUSP 101214). Additional information in the image footer.

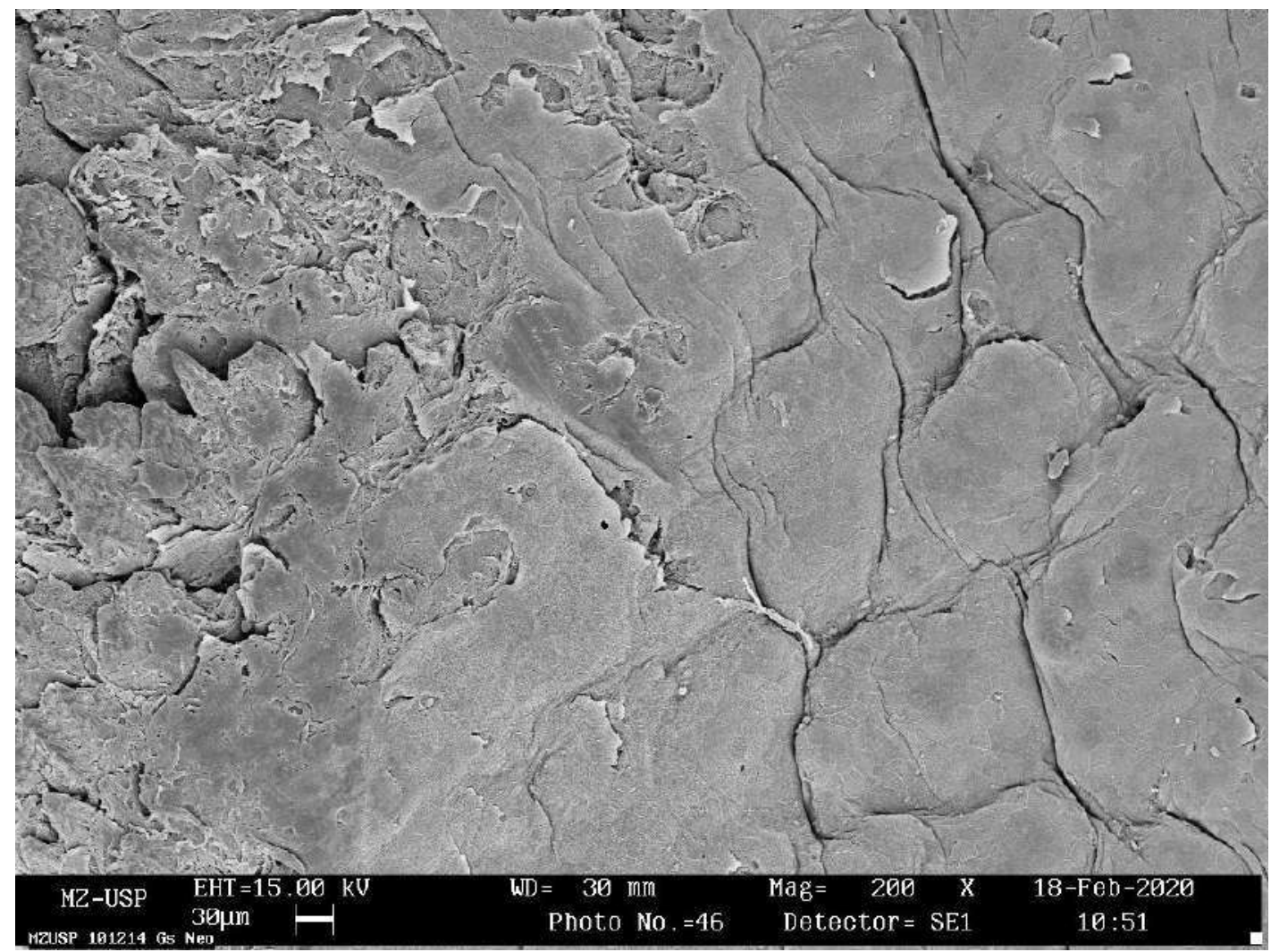

Figure 53. Dermal denticles collected from the section GS (see fig. 1) of the neonate Isogomphodon oxyrhynchus (MZUSP 101214). Additional information in the image footer. 


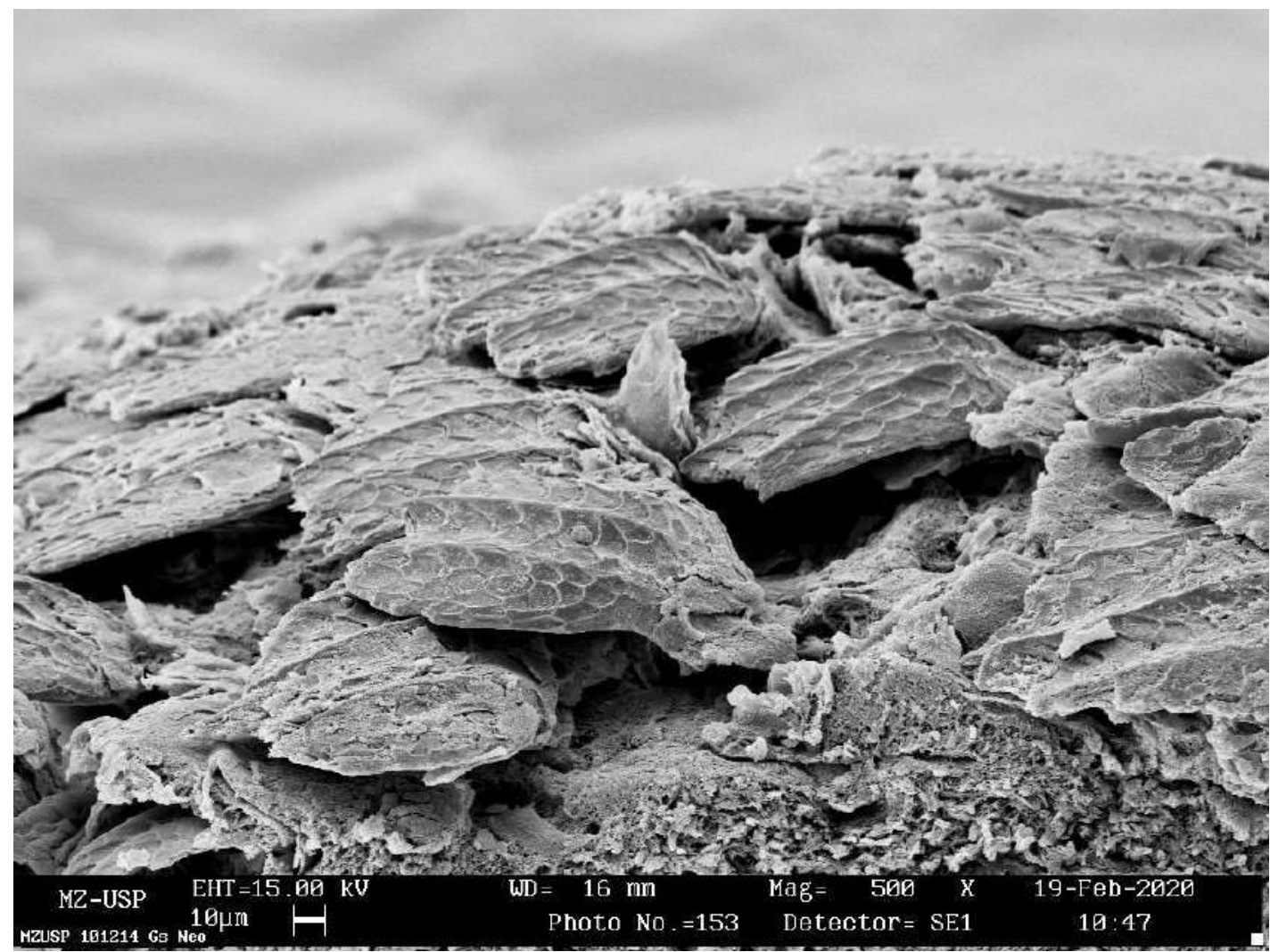

Figure 54. Dermal denticles collected from the section GS (see fig. 1) of the neonate Isogomphodon oxyrhynchus (MZUSP 101214). Additional information in the image footer.

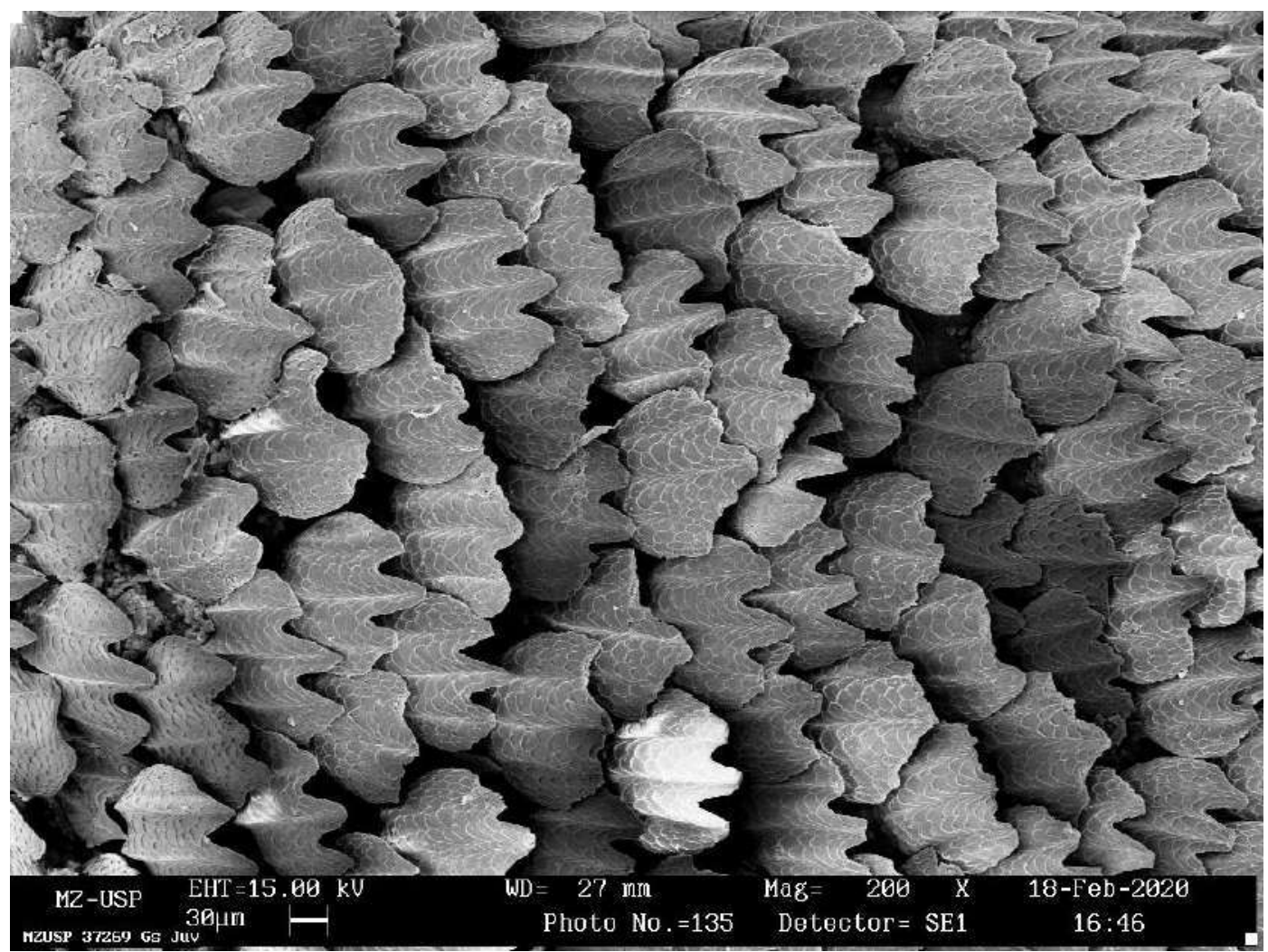

Figure 55. Dermal denticles collected from the section GS (see fig. 1) of the juvenile Isogomphodon oxyrhynchus (MZUSP 37289). Additional information in the image footer. 


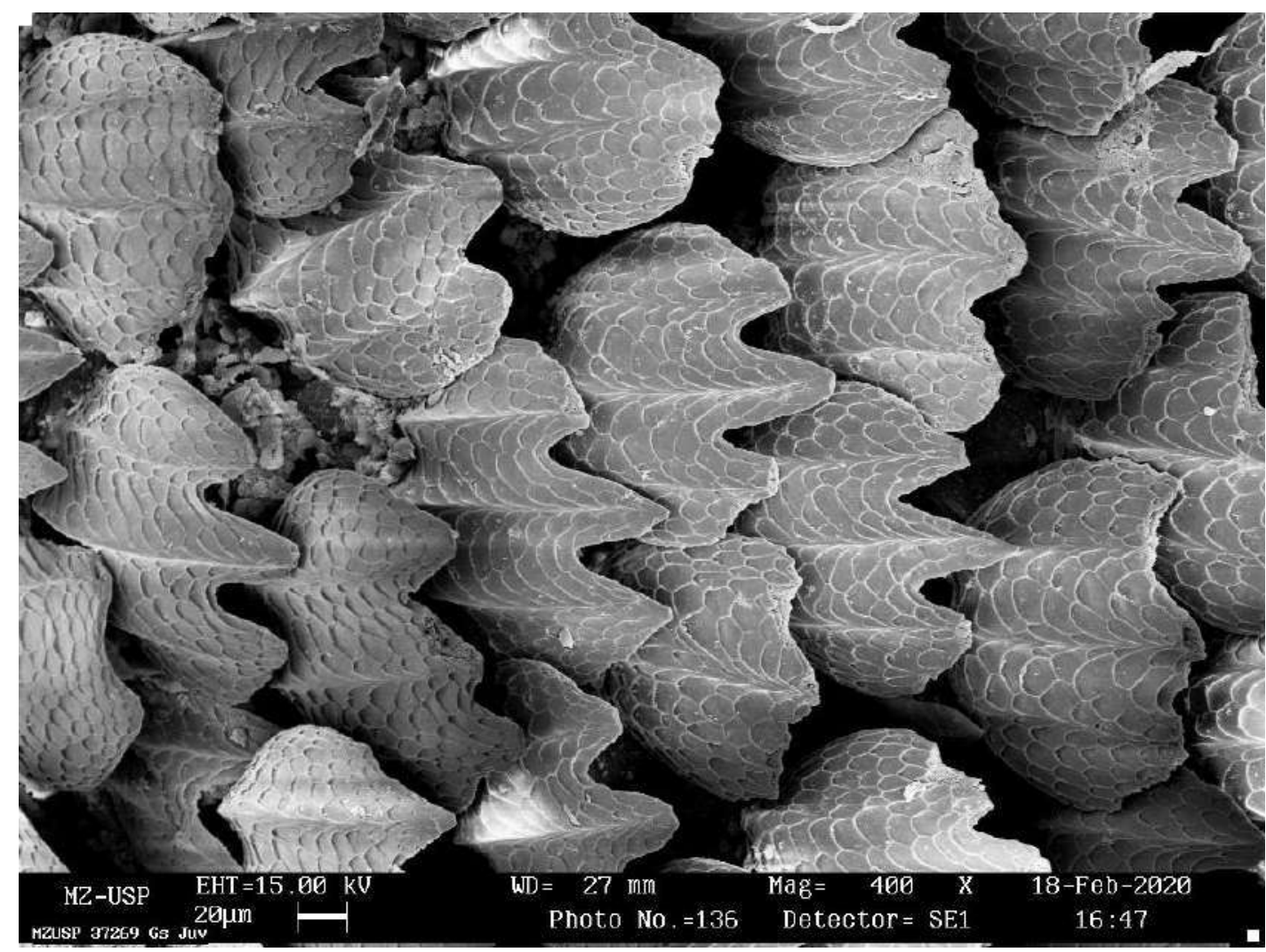

Figure 56. Dermal denticles collected from the section GS (see fig. 1) of the juvenile Isogomphodon oxyrhynchus (MZUSP 37289). Additional information in the image footer.

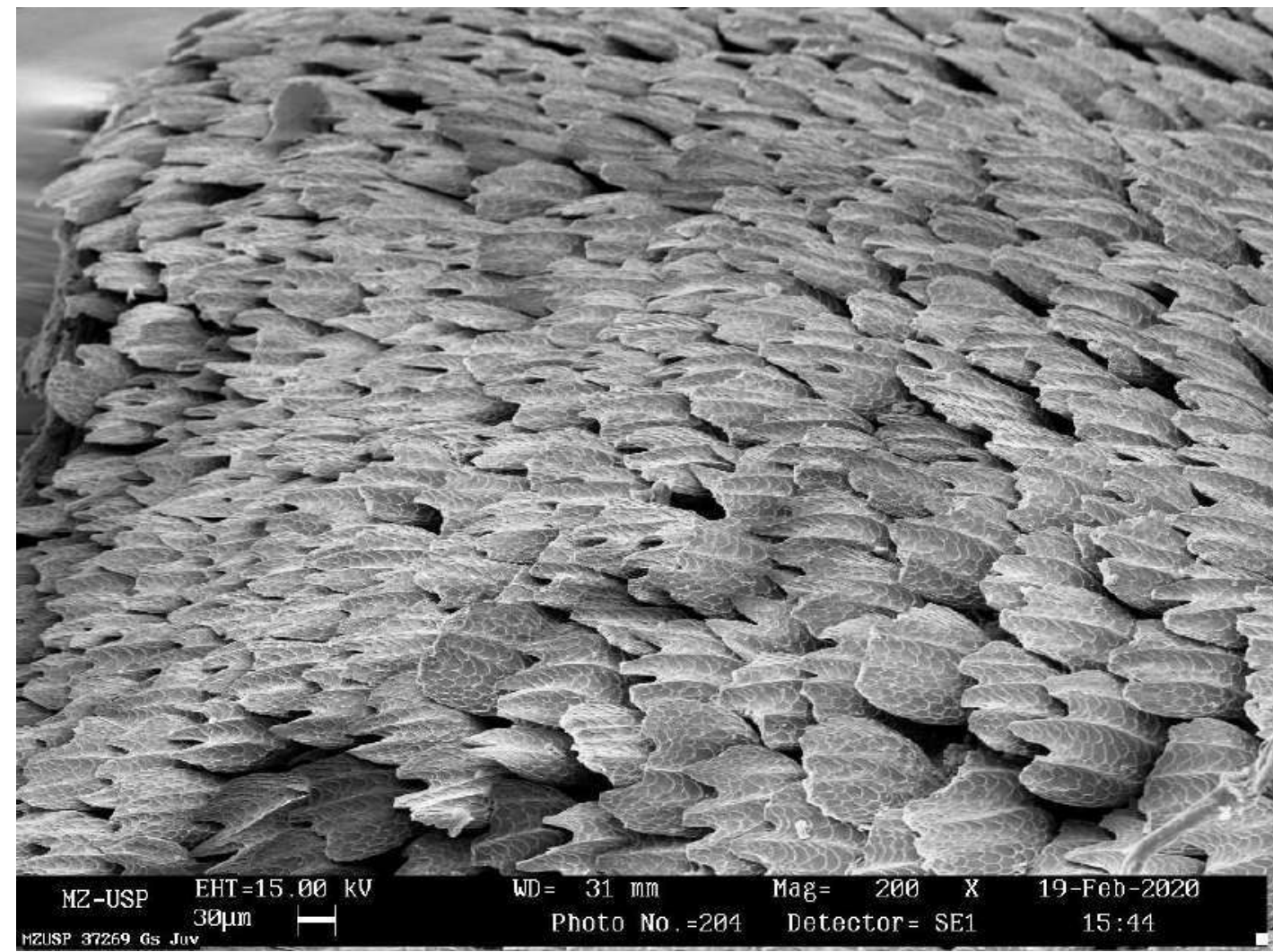

Figure 57. Dermal denticles collected from the section GS (see fig. 1) of the juvenile Isogomphodon oxyrhynchus (MZUSP 37289). Additional information in the image footer. 


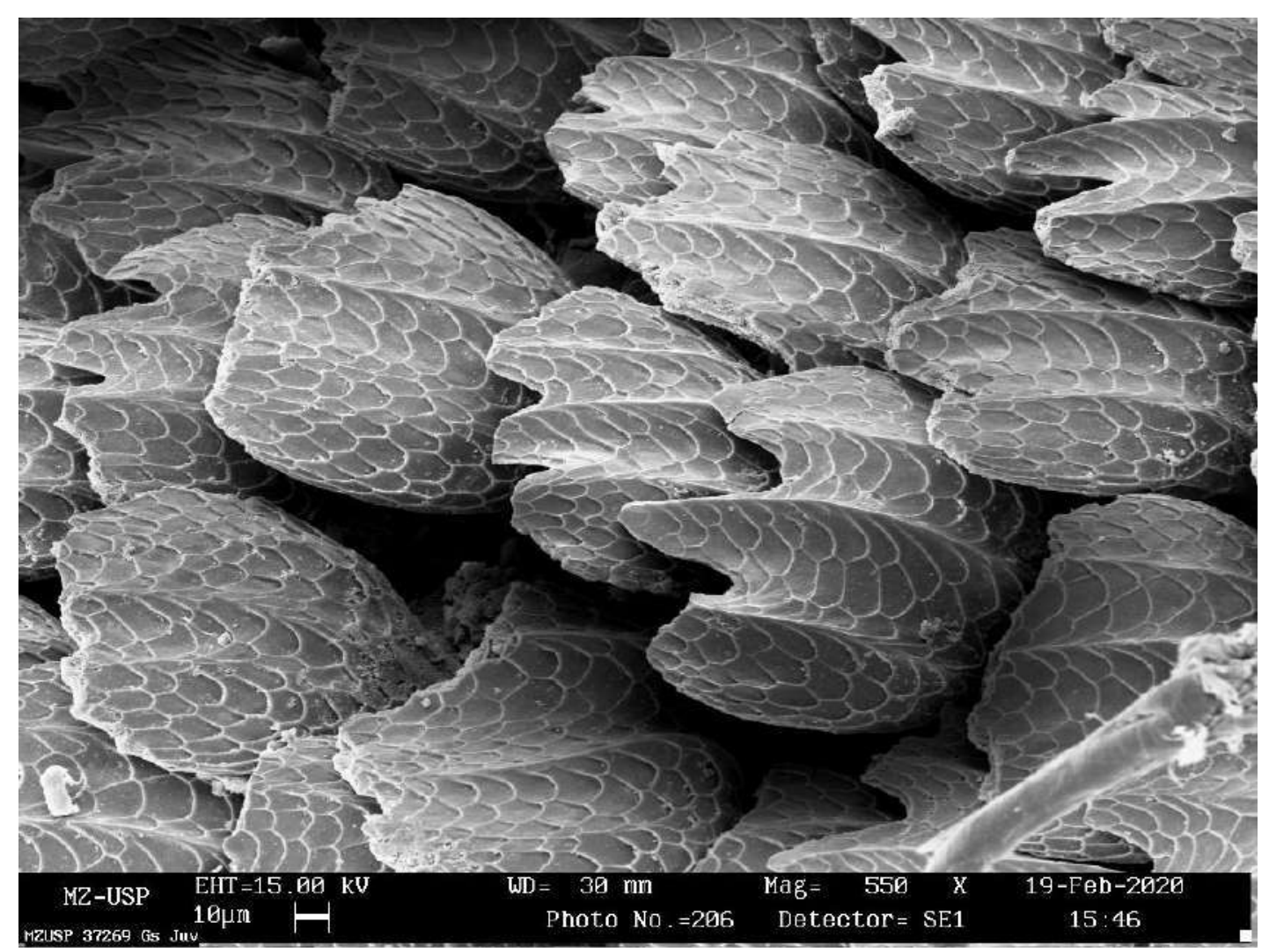

Figure 58. Dermal denticles collected from the section GS (see fig. 1) of the juvenile Isogomphodon oxyrhynchus (MZUSP 37289). Additional information in the image footer.

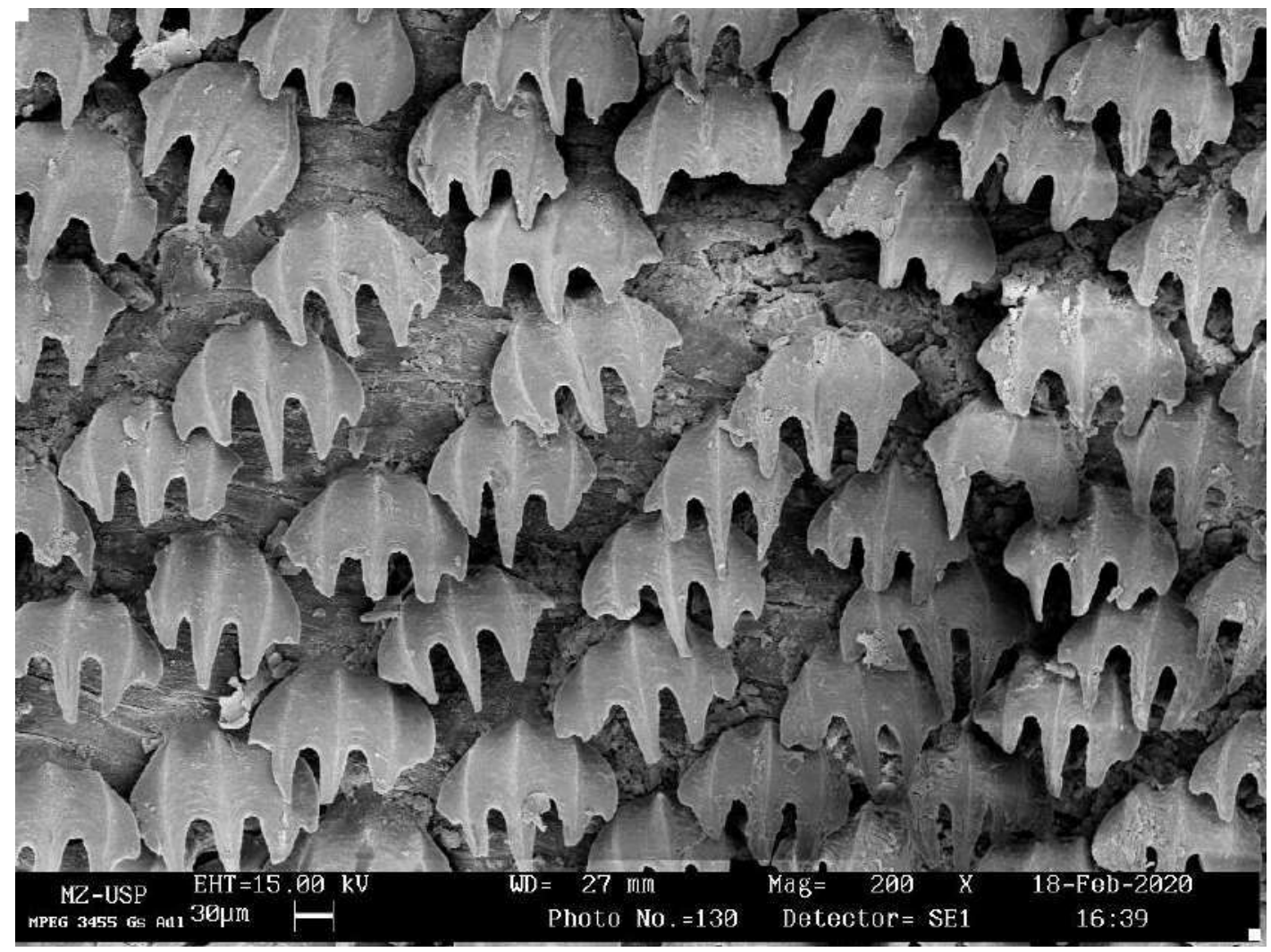

Figure 59. Dermal denticles collected from the section GS (see fig. 1) of the adult Isogomphodon oxyrhynchus (MPEG 3455). Additional information in the image footer. 


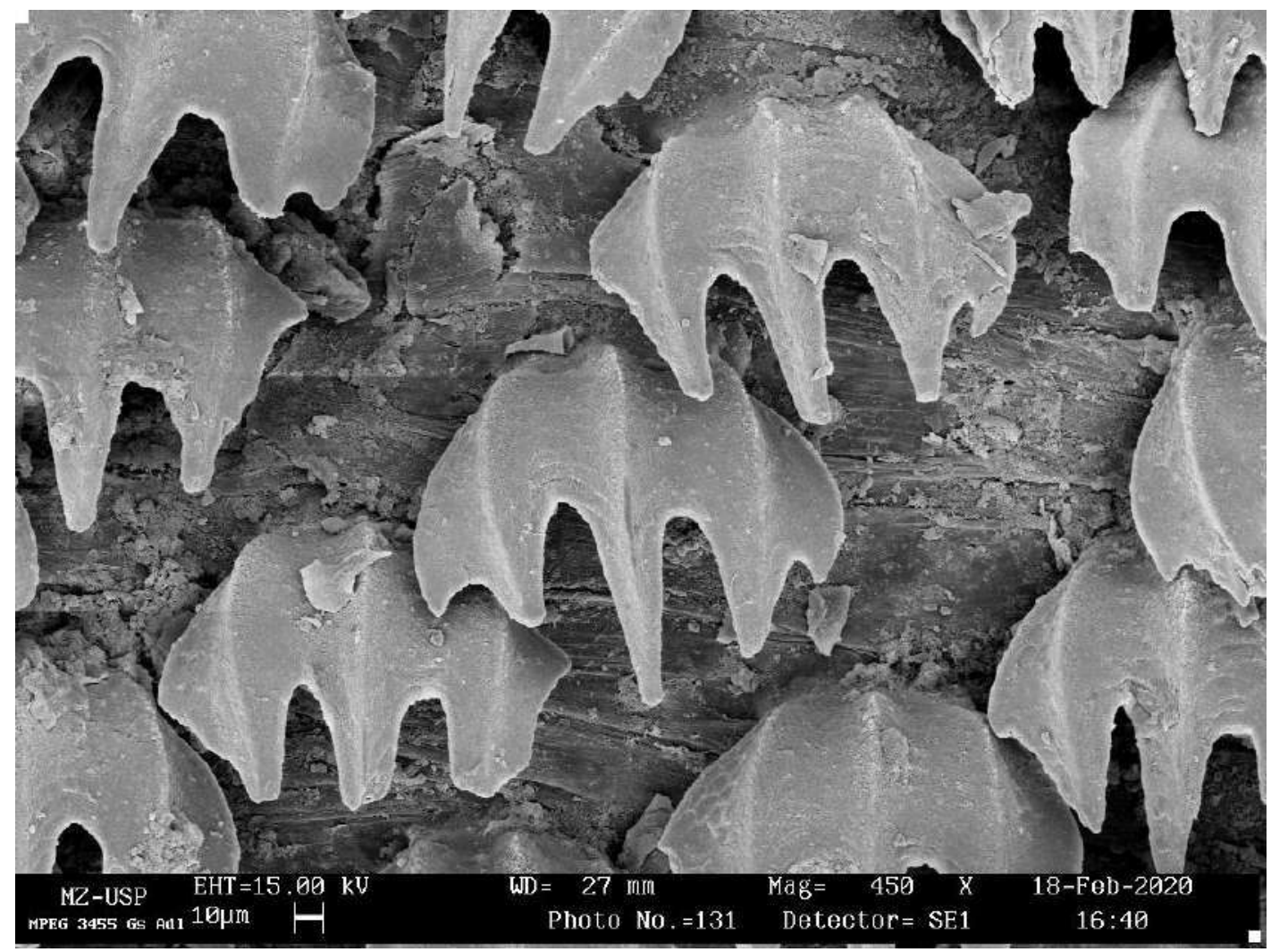

Figure 60. Dermal denticles collected from the section GS (see fig. 1) of the adult Isogomphodon oxyrhynchus (MPEG 3455). Additional information in the image footer.

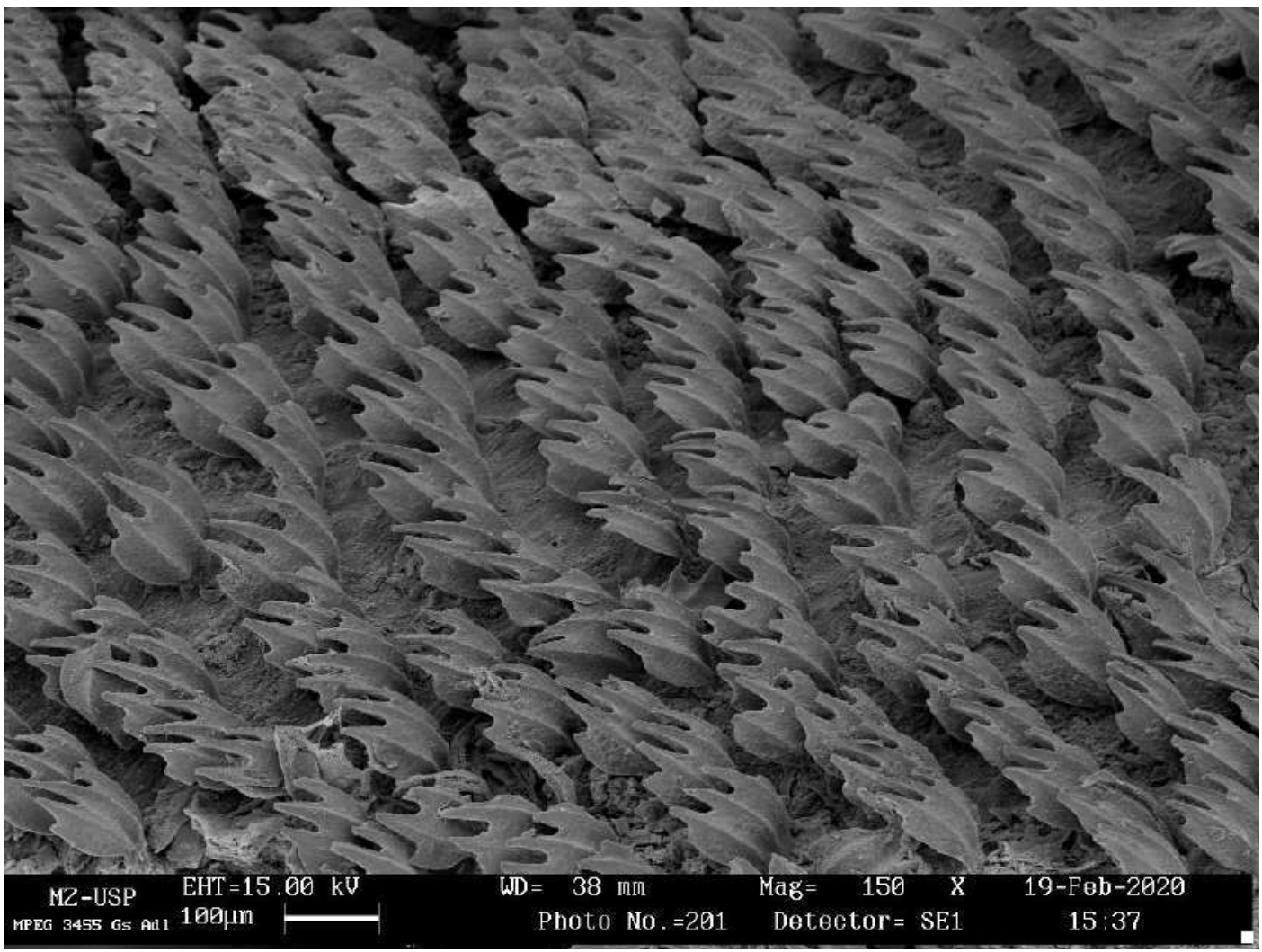

Figure 61. Dermal denticles collected from the section GS (see fig. 1) of the adult Isogomphodon oxyrhynchus (MPEG 3455). Additional information in the image footer. 


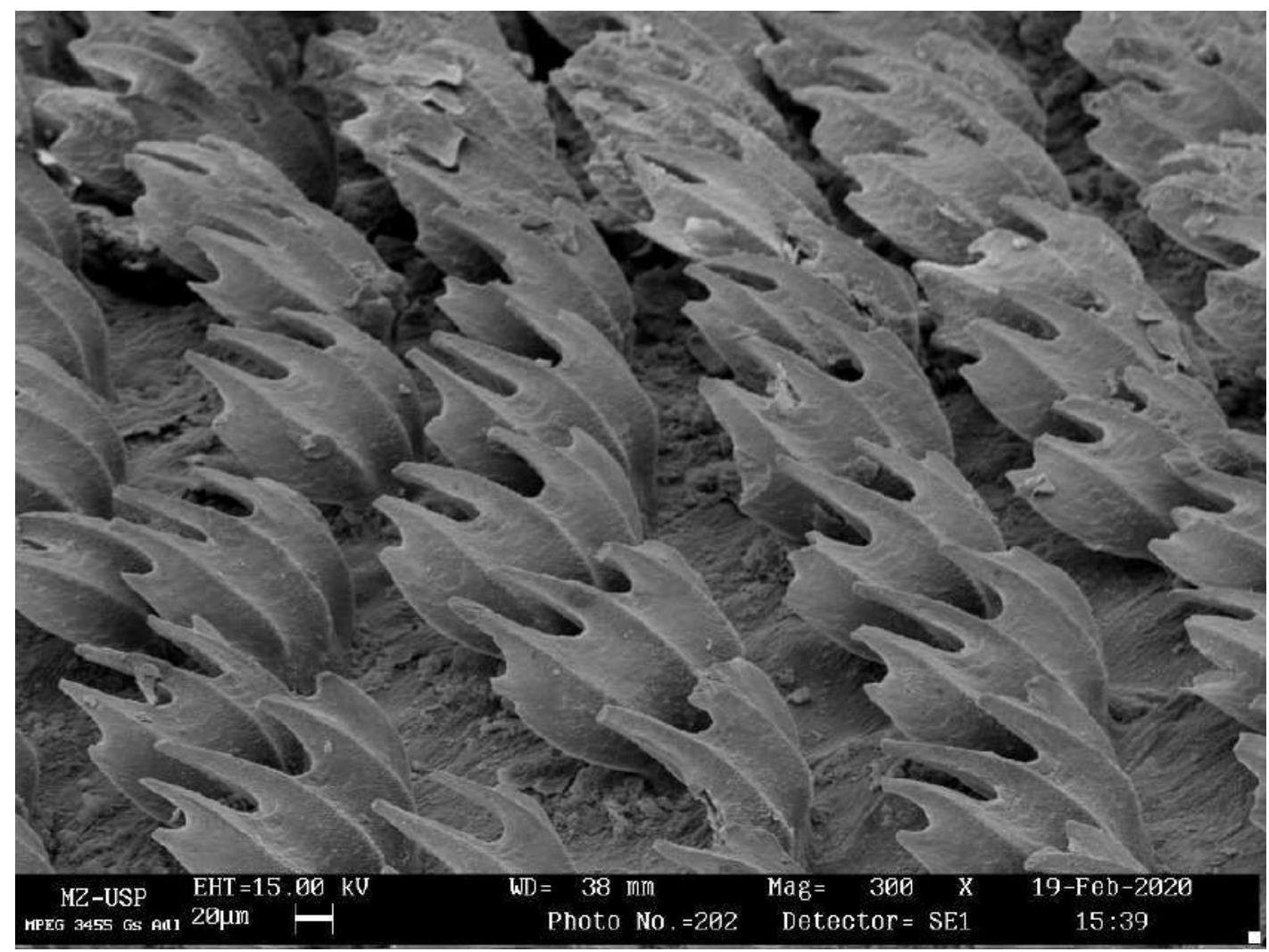

Figure 62. Dermal denticles collected from the section GS (see fig. 1) of the adult Isogomphodon oxyrhynchus (MPEG 3455). Additional information in the image footer.

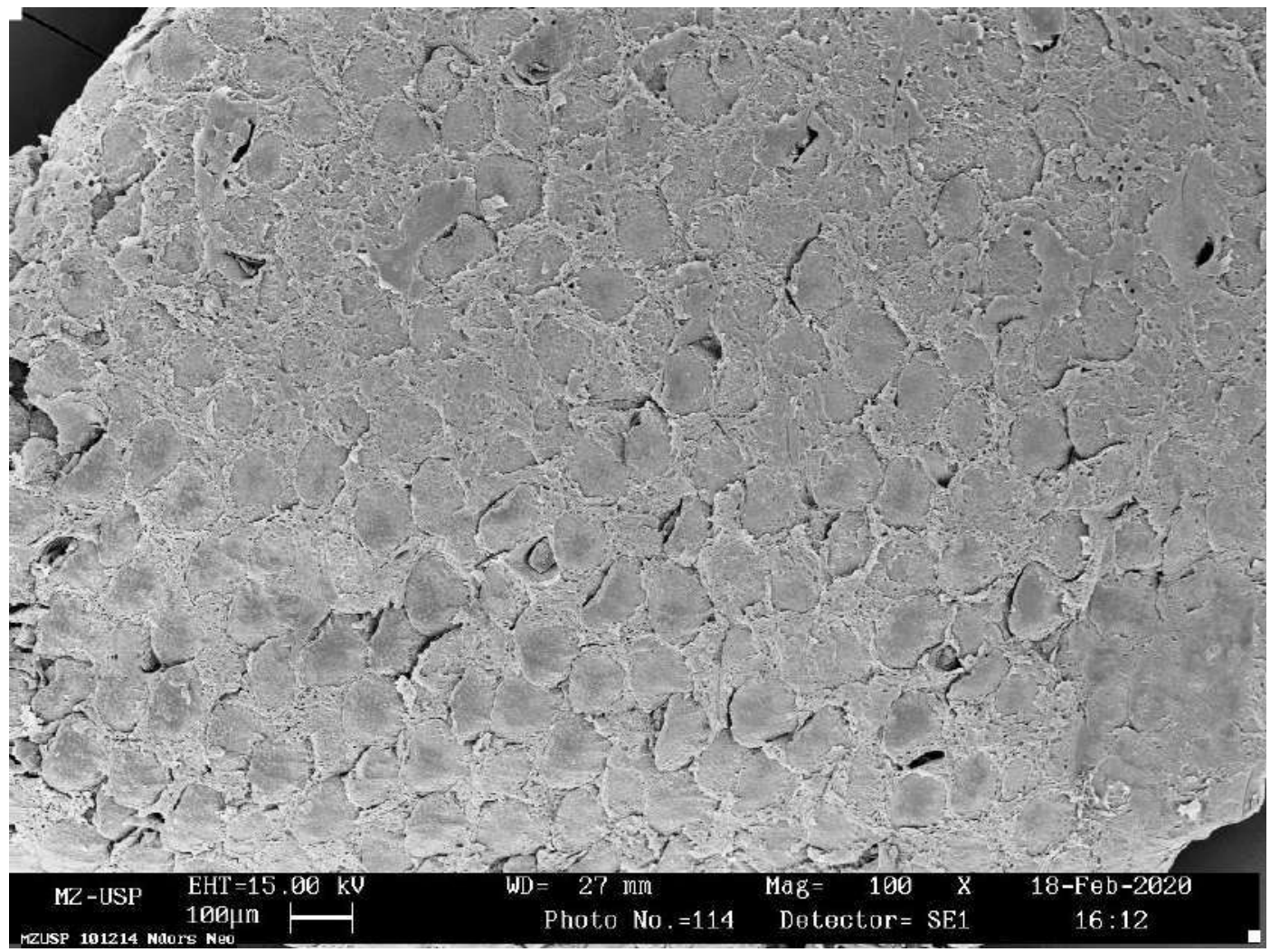

Figure 63. Dermal denticles collected from the section NDors (see fig. 1) of the neonate Isogomphodon oxyrhynchus (MZUSP 101214). Additional information in the image footer. 


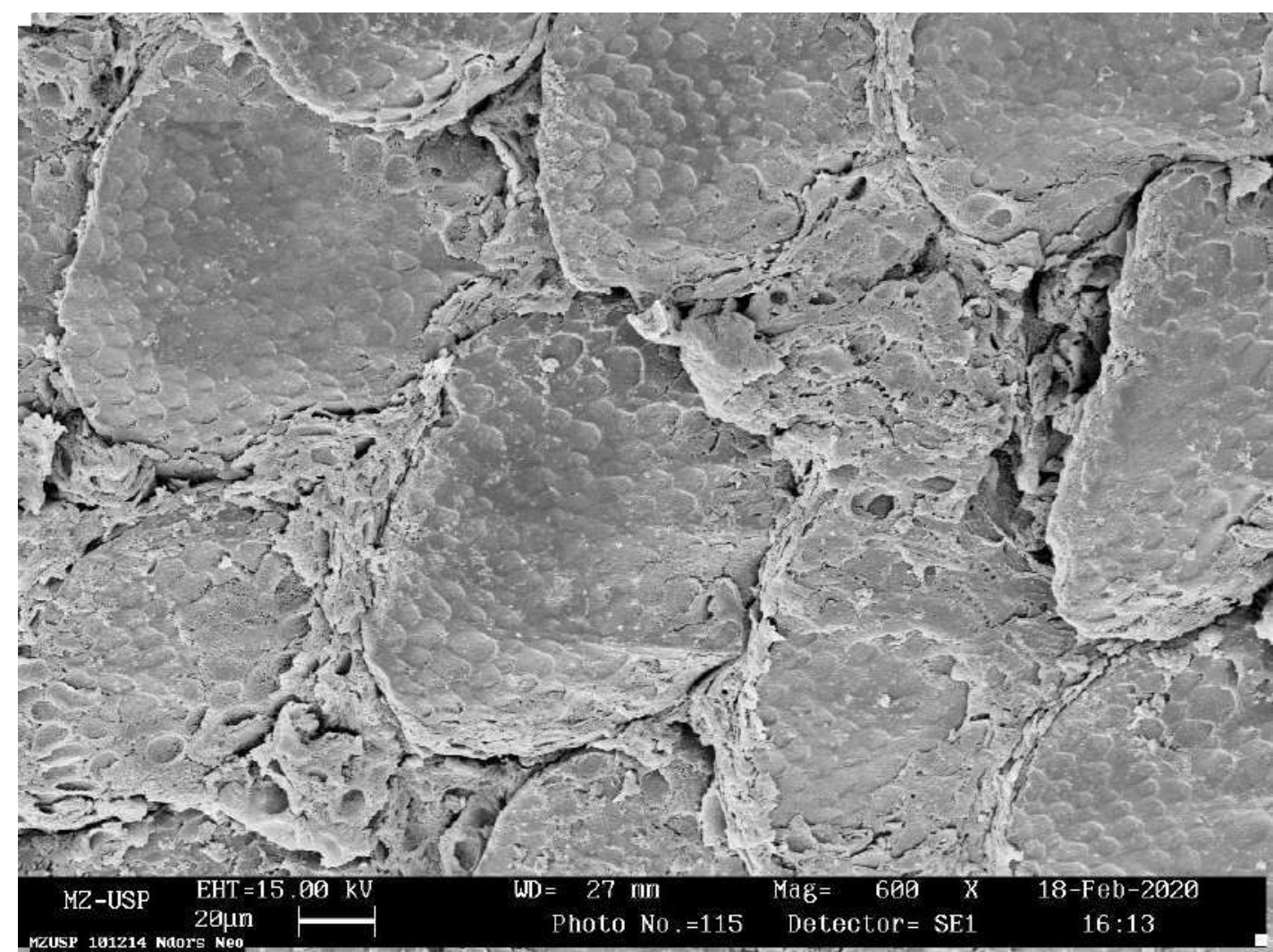

Figure 64. Dermal denticles collected from the section NDors (see fig. 1) of the neonate Isogomphodon oxyrhynchus (MZUSP 101214). Additional information in the image footer.

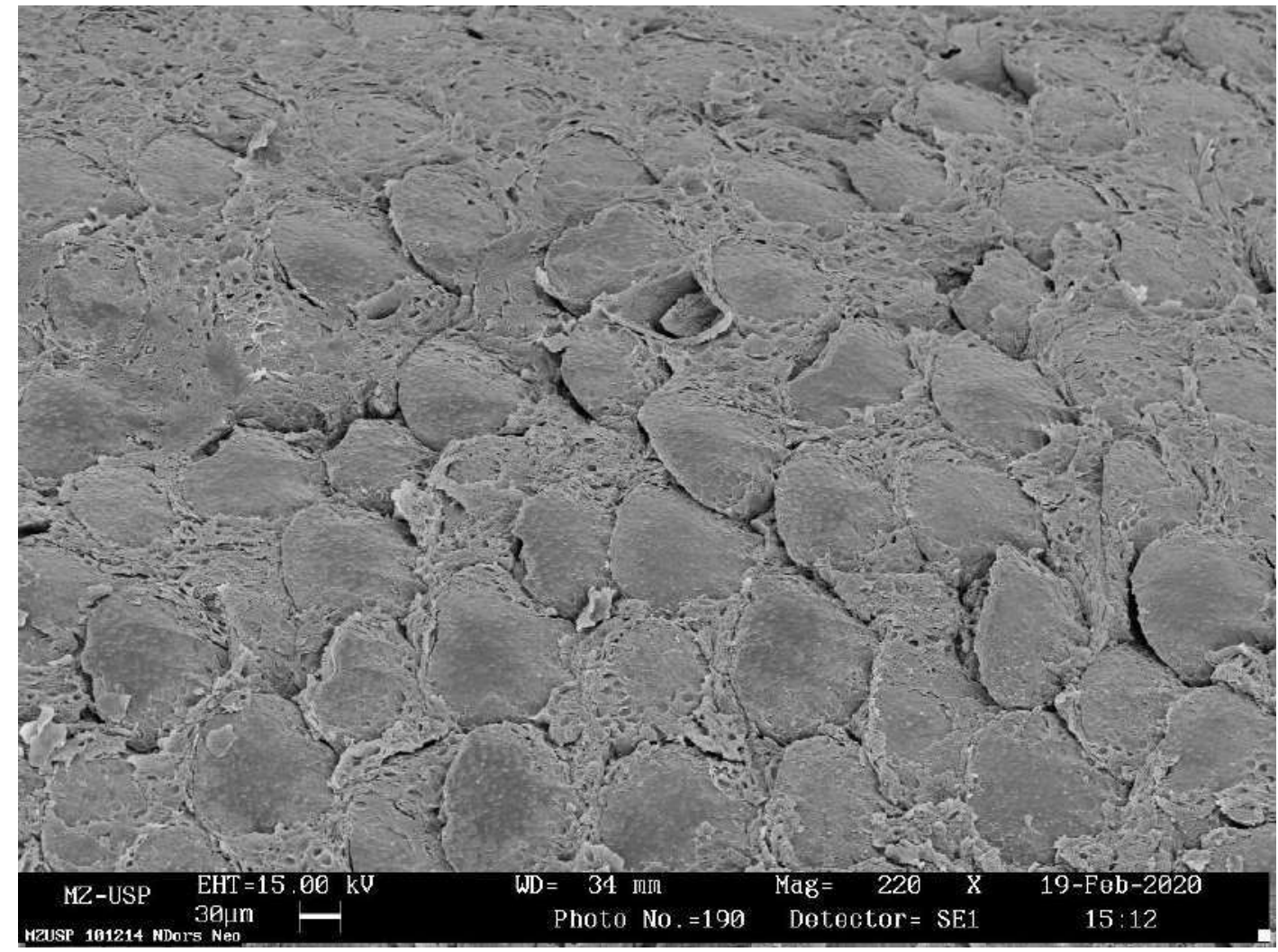

Figure 65. Dermal denticles collected from the section NDors (see fig. 1) of the neonate Isogomphodon oxyrhynchus (MZUSP 101214). Additional information in the image footer. 


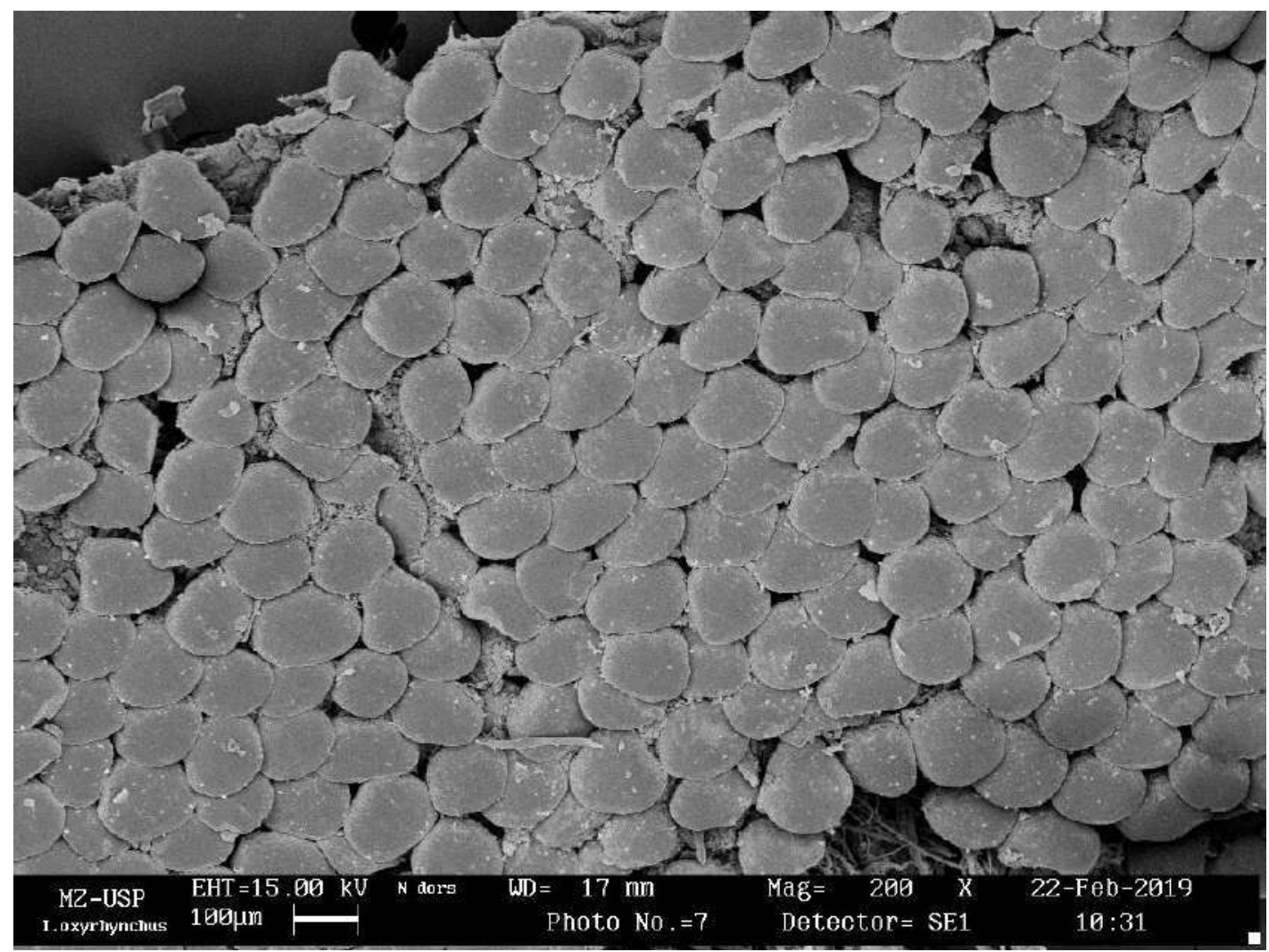

Figure 66. Dermal denticles collected from the section NDors (see fig. 1) of the juvenile Isogomphodon oxyrhynchus (MZUSP 37289). Additional information in the image footer.

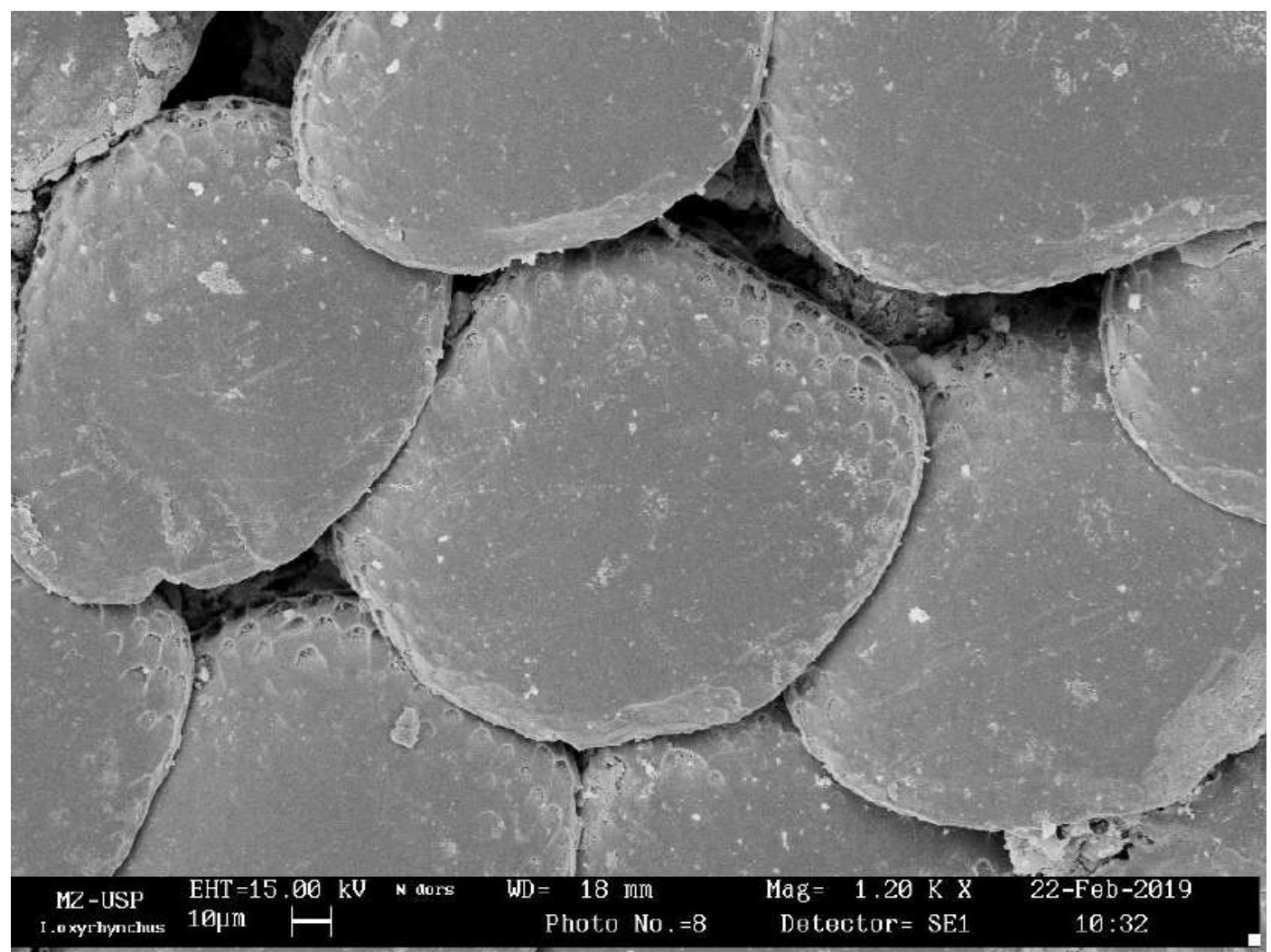

Figure 67. Dermal denticles collected from the section NDors (see fig. 1) of the juvenile Isogomphodon oxyrhynchus (MZUSP 37289). Additional information in the image footer. 


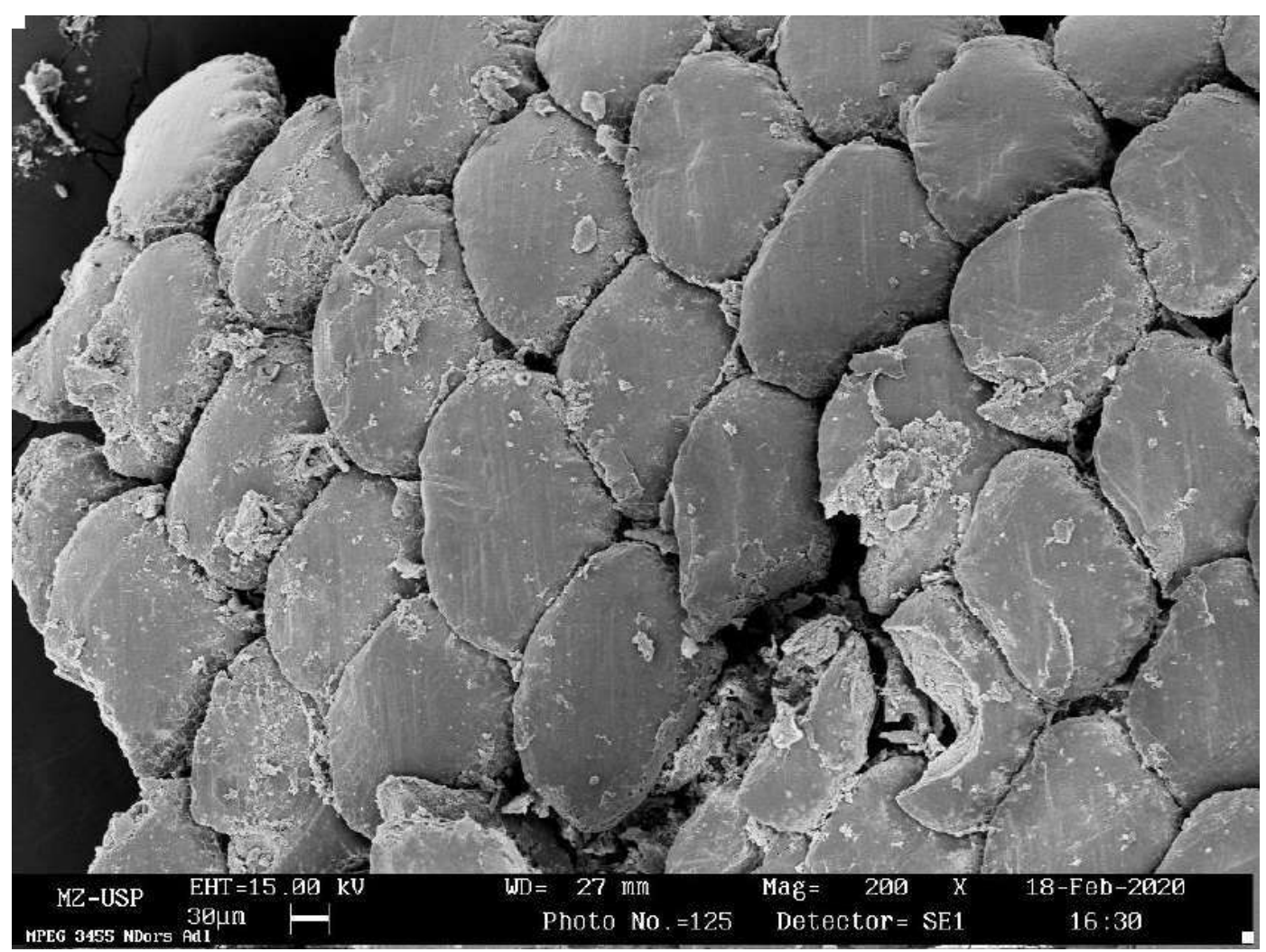

Figure 68. Dermal denticles collected from the section NDors (see fig. 1) of the adult Isogomphodon oxyrhynchus (MPEG 3455). Additional information in the image footer.

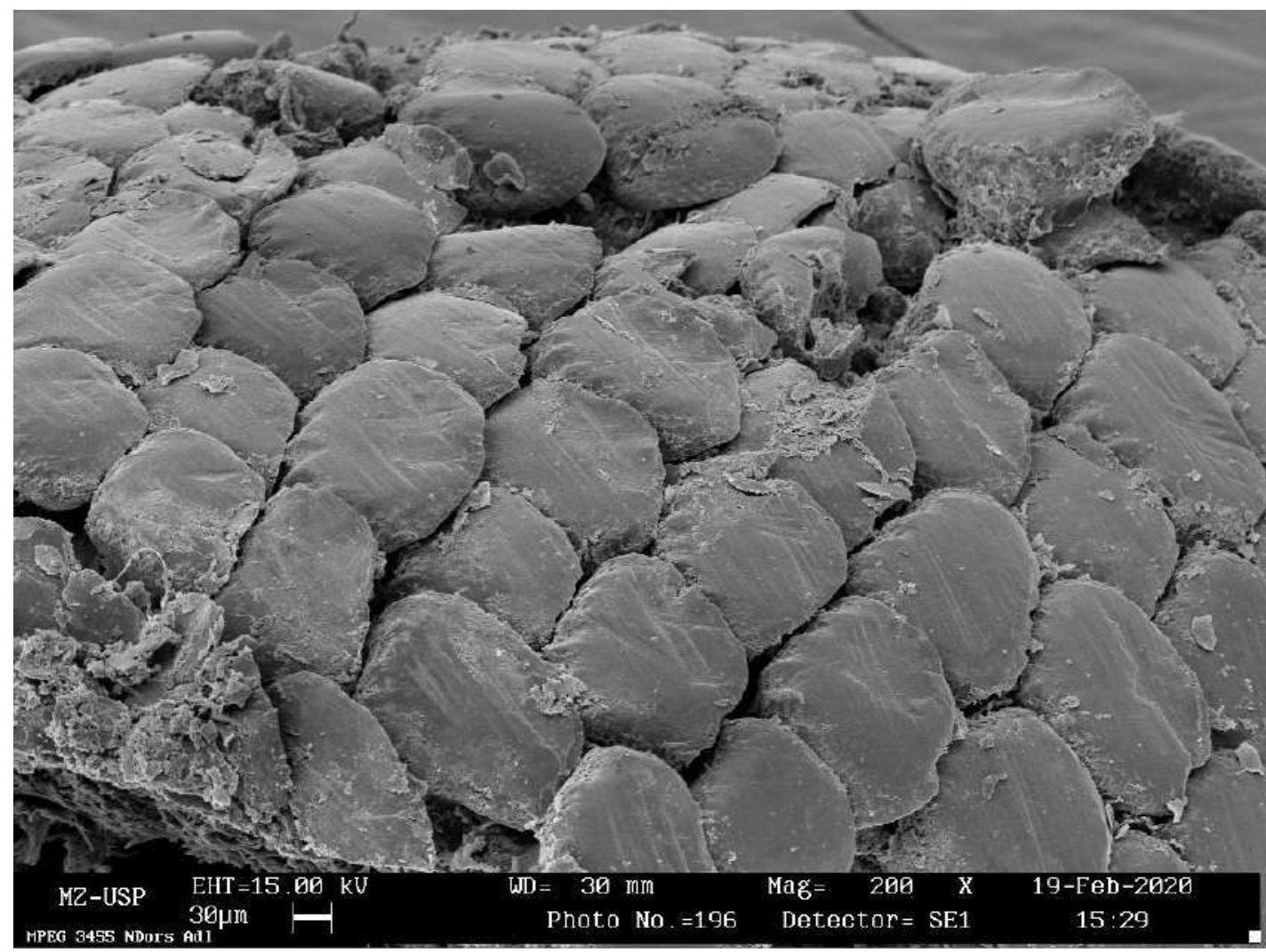

Figure 69. Dermal denticles collected from the section NDors (see fig. 1) of the adult Isogomphodon oxyrhynchus (MPEG 3455). Additional information in the image footer. 


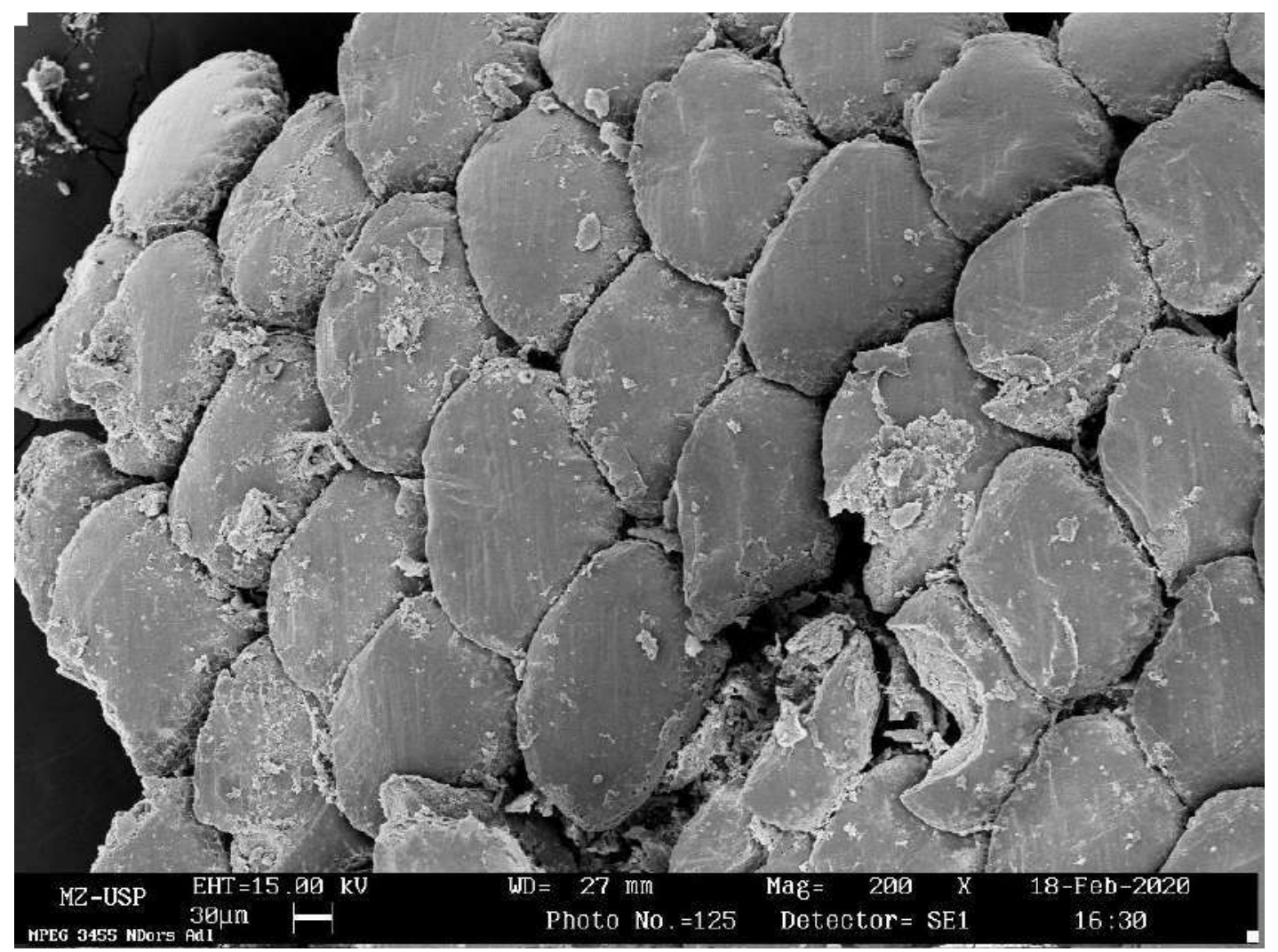

Figure 70. Dermal denticles collected from the section NDors (see fig. 1) of the adult Isogomphodon oxyrhynchus (MPEG 3455). Additional information in the image footer.

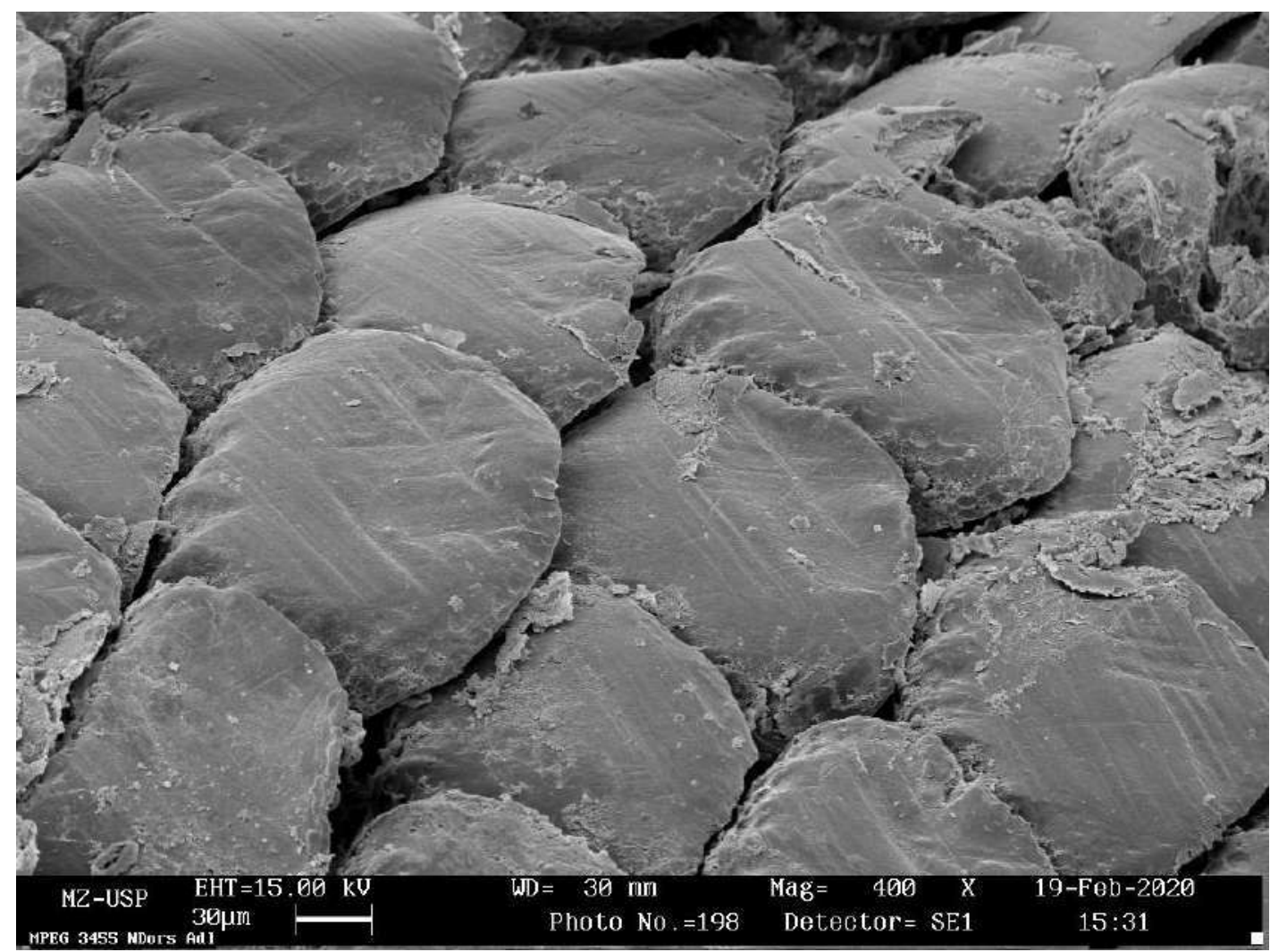

Figure 71. Dermal denticles collected from the section NDors (see fig. 1) of the adult Isogomphodon oxyrhynchus (MPEG 3455). Additional information in the image footer. 


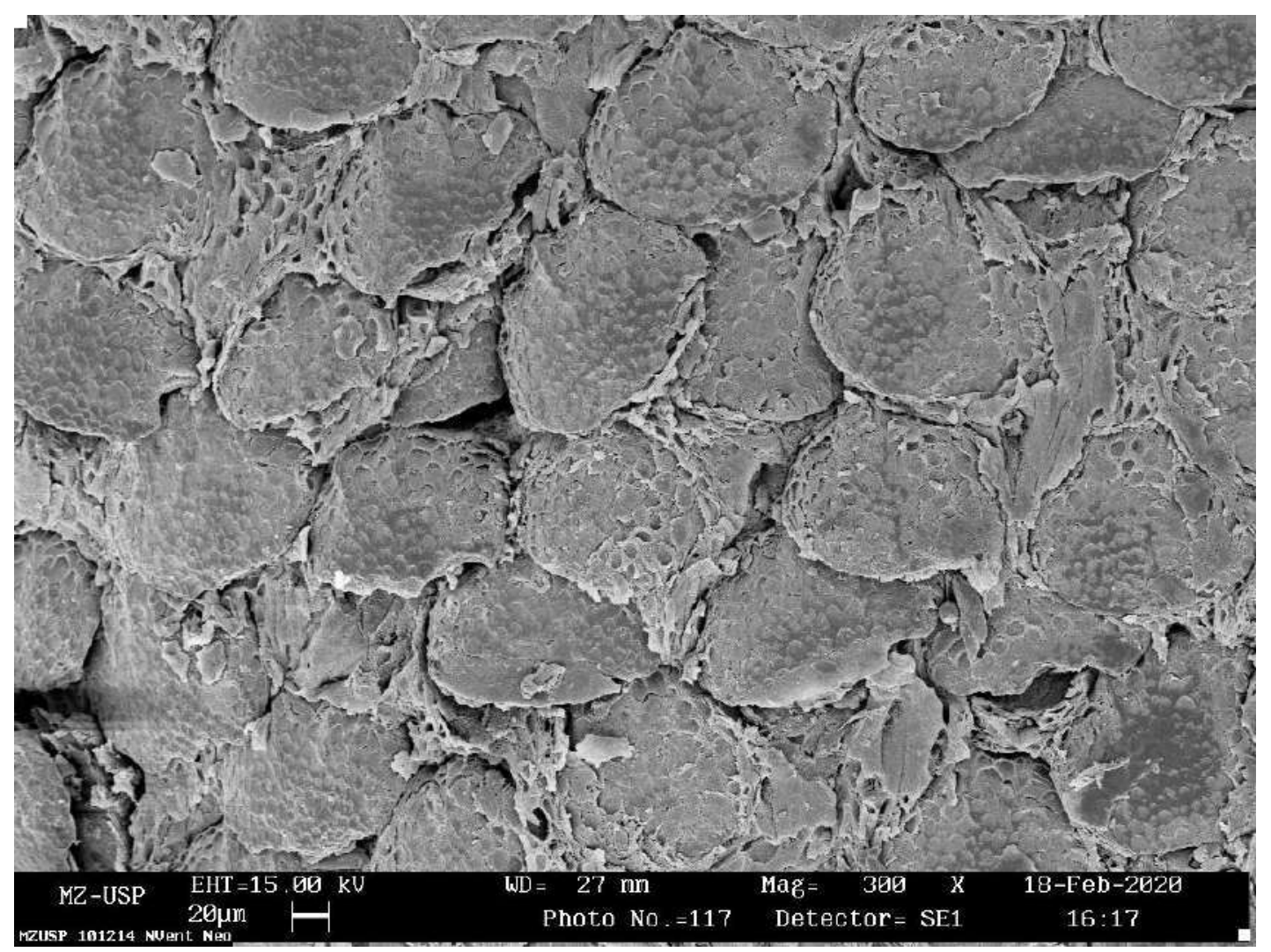

Figure 72. Dermal denticles collected from the section NVent (see fig. 1) of the neonate Isogomphodon oxyrhynchus (MZUSP 101214). Additional information in the image footer.

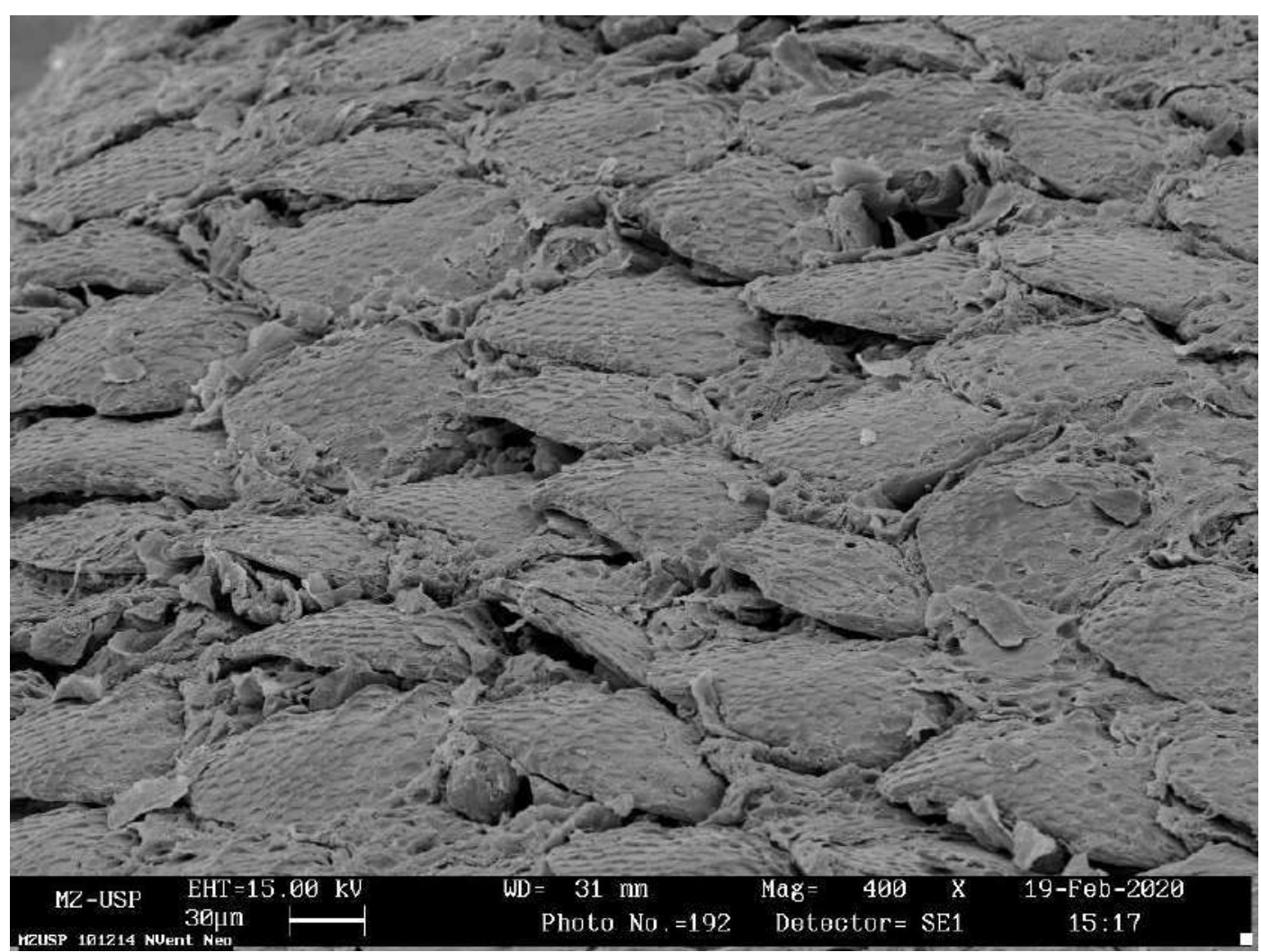

Figure 73. Dermal denticles collected from the section NVent (see fig. 1) of the neonate Isogomphodon oxyrhynchus (MZUSP 101214). Additional information in the photo footer. 


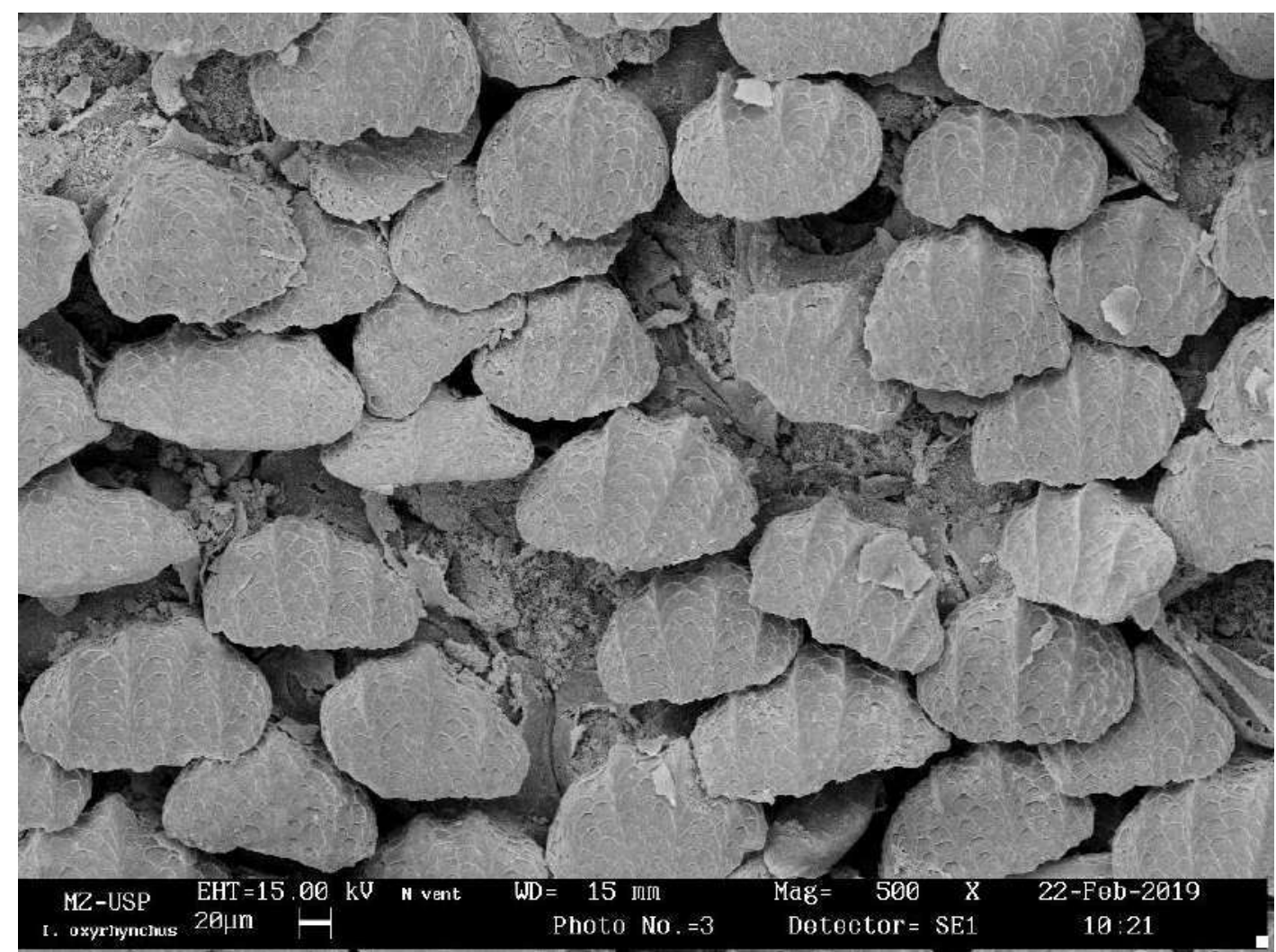

Figure 74. Dermal denticles collected from the section NVent (see fig. 1) of the juvenile Isogomphodon oxyrhynchus (MZUSP 37289). Additional information in the image footer.

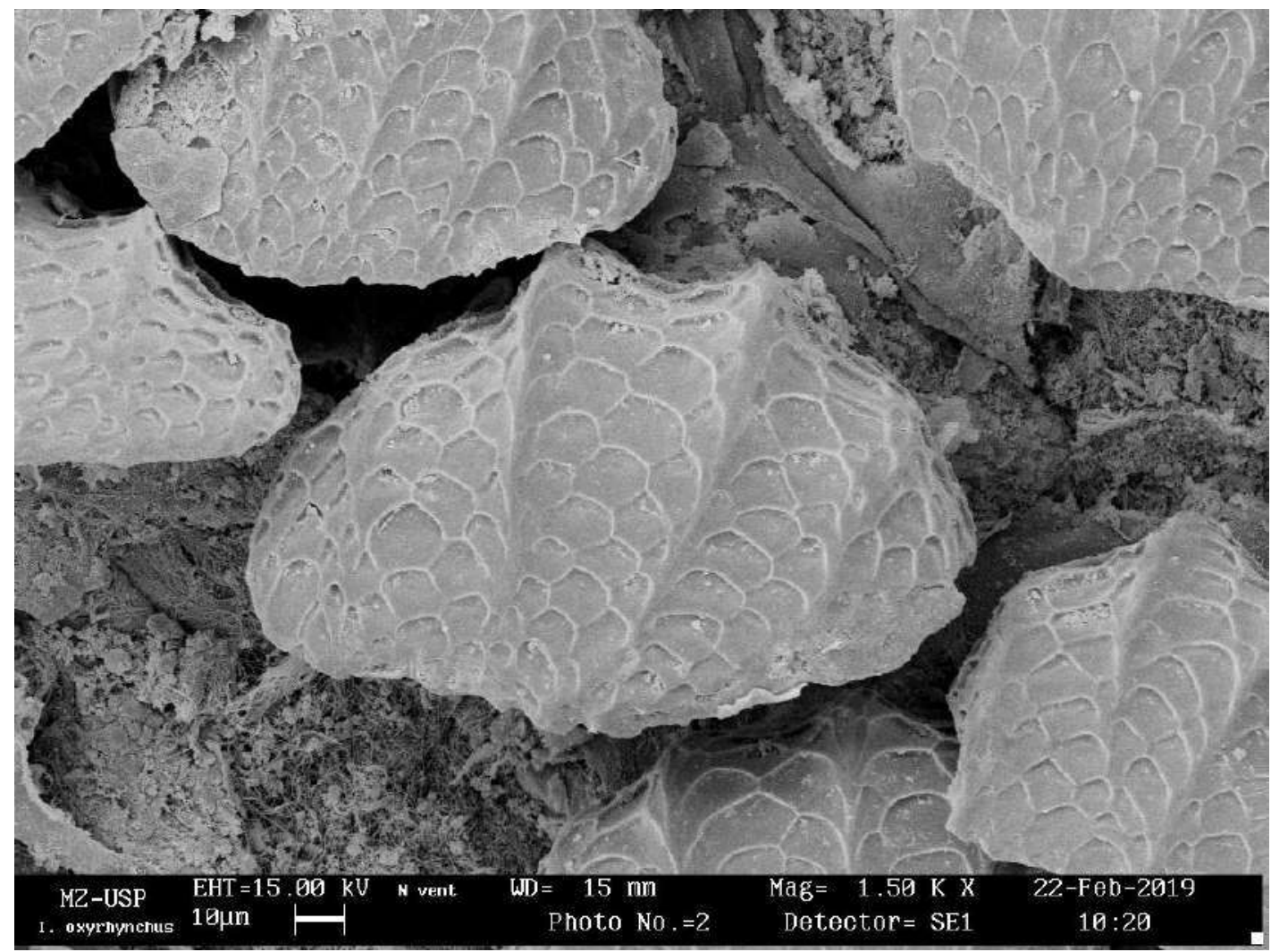

Figure 75. Dermal denticles collected from the section NVent (see fig. 1) of the juvenile Isogomphodon oxyrhynchus (MZUSP 37289). Additional information in the image footer. 


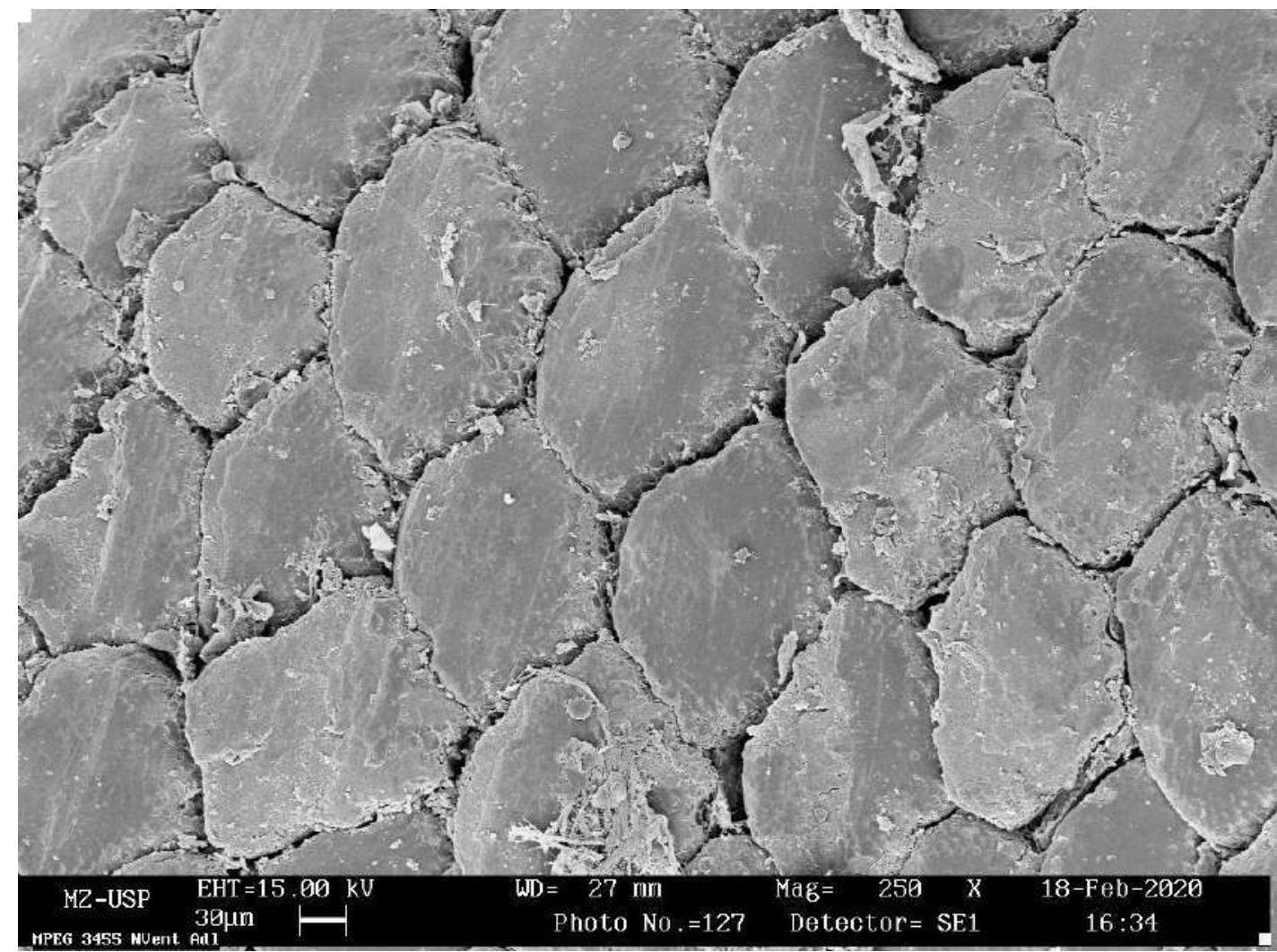

Figure 76. Dermal denticles collected from the section NVent (see fig. 1) of the adult Isogomphodon oxyrhynchus (MPEG 3455). Additional information in the image footer.

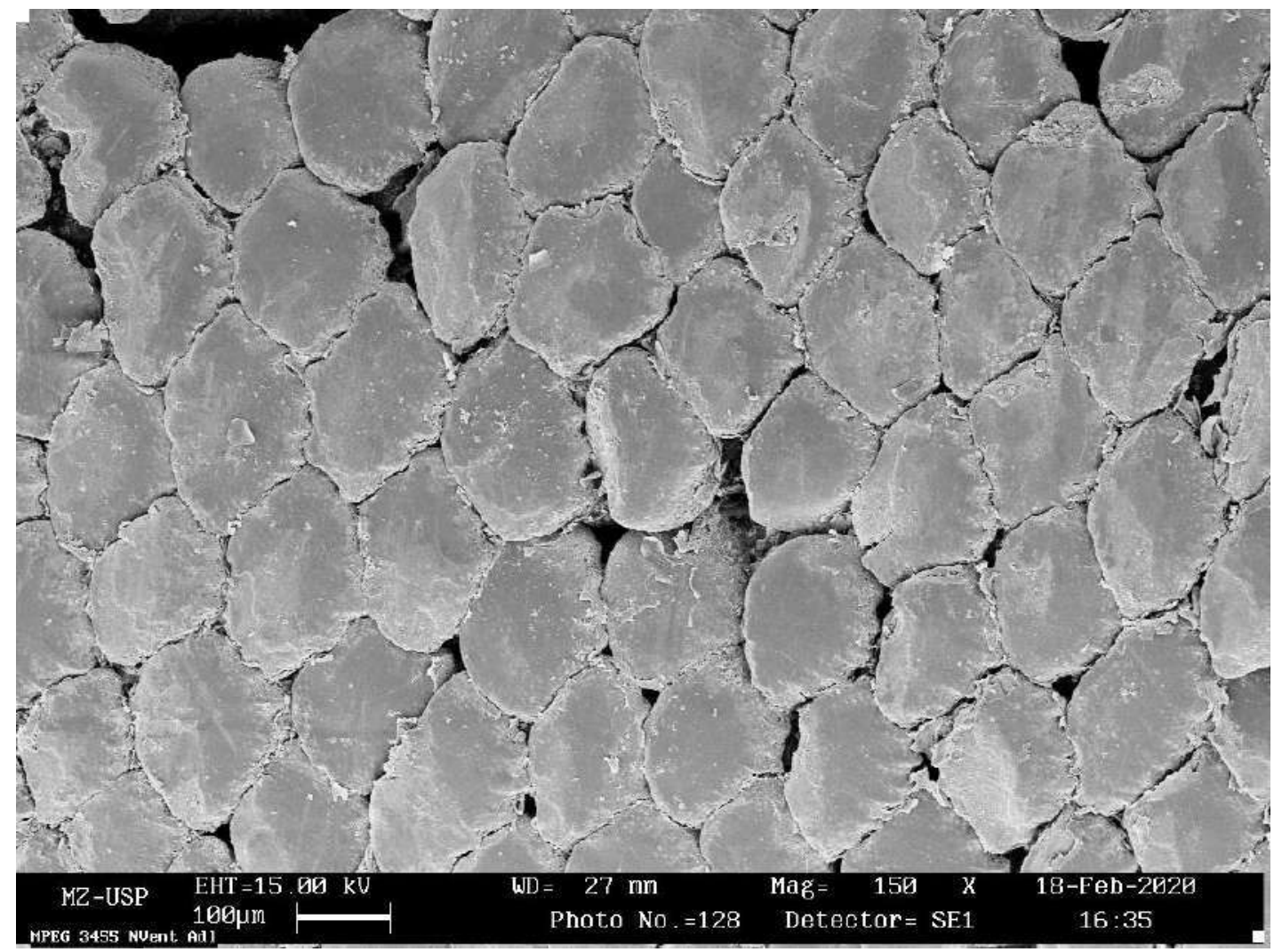

Figure 77. Dermal denticles collected from the section NVent (see fig. 1) of the adult Isogomphodon oxyrhynchus (MPEG 3455). Additional information in the image footer. 


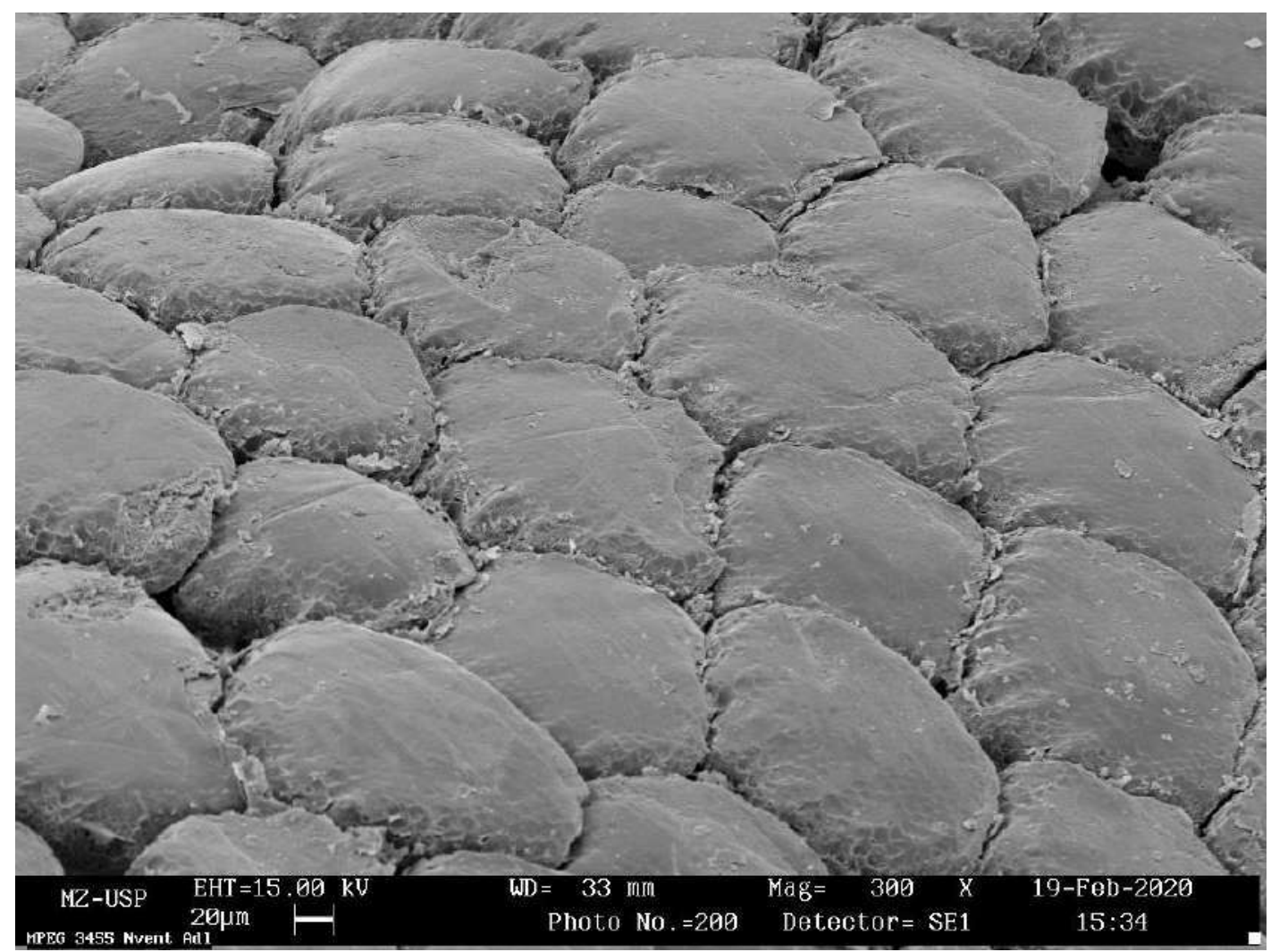

Figure 78. Dermal denticles collected from the section NVent (see fig. 1) of the adult Isogomphodon oxyrhynchus (MPEG 3455). Additional information in the image footer.

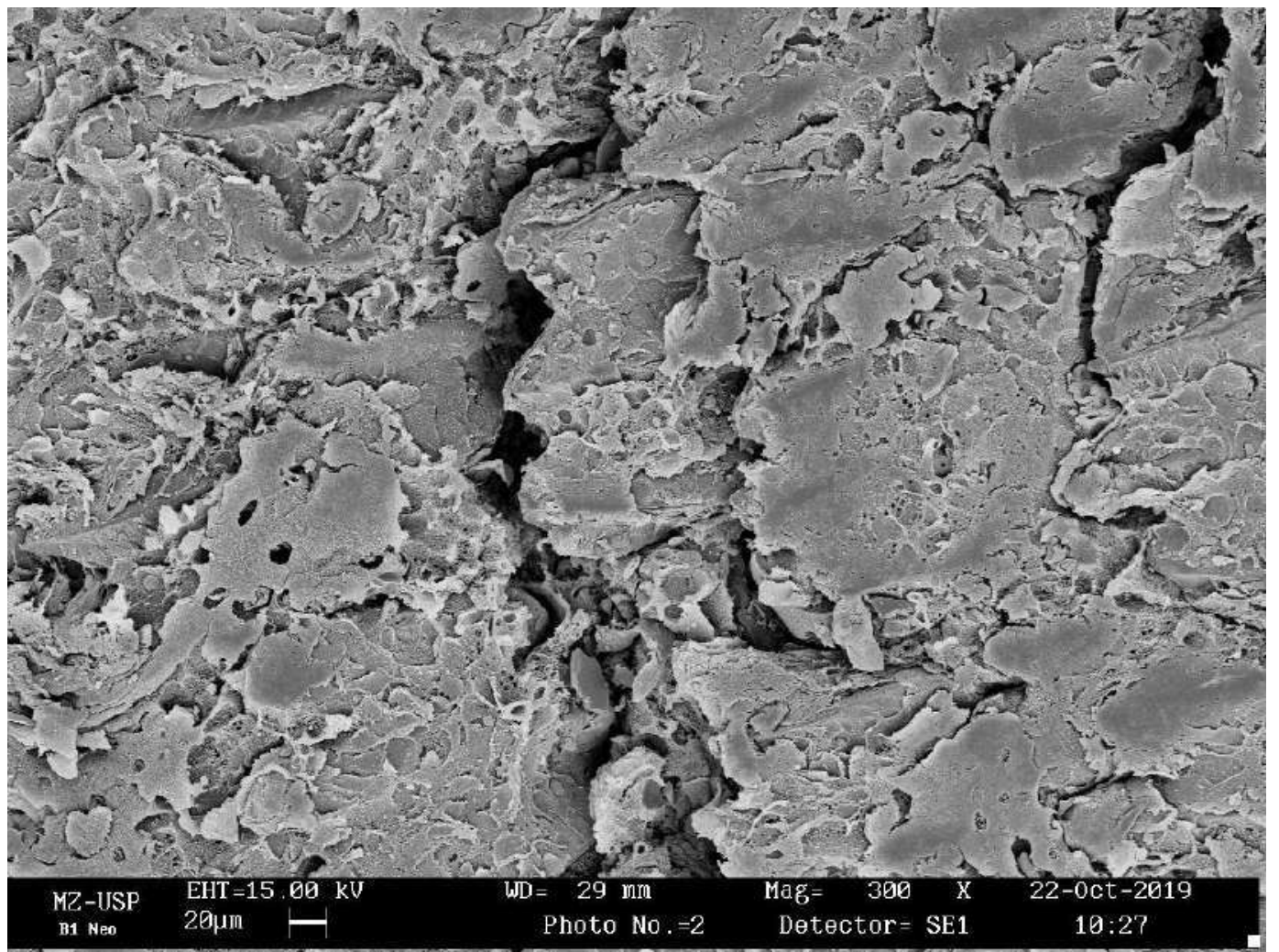

Figure 79. Dermal denticles collected from the section B1 (see fig. 1) of the neonate Isogomphodon oxyrhynchus (MZUSP 101214). Additional information in the image footer. 


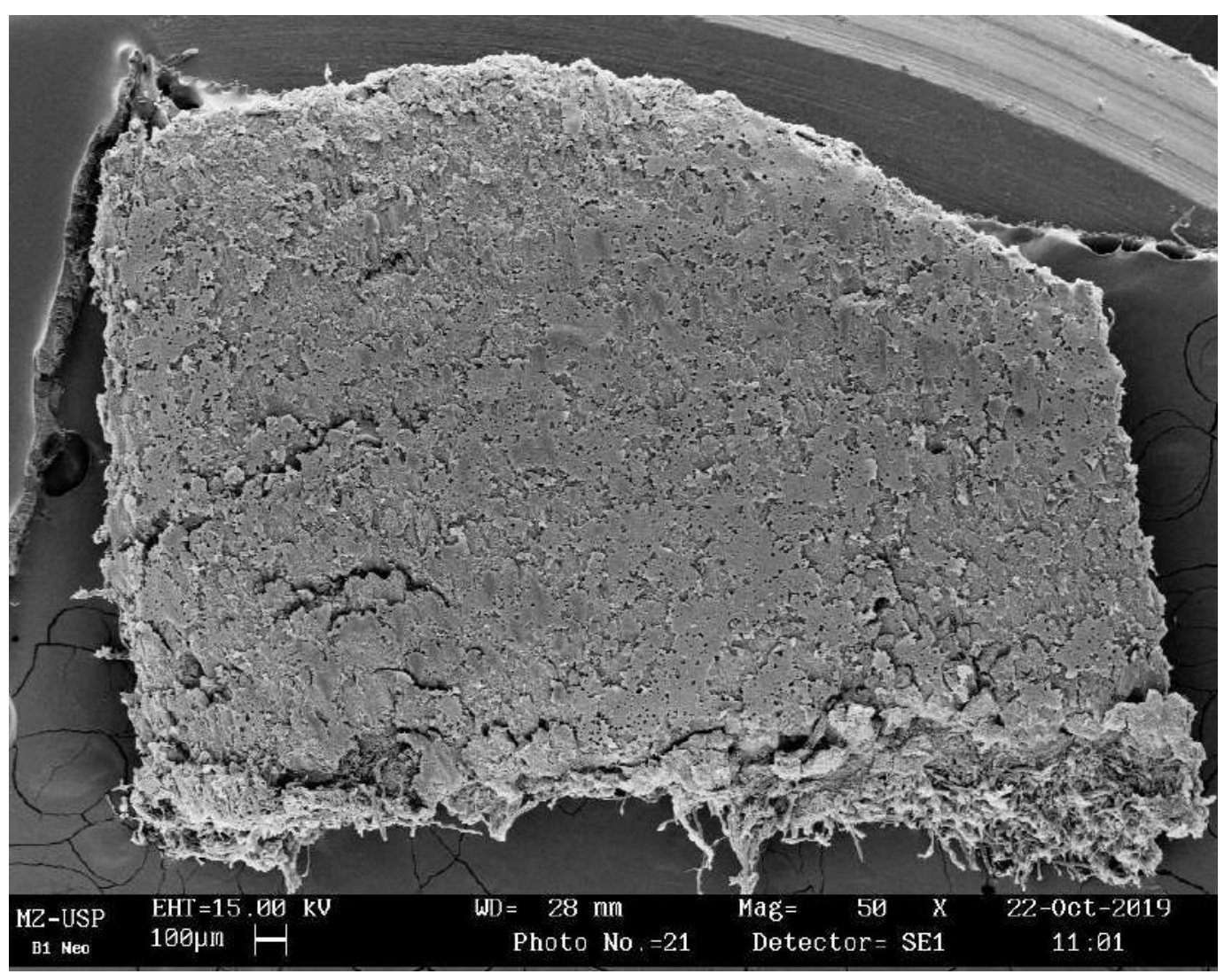

Figure 80. Dermal denticles collected from the section B1 (see fig. 1) of the neonate Isogomphodon oxyrhynchus (MZUSP 101214). Additional information in the image footer.

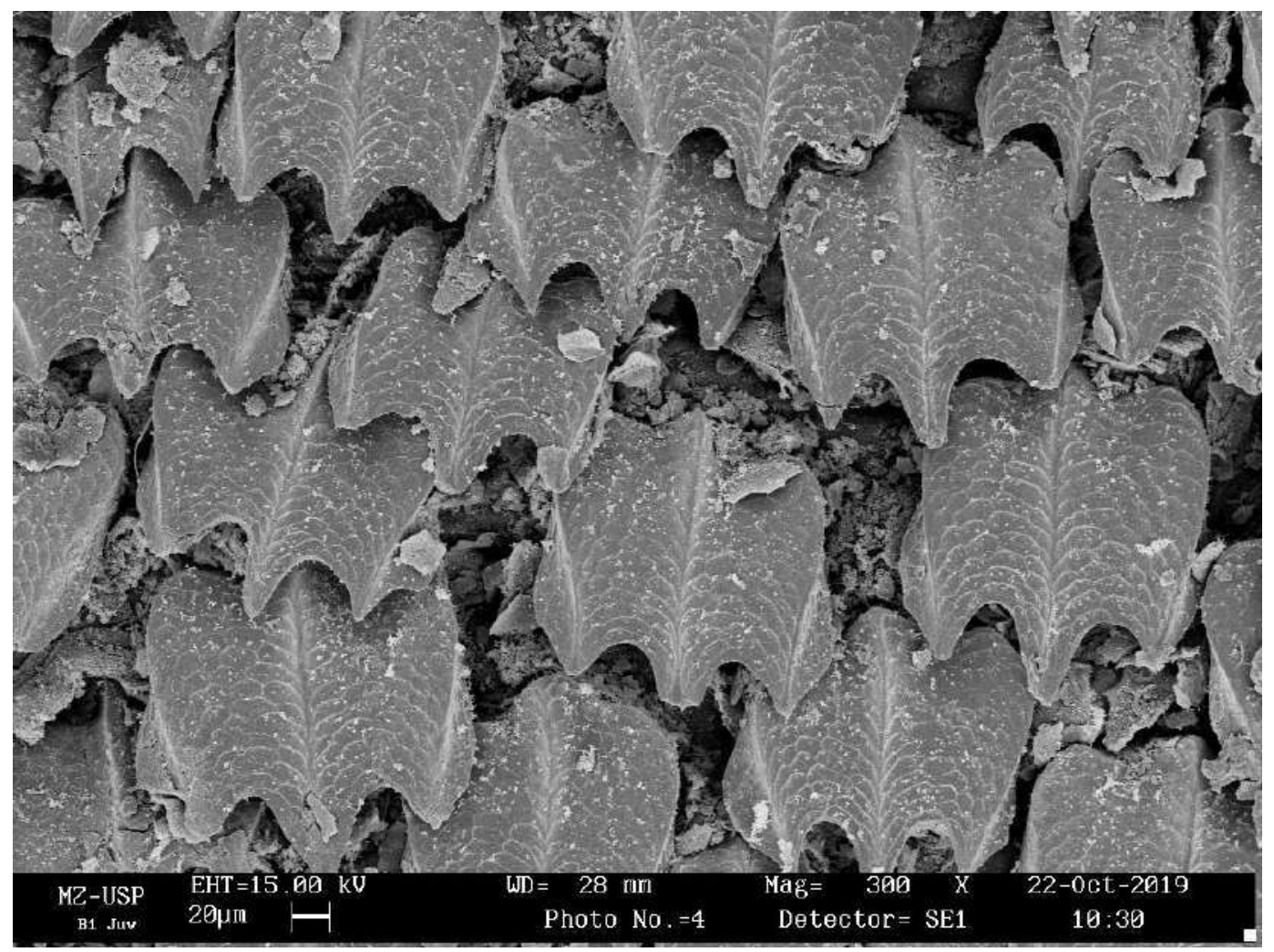

Figure 81. Dermal denticles collected from the section B1 (see fig. 1) of the juvenile Isogomphodon oxyrhynchus (MZUSP 37289). Additional information in the image footer. 


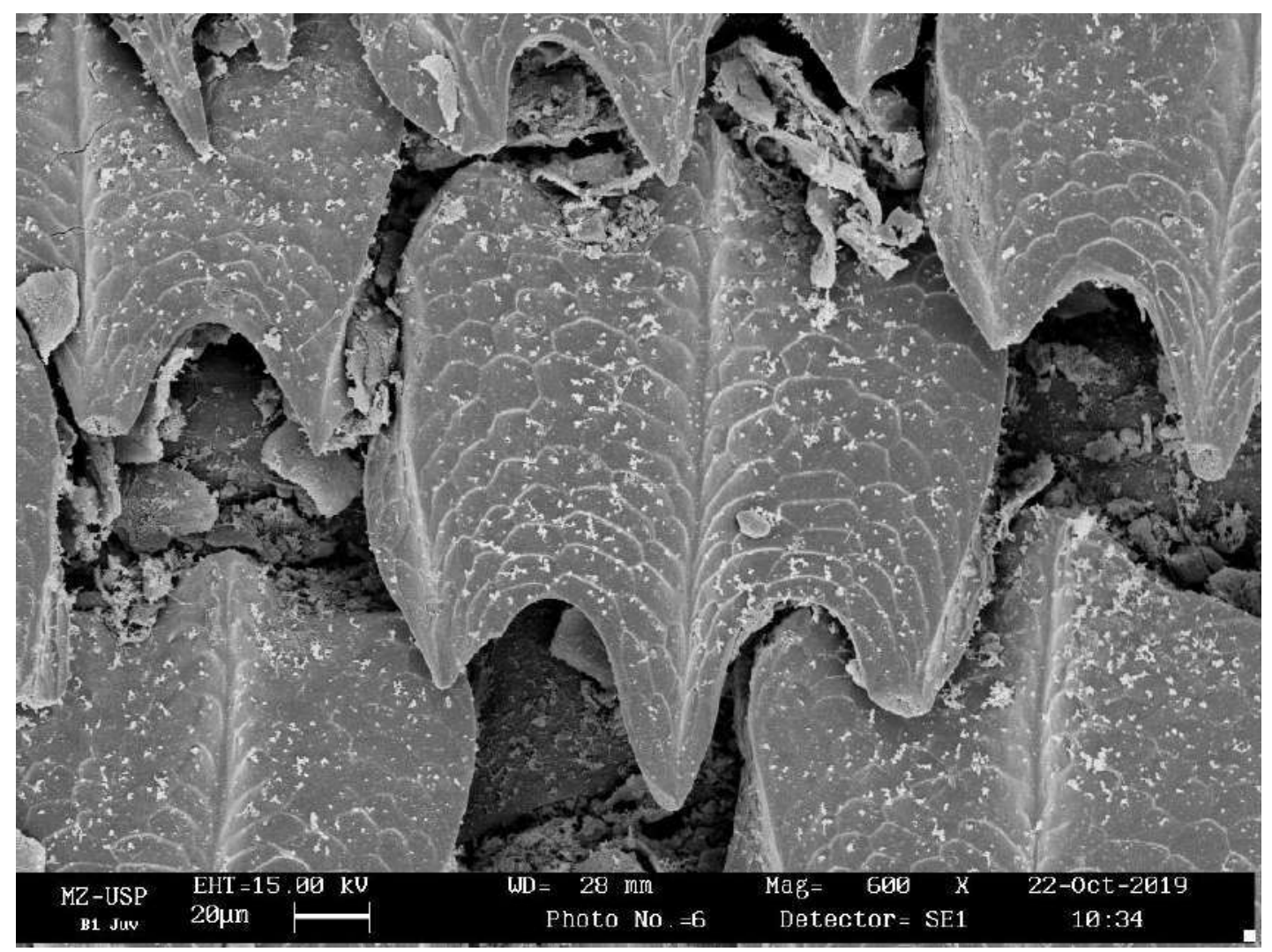

Figure 82. Dermal denticles collected from the section B1 (see fig. 1) of the juvenile Isogomphodon oxyrhynchus (MZUSP 37289). Additional information in the image footer.

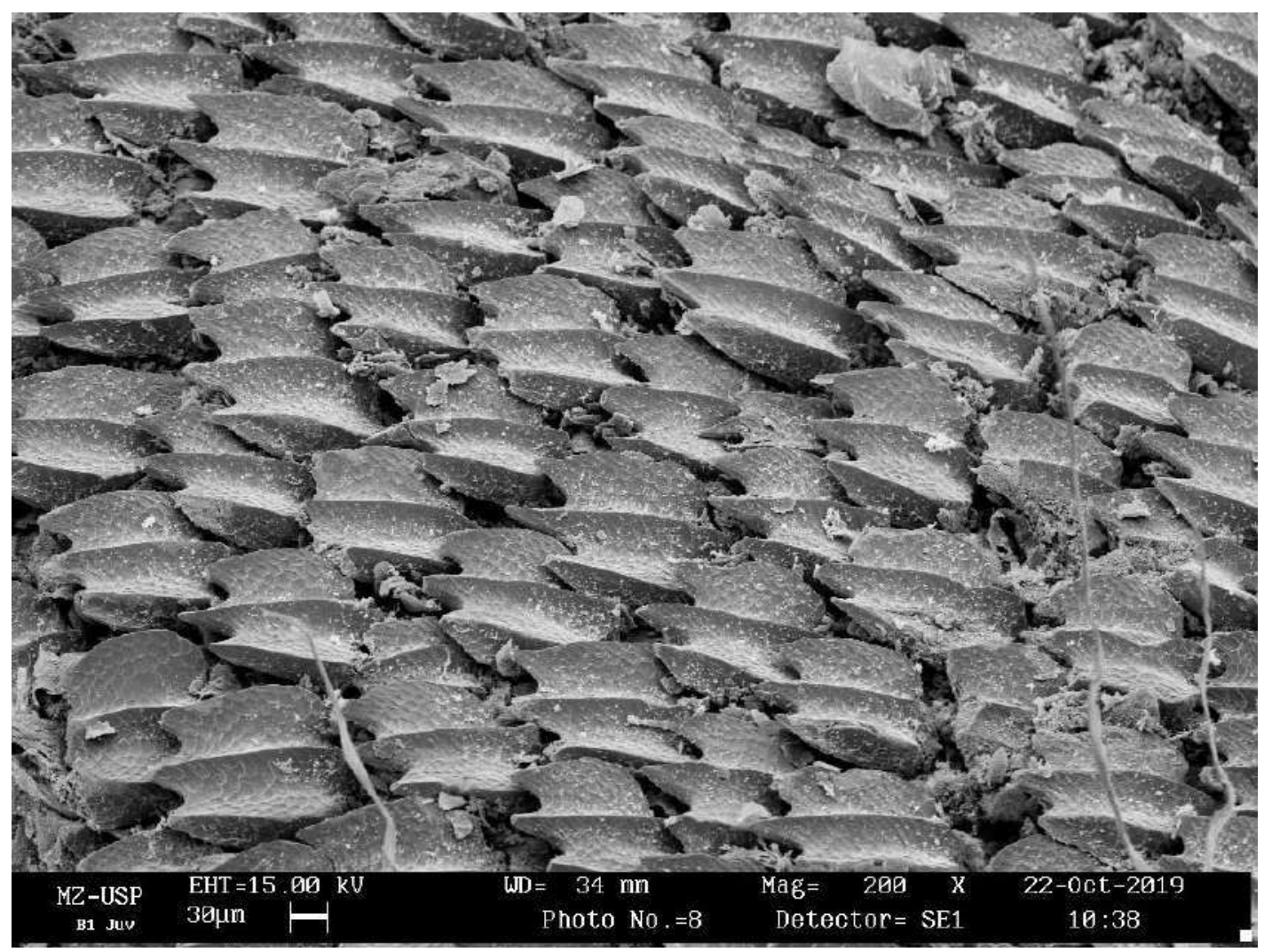

Figure 83. Dermal denticles collected from the section B1 (see fig. 1) of the juvenile Isogomphodon oxyrhynchus (MZUSP 37289). Additional information in the image footer. 


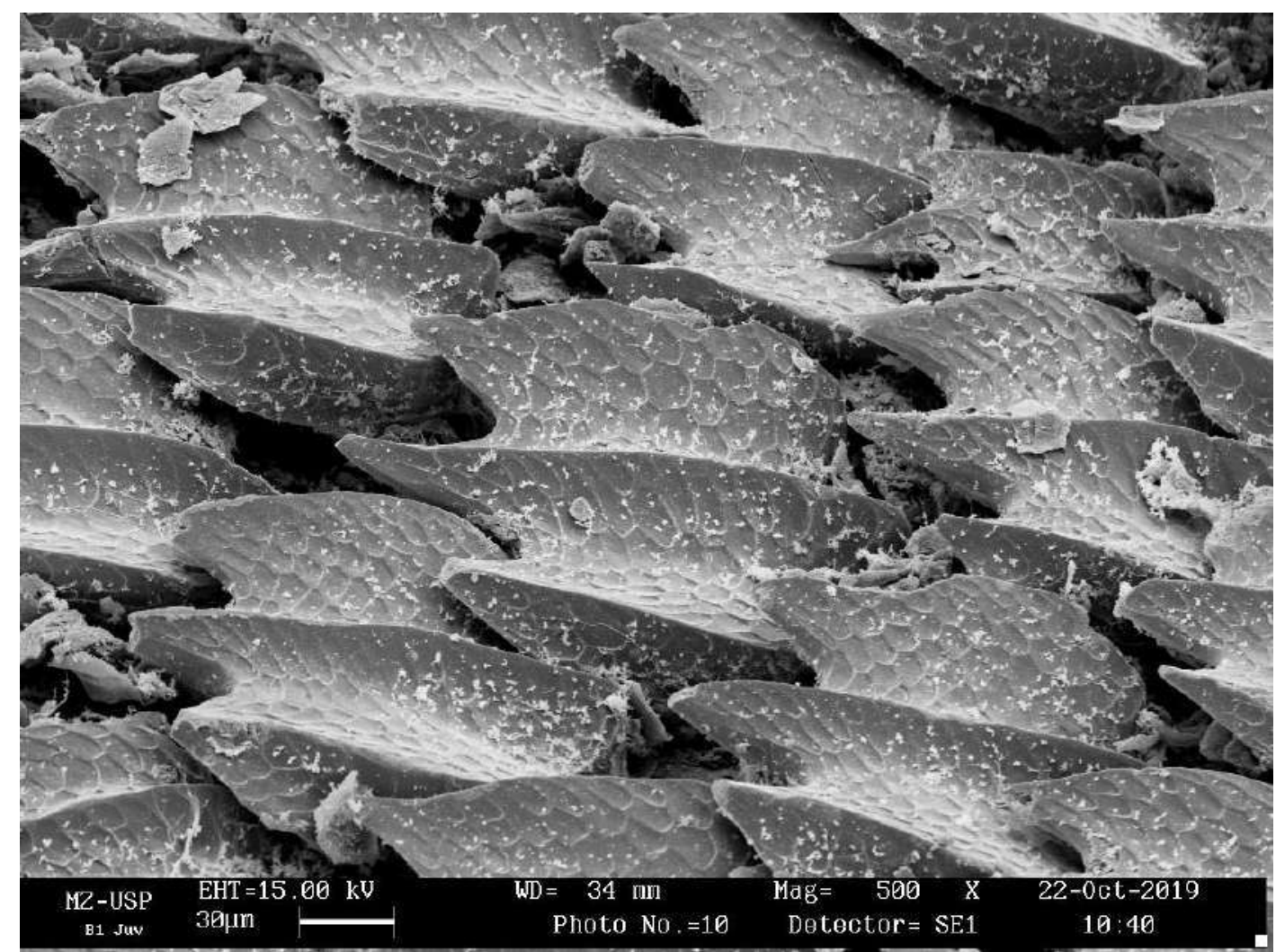

Figure 84. Dermal denticles collected from the section B1 (see fig. 1) of the juvenile Isogomphodon oxyrhynchus (MZUSP 37289). Additional information in the image footer.

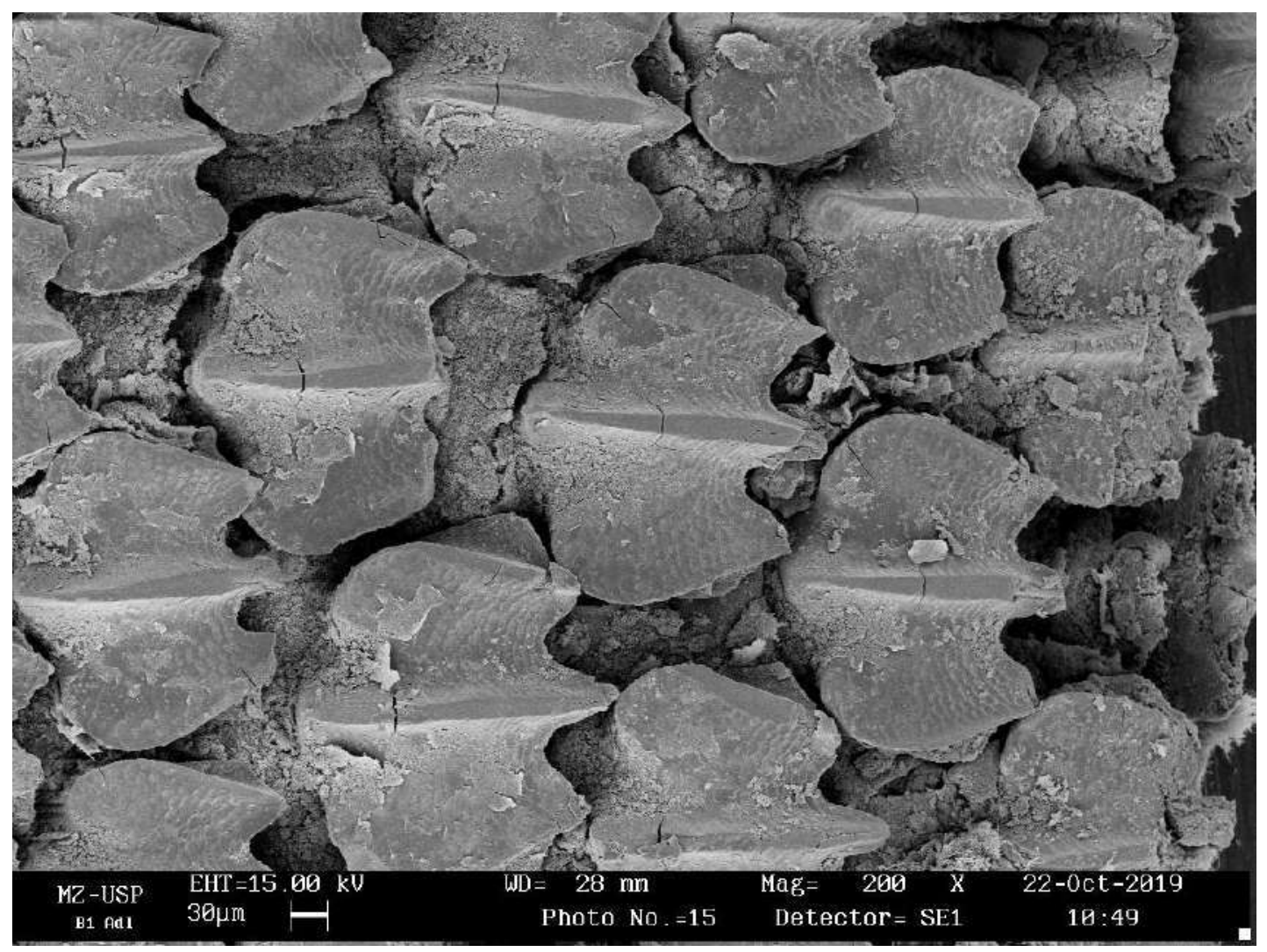

Figure 85. Dermal denticles collected from the section B1 (see fig. 1) of the adult Isogomphodon oxyrhynchus (MPEG 3455). Additional information in the image footer. 


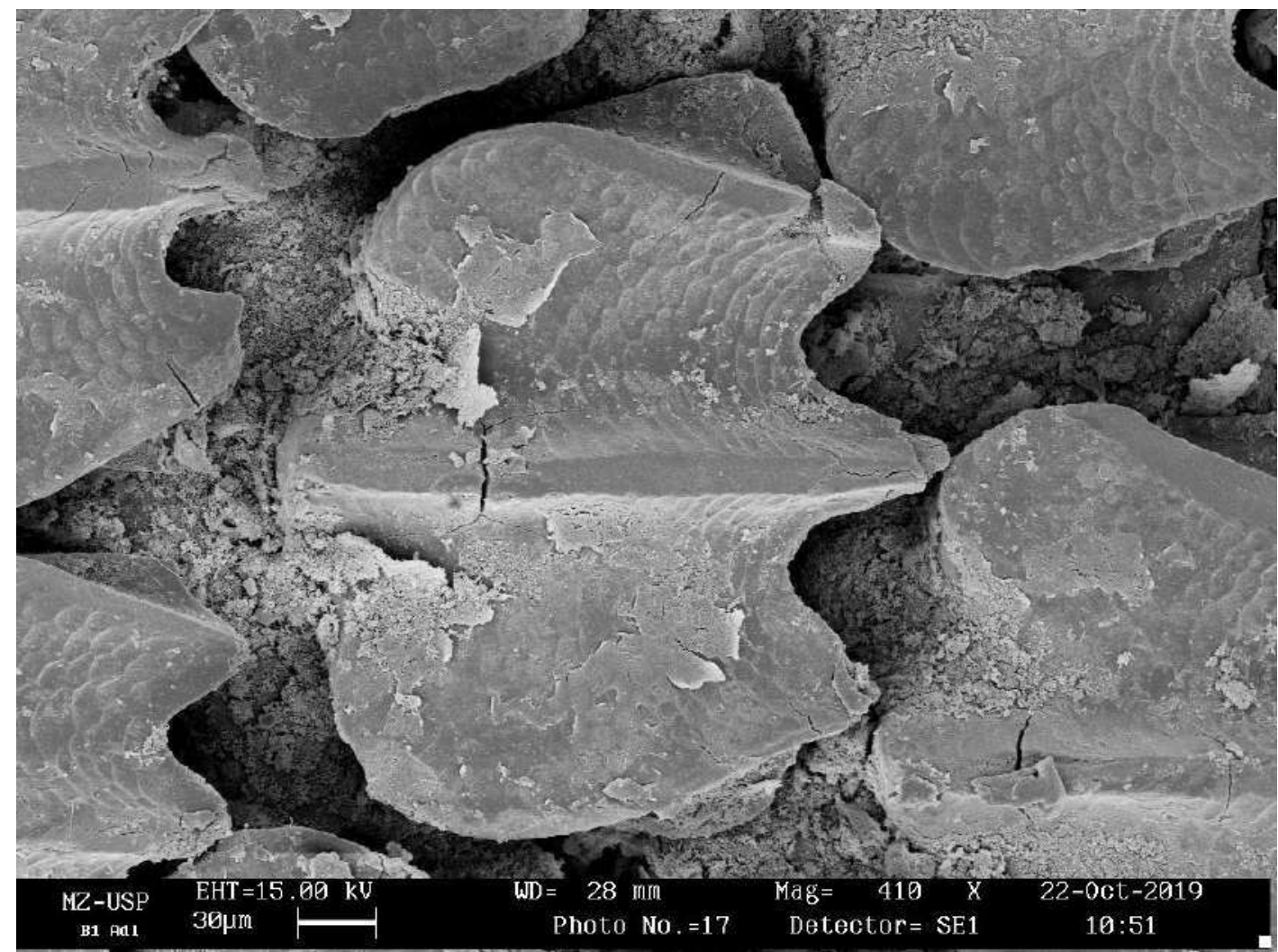

Figure 86. Dermal denticles collected from the section B1 (see fig. 1) of the adult Isogomphodon oxyrhynchus (MPEG 3455). Additional information in the image footer.

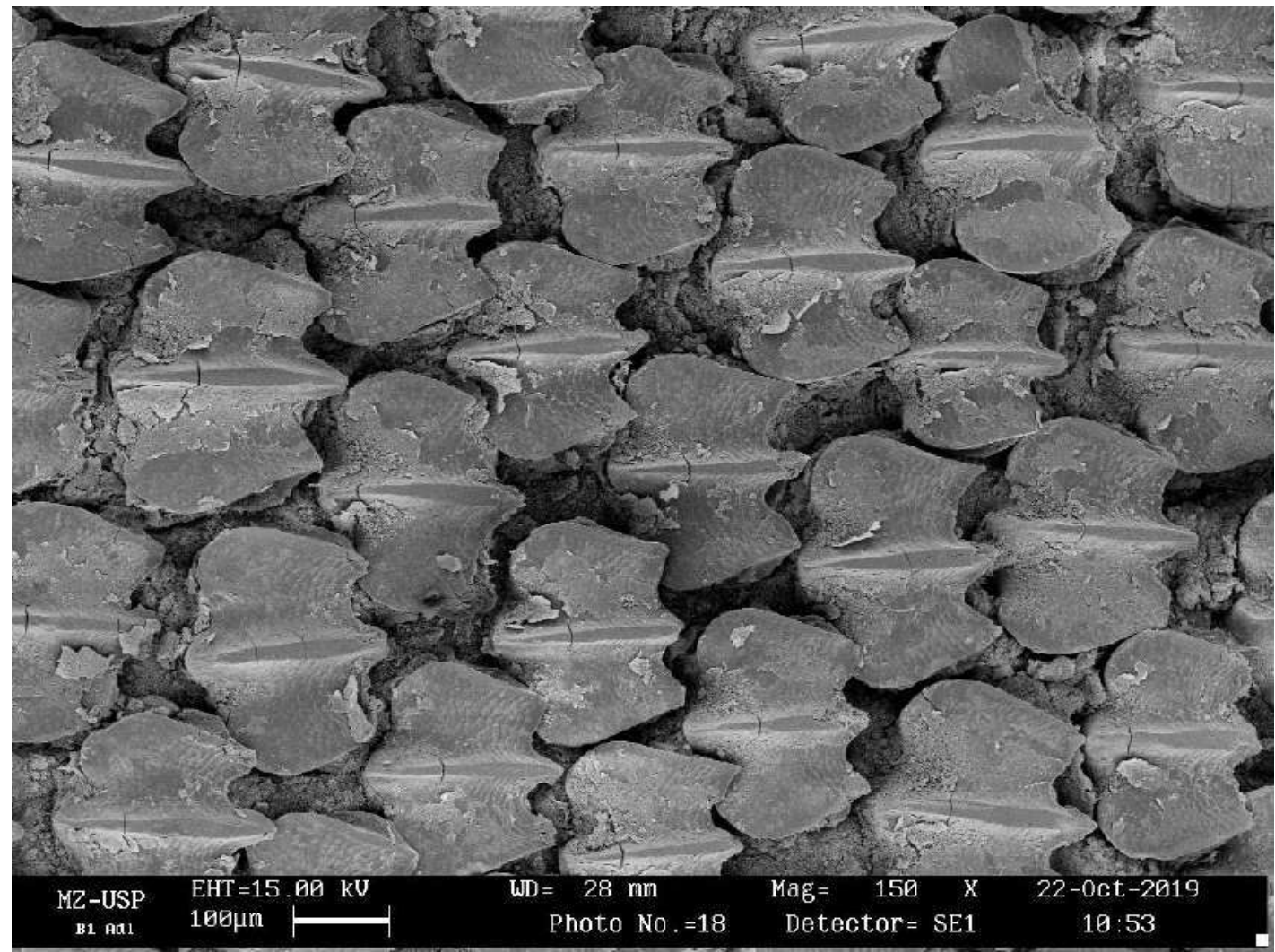

Figure 87. Dermal denticles collected from the section B1 (see fig. 1) of the adult Isogomphodon oxyrhynchus (MPEG 3455). Additional information in the image footer. 


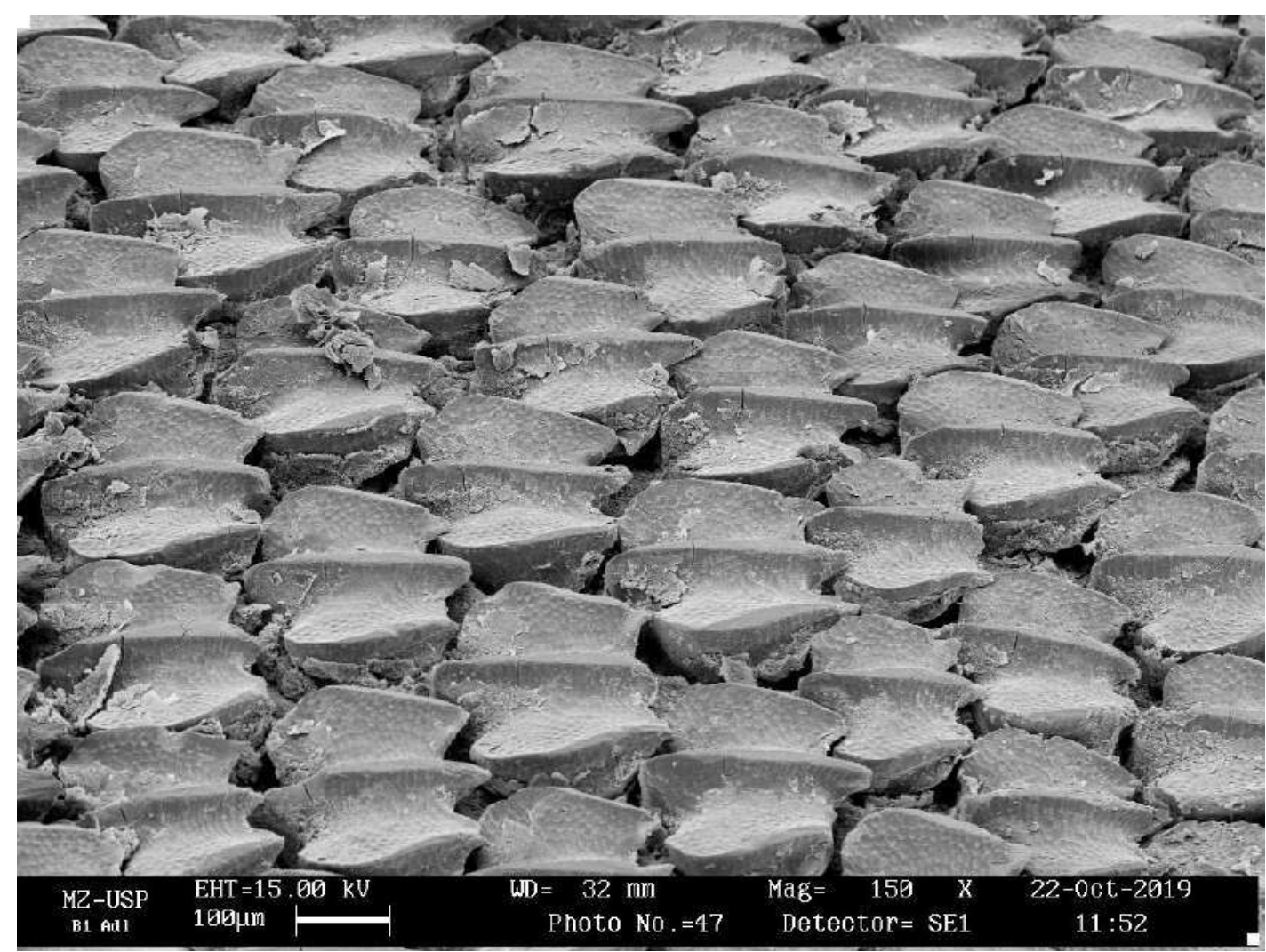

Figure 88. Dermal denticles collected from the section B1 (see fig. 1) of the adult Isogomphodon oxyrhynchus (MPEG 3455). Additional information in the image footer.

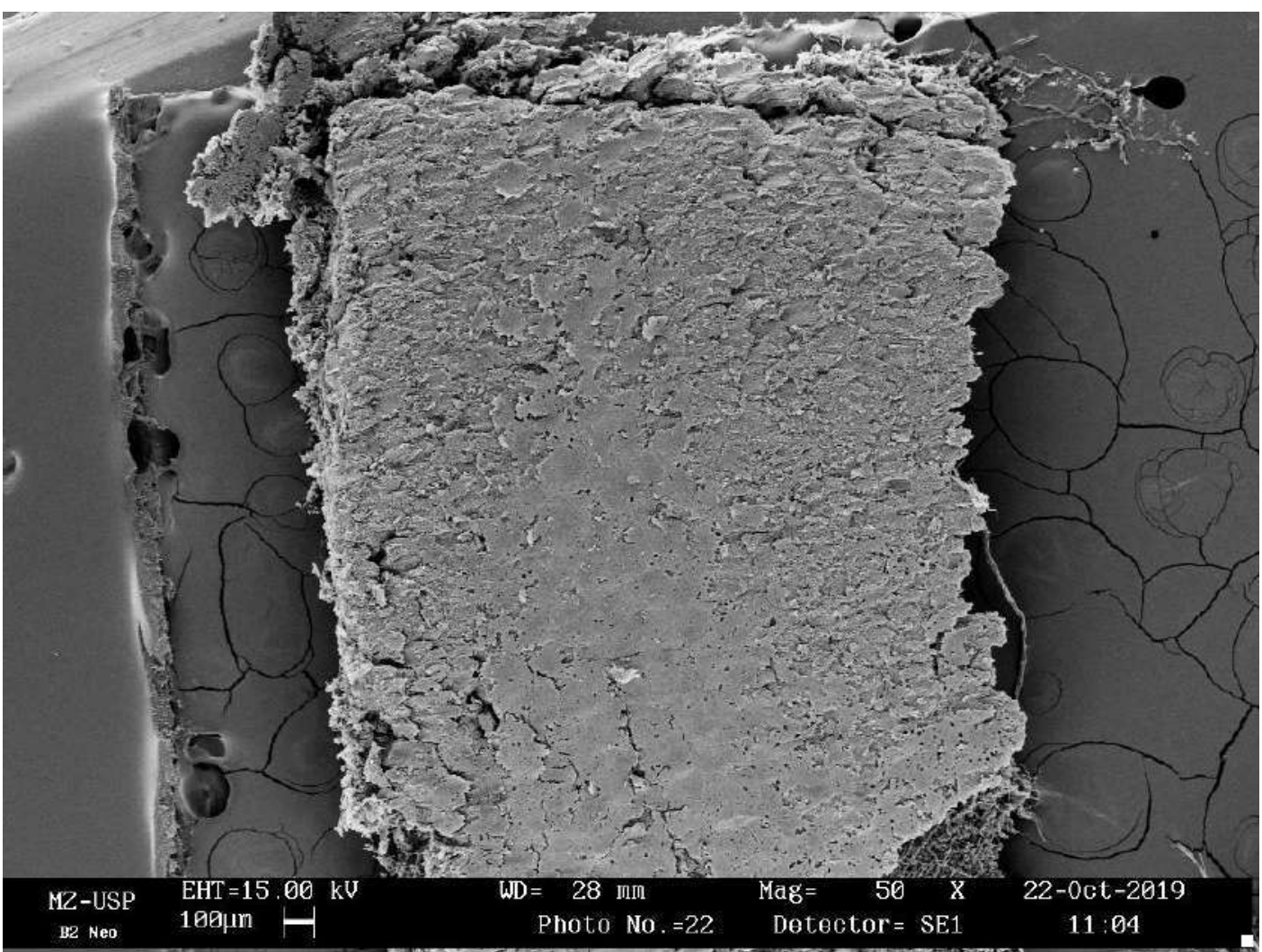

Figure 89. Dermal denticles collected from the section B2 (see fig. 1) of the neonate Isogomphodon oxyrhynchus (MZUSP 101214). Additional information in the image footer. 


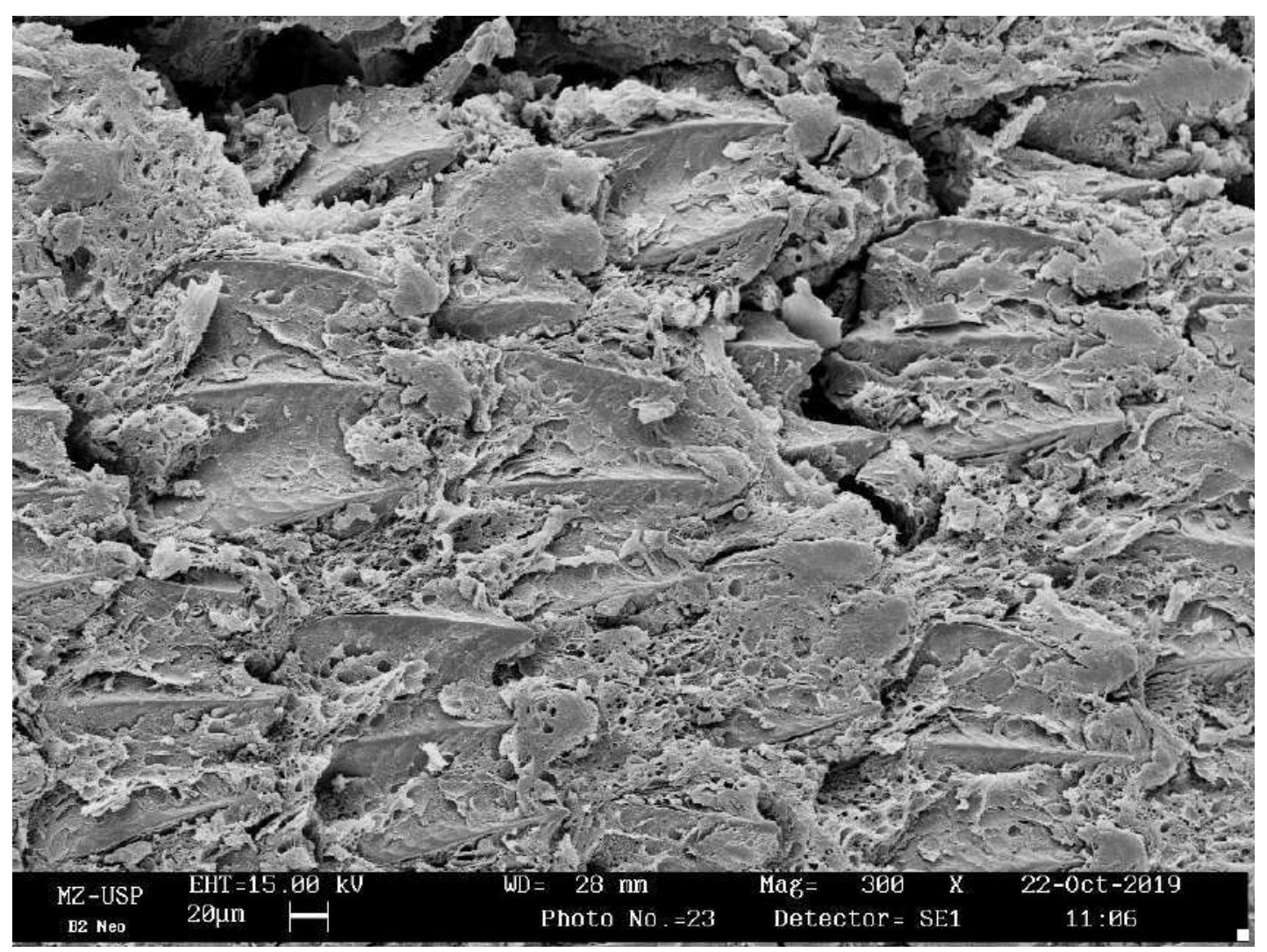

Figure 90. Dermal denticles collected from the section B2 (see fig. 1) of the neonate Isogomphodon oxyrhynchus (MZUSP 101214). Additional information in the image footer.

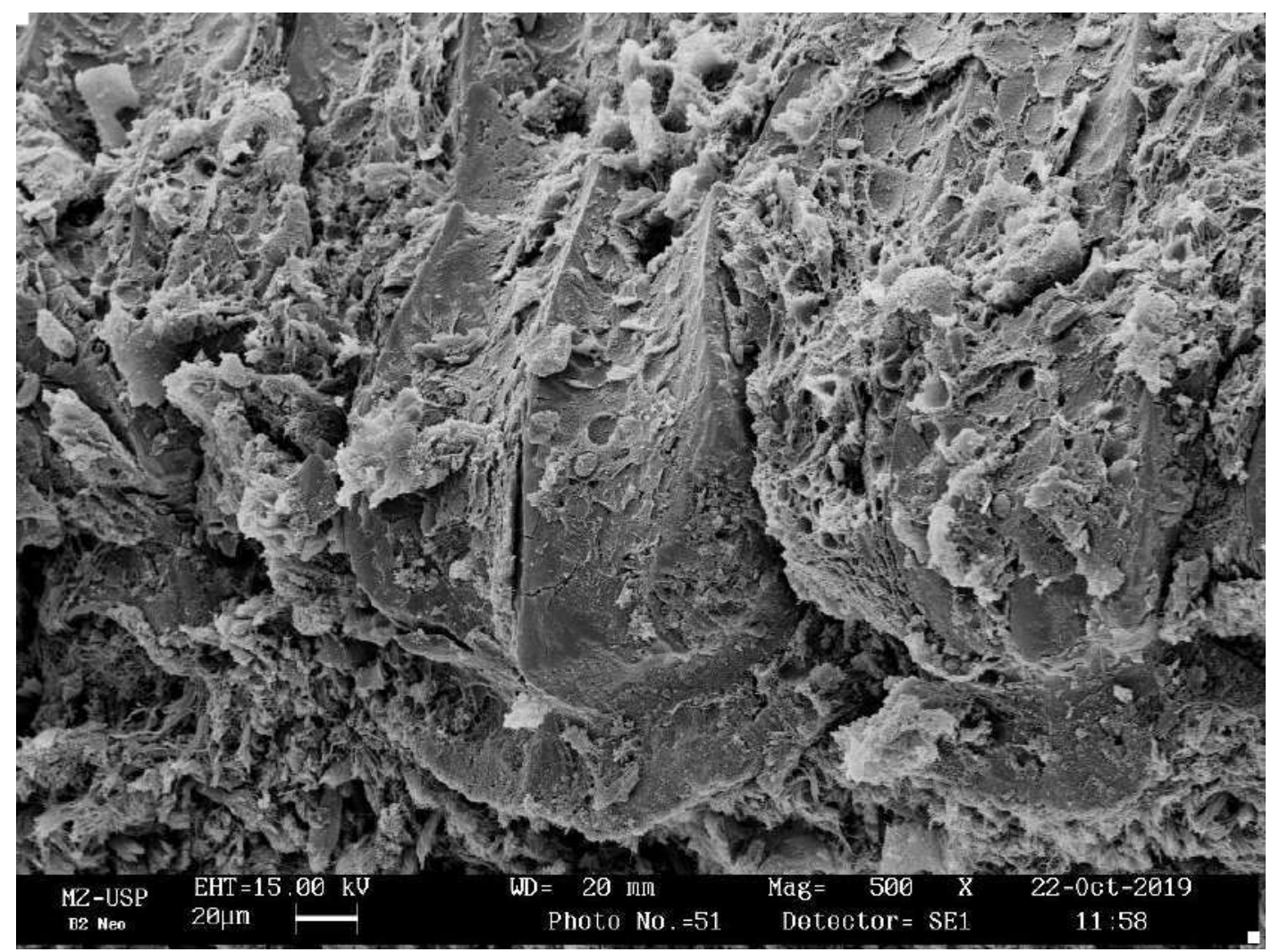

Figure 91. Dermal denticles collected from the section B2 (see fig. 1) of the neonate Isogomphodon oxyrhynchus (MZUSP 101214). Additional information in the image footer. 


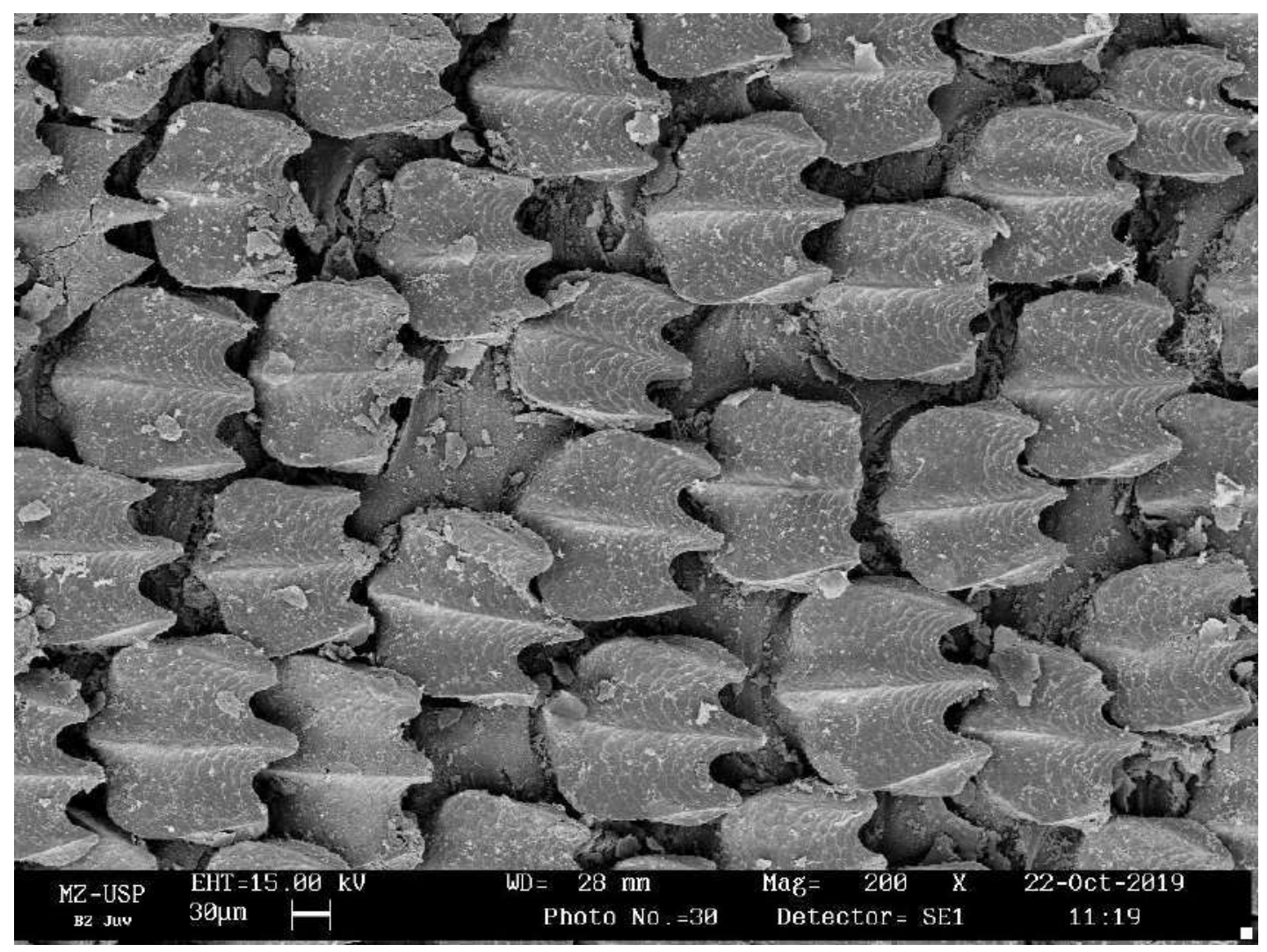

Figure 92. Dermal denticles collected from the section B2 (see fig. 1) of the juvenile Isogomphodon oxyrhynchus (MZUSP 37289). Additional information in the image footer.

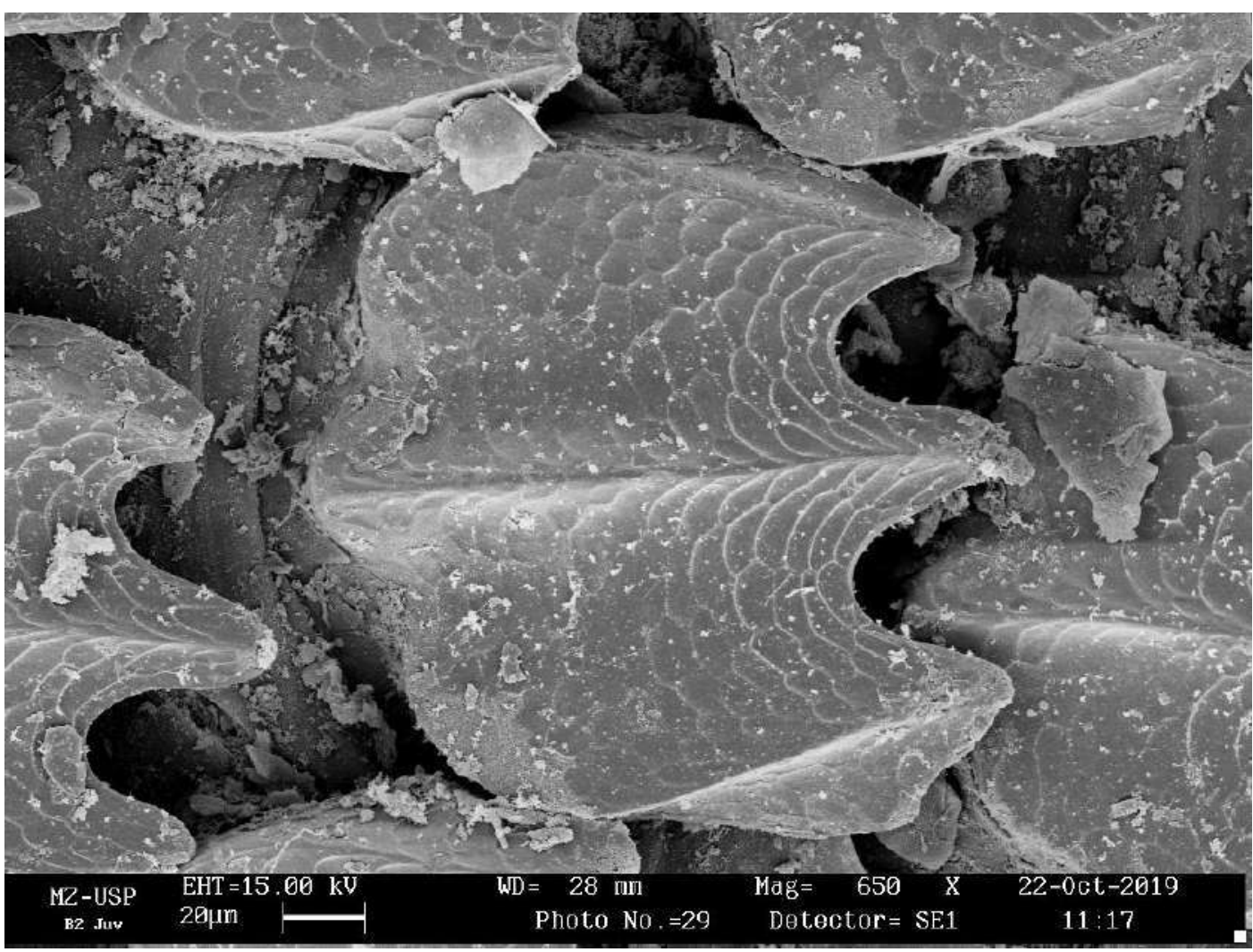

Figure 93. Dermal denticles collected from the section B2 (see fig. 1) of the juvenile Isogomphodon oxyrhynchus (MZUSP 37289). Additional information in the image footer. 


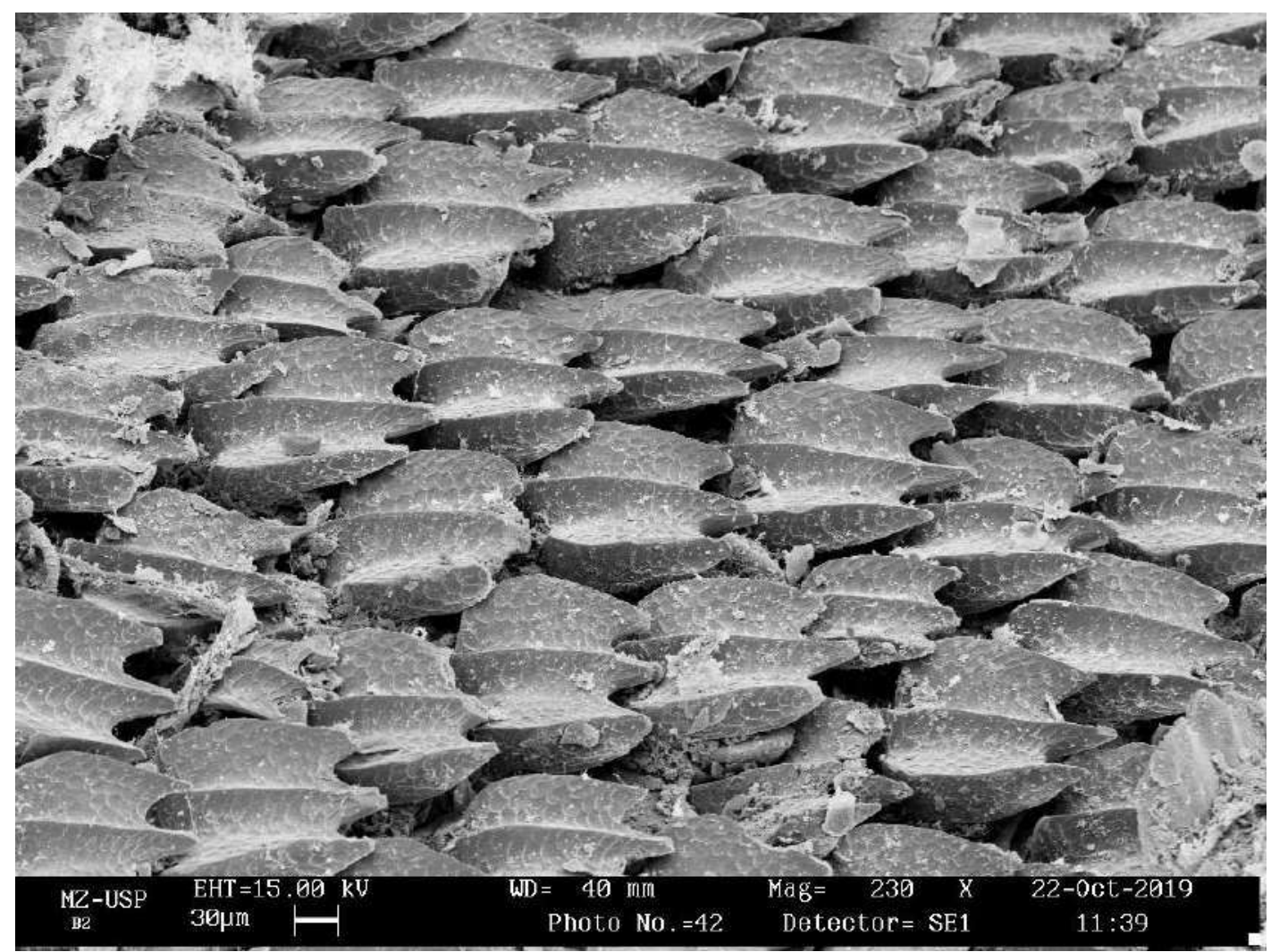

Figure 94. Dermal denticles collected from the section B2 (see fig. 1) of the juvenile Isogomphodon oxyrhynchus (MZUSP 37289). Additional information in the image footer.

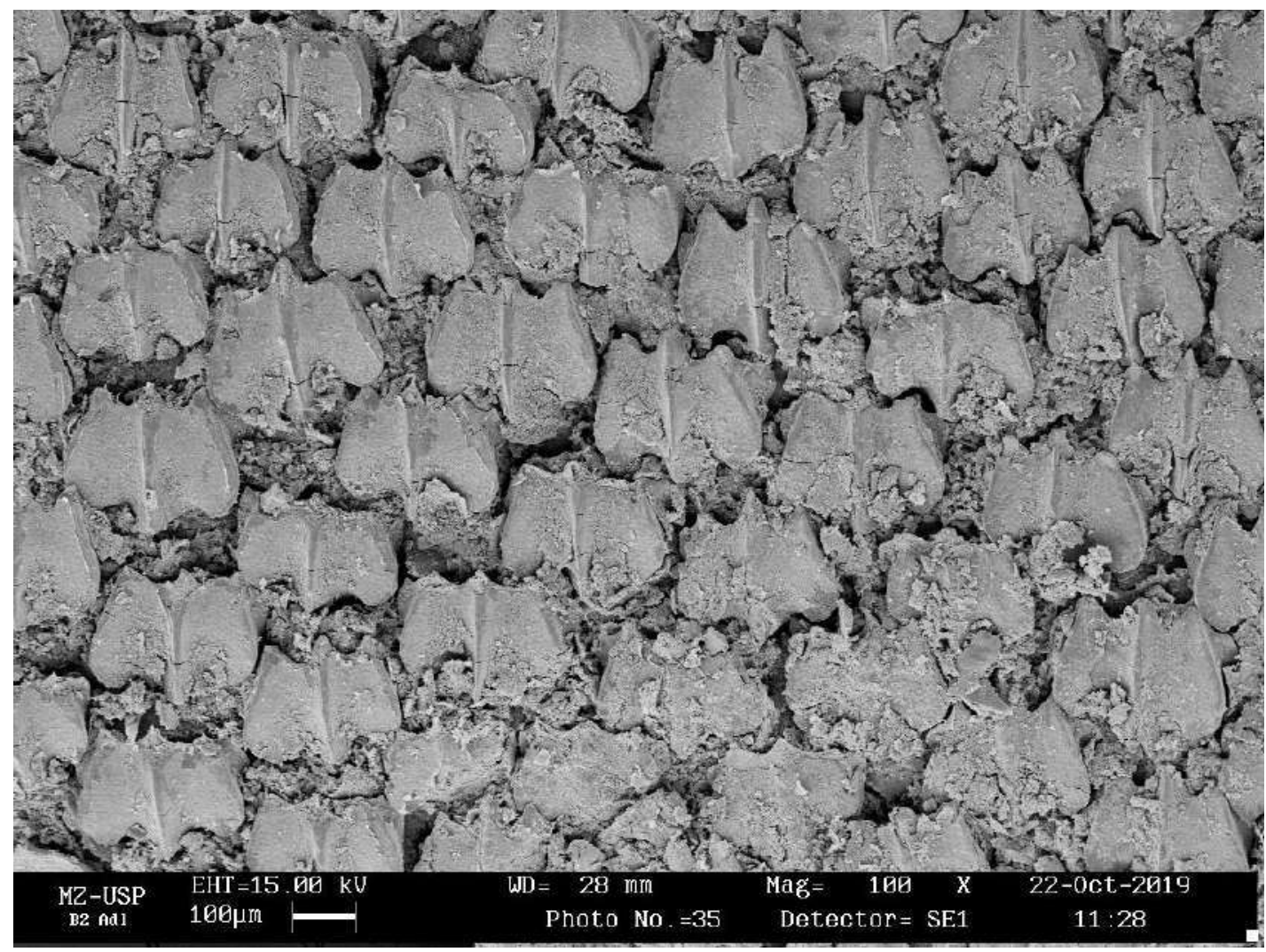

Figure 95. Dermal denticles collected from the section B2 (see fig. 1) of the adult Isogomphodon oxyrhynchus (MPEG 3455). Additional information in the image footer. 


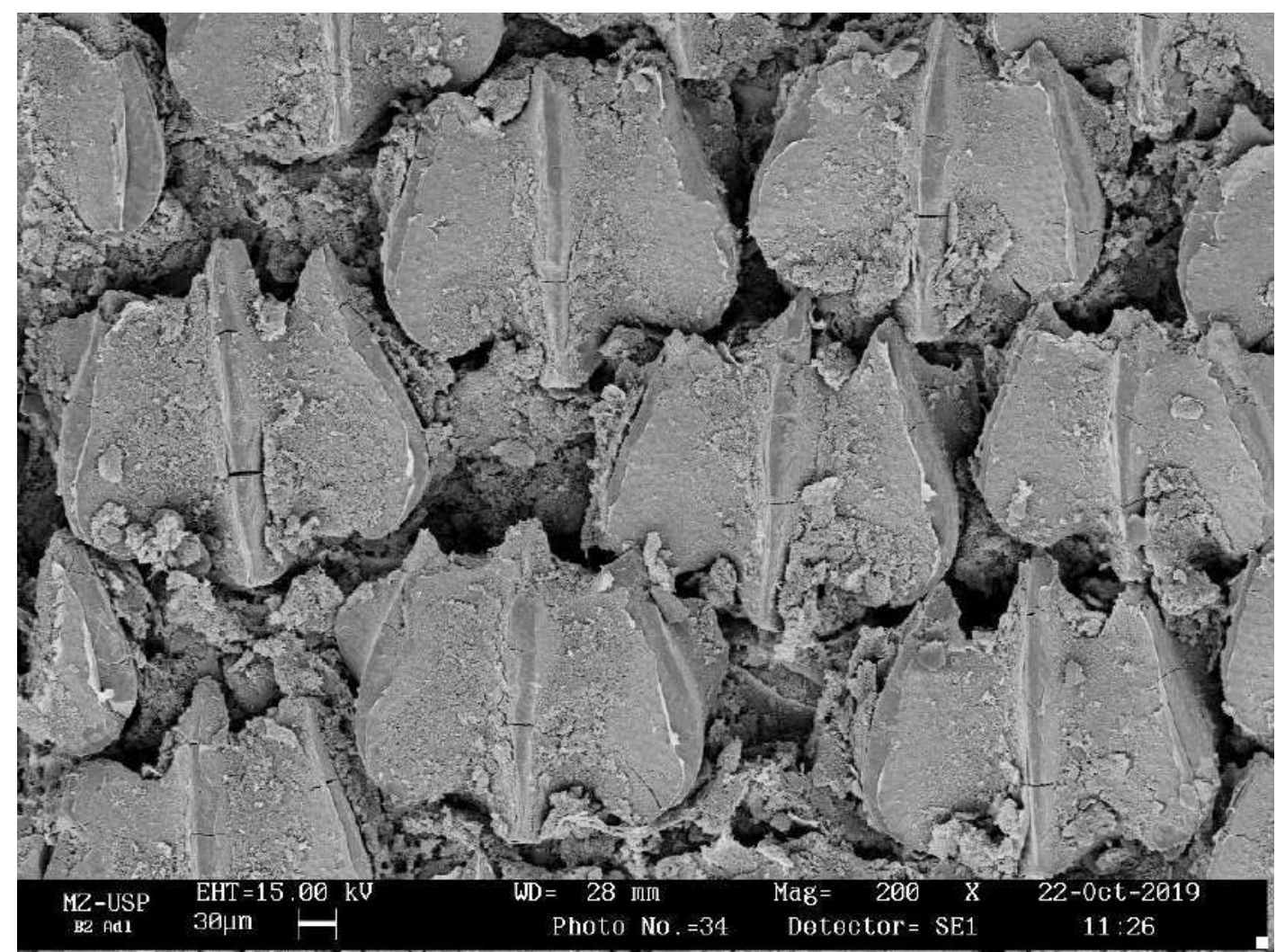

Figure 96. Dermal denticles collected from the section B2 (see fig. 1) of the adult Isogomphodon oxyrhynchus (MPEG 3455). Additional information in the image footer.

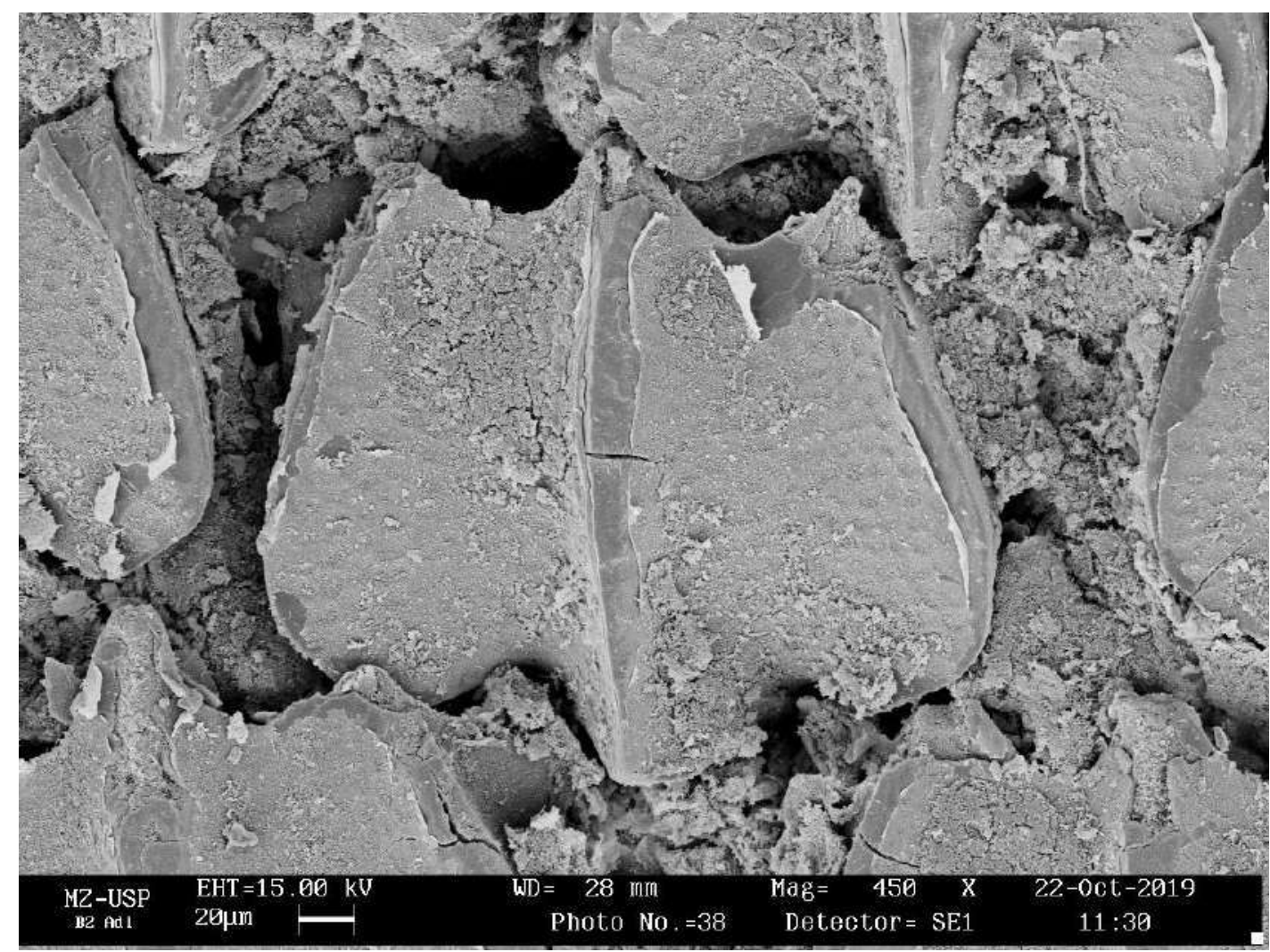

Figure 97. Dermal denticles collected from the section B2 (see fig. 1) of the adult Isogomphodon oxyrhynchus (MPEG 3455). Additional information in the image footer. 


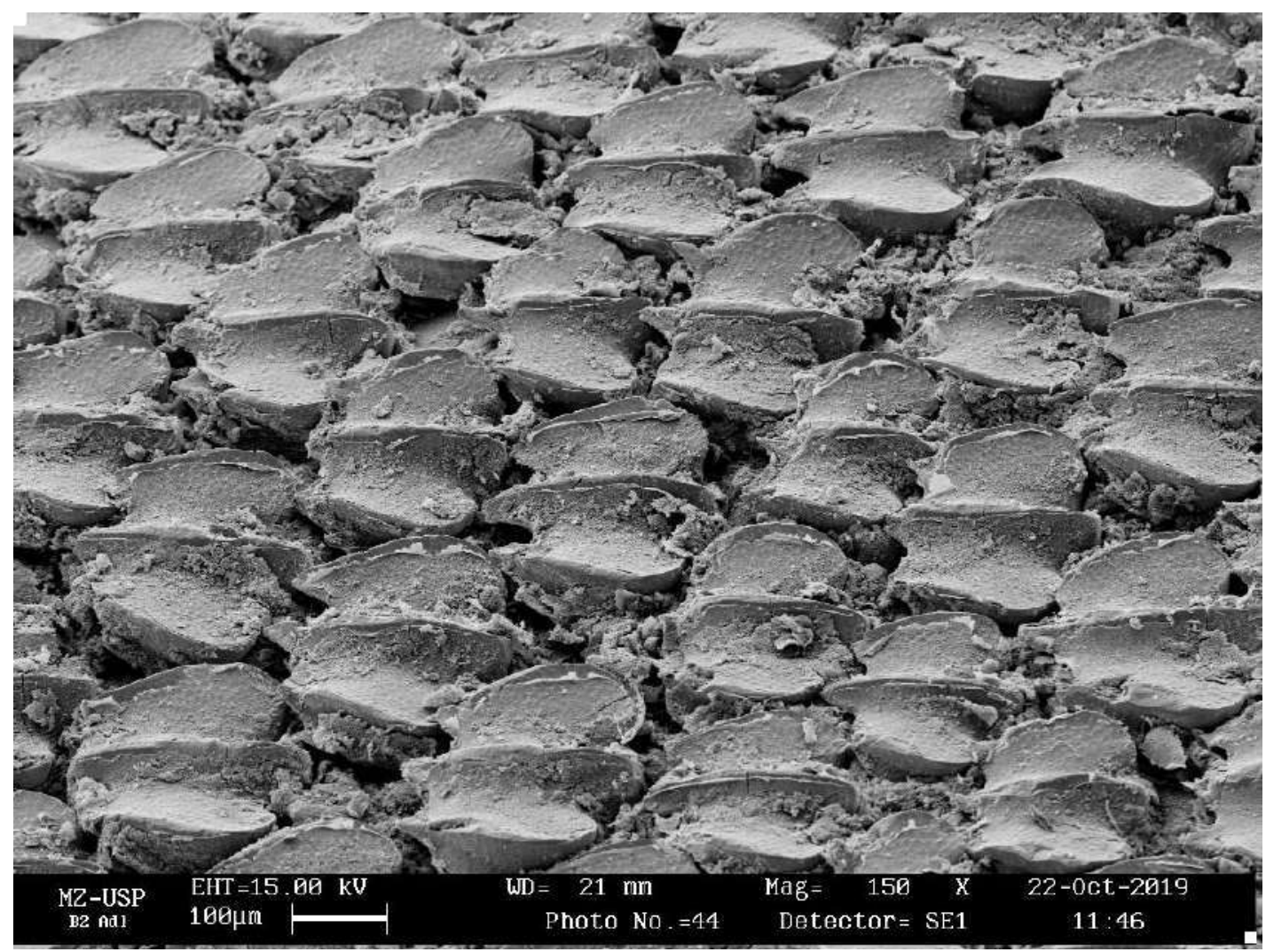

Figure 98. Dermal denticles collected from the section B2 (see fig. 1) of the adult Isogomphodon oxyrhynchus (MPEG 3455). Additional information in the image footer.

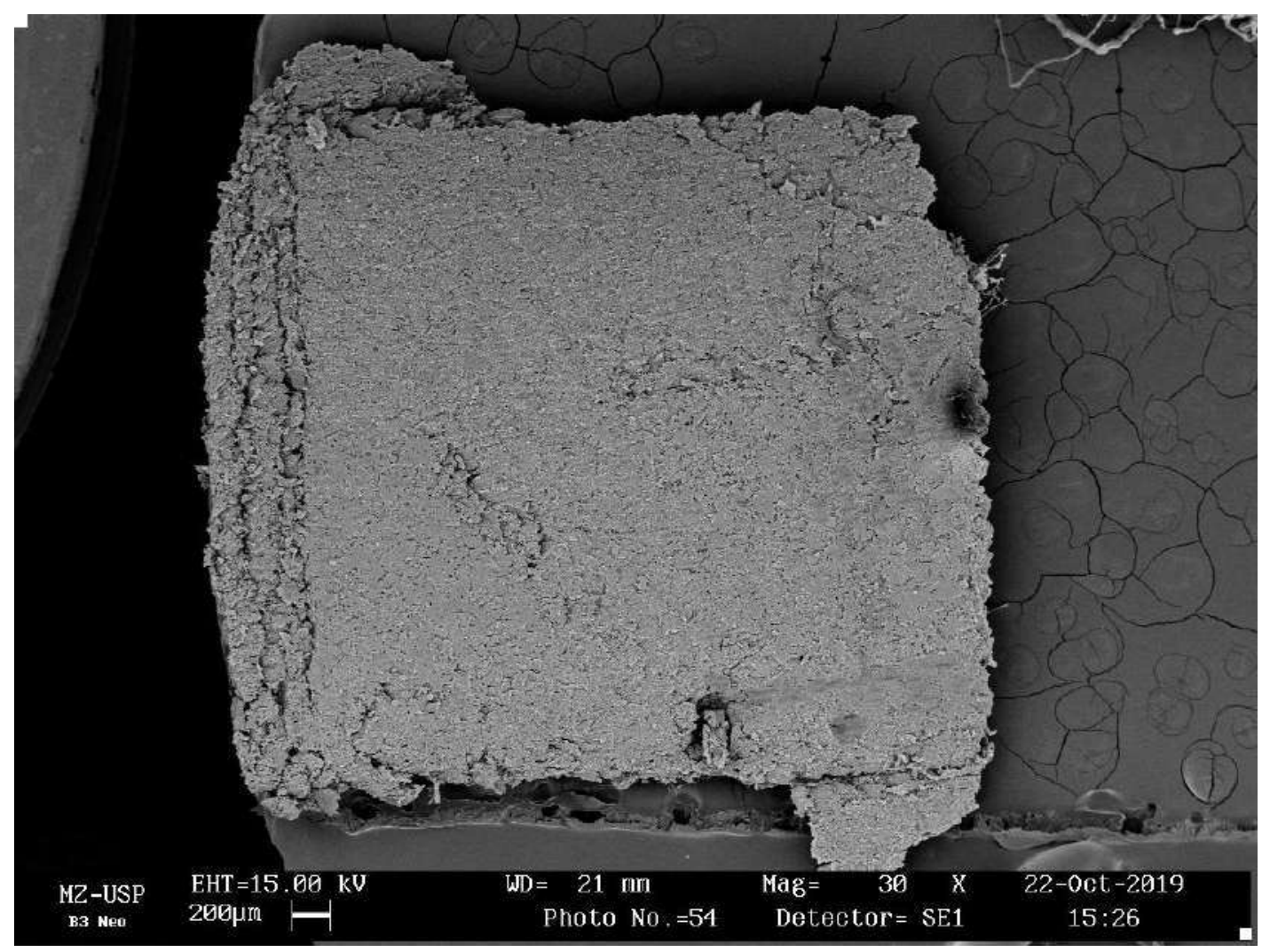

Figure 99. Dermal denticles collected from the section B3 (see fig. 1) of the neonate Isogomphodon oxyrhynchus (MZUSP 101214). Additional information in the image footer. 


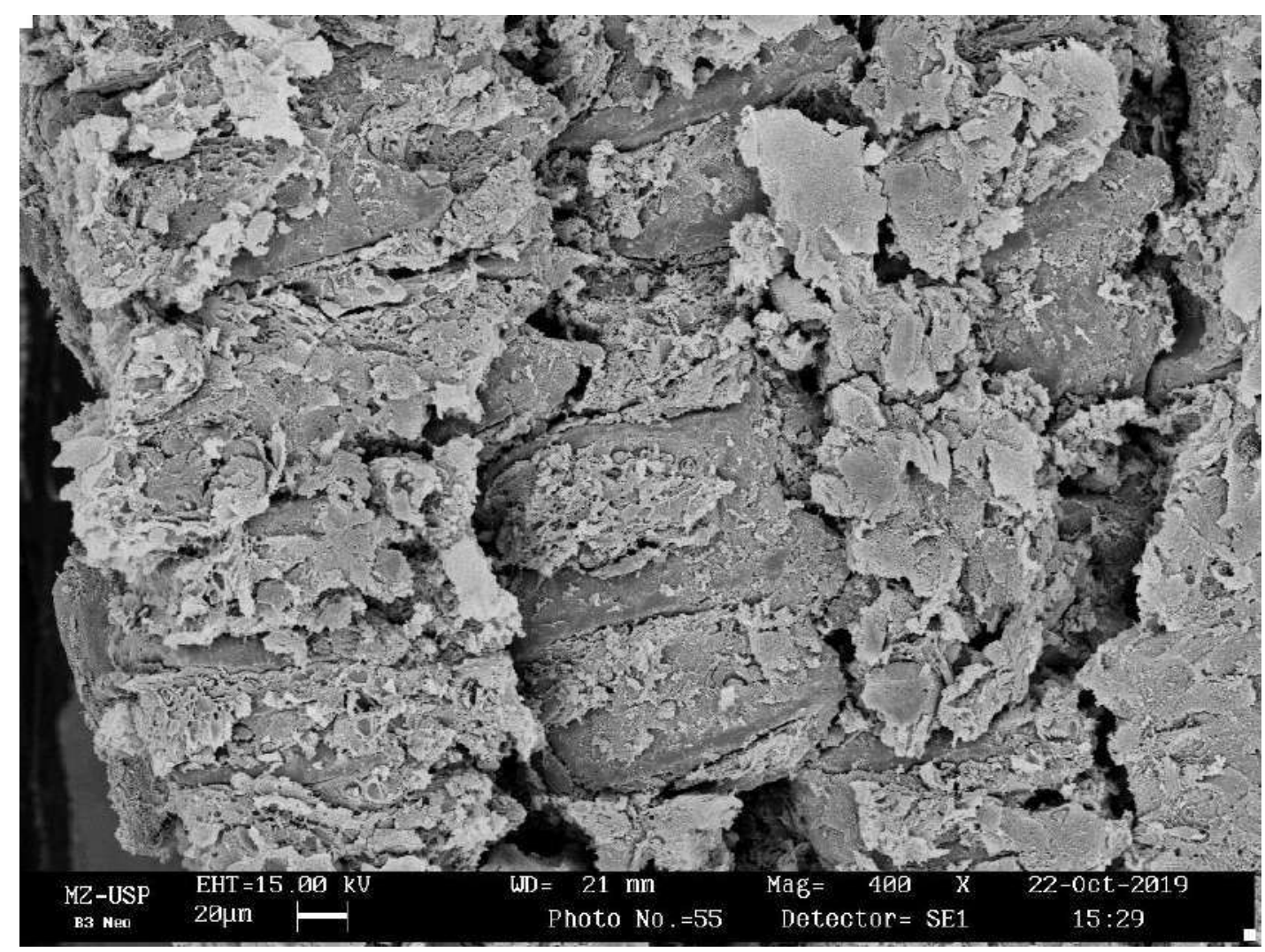

Figure 100. Dermal denticles collected from the section B3 (see fig. 1) of the neonate Isogomphodon oxyrhynchus (MZUSP 101214). Additional information in the image footer.

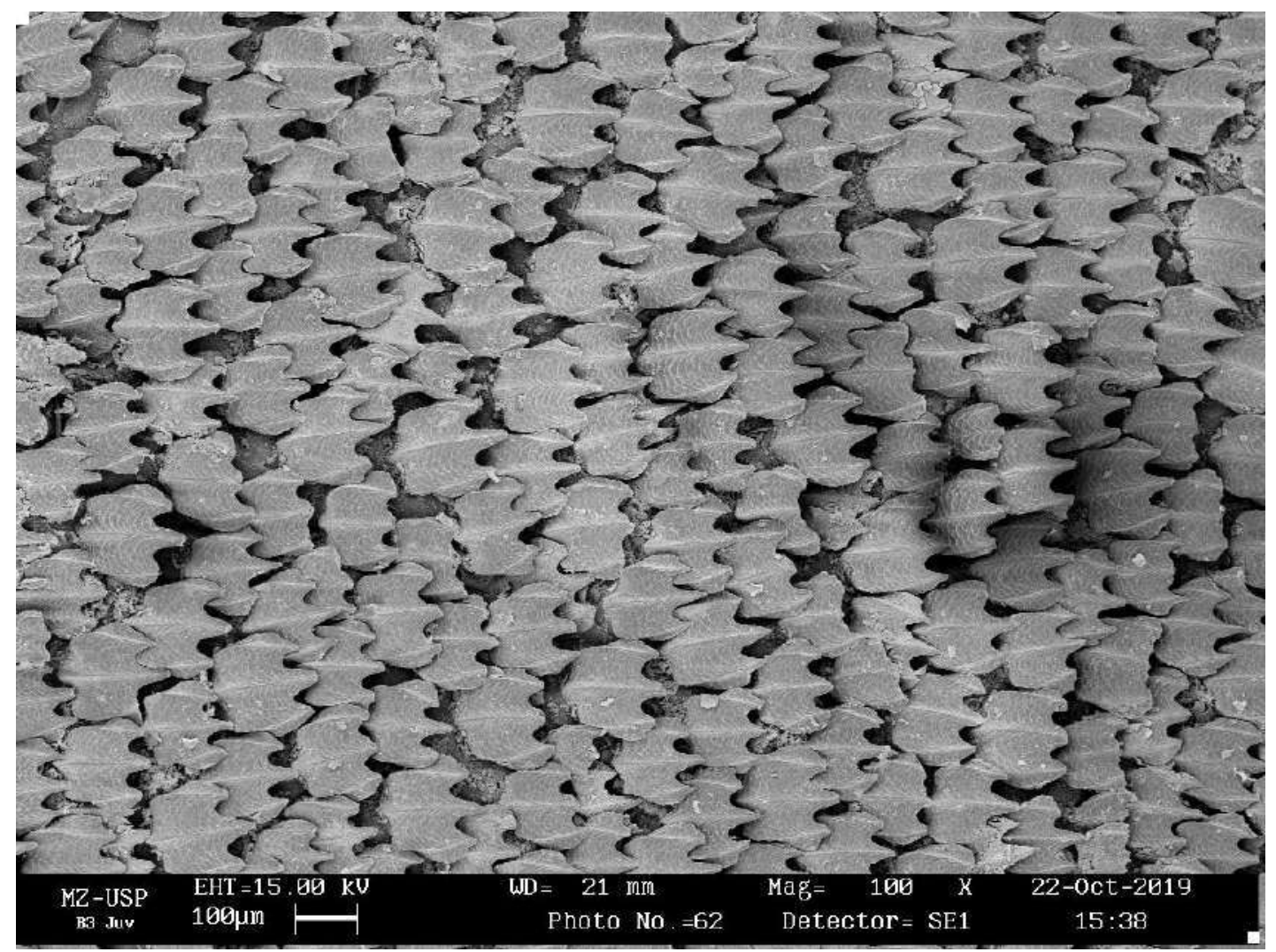

Figure 101. Dermal denticles collected from the section B3 (see fig. 1) of the juvenile Isogomphodon oxyrhynchus (MZUSP 37289). Additional information in the image footer. 


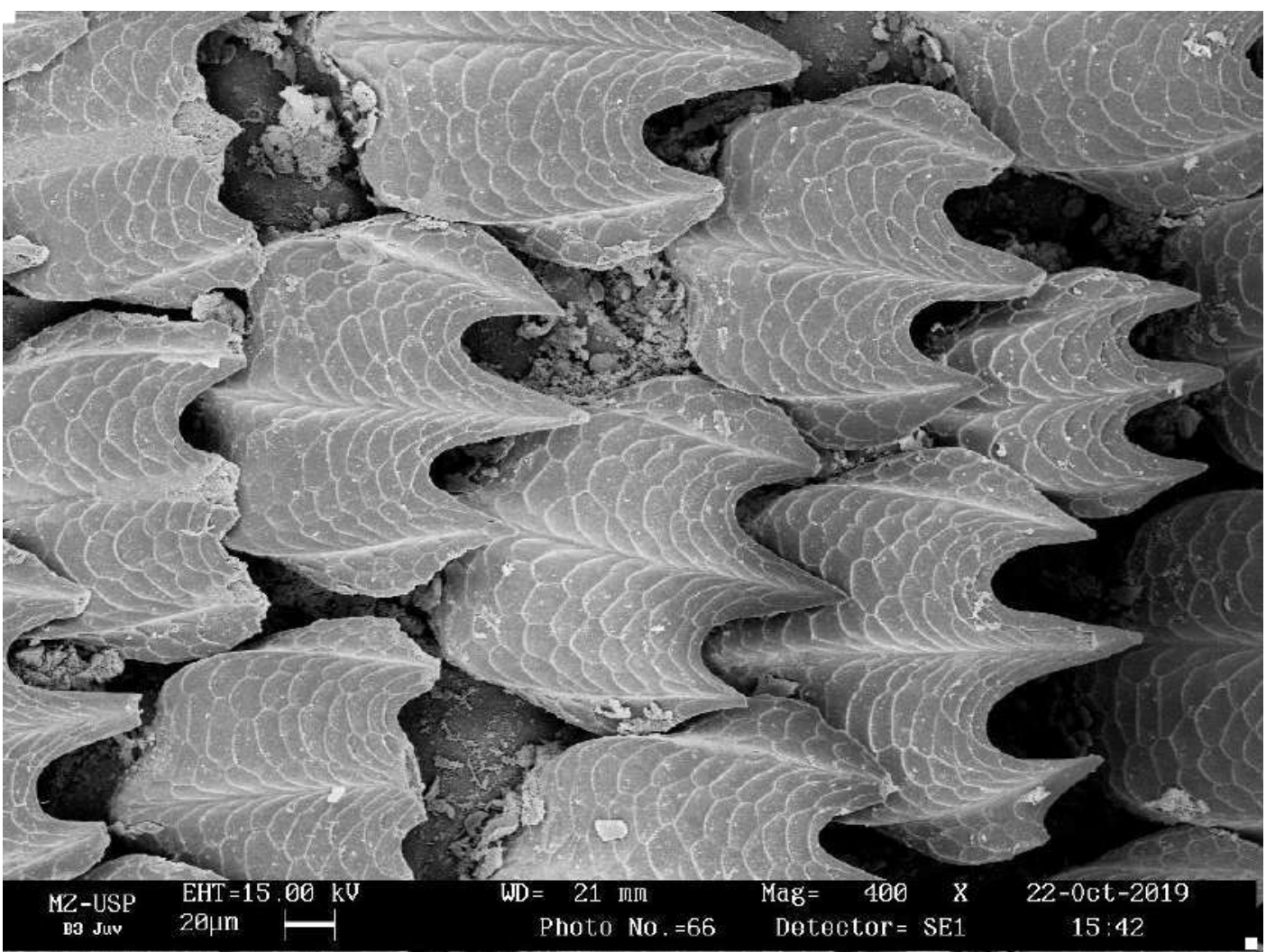

Figure 102. Dermal denticles collected from the section B3 (see fig. 1) of the juvenile Isogomphodon oxyrhynchus (MZUSP 37289). Additional information in the image footer.

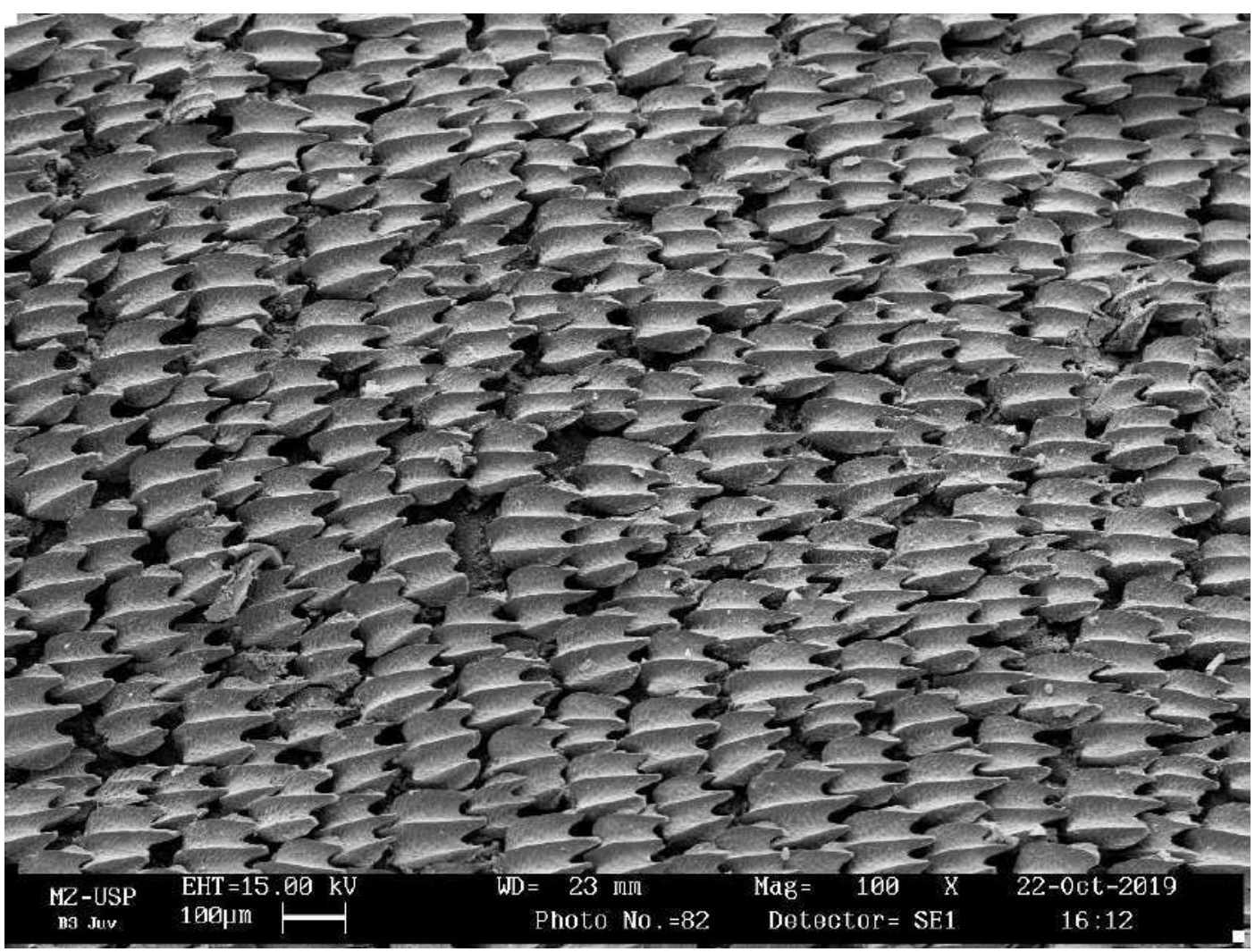

Figure 103. Dermal denticles collected from the section B3 (see fig. 1) of the juvenile Isogomphodon oxyrhynchus (MZUSP 37289). Additional information in the image footer. 


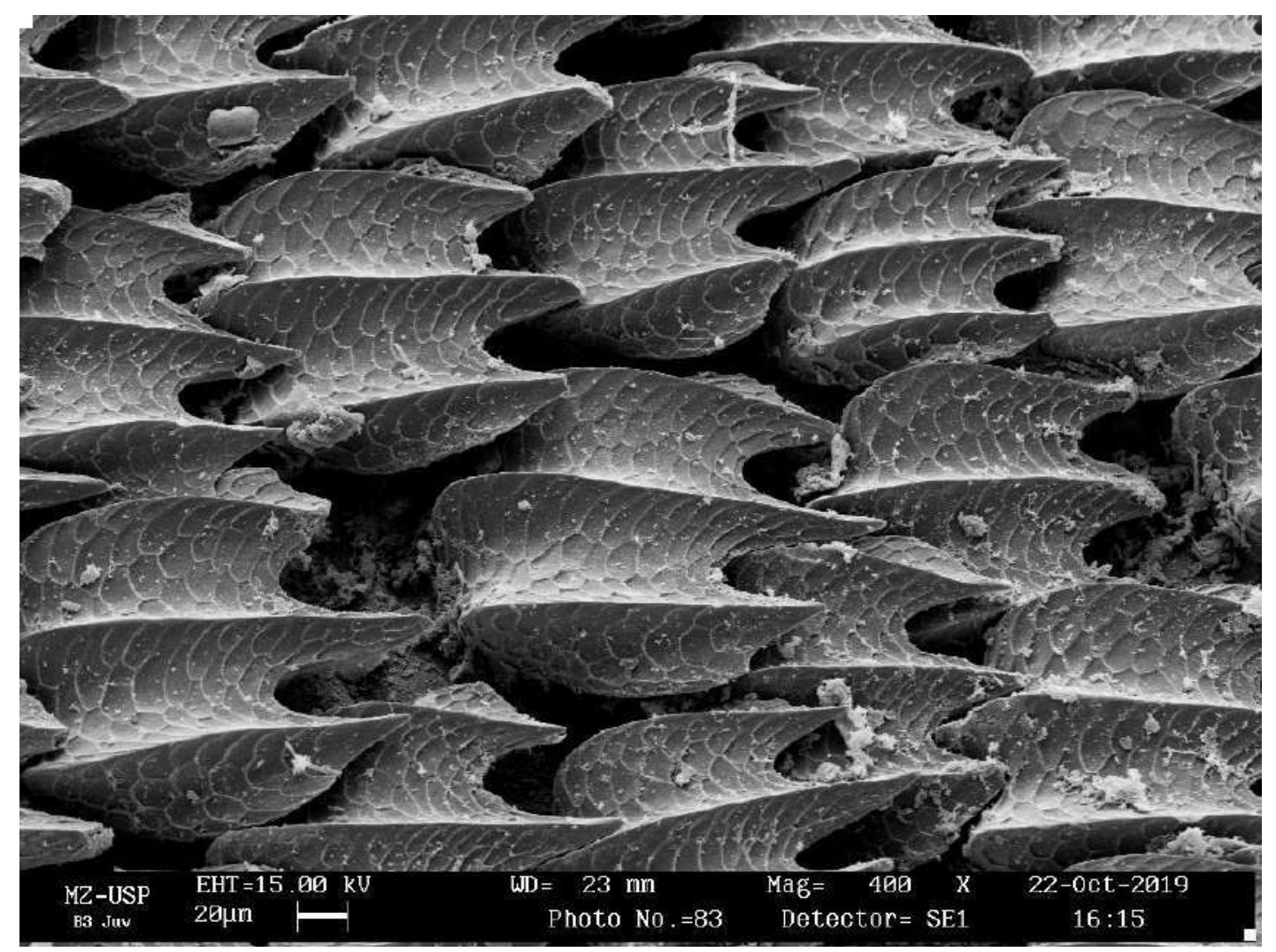

Figure 104. Dermal denticles collected from the section B3 (see fig. 1) of the juvenile Isogomphodon oxyrhynchus (MZUSP 37289). Additional information in the image footer.

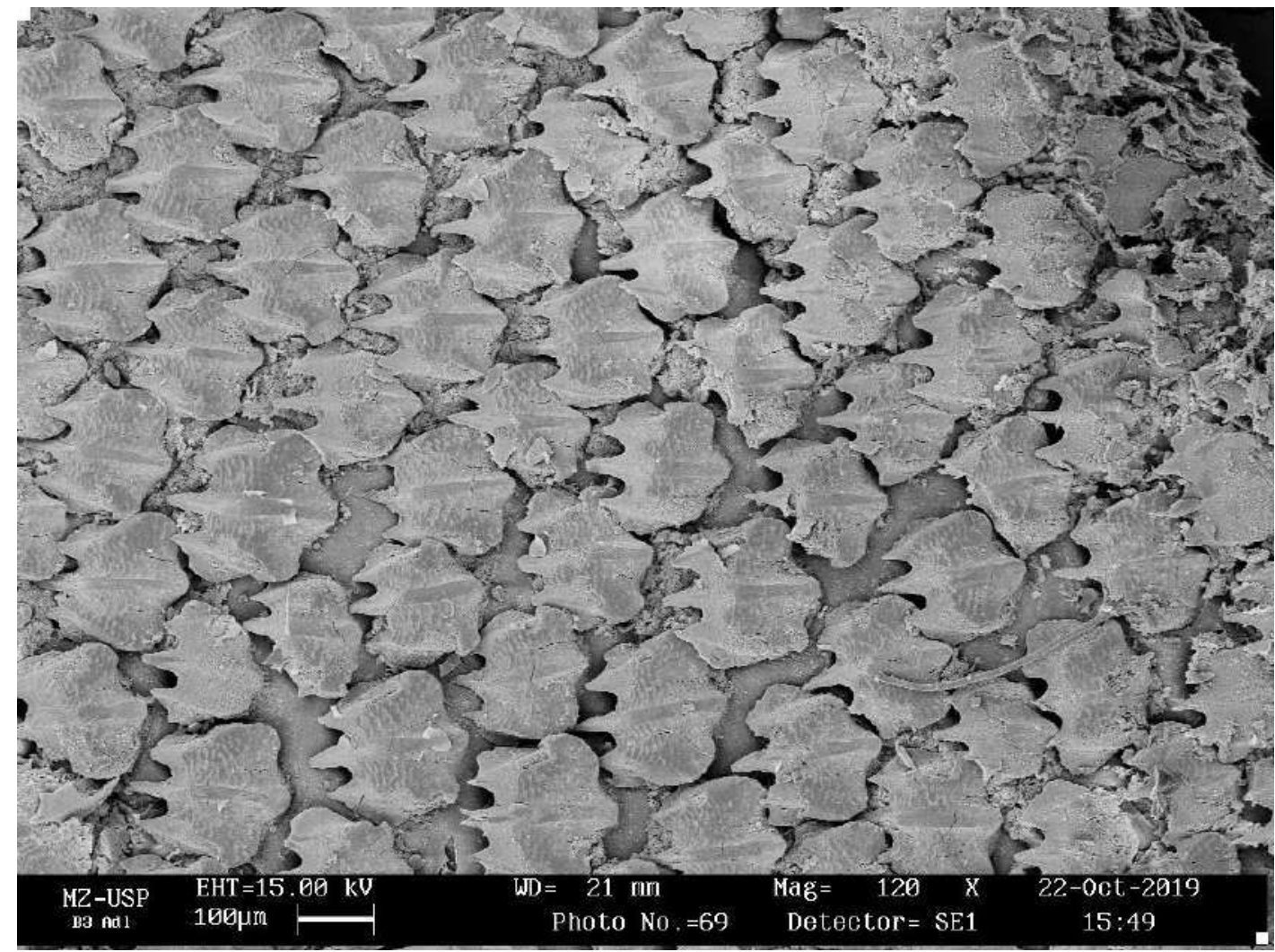

Figure 105. Dermal denticles collected from the section B3 (see fig. 1) of the adult Isogomphodon oxyrhynchus (MPEG 3455). Additional information in the image footer. 


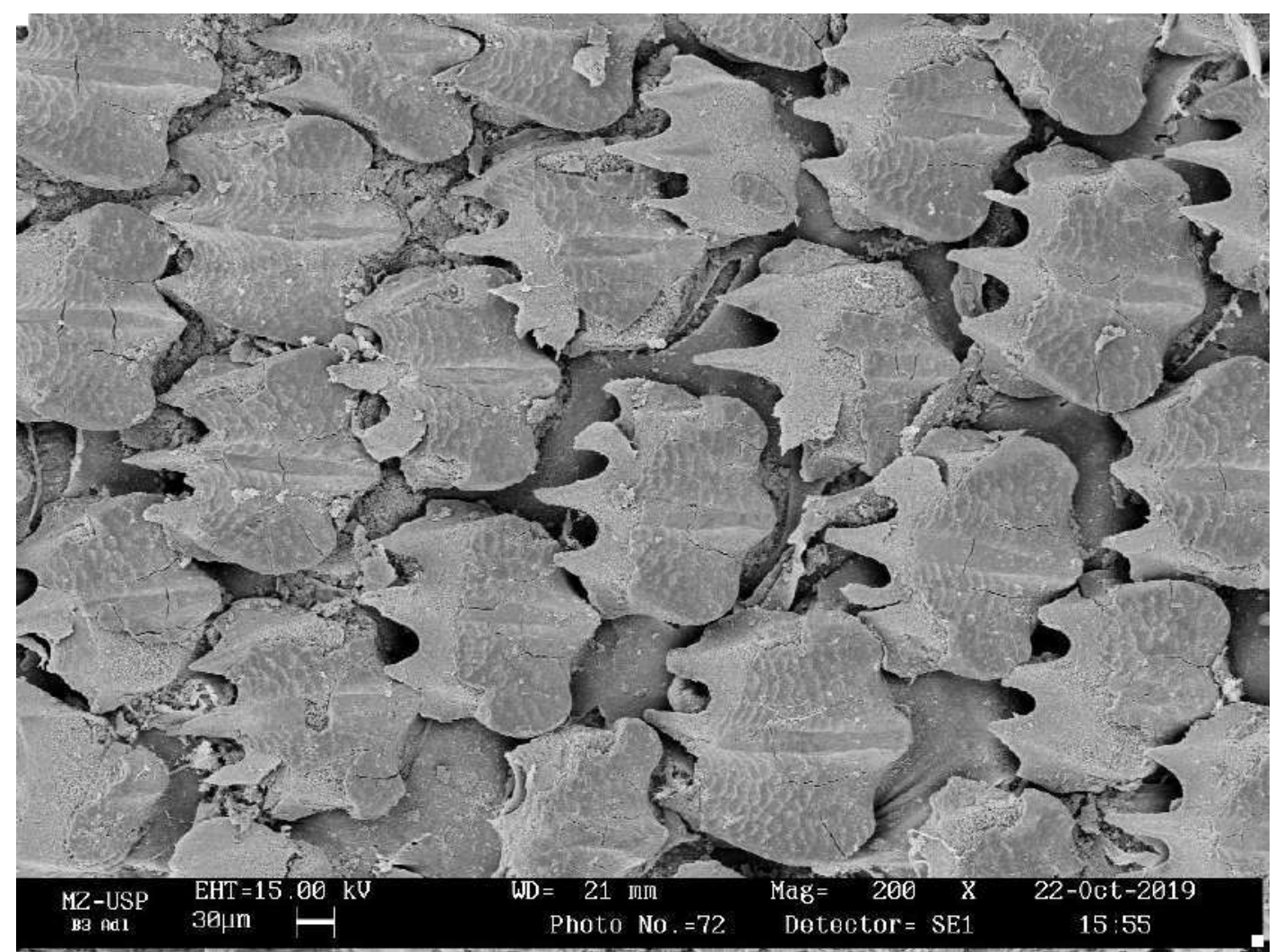

Figure 106. Dermal denticles collected from the section B3 (see fig. 1) of the adult Isogomphodon oxyrhynchus (MPEG 3455). Additional information in the image footer.

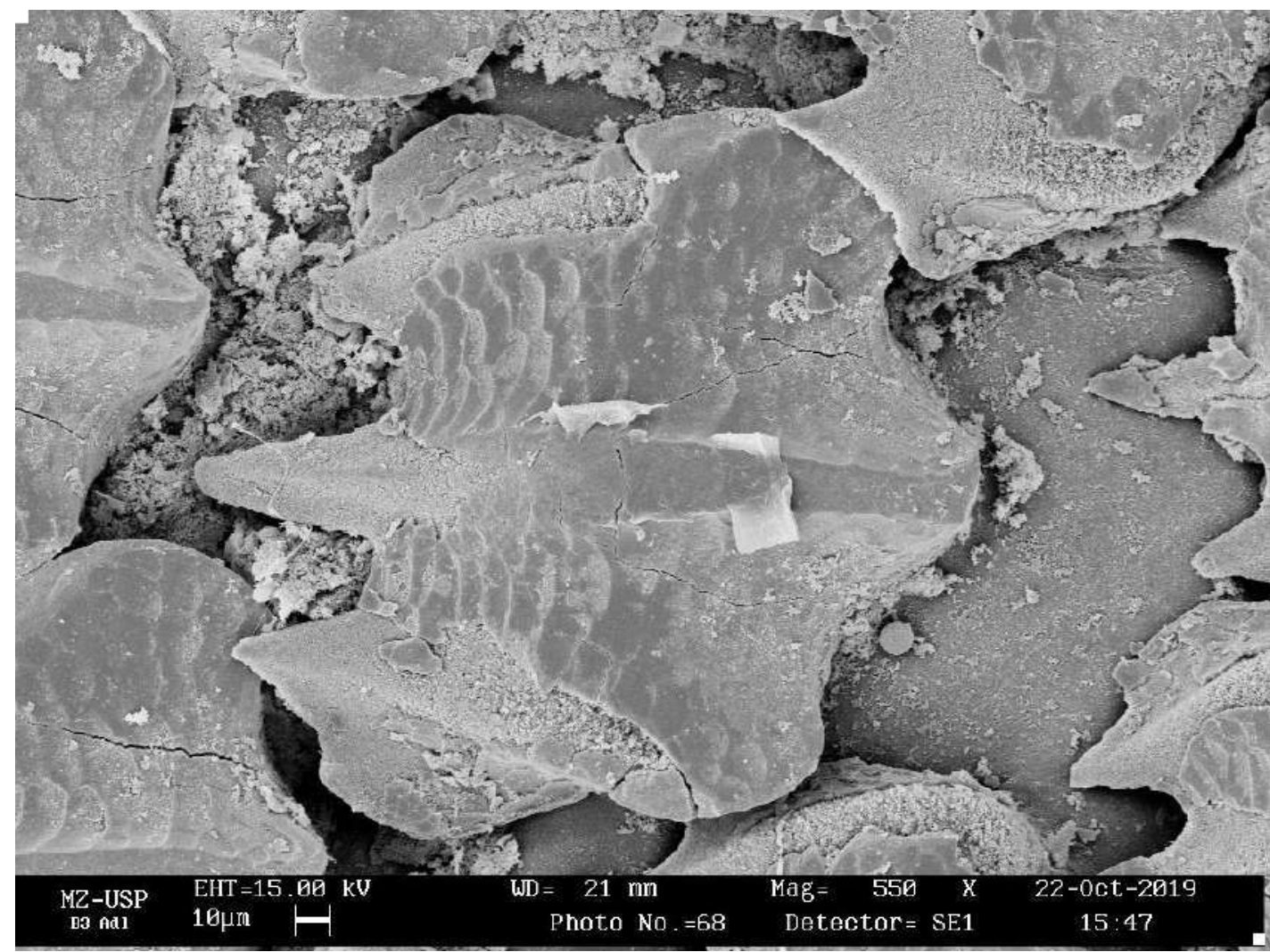

Figure 107. Dermal denticles collected from the section B3 (see fig. 1) of the adult Isogomphodon oxyrhynchus (MPEG 3455). Additional information in the image footer. 


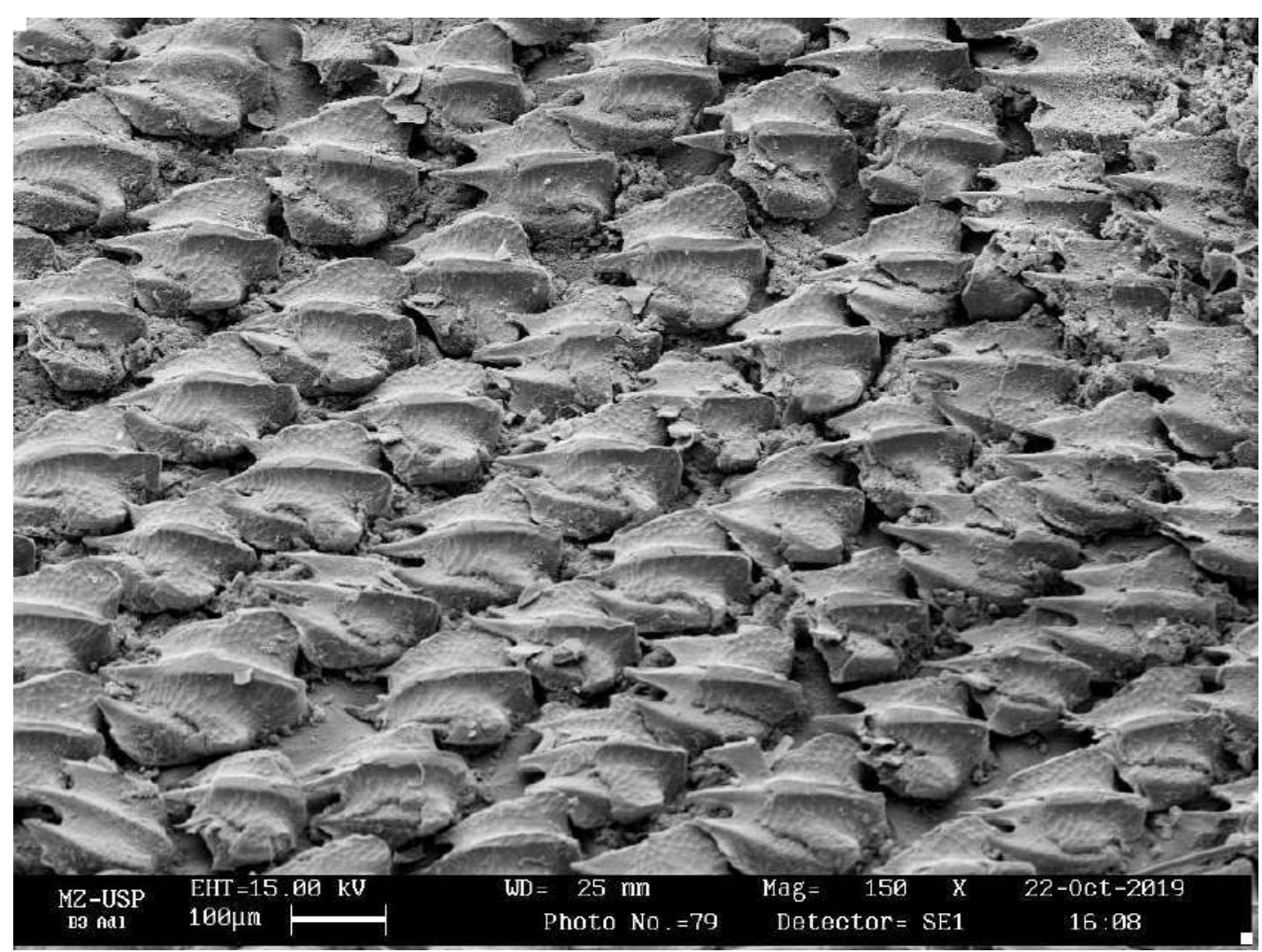

Figure 108. Dermal denticles collected from the section B3 (see fig. 1) of the adult Isogomphodon oxyrhynchus (MPEG 3455). Additional information in the image footer.

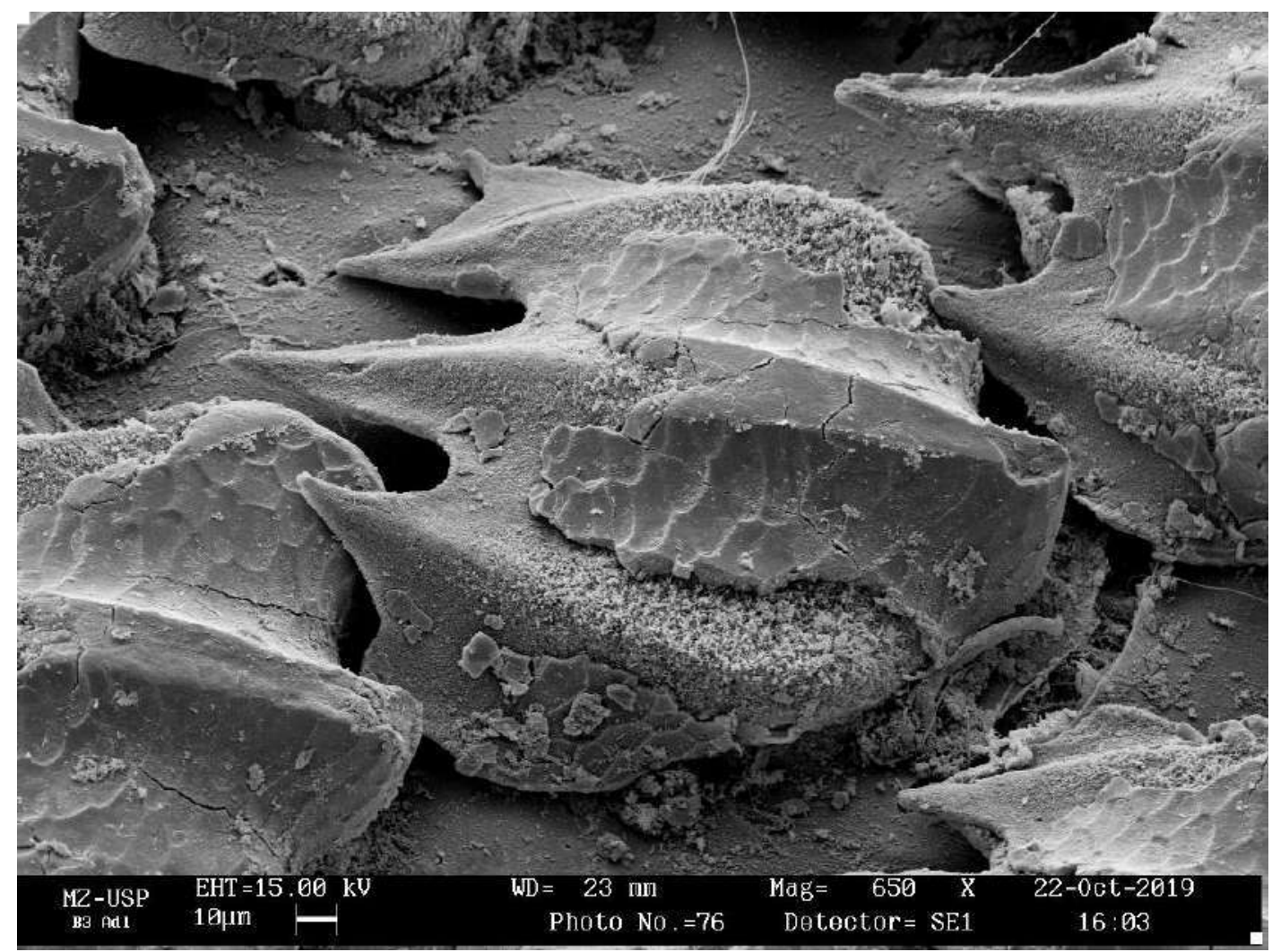

Figure 109. Dermal denticles collected from the section B3 (see fig. 1) of the adult Isogomphodon oxyrhynchus (MPEG 3455). Additional information in the image footer. 


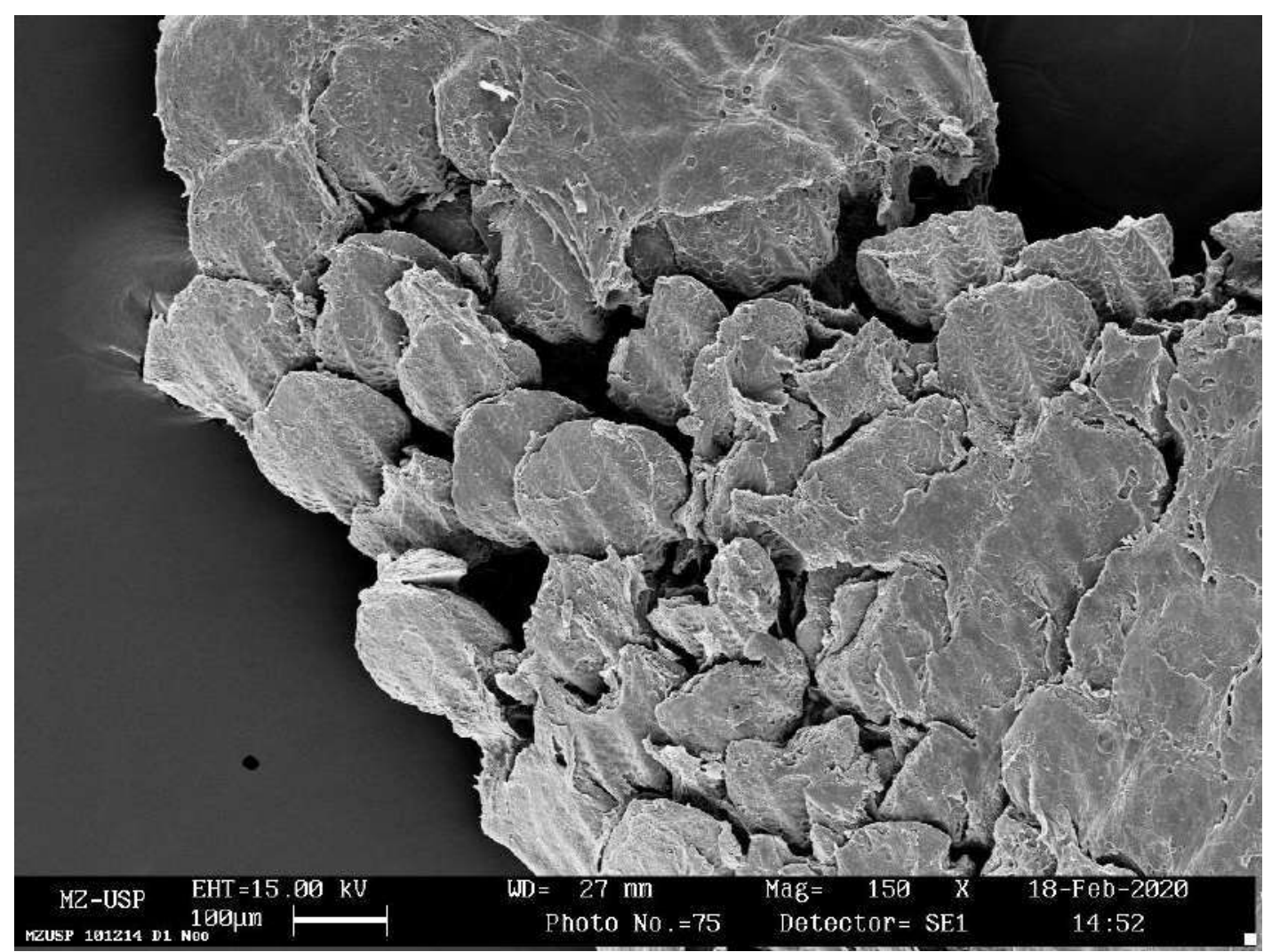

Figure 110. Dermal denticles collected from the section D1 (see fig. 1) of the neonate Isogomphodon oxyrhynchus (MZUSP 101214). Additional information in the image footer.

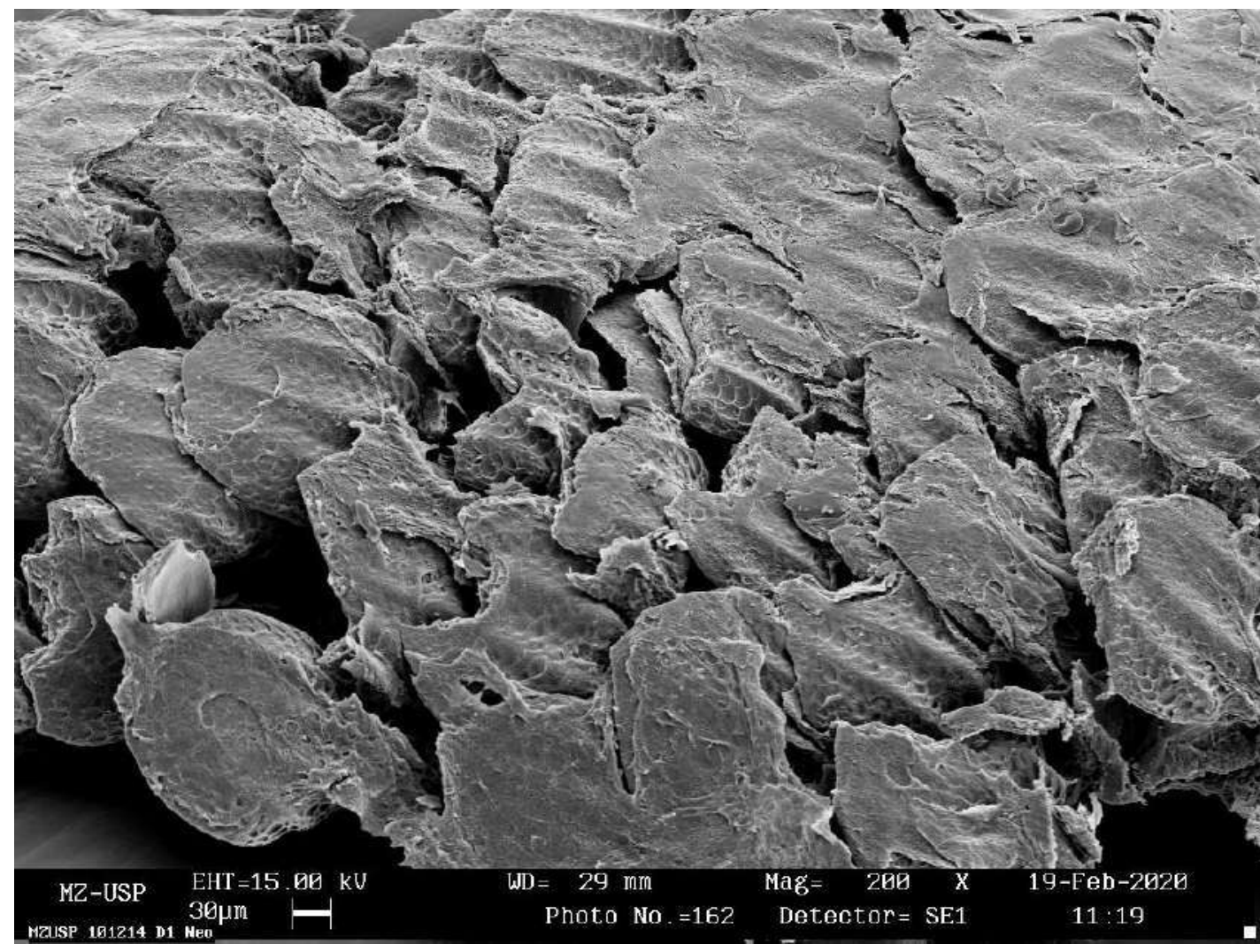

Figure 111. Dermal denticles collected from the section D1 (see fig. 1) of the neonate Isogomphodon oxyrhynchus (MZUSP 101214). Additional information in the image footer. 


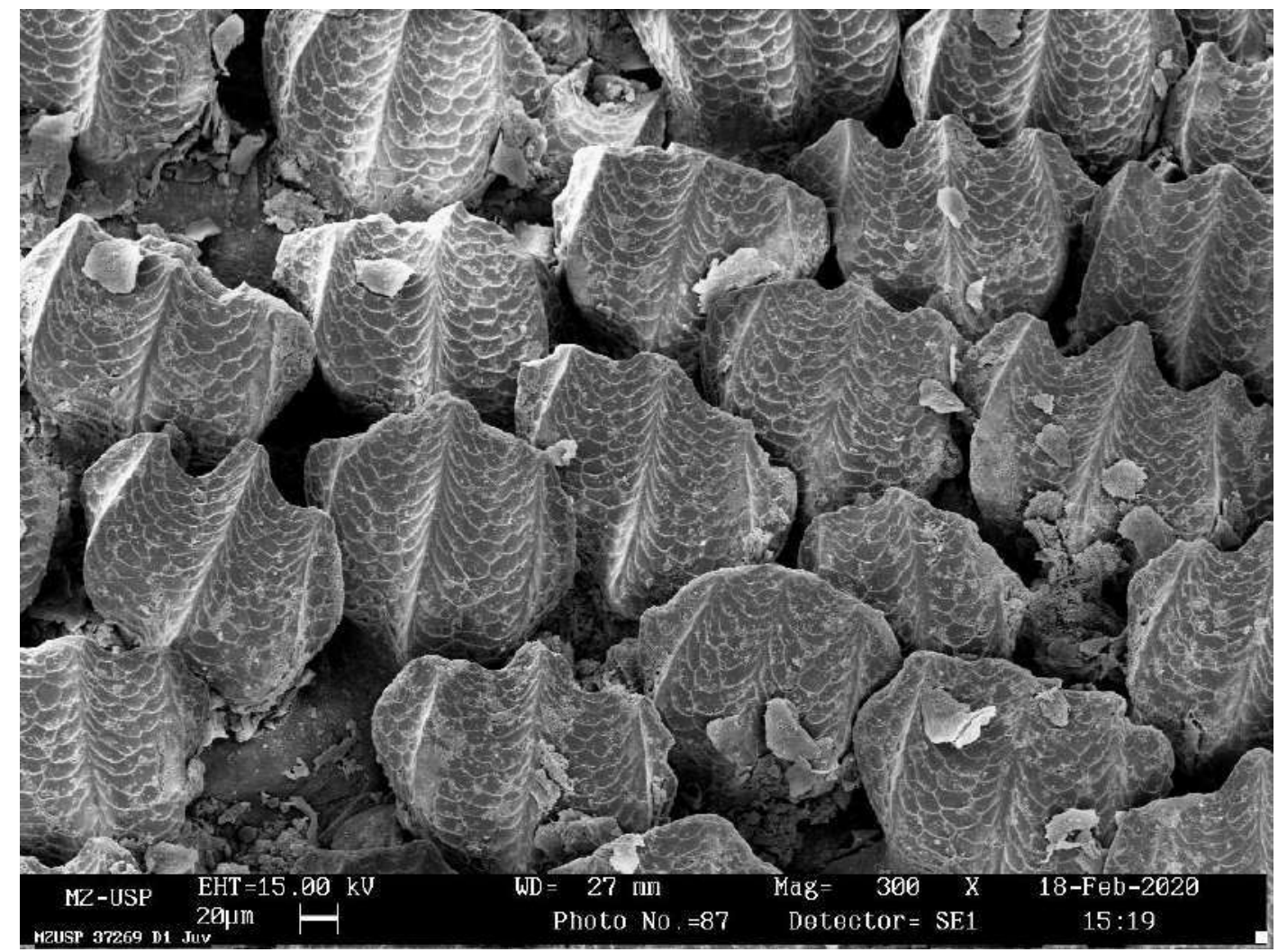

Figure 112. Dermal denticles collected from the section D1 (see fig. 1) of the juvenile Isogomphodon oxyrhynchus (MZUSP 37289). Additional information in the image footer.

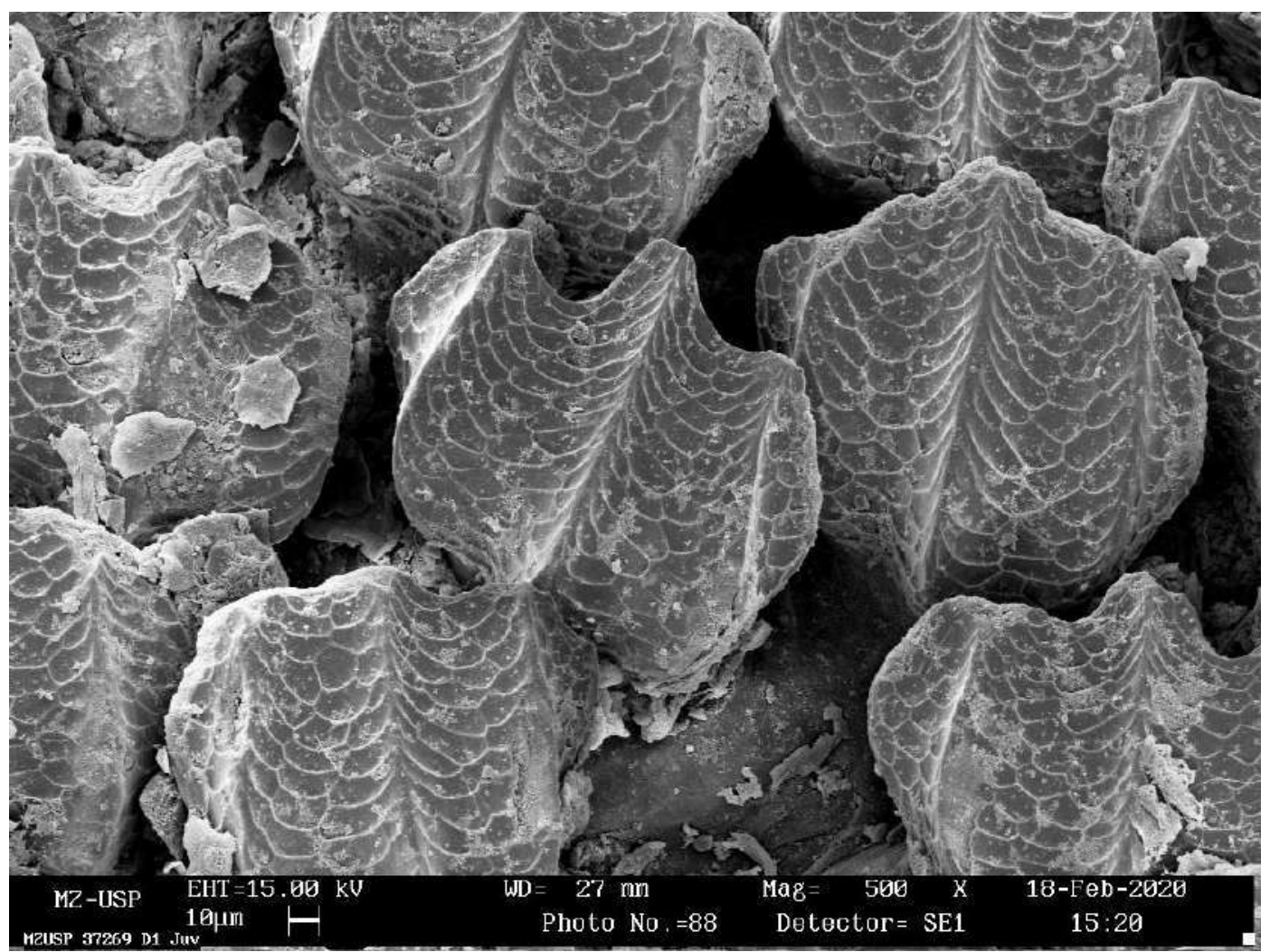

Figure 113. Dermal denticles collected from the section D1 (see fig. 1) of the juvenile Isogomphodon oxyrhynchus (MZUSP 37289). Additional information in the image footer. 


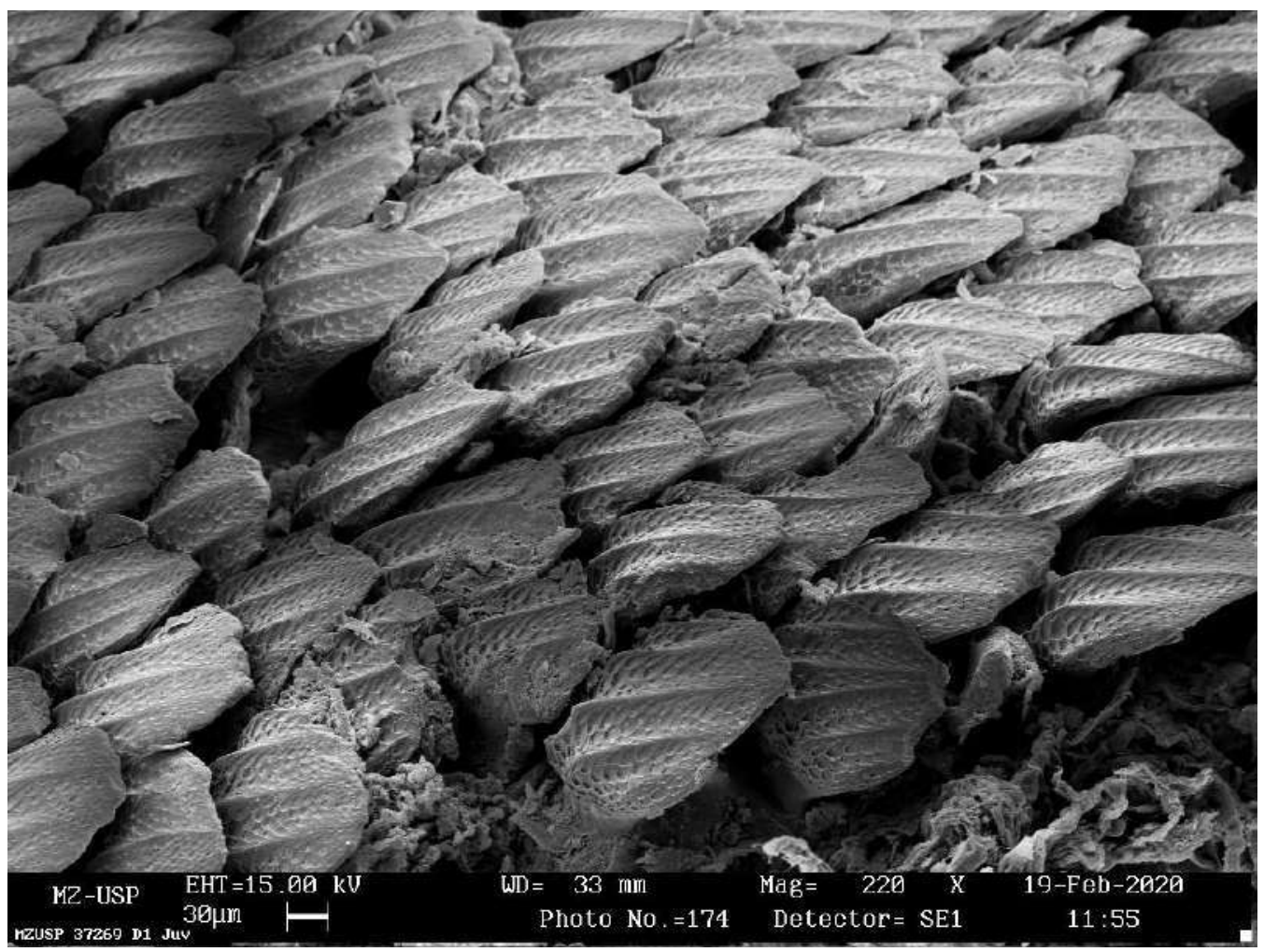

Figure 114. Dermal denticles collected from the section D1 (see fig. 1) of the juvenile Isogomphodon oxyrhynchus (MZUSP 37289). Additional information in the image footer.

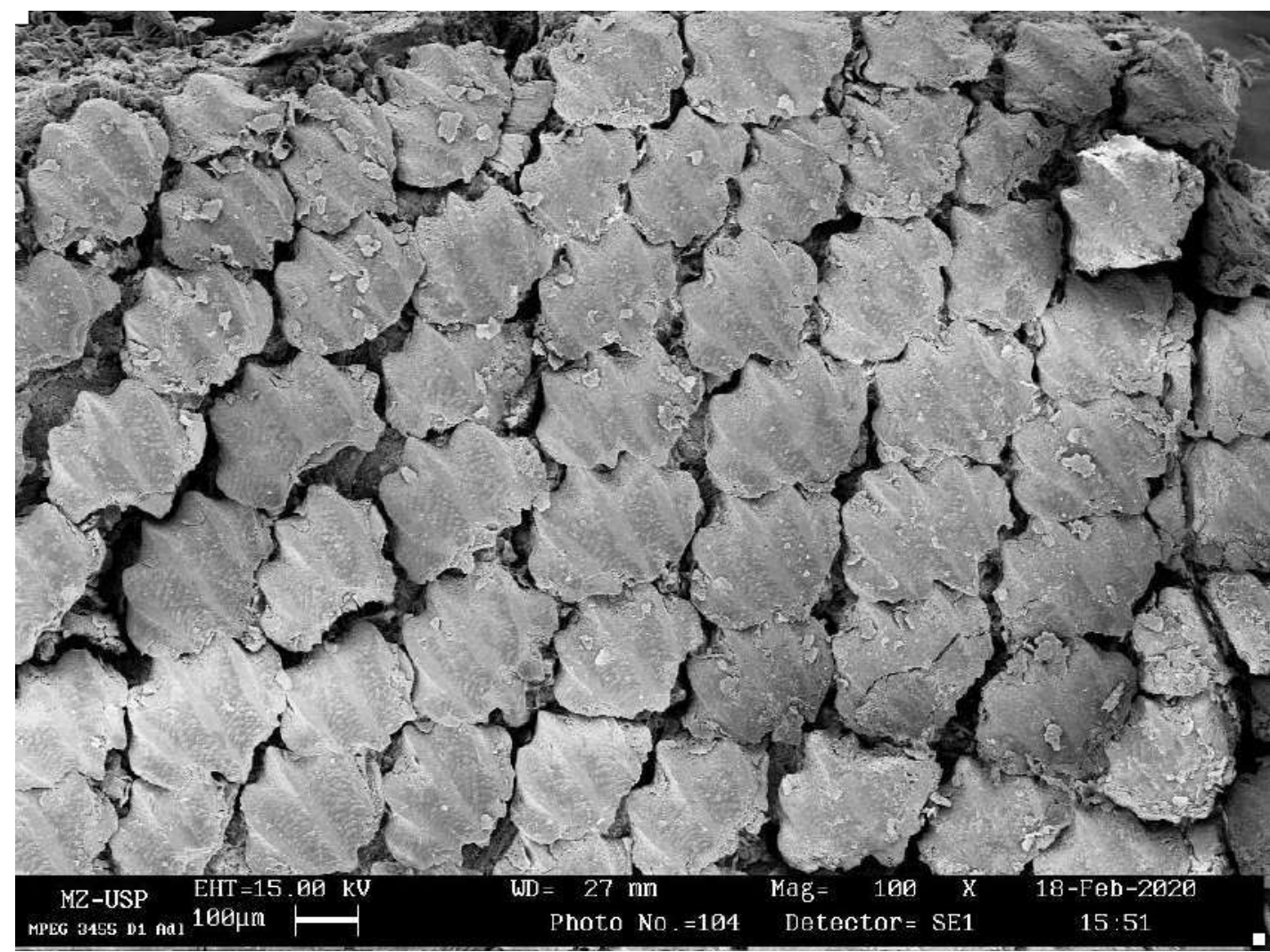

Figure 115. Dermal denticles collected from the section D1 (see fig. 1) of the adult Isogomphodon oxyrhynchus (MPEG 3455). Additional information in the image footer. 


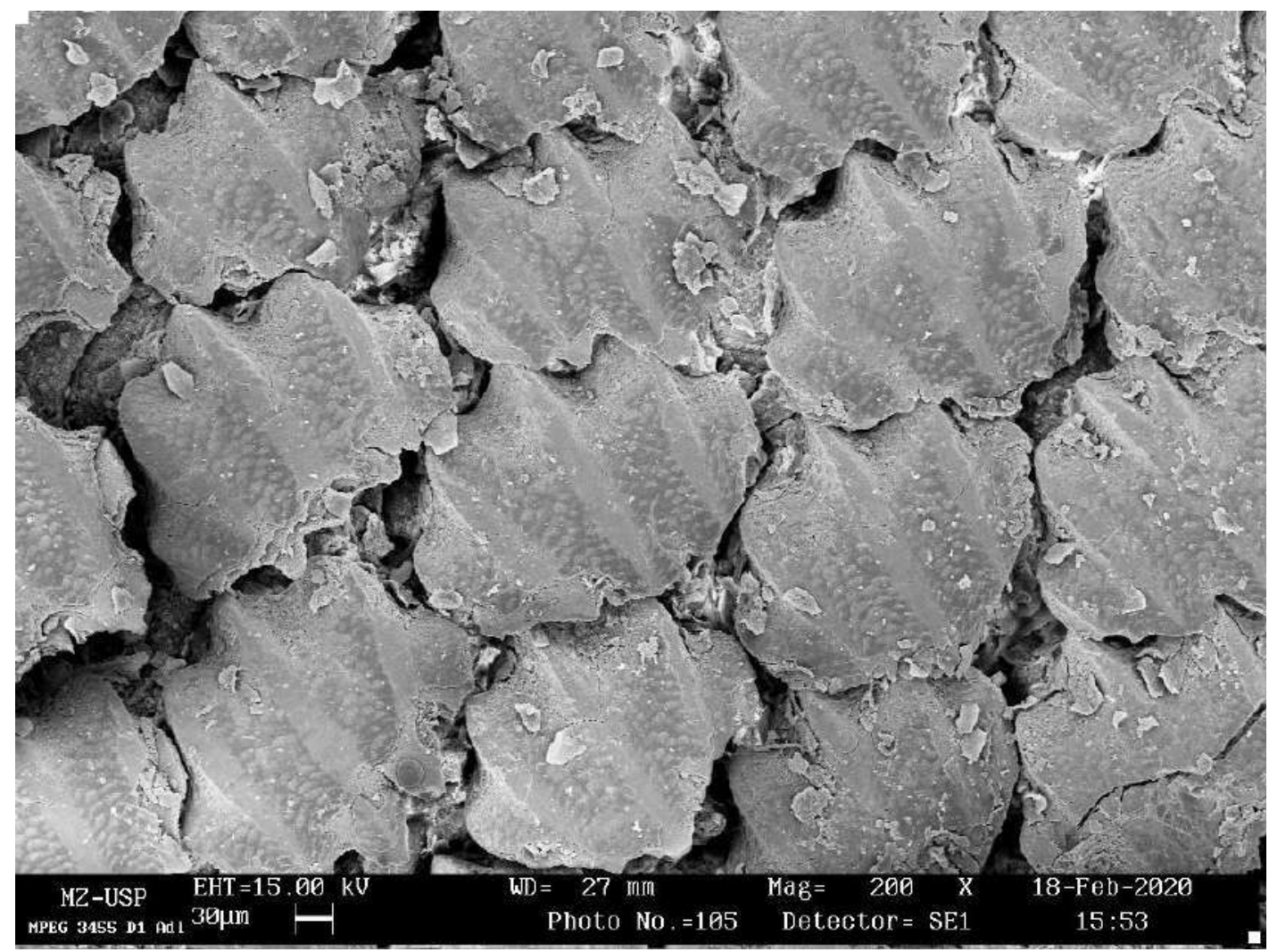

Figure 116. Dermal denticles collected from the section D1 (see fig. 1) of the adult Isogomphodon oxyrhynchus (MPEG 3455). Additional information in the image footer.

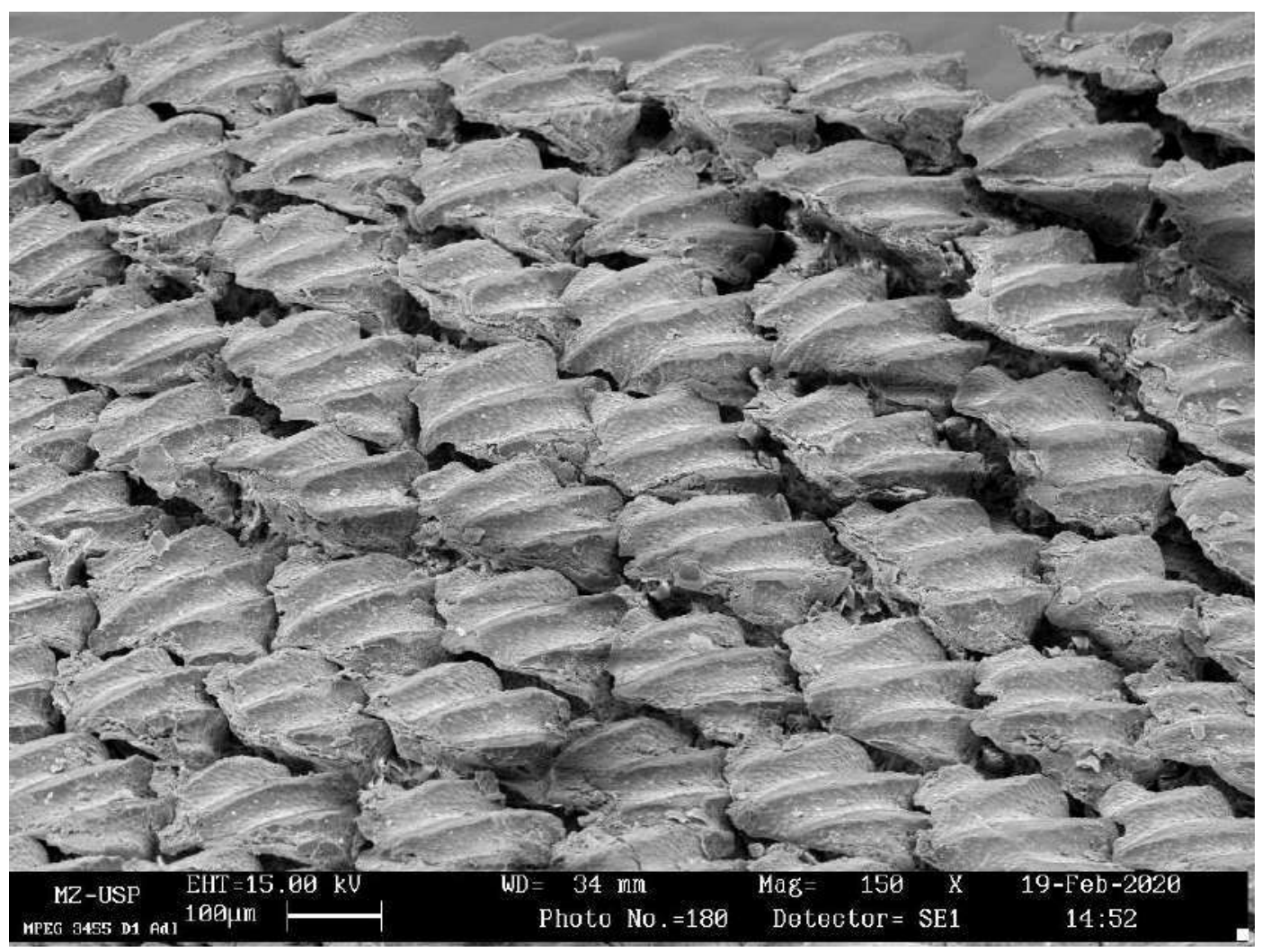

Figure 117. Dermal denticles collected from the section D1 (see fig. 1) of the adult Isogomphodon oxyrhynchus (MPEG 3455). Additional information in the image footer. 


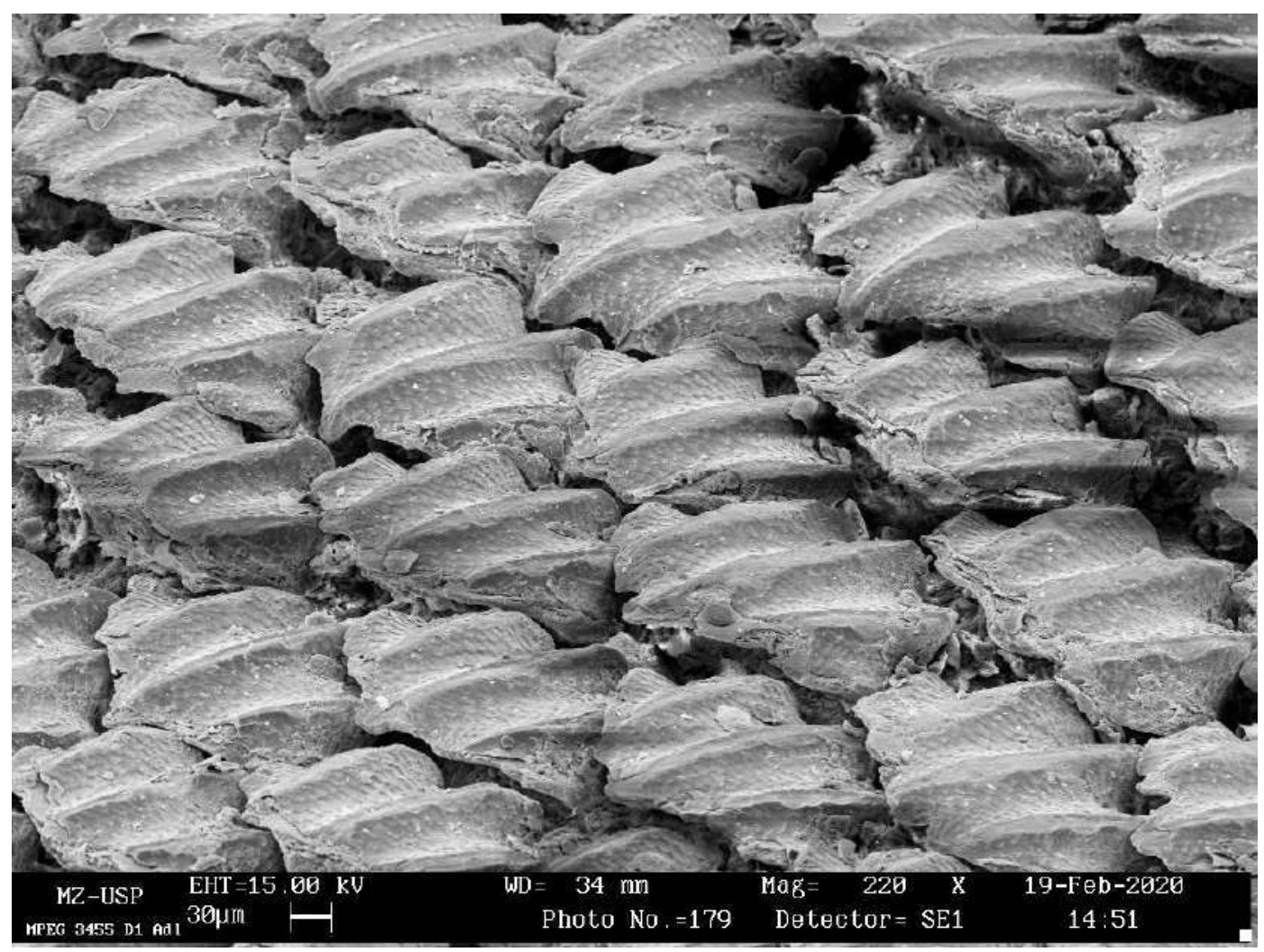

Figure 118. Dermal denticles collected from the section D1 (see fig. 1) of the adult Isogomphodon oxyrhynchus (MPEG 3455). Additional information in the image footer.

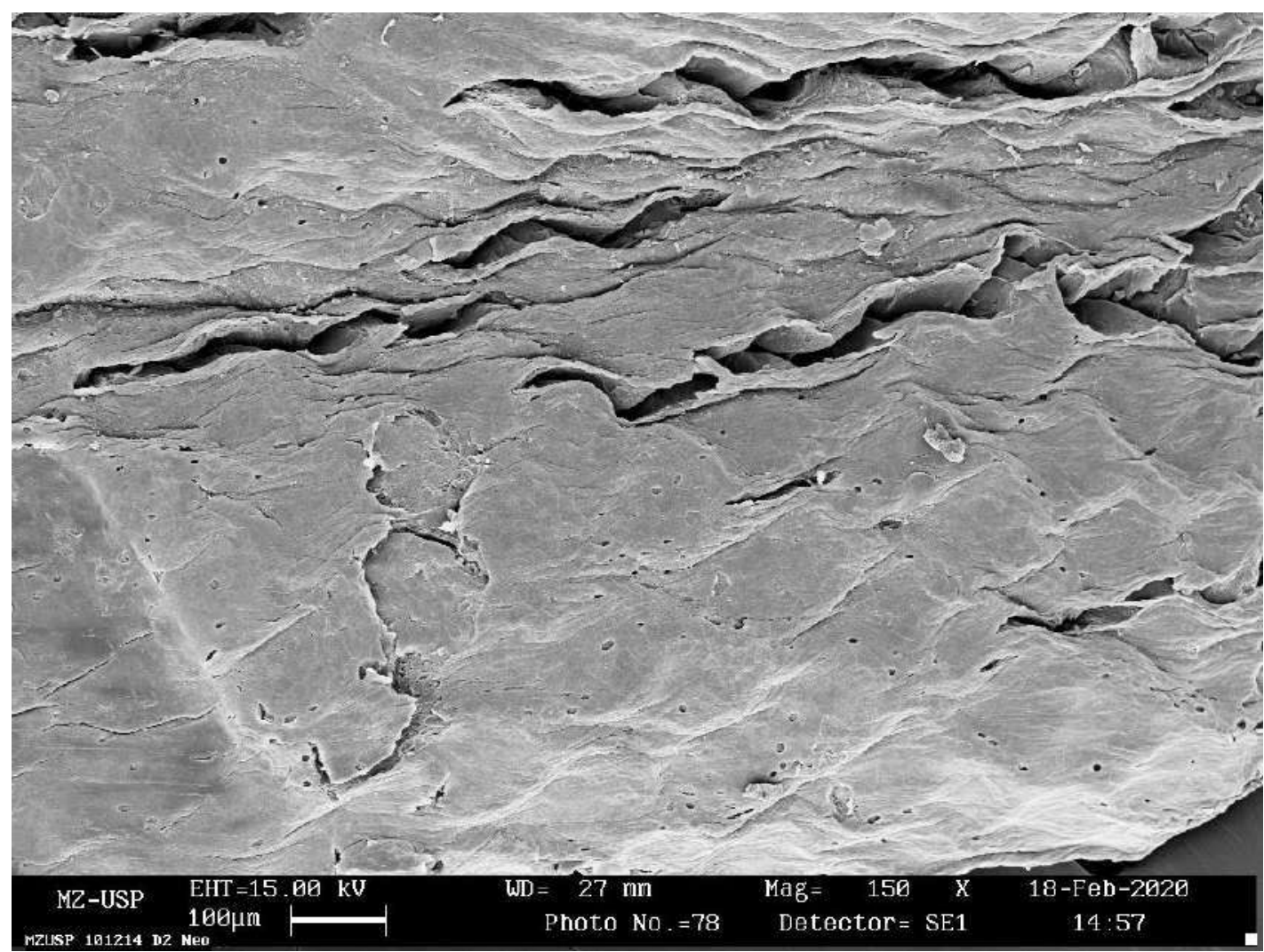

Figure 119. Dermal denticles collected from the section D2 (see fig. 1) of the neonate Isogomphodon oxyrhynchus (MZUSP 101214). Additional information in the image footer. 


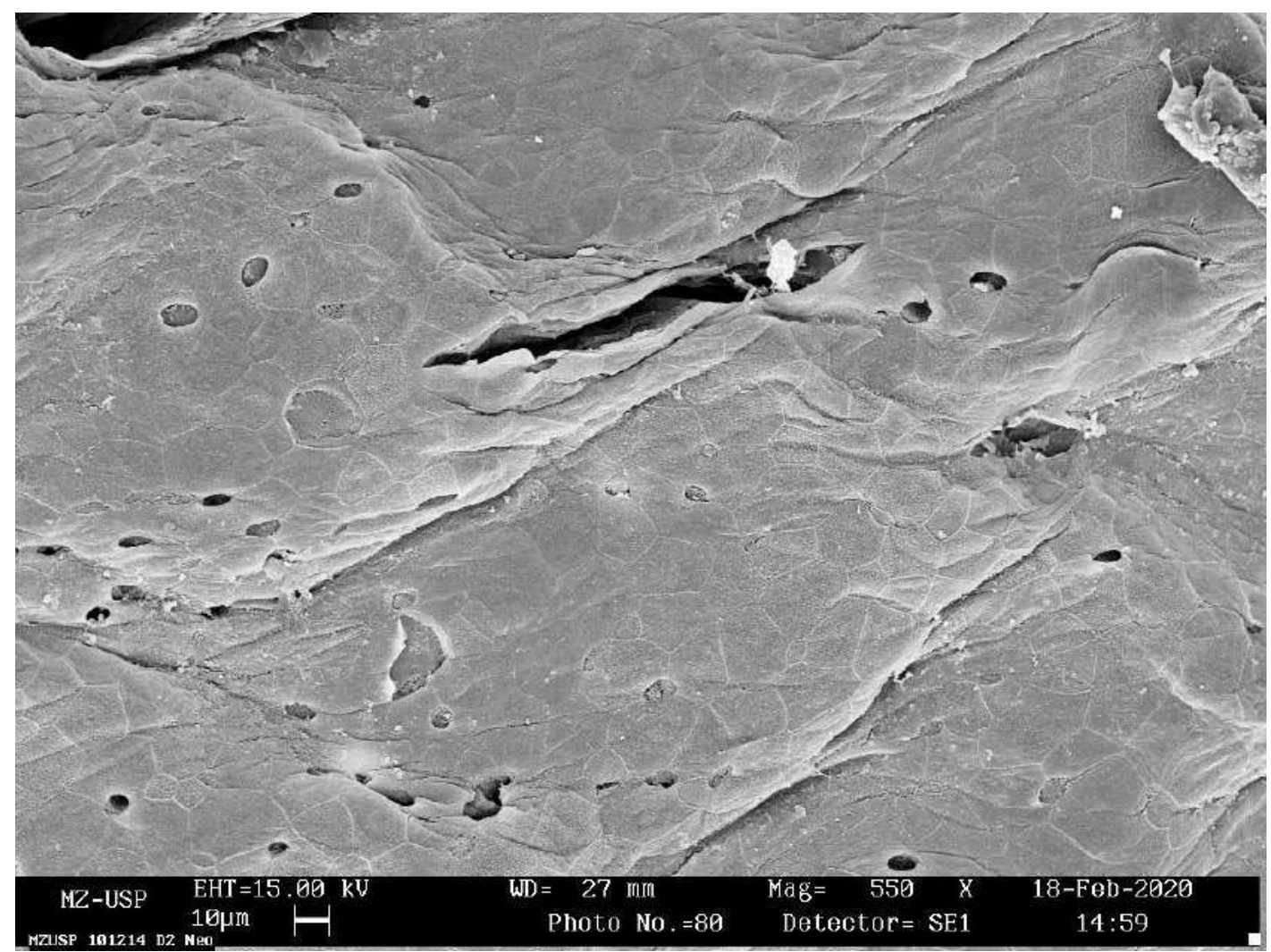

Figure 120. Dermal denticles collected from the section D2 (see fig. 1) of the neonate Isogomphodon oxyrhynchus (MZUSP 101214). Additional information in the image footer.

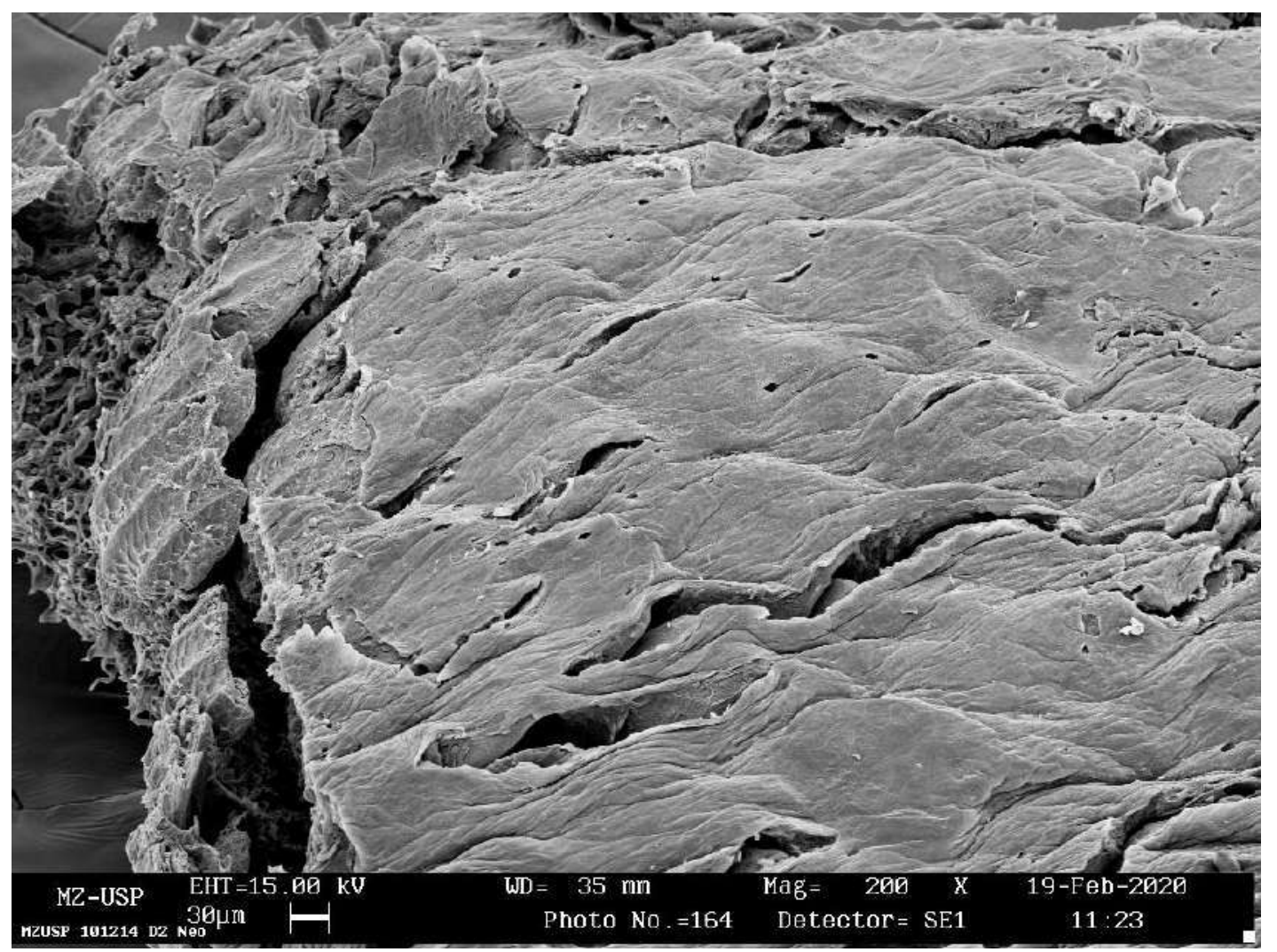

Figure 121. Dermal denticles collected from the section D2 (see fig. 1) of the neonate Isogomphodon oxyrhynchus (MZUSP 101214). Additional information in the image footer. 


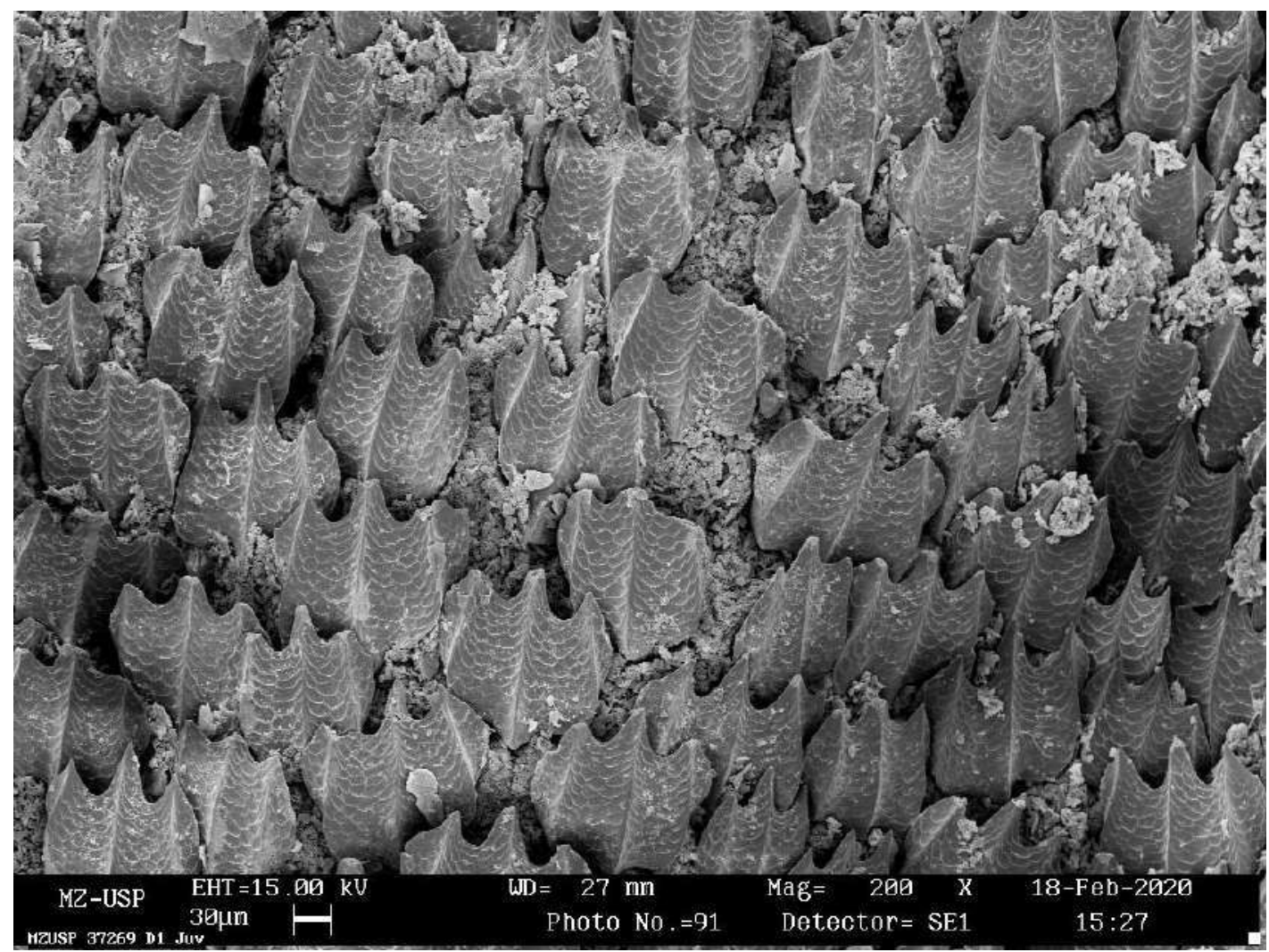

Figure 122. Dermal denticles collected from the section D2 (see fig. 1) of the juvenile Isogomphodon oxyrhynchus (MZUSP 37289). Additional information in the image footer.

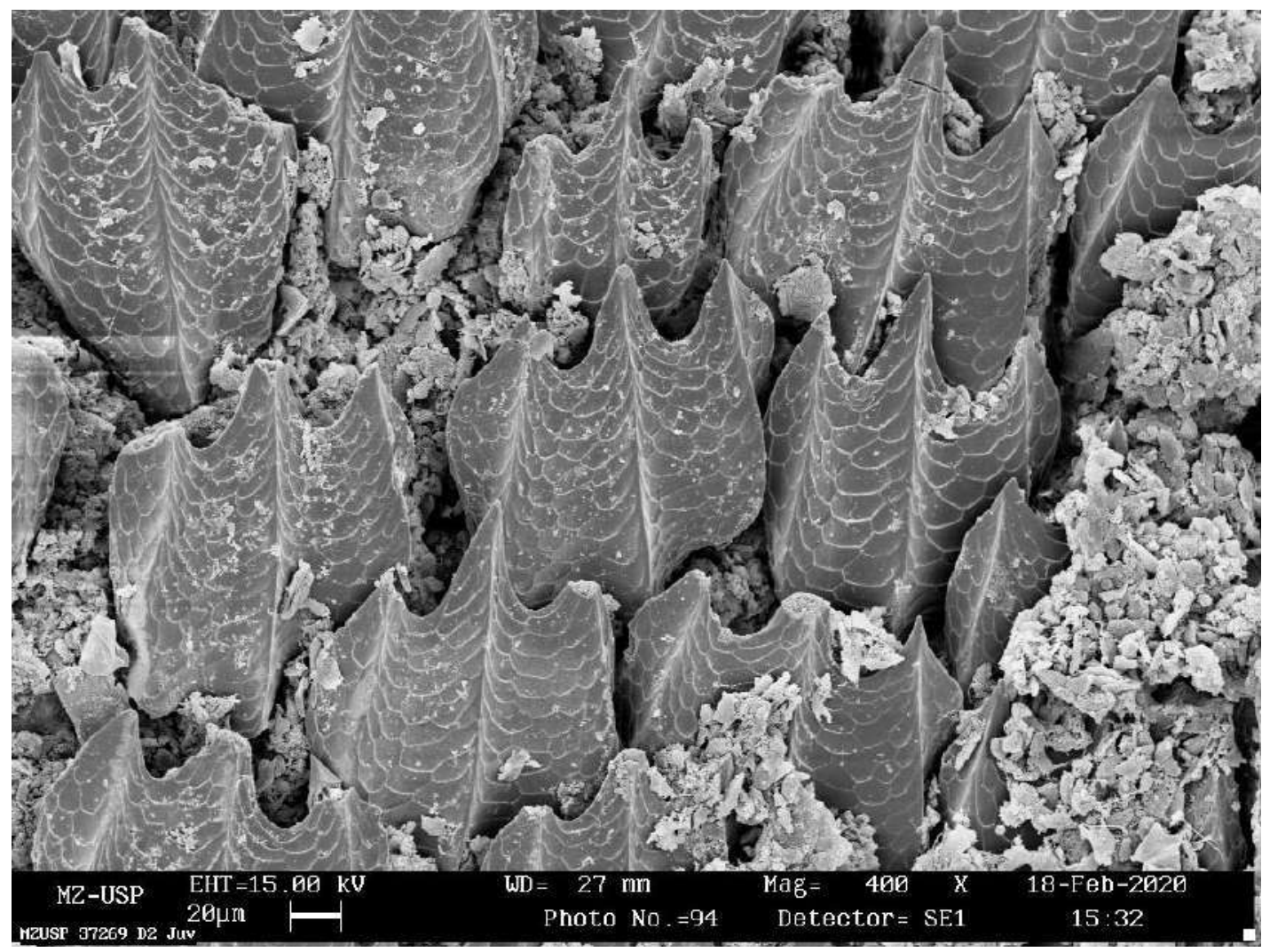

Figure 123. Dermal denticles collected from the section D2 (see fig. 1) of the juvenile Isogomphodon oxyrhynchus (MZUSP 37289). Additional information in the image footer. 


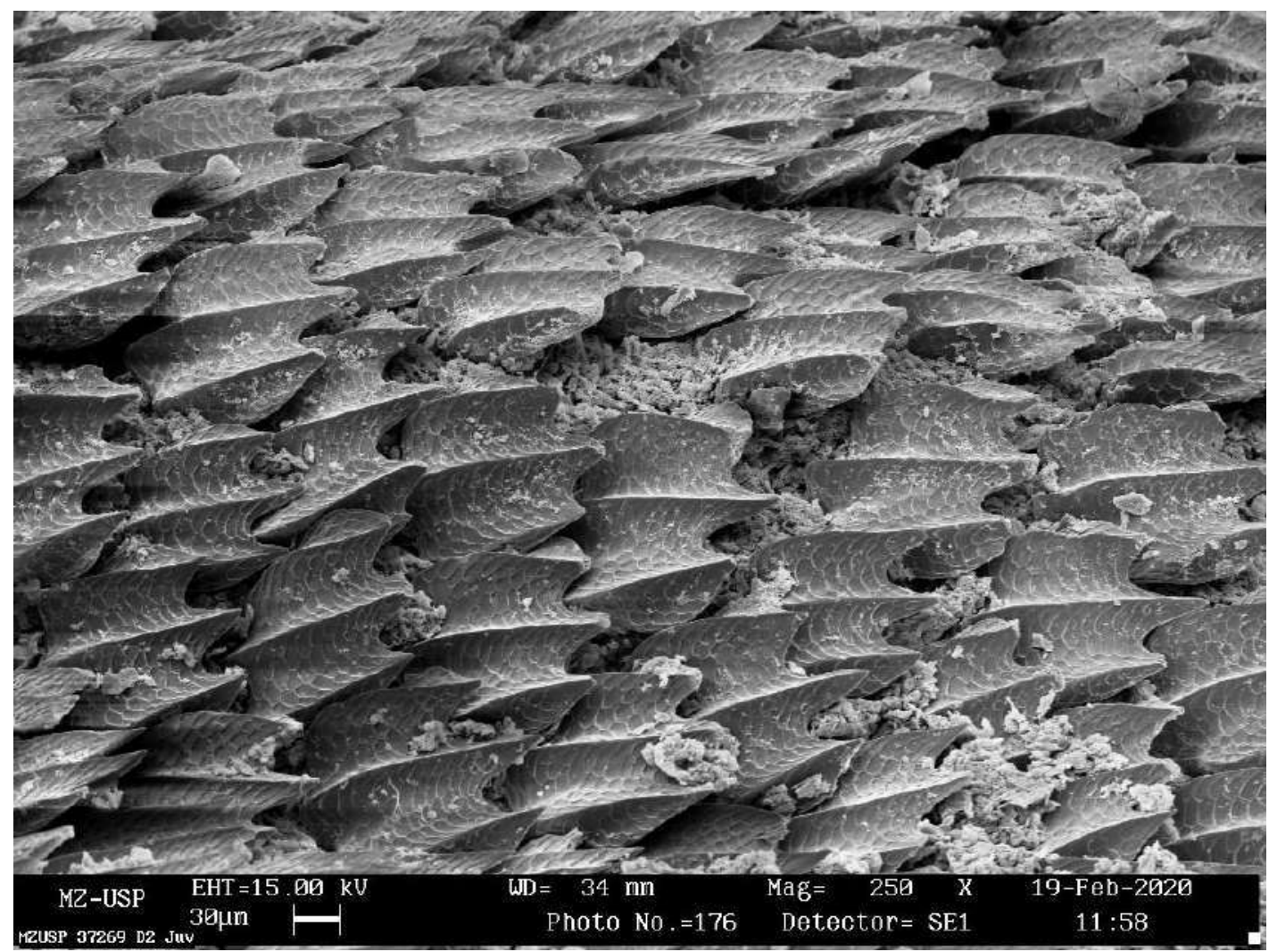

Figure 124. Dermal denticles collected from the section D2 (see fig. 1) of the juvenile Isogomphodon oxyrhynchus (MZUSP 37289). Additional information in the image footer.

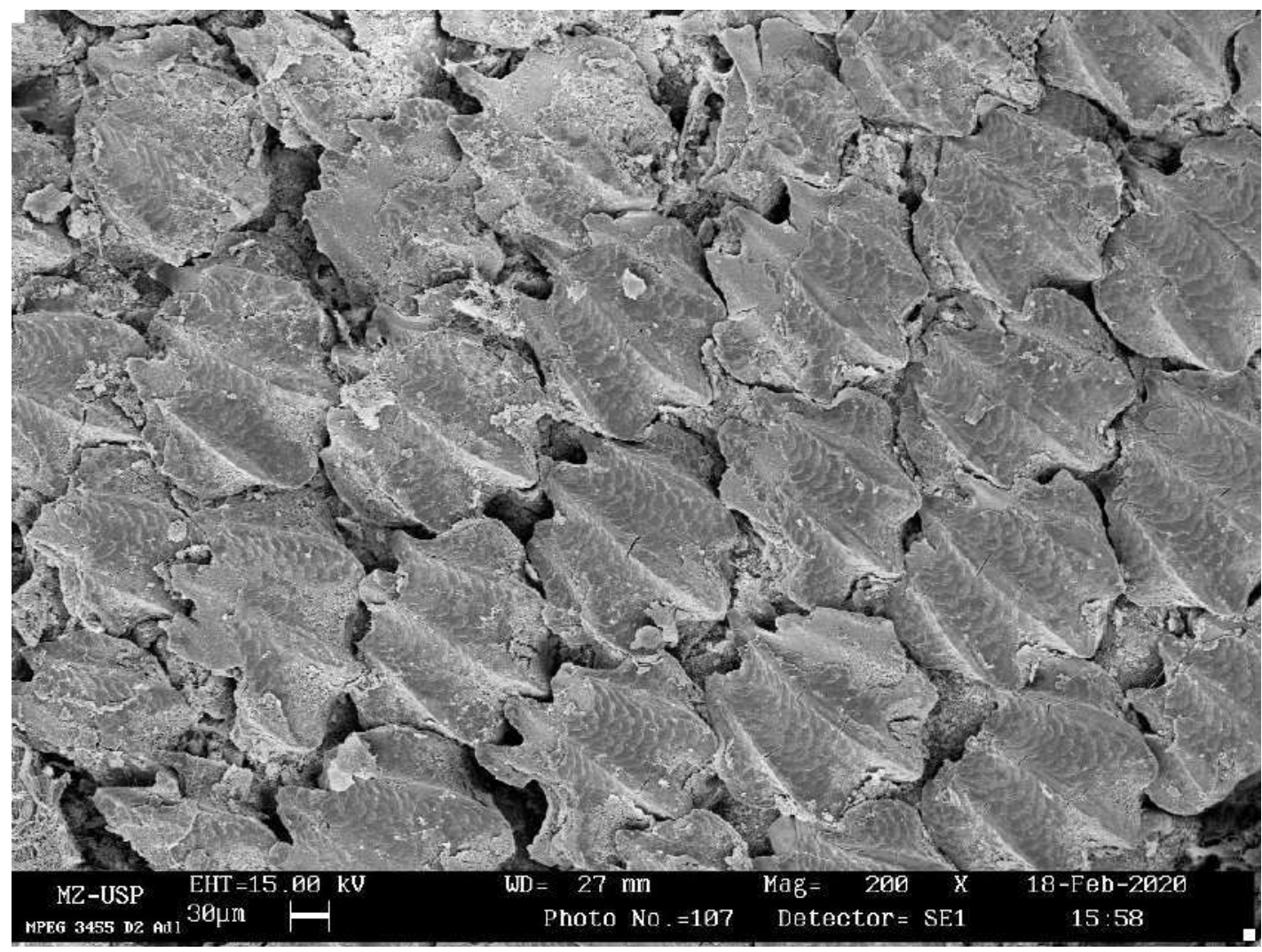

Figure 125. Dermal denticles collected from the section D2 (see fig. 1) of the adult Isogomphodon oxyrhynchus (MPEG 3455). Additional information in the image footer. 


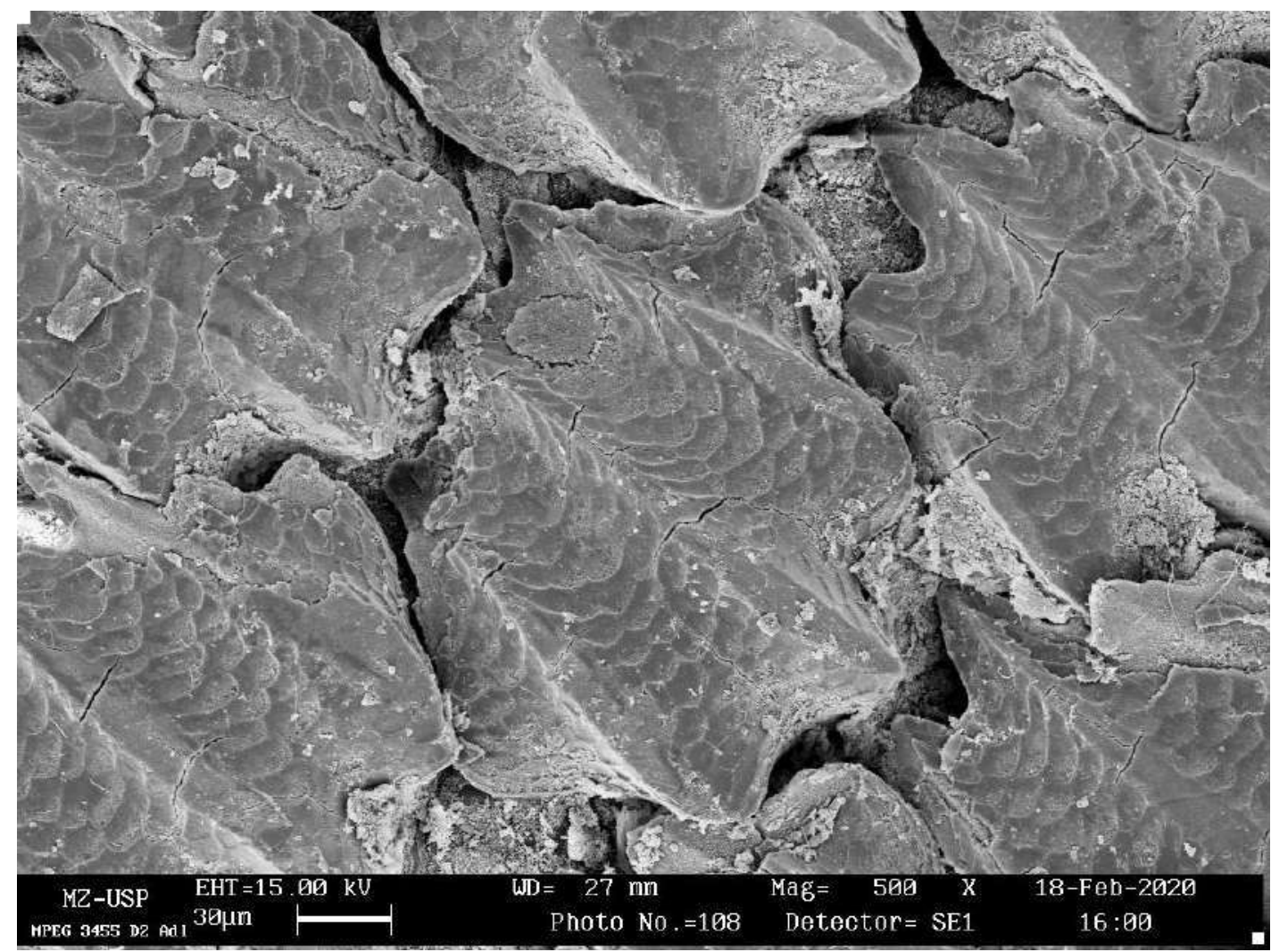

Figure 126. Dermal denticles collected from the section D2 (see fig. 1) of the adult Isogomphodon oxyrhynchus (MPEG 3455). Additional information in the image footer.

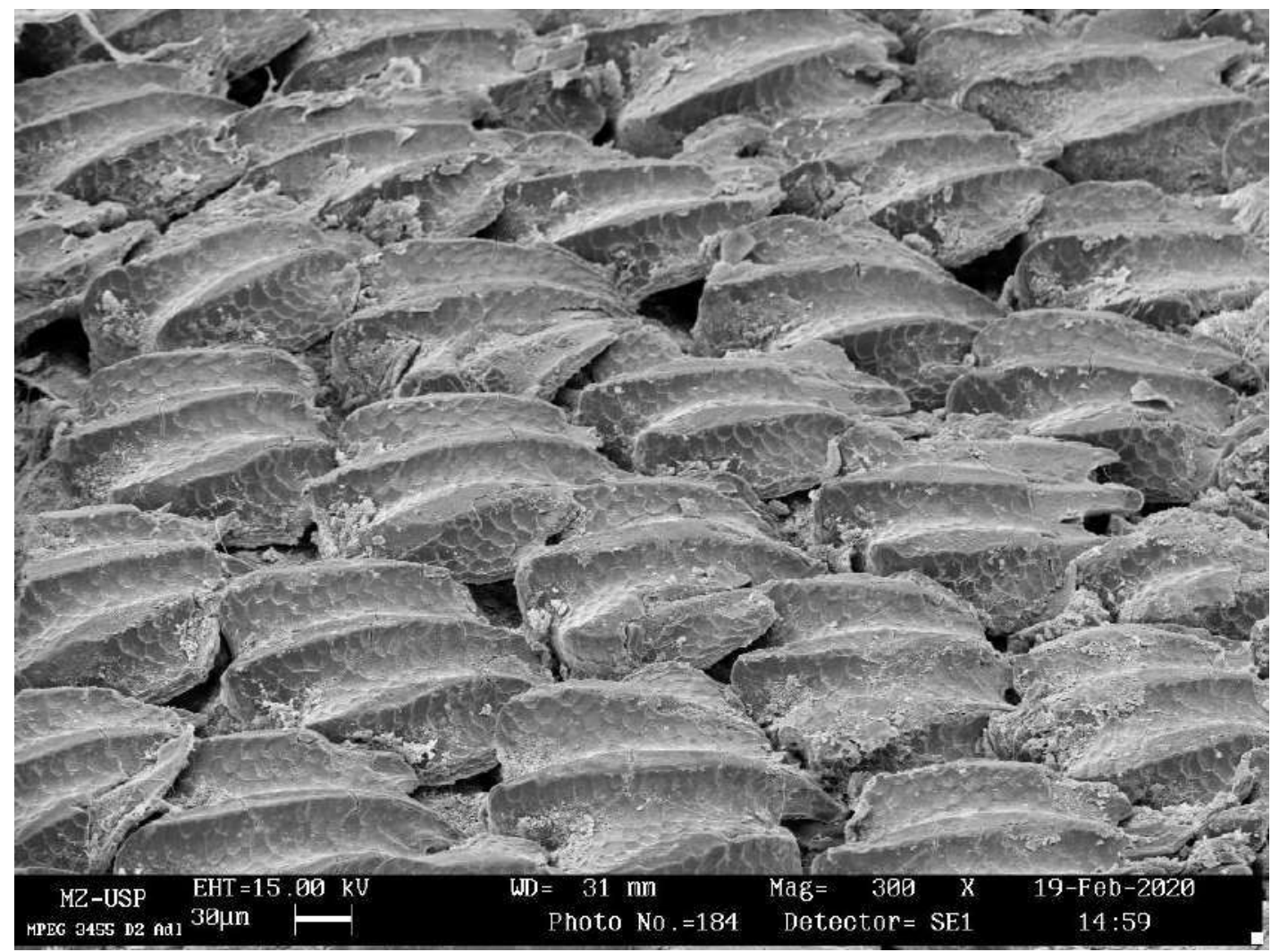

Figure 127. Dermal denticles collected from the section D2 (see fig. 1) of the adult Isogomphodon oxyrhynchus (MPEG 3455). Additional information in the image footer. 


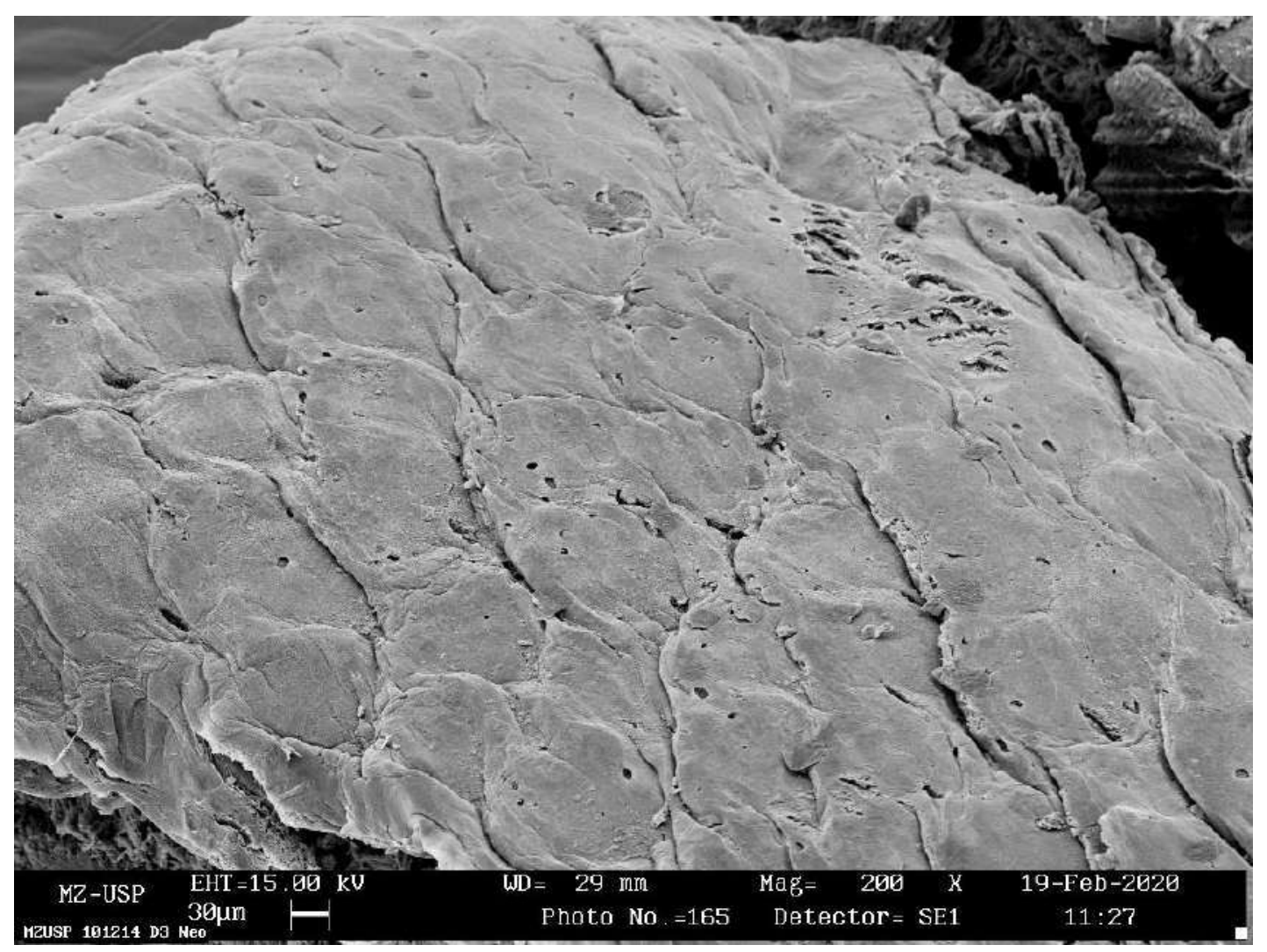

Figure 128. Dermal denticles collected from the section D3 (see fig. 1) of the neonate Isogomphodon oxyrhynchus (MZUSP 101214). Additional information in the image footer.

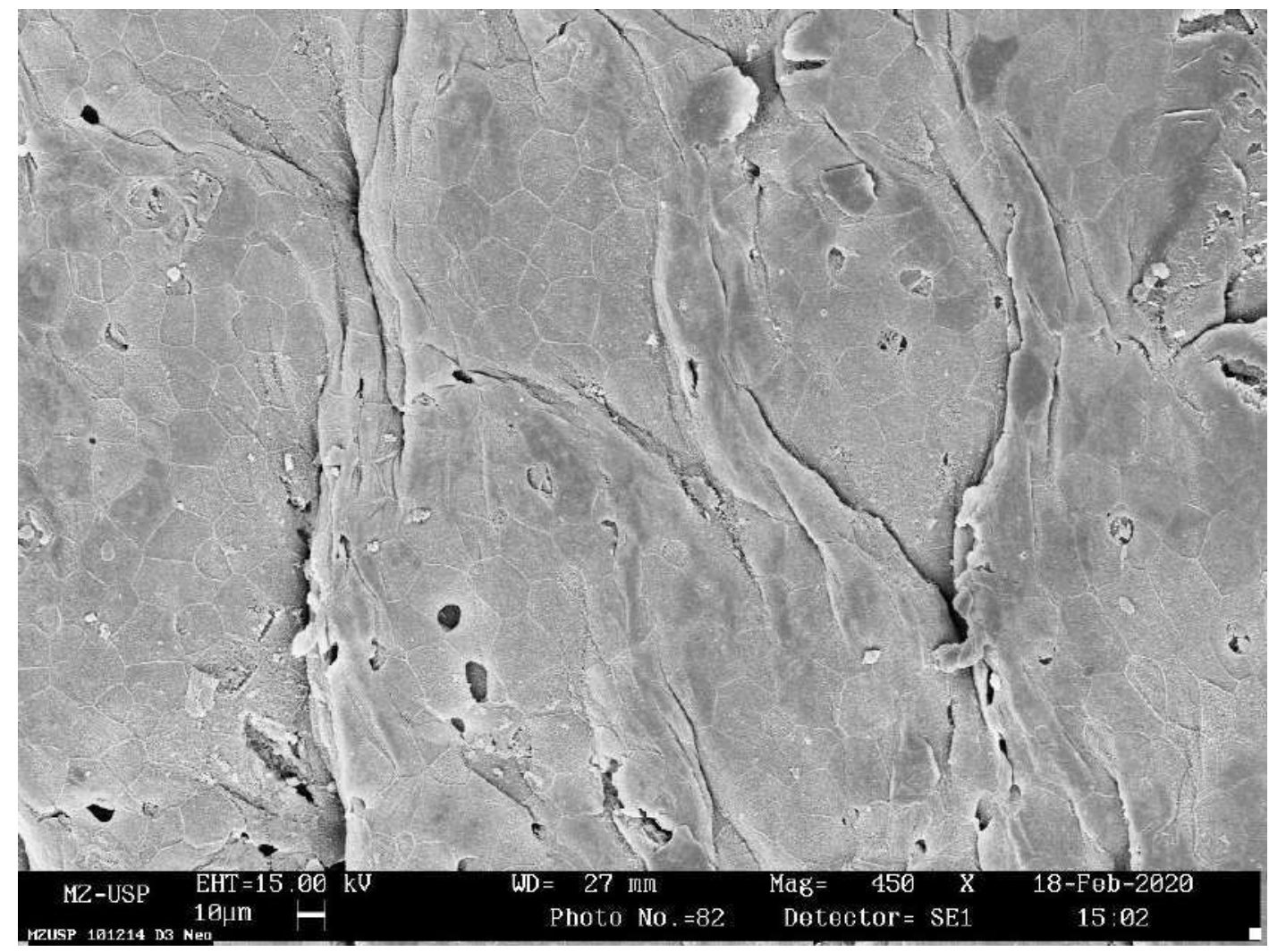

Figure 129. Dermal denticles collected from the section D3 (see fig. 1) of the neonate Isogomphodon oxyrhynchus (MZUSP 101214). Additional information in the image footer. 


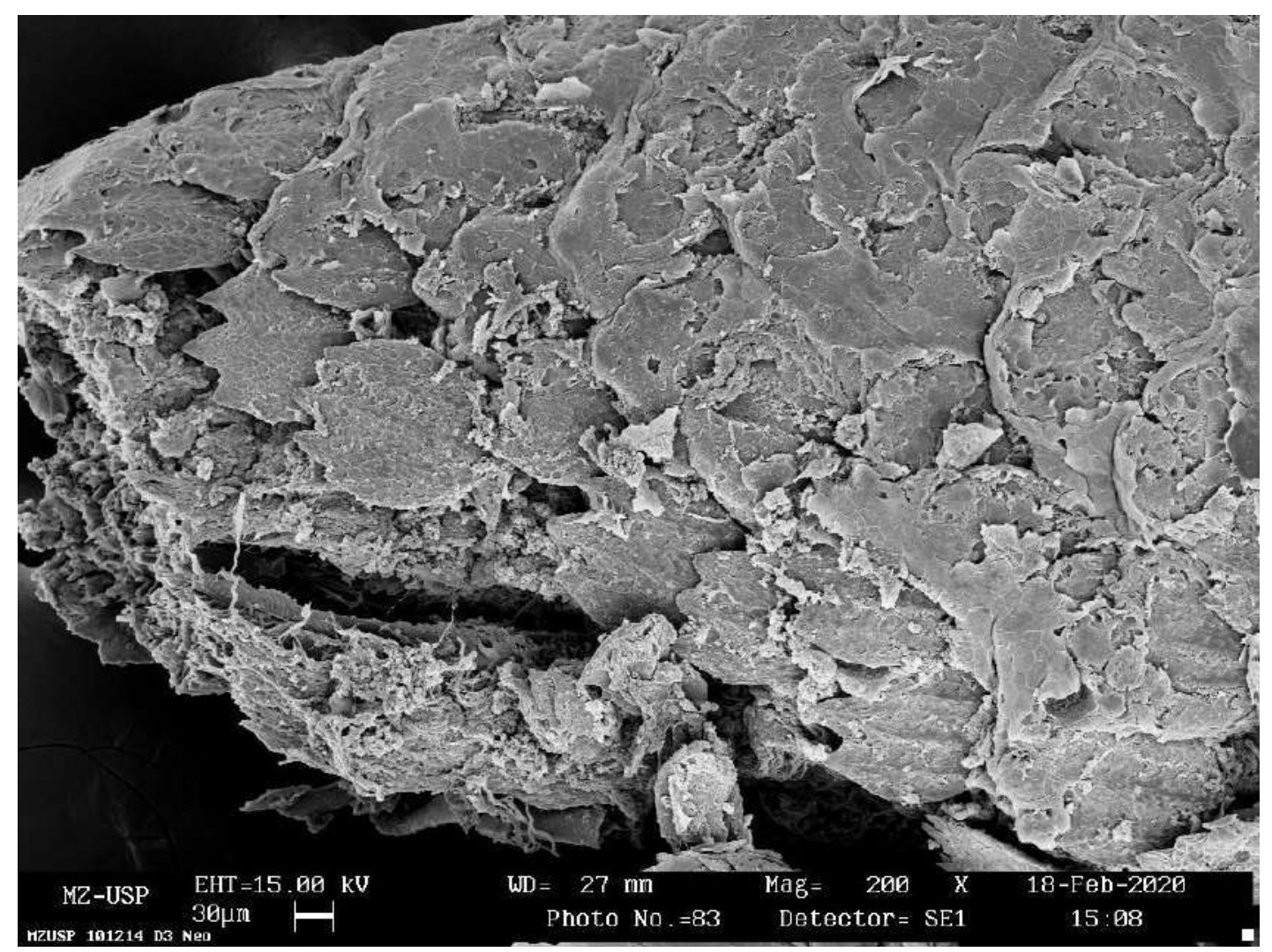

Figure 130. Dermal denticles collected from the section D3 (see fig. 1) of the neonate Isogomphodon oxyrhynchus (MZUSP 101214). Additional information in the image footer.

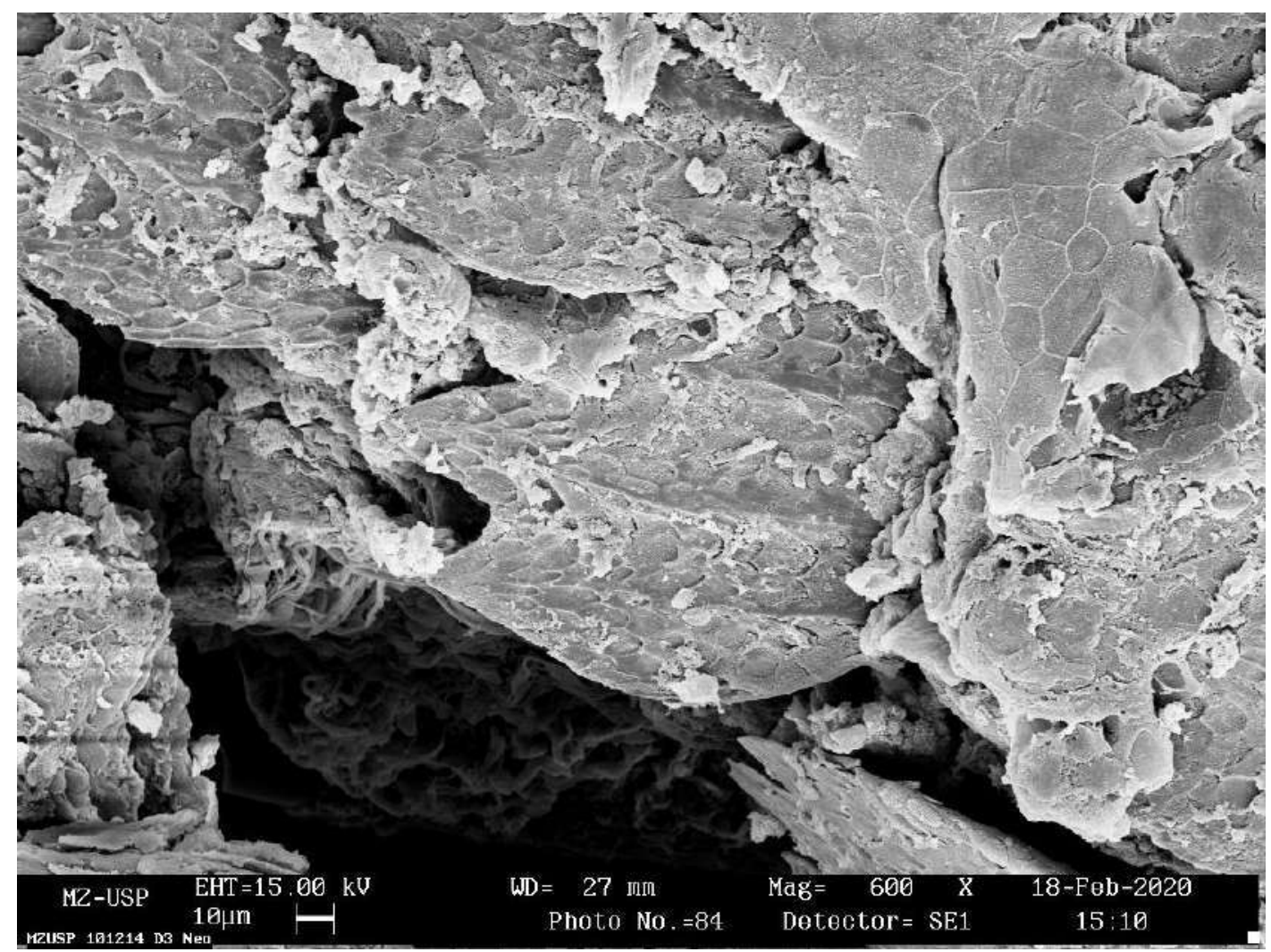

Figure 131. Dermal denticles collected from the section D3 (see fig. 1) of the neonate Isogomphodon oxyrhynchus (MZUSP 101214). Additional information in the image footer. 


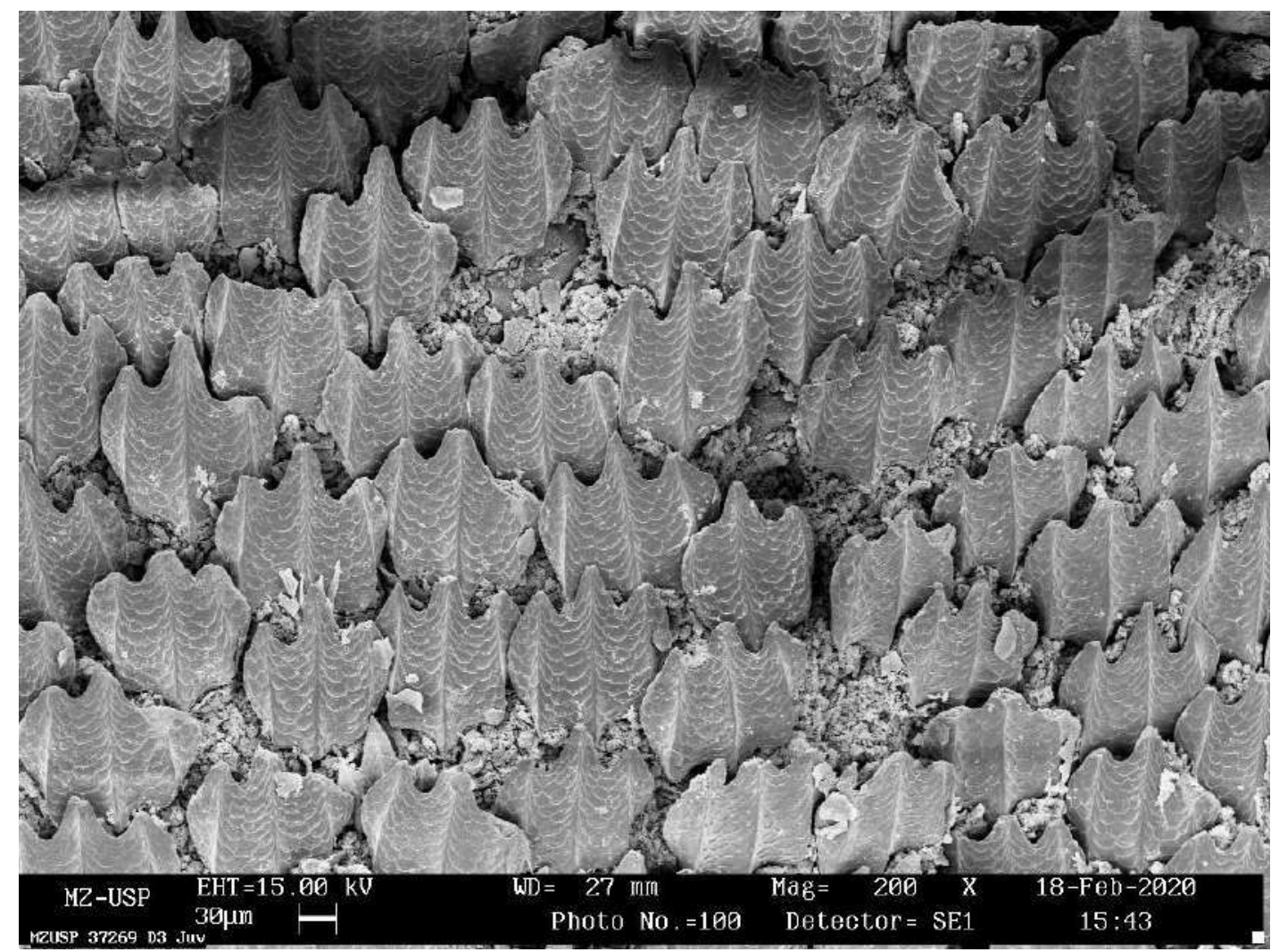

Figure 132. Dermal denticles collected from the section D3 (see fig. 1) of the juvenile Isogomphodon oxyrhynchus (MZUSP 37289). Additional information in the image footer.

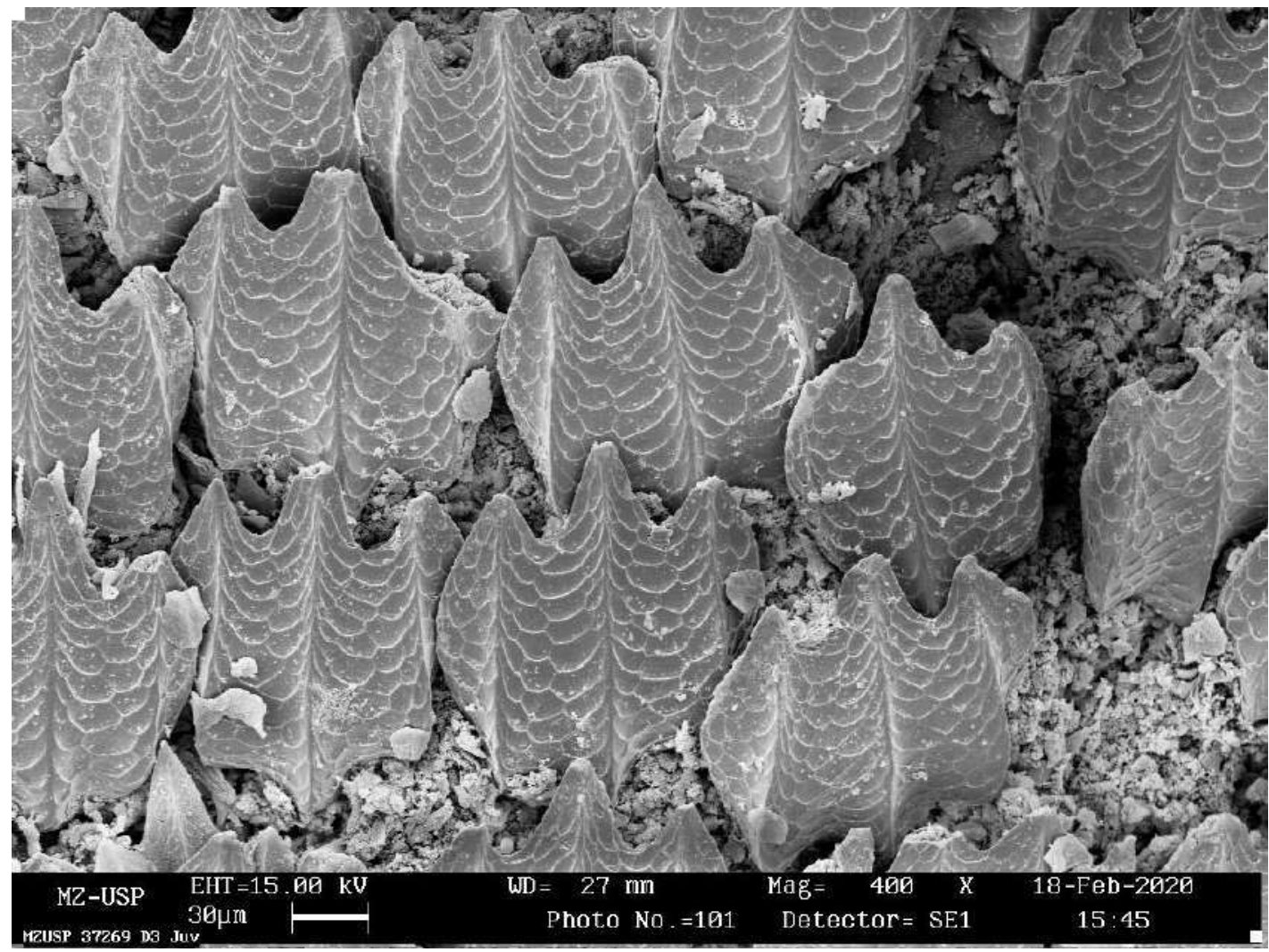

Figure 133. Dermal denticles collected from the section D3 (see fig. 1) of the juvenile Isogomphodon oxyrhynchus (MZUSP 37289). Additional information in the image footer. 


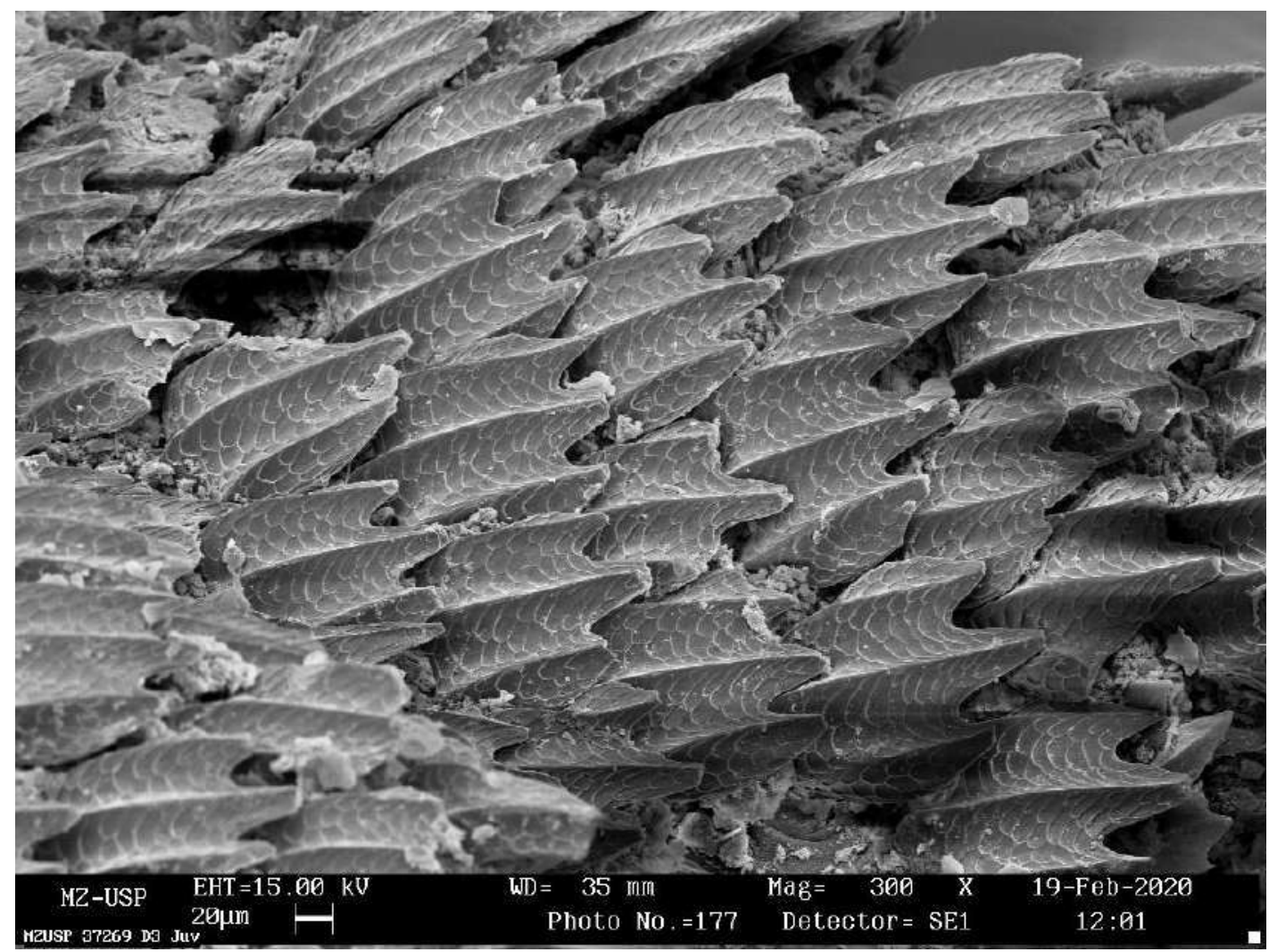

Figure 134. Dermal denticles collected from the section D3 (see fig. 1) of the juvenile Isogomphodon oxyrhynchus (MZUSP 37289). Additional information in the image footer.

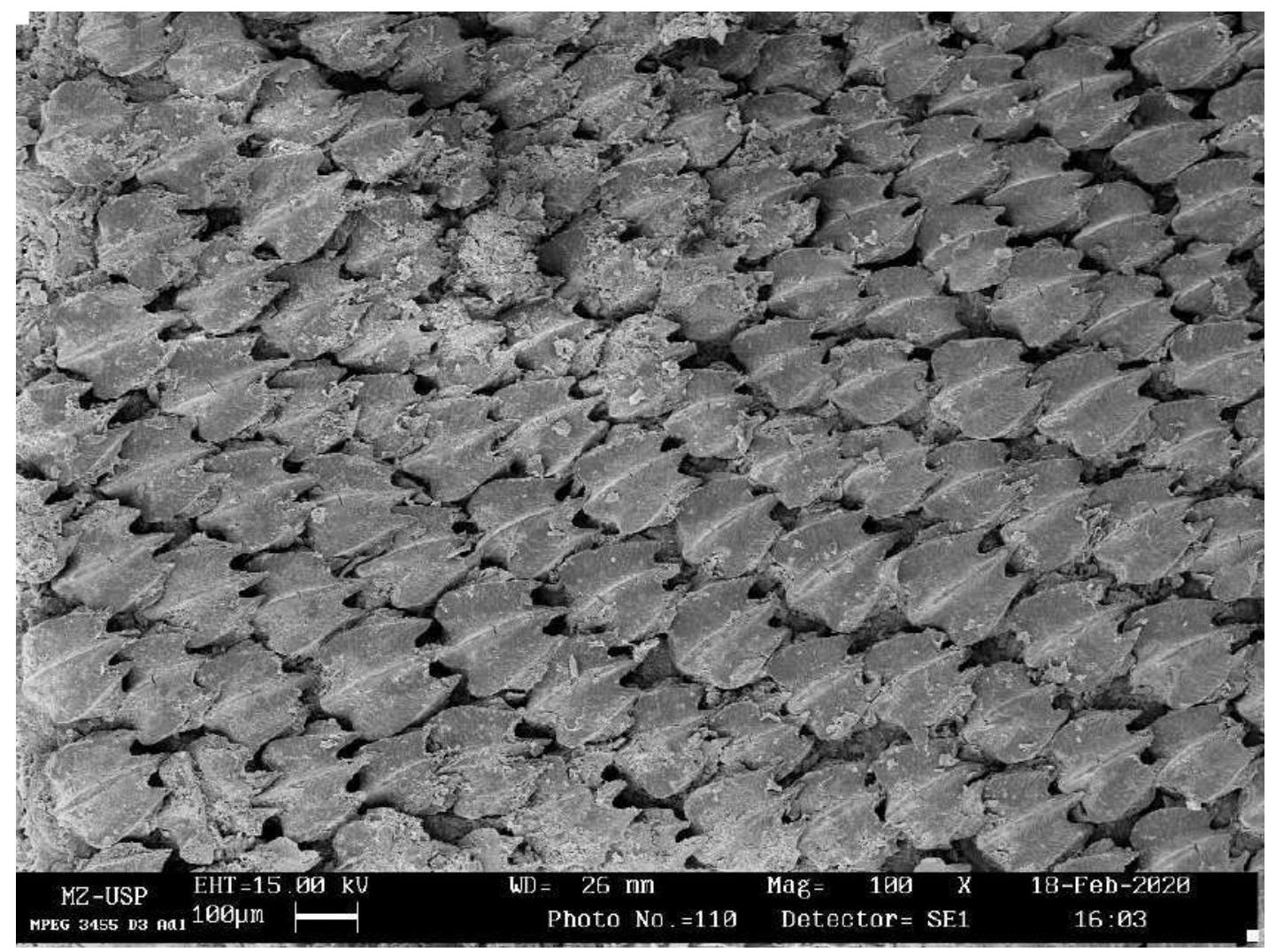

Figure 135. Dermal denticles collected from the section D3 (see fig. 1) of the adult Isogomphodon oxyrhynchus (MPEG 3455). Additional information in the image footer. 


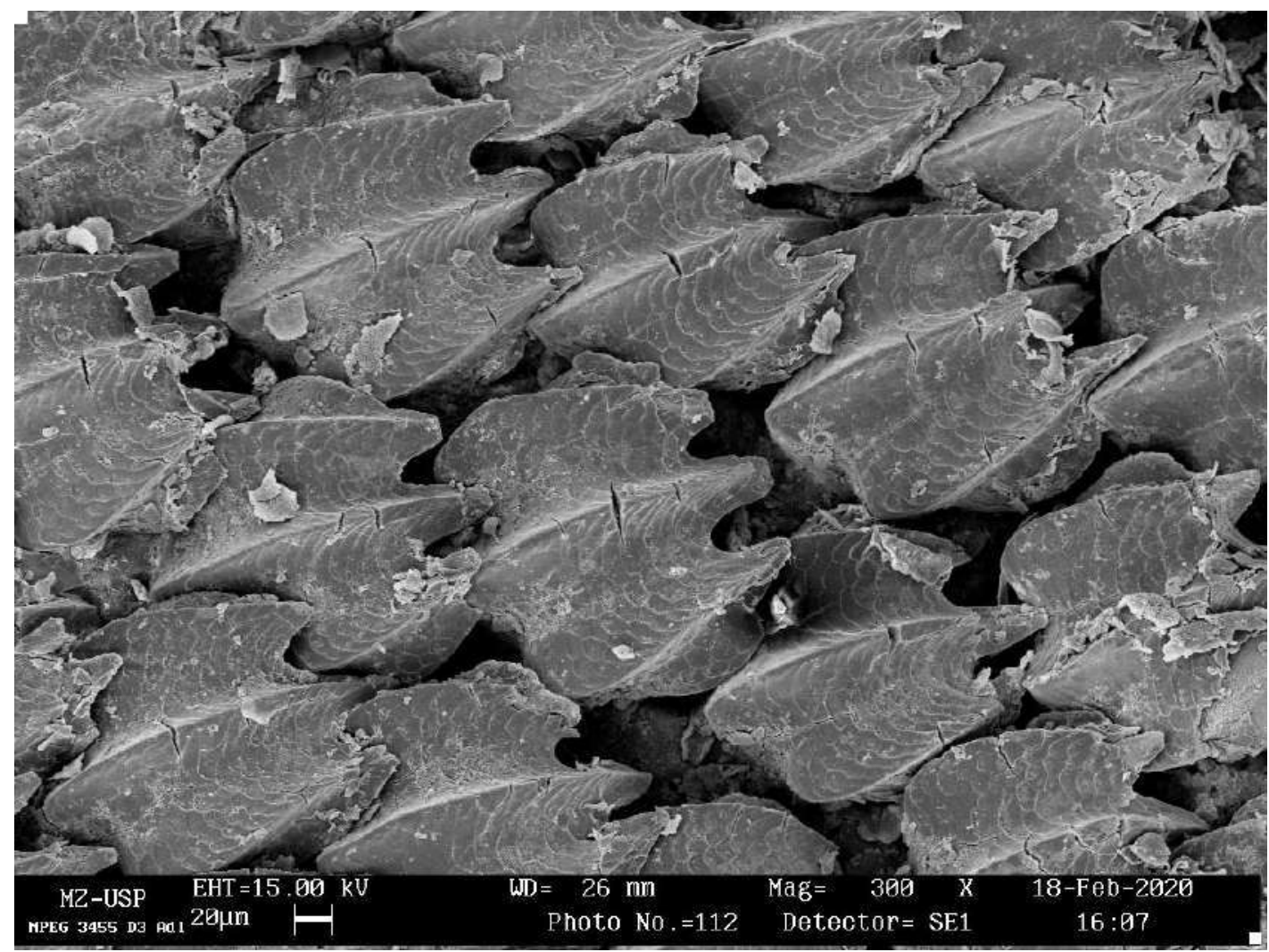

Figure 136. Dermal denticles collected from the section D3 (see fig. 1) of the adult Isogomphodon oxyrhynchus (MPEG 3455). Additional information in the image footer.

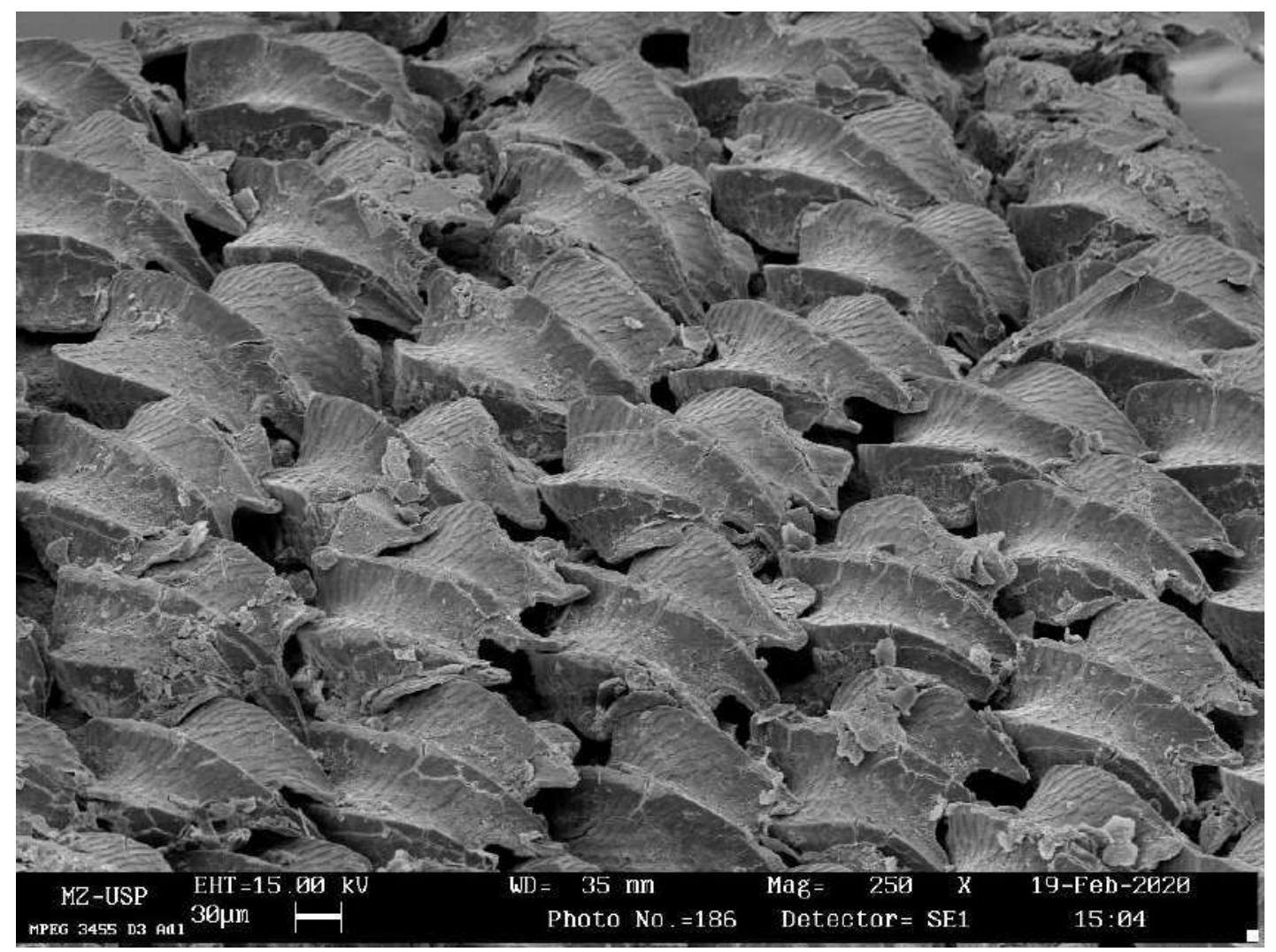

Figure 137. Dermal denticles collected from the section D3 (see fig. 1) of the adult Isogomphodon oxyrhynchus (MPEG 3455). Additional information in the image footer. 


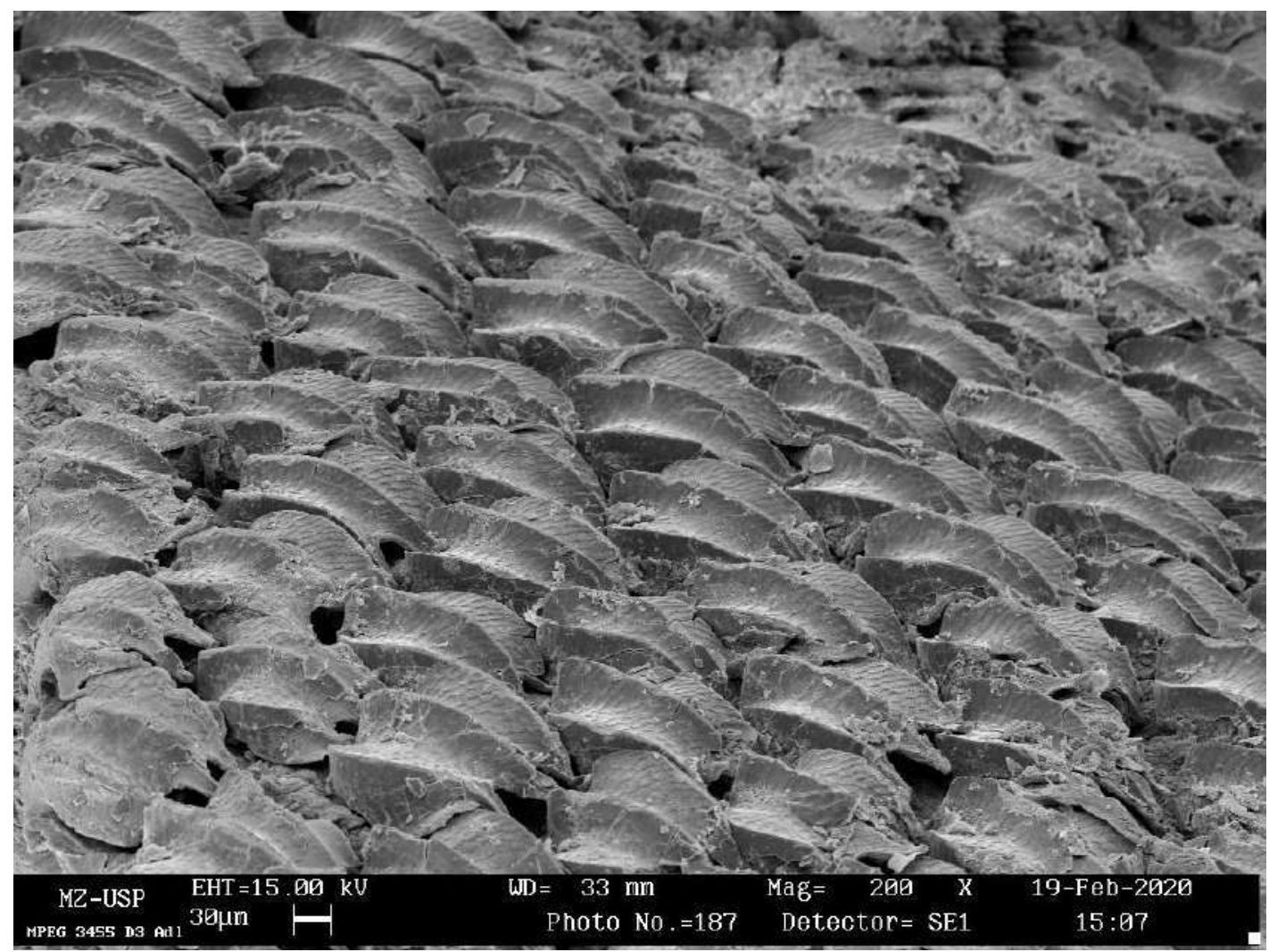

Figure 138. Dermal denticles collected from the section D3 (see fig. 1) of the adult Isogomphodon oxyrhynchus (MPEG 3455). Additional information in the image footer.

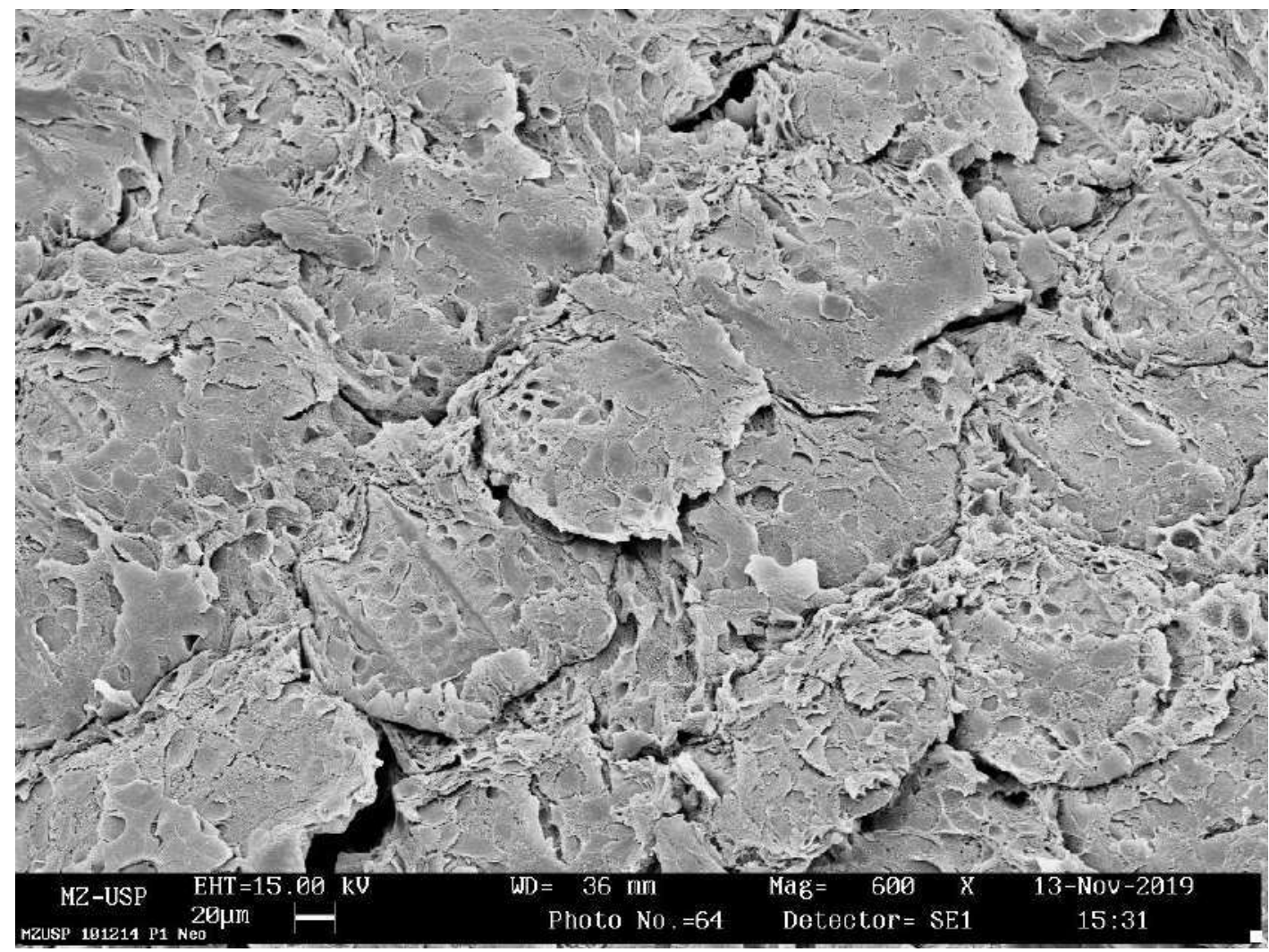

Figure 139. Dermal denticles collected from the section P1 (see fig. 1) of the neonate Isogomphodon oxyrhynchus (MZUSP 101214). Additional information in the image footer. 


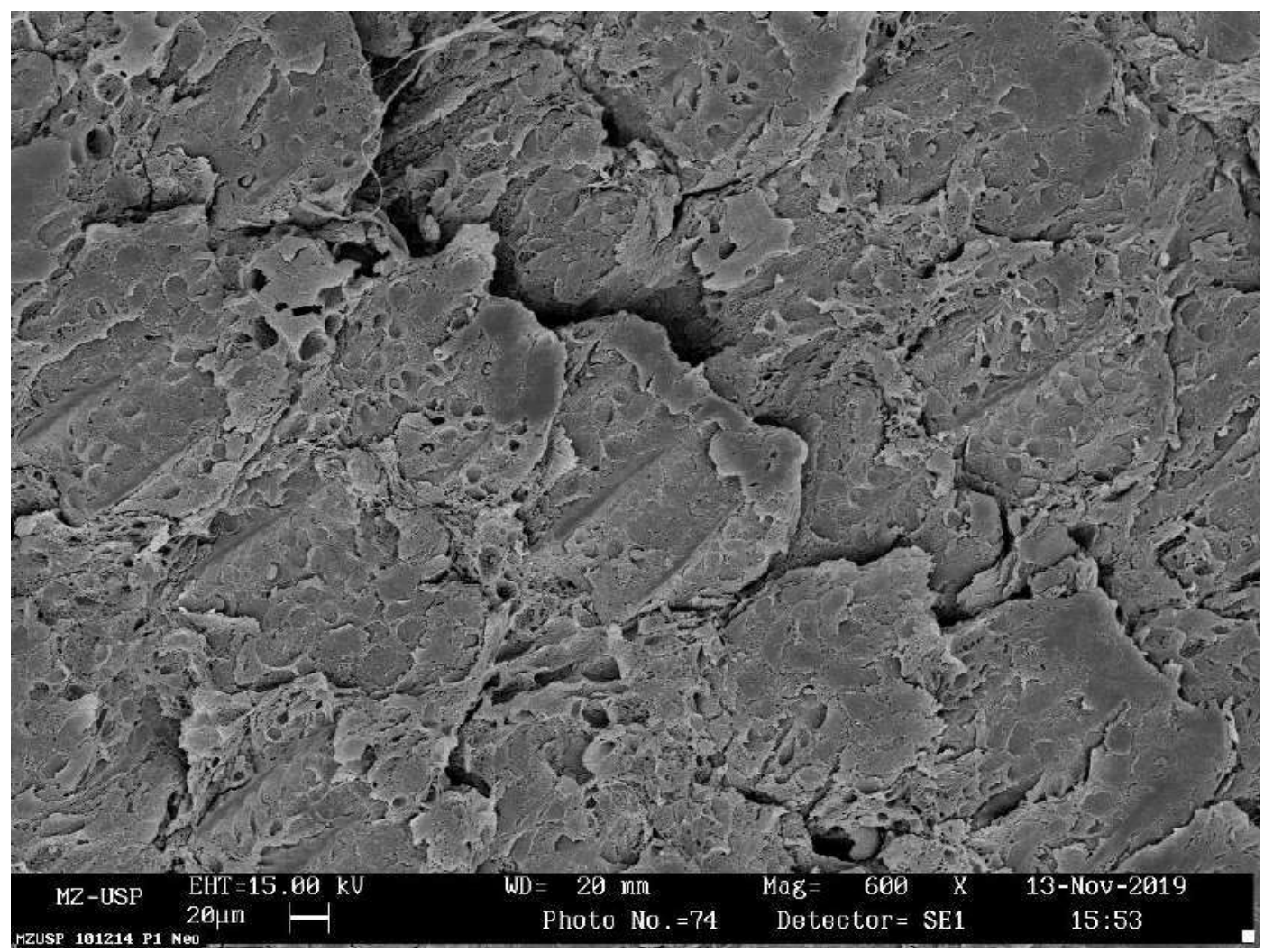

Figure 140. Dermal denticles collected from the section P1 (see fig. 1) of the neonate Isogomphodon oxyrhynchus (MZUSP 101214). Additional information in the image footer.

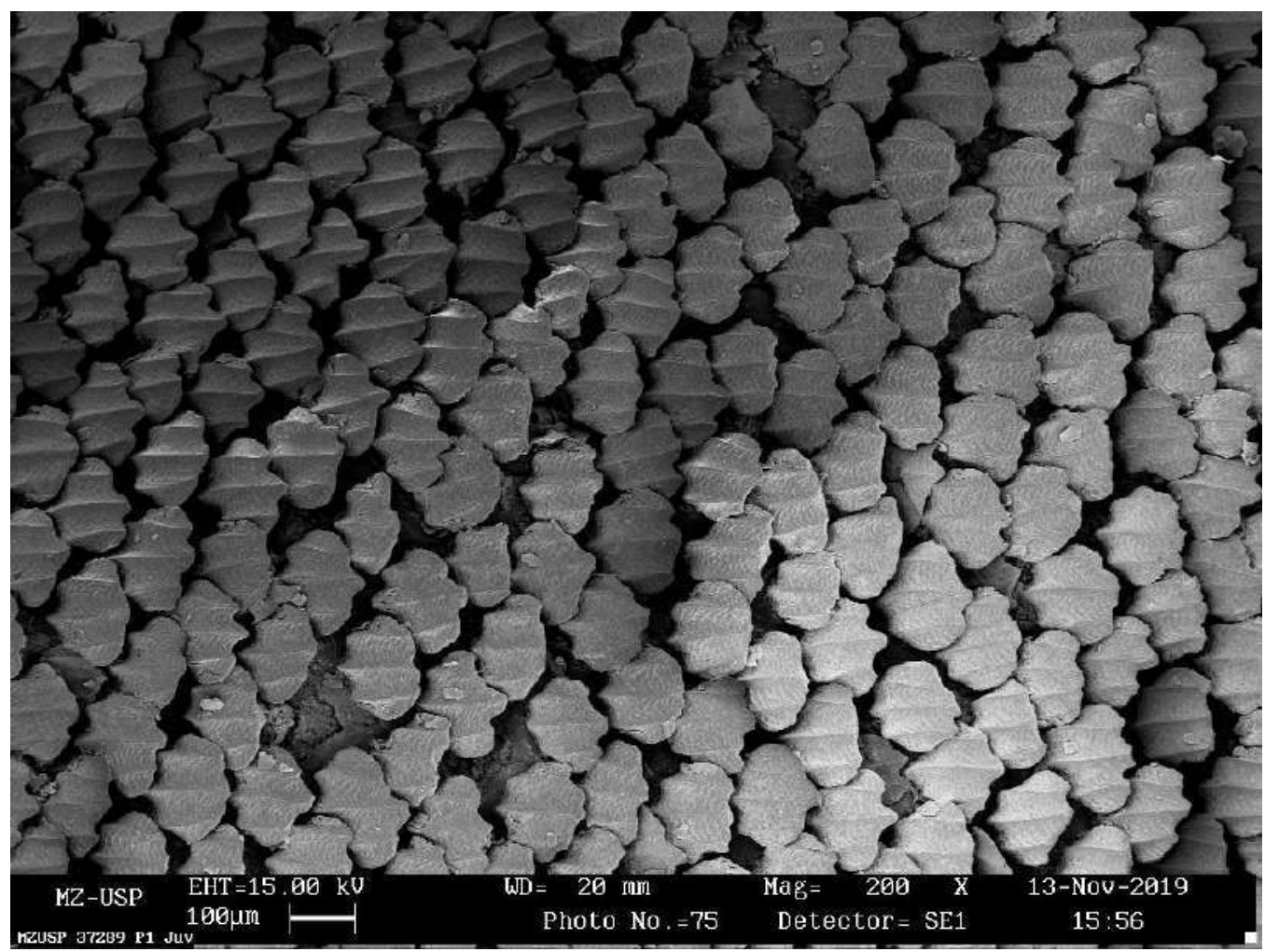

Figure 141. Dermal denticles collected from the section P1 (see fig. 1) of the juvenile Isogomphodon oxyrhynchus (MZUSP 37289). Additional information in the image footer. 


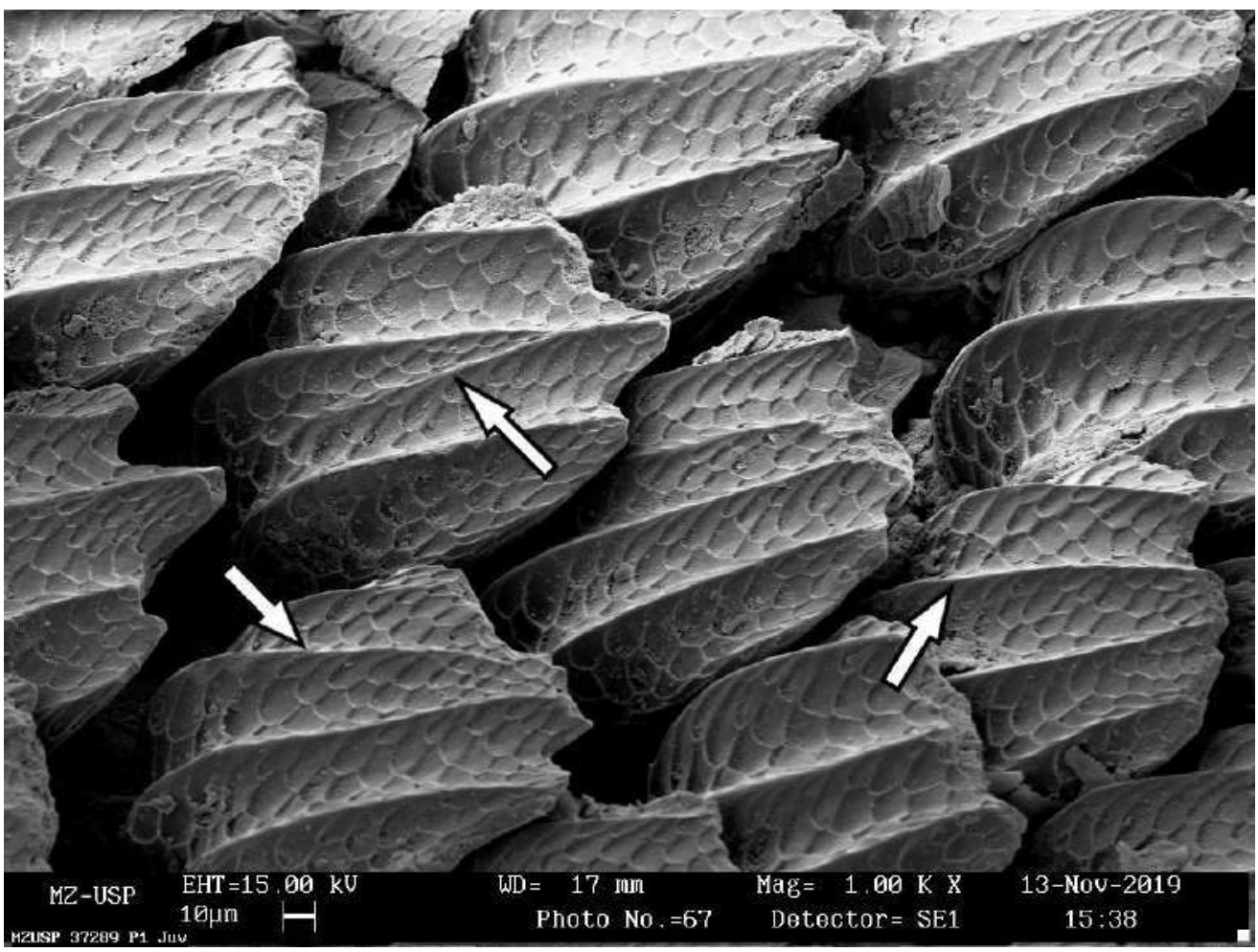

Figure 142. Dermal denticles collected from the section P1 (see fig. 1) of the juvenile Isogomphodon oxyrhynchus (MZUSP 37289). White arrows indicate the additional ridge confluent with the medial, differing from the three ridges that are common to the other scales. Additional information in the image footer.

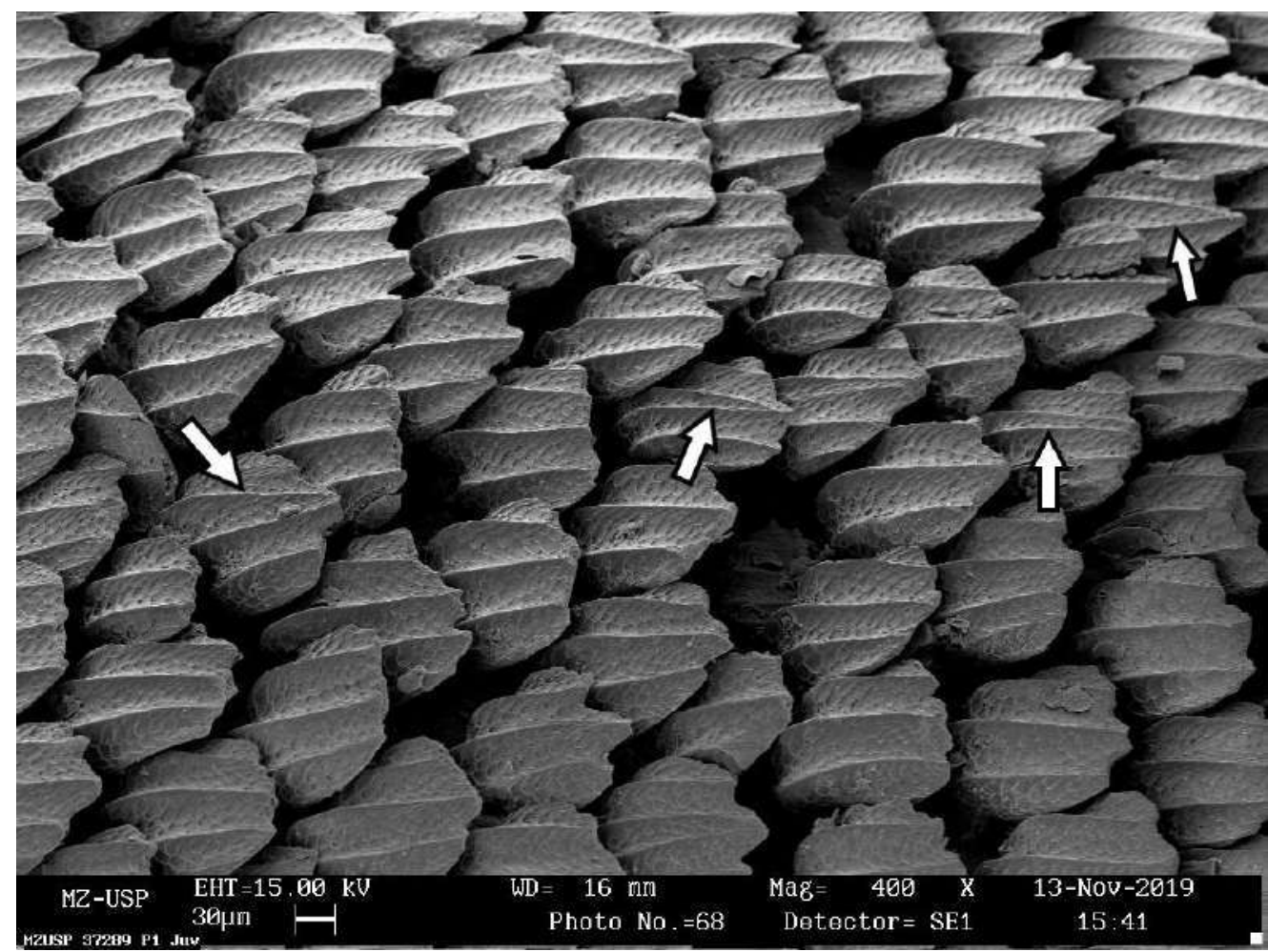

Figure 143. Dermal denticles collected from the section P1 (see fig. 1) of the juvenile Isogomphodon oxyrhynchus (MZUSP 37289). White arrows indicate the scales with additional ridge confluent with the medial, differing from the three ridges that are common to the other scales Additional information in the image footer. 


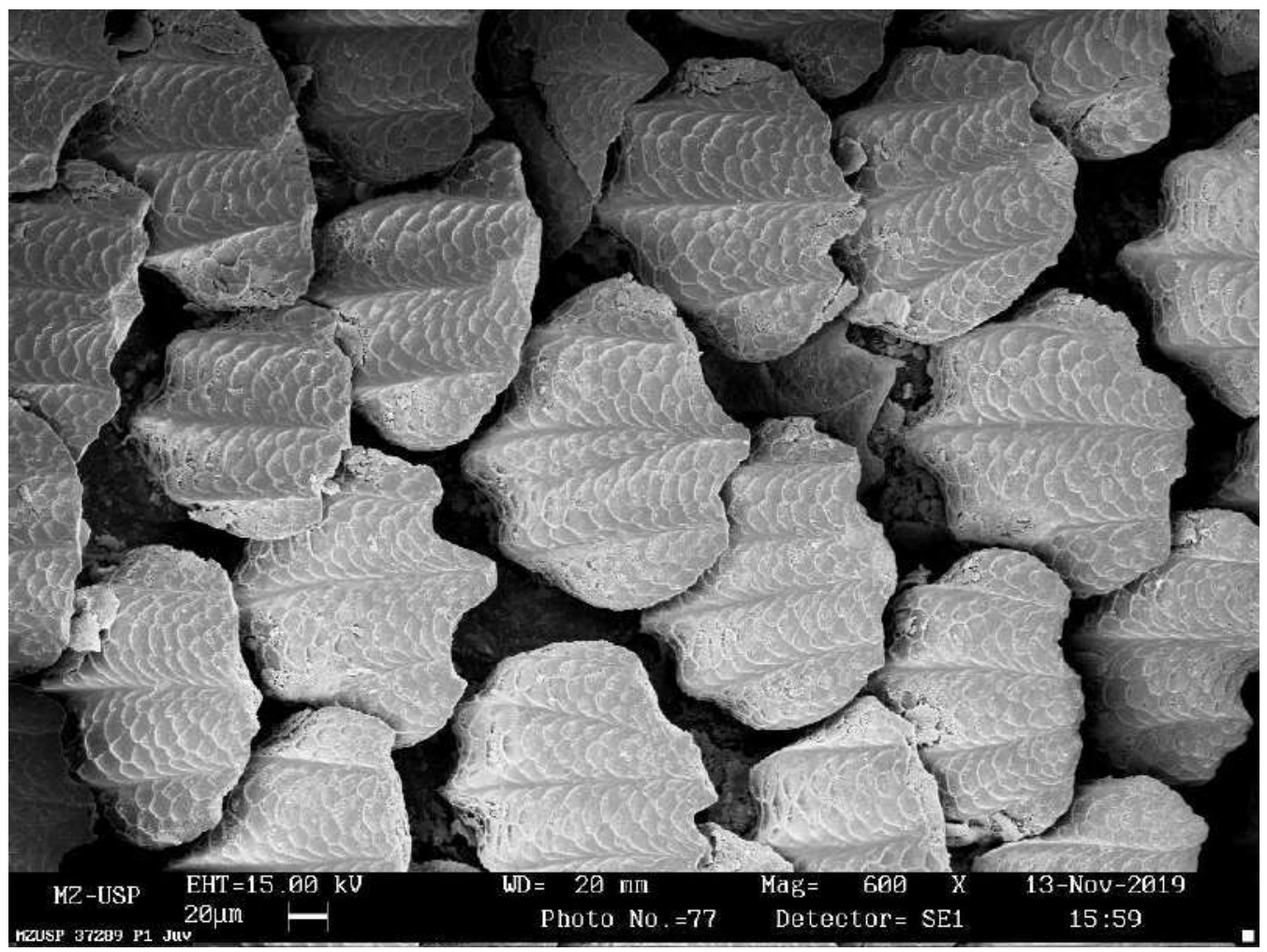

Figure 144. Dermal denticles collected from the section P1 (see fig. 1) of the juvenile Isogomphodon oxyrhynchus (MZUSP 37289). Additional information in the image footer.

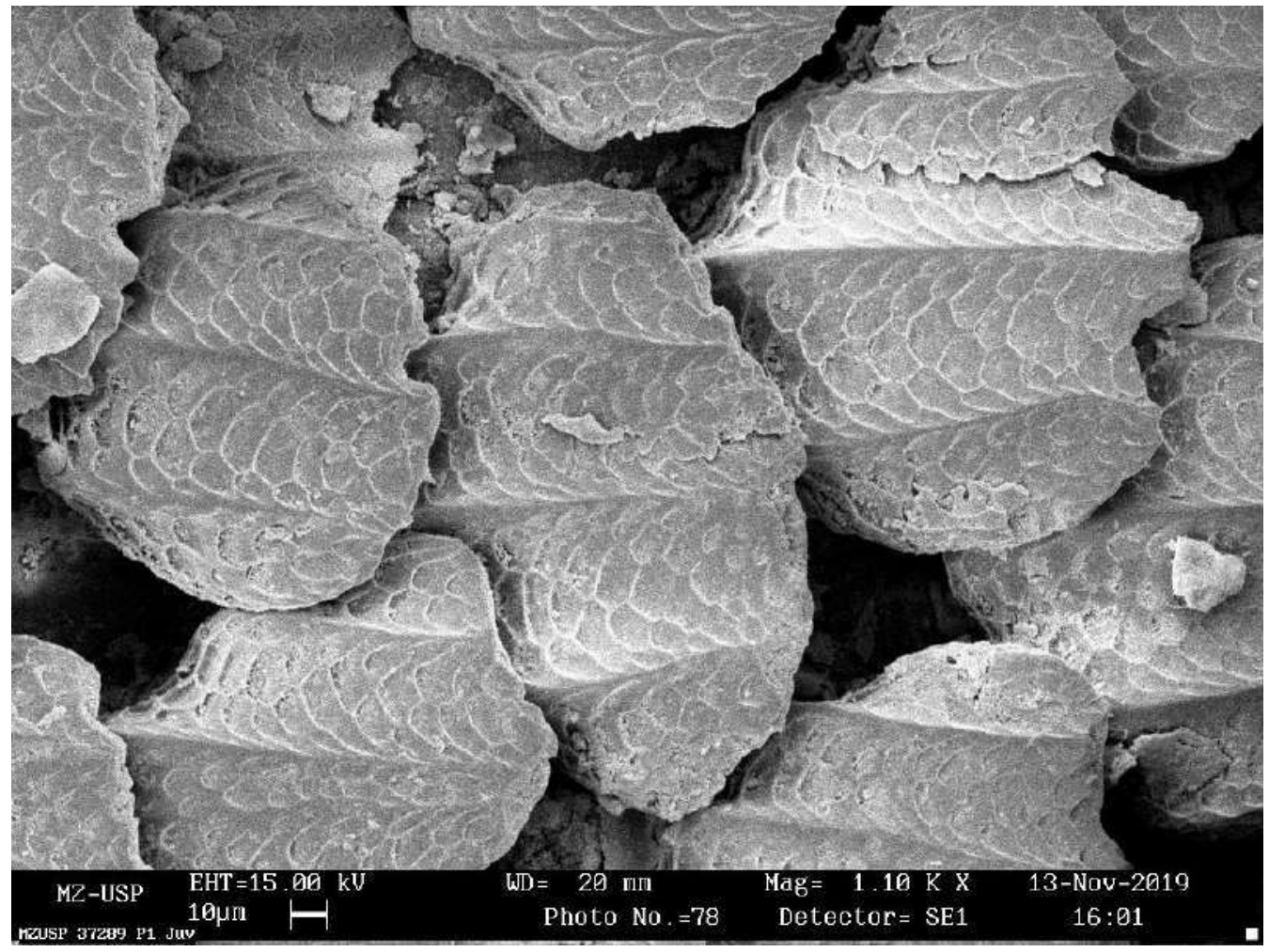

Figure 145. Dermal denticles collected from the section P1 (see fig. 1) of the juvenile Isogomphodon oxyrhynchus (MZUSP 37289). Additional information in the image footer. 


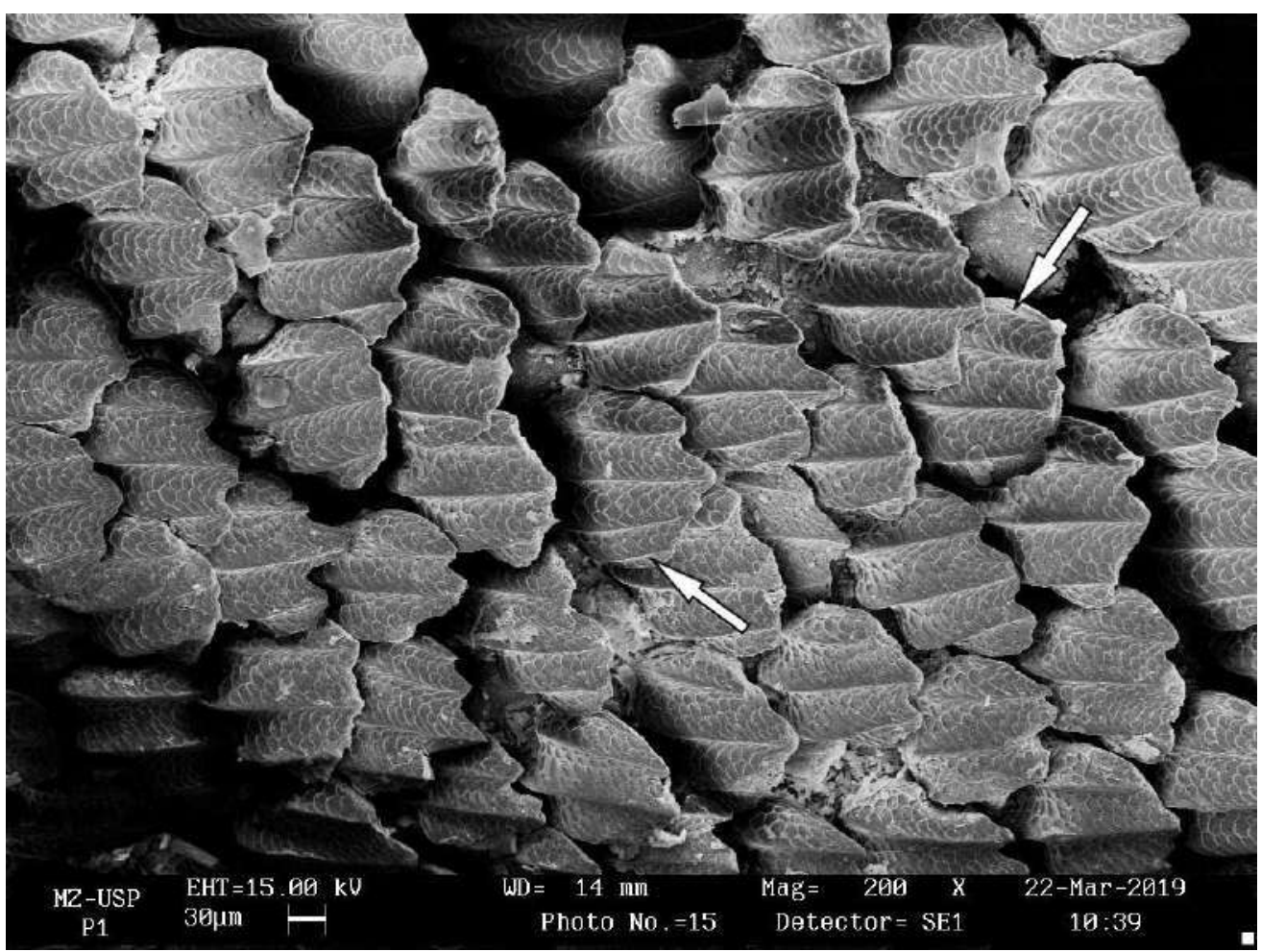

Figure 146. Dermal denticles collected from the section P1 (see fig. 1) of a juvenile Isogomphodon oxyrhynchus (MZUSP 37289). White arrows indicate the external ridge besides the standard three ridges that are common to the other scales. Additional information in the image footer.

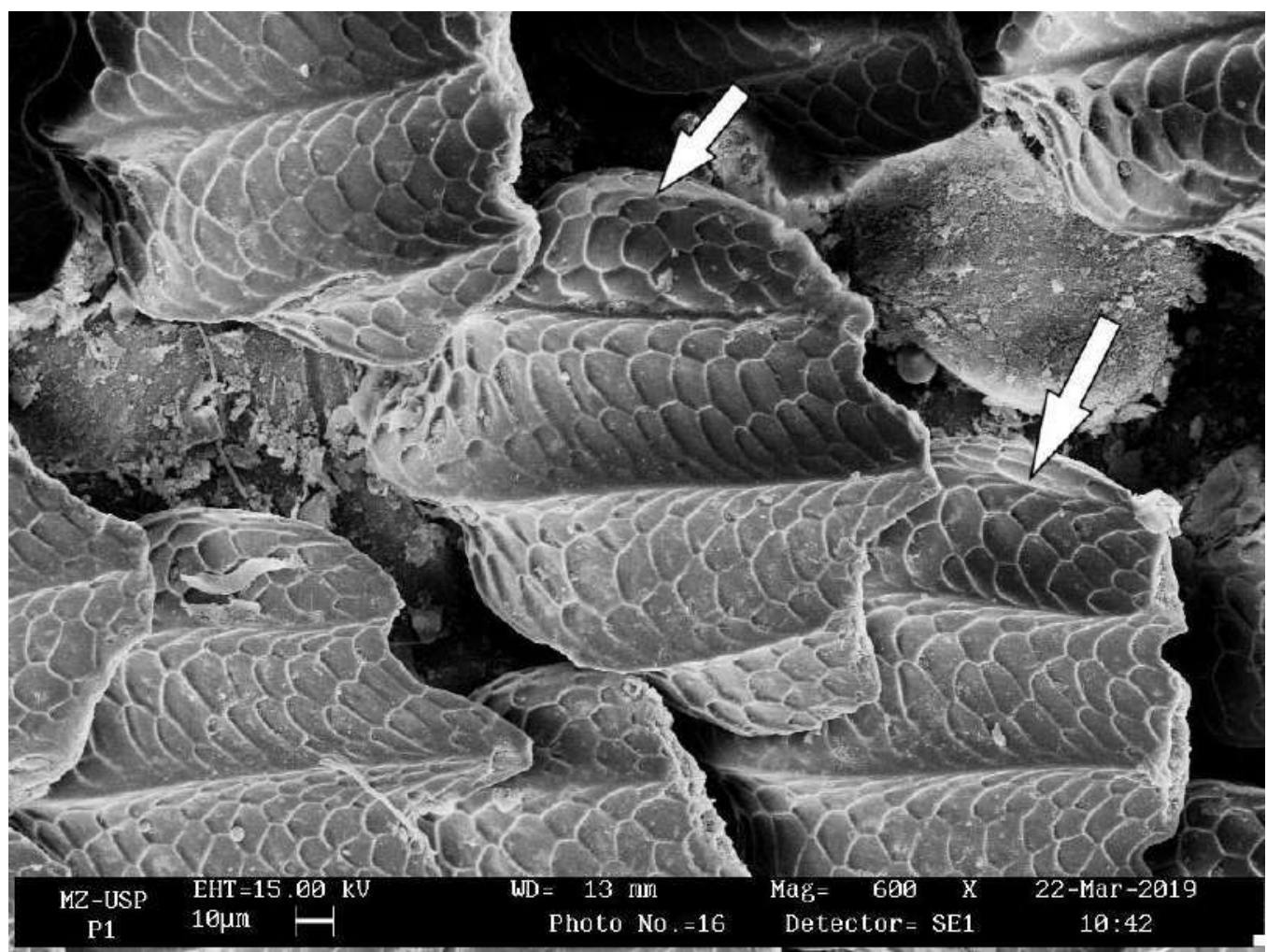

Figure 147. Dermal denticles collected from the section P1(see fig. 1) of a juvenile Isogomphodon oxyrhynchus (MZUSP 37289). White arrows indicate the external ridge besides the standard three ridges that are common to the other scales. Additional information in the image footer. 


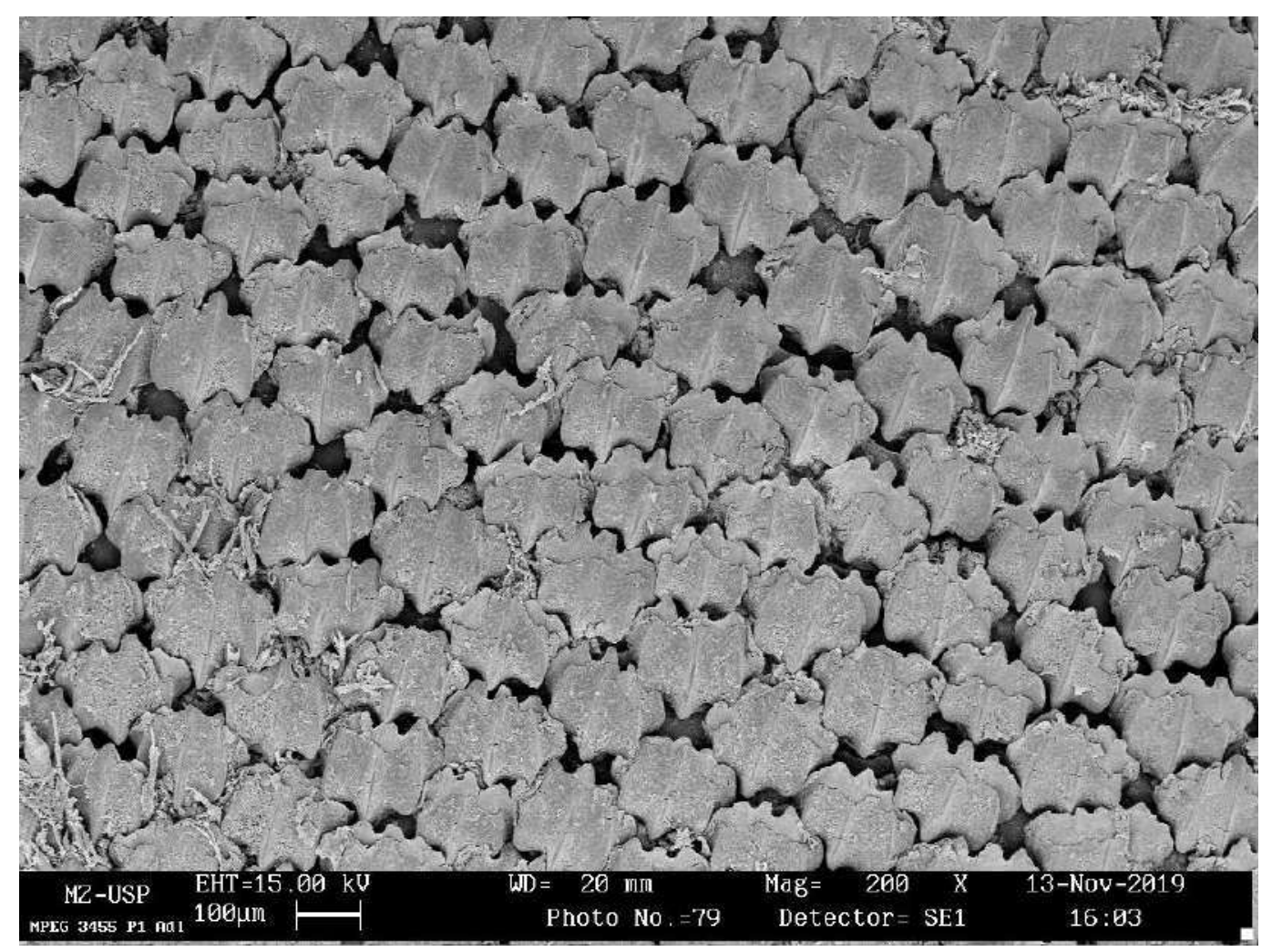

Figure 148. Dermal denticles collected from the section P1 (see fig. 1) of the adult Isogomphodon oxyrhynchus (MPEG 3455). Additional information in the image footer.

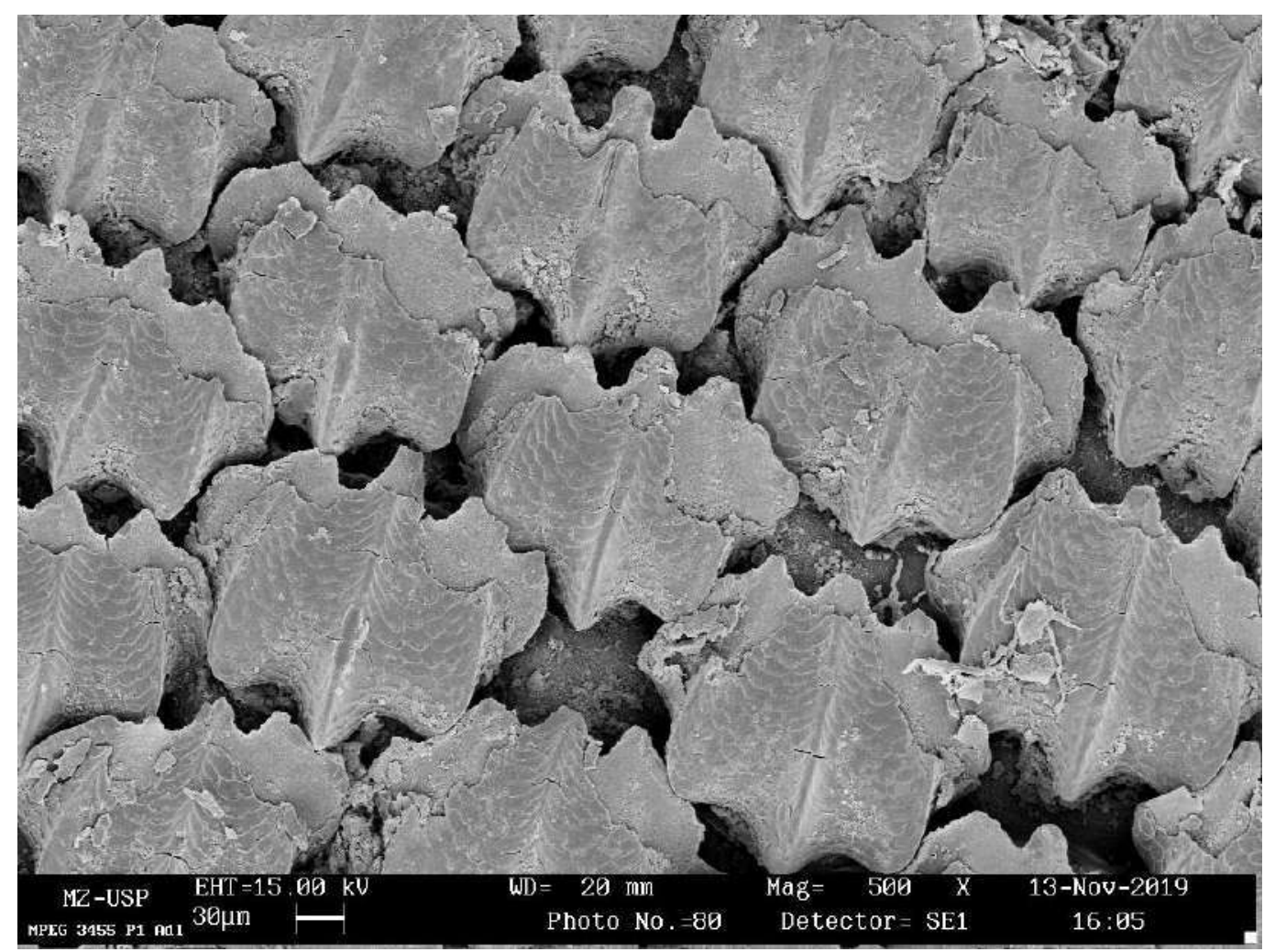

Figure 149. Dermal denticles collected from the section P1 (see fig. 1) of the adult Isogomphodon oxyrhynchus (MPEG 3455). Additional information in the image footer. 


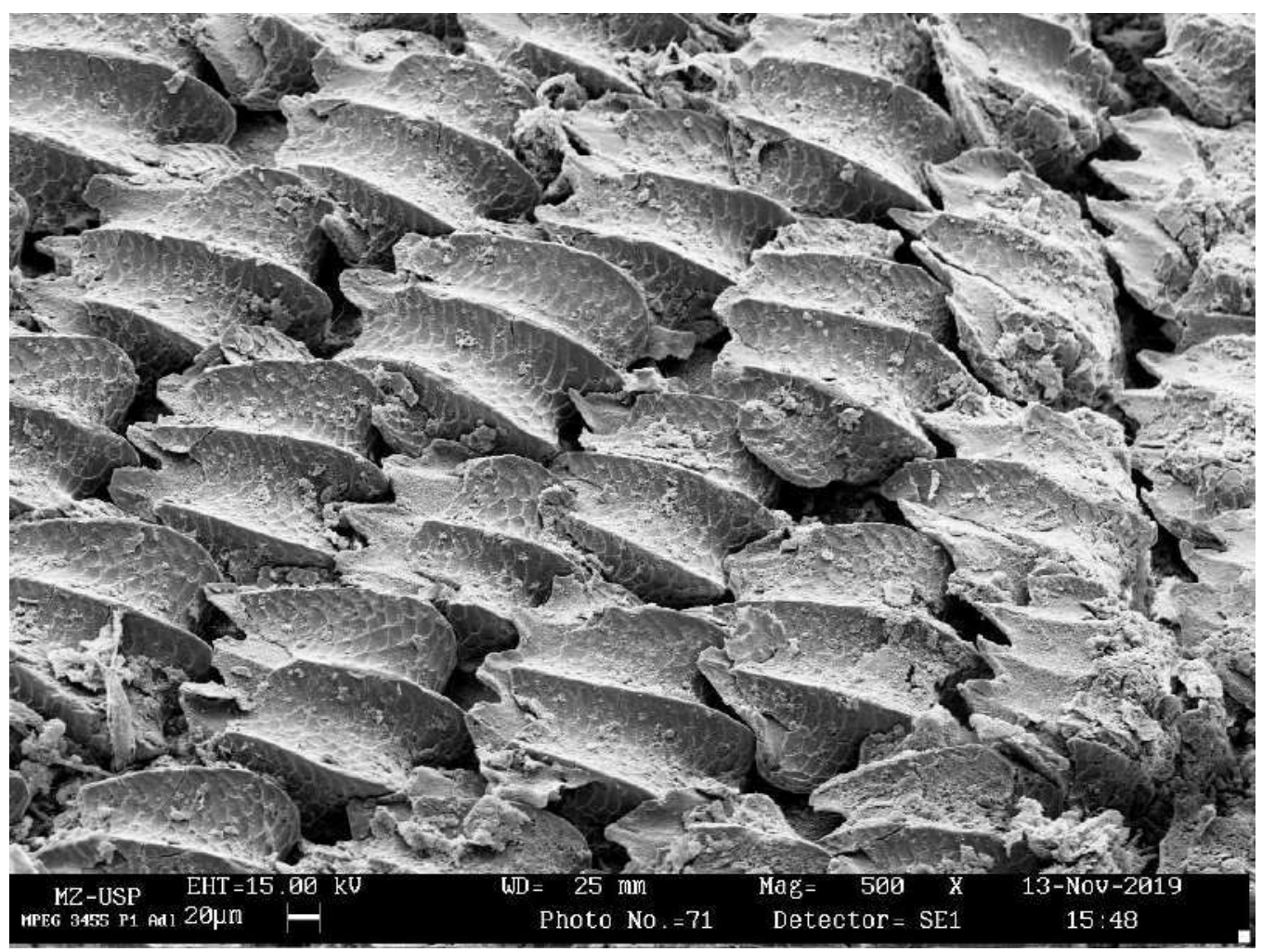

Figure 150. Dermal denticles collected from the section P1 (see fig. 1) of the adult Isogomphodon oxyrhynchus (MPEG 3455). Additional information in the image footer.

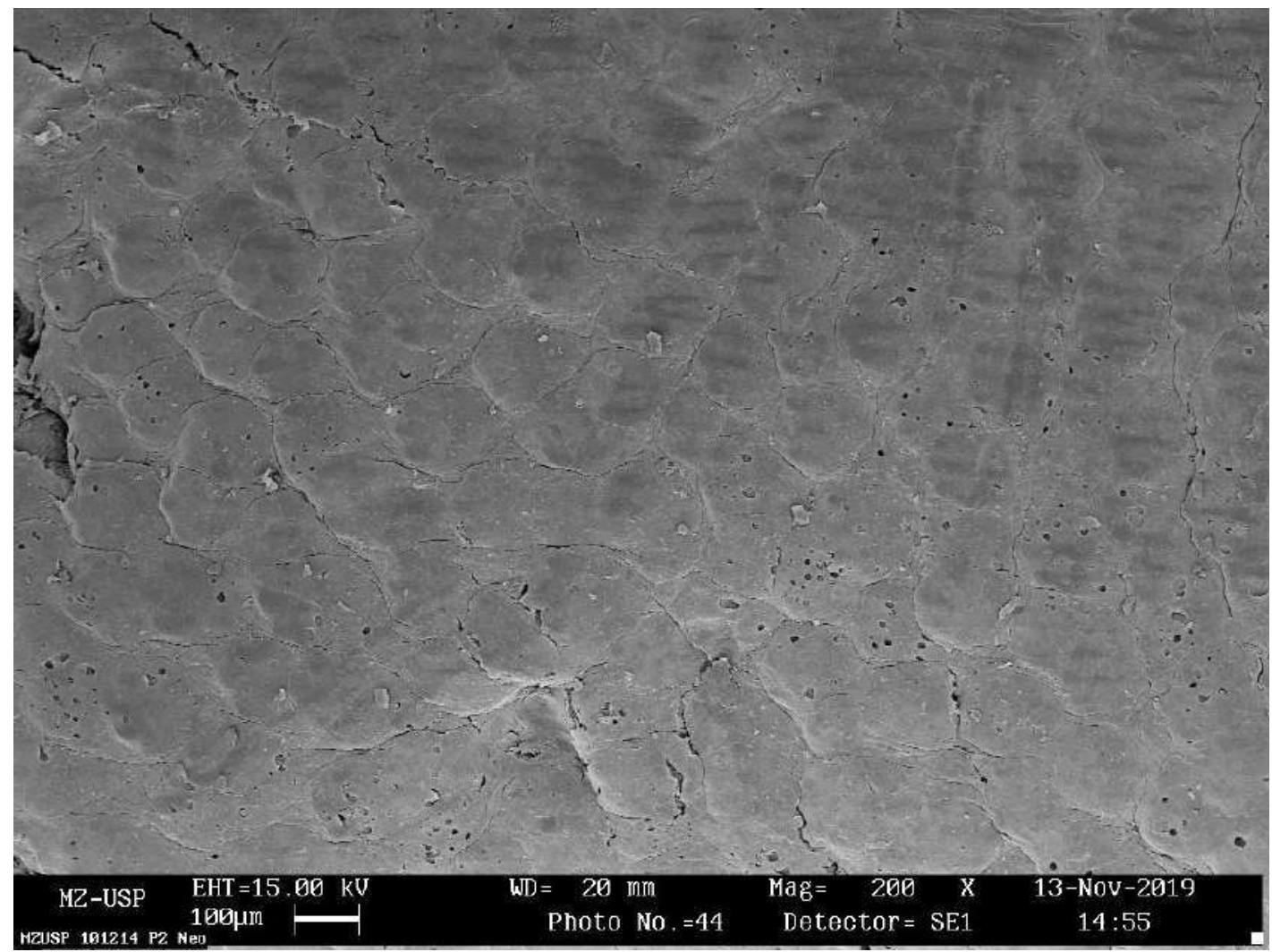

Figure 151. Dermal denticles collected from the section P2 (see fig. 1) of the neonate Isogomphodon oxyrhynchus (MZUSP 101214). Additional information in the image footer. 


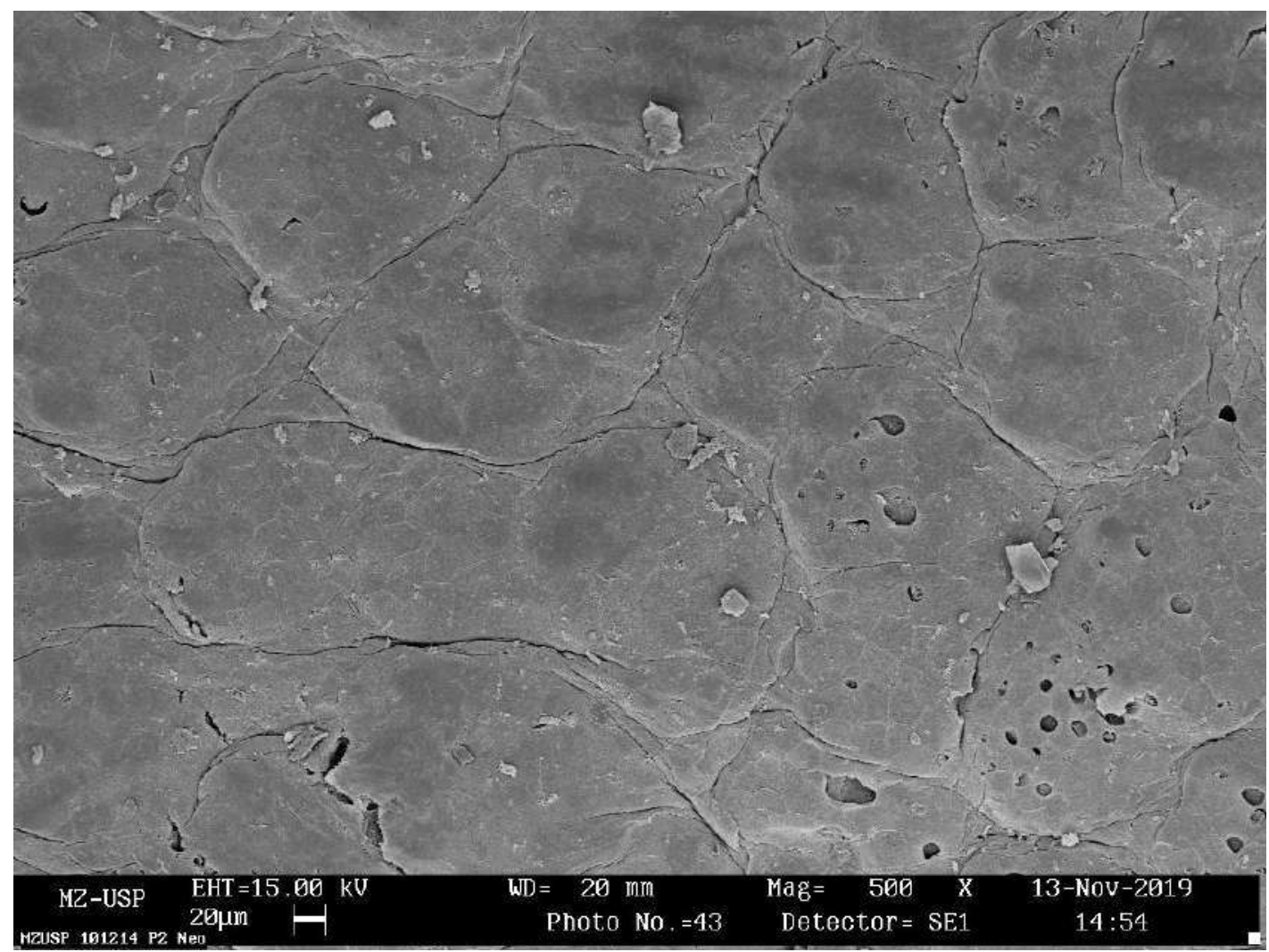

Figure 152. Dermal denticles collected from the section P2 (see fig. 1) of the neonate Isogomphodon oxyrhynchus (MZUSP 101214). Additional information in the image footer.

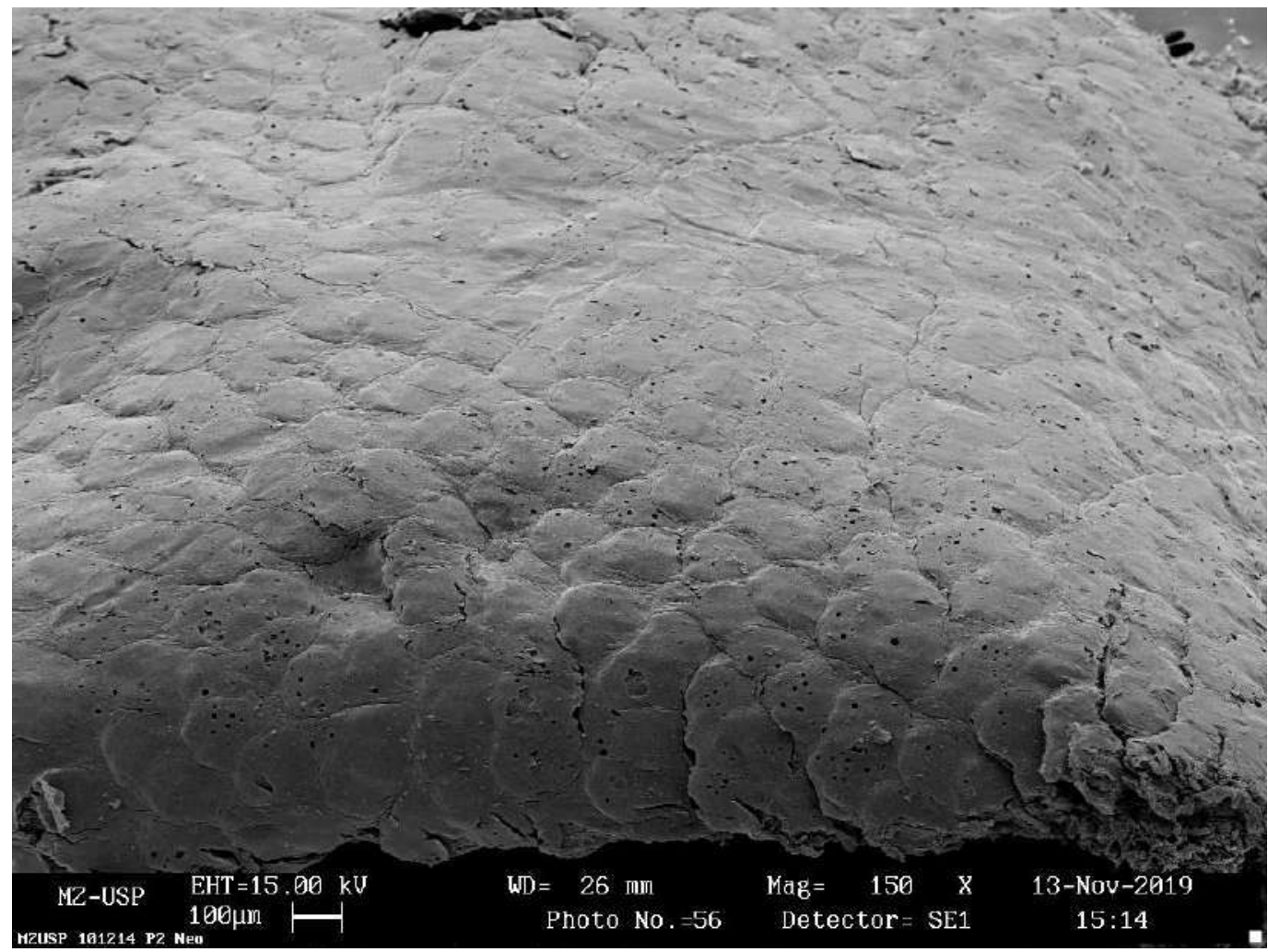

Figure 153. Dermal denticles collected from the section P2 (see fig. 1) of the neonate Isogomphodon oxyrhynchus (MZUSP 101214). Additional information in the image footer. 


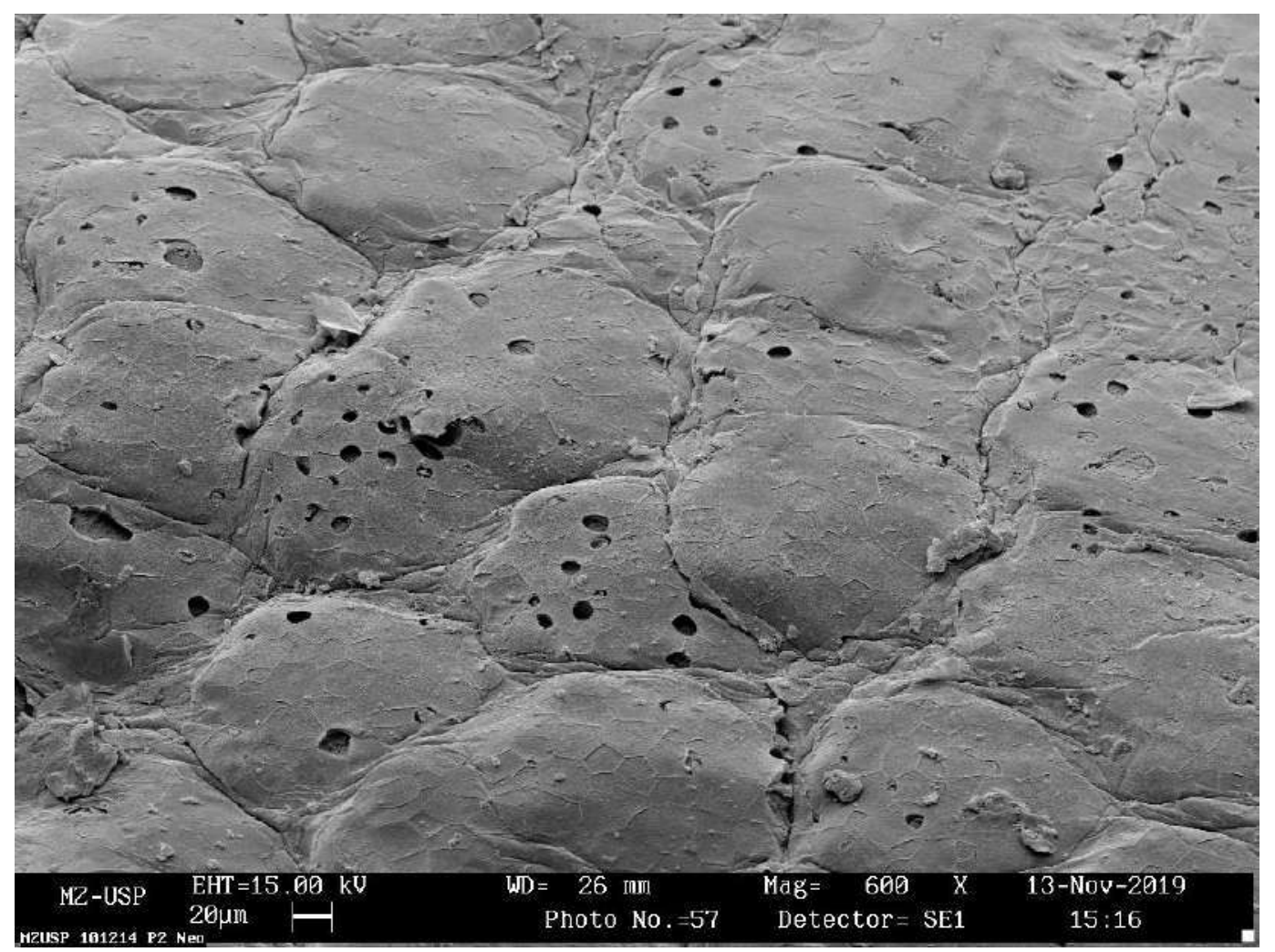

Figure 154. Dermal denticles collected from the section P2 (see fig. 1) of the neonate Isogomphodon oxyrhynchus (MZUSP 101214). Additional information in the image footer.

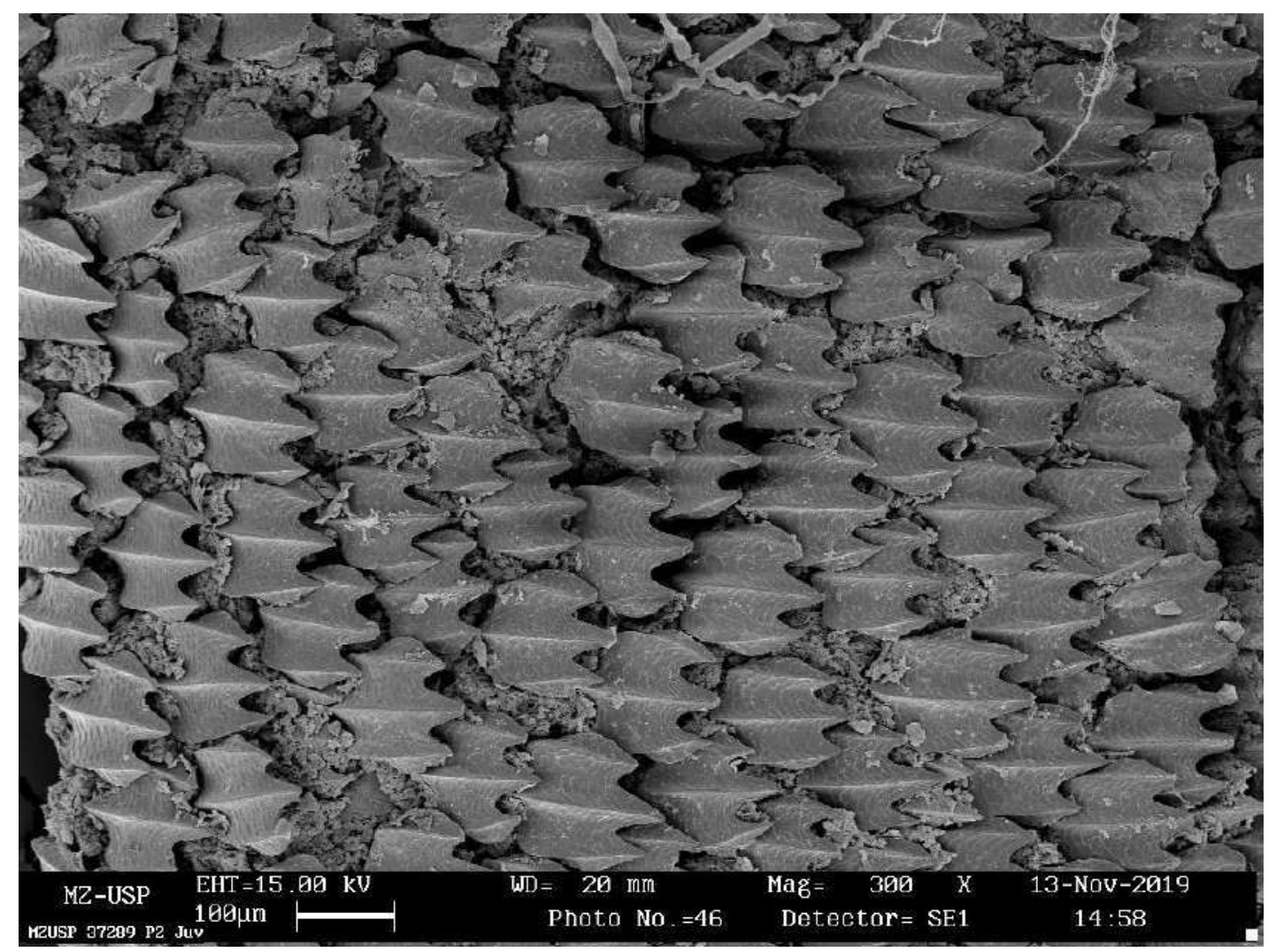

Figure 155. Dermal denticles collected from the section P2 (see fig. 1) of the juvenile Isogomphodon oxyrhynchus (MZUSP 37289). Additional information in the image footer. 


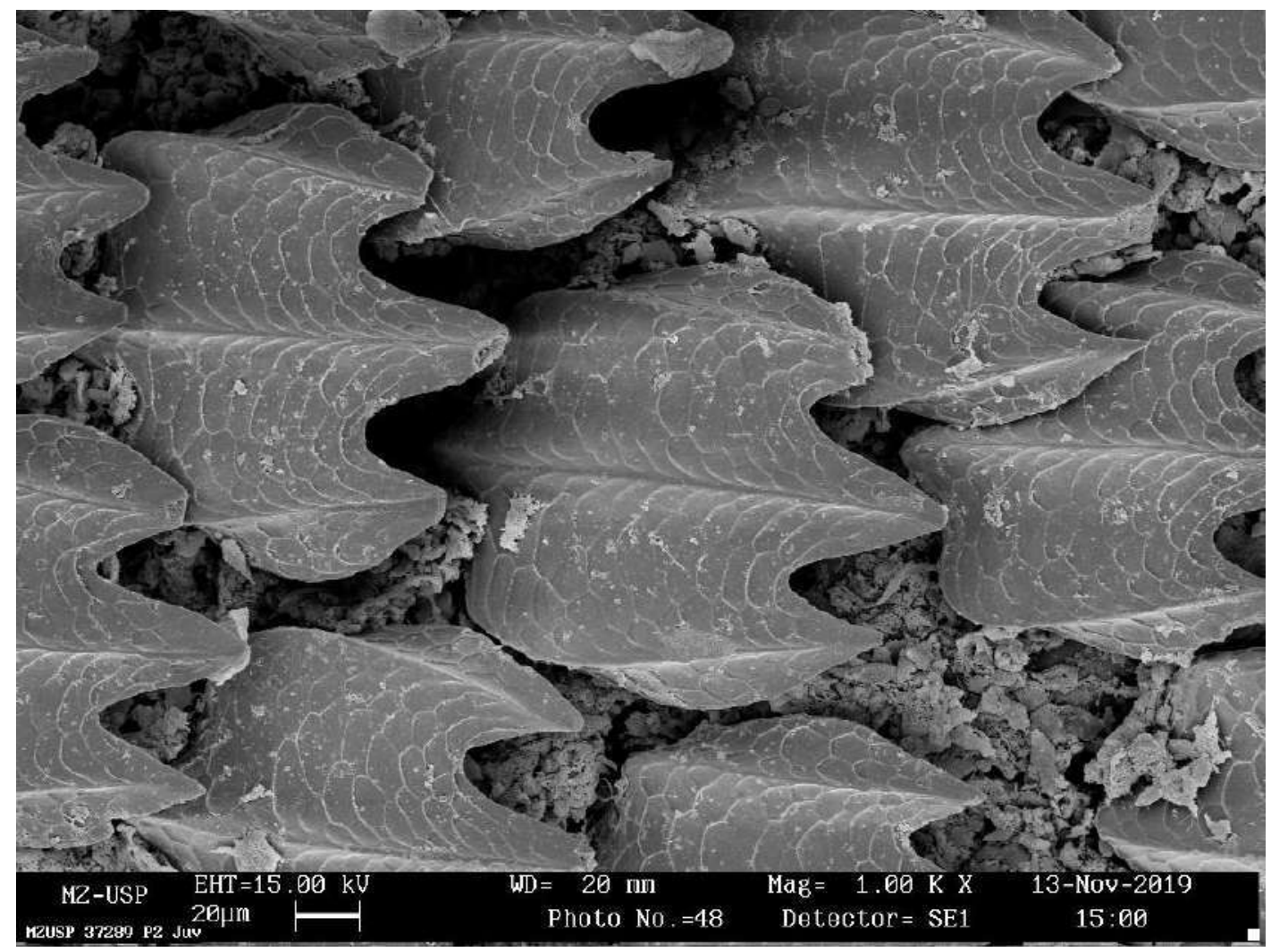

Figure 156. Dermal denticles collected from the section P2 (see fig. 1) of the juvenile Isogomphodon oxyrhynchus (MZUSP 37289). Additional information in the image footer.

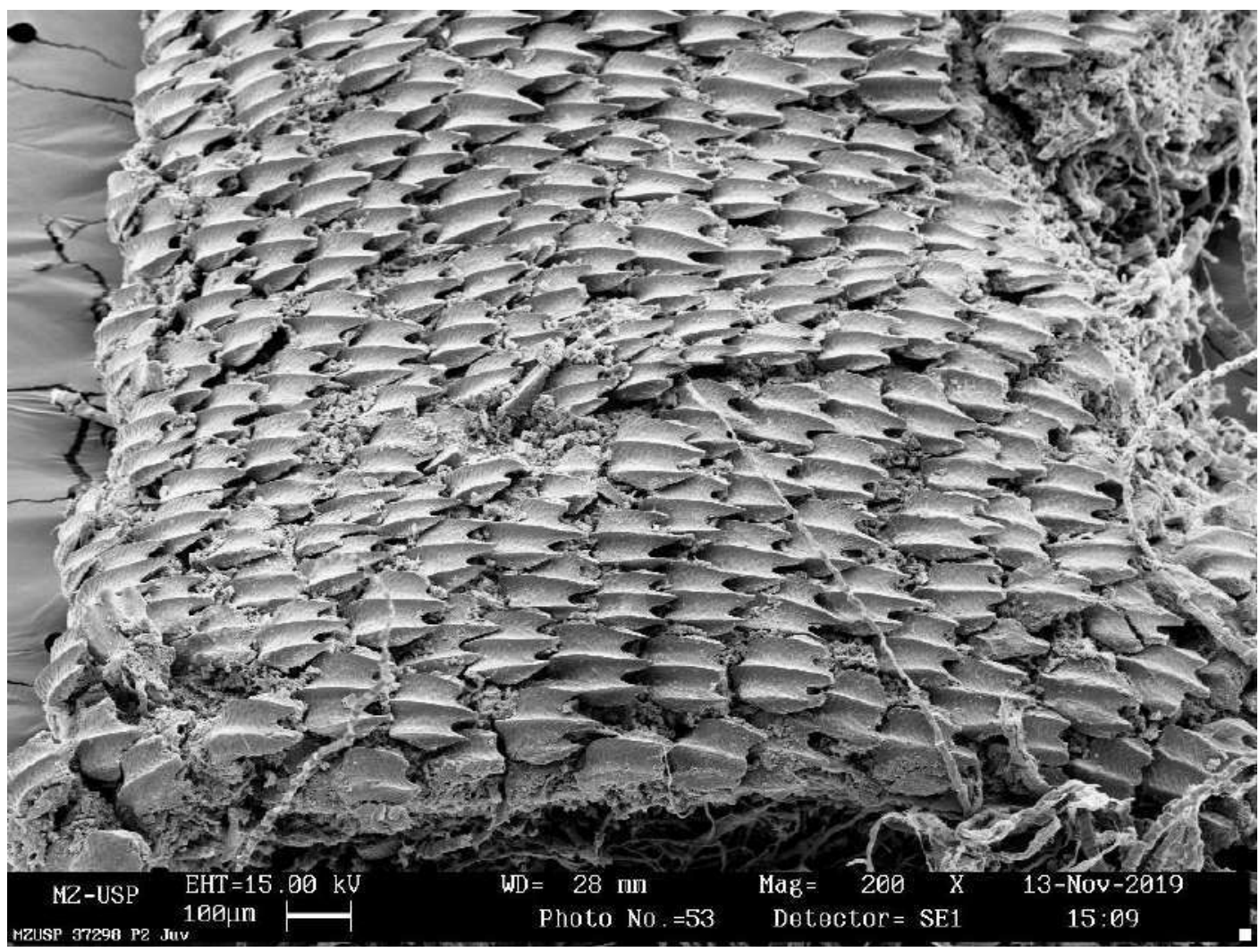

Figure 157. Dermal denticles collected from the section P2 (see fig. 1) of the juvenile Isogomphodon oxyrhynchus (MZUSP 37289). Additional information in the image footer. 


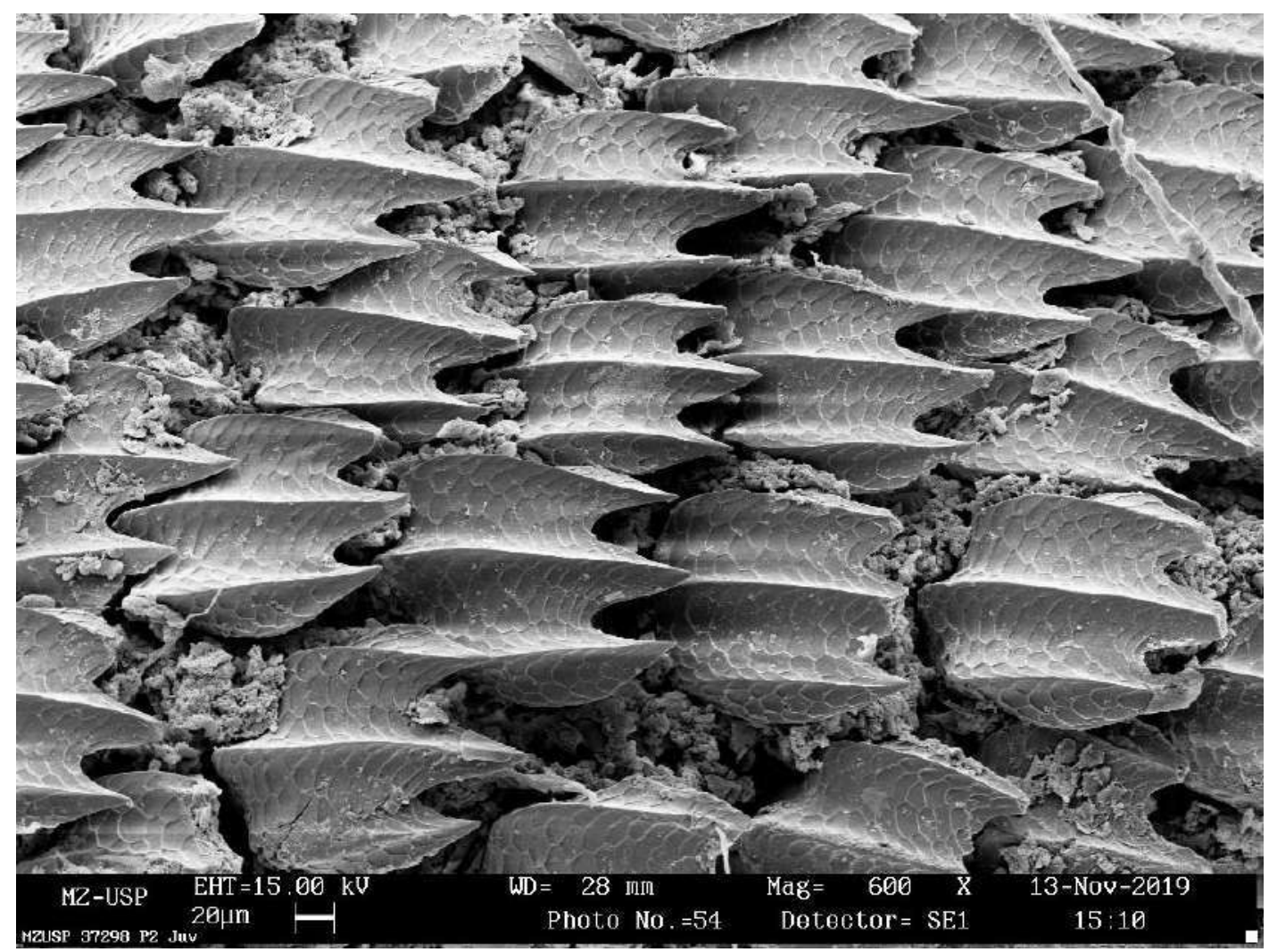

Figure 158. Dermal denticles collected from the section P2 (see fig. 1) of the juvenile Isogomphodon oxyrhynchus (MZUSP 37289). Additional information in the image footer.

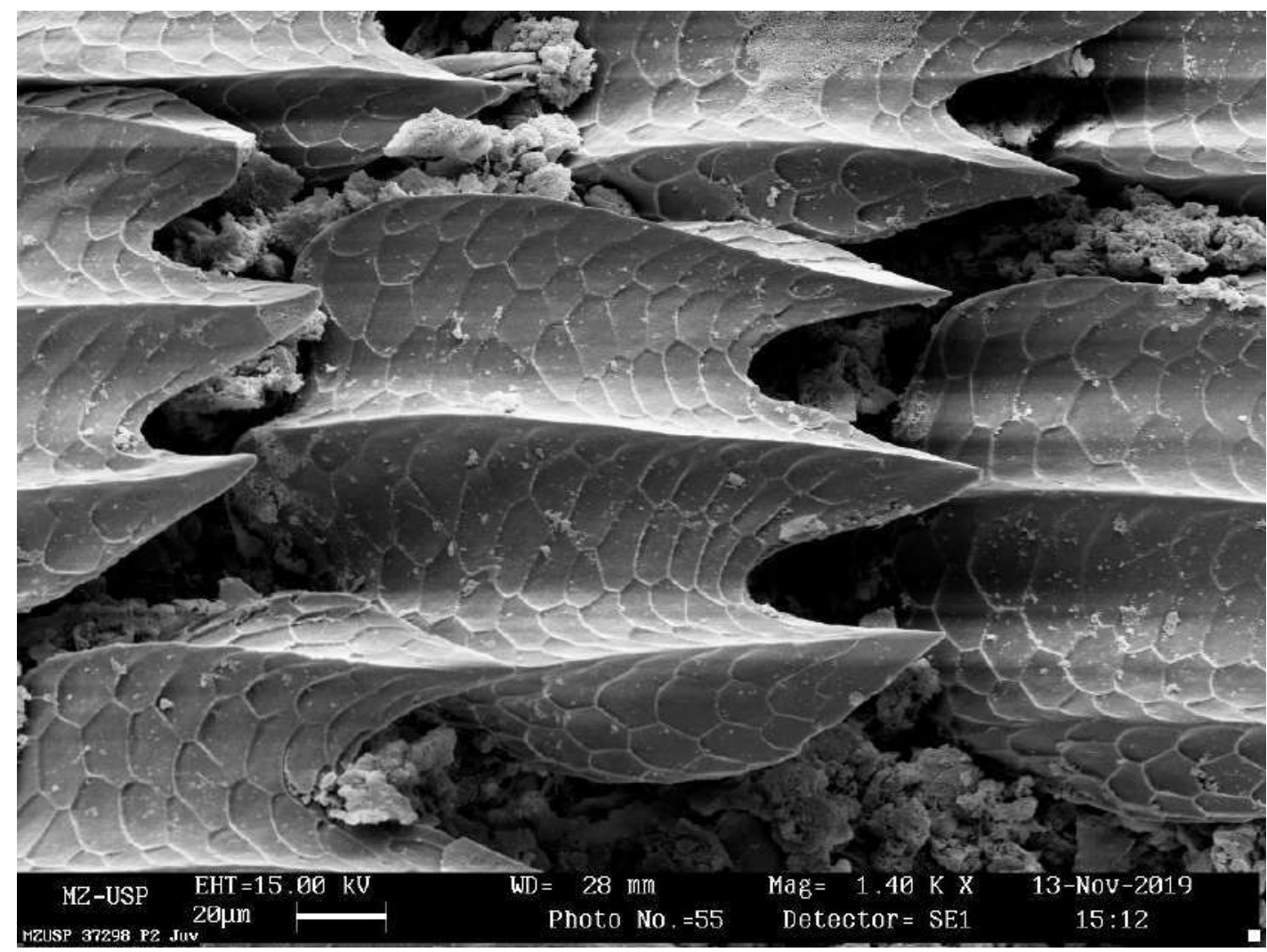

Figure 159. Dermal denticles collected from the section P2 (see fig. 1) of the juvenile Isogomphodon oxyrhynchus (MZUSP 37289). Additional information in the image footer. 


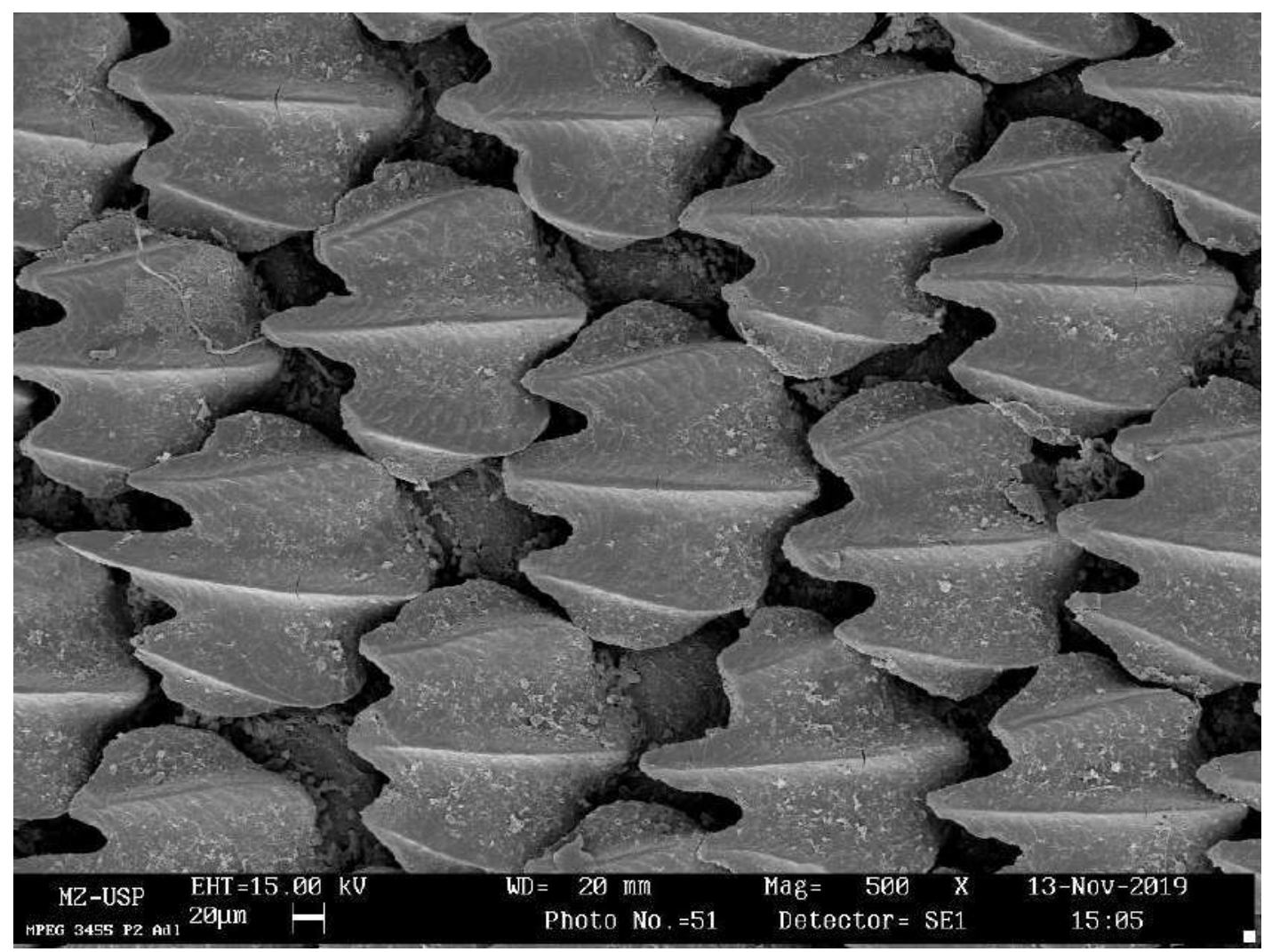

Figure 160. Dermal denticles collected from the section P2 (see fig. 1) of the adult Isogomphodon oxyrhynchus (MPEG 3455). Additional information in the image footer.

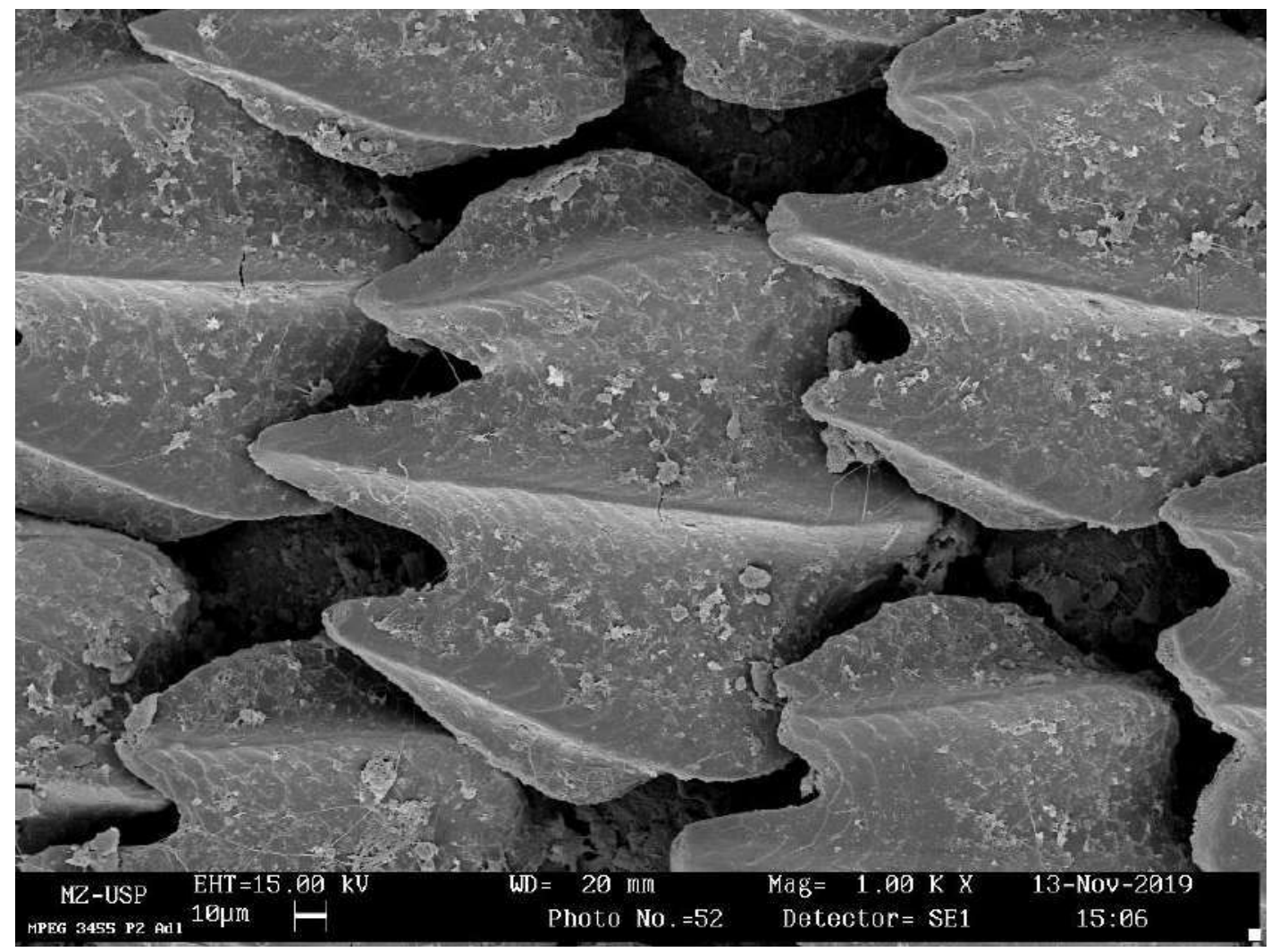

Figure 161. Dermal denticles collected from the section P2 (see fig. 1) of the adult Isogomphodon oxyrhynchus (MPEG 3455). Additional information in the image footer. 


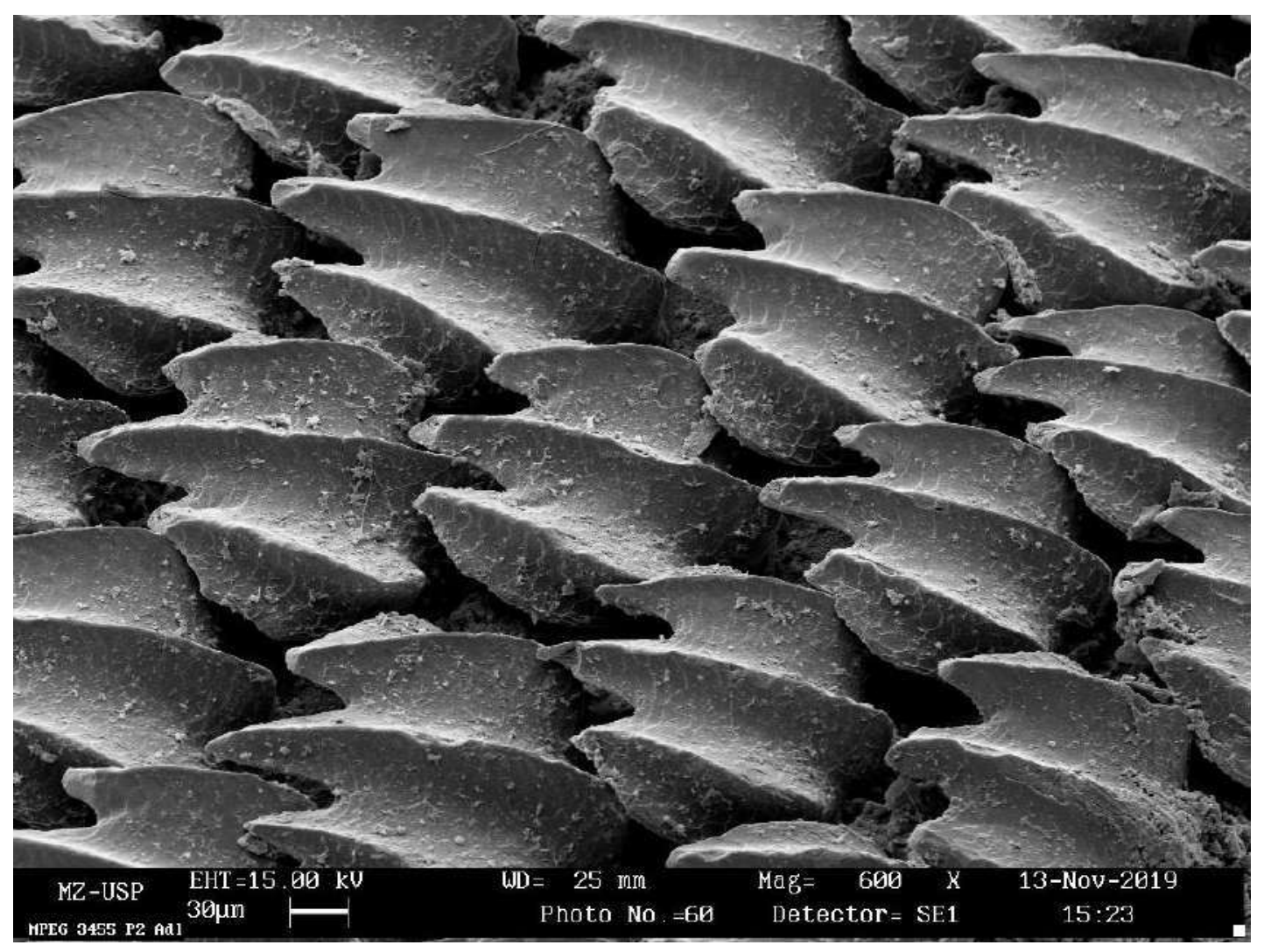

Figure 162. Dermal denticles collected from the section P2 (see fig. 1) of the adult Isogomphodon oxyrhynchus (MPEG 3455). Additional information in the image footer.

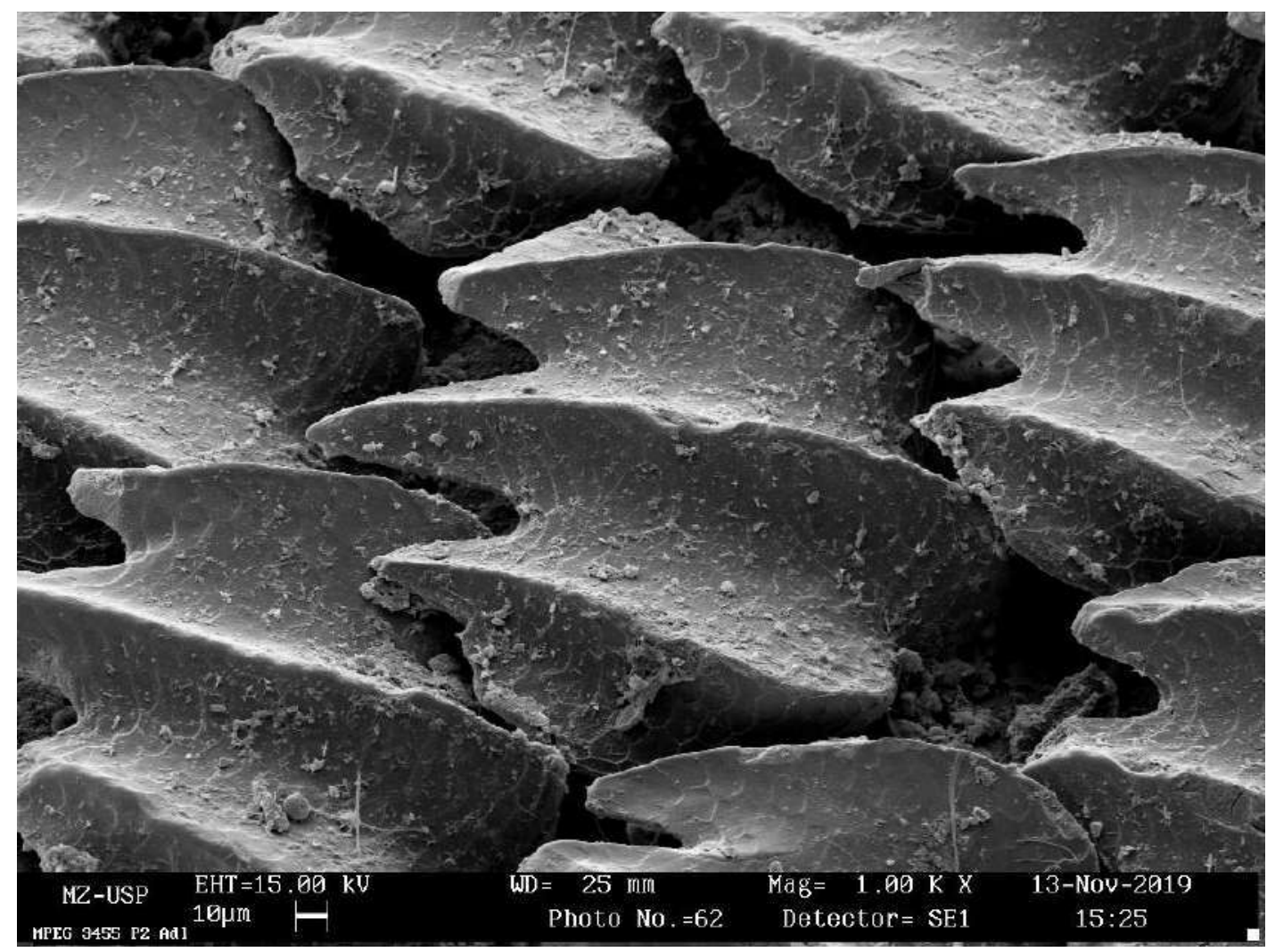

Figure 163. Dermal denticles collected from the section P2 (see fig. 1) of the adult Isogomphodon oxyrhynchus (MPEG 3455). Additional information in the image footer. 


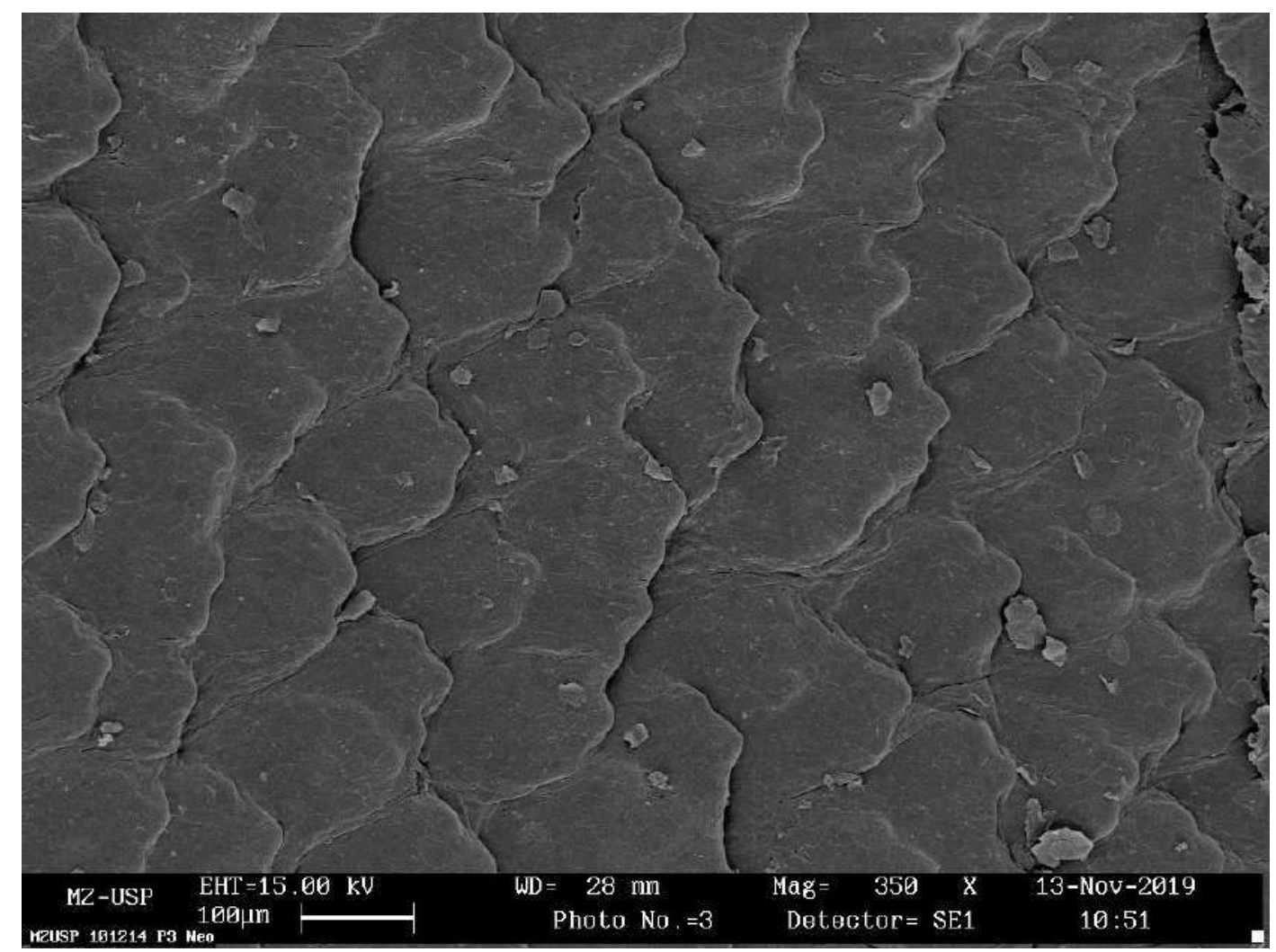

Figure 164. Dermal denticles collected from the section P3 (see fig. 1) of the neonate Isogomphodon oxyrhynchus (MZUSP 101214). Additional information in the image footer.

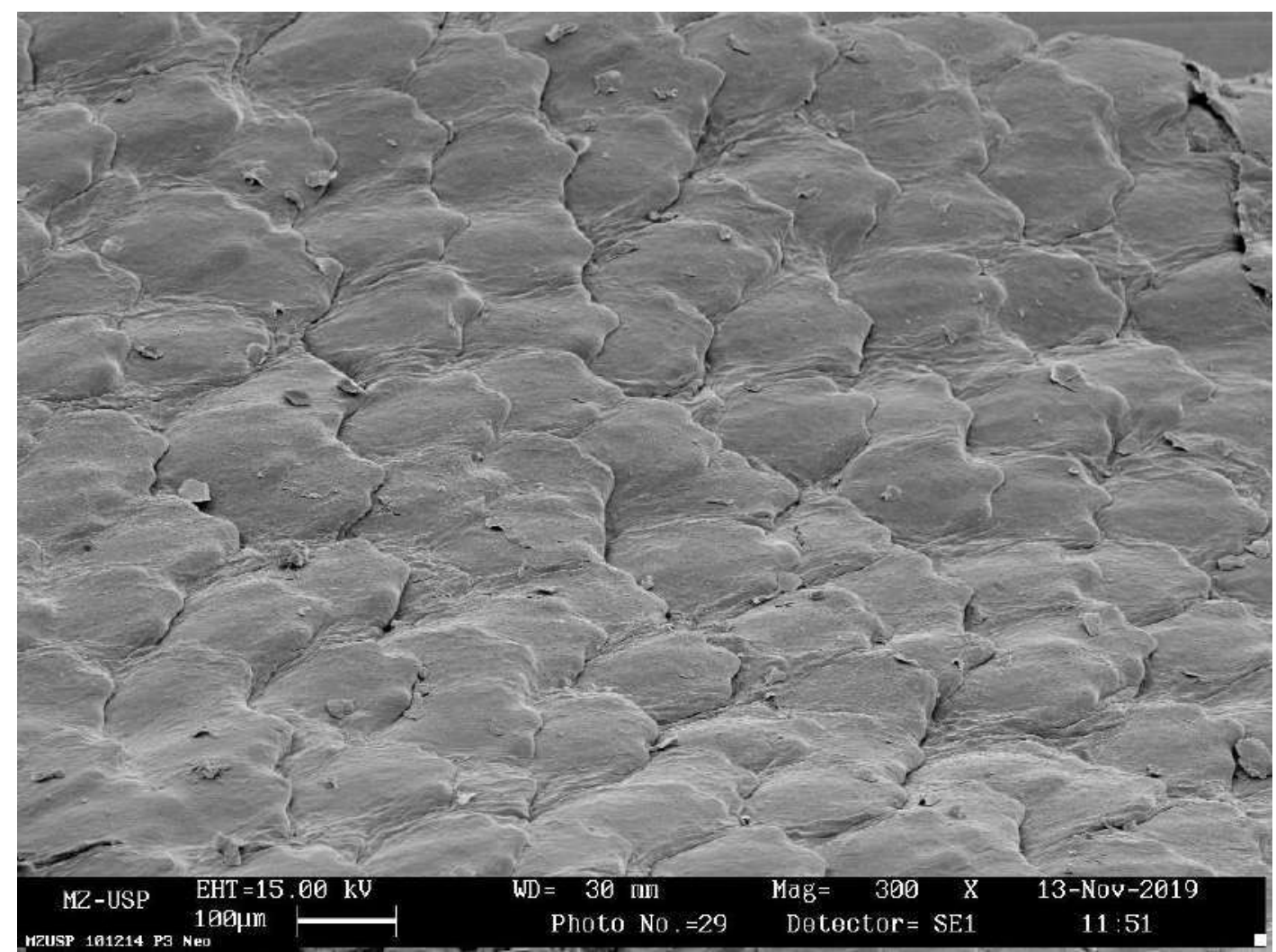

Figure 165. Dermal denticles collected from the section P3 (see fig. 1) of the neonate Isogomphodon oxyrhynchus (MZUSP 101214). Additional information in the image footer. 


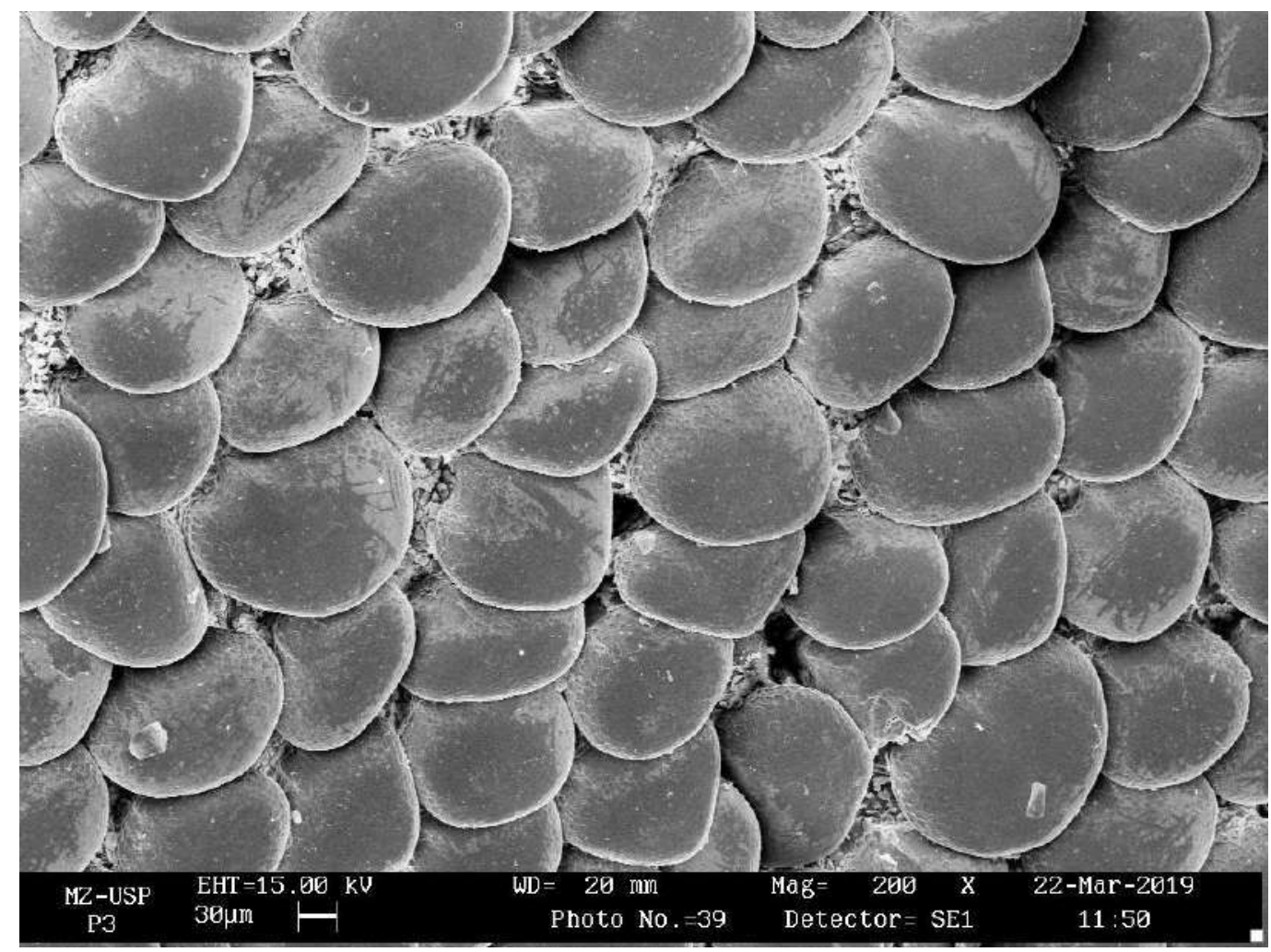

Figure 166. Dermal denticles collected from the section P3 (see fig. 1) of the juvenile Isogomphodon oxyrhynchus (MZUSP 101214). Additional information in the image footer.

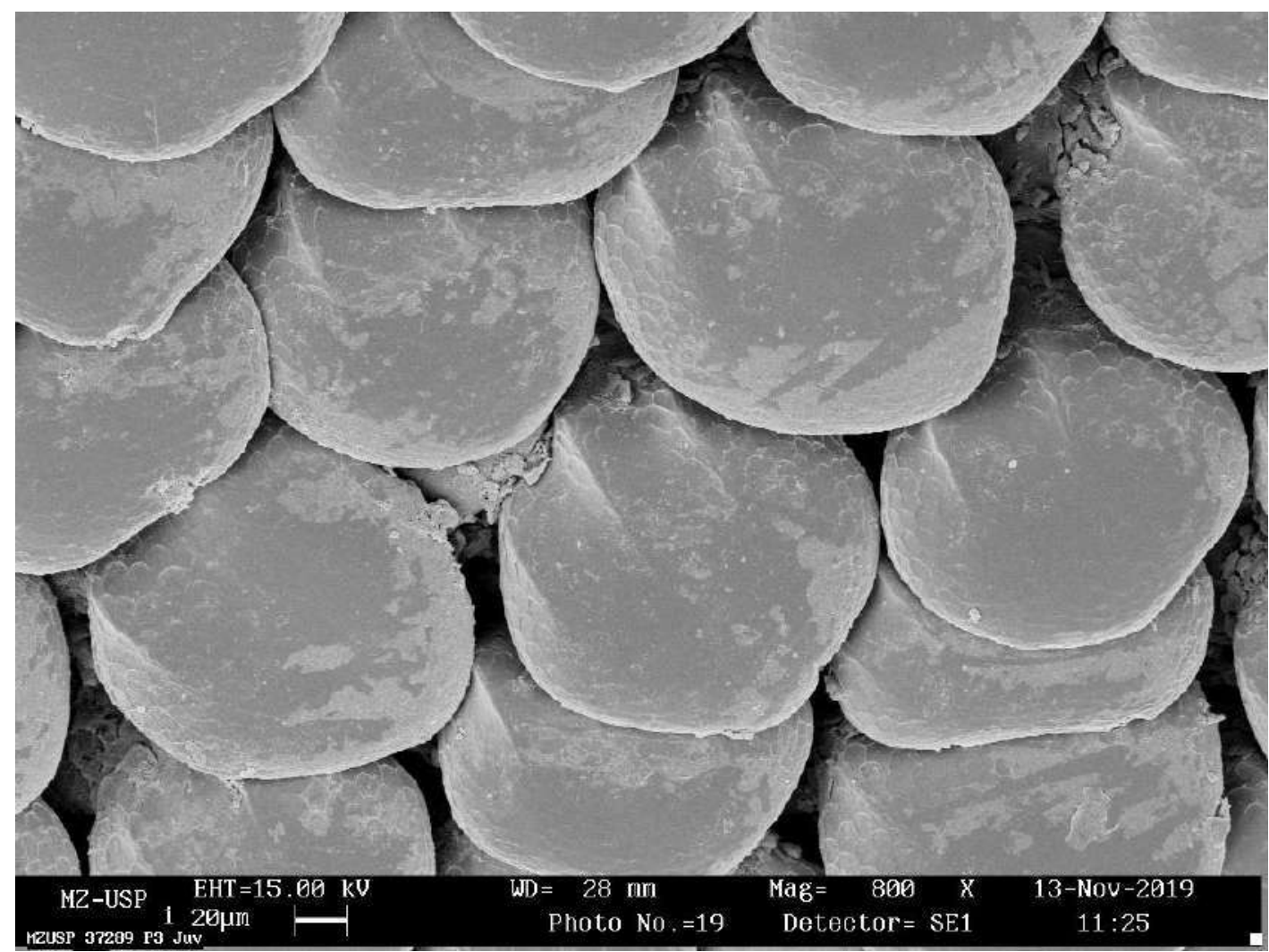

Figure 167. Dermal denticles collected from the section P3 (see fig. 1) of the juvenile Isogomphodon oxyrhynchus (MZUSP 101214). Additional information in the image footer. 


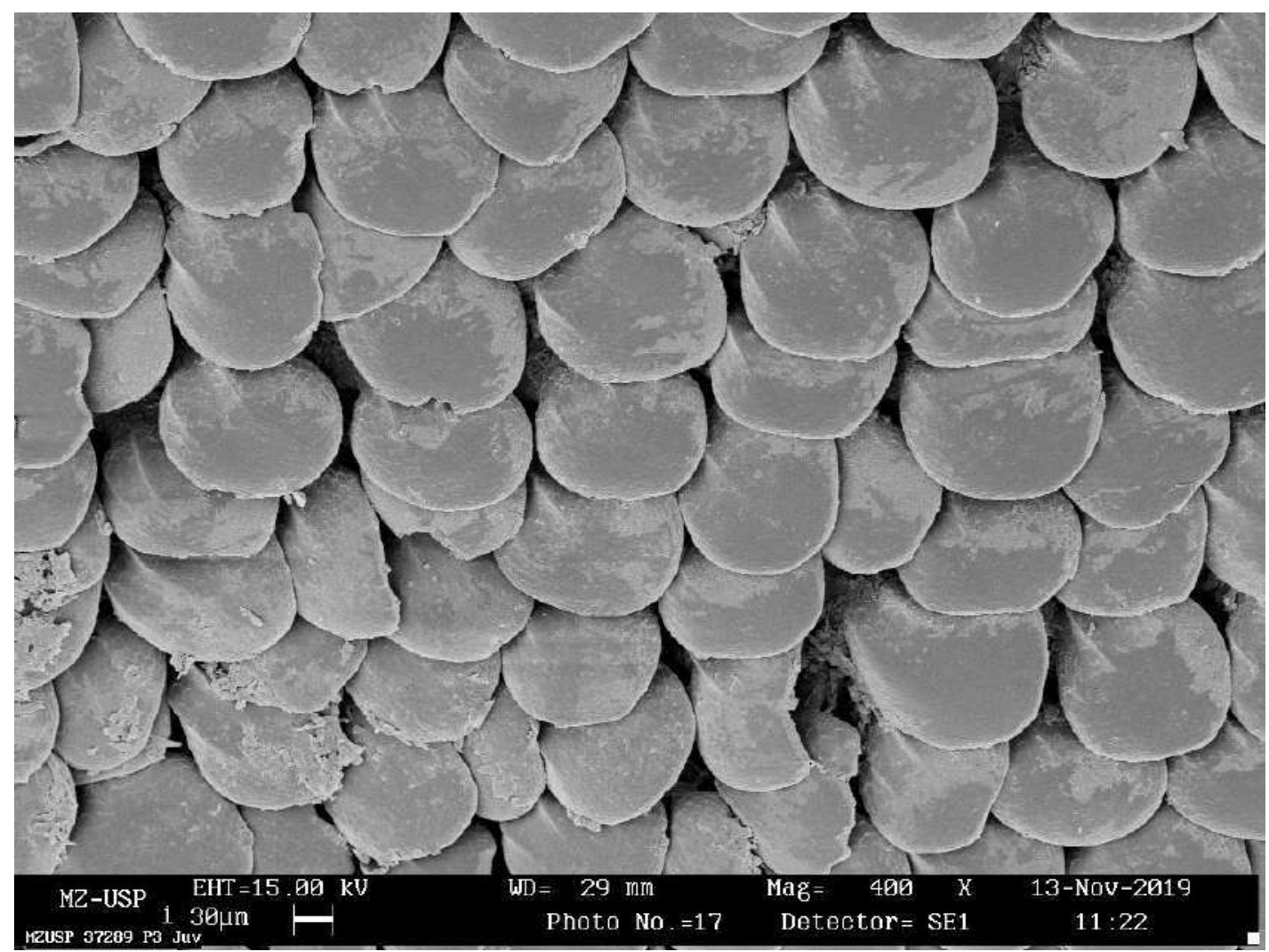

Figure 168. Dermal denticles collected from the section P3 (see fig. 1) of the juvenile Isogomphodon oxyrhynchus (MZUSP 101214). Additional information in the image footer.

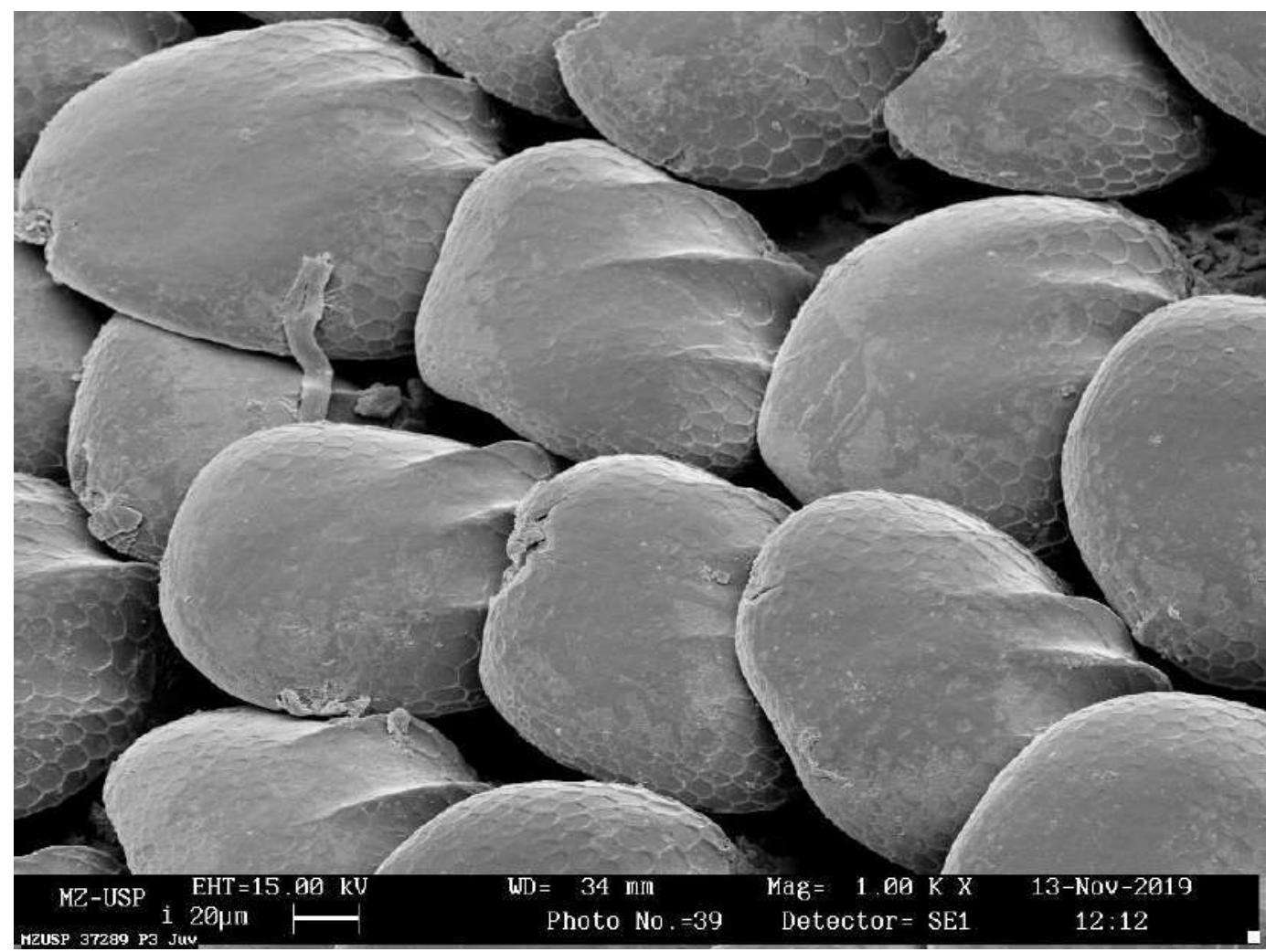

Figure 169. Dermal denticles collected from the section P3 (see fig. 1) of the juvenile Isogomphodon oxyrhynchus (MZUSP 101214). Additional information in the image footer. 


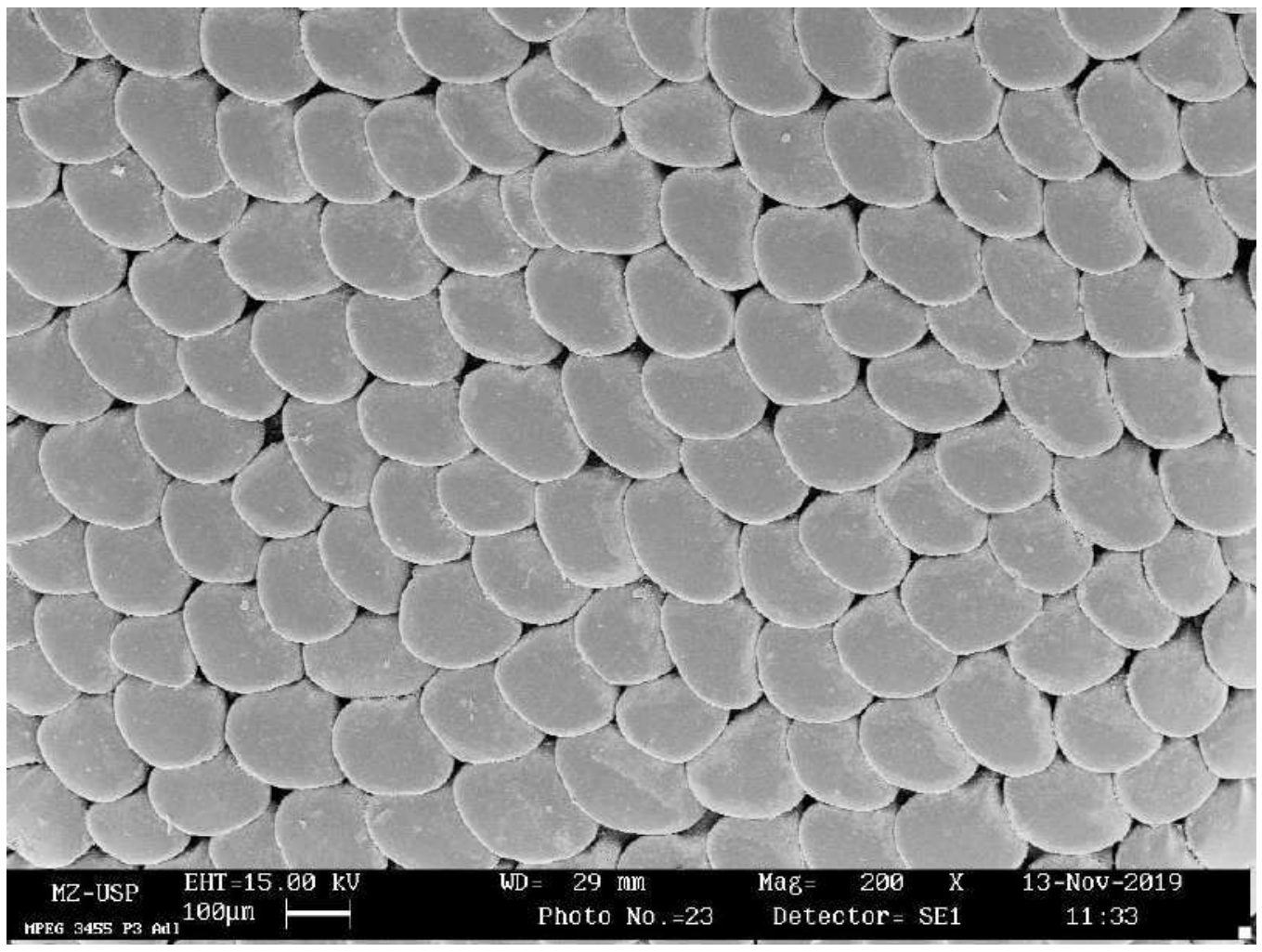

Figure 170. Dermal denticles collected from the section P3 (see fig. 1) of the adult Isogomphodon oxyrhynchus (MPEG 3455). Additional information in the image footer.

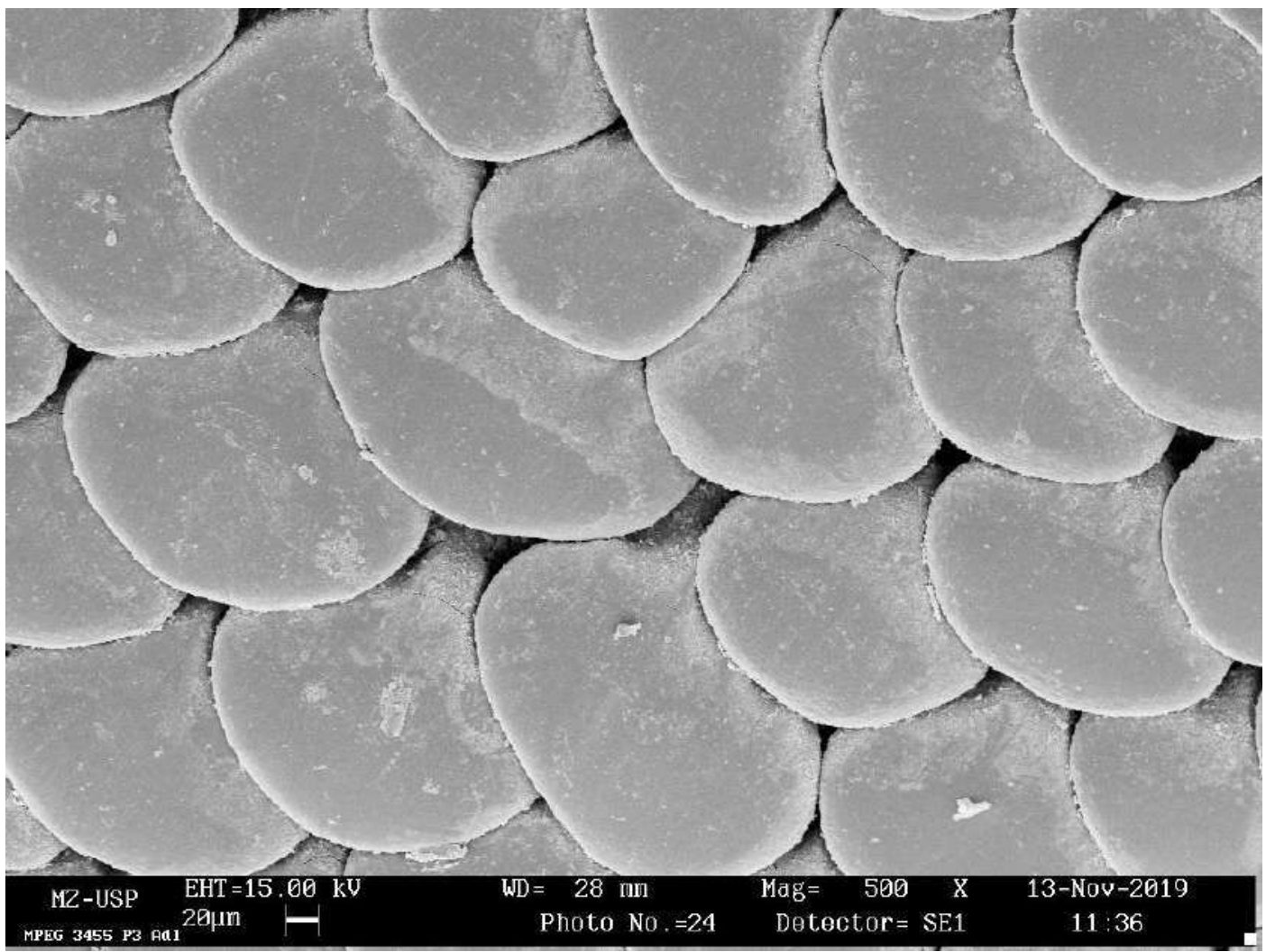

Figure 171. Dermal denticles collected from the section P3 (see fig. 1) of the adult Isogomphodon oxyrhynchus (MPEG 3455). Additional information in the image footer. 


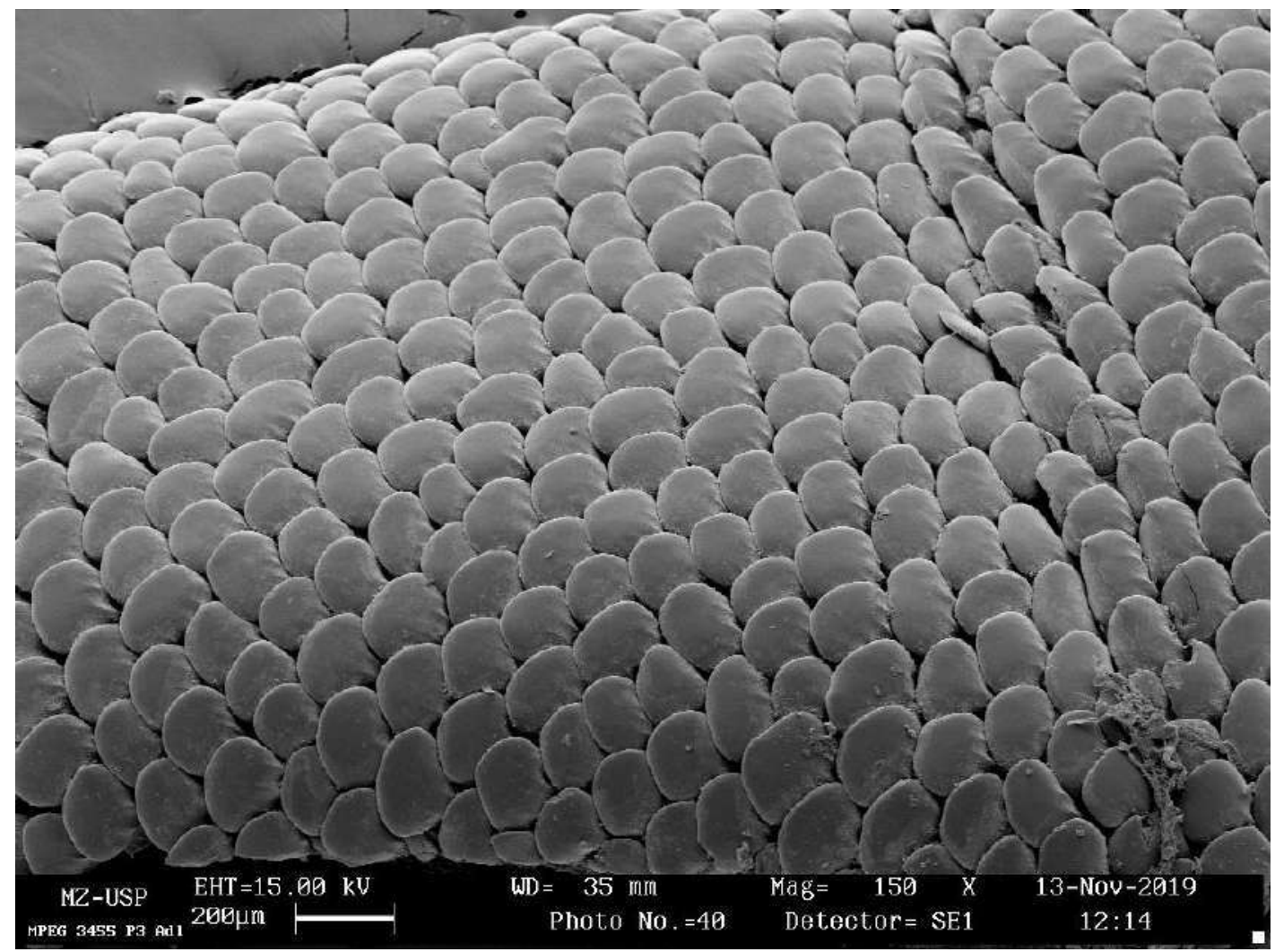

Figure 172. Dermal denticles collected from the section P3 (see fig. 1) of the adult Isogomphodon oxyrhynchus (MPEG 3455). Additional information in the image footer.

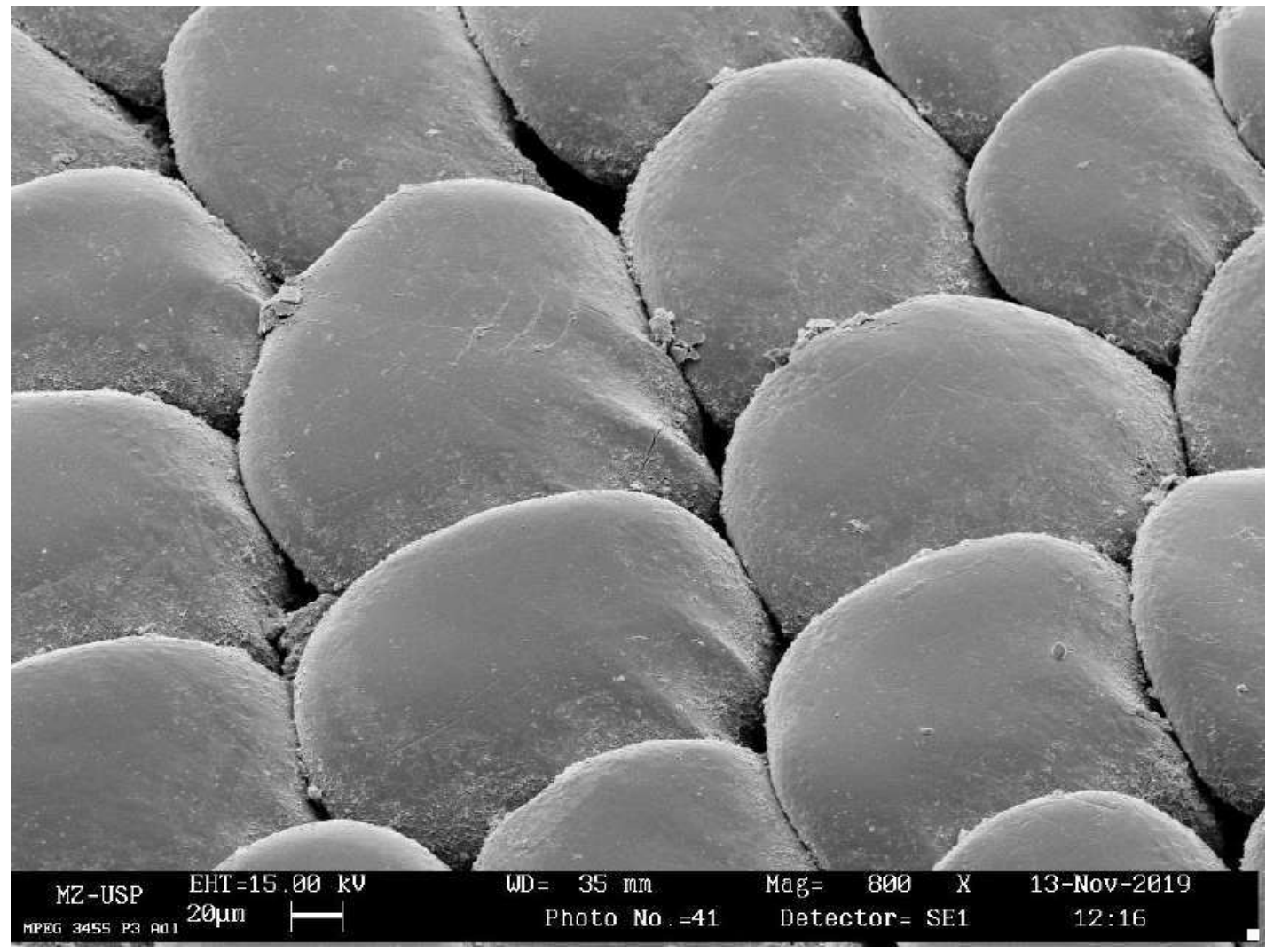

Figure 173. Dermal denticles collected from the section P3 (see fig. 1) of the adult Isogomphodon oxyrhynchus (MPEG 3455). Additional information in the image footer. 


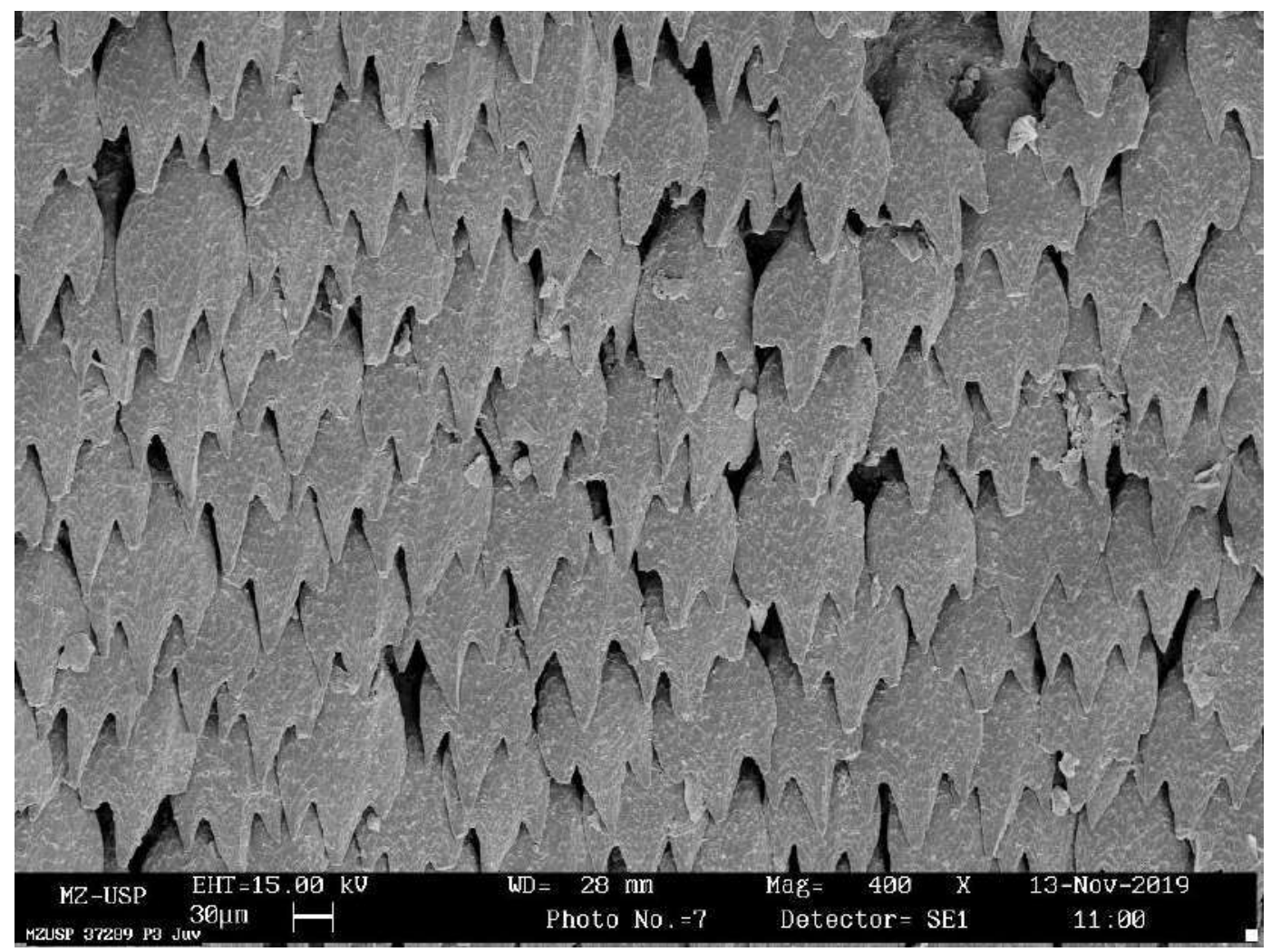

Figure 174. Dermal denticles collected from the section P3m (see fig. 1) of the juvenile Isogomphodon oxyrhynchus (MZUSP 37289). Additional information in the image footer.

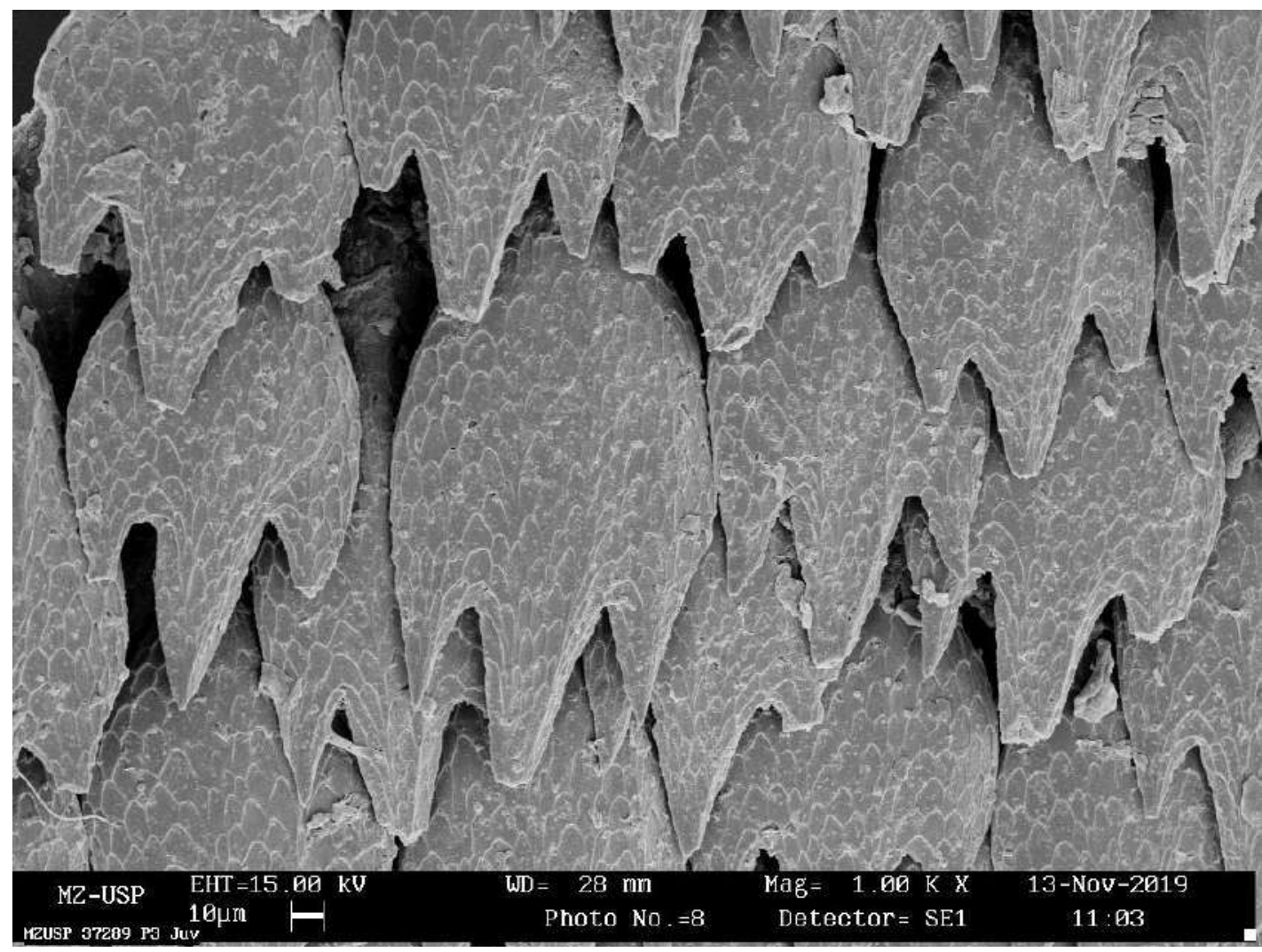

Figure 175. Dermal denticles collected from the section P3m (see fig. 1) of the juvenile Isogomphodon oxyrhynchus (MZUSP 37289). Additional information in the image footer. 


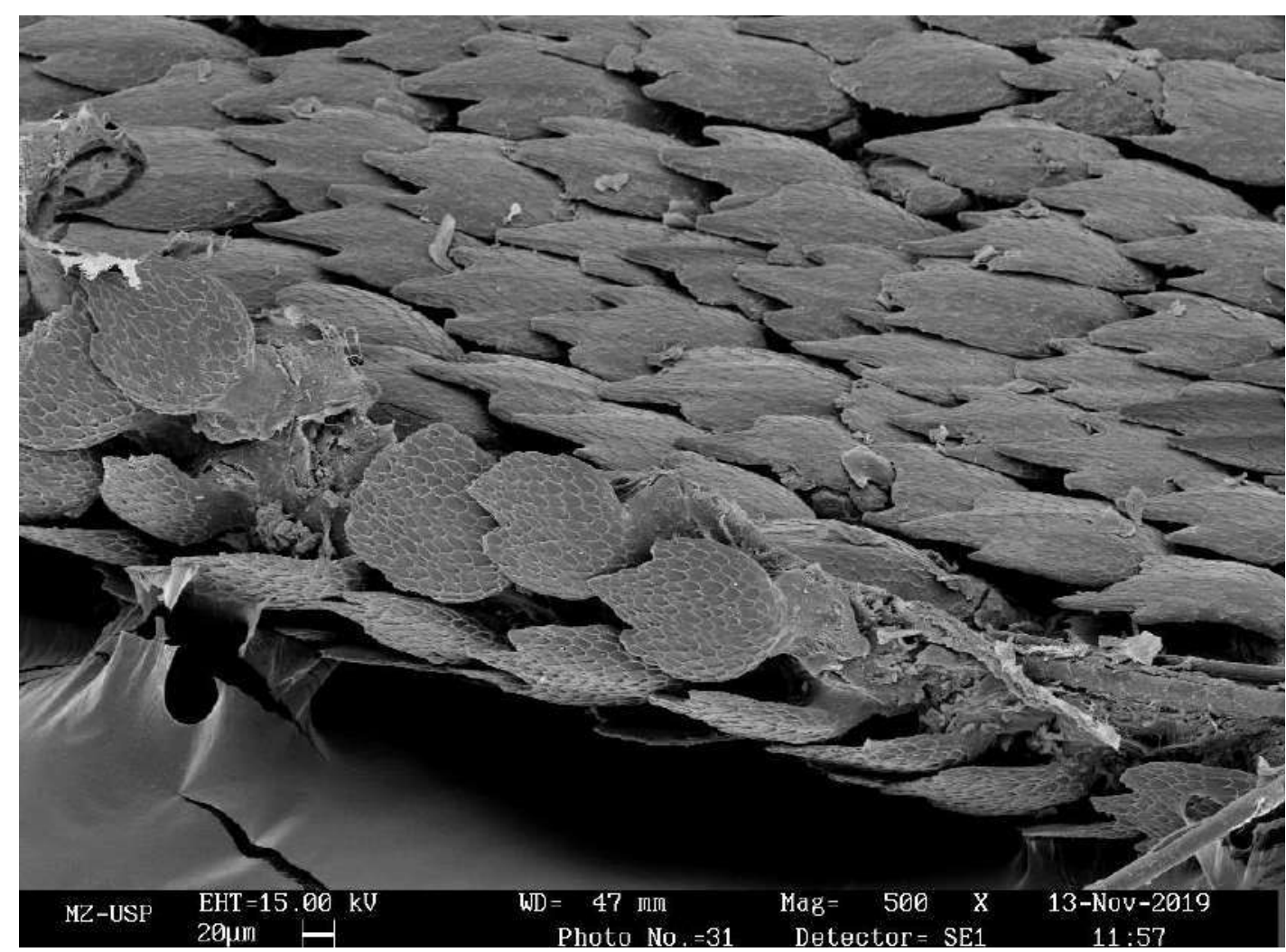

Figure 176. Dermal denticles collected from the section P3m (see fig. 1) of the juvenile Isogomphodon oxyrhynchus (MZUSP 37289). Additional information in the image footer.

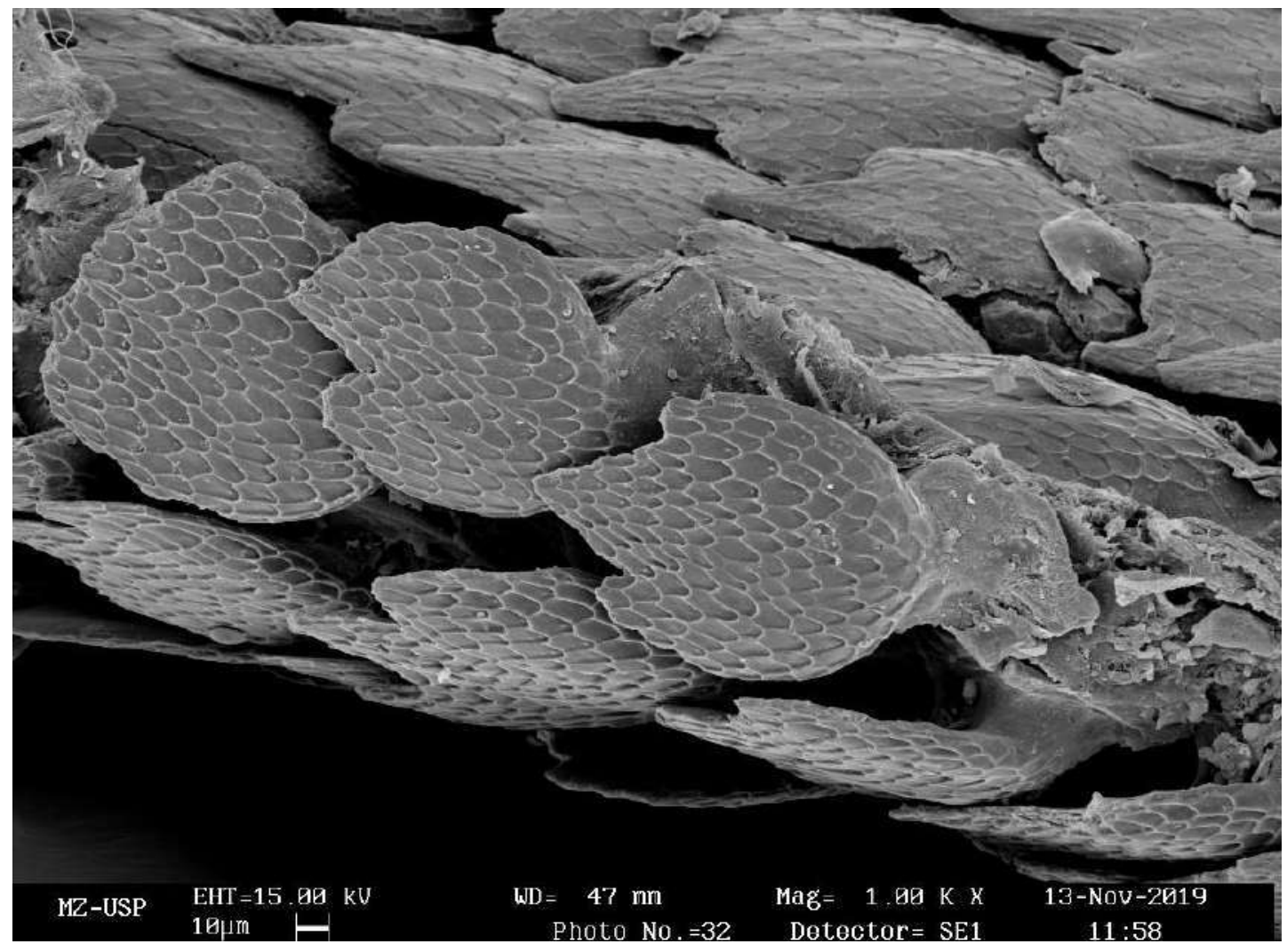

Figure 177. Dermal denticles collected from the section P3m (see fig. 1) of the juvenile Isogomphodon oxyrhynchus (MZUSP 37289). Additional information in the image footer. 


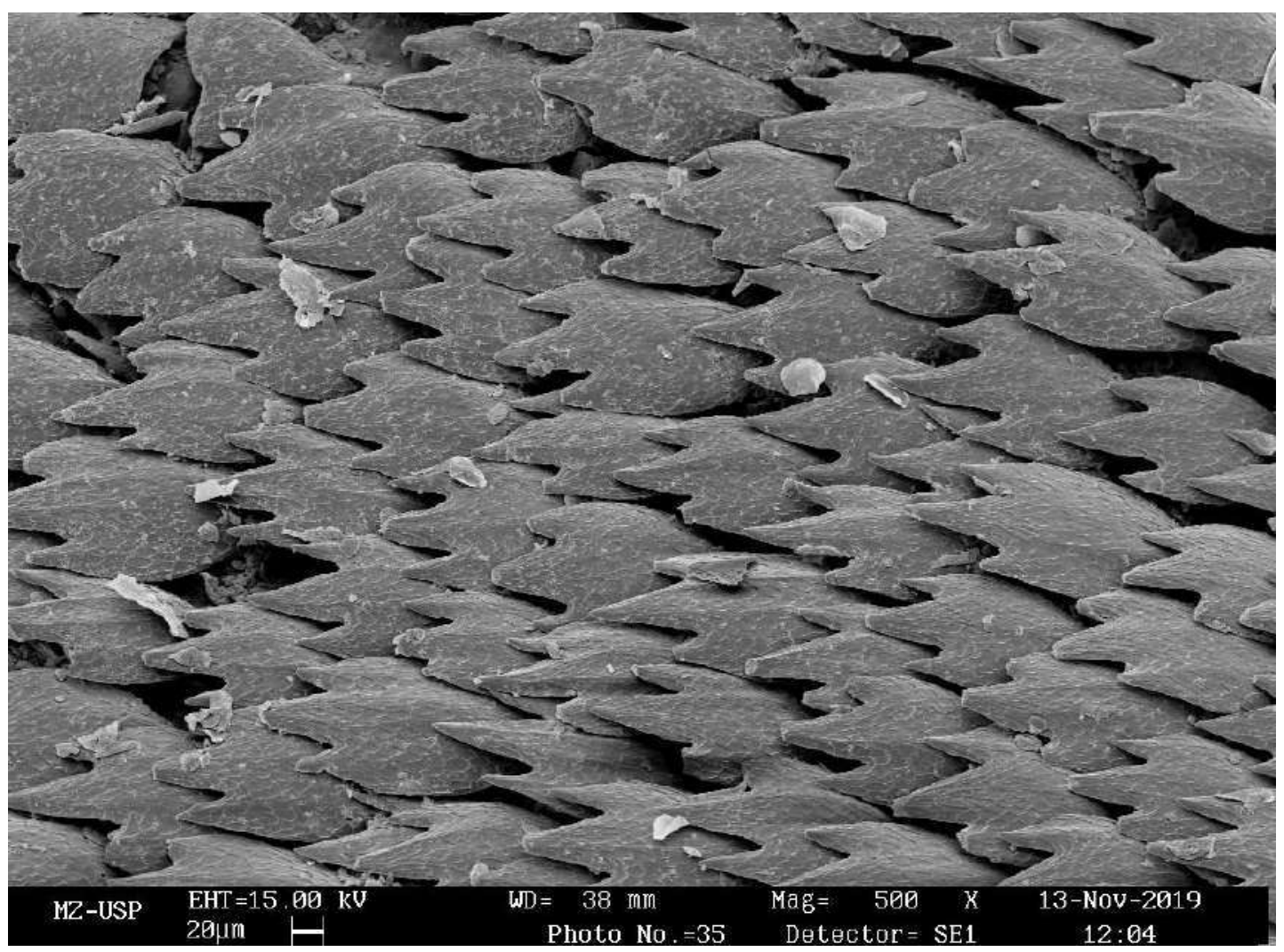

Figure 178. Dermal denticles collected from the section P3m (see fig. 1) of the juvenile Isogomphodon oxyrhynchus (MZUSP 37289). Additional information in the image footer.

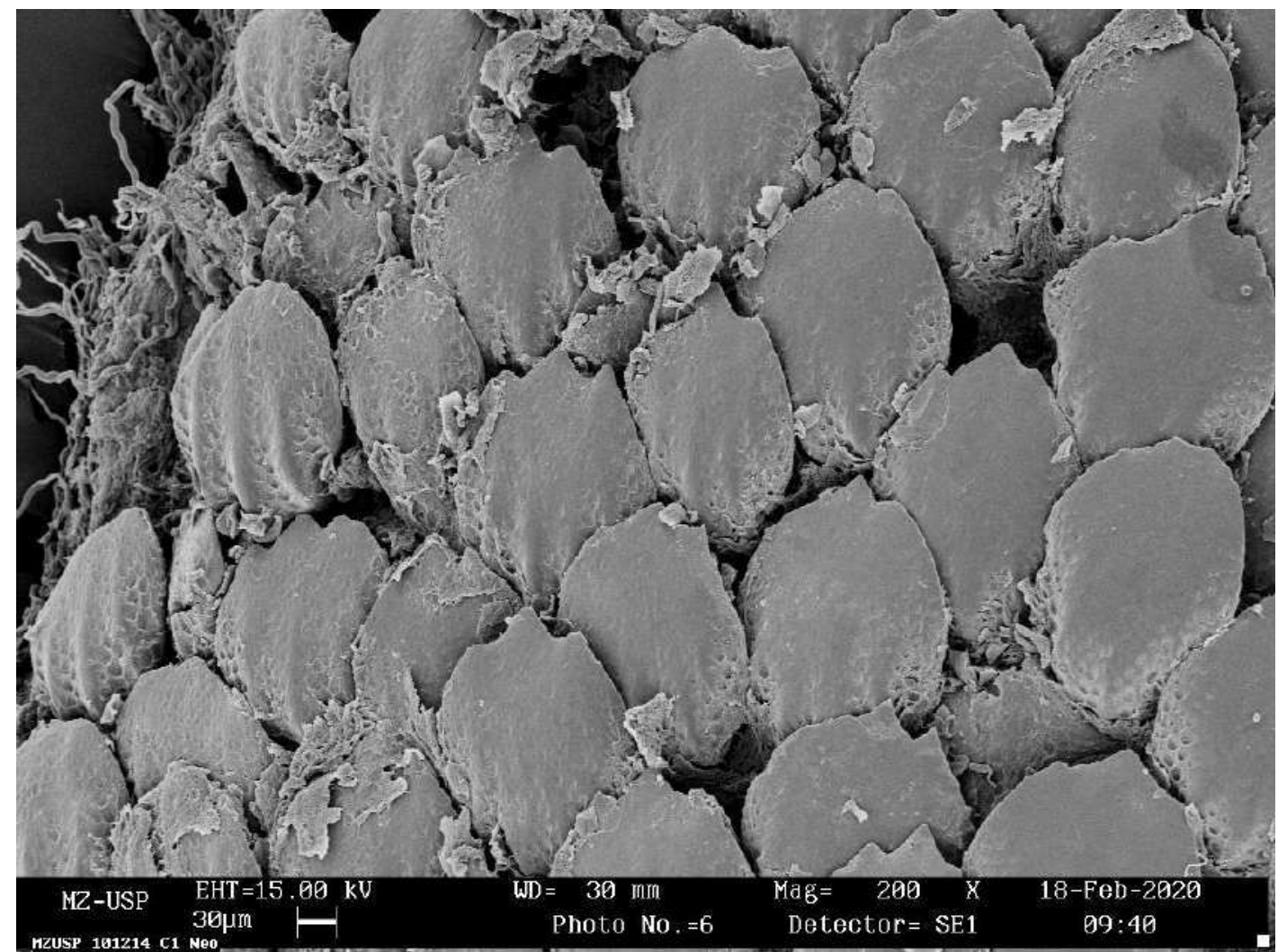

Figure 179. Dermal denticles collected from the section C1 (see fig. 1) of the neonate Isogomphodon oxyrhynchus (MZUSP 101214). Additional information in the image footer. 


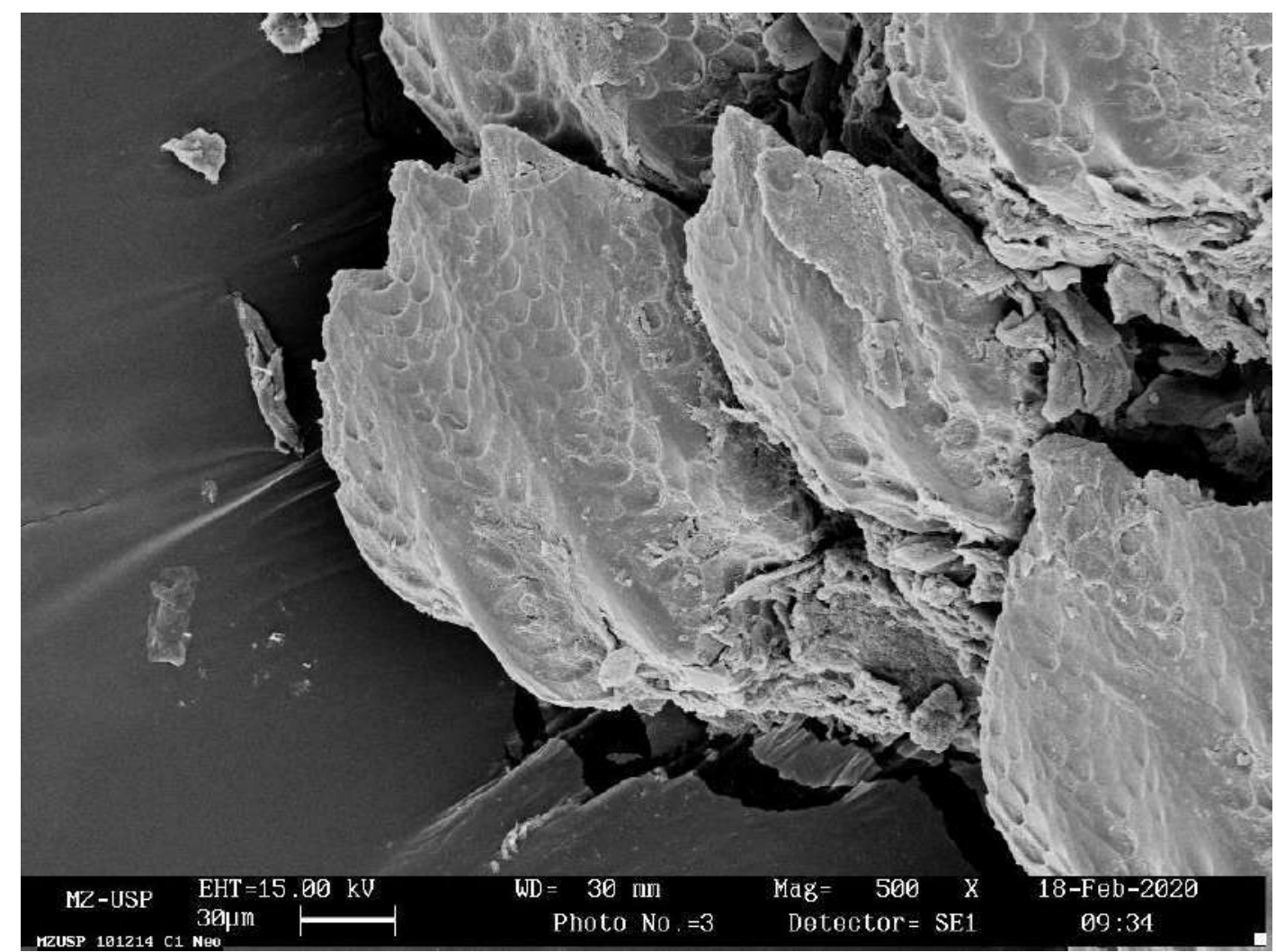

Figure 180. Dermal denticles collected from the section C1 (see fig. 1) of the neonate Isogomphodon oxyrhynchus (MZUSP 101214). Additional information in the image footer.

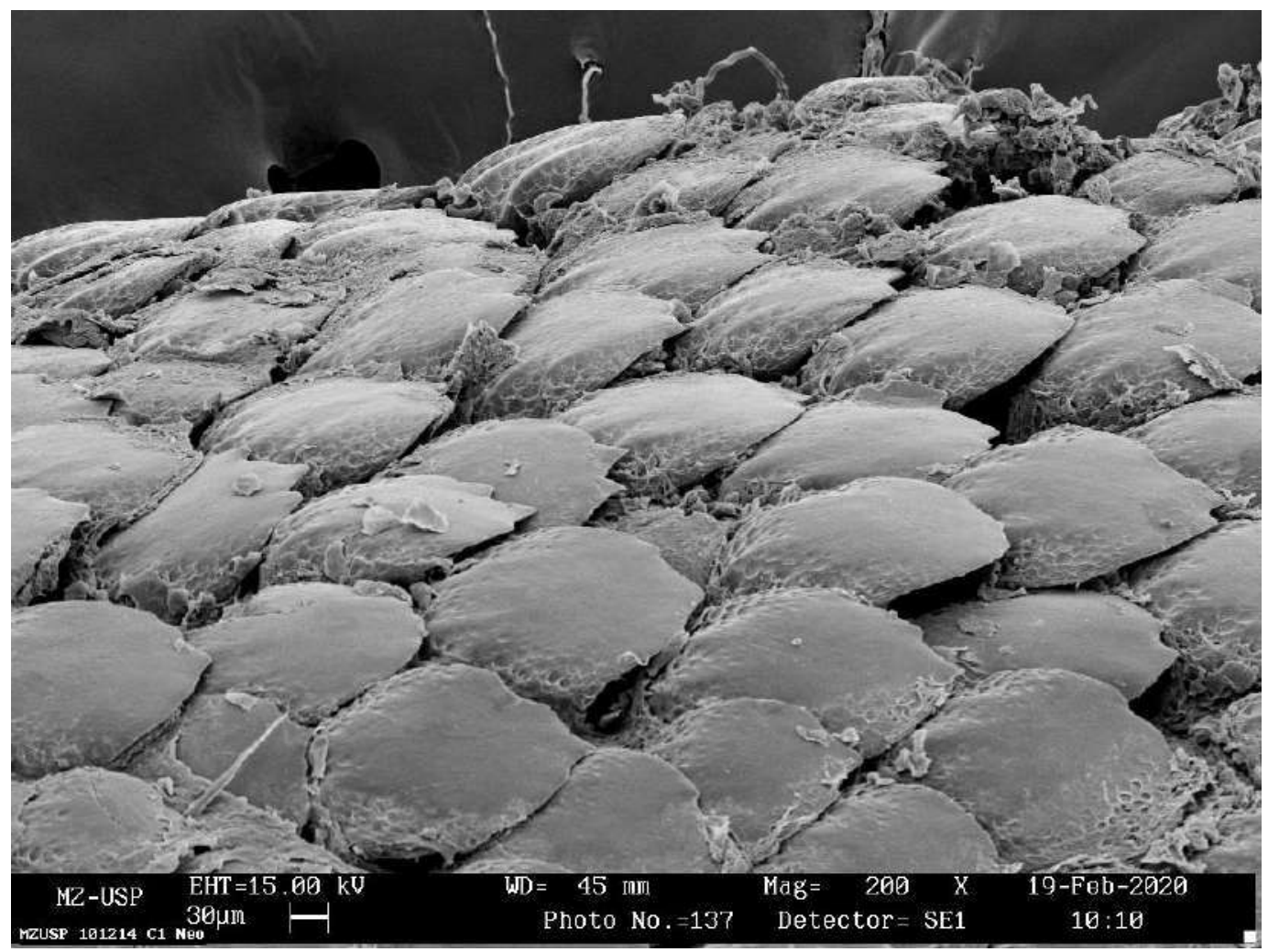

Figure 181. Dermal denticles collected from the section C1 (see fig. 1) of the neonate Isogomphodon oxyrhynchus (MZUSP 101214). Additional information in the image footer. 


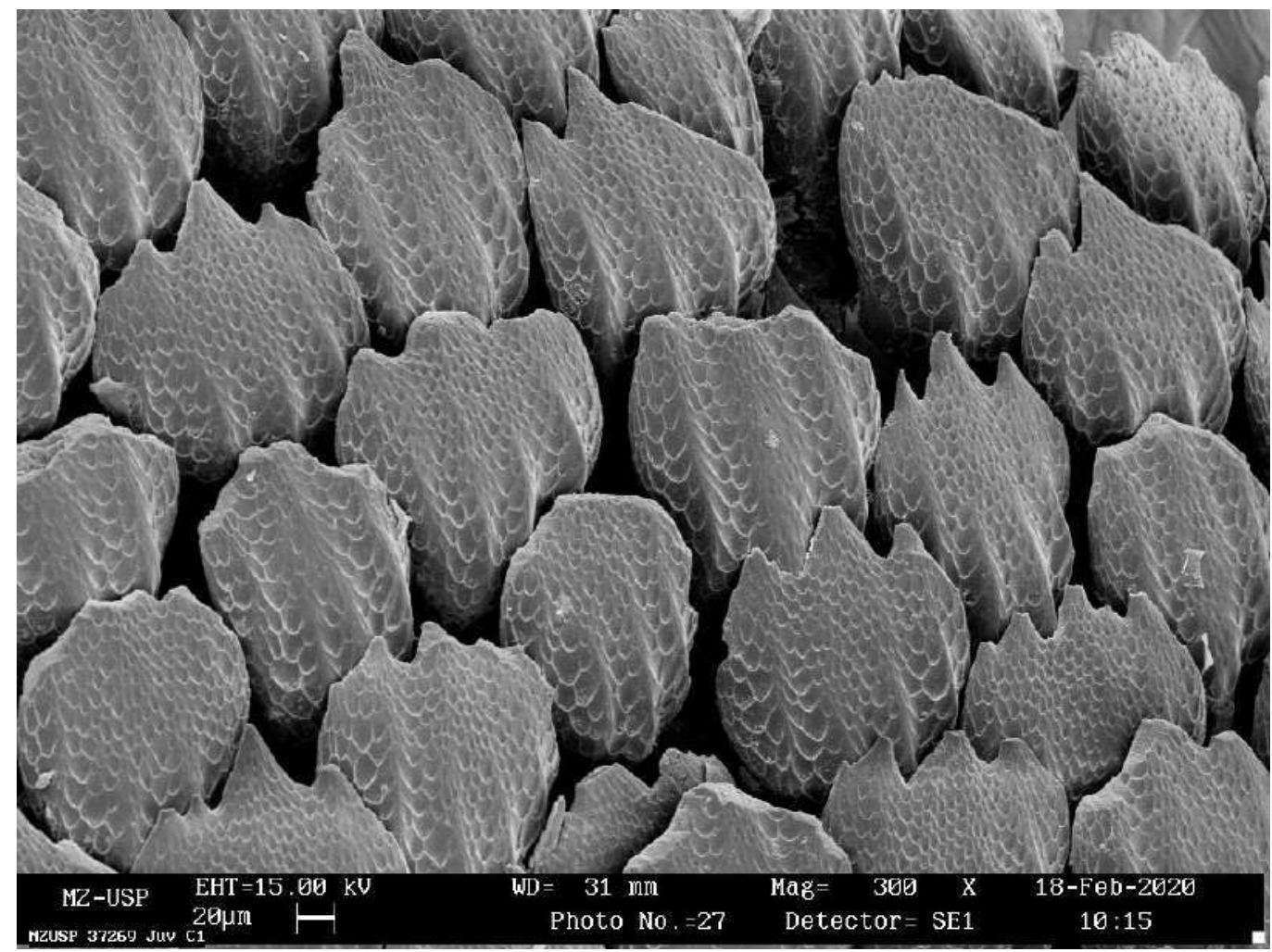

Figure 182. Dermal denticles collected from the section C1 (see fig. 1) of the juvenile Isogomphodon oxyrhynchus (MZUSP 37289). Additional information in the image footer.

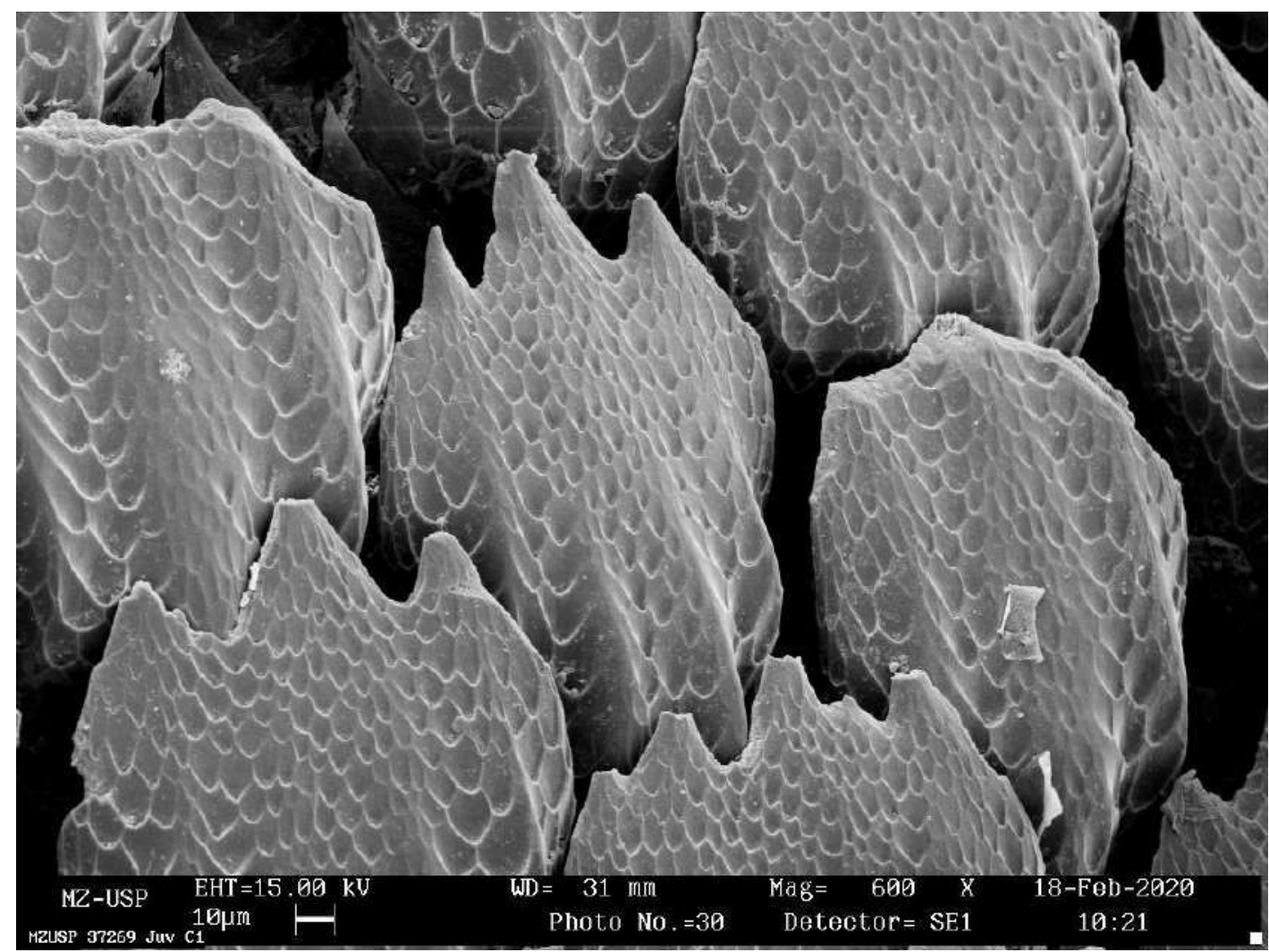

Figure 183. Dermal denticles collected from the section C1 (see fig. 1) of the juvenile Isogomphodon oxyrhynchus (MZUSP 37289). Additional information in the image footer. 


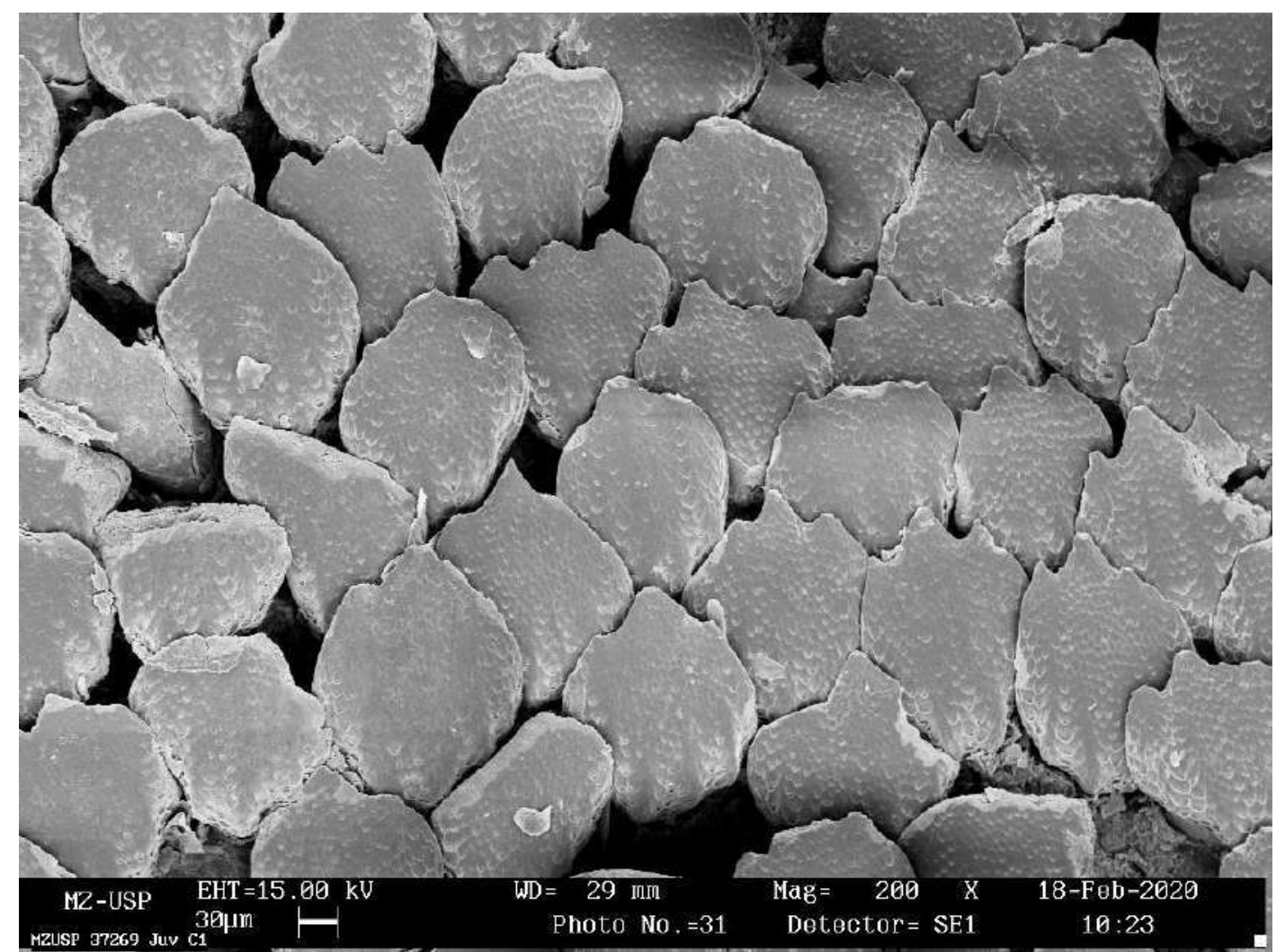

Figure 184. Dermal denticles collected from the section C1 (see fig. 1) of the juvenile Isogomphodon oxyrhynchus (MZUSP 37289). Additional information in the image footer.

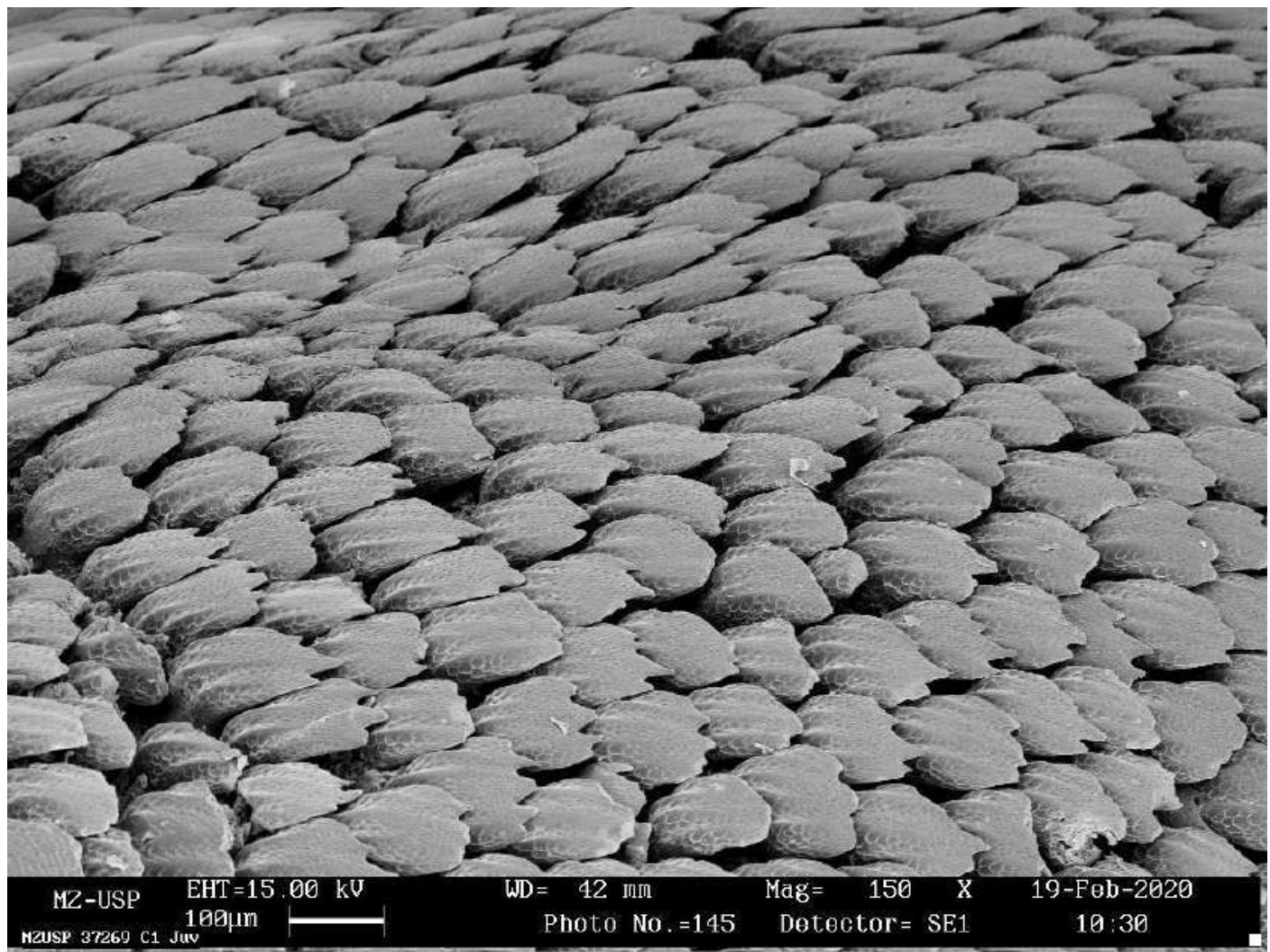

Figure 185. Dermal denticles collected from the section C1 (see fig. 1) of the juvenile Isogomphodon oxyrhynchus (MZUSP 37289). Additional information in the image footer. 


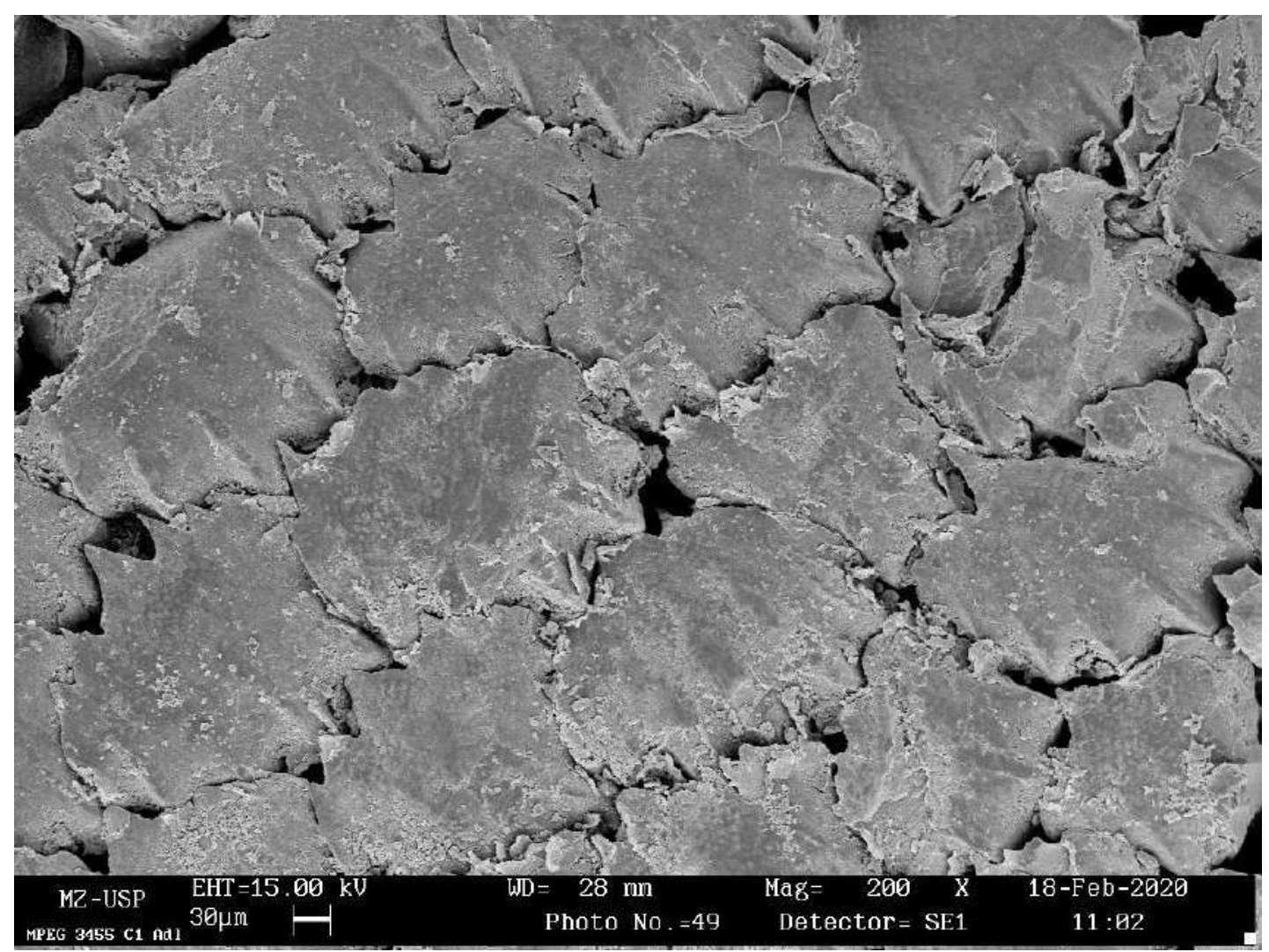

Figure 186. Dermal denticles collected from the section C1 (see fig. 1) of the adult Isogomphodon oxyrhynchus (MPEG 3455). Additional information in the image footer.

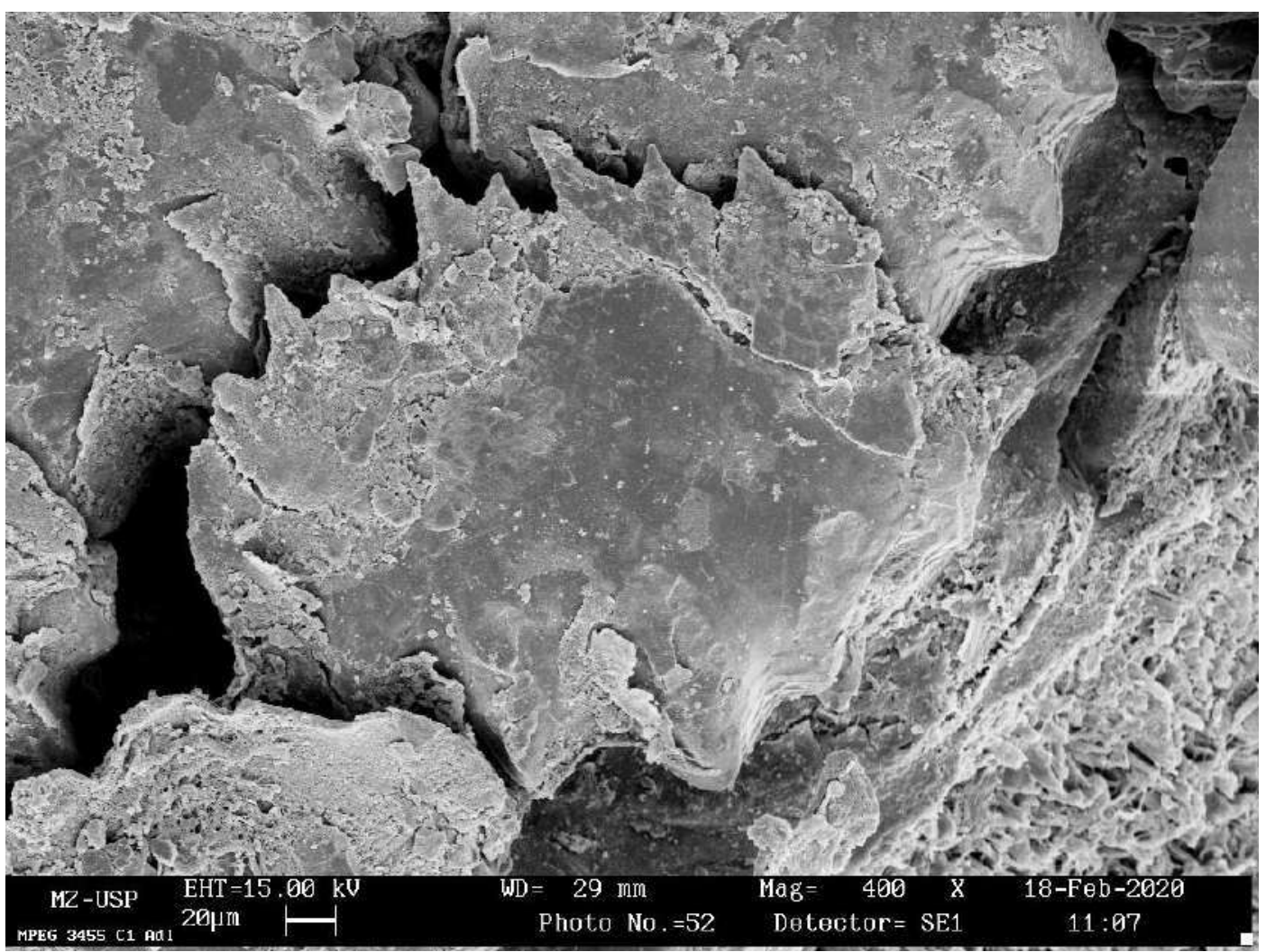

Figure 187. Dermal denticles collected from the section C1 (see fig. 1) of the adult Isogomphodon oxyrhynchus (MPEG 3455). Additional information in the image footer. 


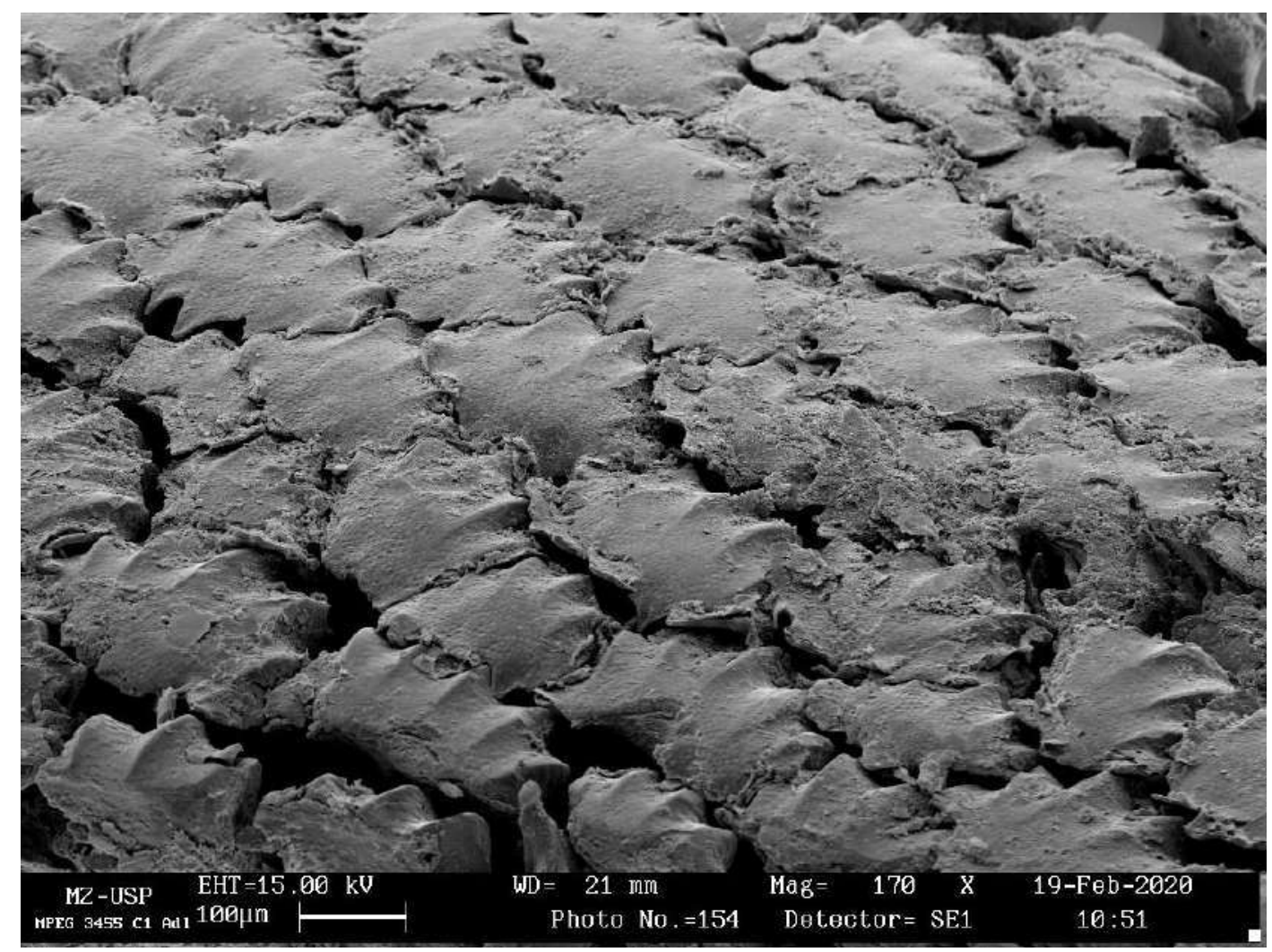

Figure 188. Dermal denticles collected from the section C1 (see fig. 1) of the adult Isogomphodon oxyrhynchus (MPEG 3455). Additional information in the image footer.

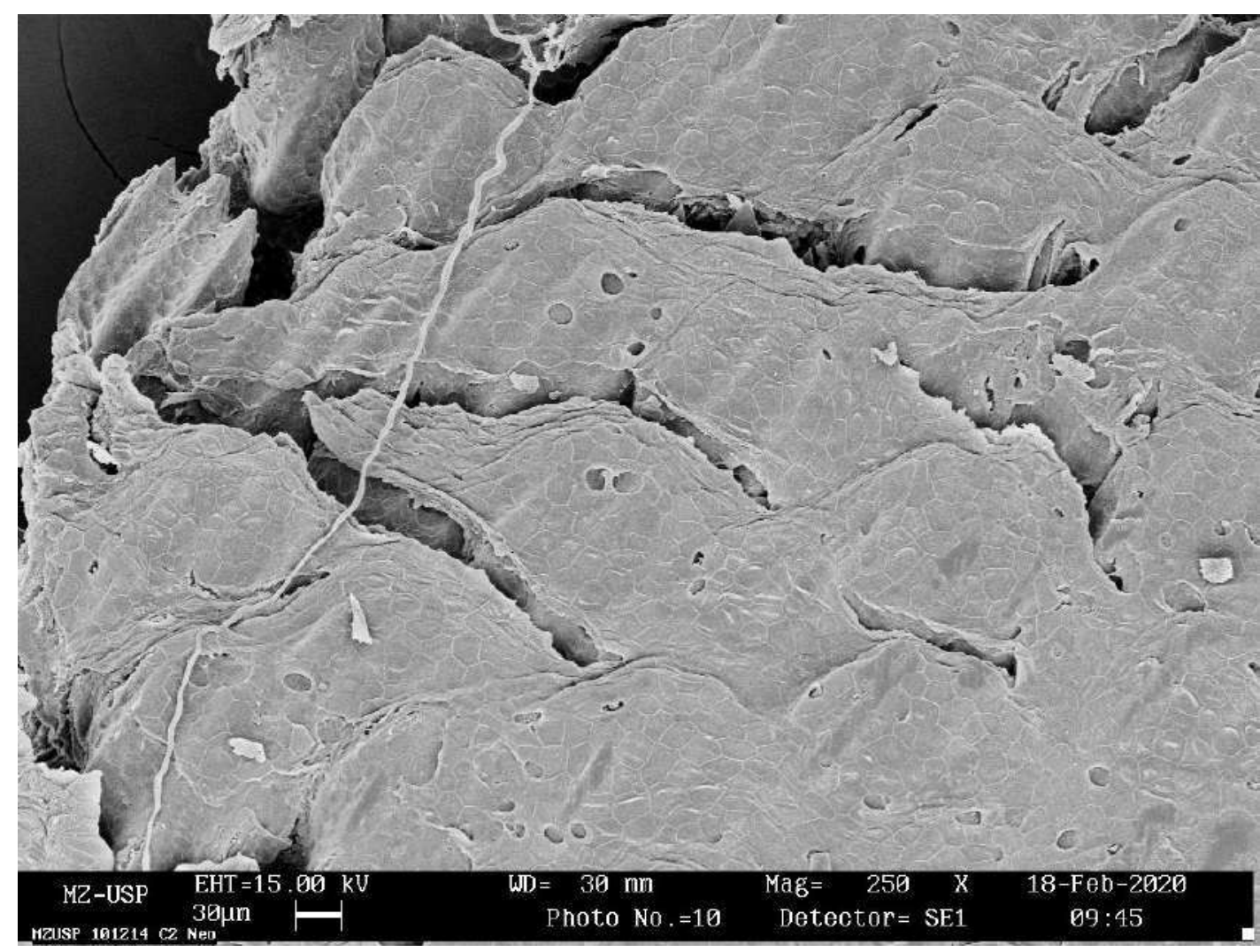

Figure 189. Dermal denticles collected from the section C2 (see fig. 1) of the neonate Isogomphodon oxyrhynchus (MZUSP 101214). Additional information in the image footer. 


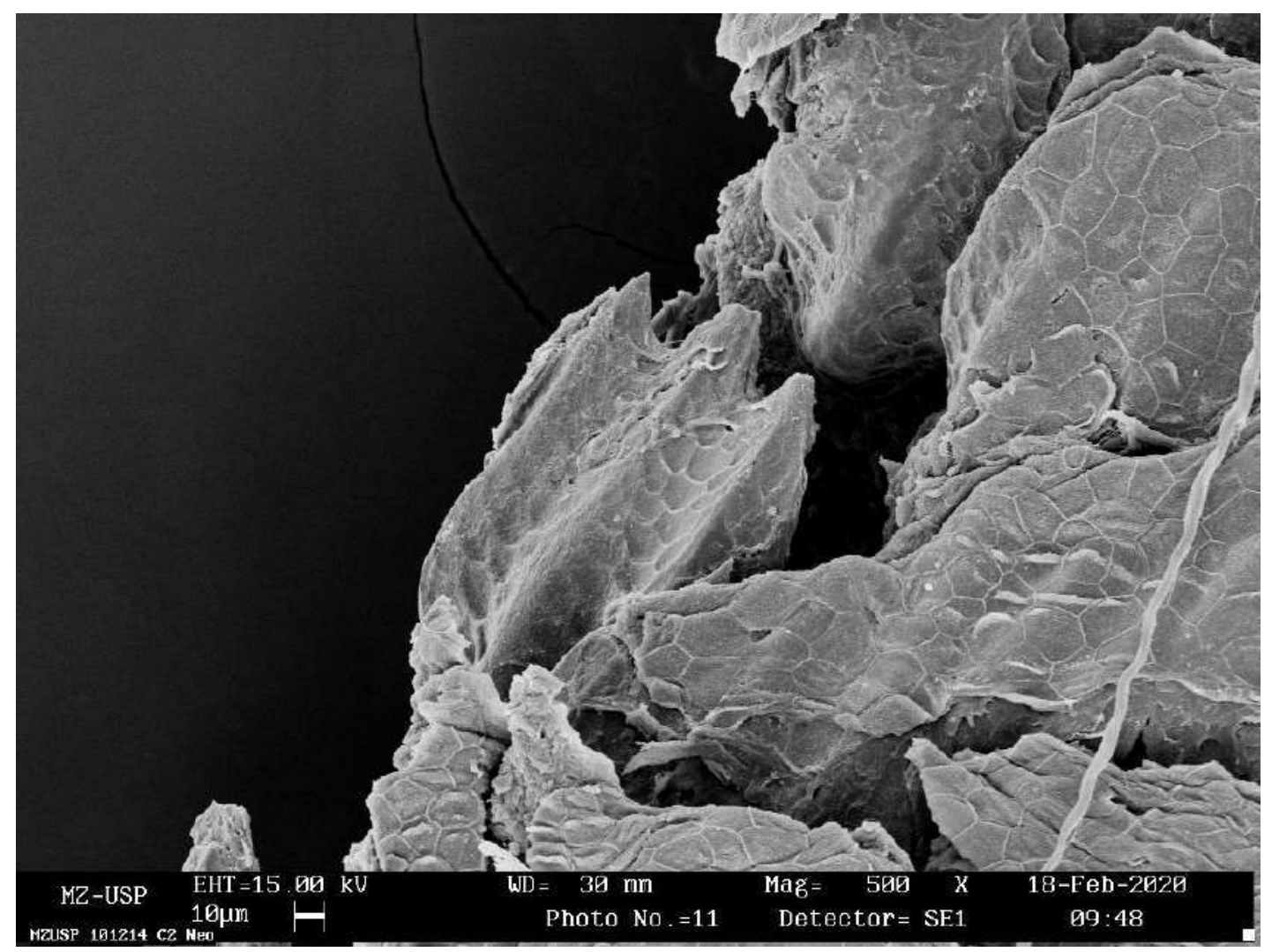

Figure 190. Dermal denticles collected from the section C2 (see fig. 1) of the neonate Isogomphodon oxyrhynchus (MZUSP 101214). Additional information in the image footer.

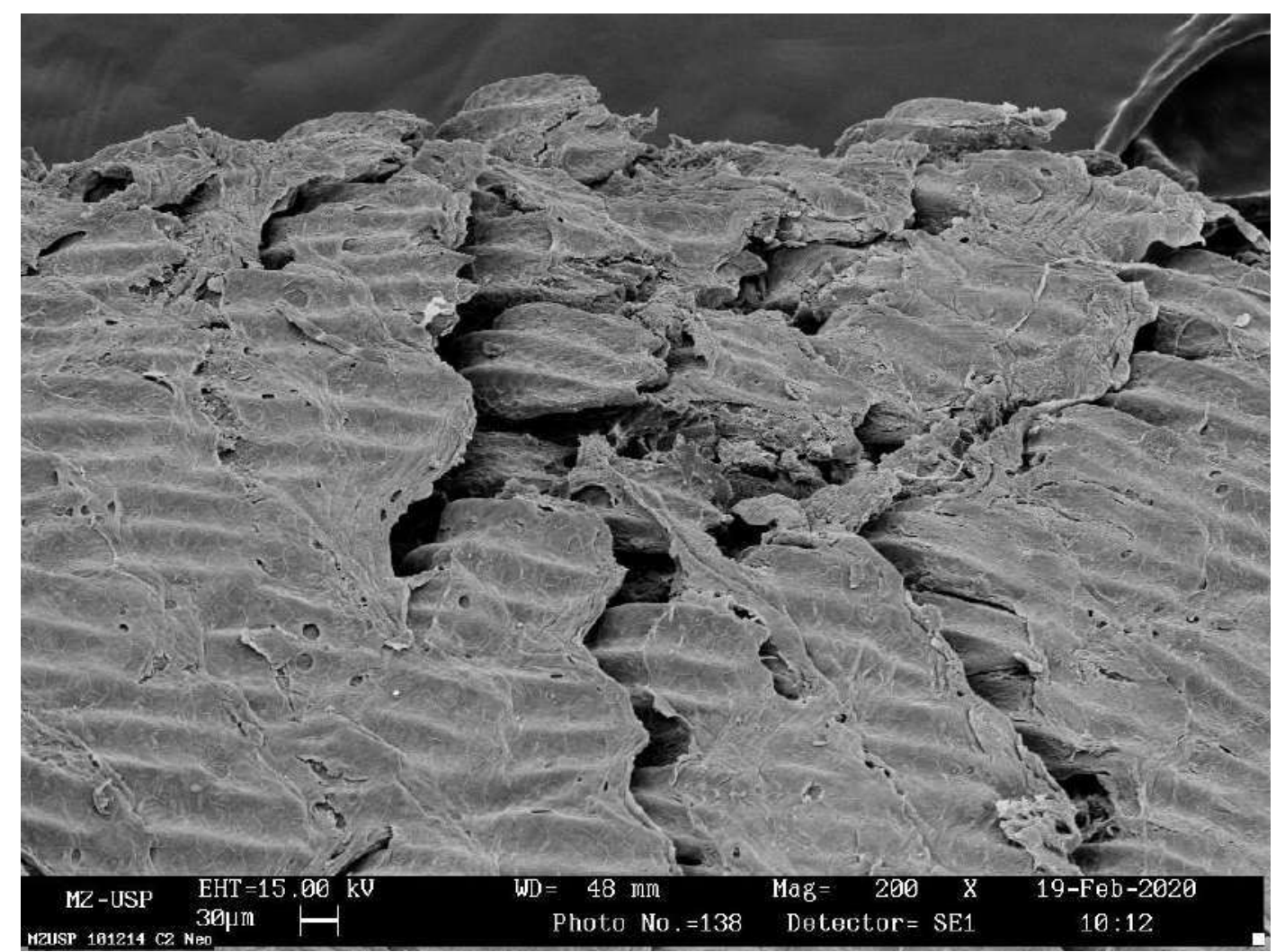

Figure 191. Dermal denticles collected from the section C2 (see fig. 1) of the neonate Isogomphodon oxyrhynchus (MZUSP 101214). Additional information in the image footer. 


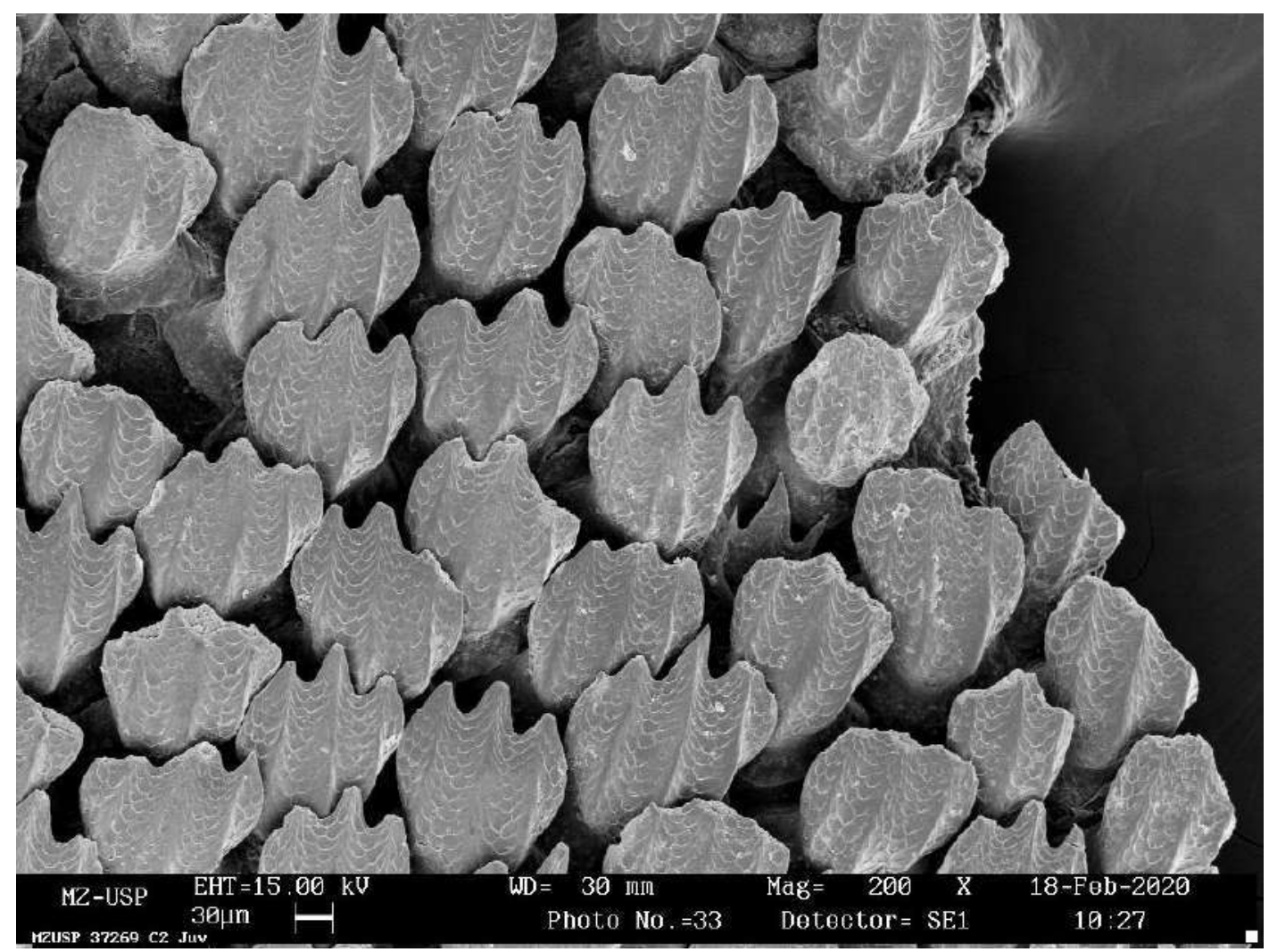

Figure 192. Dermal denticles collected from the section C2 (see fig. 1) of the juvenile Isogomphodon oxyrhynchus (MZUSP 37289). Additional information in the image footer.

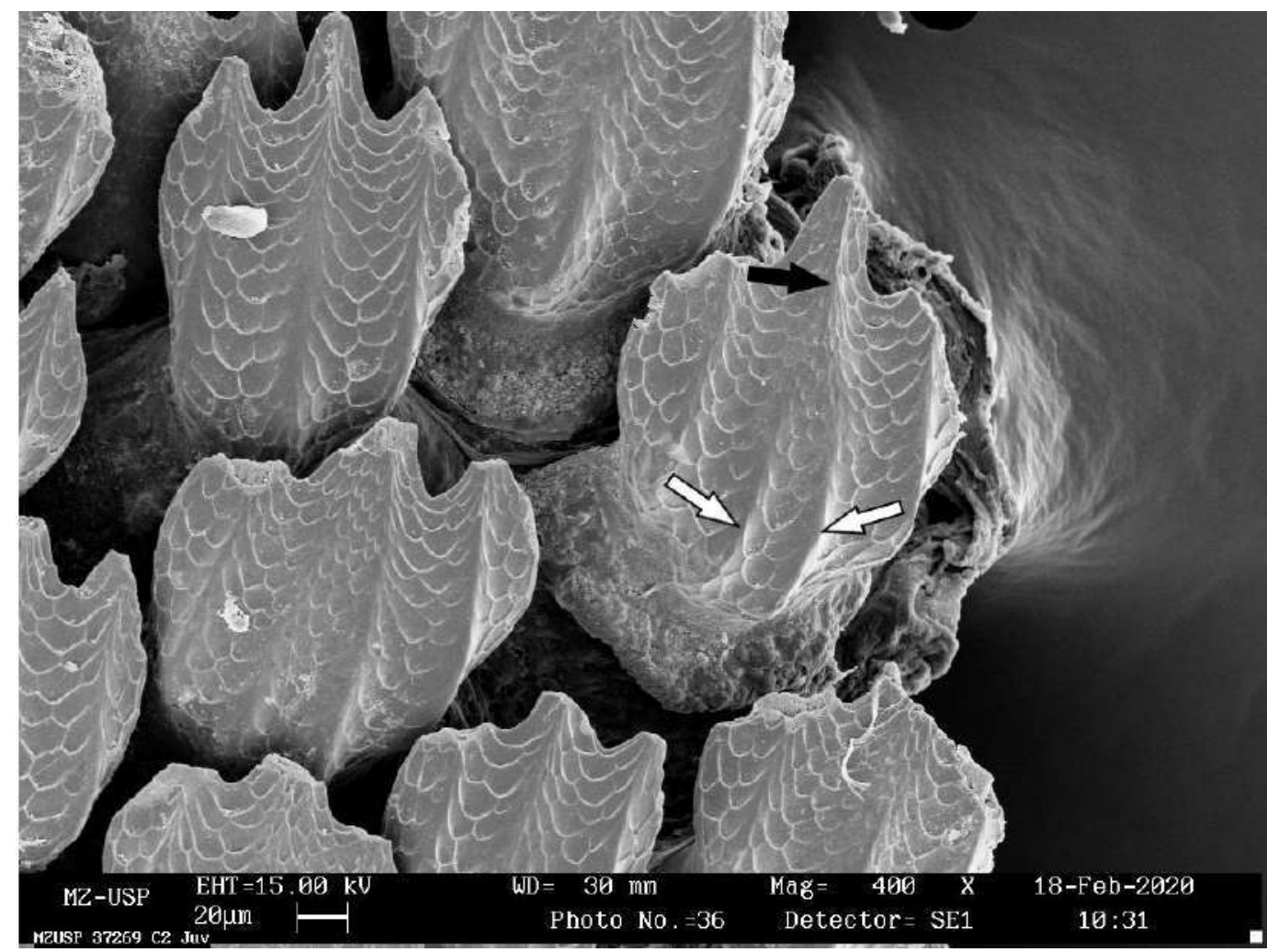

Figure 193. Dermal denticles collected from the section C2 (see fig. 1) of the juvenile Isogomphodon oxyrhynchus (MZUSP 37289). Additional information in the image footer. White arrows indicate the two primordial middle ridges converging posteriorly to the medial ridge, indicated by the black arrow. 


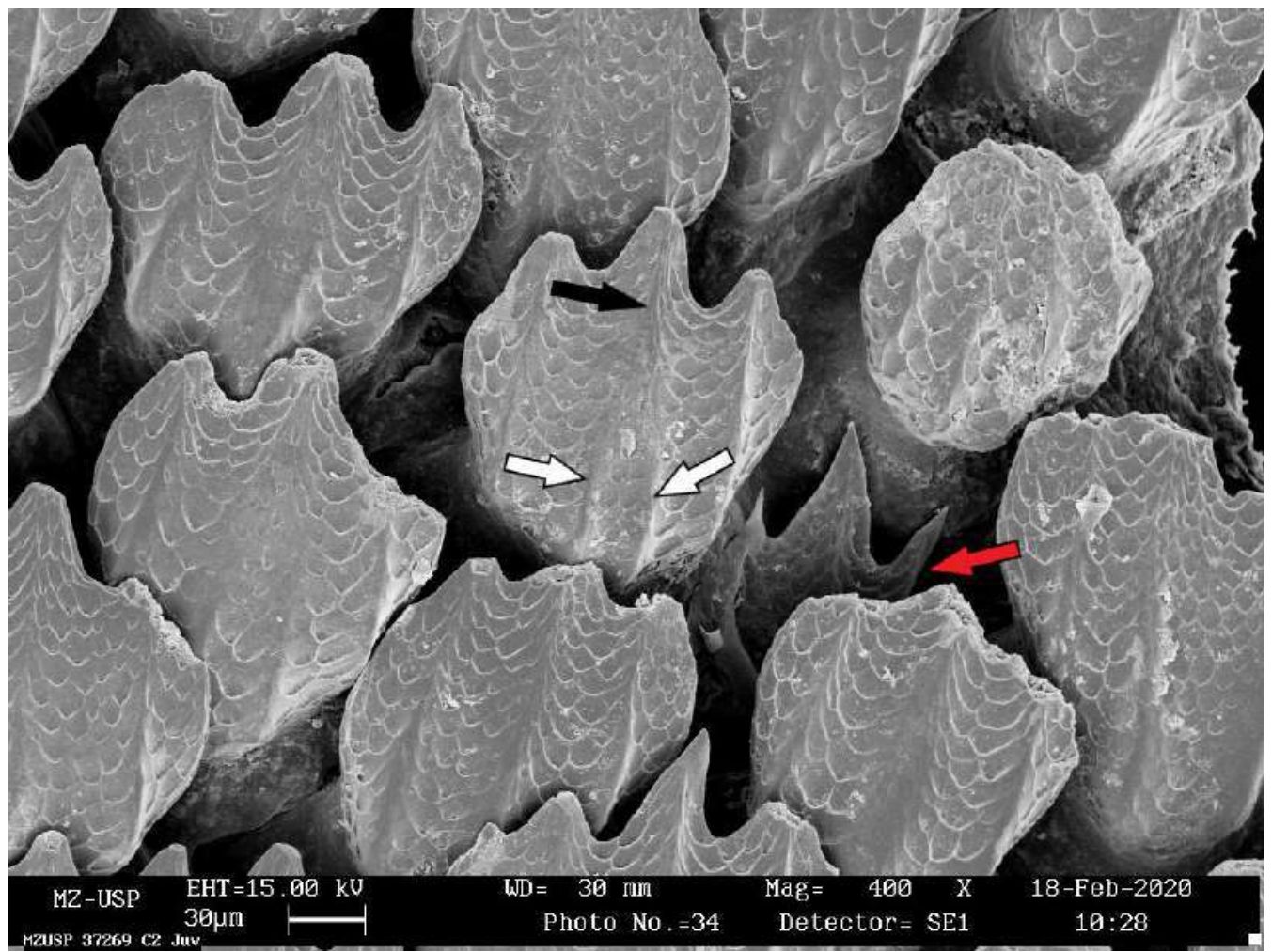

Figure 194. Dermal denticles collected from the section C2 (see fig. 1) of the juvenile Isogomphodon oxyrhynchus (MZUSP 37289). Additional information in the image footer. White arrows indicate the two primordial middle ridges converging posteriorly to the medial ridge, indicated by the black arrow. Red arrow indicates a differentiated dermal denticles.

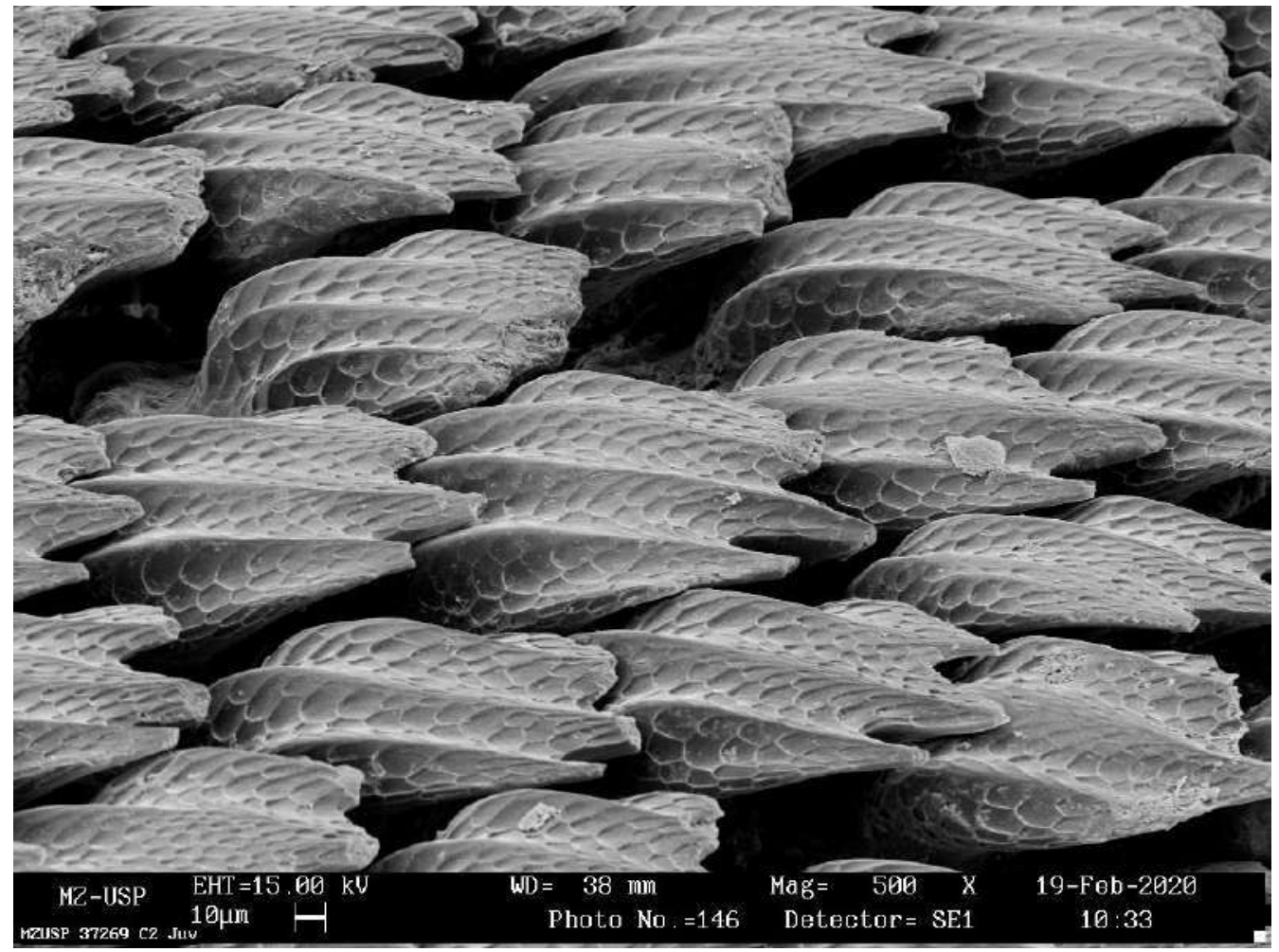

Figure 195. Dermal denticles collected from the section C2 (see fig. 1) of the juvenile Isogomphodon oxyrhynchus (MZUSP 37289). Additional information in the image footer. 


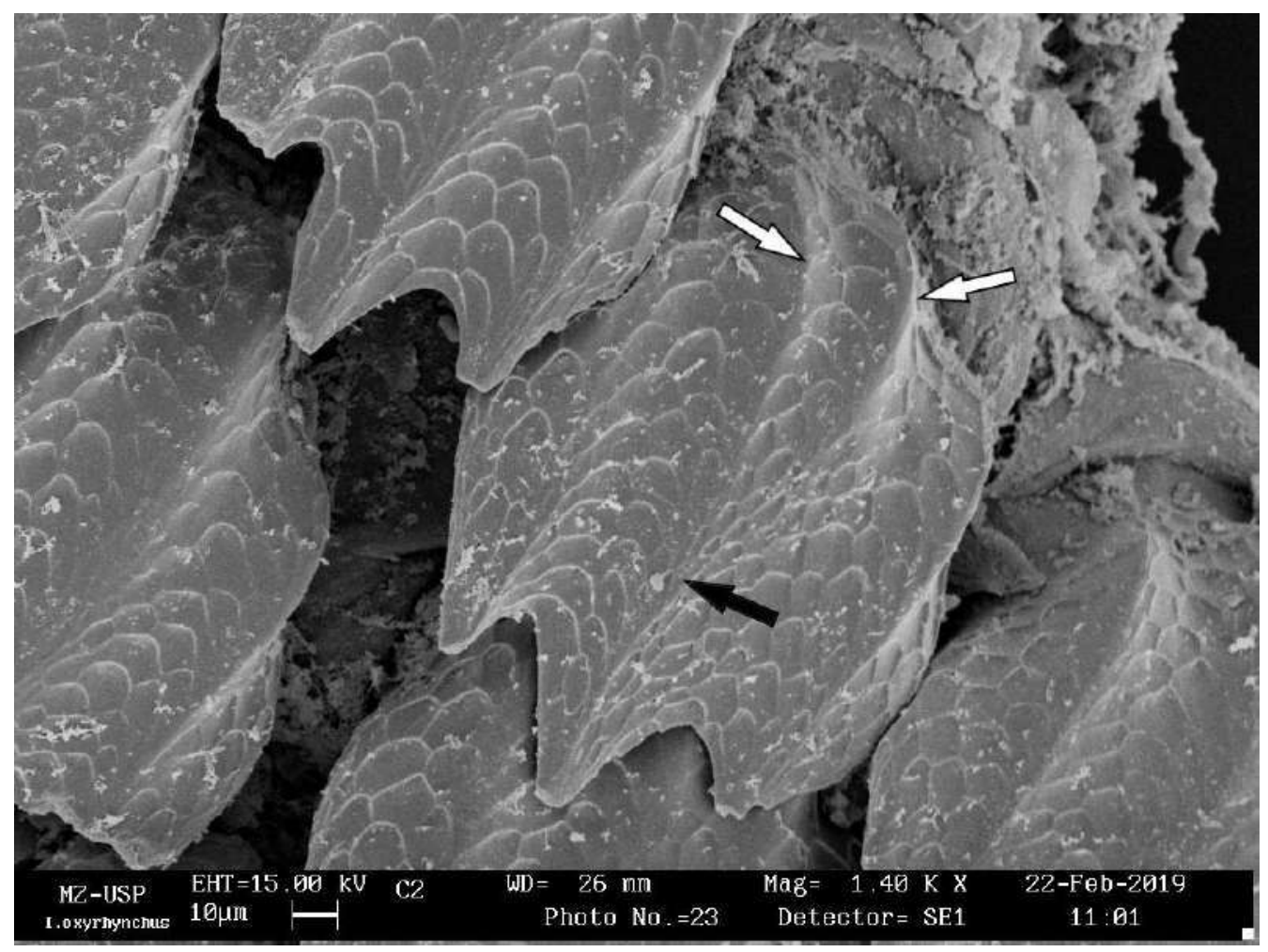

Figure 196. Dermal denticles collected from the section C2 (see fig. 1) of the juvenile Isogomphodon oxyrhynchus (MZUSP 37289). White arrows indicate the two primordial middle ridges converging posteriorly to the medial ridge, indicated by the black arrow. Additional information in the image footer.

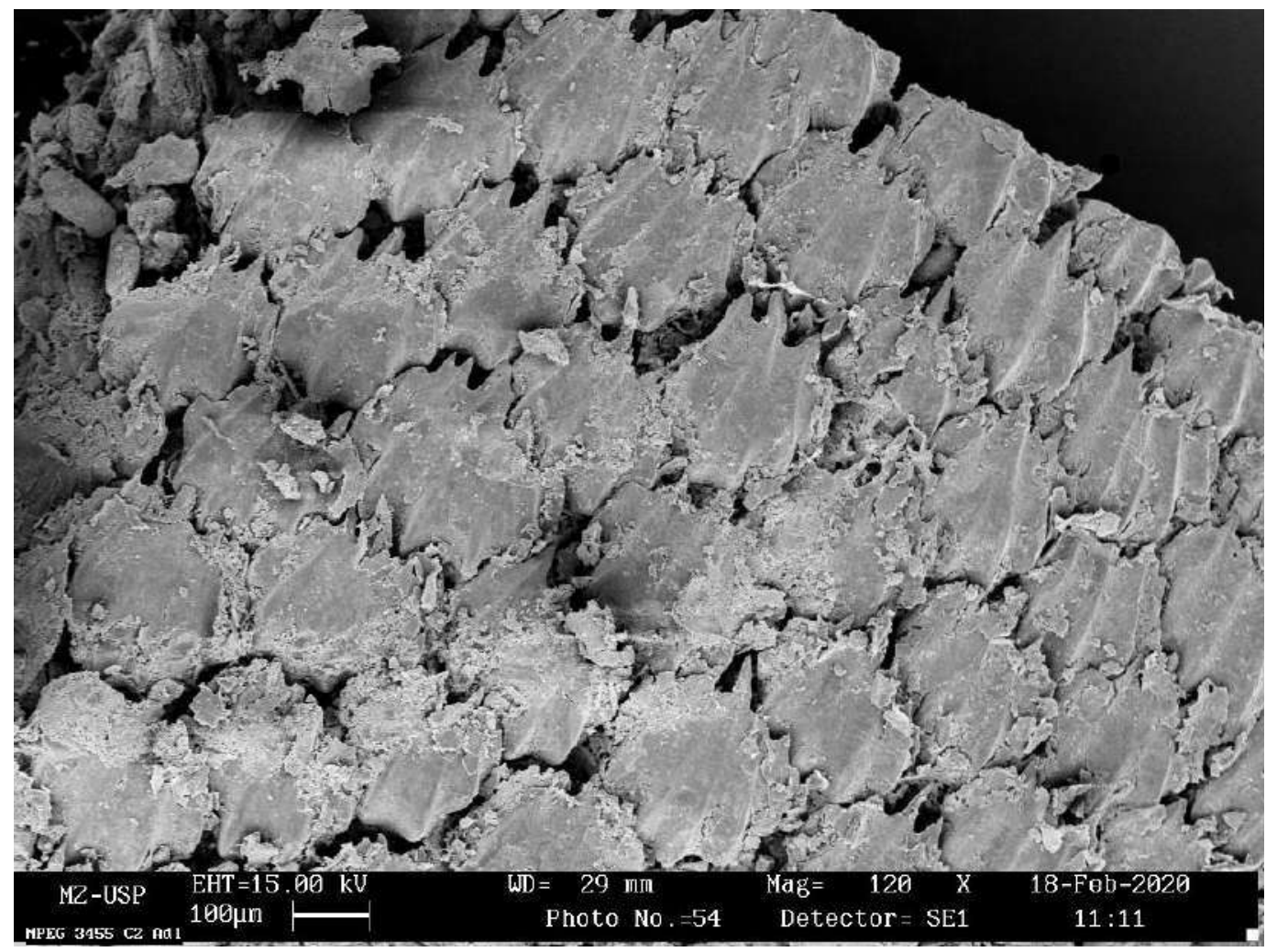

Figure 197. Dermal denticles collected from the section C2 (see fig. 1) of the adult Isogomphodon oxyrhynchus (MPEG 3455). Additional information in the image footer. 


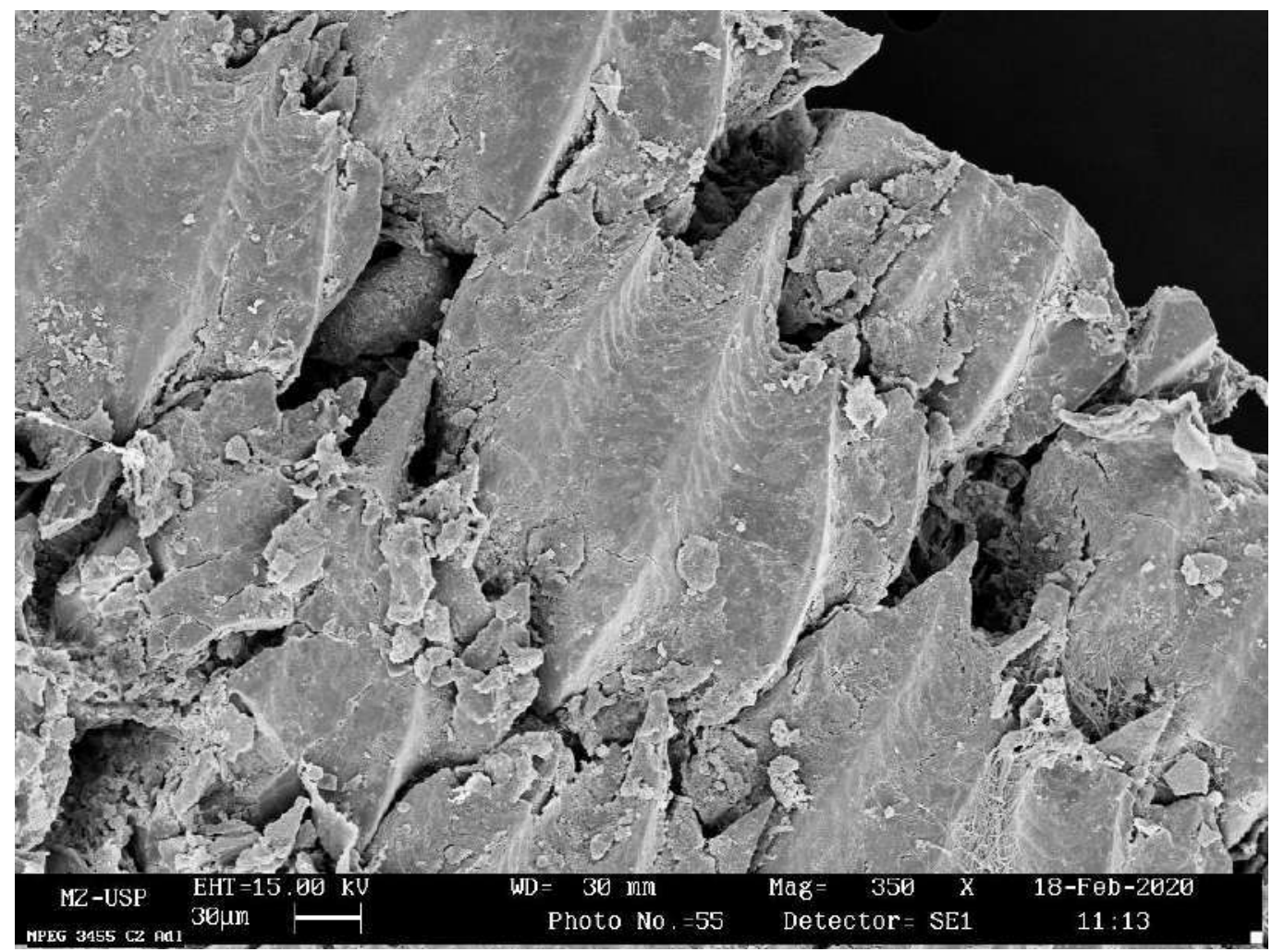

Figure 198. Dermal denticles collected from the section C2 (see fig. 1) of the adult Isogomphodon oxyrhynchus (MPEG 3455). Additional information in the image footer.

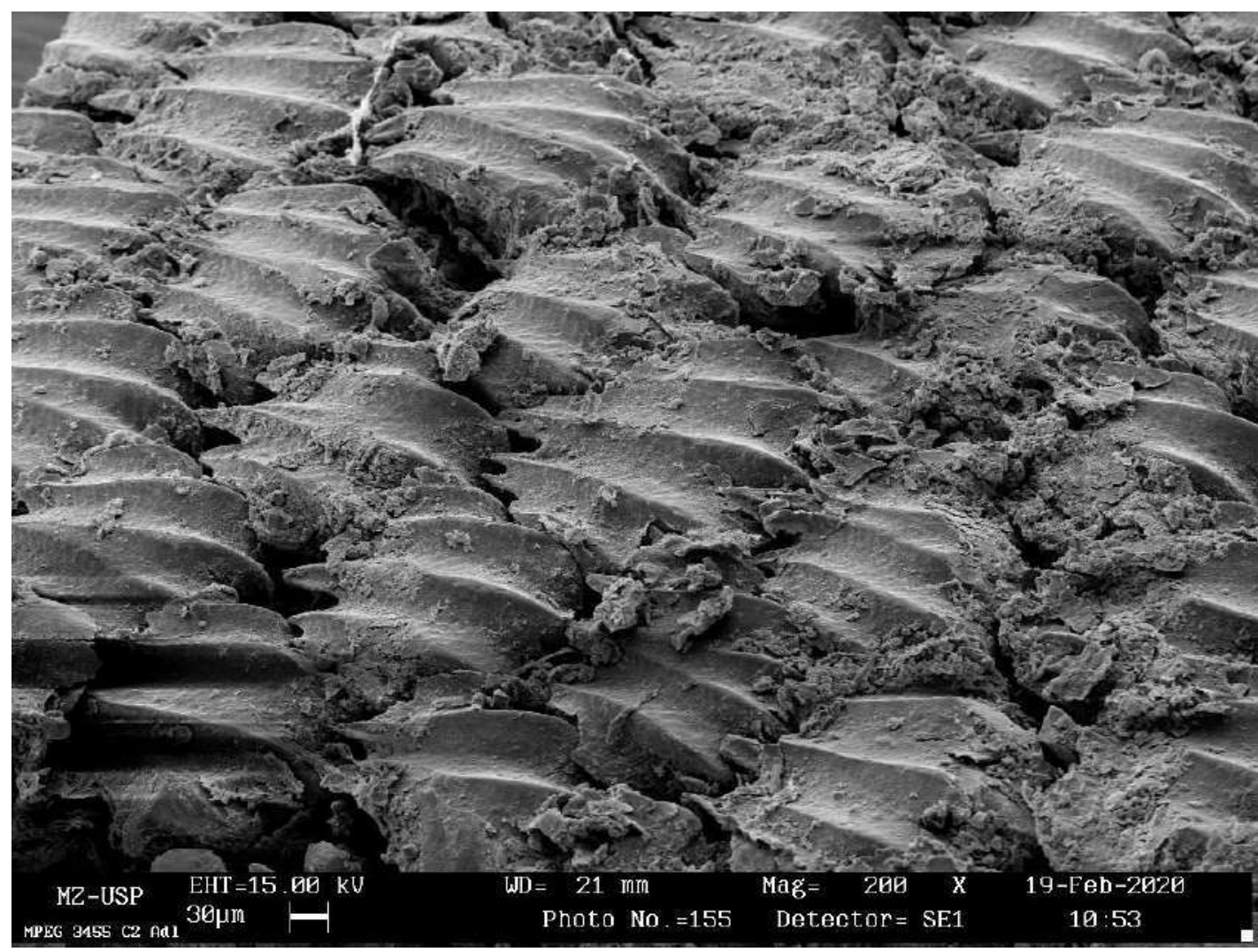

Figure 199. Dermal denticles collected from the section C2 (see fig. 1) of the adult Isogomphodon oxyrhynchus (MPEG 3455). Additional information in the image footer. 


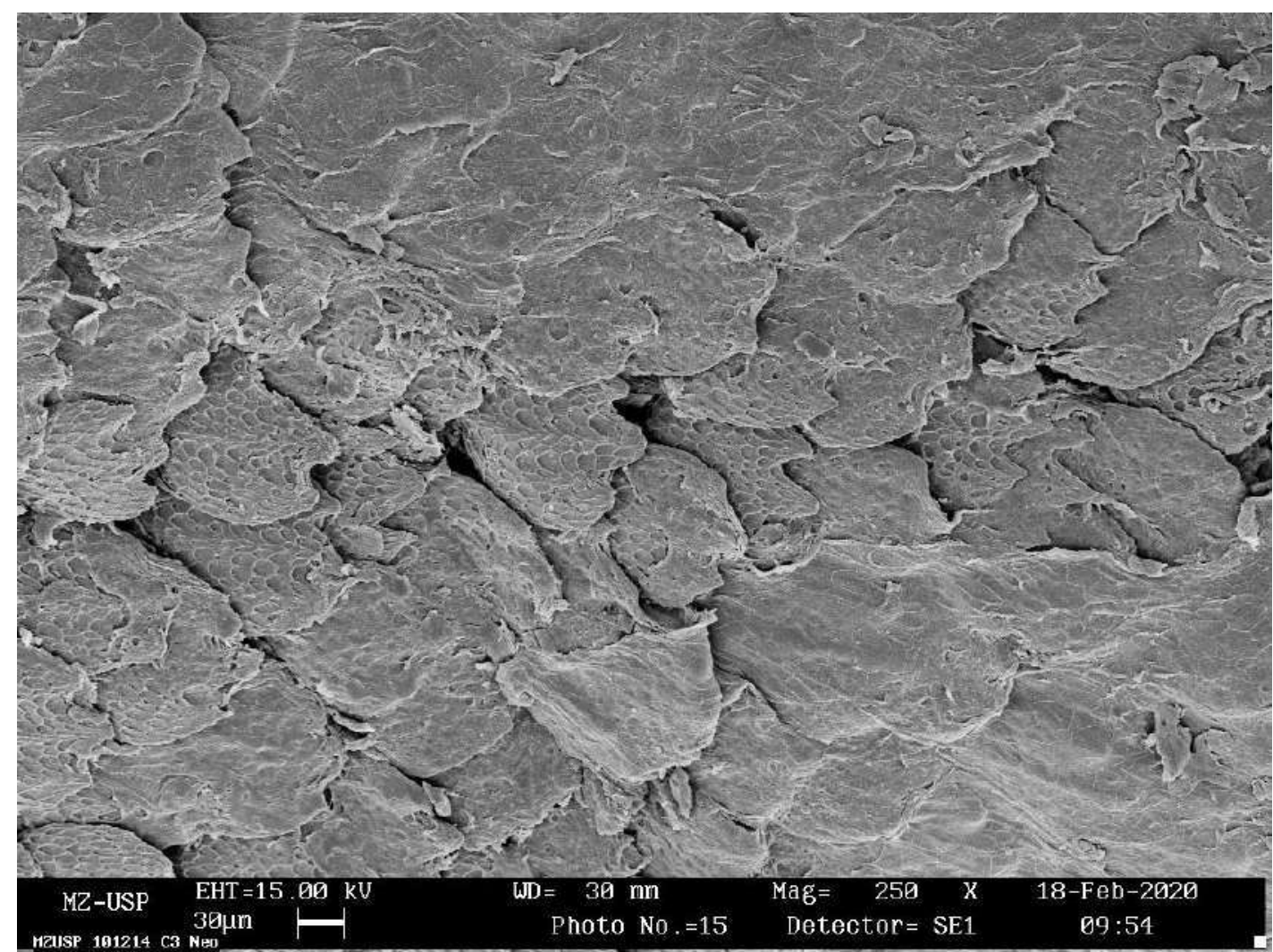

Figure 200. Dermal denticles collected from the section C3 (see fig. 1) of the neonate Isogomphodon oxyrhynchus (MZUSP 101214). Additional information in the image footer.

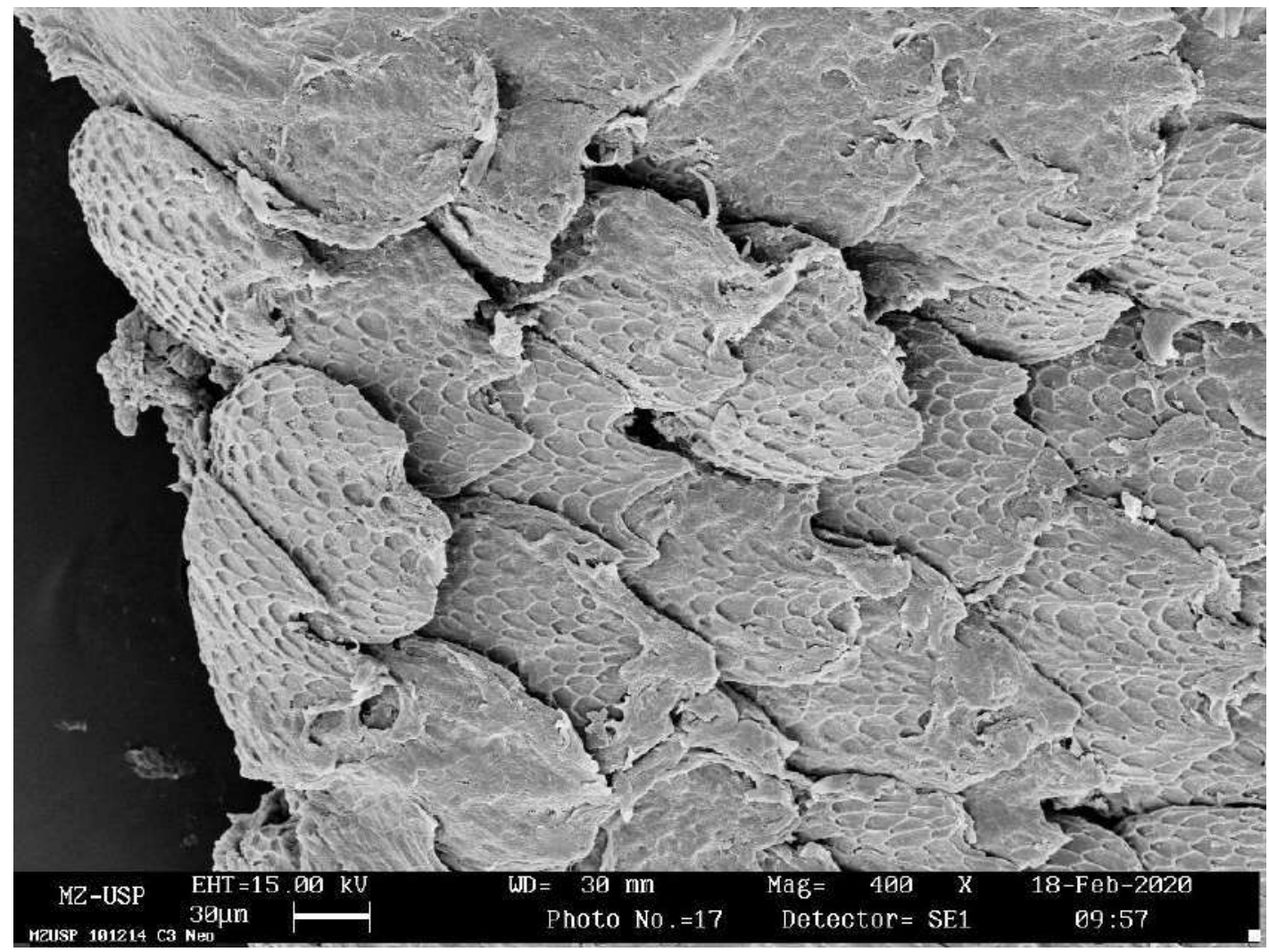

Figure 201. Dermal denticles collected from the section C3 (see fig. 1) of the neonate Isogomphodon oxyrhynchus (MZUSP 101214). Additional information in the image footer. 


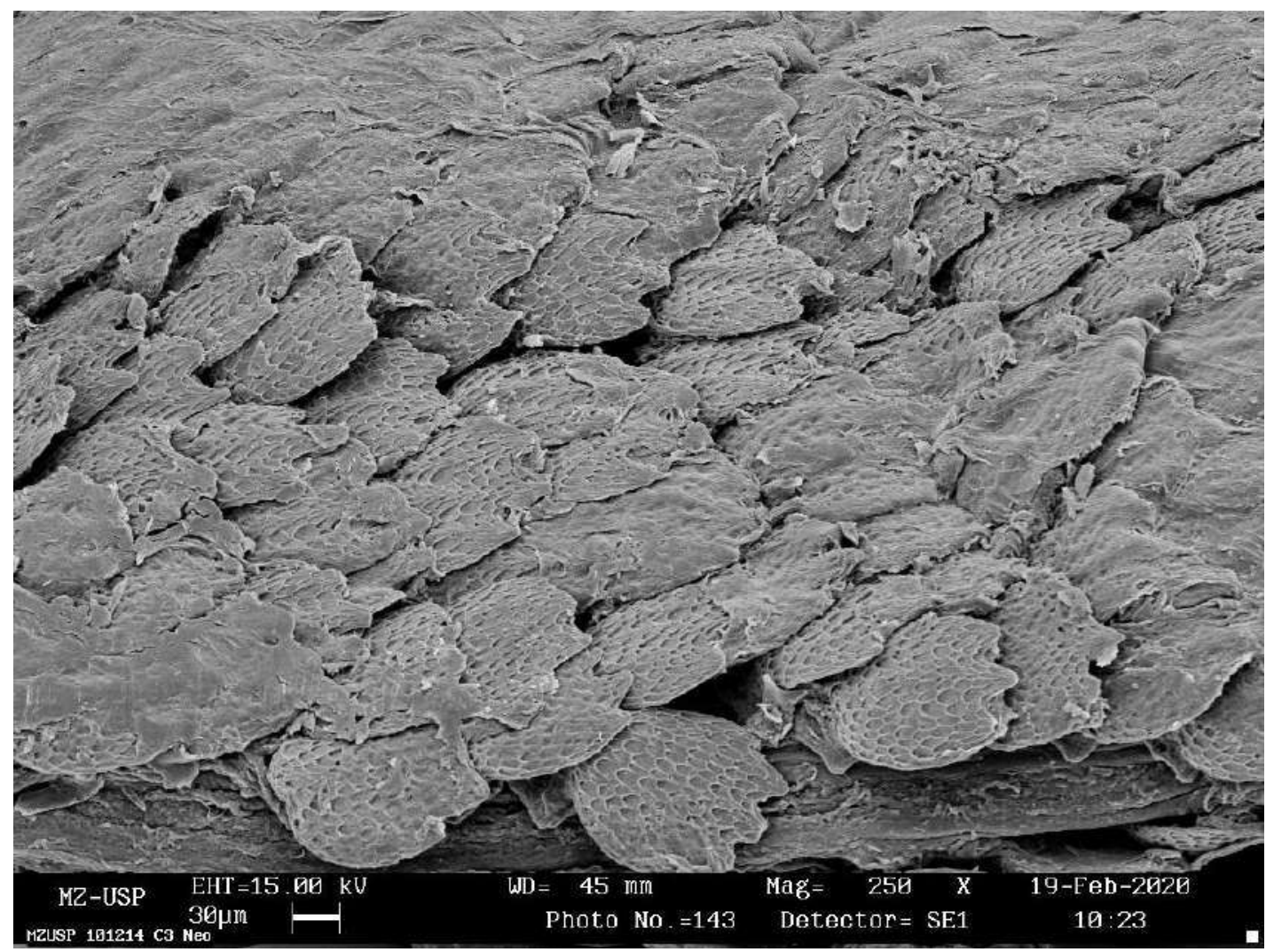

Figure 202. Dermal denticles collected from the section C3 (see fig. 1) of the neonate Isogomphodon oxyrhynchus (MZUSP 101214). Additional information in the image footer.

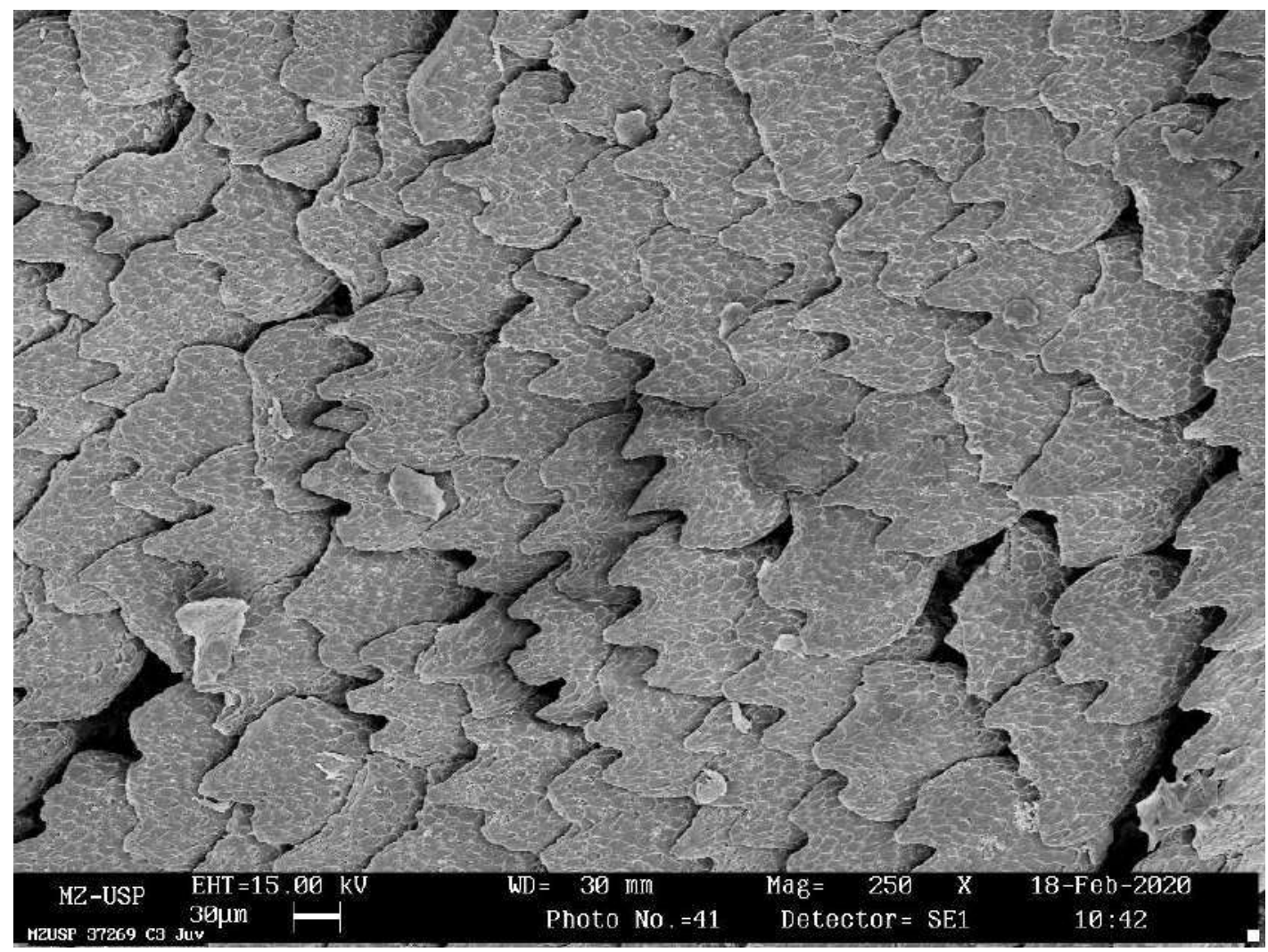

Figure 203. Dermal denticles collected from the section C3 (see fig. 1) of the juvenile Isogomphodon oxyrhynchus (MZUSP 37289). Additional information in the image footer. 


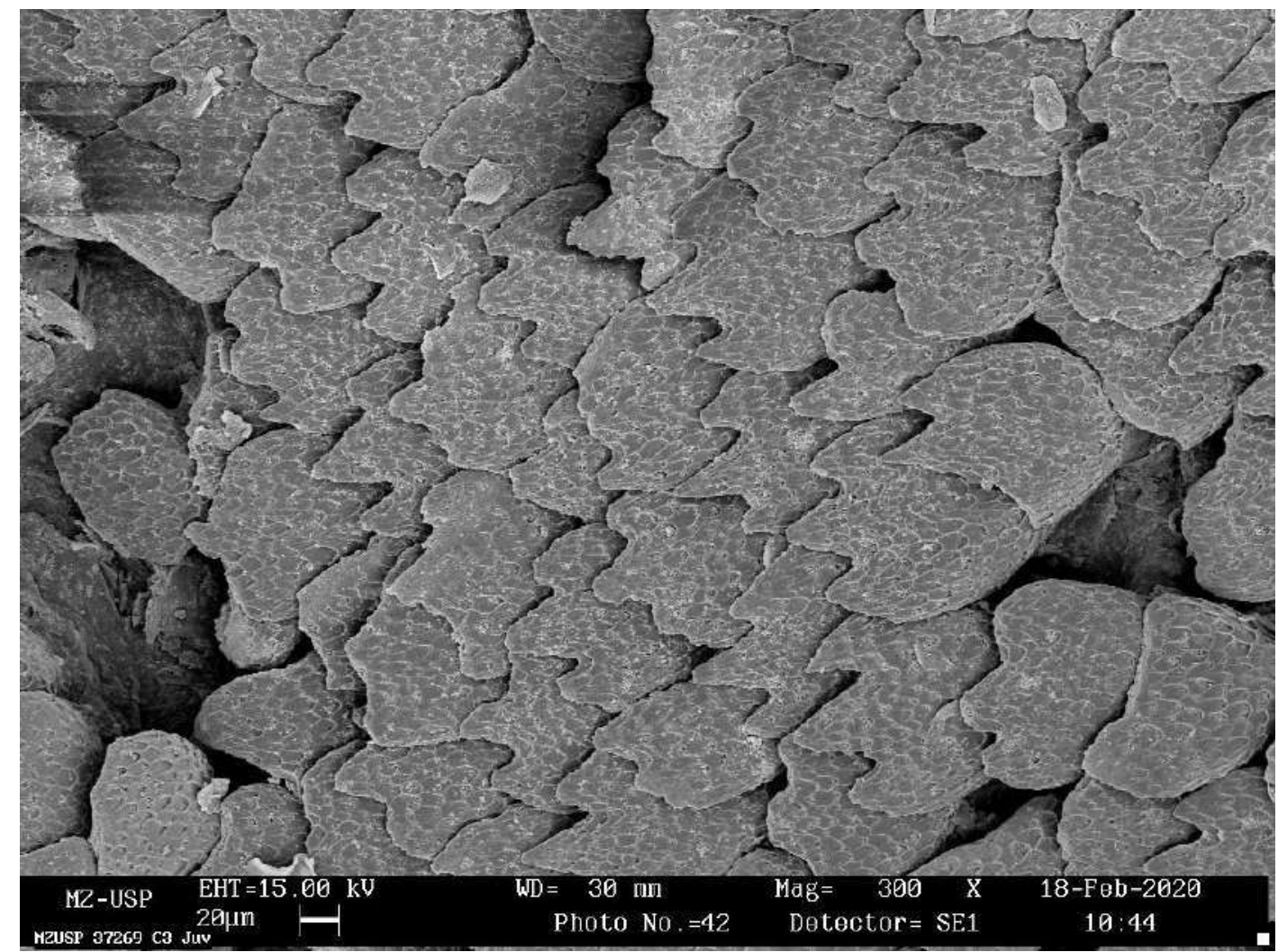

Figure 204. Dermal denticles collected from the section C3 (see fig. 1) of the juvenile Isogomphodon oxyrhynchus (MZUSP 37289). Additional information in the image footer.

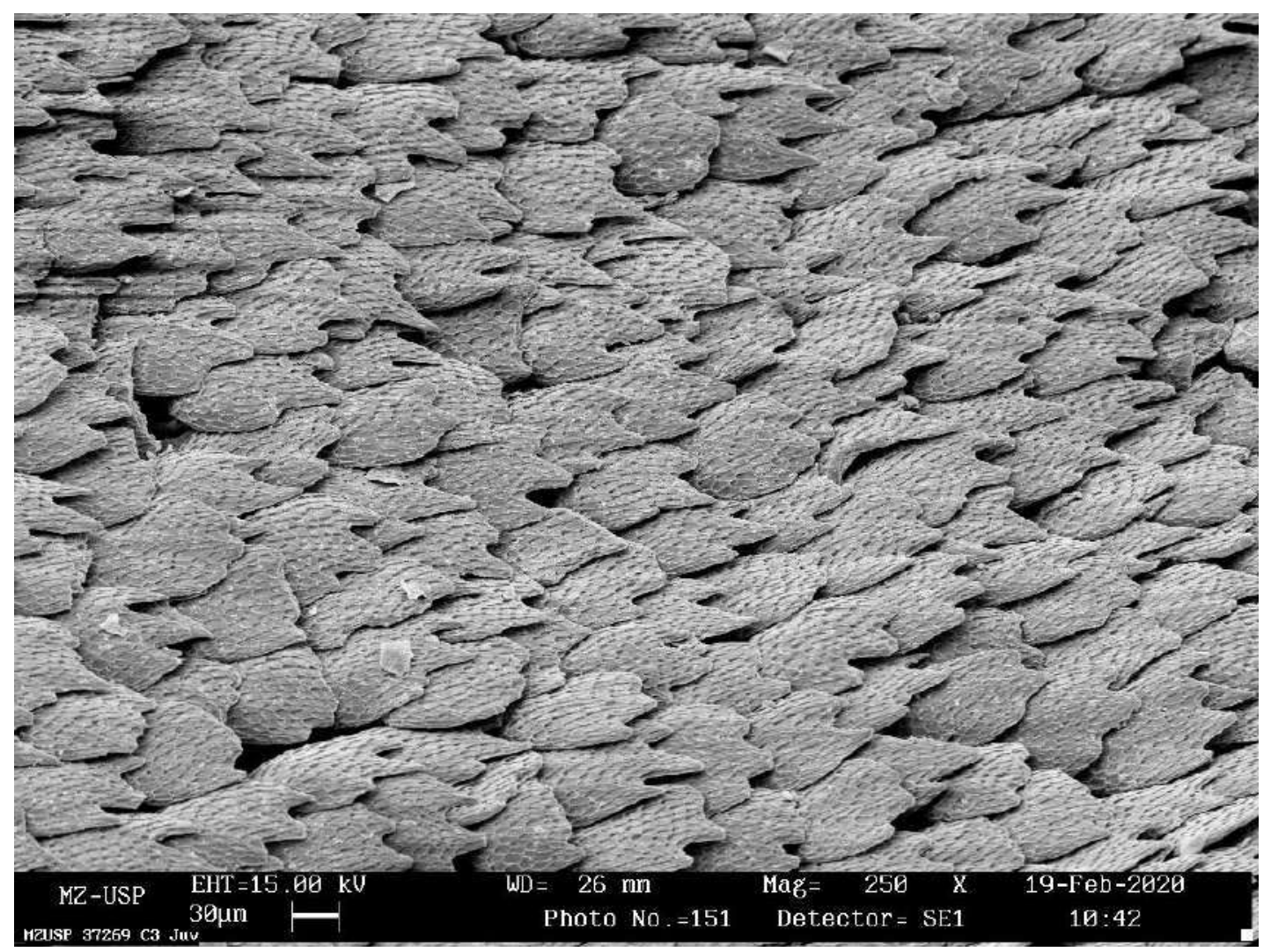

Figure 205. Dermal denticles collected from the section C3 (see fig. 1) of the juvenile Isogomphodon oxyrhynchus (MZUSP 37289). Additional information in the image footer. 


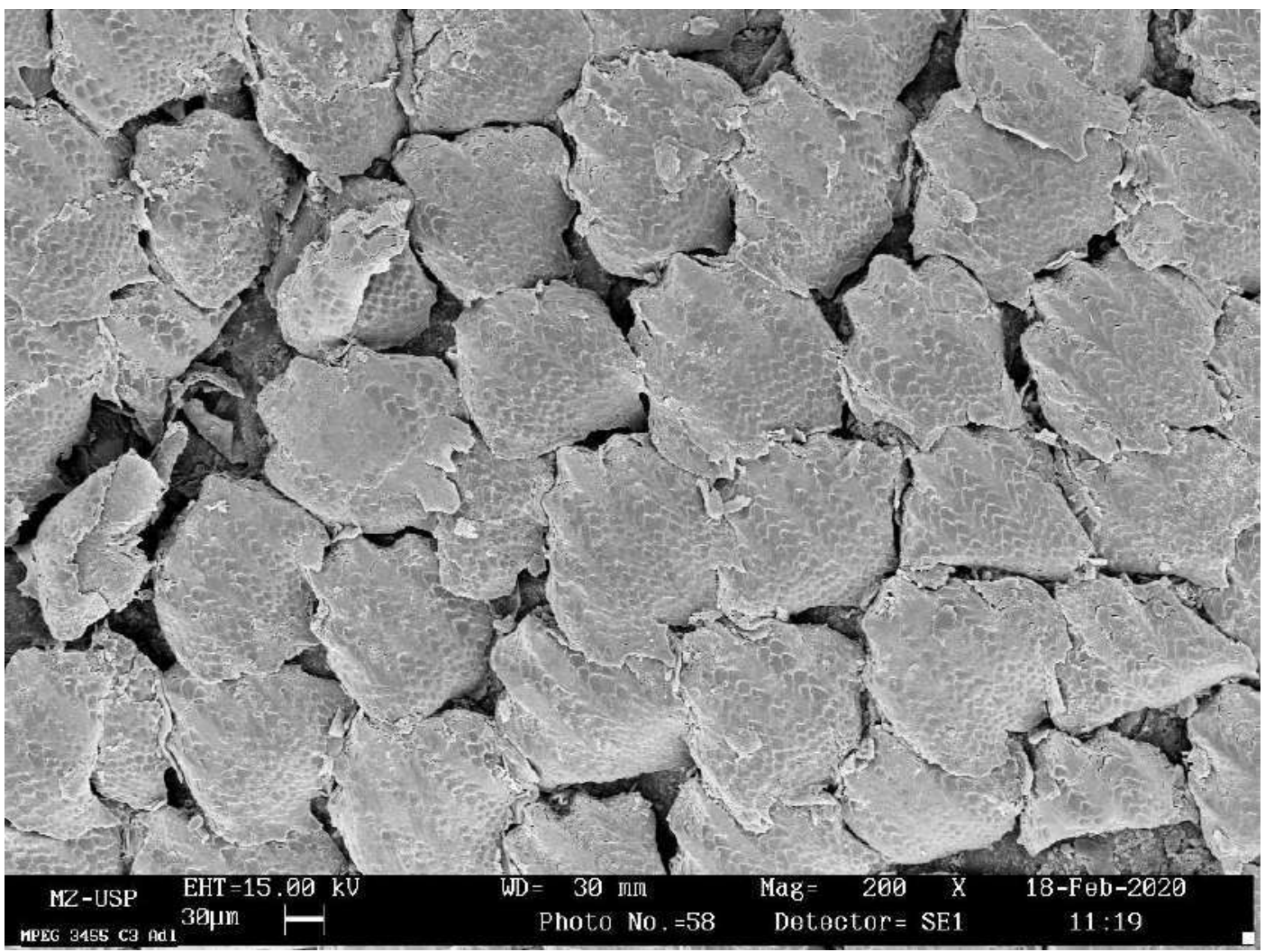

Figure 206. Dermal denticles collected from the section C3 (see fig. 1) of the adult Isogomphodon oxyrhynchus (MPEG 3455). Additional information in the image footer.

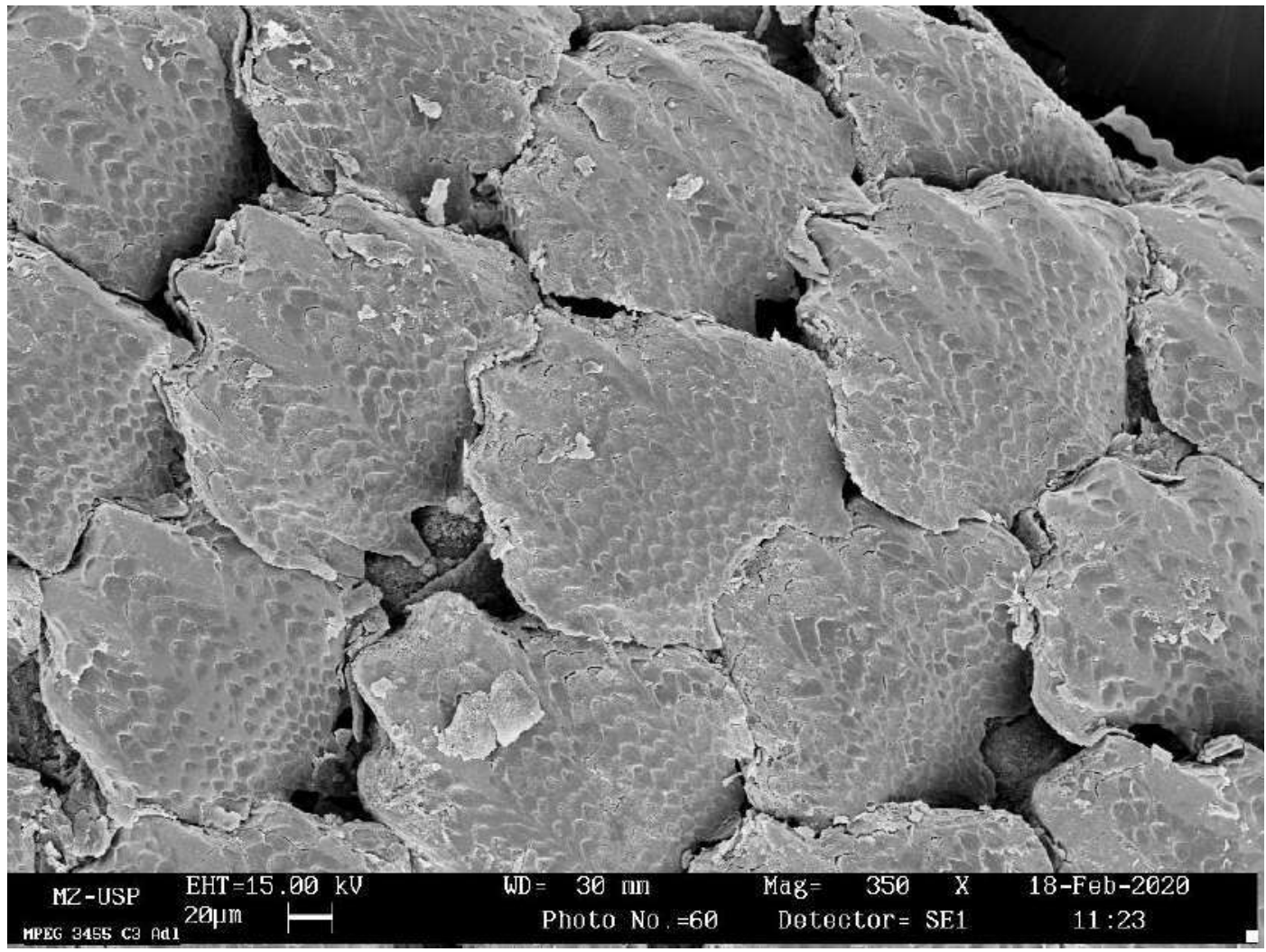

Figure 207. Dermal denticles collected from the section C3 (see fig. 1) of the adult Isogomphodon oxyrhynchus (MPEG 3455). Additional information in the image footer. 


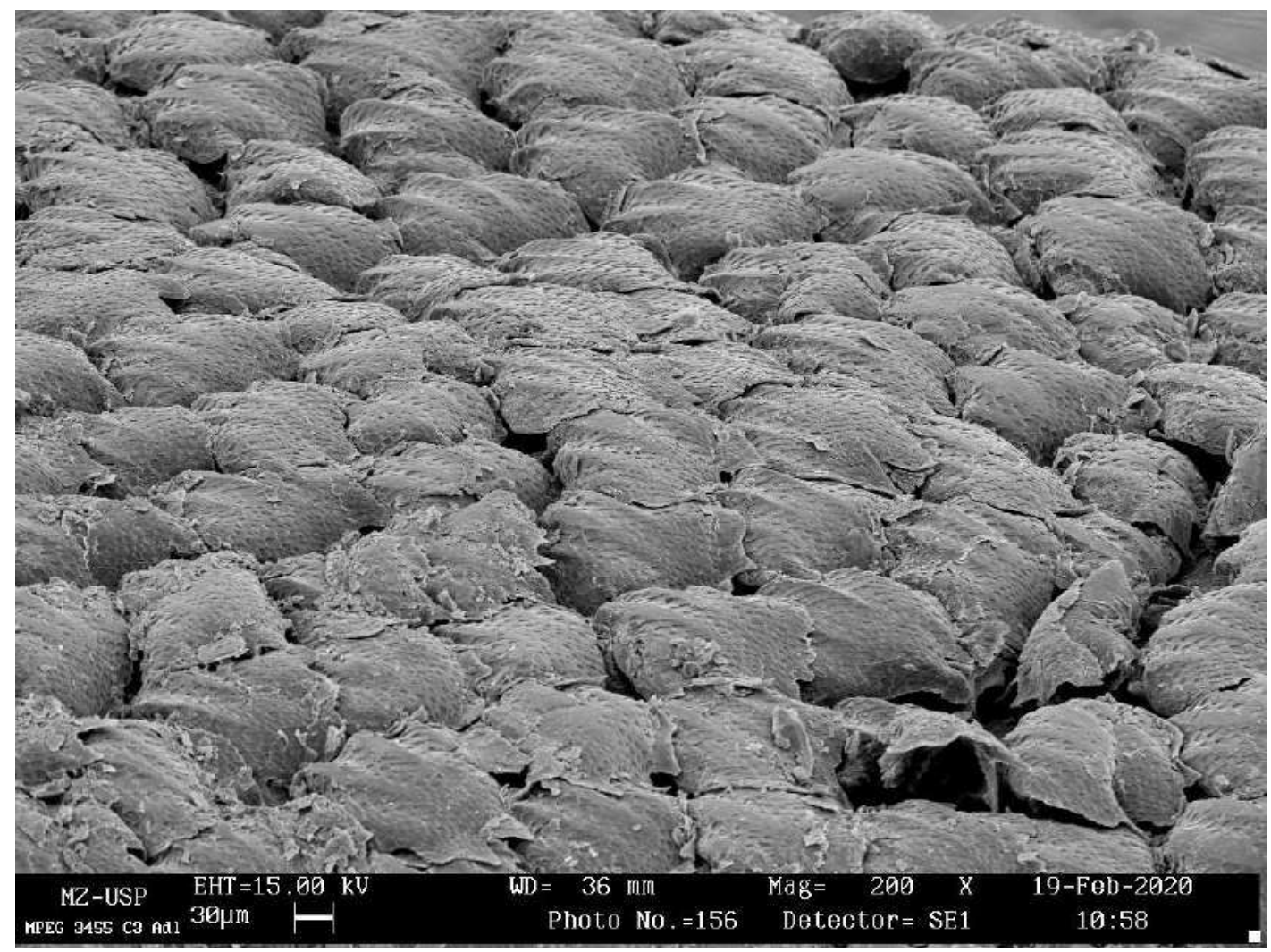

Figure 208. Dermal denticles collected from the section C3 (see fig. 1) of the adult Isogomphodon oxyrhynchus (MPEG 3455). Additional information in the image footer.

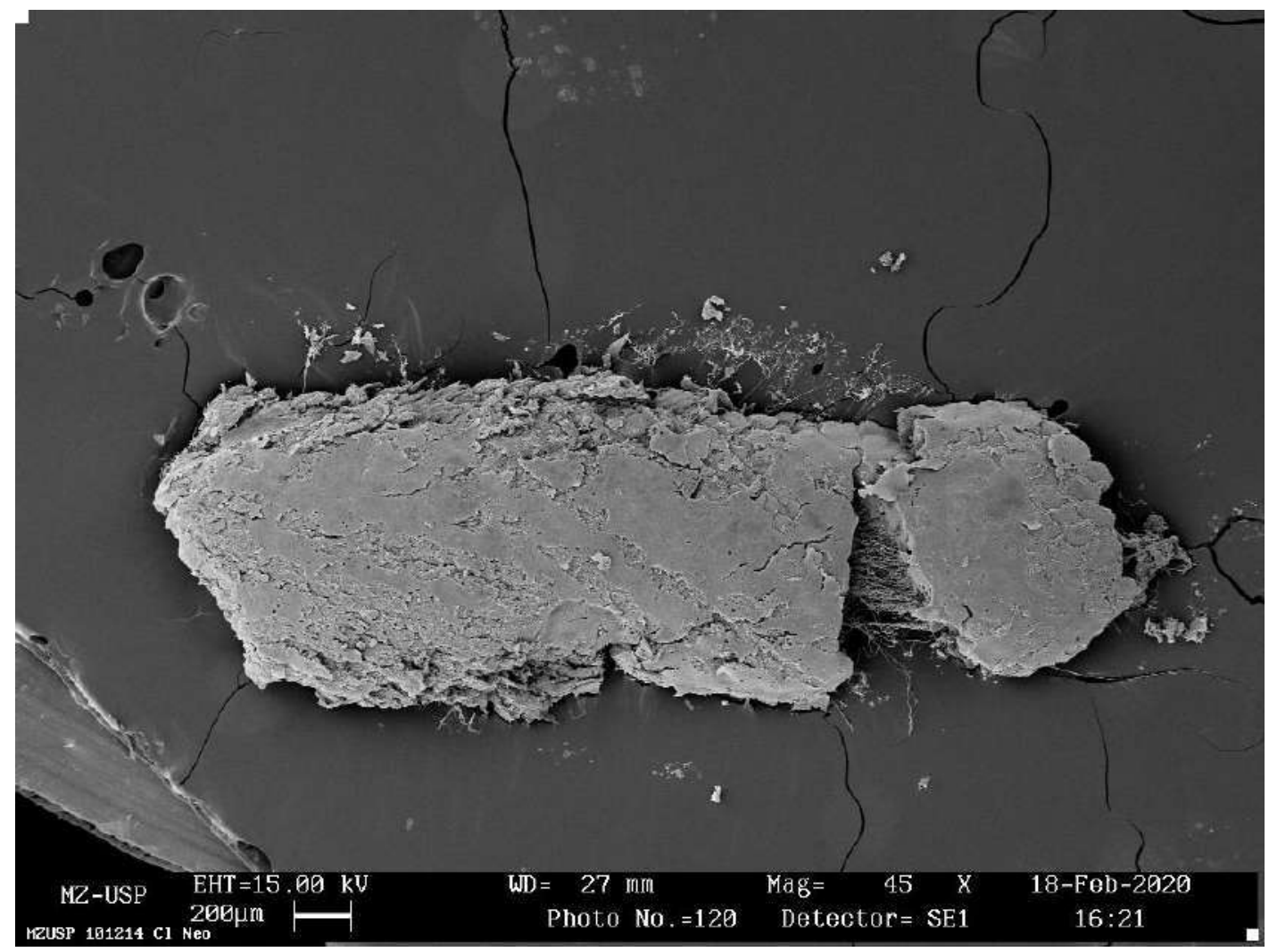

Figure 209. Dermal denticles collected from the section CL (see fig. 1) of the neonate Isogomphodon oxyrhynchus (MZUSP 101214). Additional information in the image footer. 


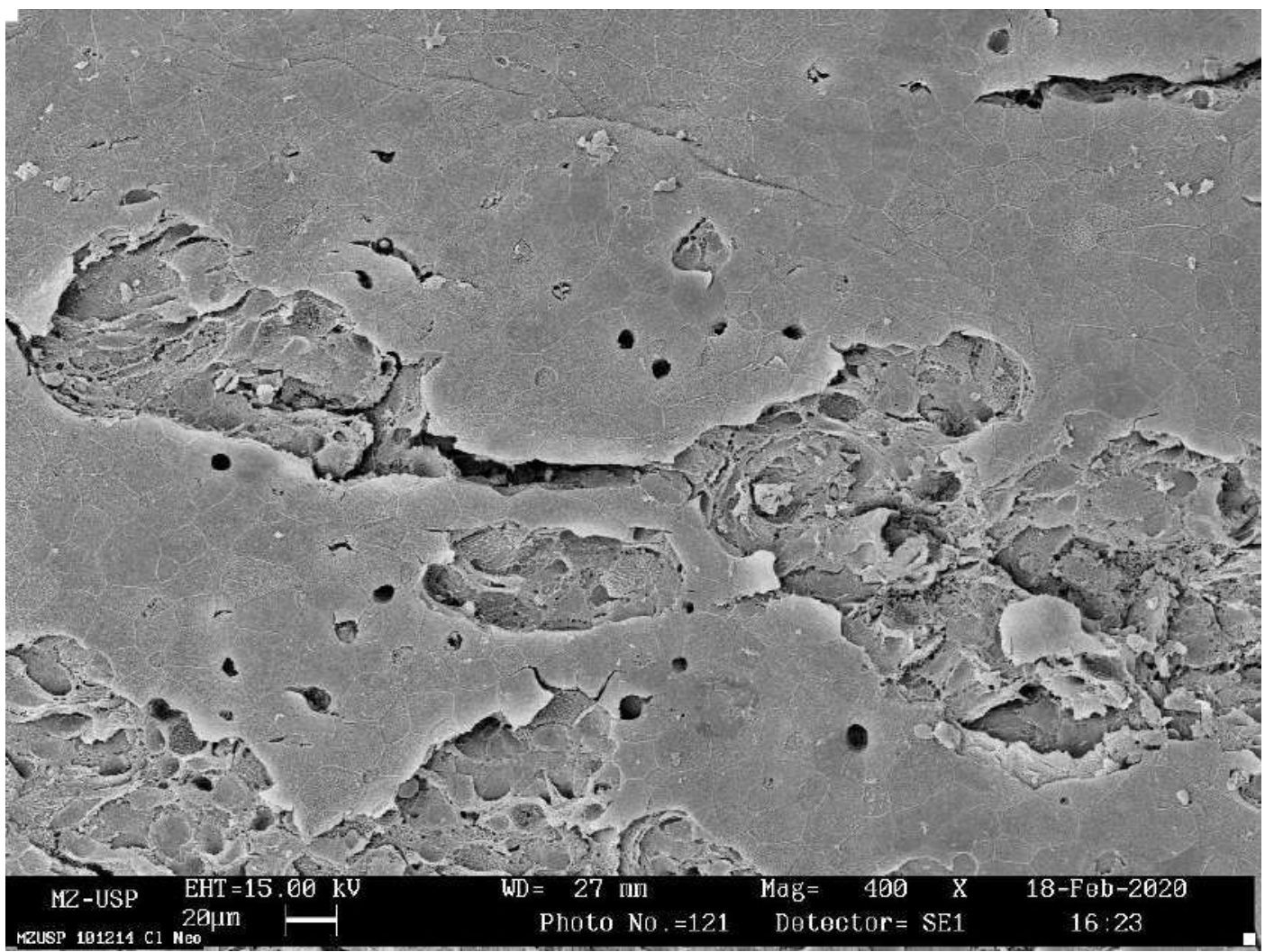

Figure 210. Dermal denticles collected from the section CL (see fig. 1) of the neonate Isogomphodon oxyrhynchus (MZUSP 101214). Additional information in the image footer.

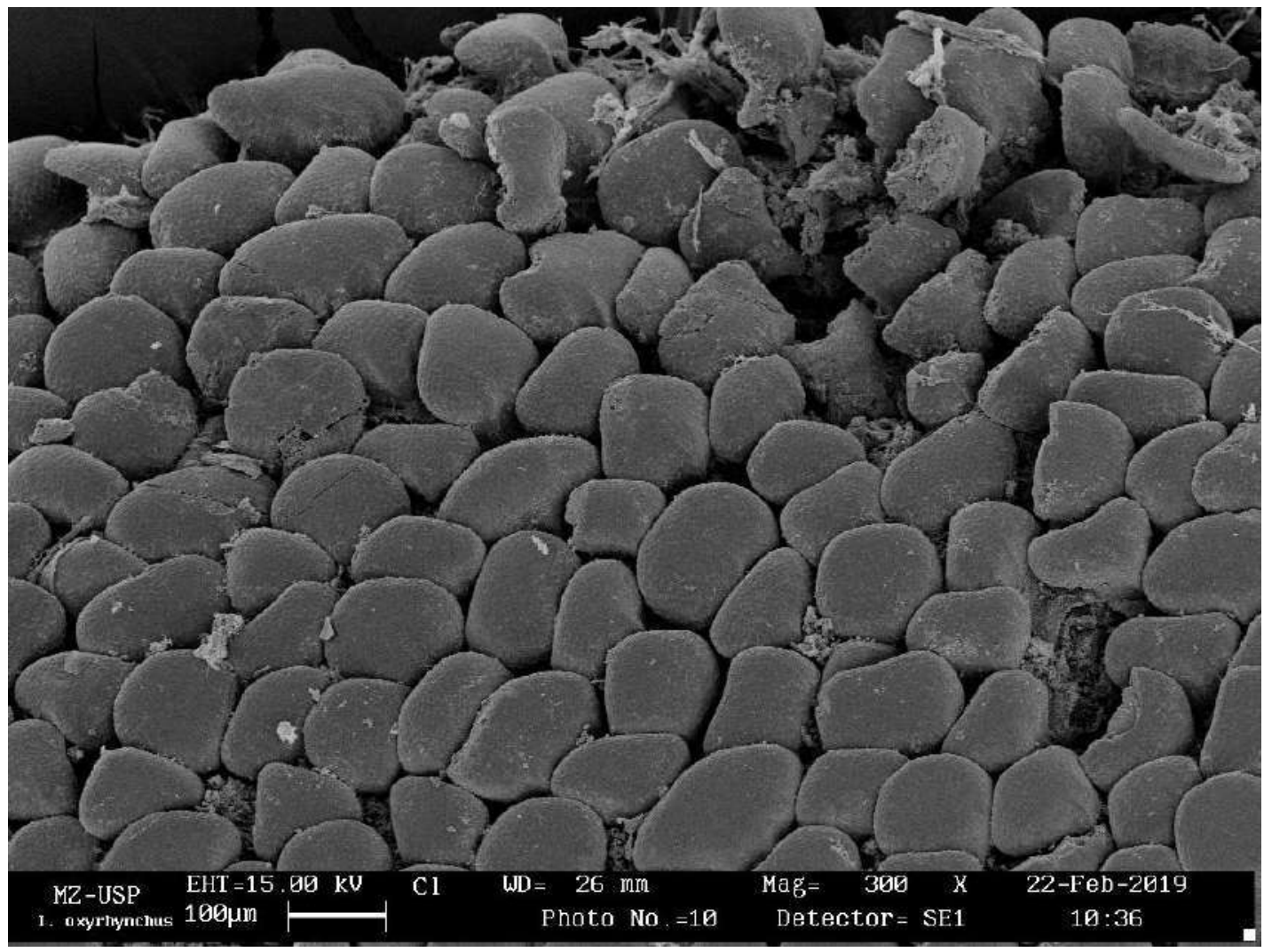

Figure 211. Dermal denticles collected from the section CL (see fig. 1) of the juvenile Isogomphodon oxyrhynchus (MZUSP 37289). Additional information in the image footer. 


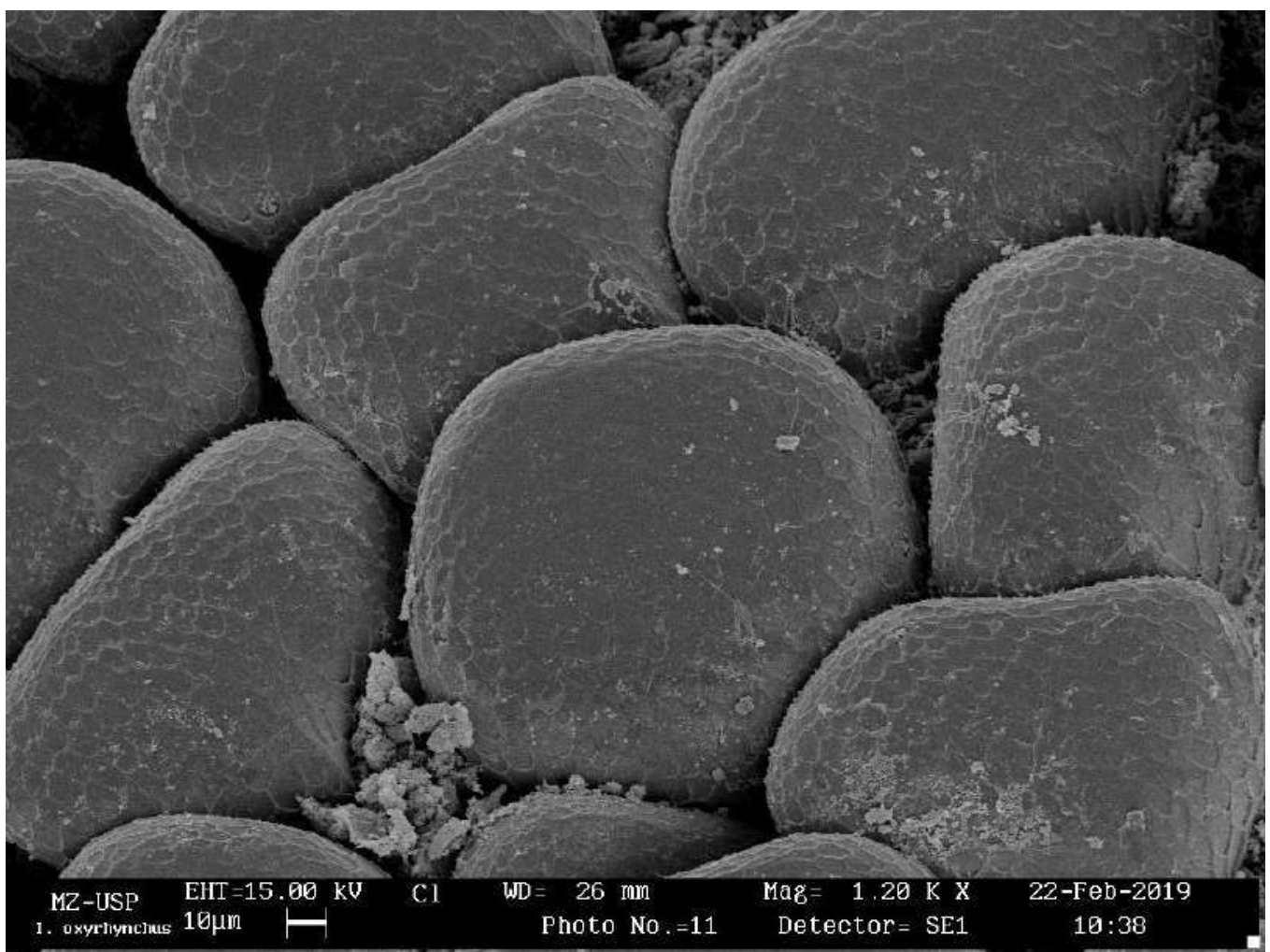

Figure 212. Dermal denticles collected from the section CL (see fig. 1) of the juvenile Isogomphodon oxyrhynchus (MZUSP 37289). Additional information in the image footer.

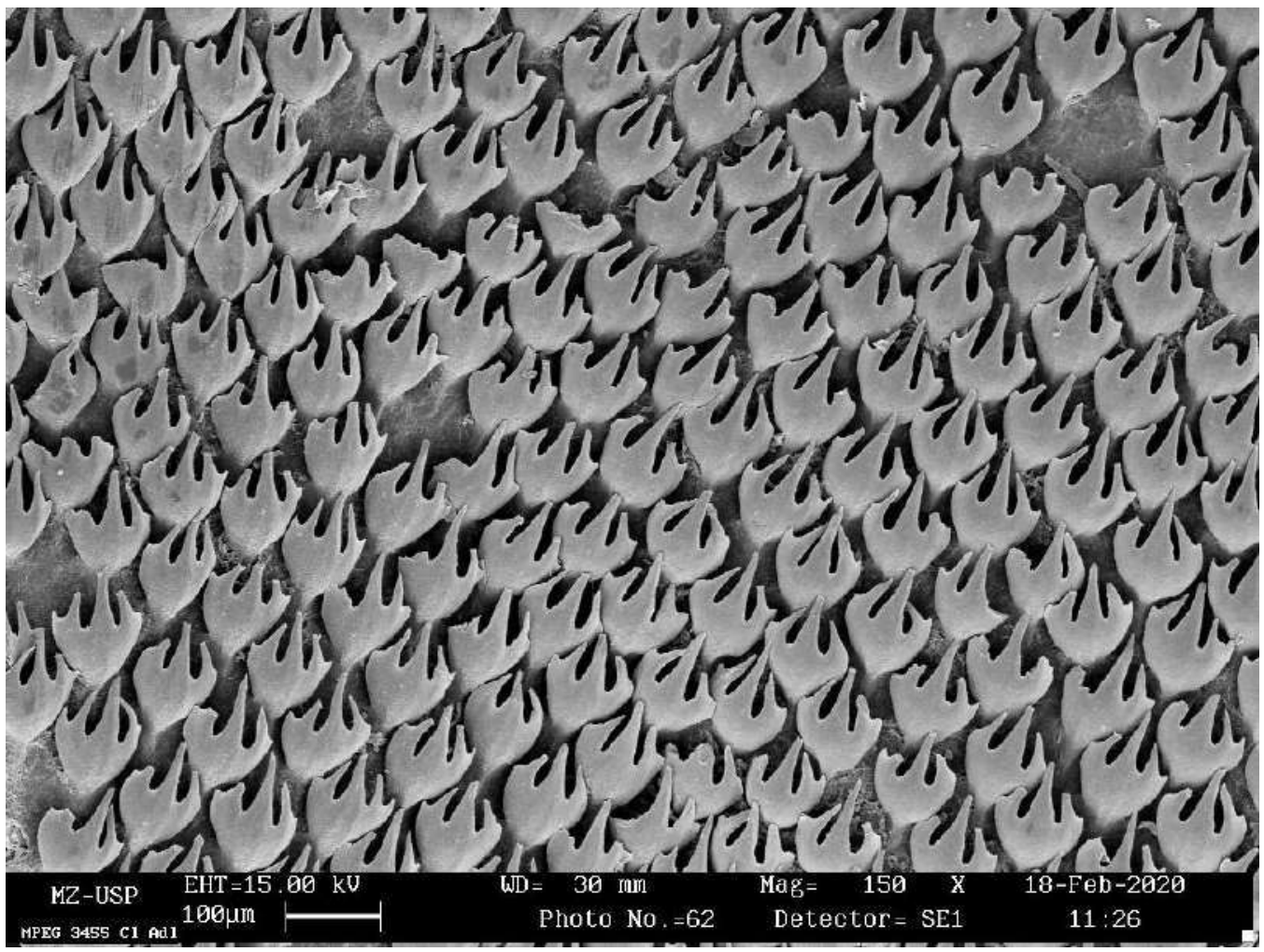

Figure 213. Dermal denticles collected from the section CL (see fig. 1) of the adult Isogomphodon oxyrhynchus (MPEG 3455). The sample on this specimen was collected from the anterior portion of the clasper. Additional information in the image footer. 


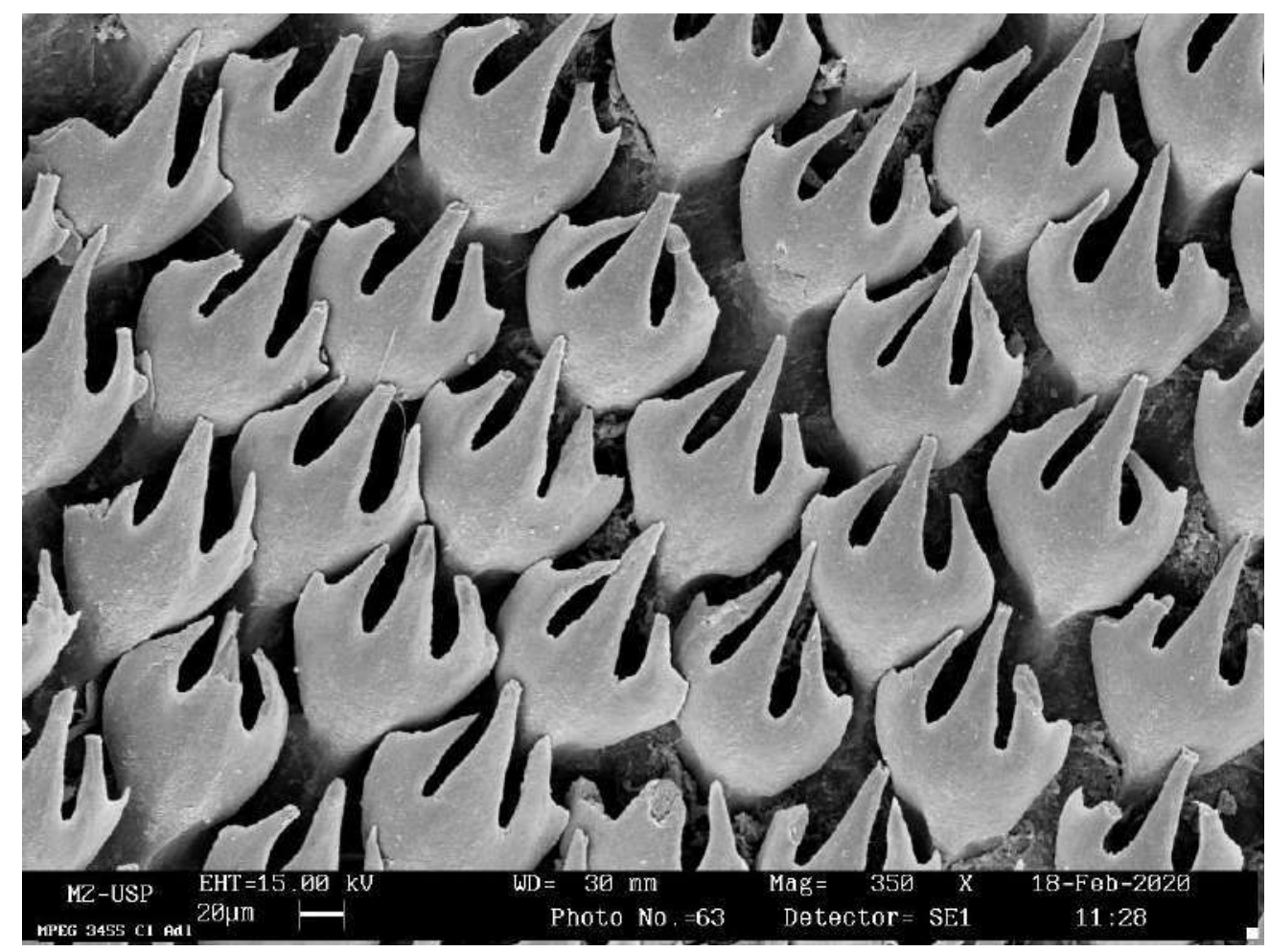

Figure 214. Dermal denticles collected from the section CL (see fig. 1) of the adult Isogomphodon oxyrhynchus (MPEG 3455). The sample on this specimen was collected from the anterior portion of the clasper. Additional information in the image footer.

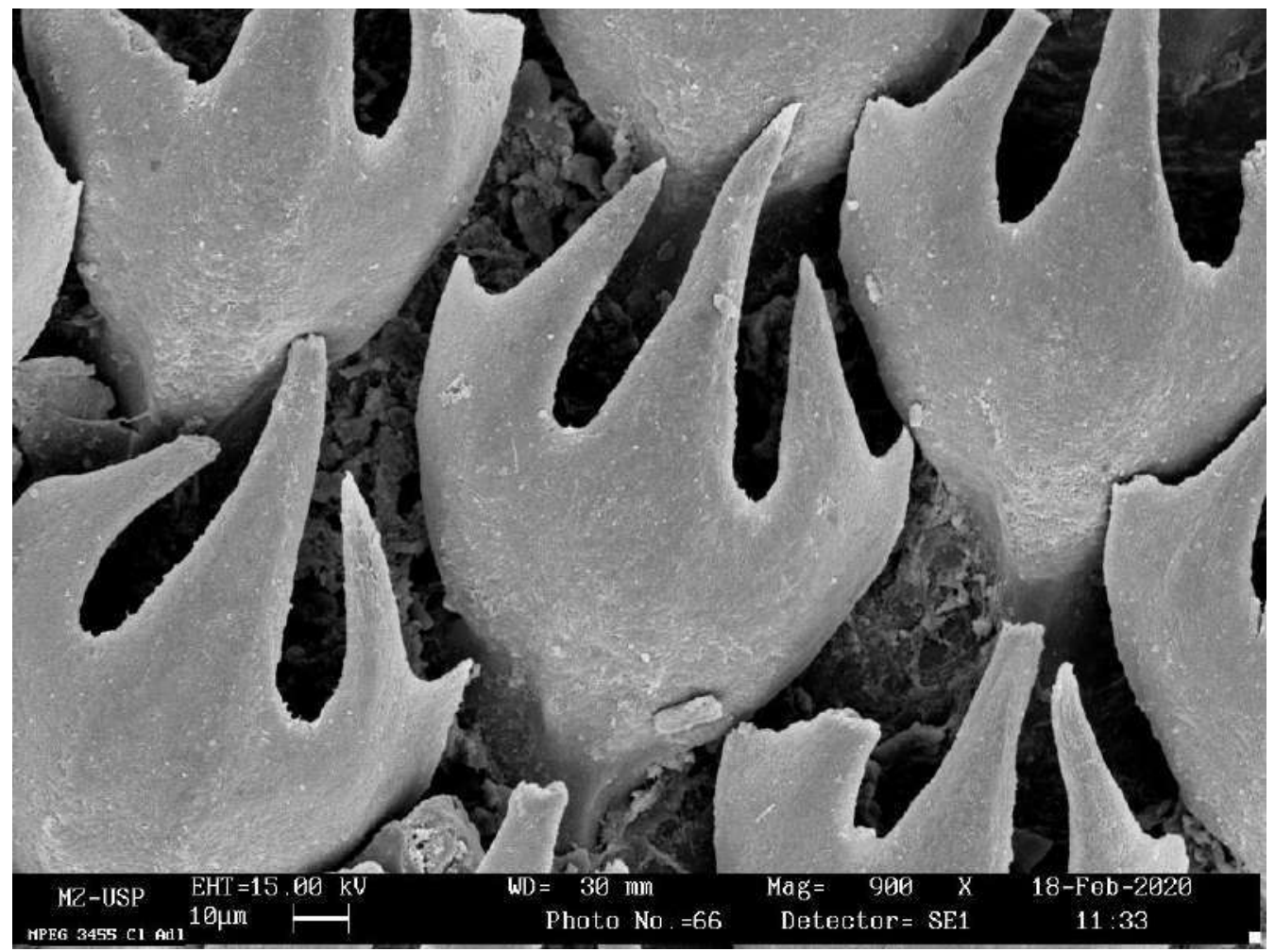

Figure 215. Dermal denticles collected from the section CL (see fig. 1) of the adult Isogomphodon oxyrhynchus (MPEG 3455). The sample on this specimen was collected from the anterior portion of the clasper. Additional information in the image footer. 


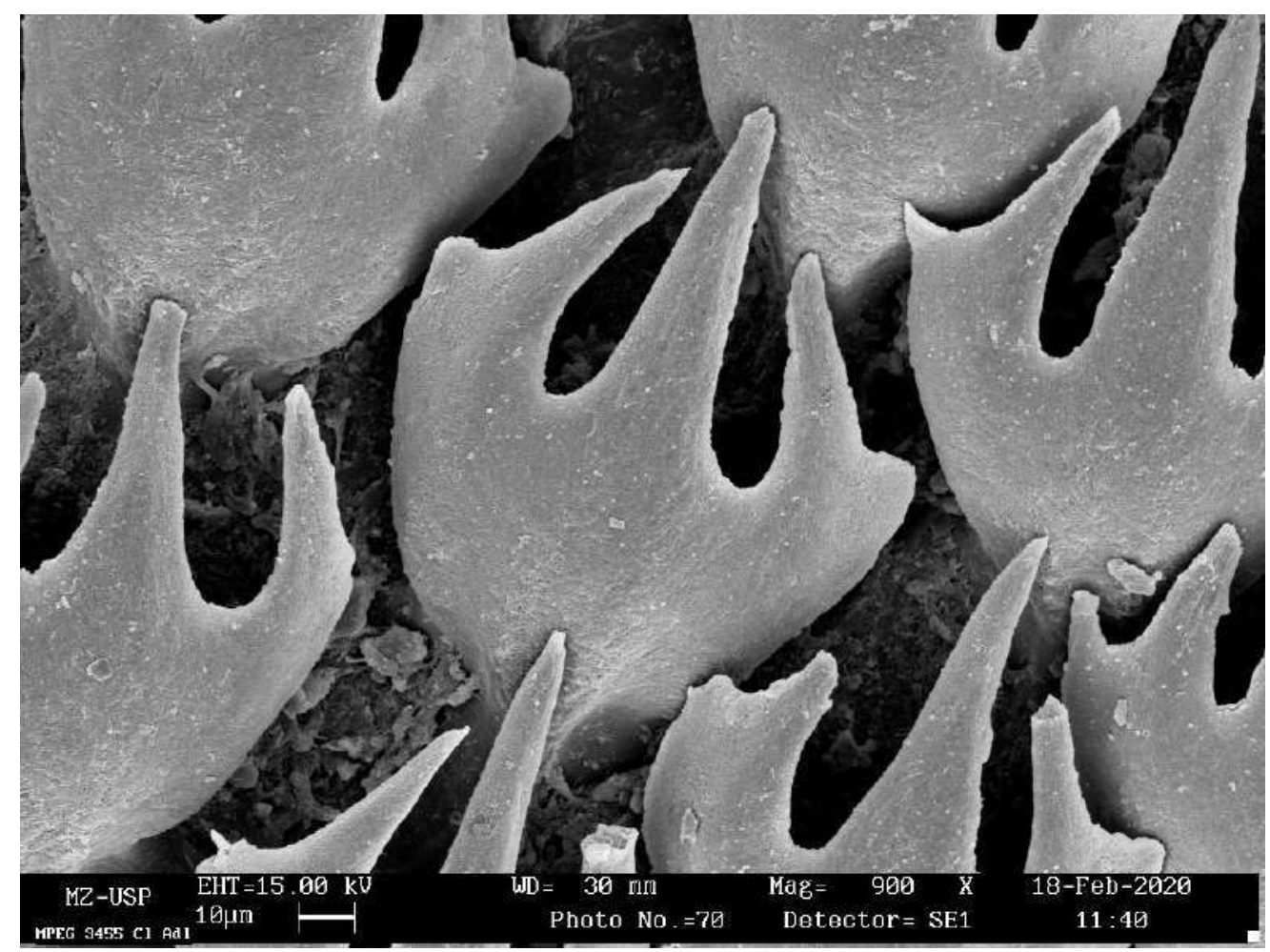

Figure 216. Dermal denticles collected from the section CL (see fig. 1) of the adult Isogomphodon oxyrhynchus (MPEG 3455). The sample on this specimen was collected from the anterior portion of the clasper. Additional information in the image footer.

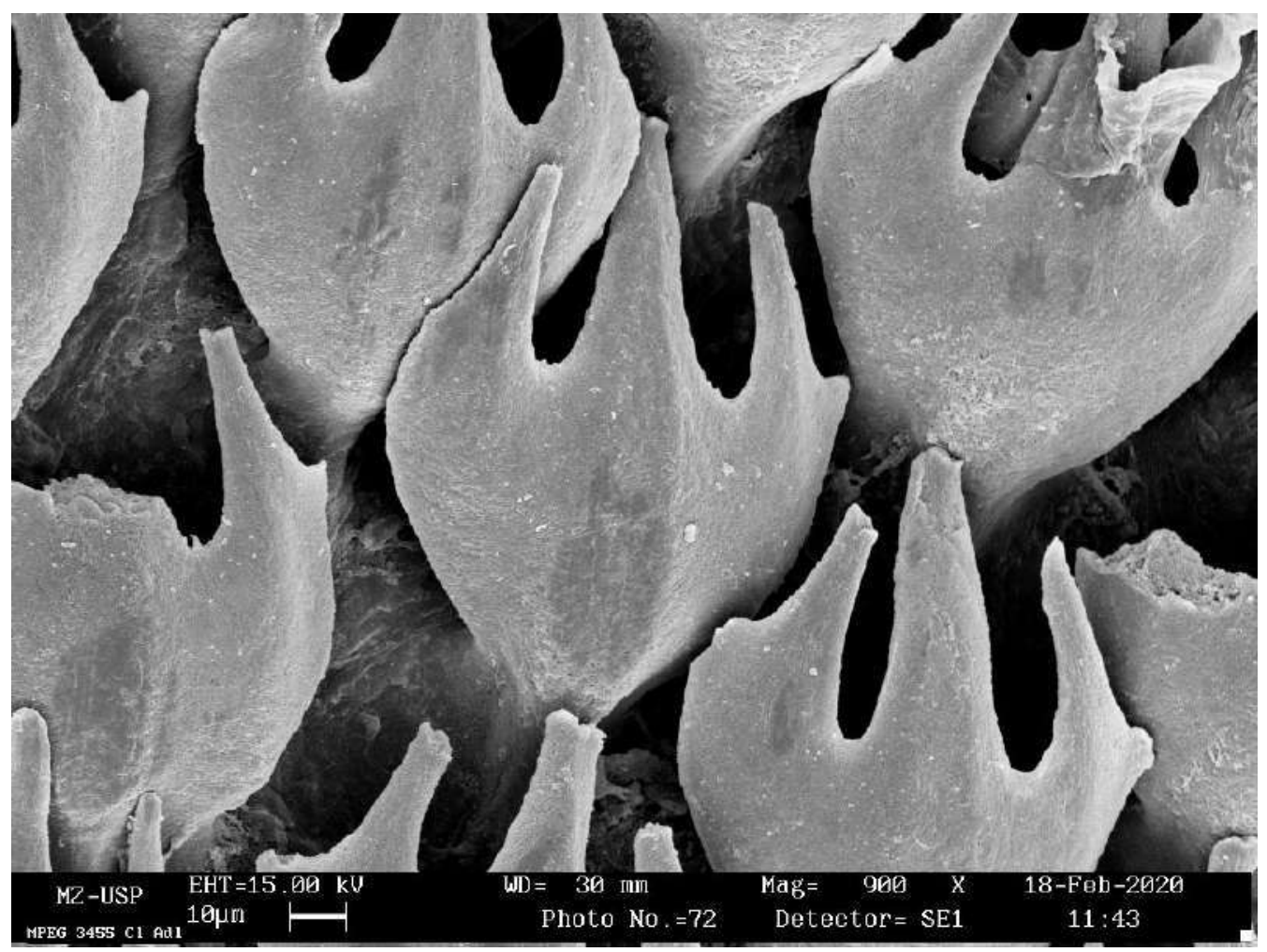

Figure 217. Dermal denticles collected from the section CL (see fig. 1) of the adult Isogomphodon oxyrhynchus (MPEG 3455). The sample on this specimen was collected from the anterior portion of the clasper. Additional information in the image footer. 


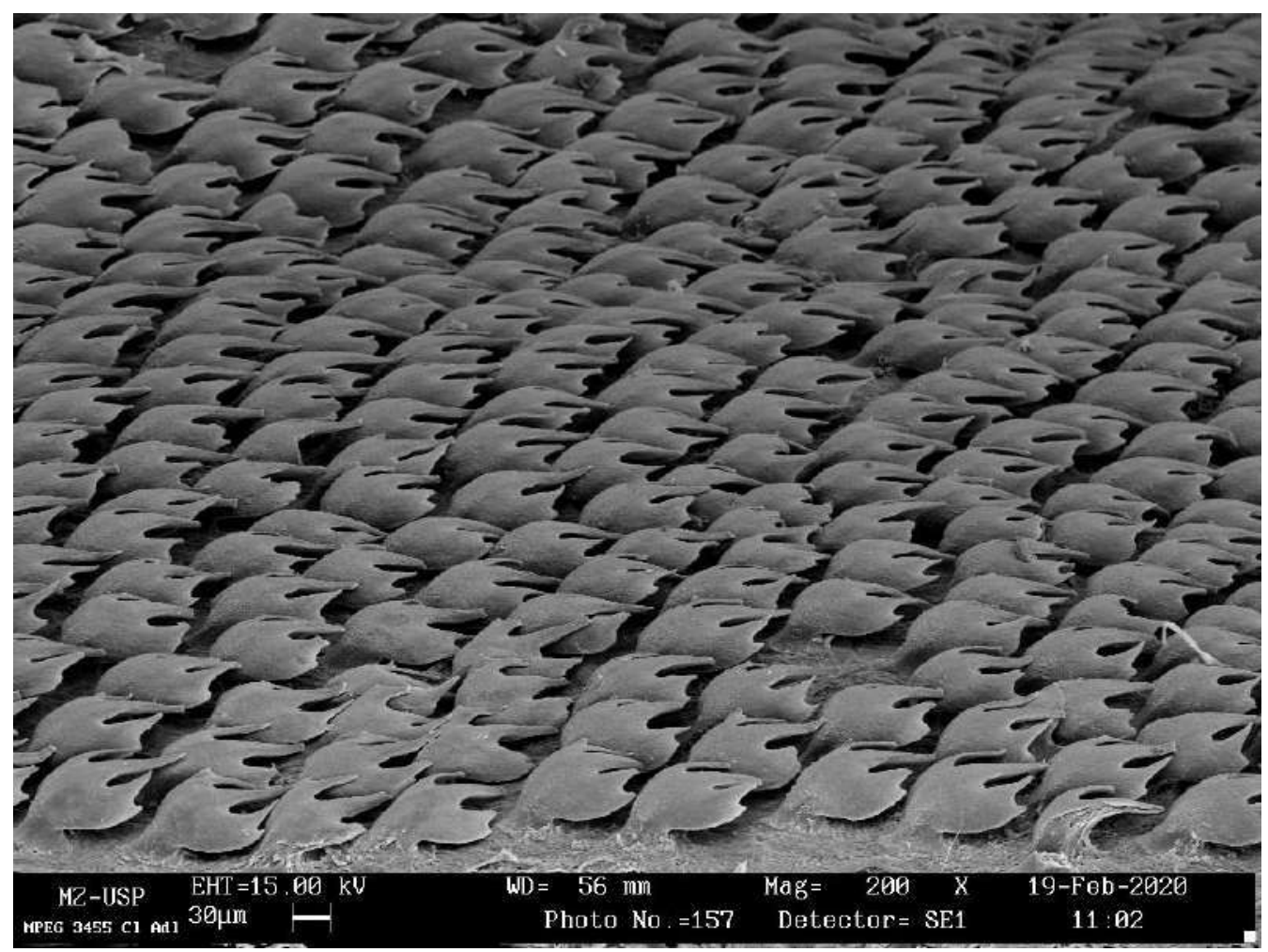

Figure 218. Dermal denticles collected from the section CL (see fig. 1) of the adult Isogomphodon oxyrhynchus (MPEG 3455). The sample on this specimen was collected from the anterior portion of the clasper. Additional information in the image footer.

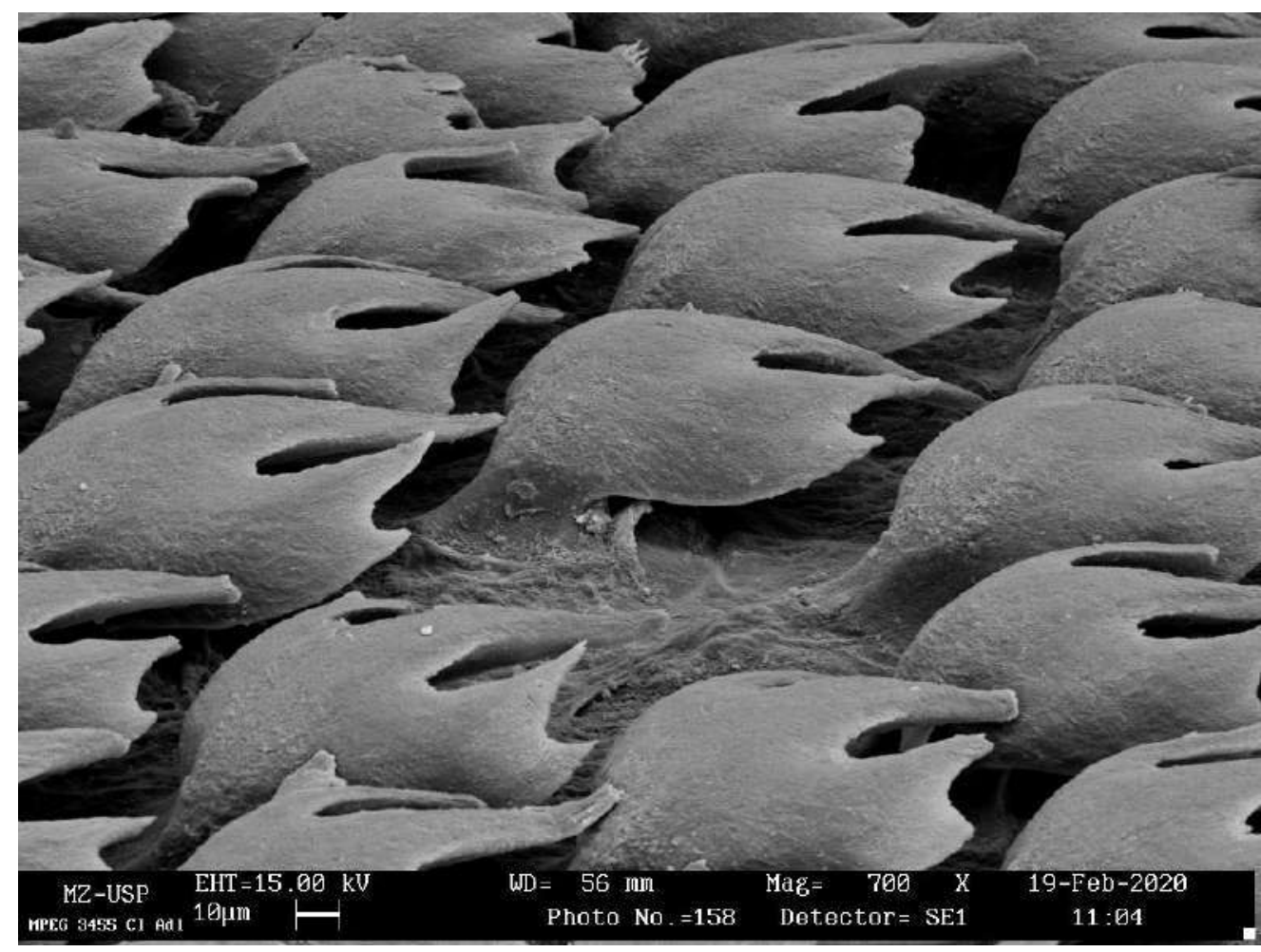

Figure 219. Dermal denticles collected from the section CL (see fig. 1) of the adult Isogomphodon oxyrhynchus (MPEG 3455). The sample on this specimen was collected from the anterior portion of the clasper. Additional information in the image footer. 


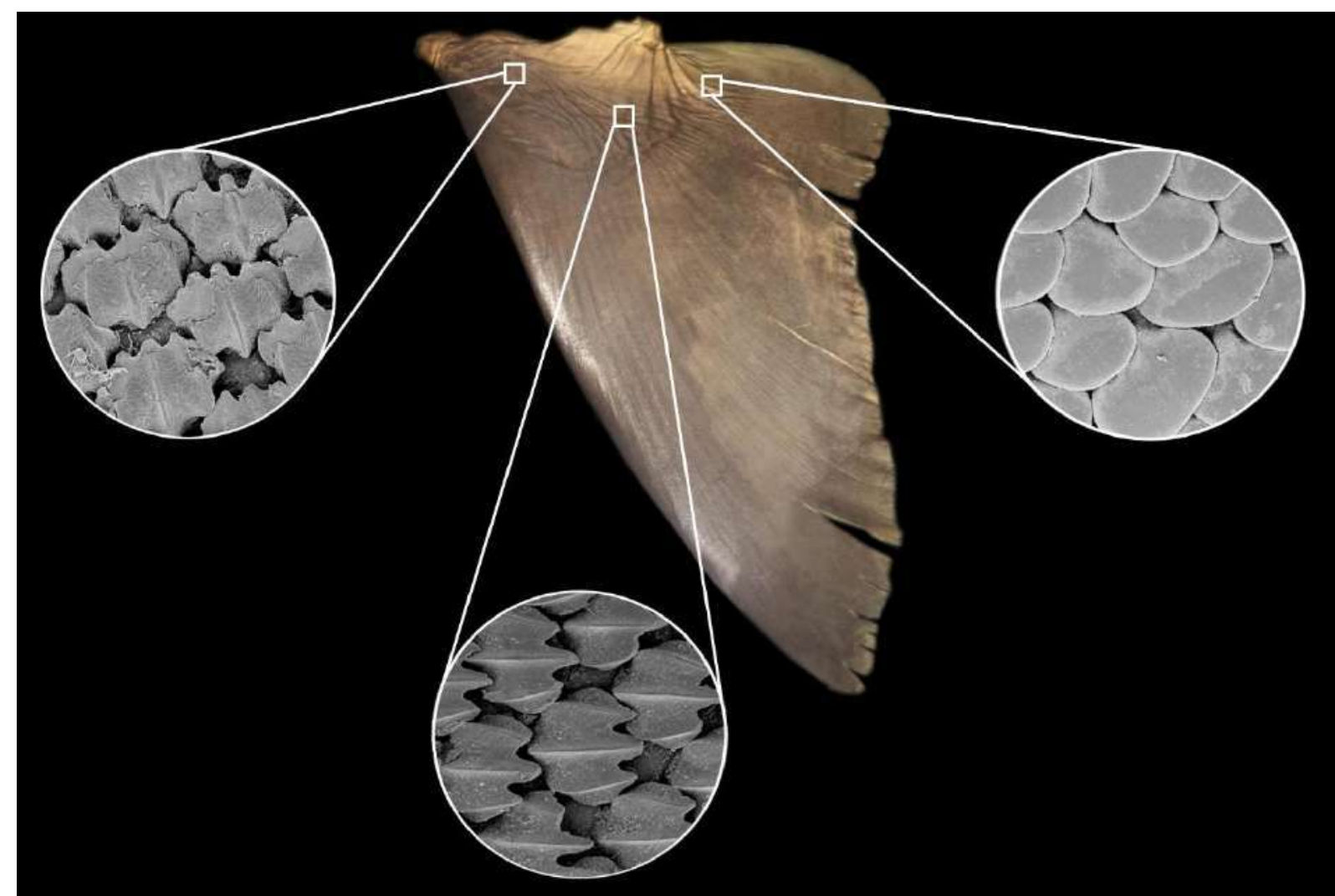

Figure 220. Illustration of the dermal denticles on the right pectoral fin (Image flipped for schematic purposes). P1 - Left; P2 - Middle; P3 - Right

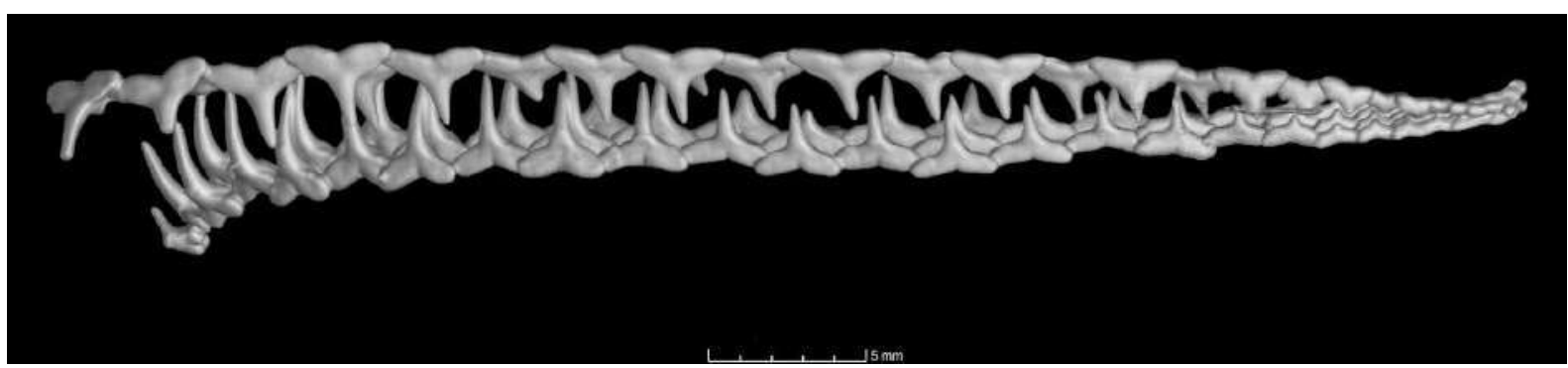

Figure 221. Labial-lateral view of the dentition of Isogomphodon oxyrhynchus (MZUSP 101214) obtained with $3 \mathrm{D}$ reconstruction from the CT-Scan imaging.

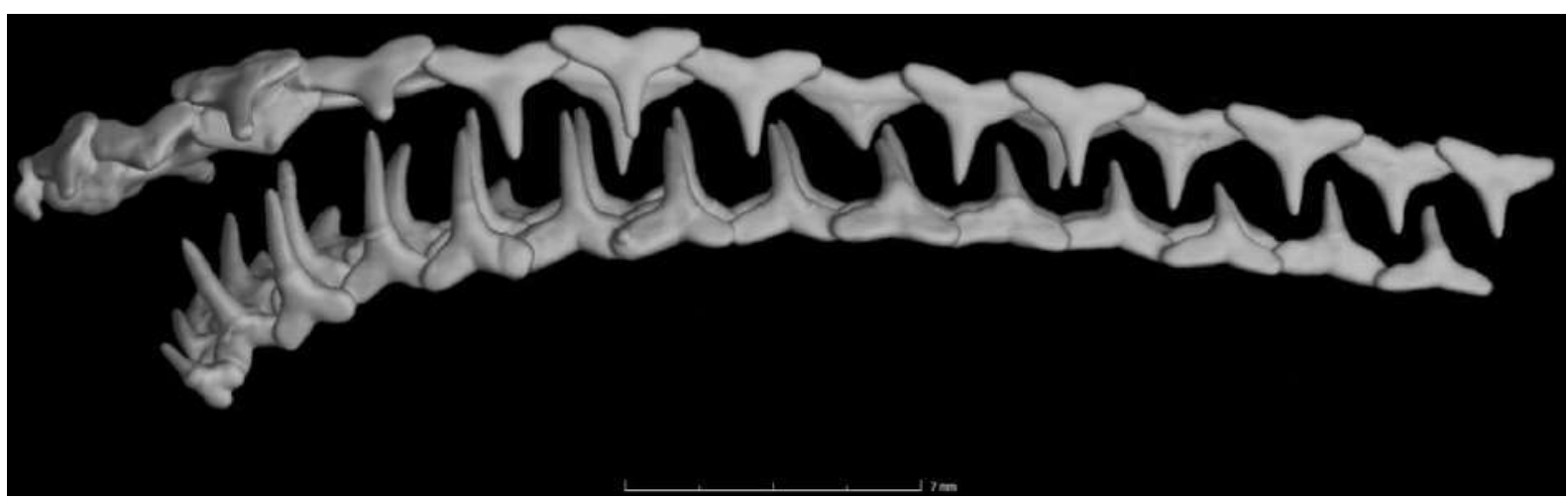

Figure 222. Labial view of the dentition of Isogomphodon oxyrhynchus (MZUSP 101214), showing anterior teeth rows, obtained with 3D reconstruction from the CT-Scan imaging. 


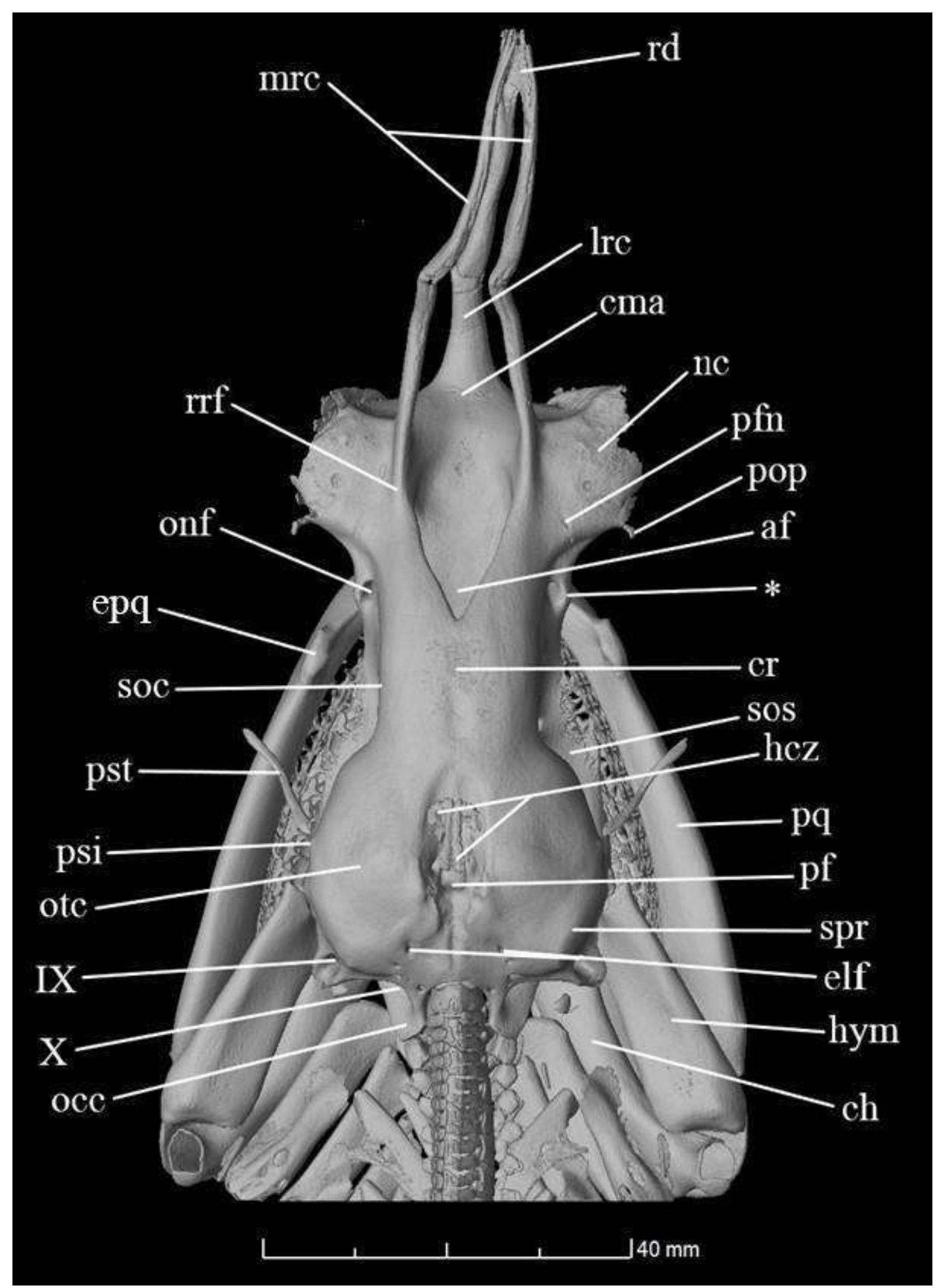

Figure 223. Dorsal view of the anterior portion of the neurocranium + splanchnocranium of Isogomphodon oxyrhynchus (MZUSP 101214) obtained with 3D reconstruction from the CT-Scan imaging. Anatomical abbreviations: af, anterior fontanelle; ch, ceratohyal; cr, cranial roof; elf, endolymphatic foramen; epq, ethmoid process of the palatoquadrate; IX, glossopharyngeal foramen; hcz, hypercalcified zones; hym, hyomandibula; lrc, lateral rostral cartilage; mrc, medial rostral cartilage; nc, nasal capsule; ob, orbit; occ, occipital condyle; onf, 
orbitonasal foramen; otc, otic capsule; pf, parietal fossa; plf, perilymphatic fenestrae; pop, preorbital process; pq, palatoquadrate; psi, postorbital process insertion; pst, postorbital process; rd, rostral node; rrf, ridge connecting base of lateral rostral cartilage with edge of anterior fontanelle; soc, supraorbital crest; sos, suborbital shelf; spr, sphenopterotic ridge; $\mathrm{X}$, vagus nerve foramen; *Lateral dilatations of the orbitonasal foramen;

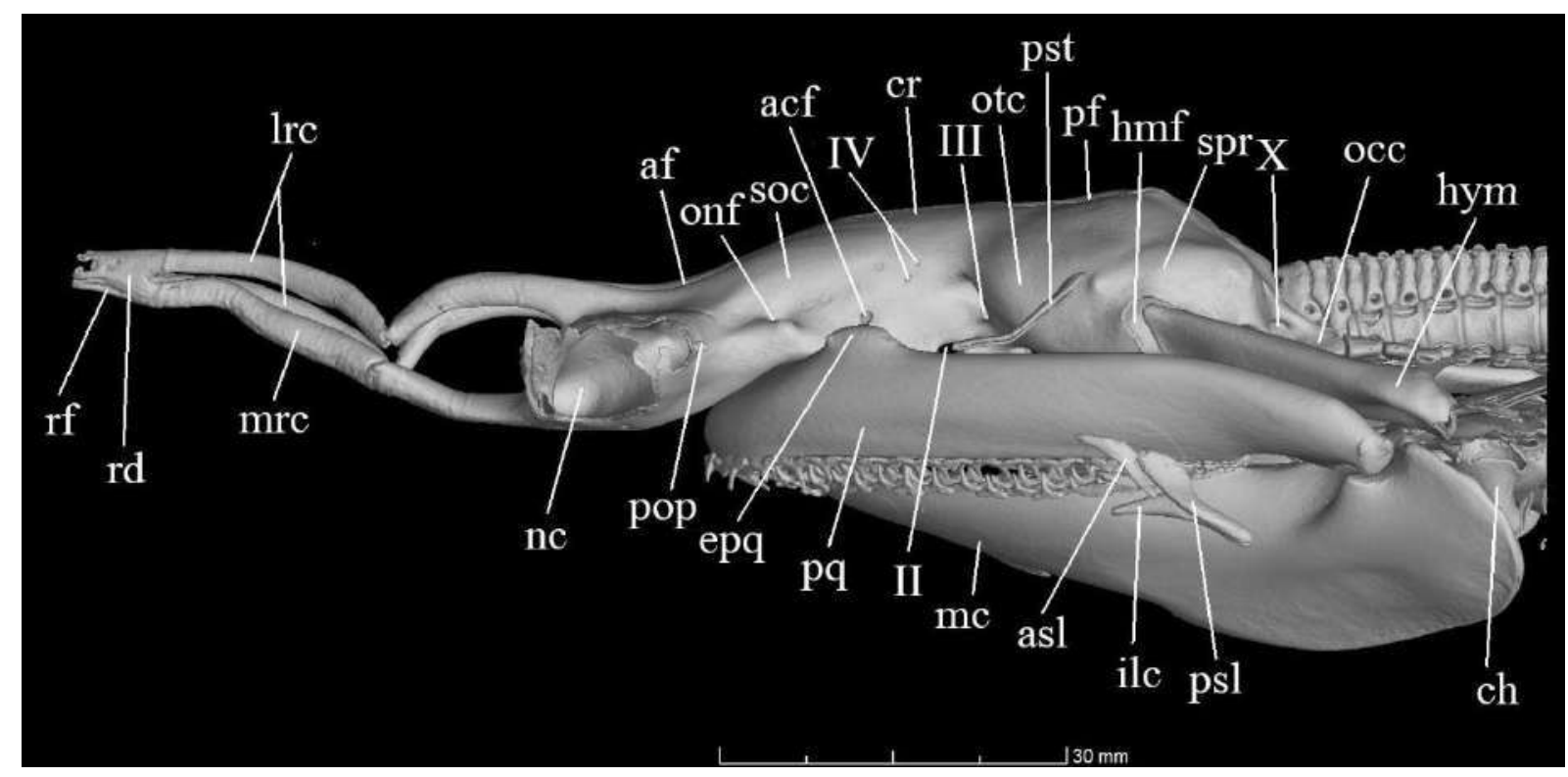

Figure 224. Lateral view of the neurocranium + splanchnocranium of Isogomphodon oxyrhynchus (MZUSP 101214) obtained with 3D reconstruction from the CT-Scan imaging. Anatomical abbreviations: acf, anterior cerebral foramen; af, anterior fontanelle; asl, anterior superior labial cartilage; ch, ceratohyal; cr, cranial roof; epq, ethmoid process of the palatoquadrate; hmf, hyomandibular facet; hym, hyomandibula; ilc, inferior labial cartilage; lrc, lateral rostral cartilage; mc, Meckel's cartilage; mrc, medial rostral cartilage; nc, nasal capsule; occ, occipital condyle; II, otic foramen; onf, orbitonasal foramen; otc, otic capsule; pf, parietal fossa; pop, preorbital process; pq, palatoquadrate; psl, posterior superior labial cartilage; pst, postorbital process; rd, rostral node; rf, rostral fenestrae; soc, supraorbital crest; spr, sphenopterotic ridge; III, oculomotor nerve foramen; IV, trochlear foramen; $\mathrm{X}$, vagus nerve foramen; 


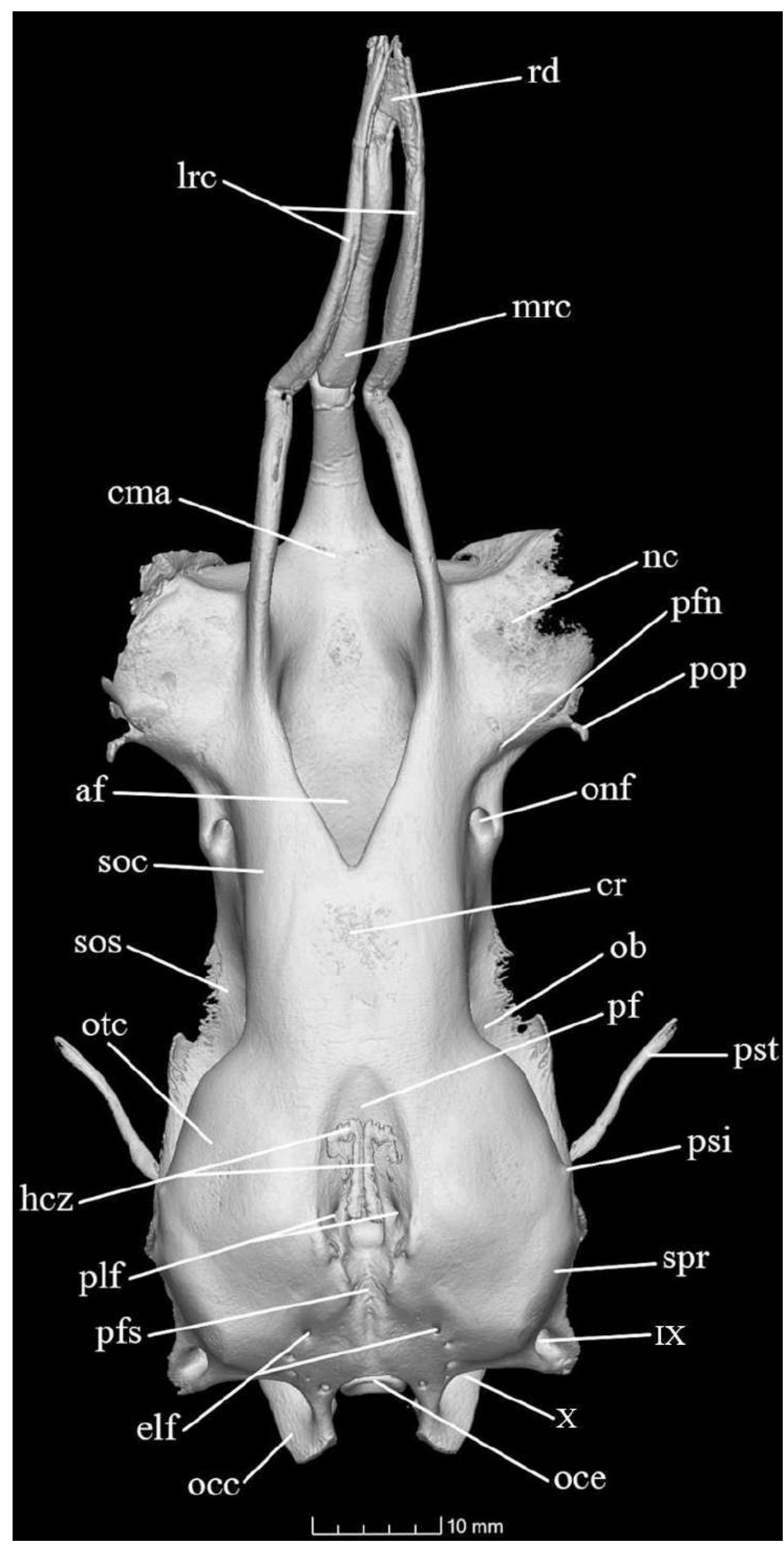


Figure 225. Dorsal view of the Neurocranium of juvenile Isogomphodon oxyrhynchus (MZUSP 101214) obtained with 3D reconstruction from the CT-Scan imaging. Anatomical abbreviations: af, anterior fontanelle; cma, confluence between the medial rostral cartilage and anterior fontanelle; cr, cranial roof; elf, endolymphatic foramen; IX, glossopharyngeal foramen; hcz, hypercalcified zones; lrc, lateral rostral cartilage; mrc, medial rostral cartilage; nc, nasal capsule; ob, orbit; occ, occipital condyle; oce, occipital centrum; onf, orbitonasal foramen; otc, otic capsule; pf, parietal fossa; pfn, profundus nerve foramen; pfs, parietal fossa sulcus; plf, perilymphatic fenestrae; pop, preorbital process; psi, postorbital process insertion; psl, posterior superior labial cartilage; pst, postorbital process; rd, rostral node; soc, supraorbital crest; sos, suborbital shelf; spr, sphenopterotic ridge; X, vagus nerve foramen; 


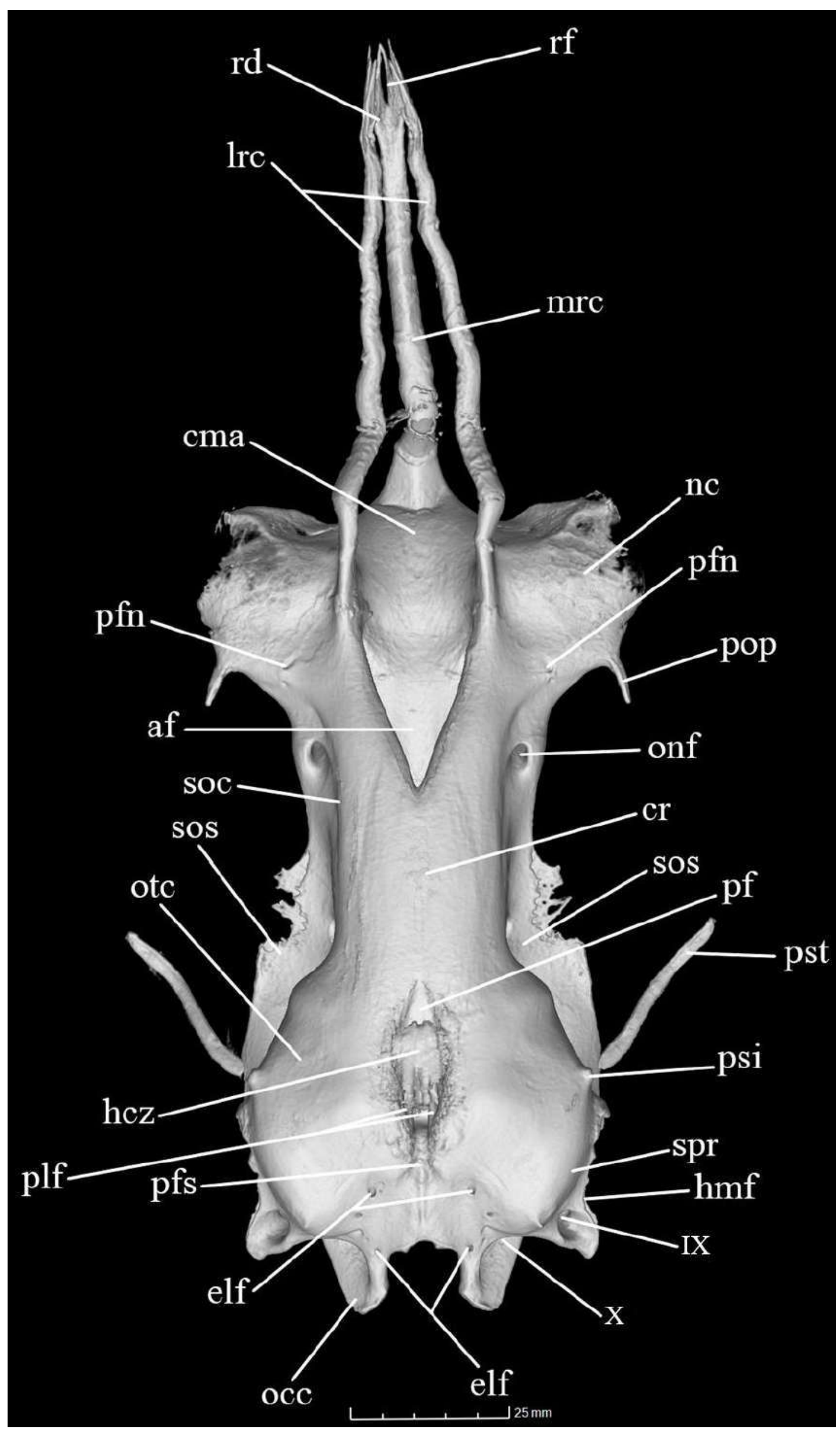


Figure 226. Dorsal view of the Neurocranium of adult Isogomphodon oxyrhynchus (MPEG3455) obtained with 3D reconstruction from the CT-Scan imaging. Anatomical abbreviations: af, anterior fontanelle; cma, confluence between the medial rostral cartilage and anterior fontanelle; cr, cranial roof; elf, endolymphatic foramen; IX, glossopharyngeal foramen; hcz, hypercalcified zones; lrc, lateral rostral cartilage; mrc, medial rostral cartilage; nc, nasal capsule; ob, orbit; occ, occipital condyle; oce, occipital centrum; onf, orbitonasal foramen; otc, otic capsule; pf, parietal fossa; pfn, profundus nerve foramen; pfs, parietal fossa sulcus; plf, perilymphatic fenestrae; pop, preorbital process; psi, postorbital process insertion; psl, posterior superior labial cartilage; pst, postorbital process; rd, rostral node; soc, supraorbital crest; sos, suborbital shelf; spr, sphenopterotic ridge; X, vagus nerve foramen; 


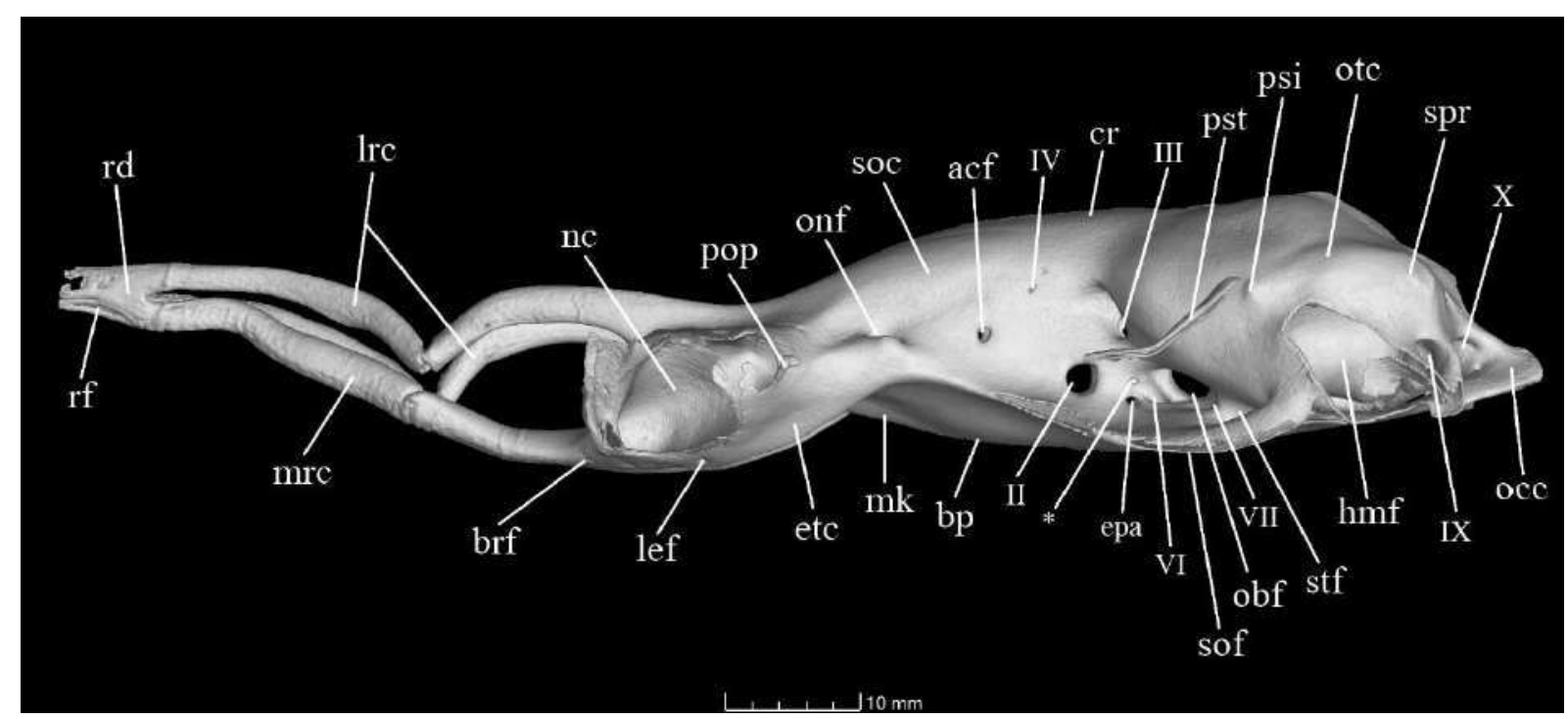

Figure 227. Lateral view of the Neurocranium of juvenile Isogomphodon oxyrhynchus (MZUSP 101214) obtained with 3D reconstruction from the CT-Scan imaging. Anatomical abbreviations: acf, anterior cerebral vein foramen; anf, abducens nerve foramen; bp, basal plate; brf, basirostral fenestrae; cr, cranial roof; elf, endolymphatic foramen; epa, efferent pseudobranchial artery foramen; etc, ectethmoid condyle; hmf, hyomandibular facet; lef, lateral ectethmoid foramen; lrc, lateral rostral cartilage; mk, medial keel; mrc, medial rostral cartilage; nc, nasal capsule; ob, orbit; obf, orbital fissure; occ, occipital condyle; oce, occipital centrum; onf, orbitonasal foramen; otc, otic capsule; pf, parietal fossa; pfn, profundus nerve foramen; pfs, parietal fossa sulcus; plf, perilymphatic fenestrae; pop, preorbital process; psi, postorbital process insertion; pst, postorbital process; rd, rostral node; rf, rostral fenestrae; soc, supraorbital crest; sos, suborbital shelf; spr, sphenopterotic ridge; stf, stapedial foramen; II, otic foramen; III, oculomotor nerve foramen; IV, trochlear nerve foramen; VI, abducens nerve foramen; VII; facial nerve foramen; IX, glossopharyngeal foramen; X, vagus nerve foramen; *, artifactual foramen; 


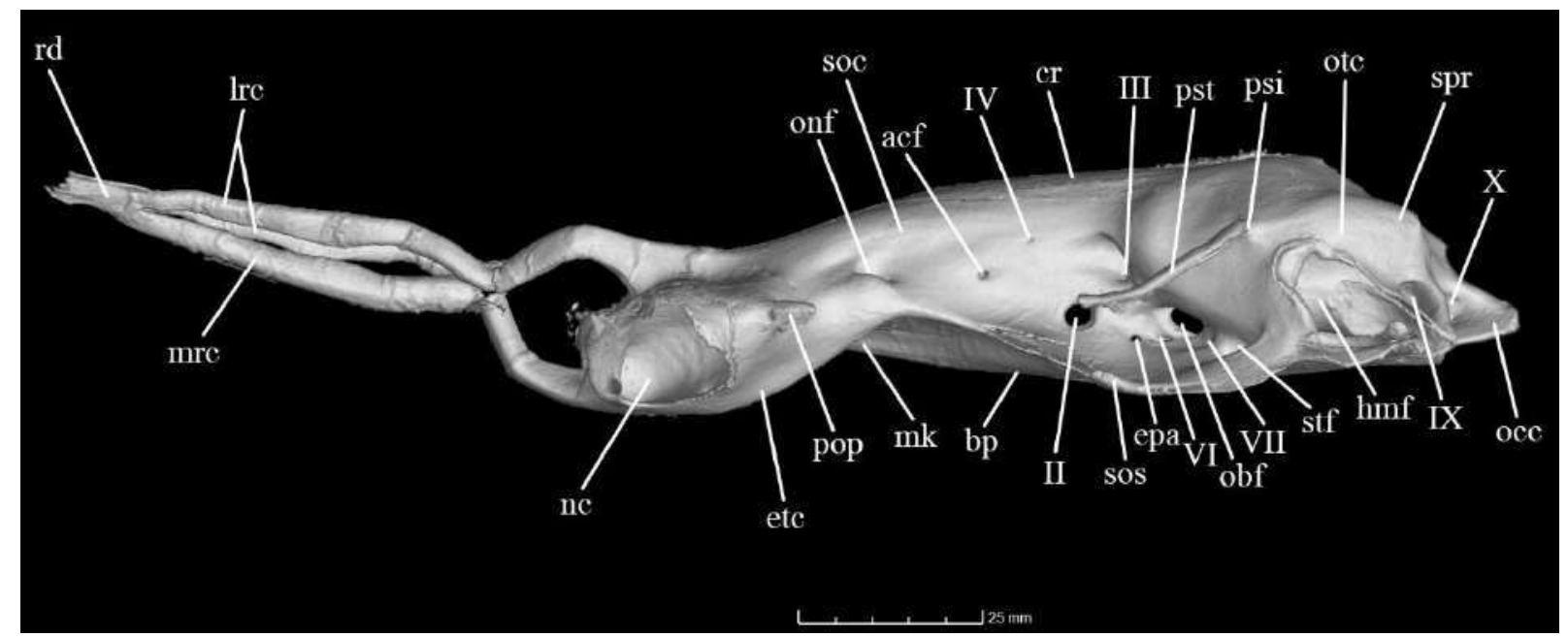

Figure 228. Lateral view of the Neurocranium of adult Isogomphodon oxyrhynchus (MPEG3455) obtained with 3D reconstruction from the CT-Scan imaging. Anatomical abbreviations: acf, anterior cerebral vein foramen; bp, basal plate; brf, basirostral fenestrae; cr, cranial roof; elf, endolymphatic foramen; epa, efferent pseudobranchial artery foramen; etc, ectethmoid condyle; hmf, hyomandibular facet; icv, interorbital canal for posterior vein; lef, lateral ectethmoid foramen; lrc, lateral rostral cartilage; mk, medial keel; mrc, medial rostral cartilage; nc, nasal capsule; ob, orbit; obf, orbital fissure; occ, occipital condyle; oce, occipital centrum; onf, orbitonasal foramen; otc, otic capsule; pf, parietal fossa; pfn, profundus nerve foramen; pfs, parietal fossa sulcus; plf, perilymphatic fenestrae; pop, preorbital process; psi, postorbital process insertion; pst, postorbital process; rd, rostral node; rf, rostral fenestrae; soc, supraorbital crest; sos, suborbital shelf; spr, sphenopterotic ridge; stf, stapedial foramen; II, otic foramen; III, oculomotor nerve foramen; IV, trochlear nerve foramen; VI, abducens nerve foramen; VII; facial nerve foramen; IX, glossopharyngeal foramen; X, vagus nerve foramen; 


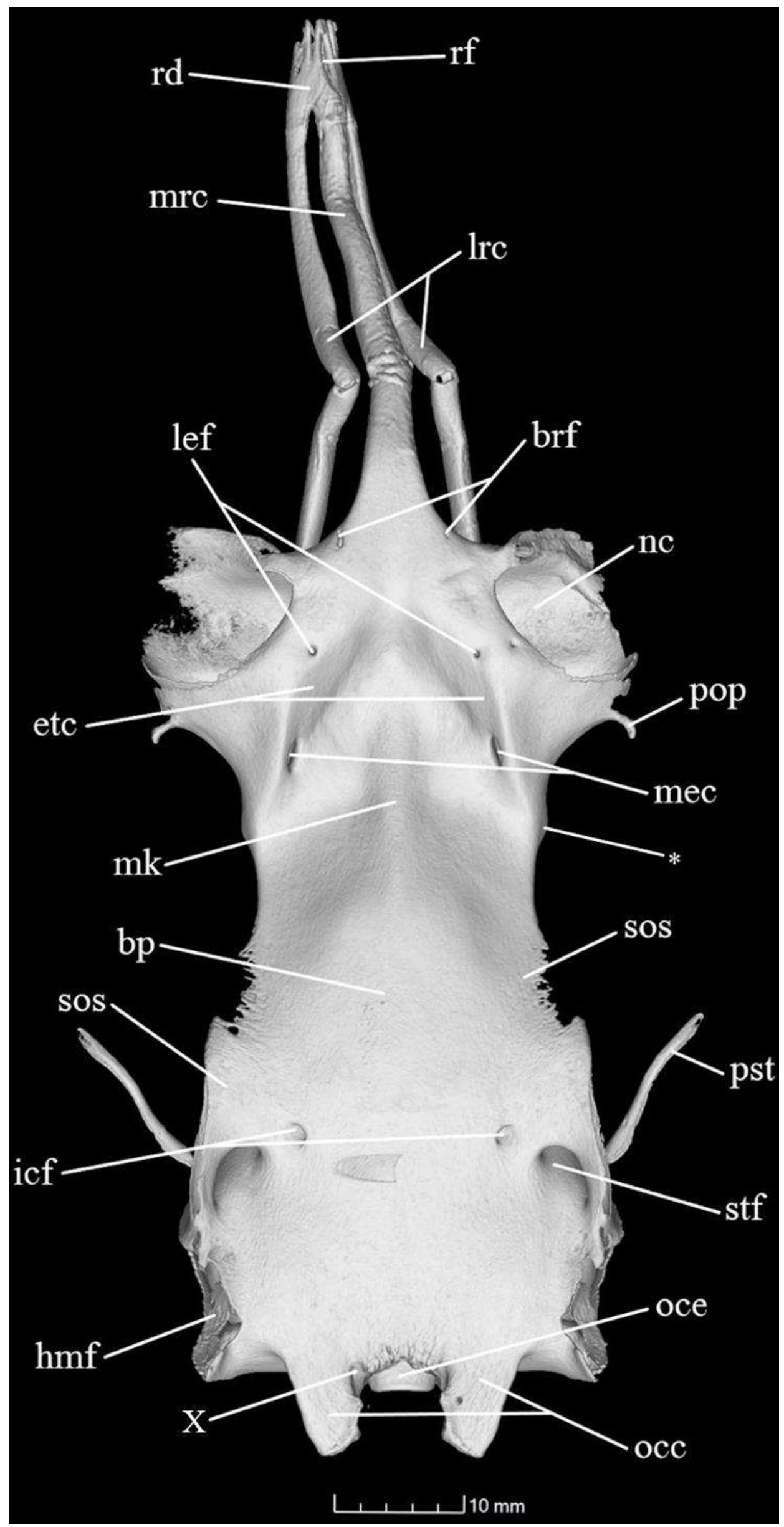


Figure 229. Ventral view of the Neurocranium of juvenile Isogomphodon oxyrhynchus (MZUSP 101214) obtained with 3D reconstruction from the CT-Scan imaging. Anatomical abbreviations: bp, basal plate; brf, basirostral fenestrae; etc, ectethmoid condyle; hmf, hyomandibular facet; icf, internal carotid foramen; lef, lateral ectethmoid foramen; lrc, lateral rostral cartilage; mec, medial ectethmoid foramen; mk, medial keel; mrc, medial rostral cartilage; nc, nasal capsule; occ, occipital condyle; oce, occipital centrum; pop, preorbital process; pst, postorbital process; rd, rostral node; rf, rostral fenestrae; sos, suborbital shelf; stf, stapedial foramen; X, vagus nerve foramen; *Lateral dilatations of the orbitonasal foramen 


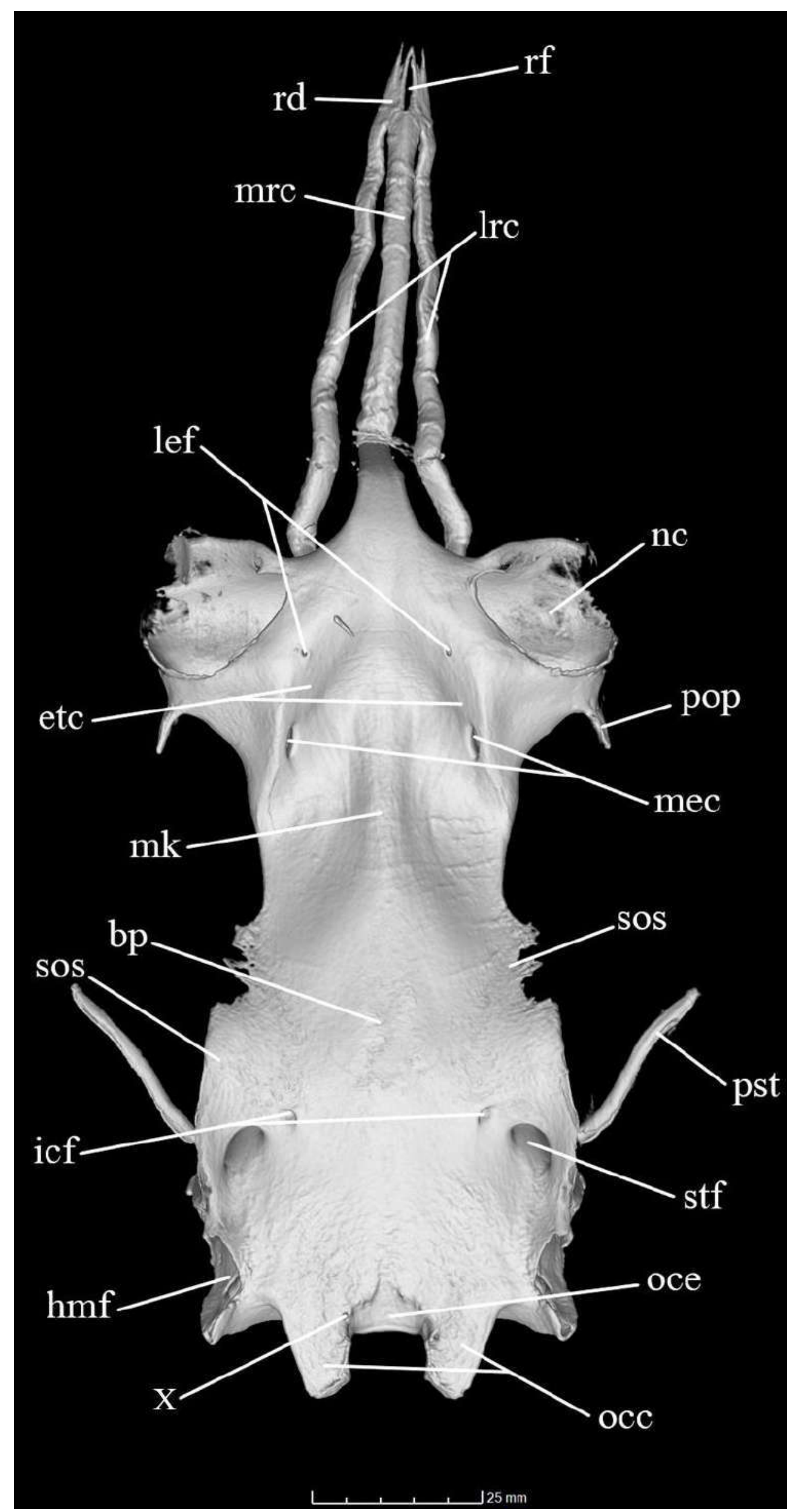


Figure 230. Ventral view of the Neurocranium of adult Isogomphodon oxyrhynchus (MPEG3455) obtained with 3D reconstruction from the CT-Scan imaging. Anatomical abbreviations: bp, basal plate; brf, basirostral fenestrae; etc, ectethmoid condyle; hmf, hyomandibular facet; icf, internal carotid foramen; lef, lateral ectethmoid foramen; lrc, lateral rostral cartilage; mec, medial ectethmoid foramen; mk, medial keel; mrc, medial rostral cartilage; nc, nasal capsule; occ, occipital condyle; oce, occipital centrum; pop, preorbital process; pst, postorbital process; rd, rostral node; rf, rostral fenestrae; sos, suborbital shelf; stf, stapedial foramen; vnf, vagus nerve foramen;

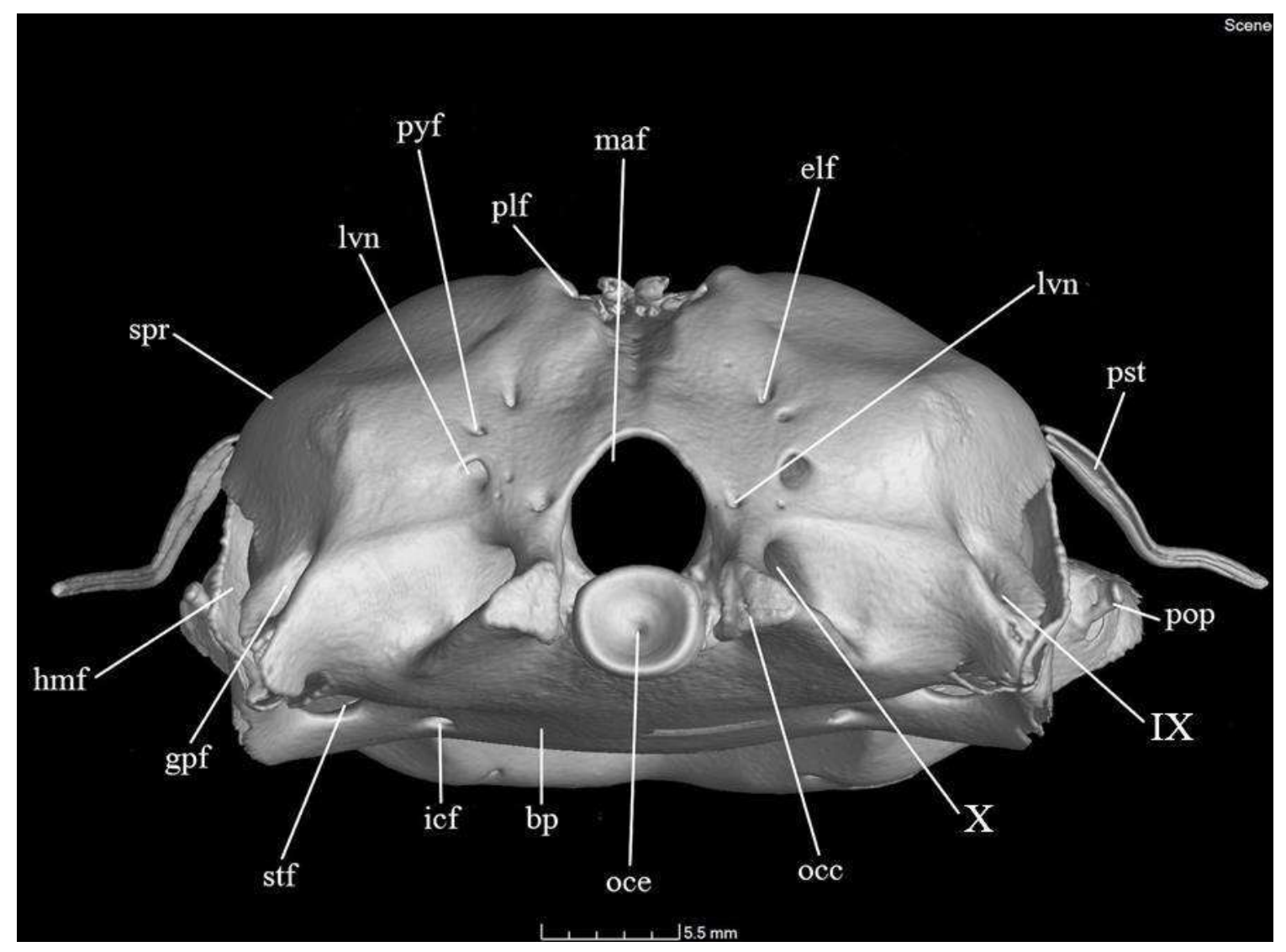

Figure 231. Caudal view of the Neurocranium of juvenile Isogomphodon oxyrhynchus (MZUSP 101214) obtained with 3D reconstruction from the CT-Scan imaging. Anatomical abbreviations: bp, basal plate; elf, endolymphatic foramen; IX, glossopharyngeal foramen; hmf, hyomandibular facet; icf, internal carotid foramen; lvn, foramen for lateral branch of vagus nerve; maf, magnum foramen; occ, occipital condyle; oce, occipital centrum; onf, orbitonasal foramen; plf, perilymphatic fenestrae; pop, preorbital process; pst, postorbital process; pyf, perilymphatic foramen; spr, sphenopterotic ridge; stf, stapedial foramen; X, vagus nerve foramen. 


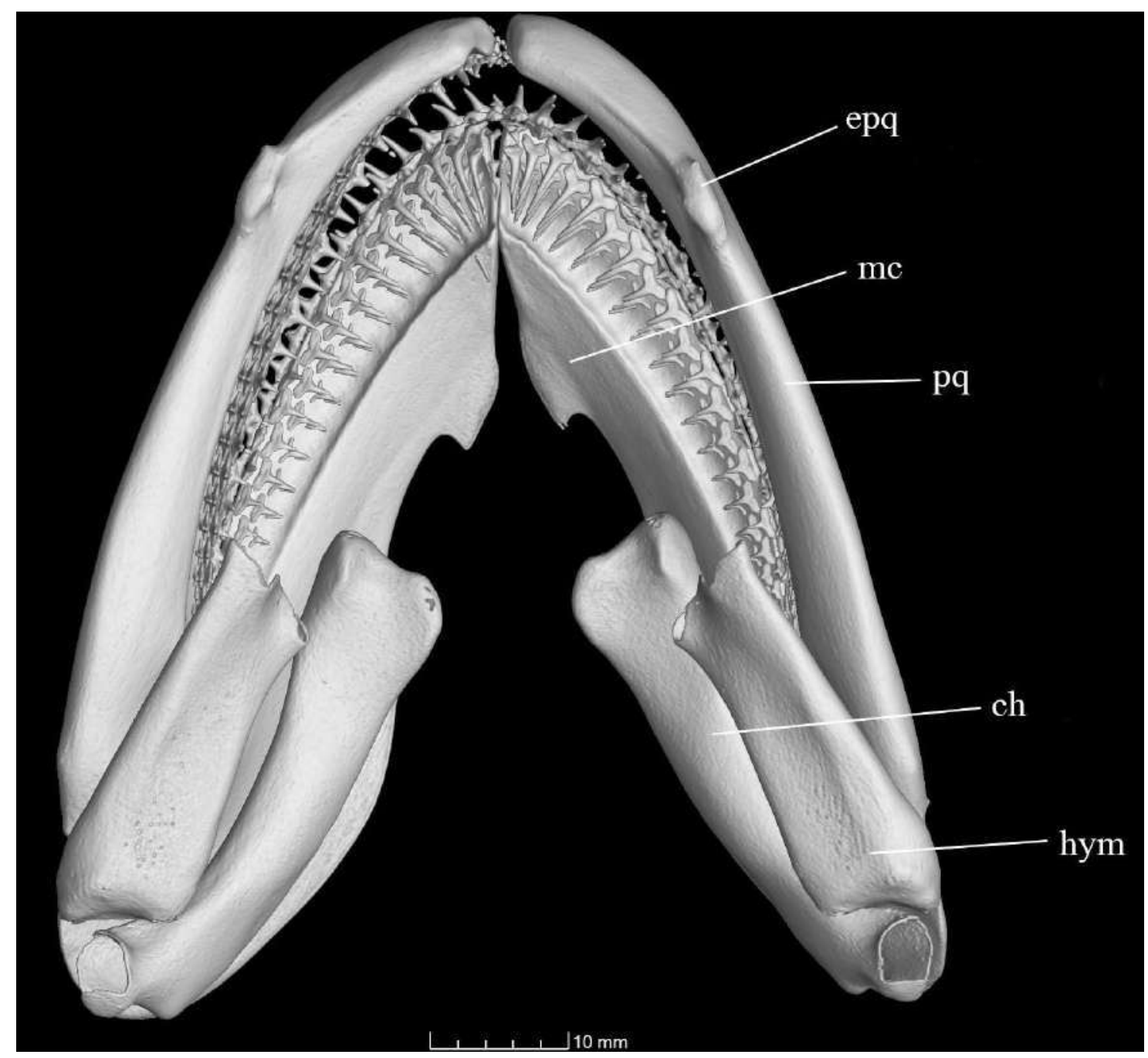

Figure 232. Dorsal view of the Mandibular arch, with the hyoid arch, of juvenile Isogomphodon oxyrhynchus (MZUSP 101214) obtained with 3D reconstruction from the CT-Scan imaging. Anatomical abbreviations: ch, ceratohyal; epq, ethmoid process of the palatoquadrate; hym, hyomandibula; mc, Meckel's cartilage; 


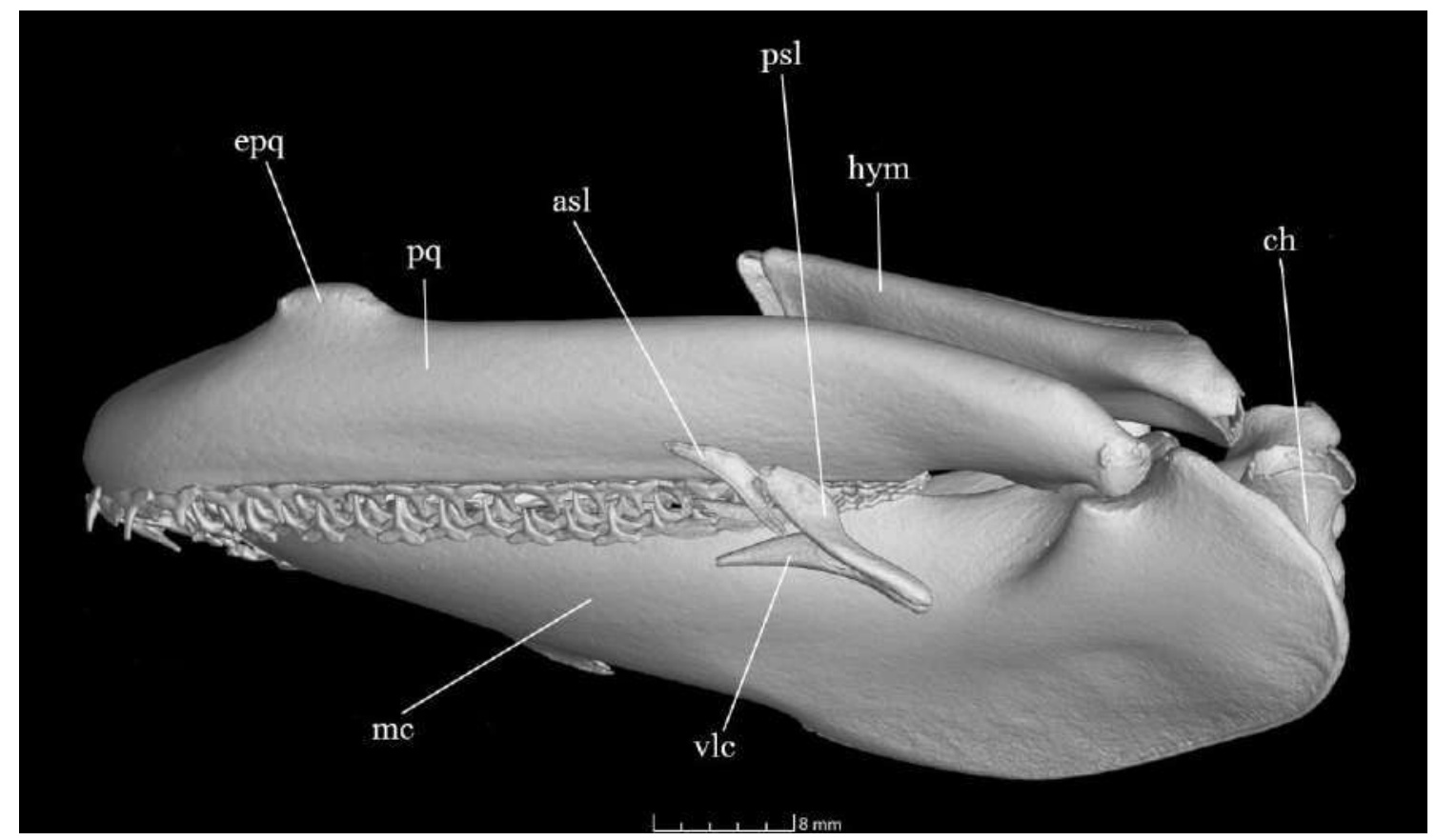

Figure 233. Lateral view of the Mandibular arch, with the hyoid arch, of juvenile Isogomphodon oxyrhynchus (MZUSP 101214) obtained with 3D reconstruction from the CT-Scan imaging. Anatomical abbreviations: asl, anterodorsal labial cartilage; ch, ceratohyal; epq, ethmoid process of the palatoquadrate; hym, hyomandibula; vlc, ventral labial cartilage; mc, Meckel's cartilage; pq, palatoquadrate; psl, posterodorsal labial cartilage. 


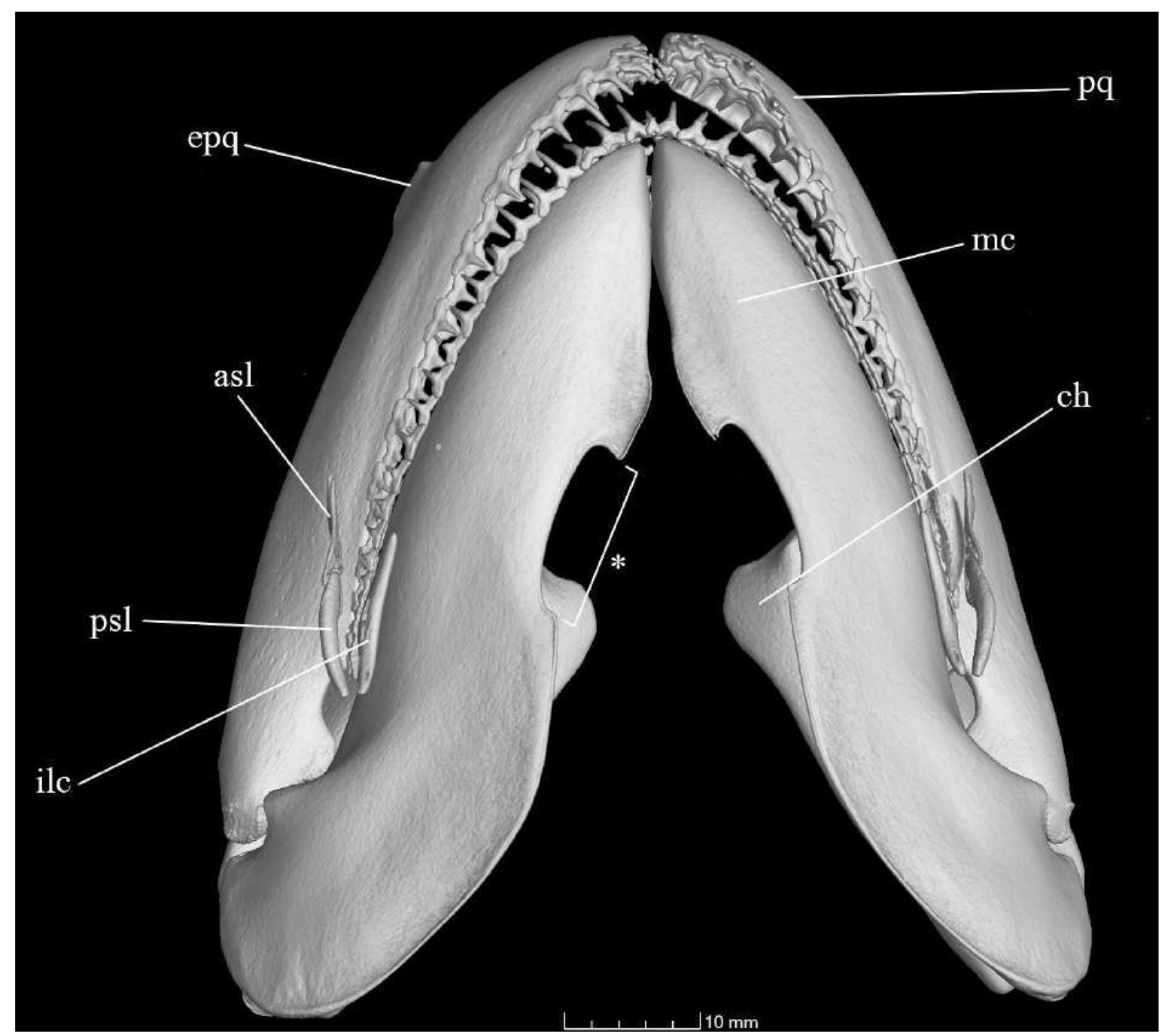

Figure 234. Ventral view of the Mandibular arch, with the hyoid arch, of juvenile Isogomphodon oxyrhynchus (MZUSP 101214) obtained with 3D reconstruction from the CT-Scan imaging. Anatomical abbreviations: asl, anterior superior labial cartilage; ch, ceratohyal; epq, ethmoid process of the palatoquadrate; hym, hyomandibula; ilc, inferior labial cartilage; mc, Meckel's cartilage; pq, palatoquadrate; psl, posterior superior labial cartilage; *Cshaped recess. 


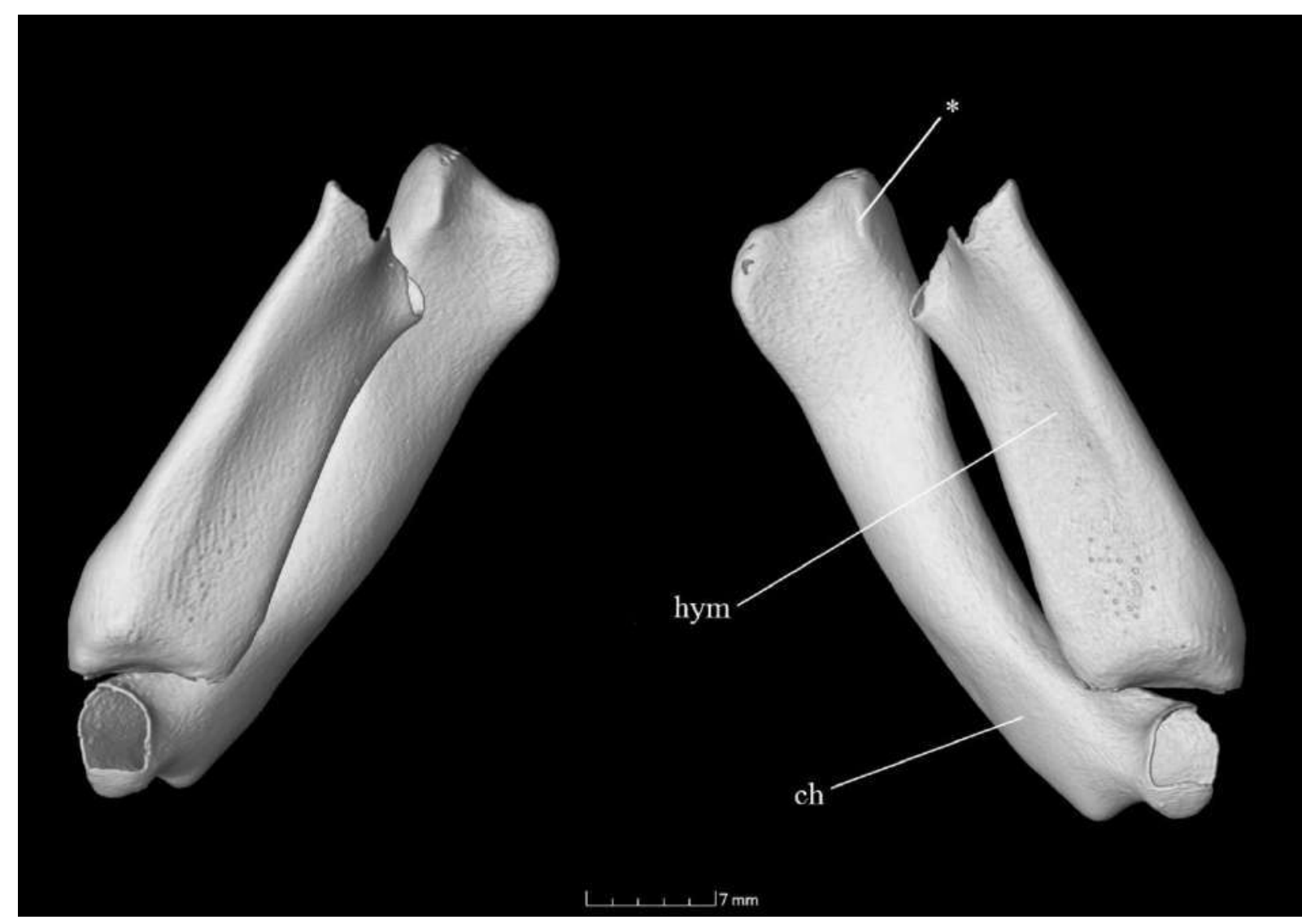

Figure 235. Dorsal view of the Hyoid arch, of juvenile Isogomphodon oxyrhynchus (MZUSP 101214) obtained with 3D reconstruction from the CT-Scan imaging. Anatomical abbreviations: ch, ceratohyal; hym, hyomandibula; *Anterior dilatation on ceratohyal. 


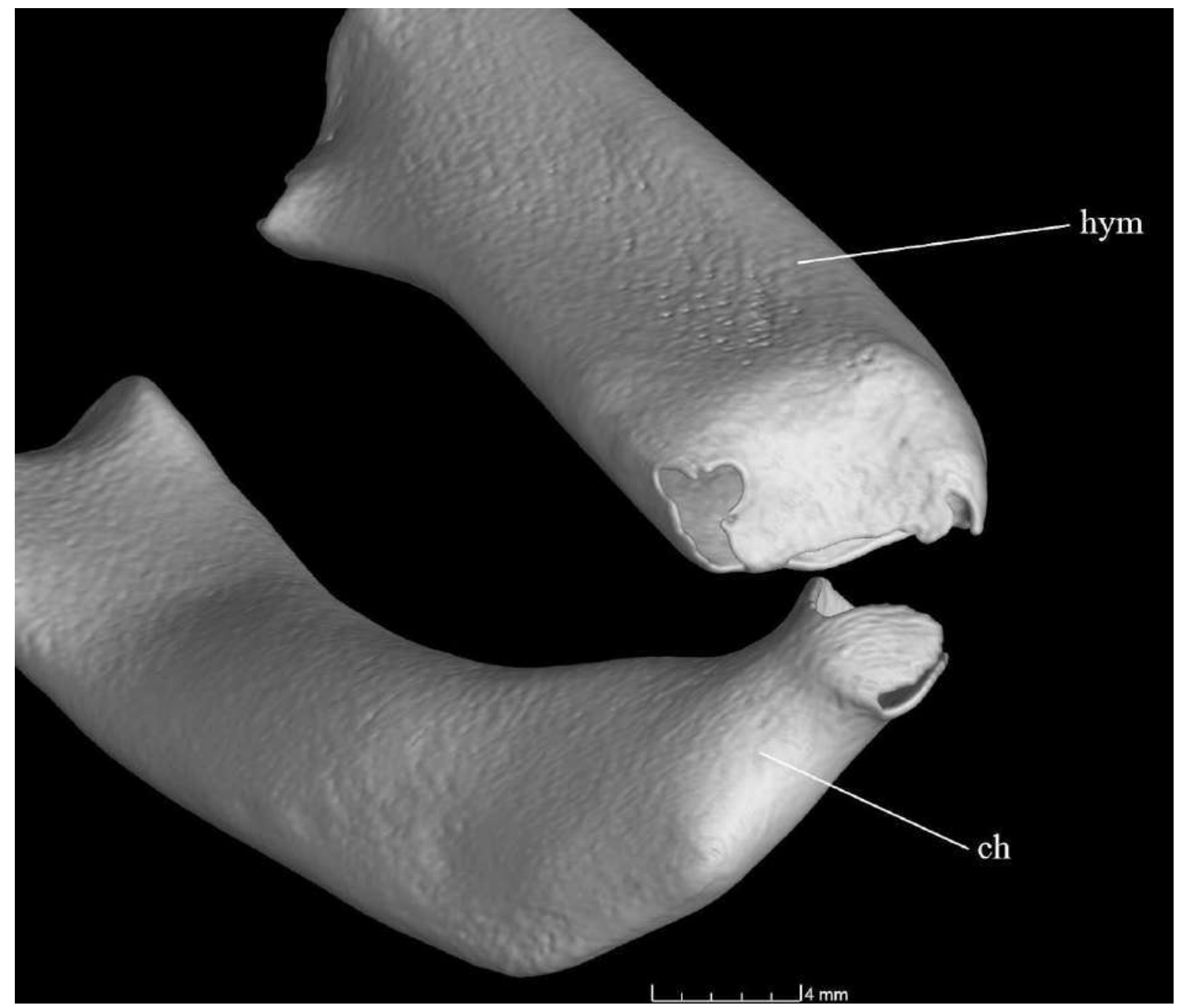

Figure 236. Caudal view of the Hyoid arch, focusing on the articular region, of juvenile Isogomphodon oxyrhynchus (MZUSP 101214) obtained with 3D reconstruction from the CT-Scan imaging. Anatomical abbreviations: ch, ceratohyal; hym, hyomandibula. 


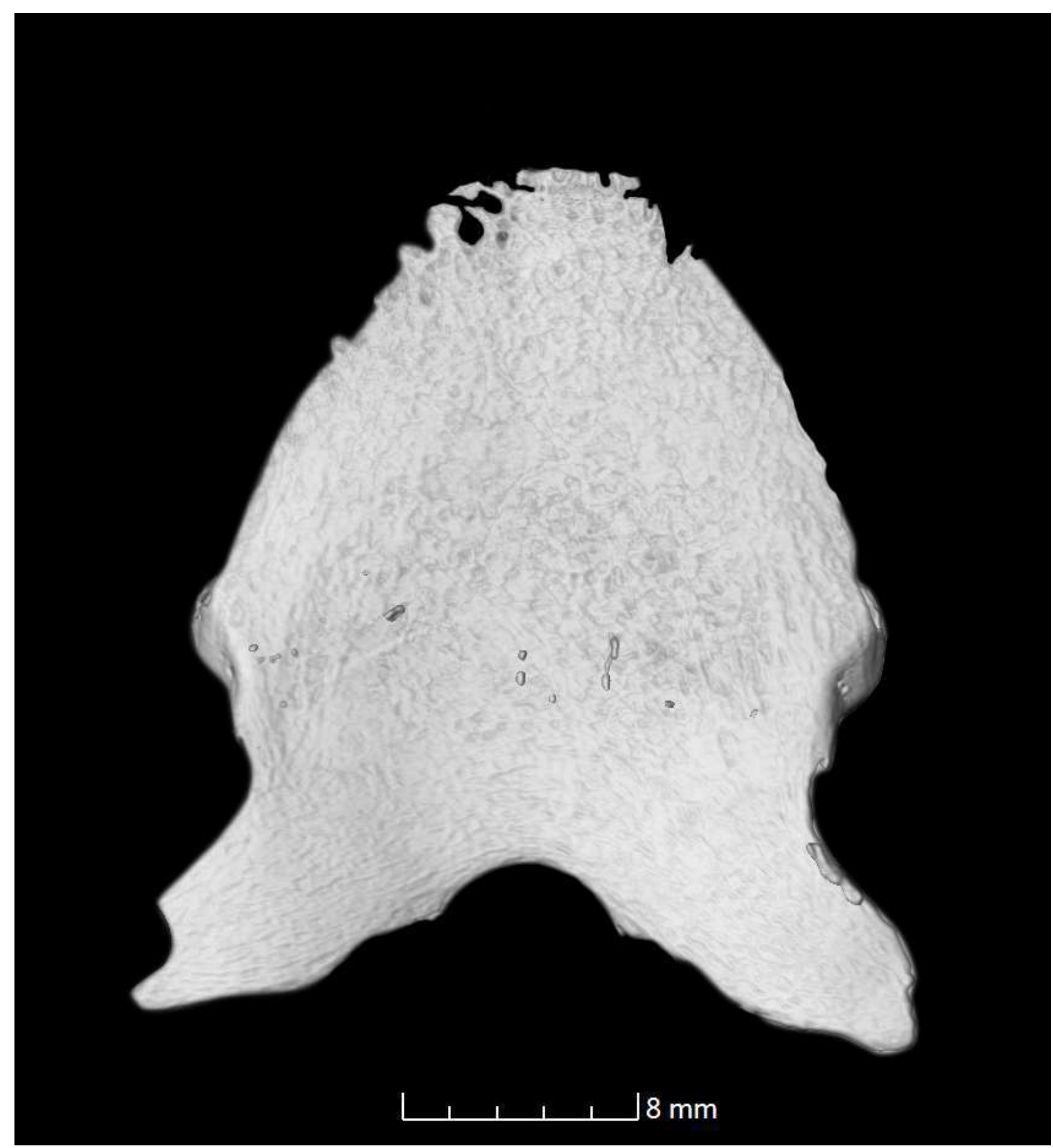

Figure 237. Dorsal view of the basihyal of Isogomphodon oxyrhynchus (MZUSP 101214) obtained with 3D reconstruction from the CT-Scan imaging. 


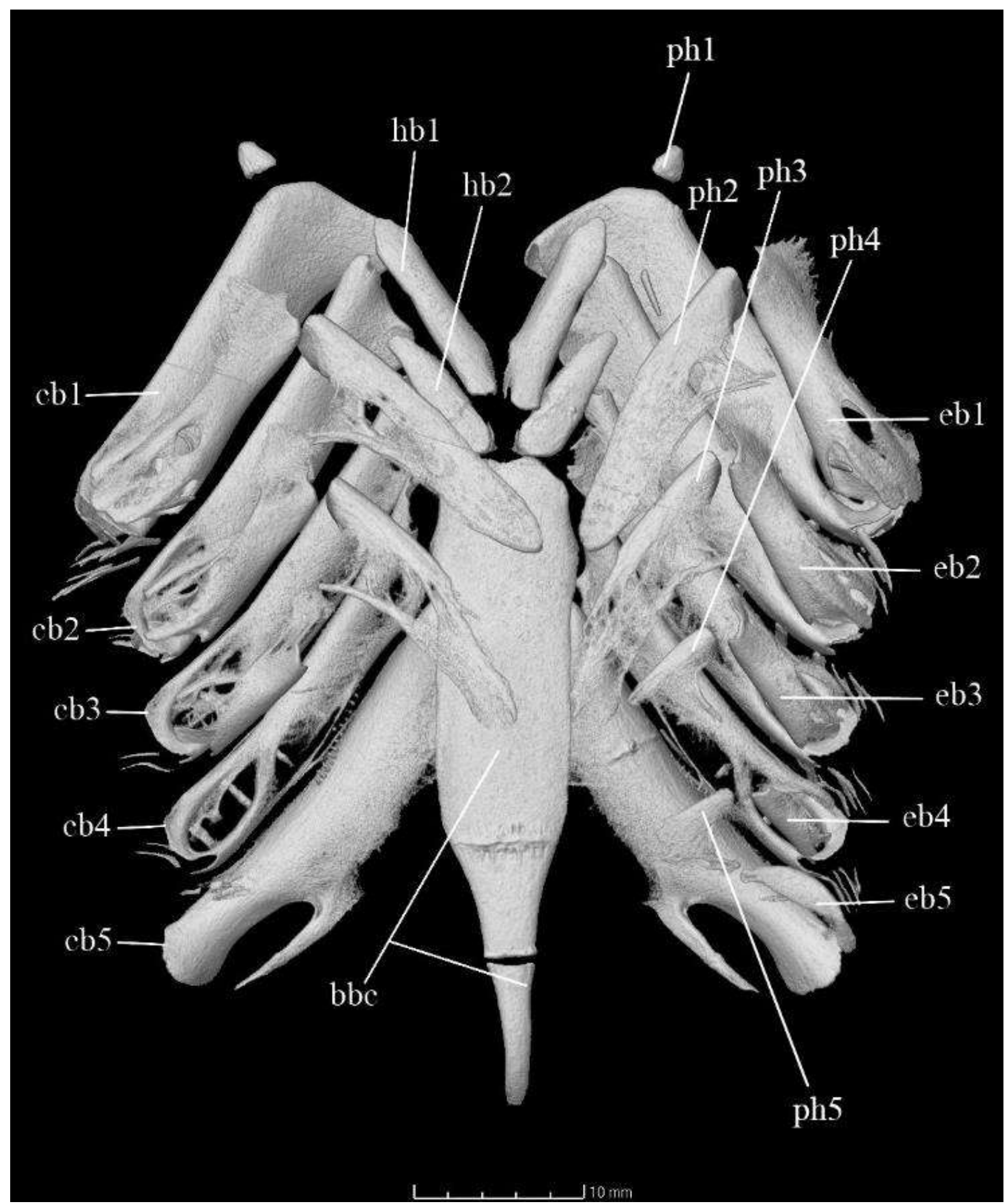

Figure 238. Dorsal view of the branchial arches of a juvenile Isogomphodon oxyrhynchus (MZUSP 101214) obtained with 3D reconstruction from the CT-Scan imaging. Anatomical abbreviations: cb (1-5), ceratobranchial; eb (1-5), epibranchial; bbc, basibranchial copula; hb (1-2), hypobranchial; ph (1-5), pharyngobranchial; 


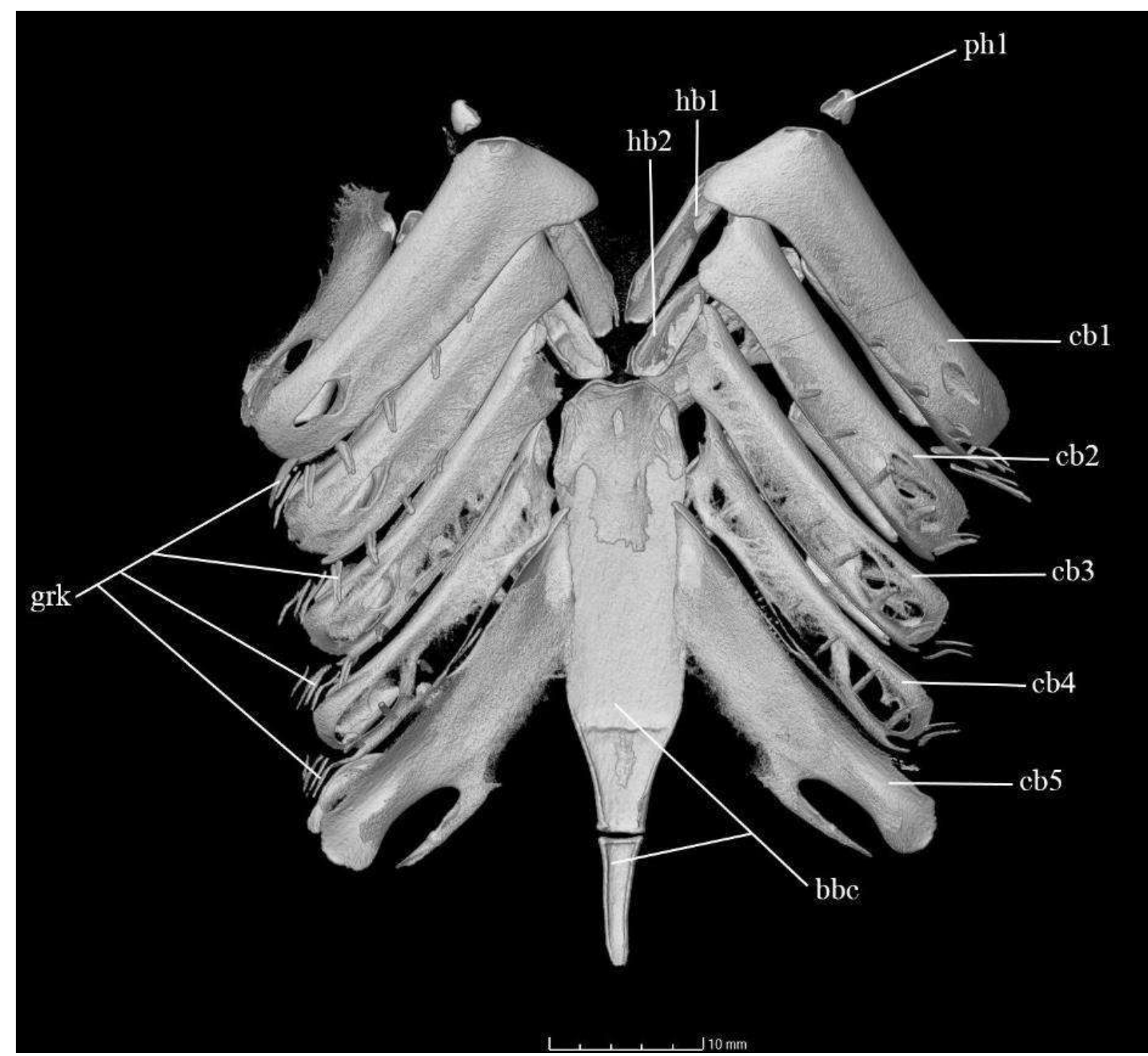

Figure 239. Ventral view of the branchial arches of a juvenile Isogomphodon oxyrhynchus (MZUSP 101214) obtained with 3D reconstruction from the CT-Scan imaging. Anatomical abbreviations: ph1, pharyngobranchial 1; eb (1-5), epibranchial; bbc, basibranchial copula; grk, gill rakers; hb (1-2), hypobranchial. 


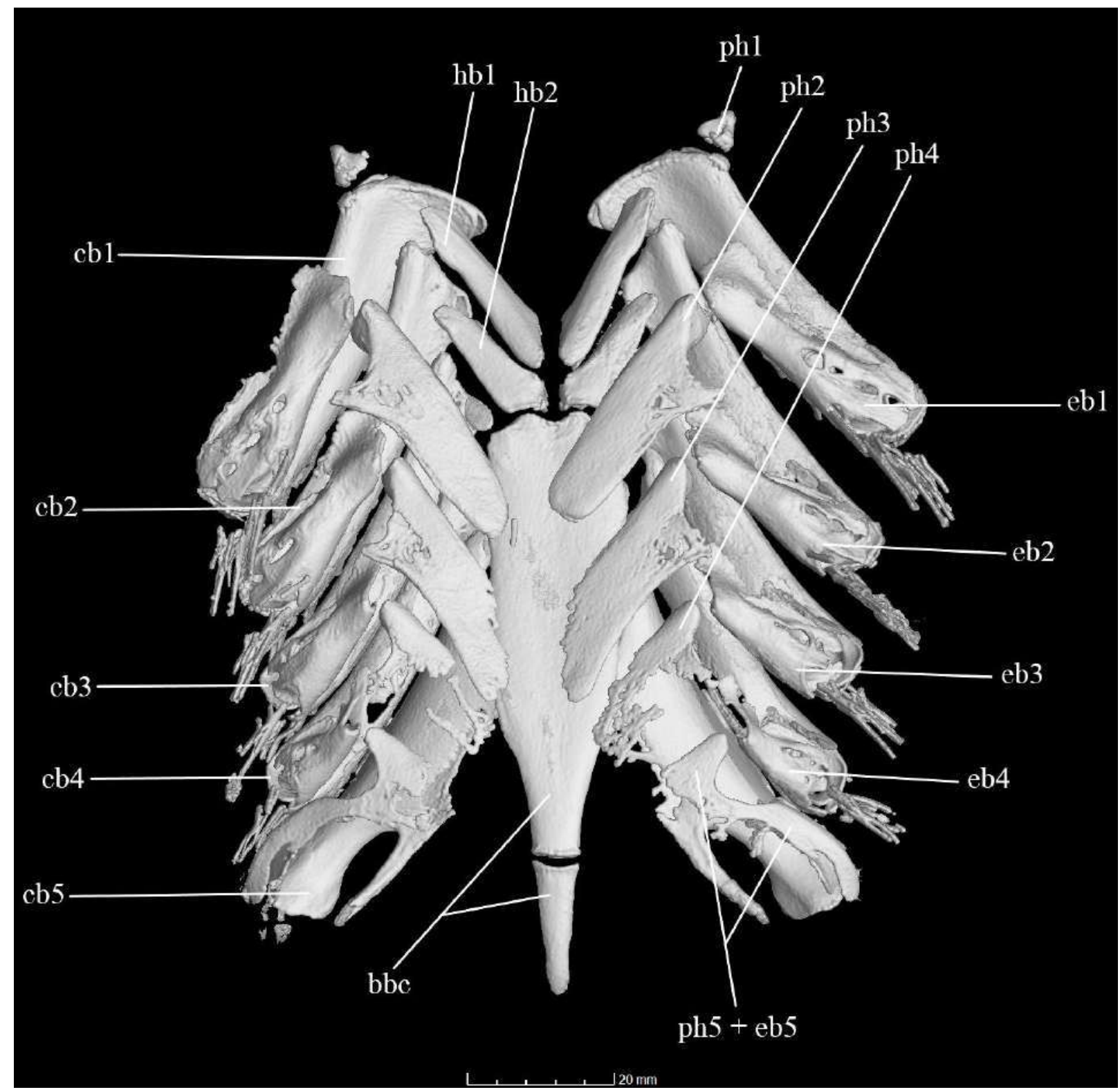

Figure 240. Dorsal view of the branchial arches of an adult Isogomphodon oxyrhynchus (MPEG3455) obtained with 3D reconstruction from the CT-Scan imaging. Anatomical abbreviations: cb (1-5), ceratobranchial; eb (1-5), epibranchial; bbc, basibranchial copula; hb (1-2), hypobranchial; ph (1-5), pharyngobranchial; 


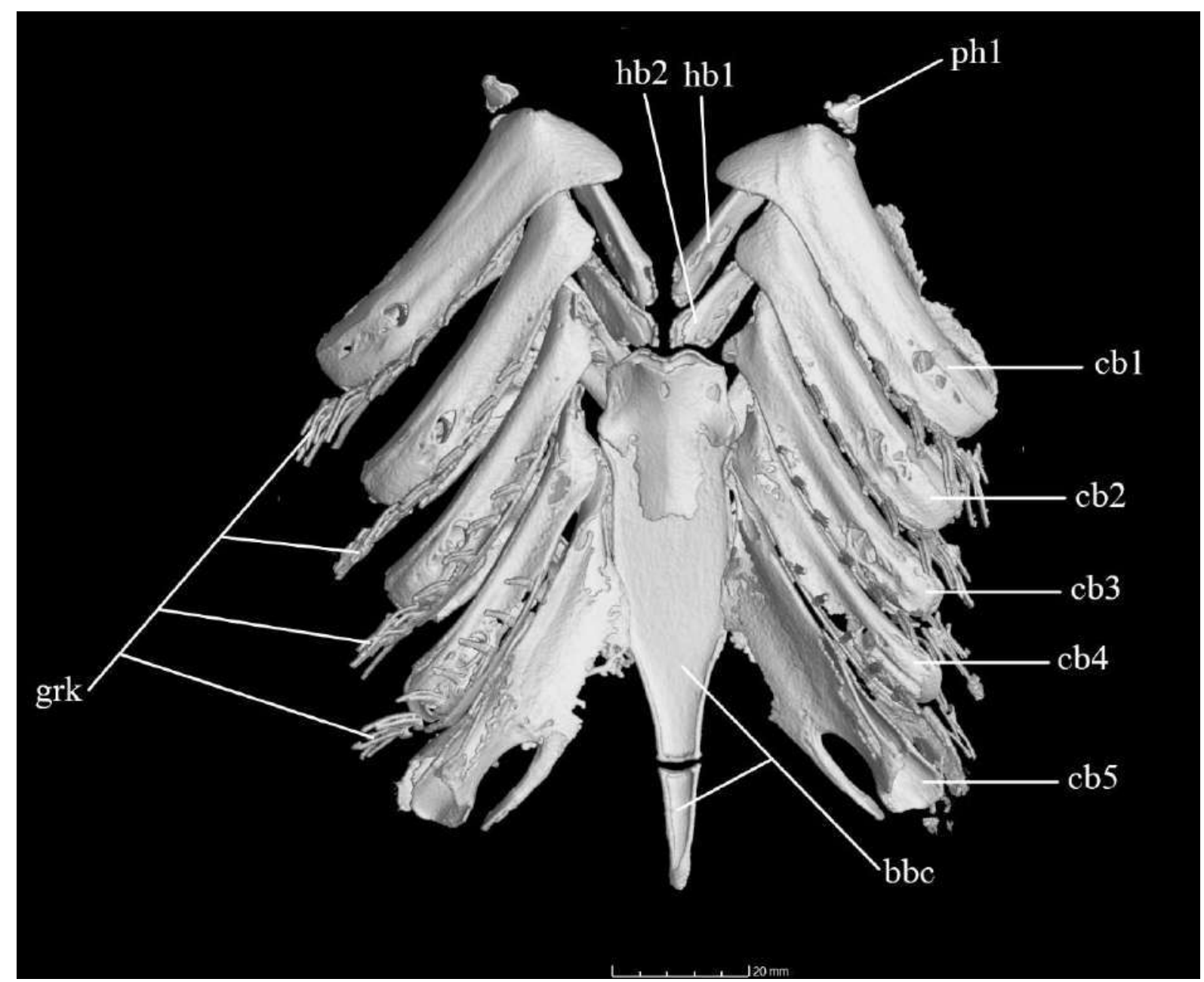

Figure 241. Ventral view of the branchial arches of an adult Isogomphodon oxyrhynchus (MPEG3455) obtained with 3D reconstruction from the CT-Scan imaging. Anatomical abbreviations: ph1, pharyngobranchial 1; eb (15), epibranchial; bbc, basibranchial copula; grk, gill rakers; hb (1-2), hypobranchial 


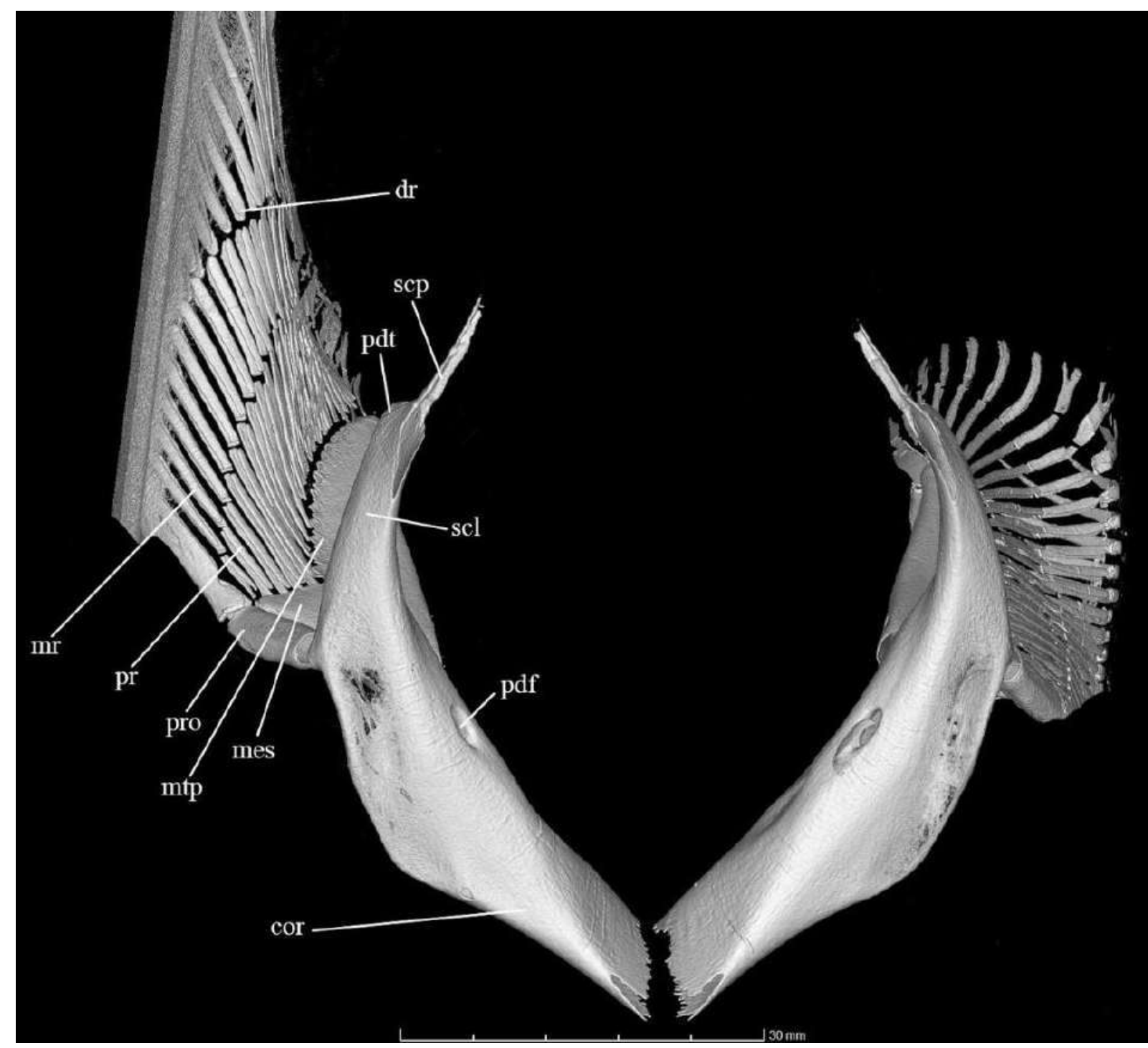

Figure 242. Dorso-frontal view of the pectoral girdle of Isogomphodon oxyrhynchus (MZUSP 101214) obtained with 3D reconstruction from the CT-Scan imaging. Anatomical abbreviations: cor, coracoid bar; df, diazonal foramen; dr, distal radial; fms, facet for mesopterygium; fpr, facet for propterygium; mes, mesopterygium; mr, medial radial; mtp, metapterygium; pdf, post dorsal foramen; pdt, posterodorsal triangular process of scapula; pr, proximal radial; pro, propterygium; scl, scapula; scp, scapular process. 


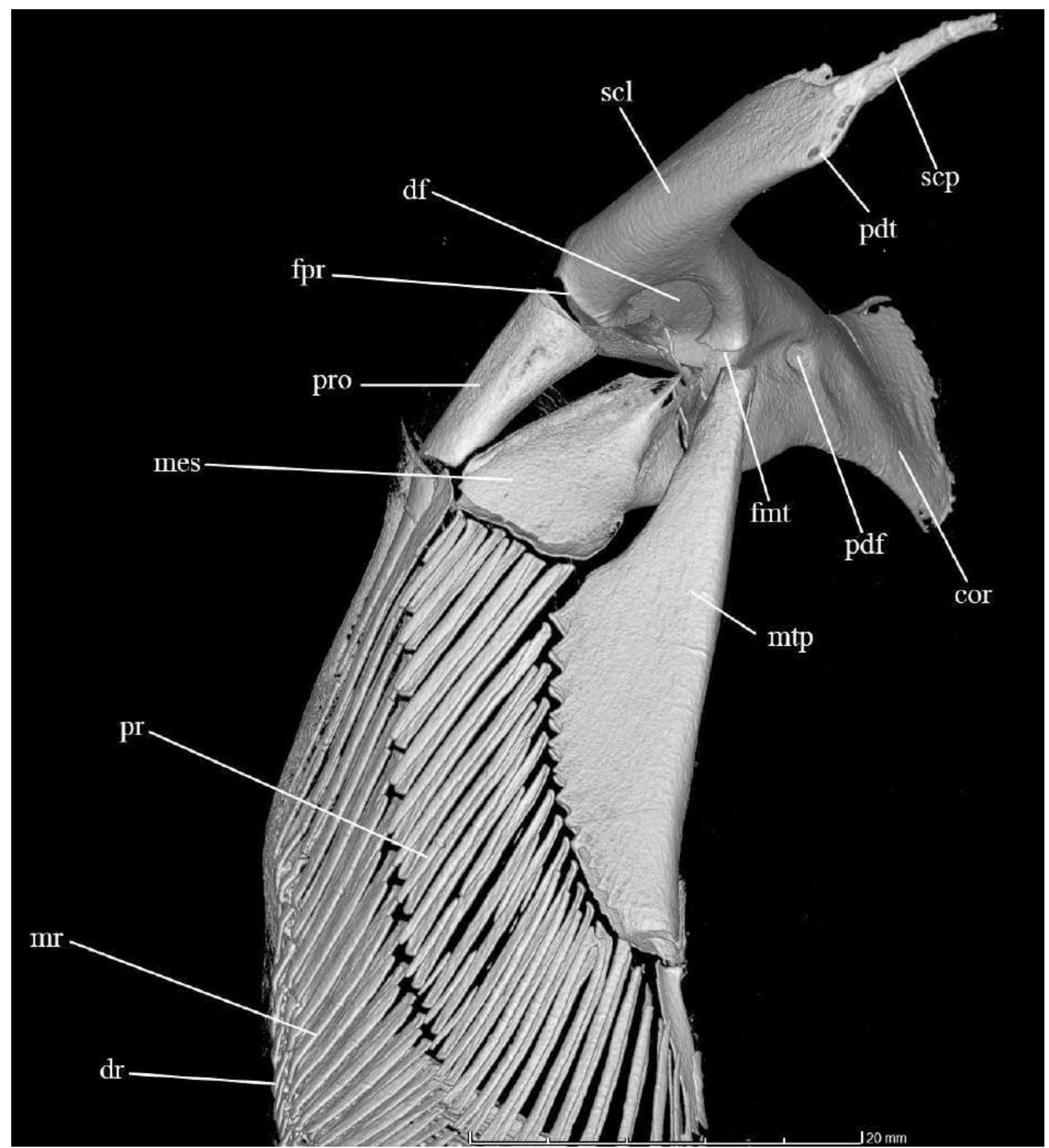

Figure 243. Dorso caudal view of the pectoral girdle of Isogomphodon oxyrhynchus (MZUSP 101214) obtained with 3D reconstruction from the CT-Scan imaging. Anatomical abbreviations: cor, coracoid bar; df, diazonal foramen; dr, distal radial; fmt, facet for metapterygium; fpr, facet for propterygium; mes, mesopterygium; mr, medial radial; mtp, metapterygium; pdf, posterodorsal foramen; pdt, posterodorsal triangular process of scapula; pr, proximal radial; pro, propterygium; scl, scapula; scp, scapular process. 


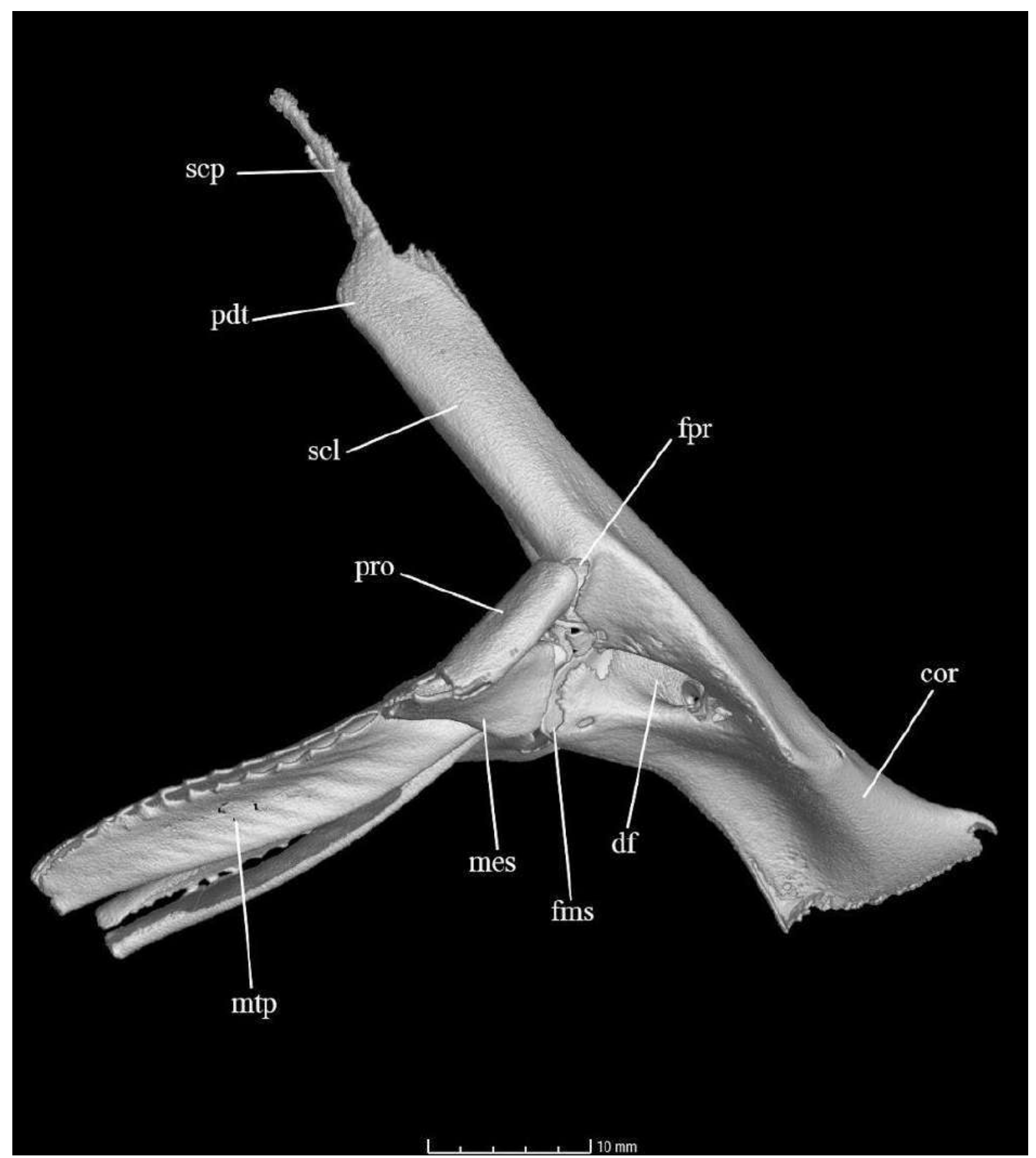

Figure 244. Lateral view of the pectoral girdle of Isogomphodon oxyrhynchus (MZUSP 101214) obtained with 3D reconstruction from the CT-Scan imaging. Anatomical abbreviations: cor, coracoid bar; df, diazonal foramen; fms, facet for mesopterygium; fpr, facet for propterygium; mes, mesopterygium; mtp, metapterygium; pdt, posterodorsal triangular process of scapula; pro, propterygium; scl, scapula; scp, scapular process. 


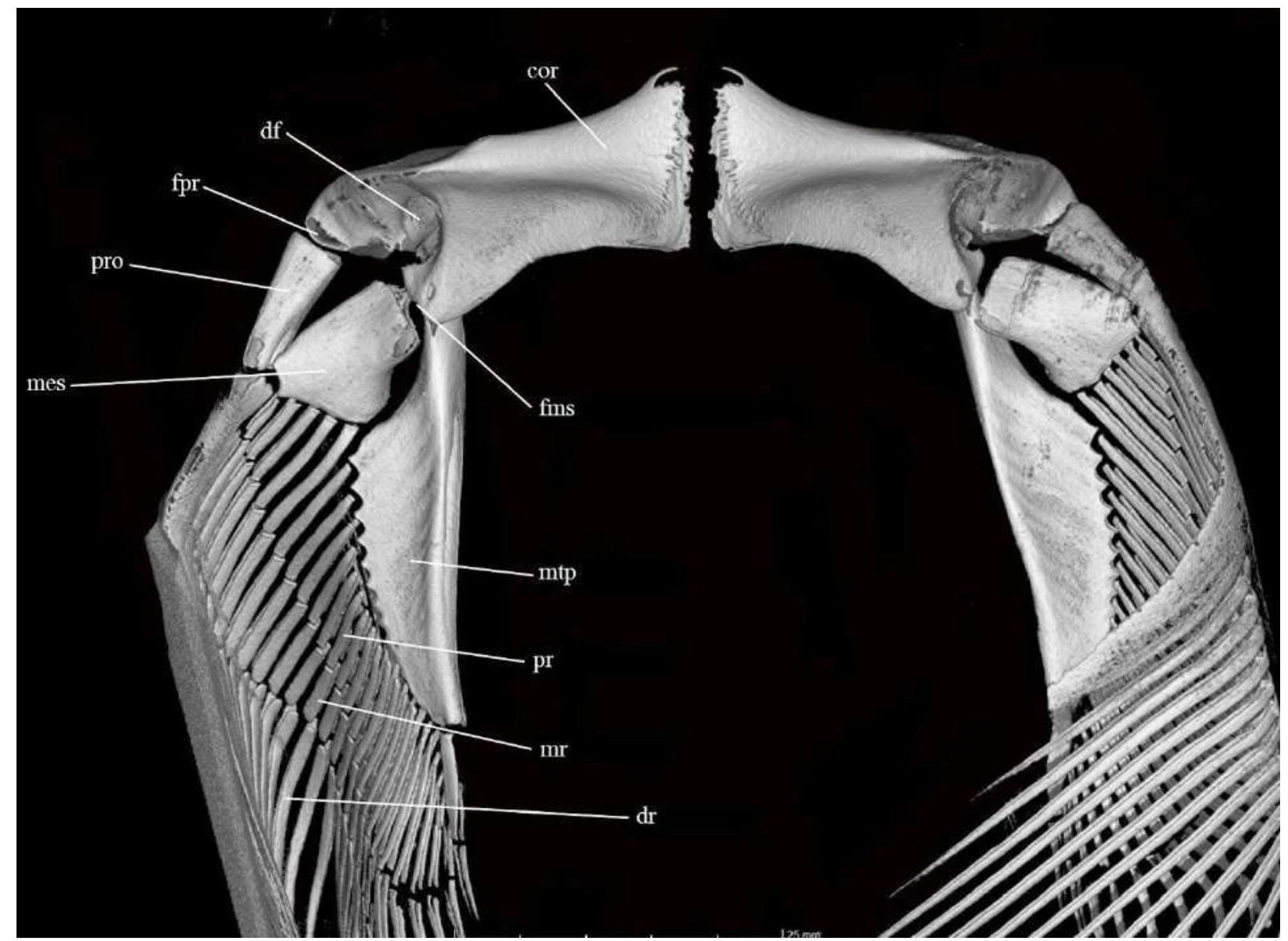

Figure 245. Ventral view of the pectoral girdle of Isogomphodon oxyrhynchus (MZUSP 101214) obtained with 3D reconstruction from the CT-Scan imaging. Anatomical abbreviations: cor, coracoid bar; df, diazonal foramen; dr, distal radial; fms, facet for mesopterygium; fpr, facet for propterygium; mes, mesopterygium; mr, medial radial; mtp, metapterygium; pr, proximal radial; pro, propterygium; 


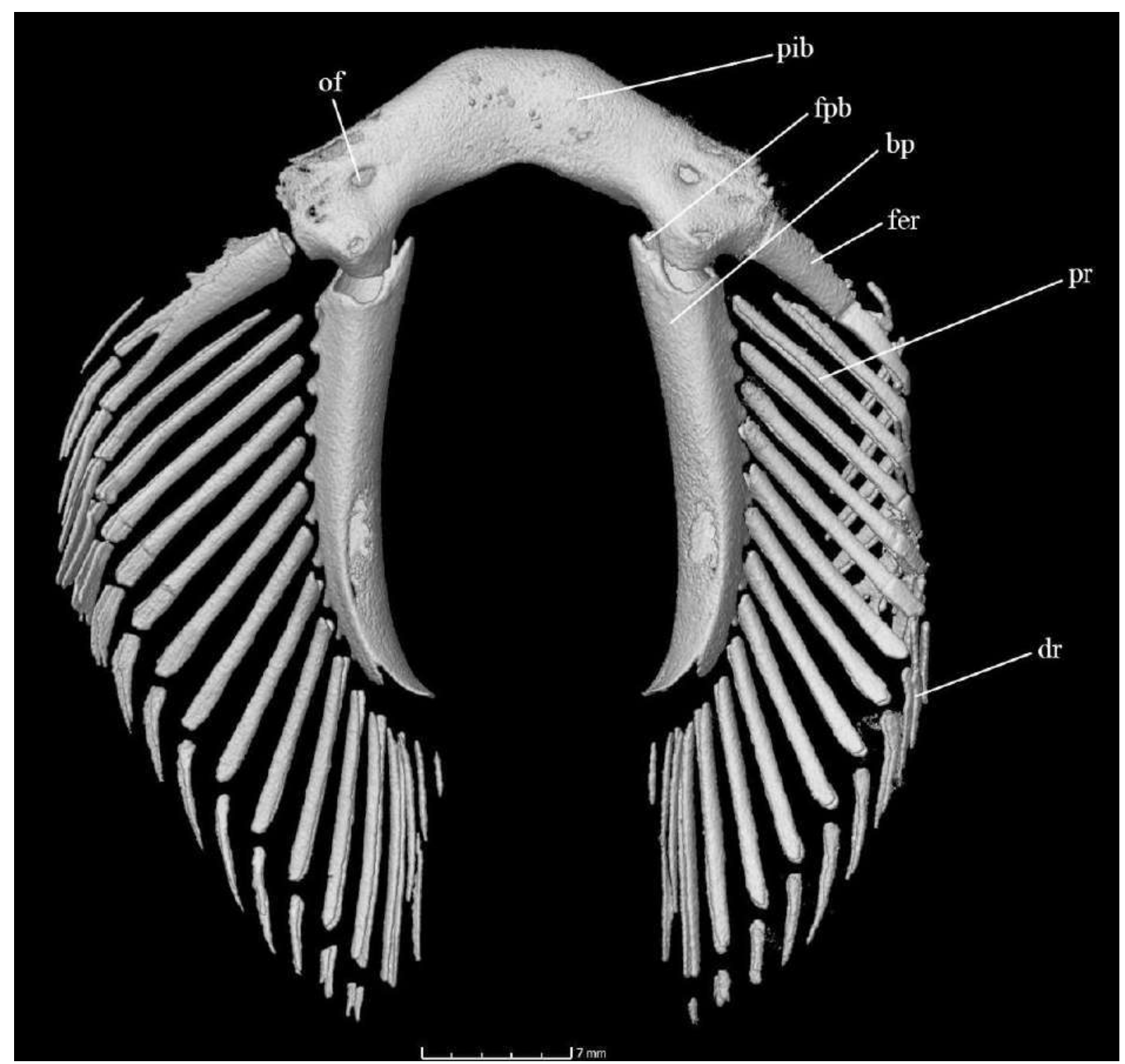

Figure 246. Dorsal view of the pelvic girdle of Isogomphodon oxyrhynchus (MZUSP 101214) obtained with 3D reconstruction from the CT-Scan imaging. Anatomical abbreviations: bp, basipterygium; dr, distal radial; fpb, facet for puboischial bar; fer, first enlarged proximal radial; of, obturator foramen; pib, puboischial bar; pr, proximal radial. 


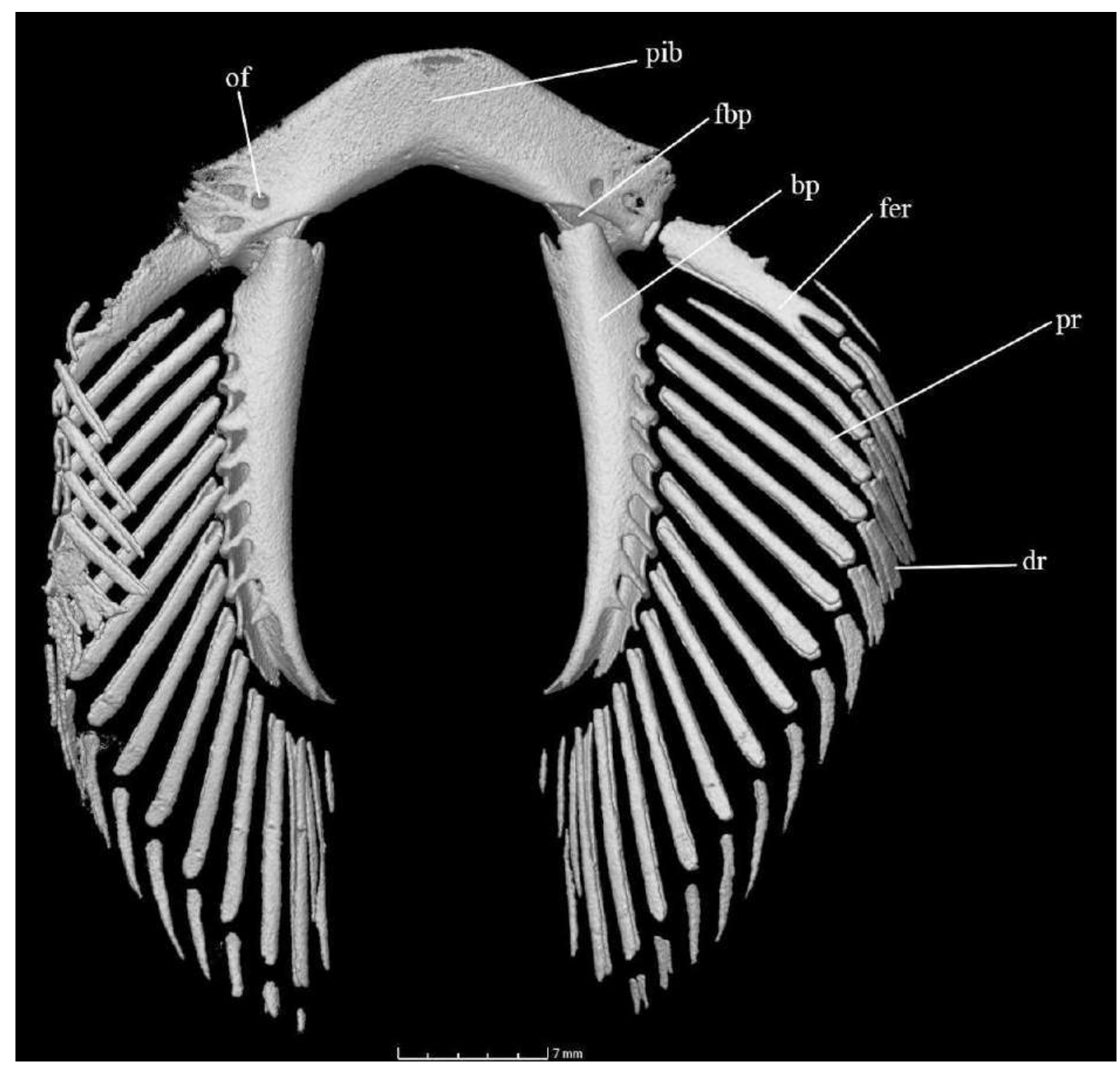

Figure 247. Ventral view of the pelvic girdle of Isogomphodon oxyrhynchus (MZUSP 101214) obtained with 3D reconstruction from the CT-Scan imaging. Anatomical abbreviations: bp, basipterygium; dr, distal radial; fbp, facet for basipterygium; fer, first enlarged proximal radial; of, obturator foramen; pib, puboischial bar; pr, proximal radial. 


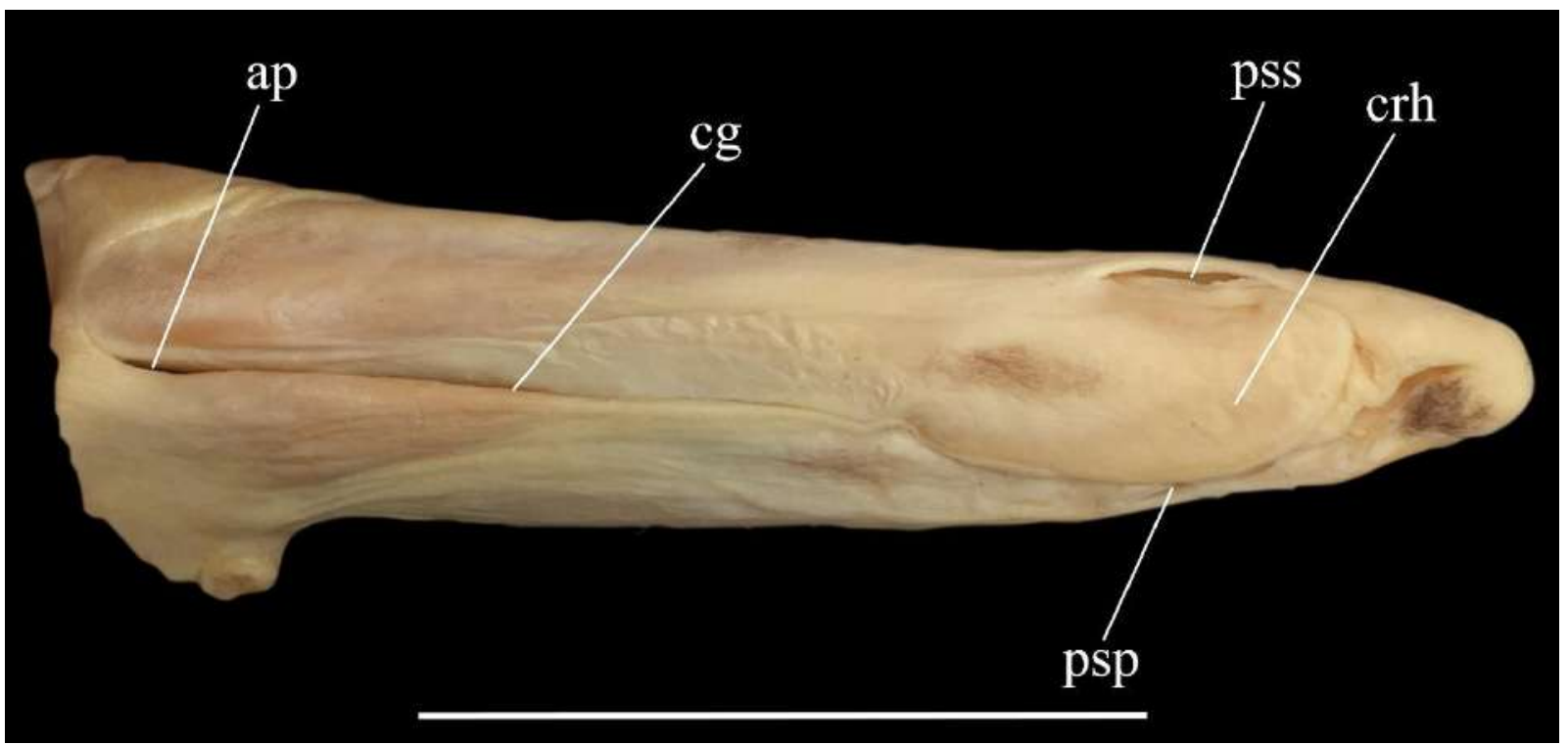

Figure 248. Dorsal view of the external structure of the left clasper of Isogomphodon oxyrhynchus (MZUSP 101214) with glans not dilated. Anatomical abbreviations: ap, apopyle; cg, clasper groove; crh, cover rhipidion; psp, pseudopera; pss, pseudoshiphon; (Scale bar $=475 \mathrm{~mm}$ )

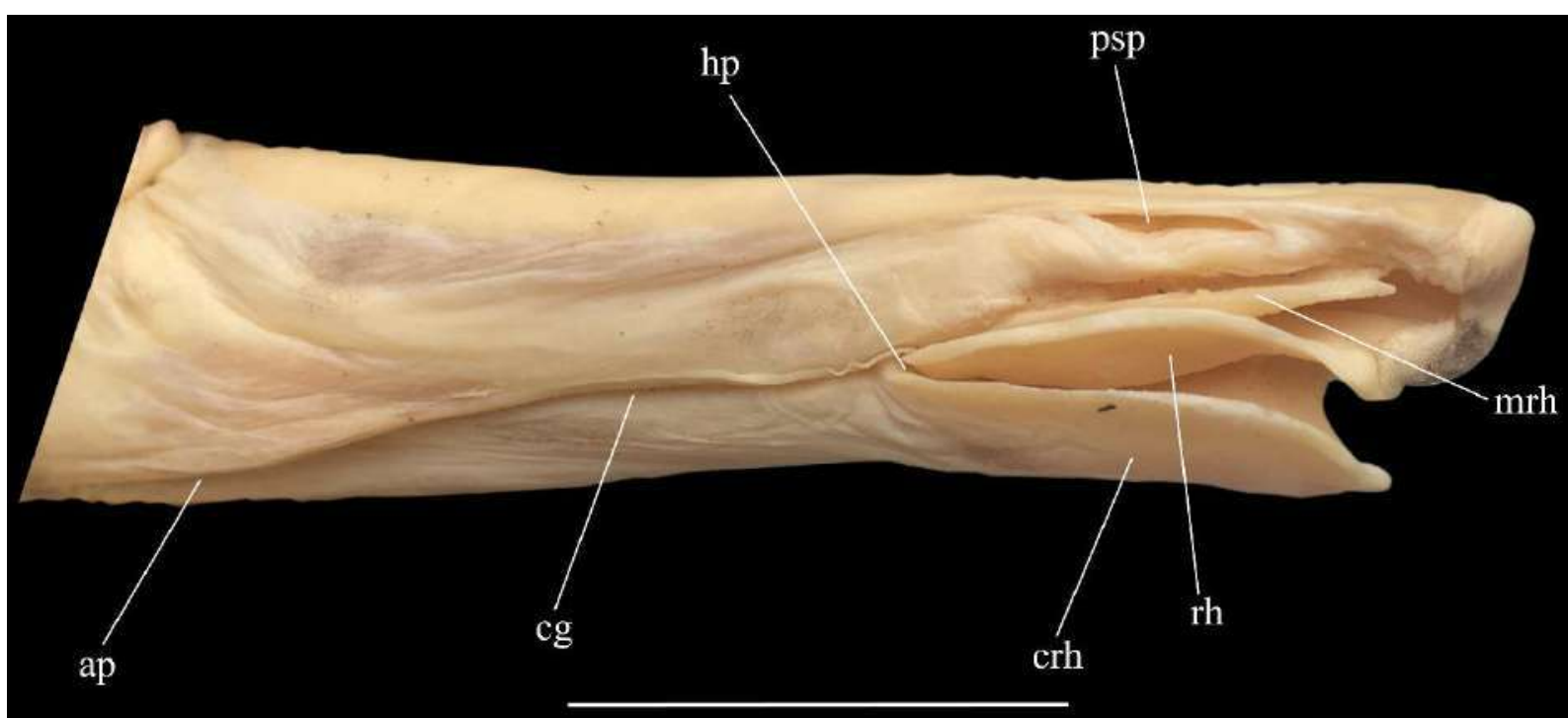

Figure 249. Dorsal view of the external structure of the right clasper of Isogomphodon oxyrhynchus (MZUSP 101214) illustrating the dilated glans. Anatomical abbreviations: ap, apopyle; cg, clasper groove; crh, cover rhipidion; hp, hypopyle; mrh, mesorhipidion; psp, pseudopera; rh, rhipidion. (Scale bar $=250 \mathrm{~mm}$ ) 


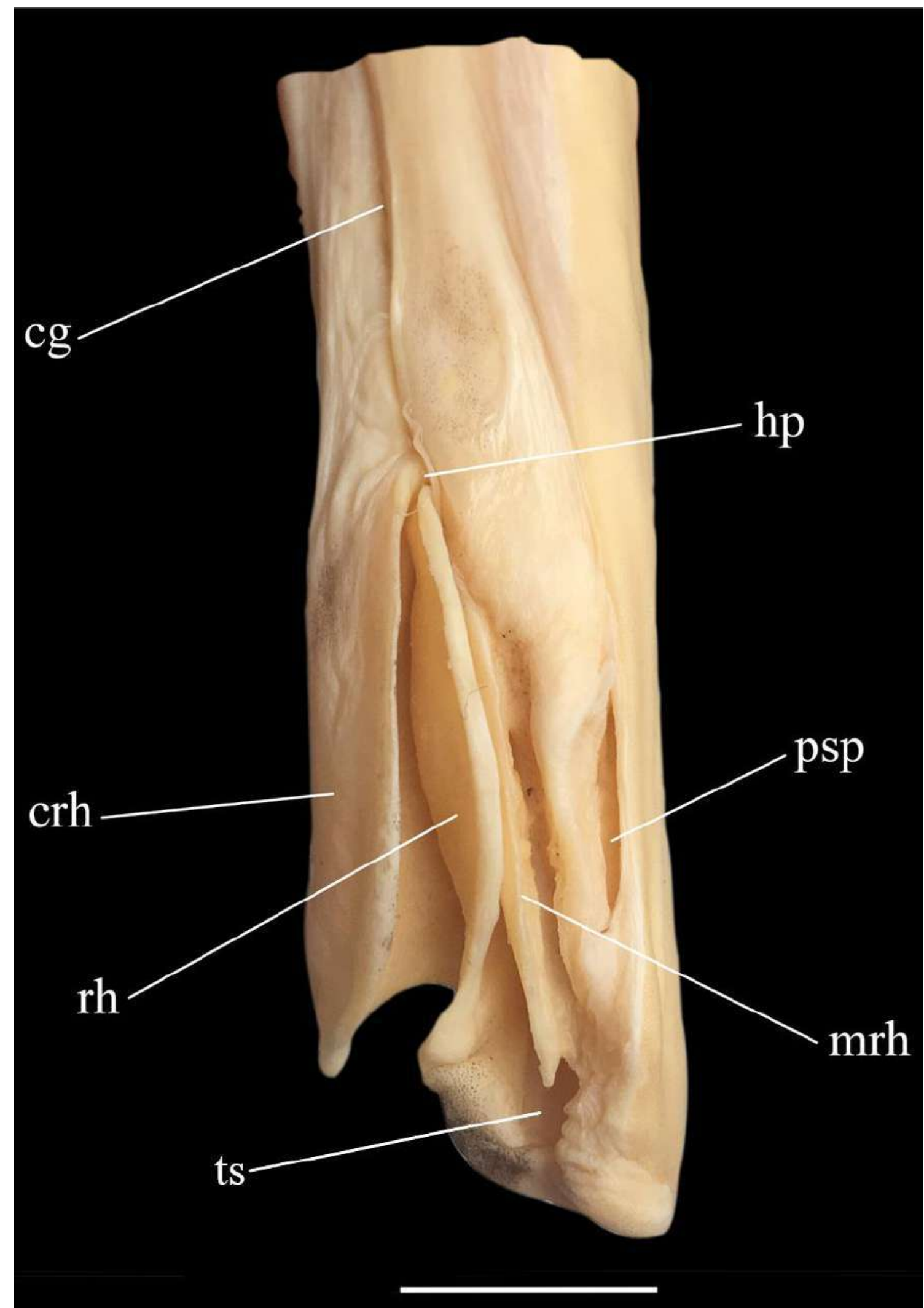

Figure 250. Dorsal view of the external structure of the right clasper of Isogomphodon oxyrhynchus (MZUSP 101214) focusing on the dilated glans. Anatomical abbreviations: ap, apopyle; cg, clasper groove; crh, cover rhipidion; hp, hypopyle; mrh, mesorhipidion; psp, pseudopera; rh, rhipidion; ts, 'terminal sulcus'. (Scale bar = 100 $\mathrm{mm}$ ) 


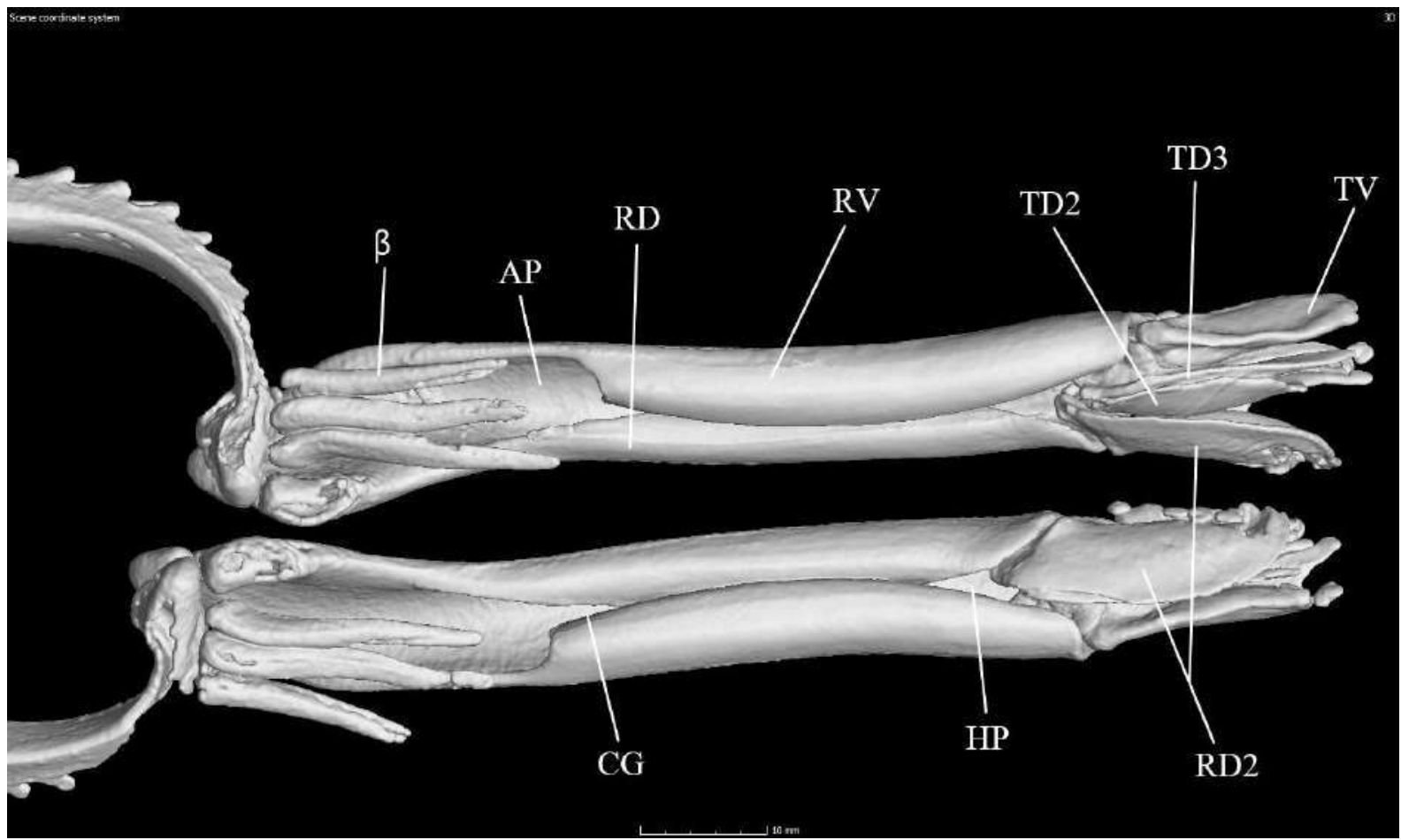

Figure 251. Dorsal view of the internal structure of the clasper of Isogomphodon oxyrhynchus (MZUSP 101214) obtained with 3D reconstruction from the CT-Scan imaging. Anatomical abbreviations: AP, apopyle; CG, clasper groove; HP, hypopyle; RD, dorsal marginal cartilage; RD2, accessory dorsal marginal cartilage (inside cover rhipidion); RV, ventral marginal cartilage; TD2, dorsal terminal 2 cartilage; TD3, accessory terminal cartilage (inside mesorhipidion); TV, ventral terminal cartilage; $\beta$, beta cartilage; 


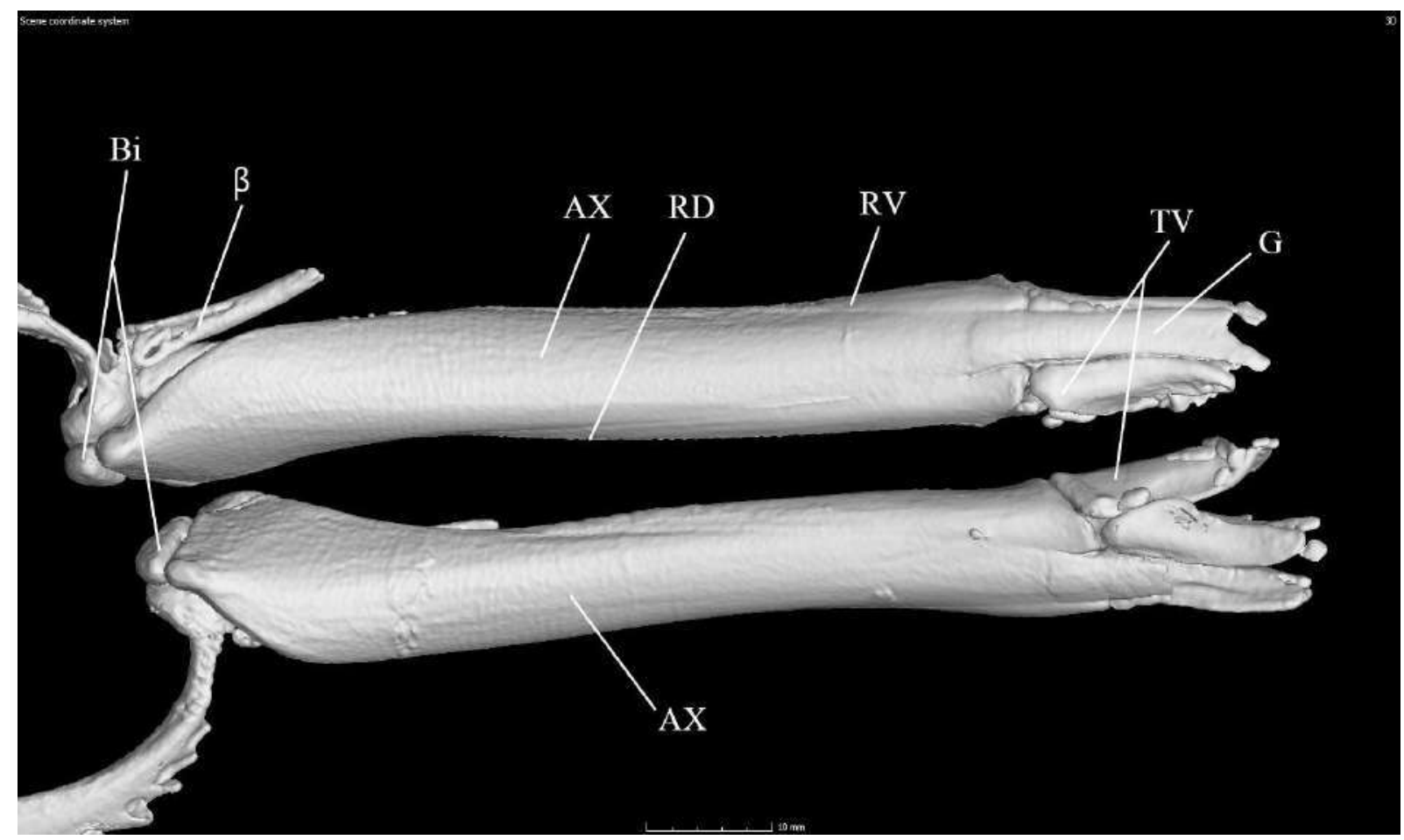

Figure 252. Ventral view of the internal structure of the clasper of Isogomphodon oxyrhynchus (MZUSP 101214) obtained with 3D reconstruction from the CT-Scan imaging. Anatomical abbreviations: AX, axial cartilage or appendix stem; Bi, Basipterygium insertion; G, end-style; RD, dorsal marginal cartilage; RV, ventral marginal cartilage; TV, ventral terminal cartilage; $\beta$, beta cartilage;

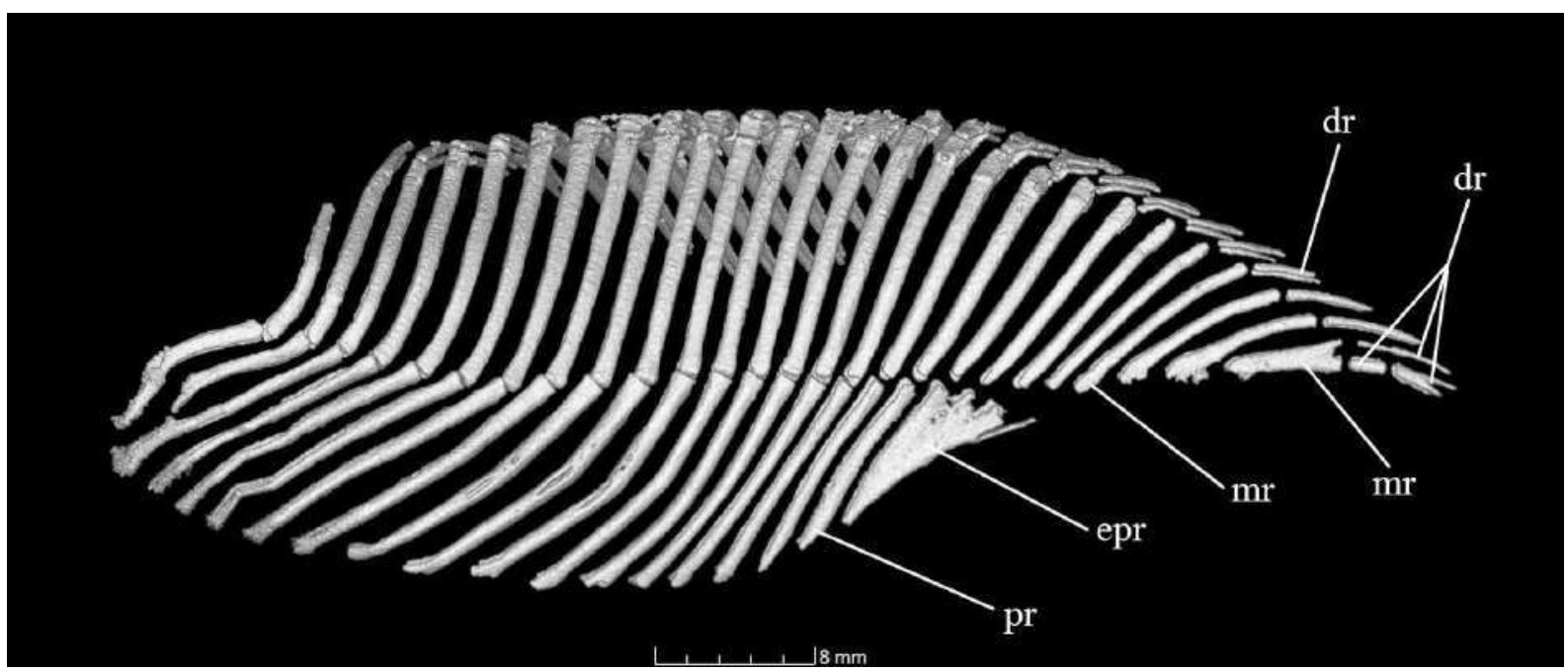

Figure 253. First dorsal fin of Isogomphodon oxyrhynchus (MZUSP 101214) obtained with 3D reconstruction from the CT-Scan imaging. Anatomical abbreviations: dr, distal radial; epr, expanded proximal radial; mr, medial radial; pr, proximal radial; 


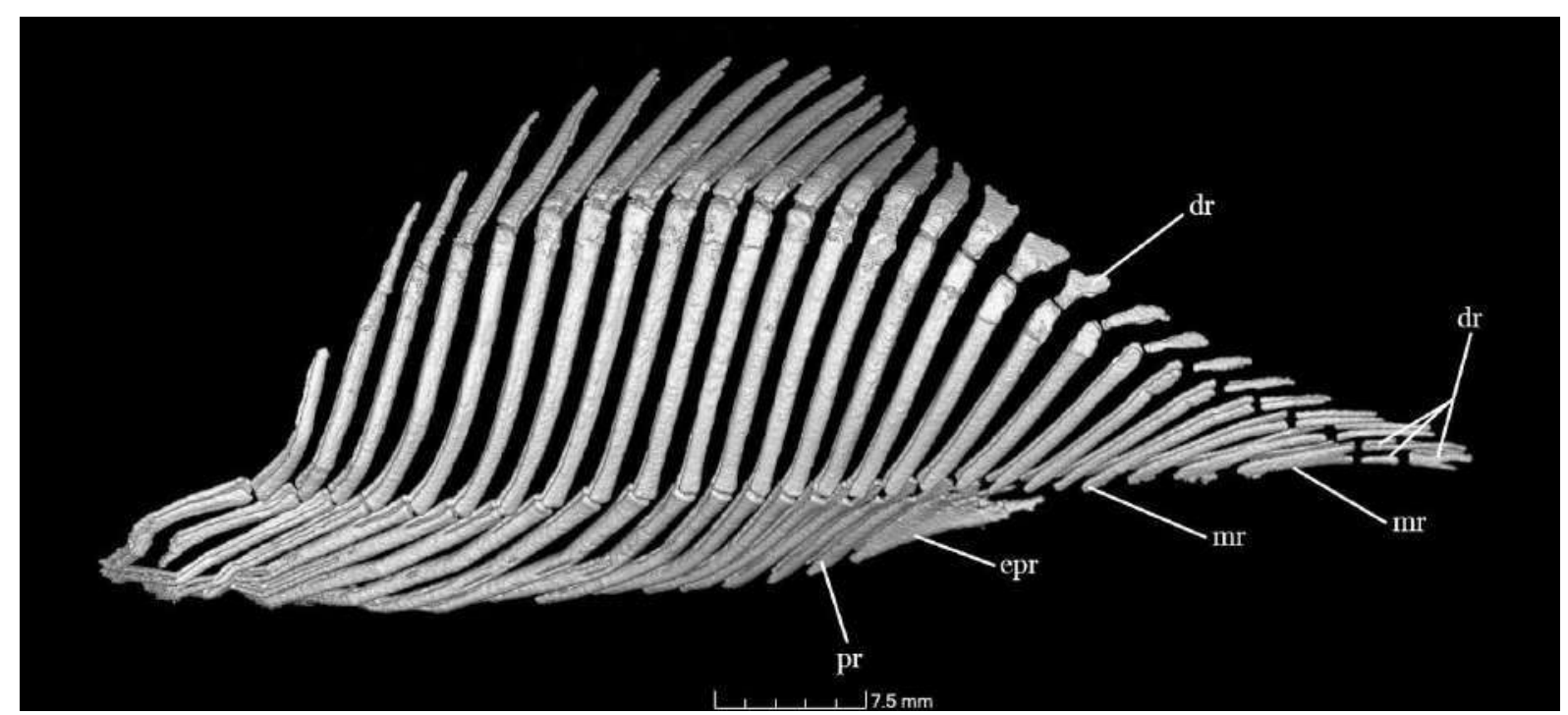

Figure 254. First dorsal fin of Isogomphodon oxyrhynchus (MZUSP 101214) obtained with 3D reconstruction from the CT-Scan imaging. Anatomical abbreviations: dr, distal radial; epr, expanded proximal radial; mr, medial radial; pr, proximal radial;

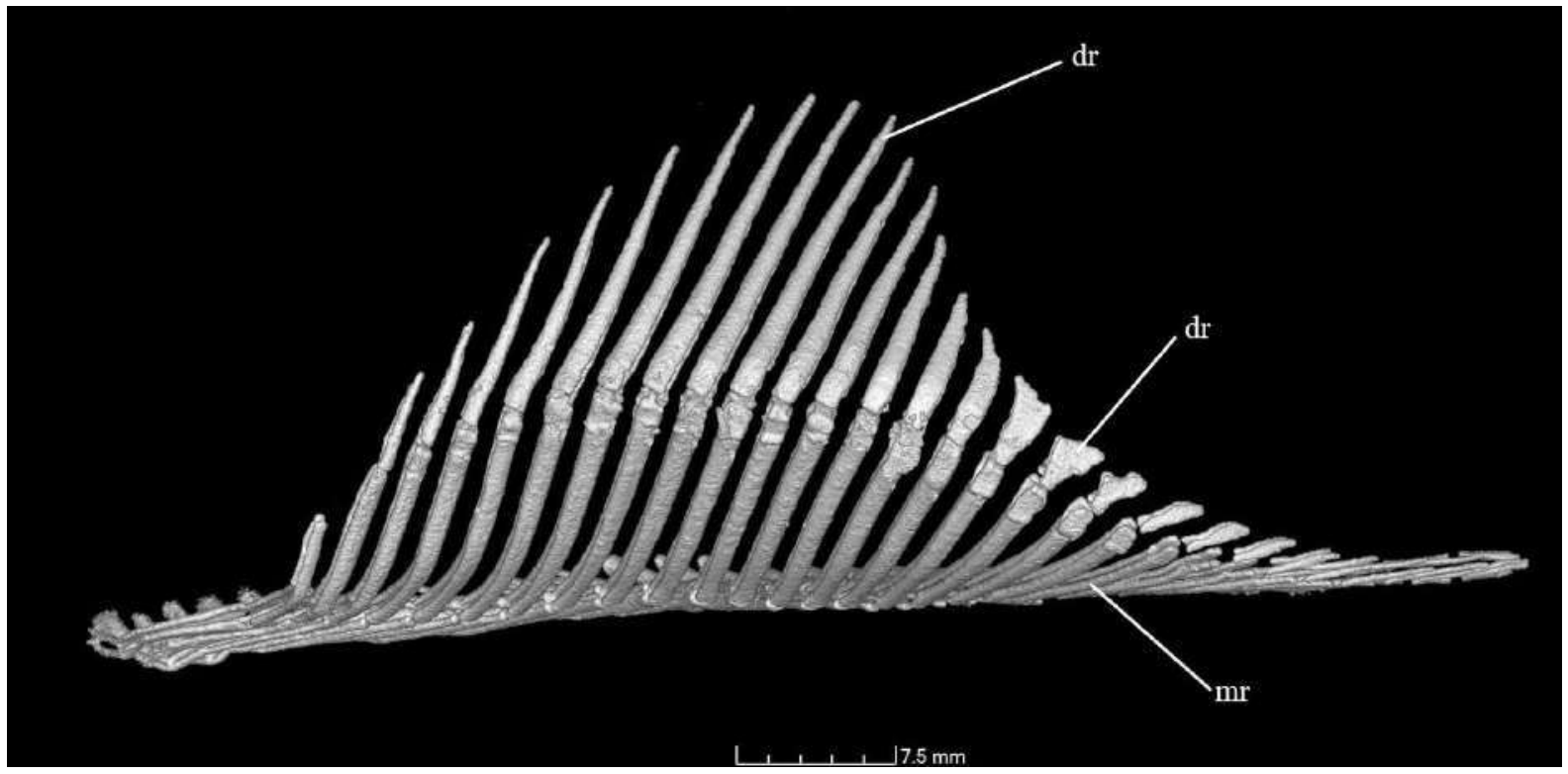

Figure 255. First dorsal fin of Isogomphodon oxyrhynchus (MZUSP 101214) obtained with 3D reconstruction from the CT-Scan imaging. Anatomical abbreviations: dr, distal radial; mr, medial radial; 


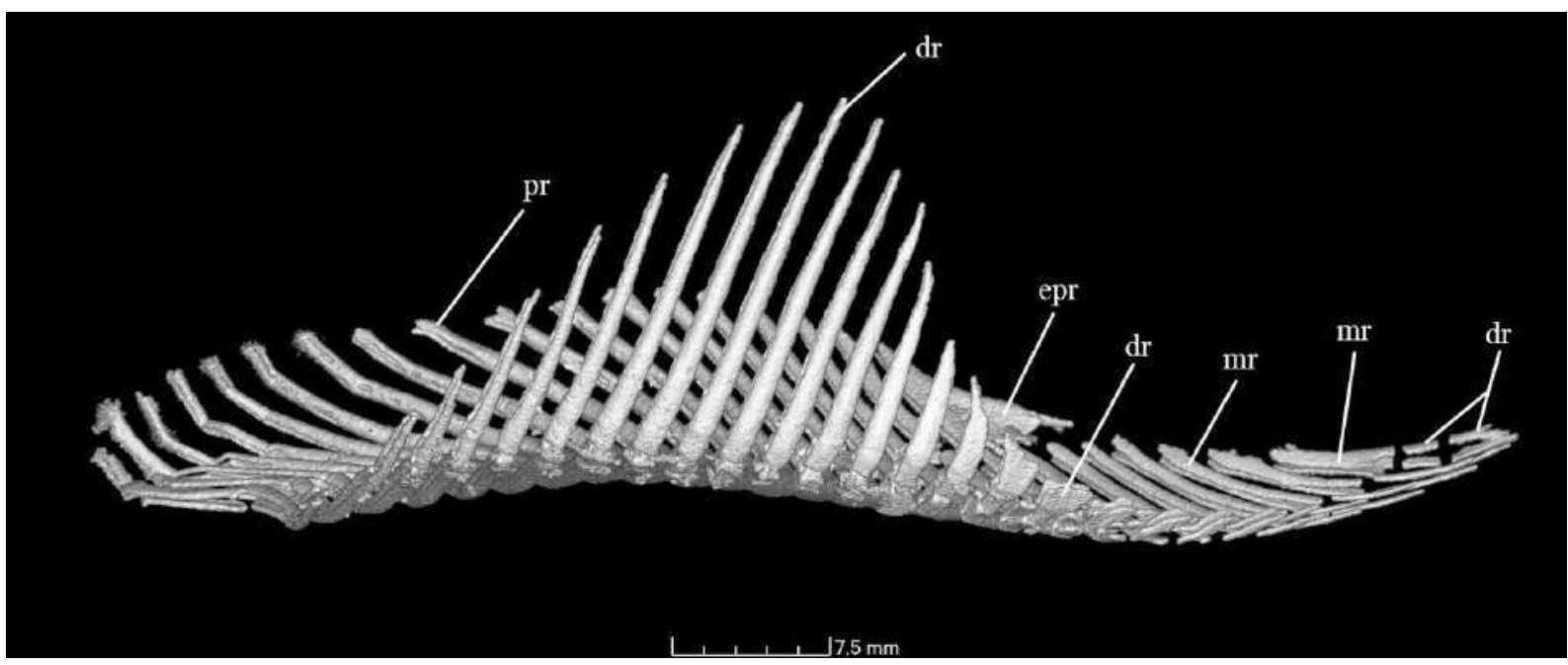

Figure 256. First dorsal fin of Isogomphodon oxyrhynchus (MZUSP 101214) obtained with 3D reconstruction from the CT-Scan imaging. Anatomical abbreviations: dr, distal radial; epr, expanded proximal radial; mr, medial radial; pr, proximal radial;

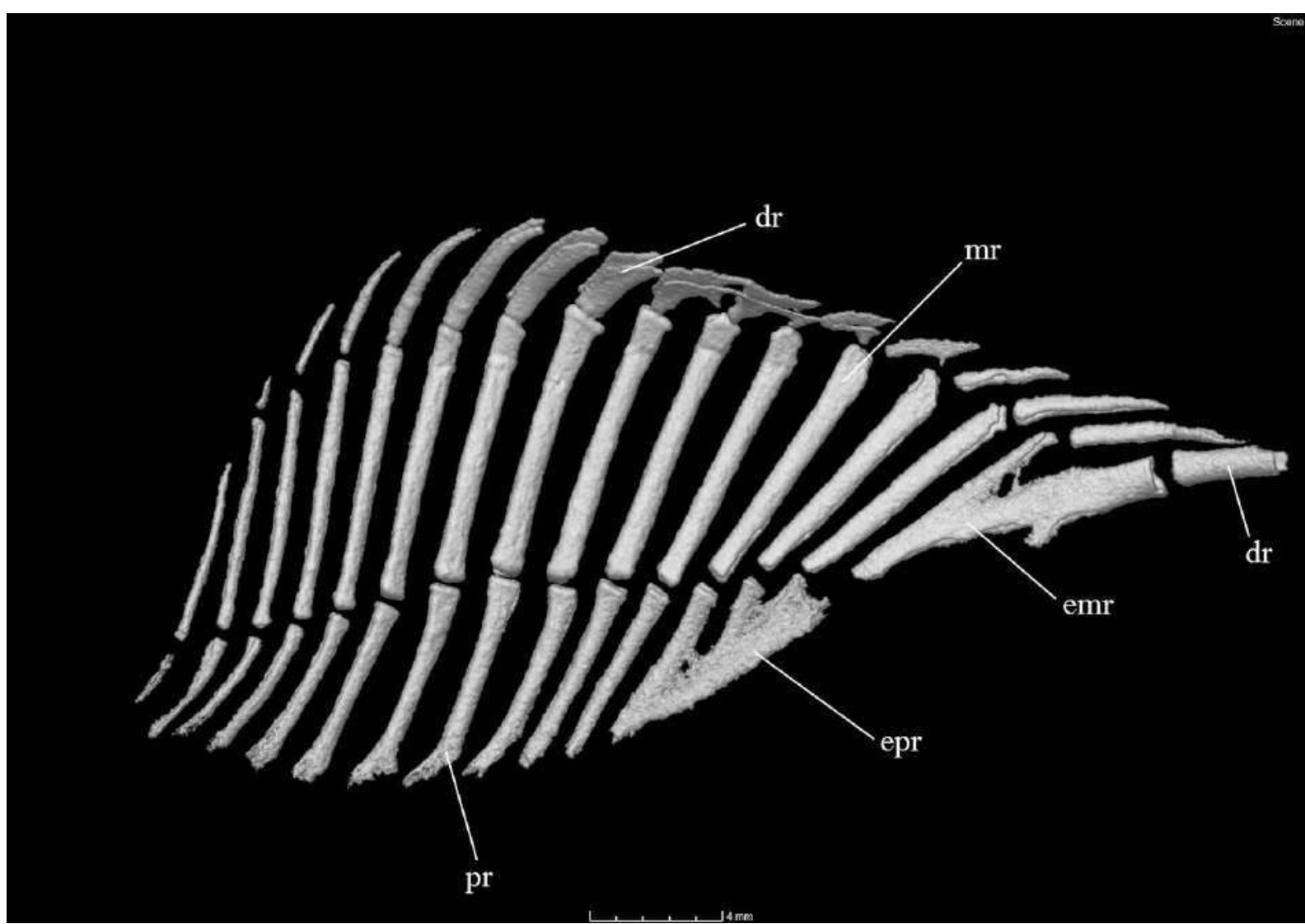

Figure 257. Second dorsal fin of Isogomphodon oxyrhynchus (MZUSP 101214) obtained with 3D reconstruction from the CT-Scan imaging. Anatomical abbreviations: dr, distal radial; emr, expanded medial radial; epr, expanded proximal radial; mr, medial radial; pr, proximal radial; 


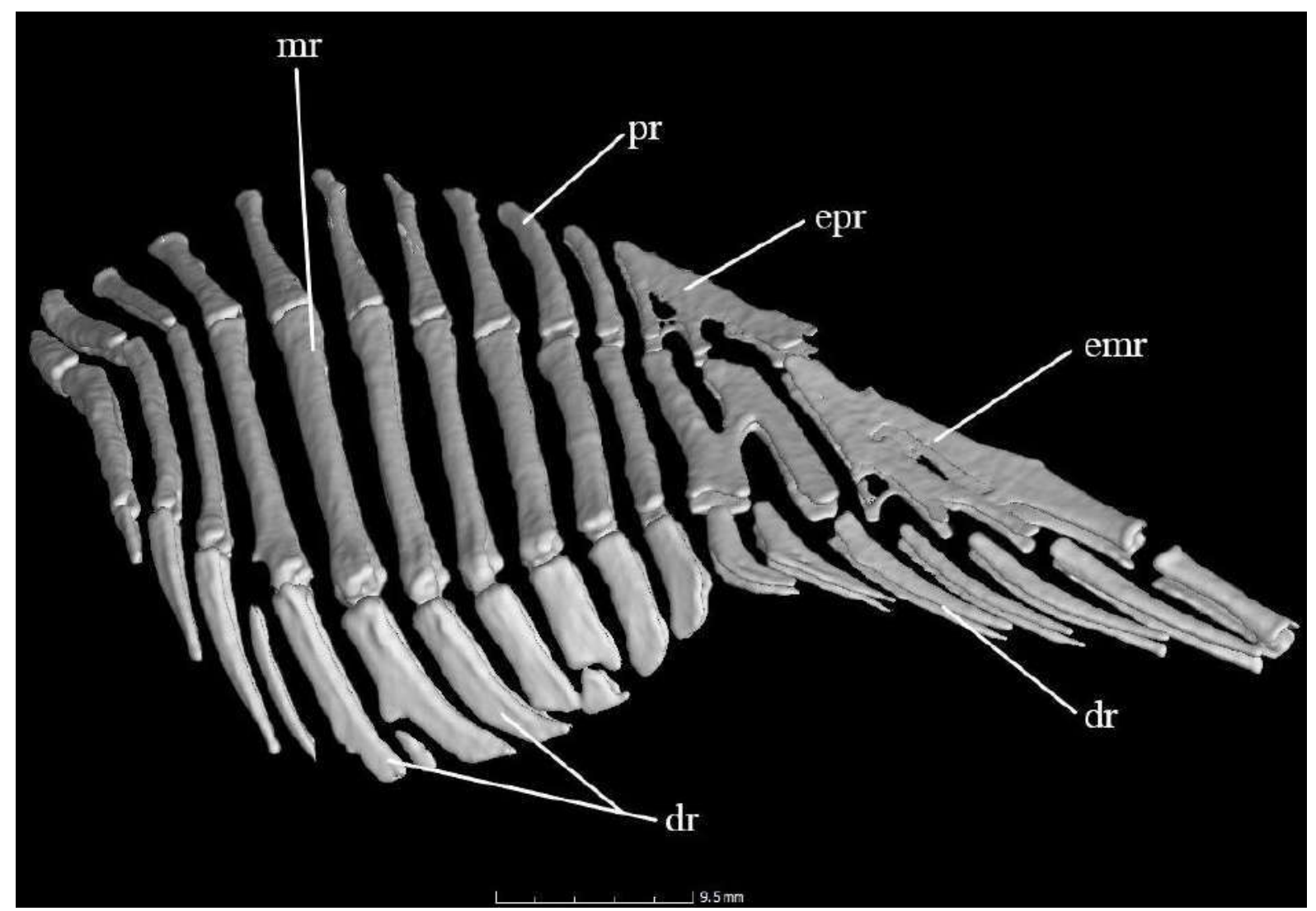

Figure 258. Anal fin of an adult Isogomphodon oxyrhynchus (MPEG 3455) obtained with 3D reconstruction from the CT-Scan imaging. Anatomical abbreviations: dr, distal radial; emr, expanded medial radial; epr, expanded proximal radial; mr, medial radial; pr, proximal radial;

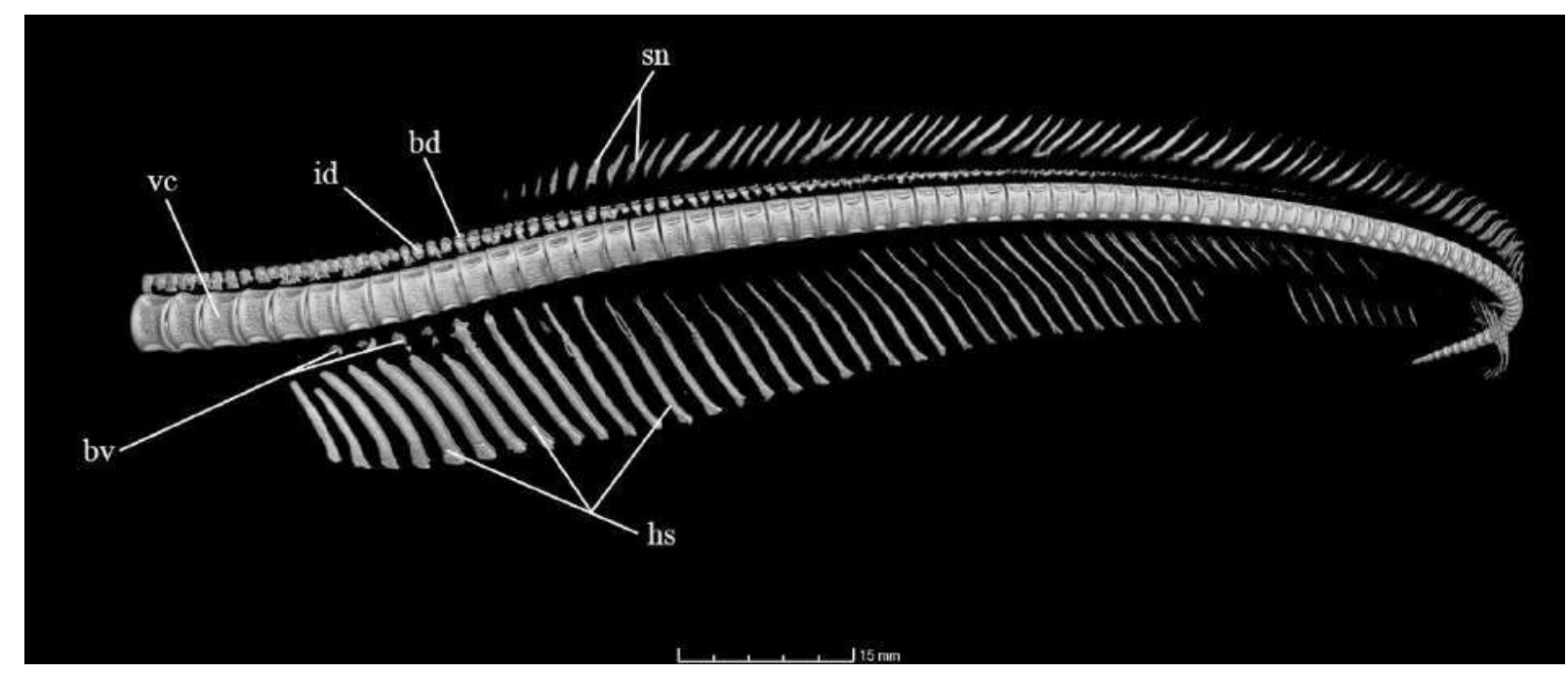

Figure 259. Caudal fin of Isogomphodon oxyrhynchus (MZUSP 101214) obtained with 3D reconstruction from the CT-Scan imaging. Anatomical abbreviations: bd, basidorsal; bv, basiventral; hs, haemal spine; id, interdorsal; sn, supraneural; vc, vertebral centrum. 


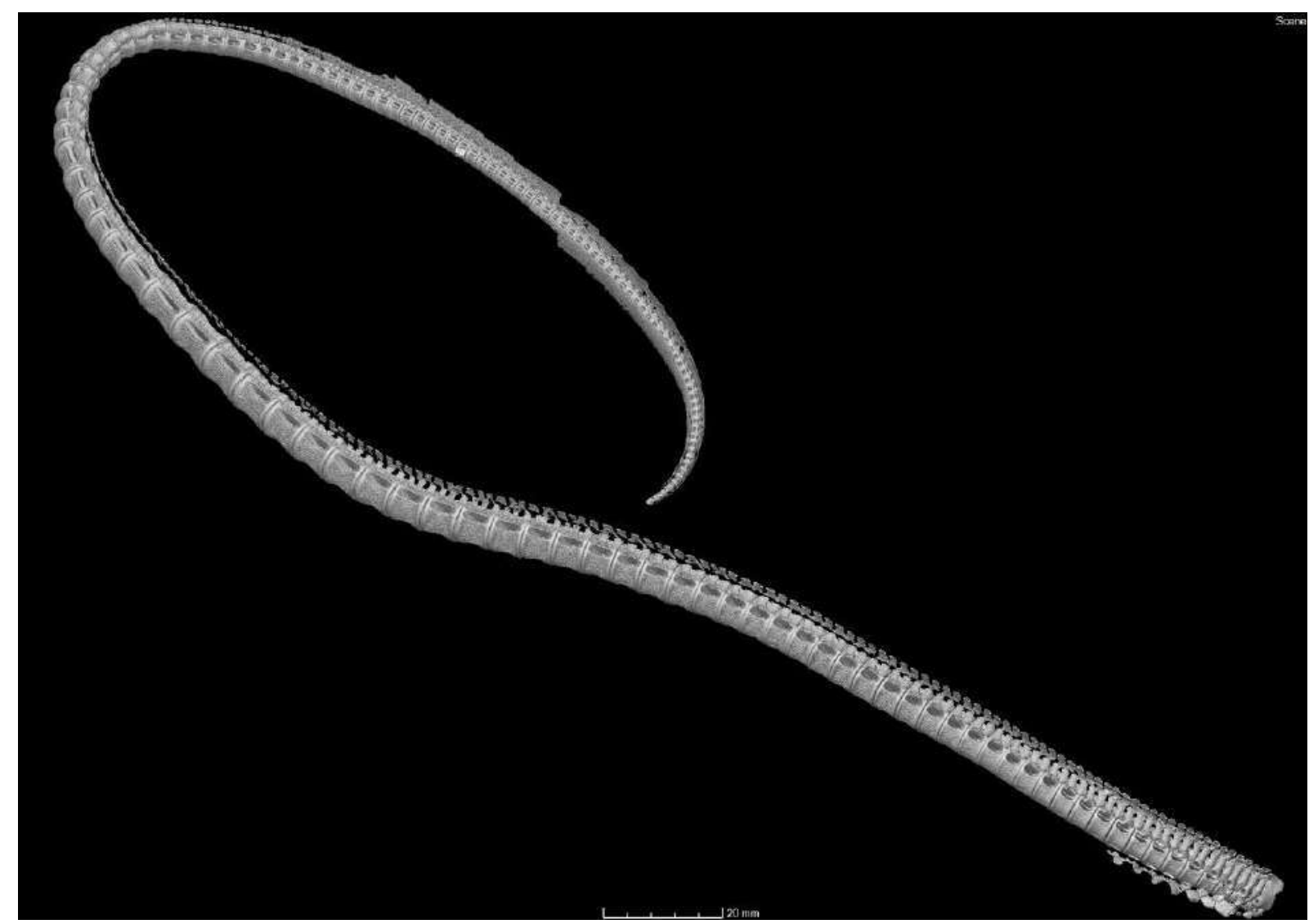

Figure 260. General view of the vertebral column of Isogomphodon oxyrhynchus (MZUSP 101214) obtained with $3 \mathrm{D}$ reconstruction from the CT-Scan imaging. 


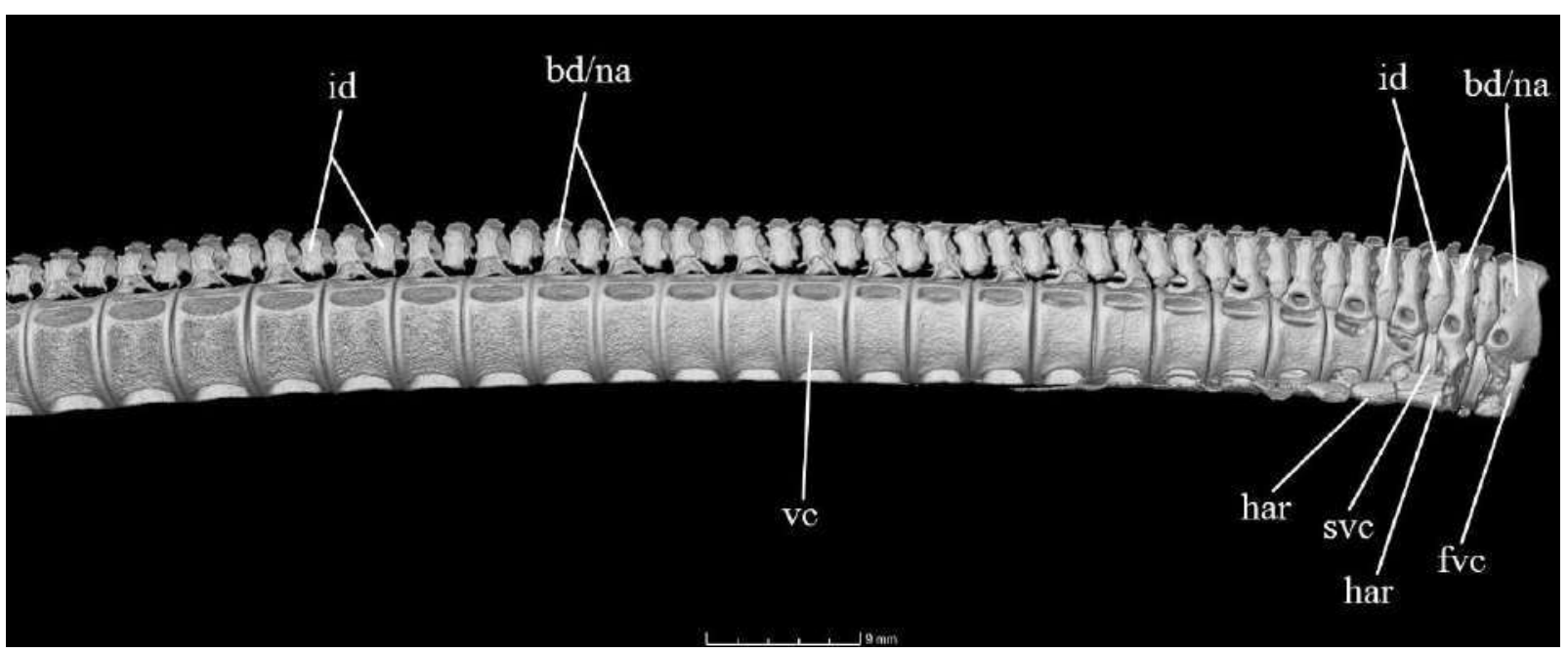

Figure 261. Caudal fin of Isogomphodon oxyrhynchus (MZUSP 101214) obtained with 3D reconstruction from the CT-Scan imaging. Anatomical abbreviations: bd, basidorsal; fvc, first vertebral centrum; har, haemal arch; hs, haemal spine; id, interdorsal; na, neural arch; svc, second vertebral centrum; vc, vertebral centrum;

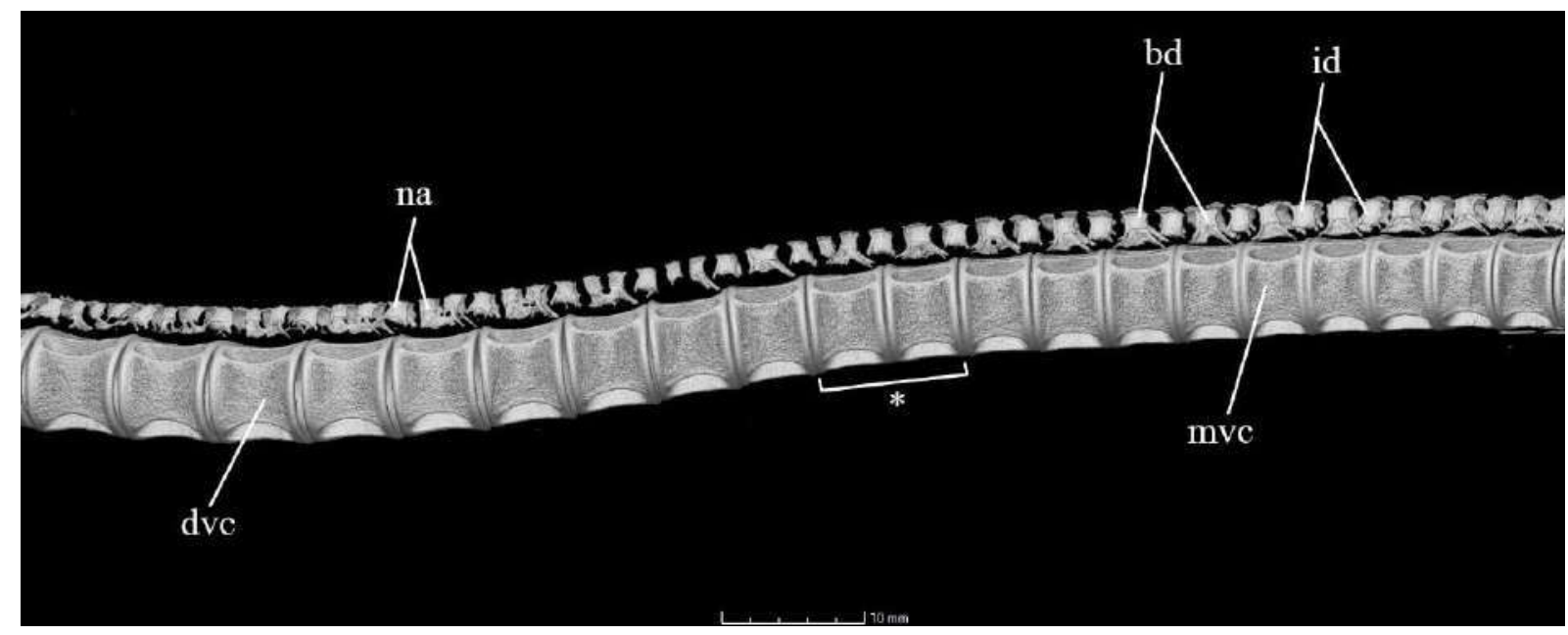

Figure 262. Caudal fin of Isogomphodon oxyrhynchus (MZUSP 101214) obtained with 3D reconstruction from the CT-Scan imaging. Anatomical abbreviations: bd, basidorsal; dvc, diplospondylic vertebral centrum; id, interdorsal; mvc, monospondylic vertebral centrum; na, neural arch; vc, vertebral centrum. 


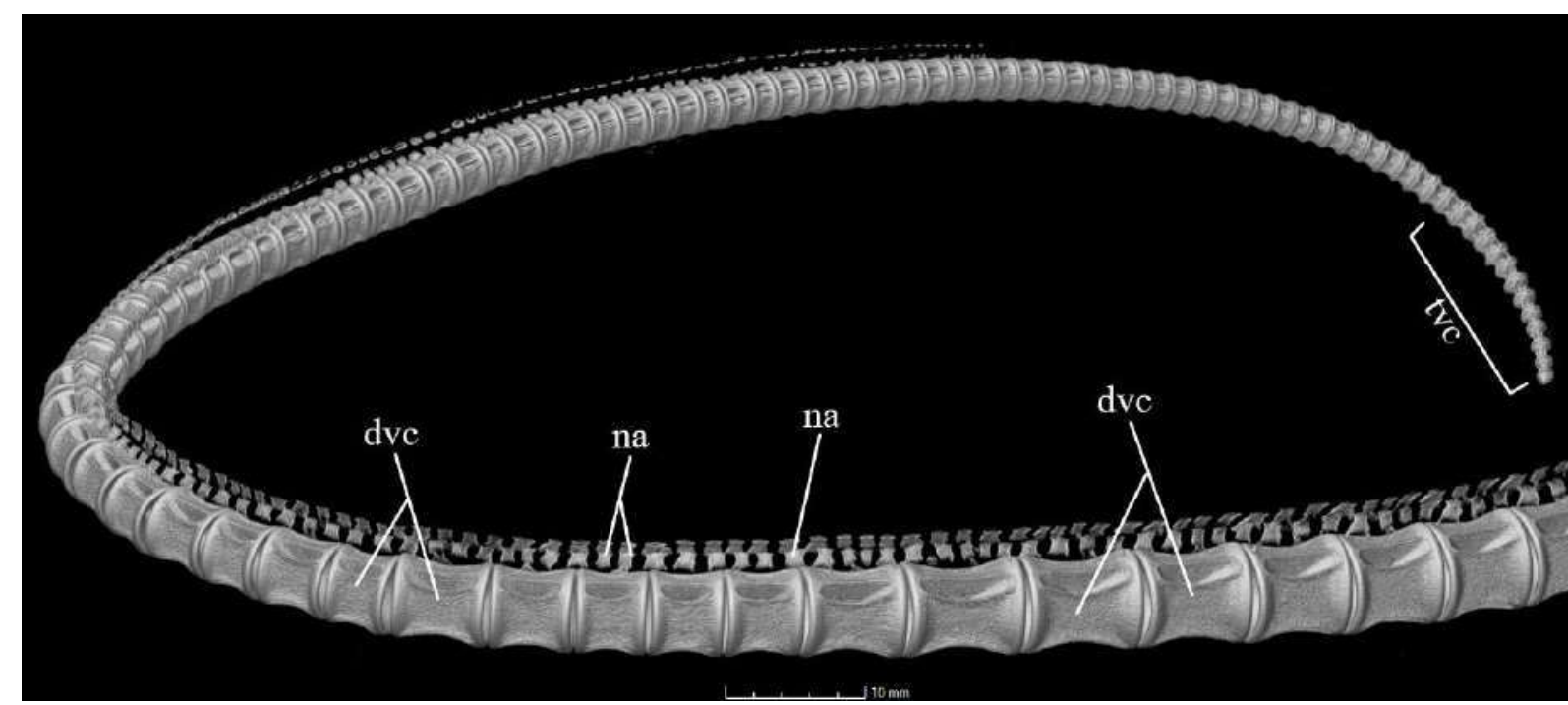

Figure 263. Caudal fin of Isogomphodon oxyrhynchus (MZUSP 101214) obtained with 3D reconstruction from the CT-Scan imaging. Anatomical abbreviations: bd, basidorsal; bv, basiventral; hs, haemal spine; id, interdorsal; sn, supraneural; vc, vertebral centrum 


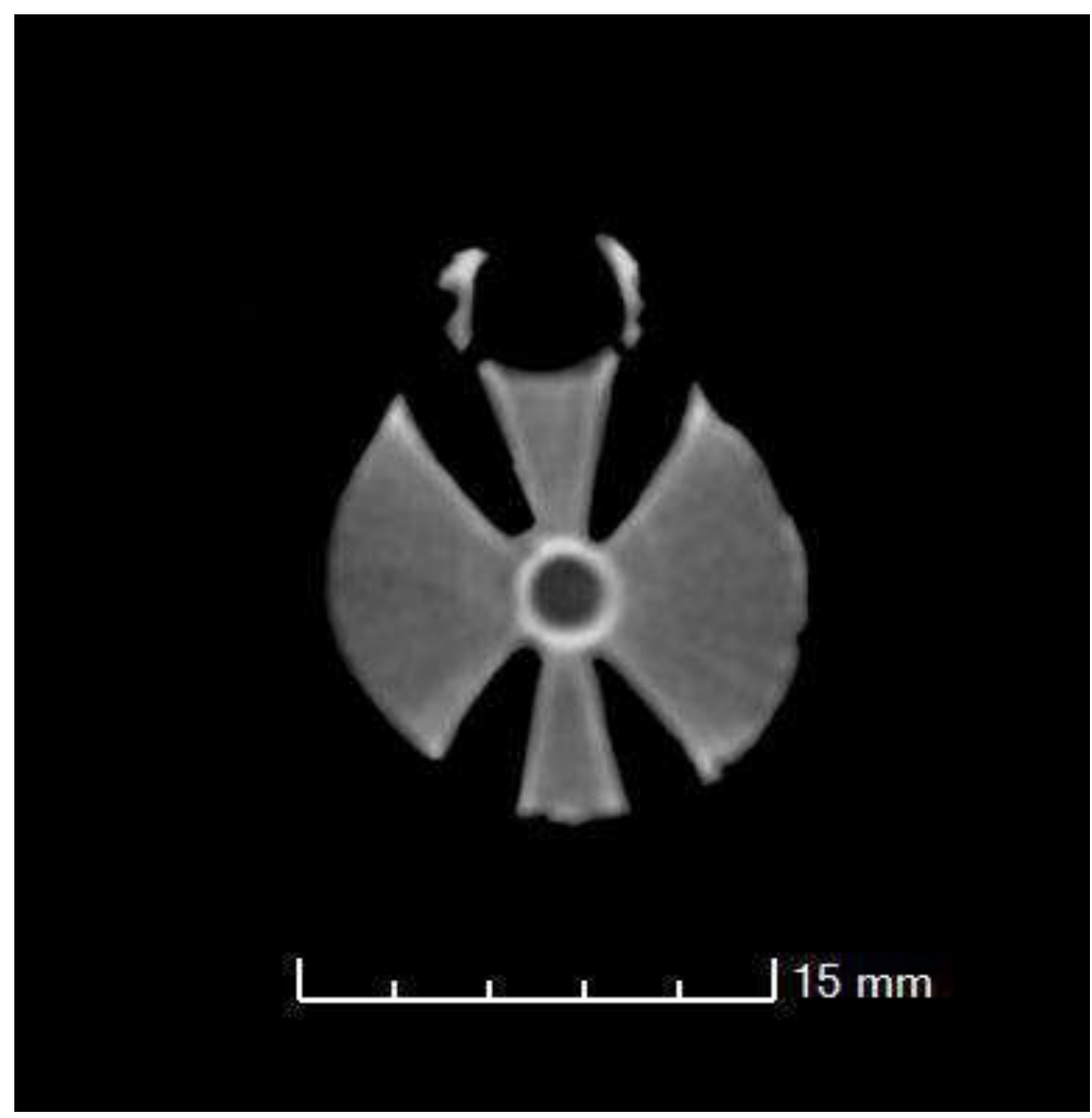

Figure 264. Vertebral calcification pattern of a diplospondylic centrum of Isogomphodon oxyrhynchus (MPEG $3455)$. 


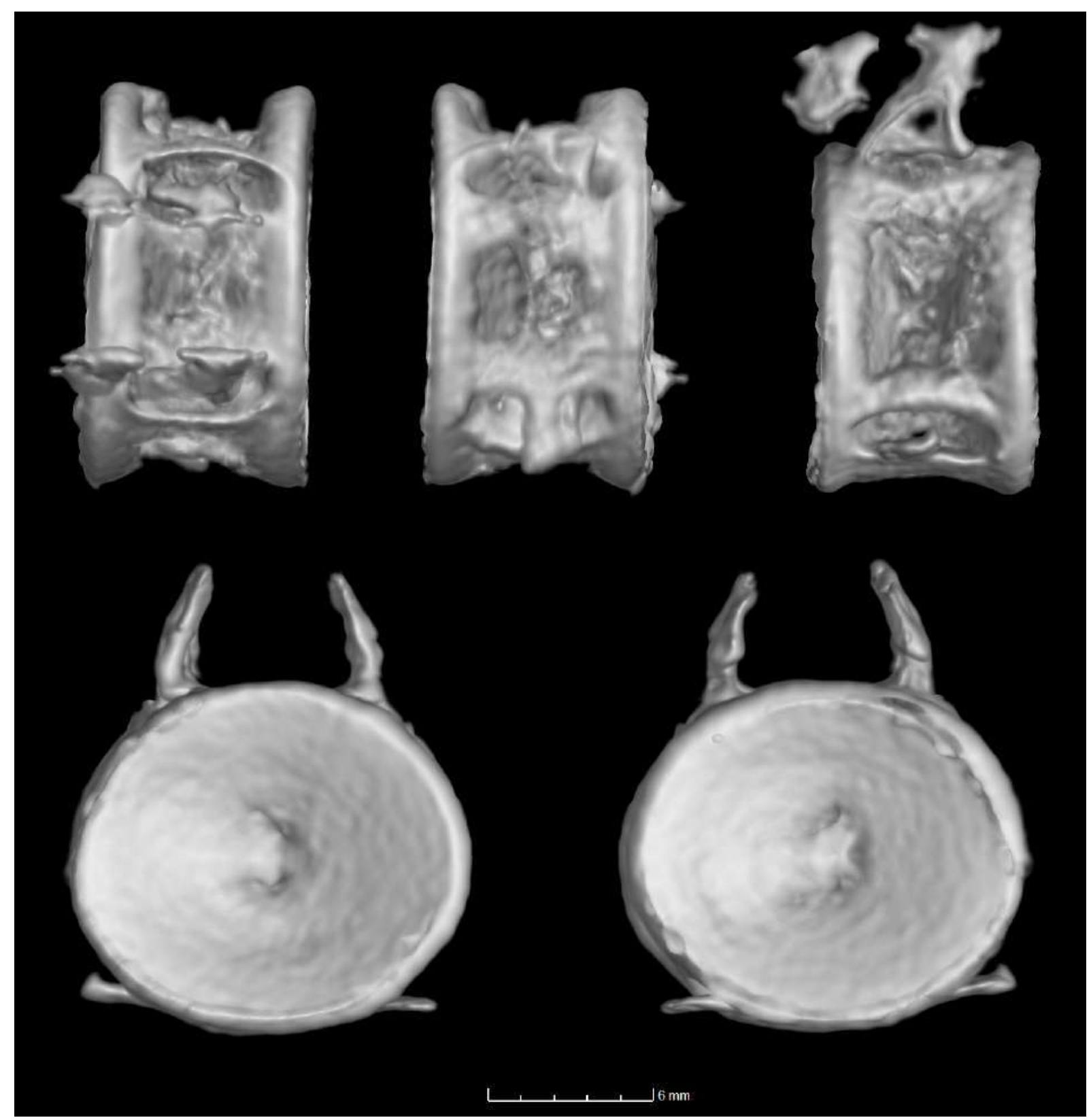

Figure 265. Monospondylic vertebral centrum of Isogomphodon oxyrhynchus (MPEG 3455) obtained with 3D reconstruction from the CT-Scan imaging. Upper left - dorsal view; Upper middle - ventral view; Upper right lateral view; Lower left - anterior view; Lower right - posterior view; 


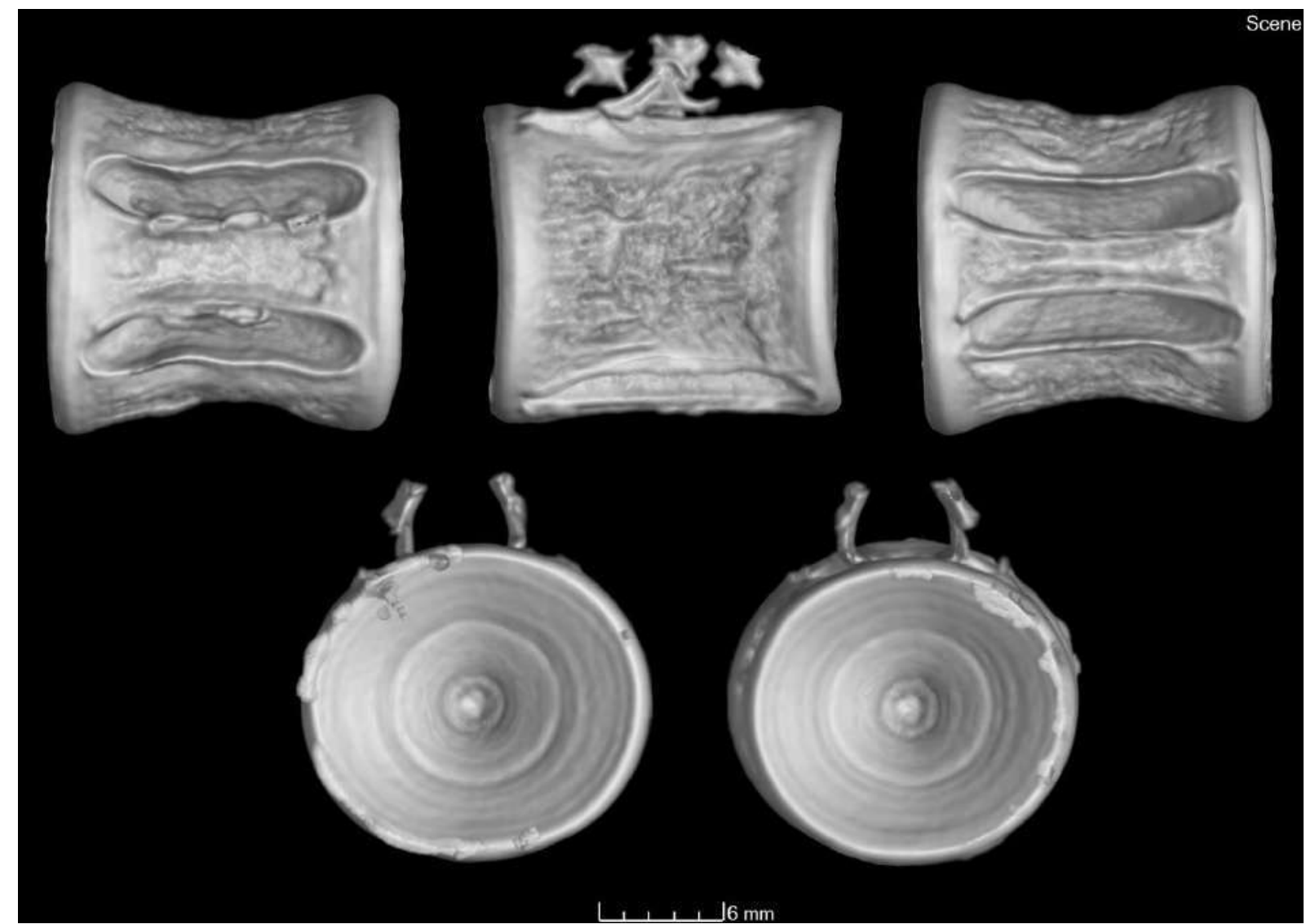

Figure 266. Diplospondylic vertebral centrum of Isogomphodon oxyrhynchus (MPEG 3455) obtained with 3D reconstruction from the CT-Scan imaging. Upper left - dorsal view; Upper middle - lateral view; Upper right ventral view; Lower left - anterior view; Lower right - posterior view; 
Chapter 2

Isogomphodon oxyrhynchus within Carcharhinidae: Comparative Anatomy and Phylogenetic implications 


\section{Introduction}

\subsection{Morphological complexes and their significance in Selachii phylogeny.}

The decade of 1940 was marked by a decadency of the systematics among other fields in biological sciences, due to the evident lack of a reliable method to rationally allocate the organisms in an ordered system (Hennig 1966). The cladistic method, as also postulated by Hennig (1950), was created not only to fill such gap but to also investigate the evolutionary relationships between different organisms through an efficient methodology strongly based on the term 'homology', which can be simply defined as "equivalence of parts" (de Pinna, 1991). As the method became established and widespread, the organisms were categorized and allocated in their evolutionary trees based on the essential piece that builds the phylogenetic hypothesis: the character state. The concept of character in a cladistic perspective of what morphology can say about the relationships of the organisms was initially discussed in Patterson (1982). Many authors proposed solid definitions for the term 'character' (see Sereno 2007 Table 1), where most are connected, if not synonymized, to the definition of 'homology' or 'primary homology'. Based on such concepts, the phylogenetic systematics takes places to elaborate hypothesis to explain the evolutionary history of the organisms. It is through the comparative anatomy between taxa that characters are proposed and coded, which is an essential key to a phylogenetic analysis (Sereno 2007). Moreover, the detection of similarities and differences is rather useful to give insights in how character might group or separate different clades.

Chondrichthyes are an ancient group whose oldest fossil records, which are restricted to scales dated from the Middle Ordovician, 467.3 \pm 1.1 million years ago (Andreev et al. 2015). Elasmobranchii, in turn, comprise three main lineages: groups related to Cladoselache $†$ from the Devonian, Euselachii from the Triassic and Neoselachii from the Mezosoic. The Carcharhinidae, the most diverse family within the order Carcharhiniformes, includes 59 species, 12 valid genera, widely distributed around the planet except on the poles (Compagno 1988; Fricke, Eschmeyer \& Van der Laan 2020). Available hypotheses of relationships in Carcharhinidae are mostly based on molecular data (e.g., Vélez-Zuazo \& Agnarson 2011; Naylor et al. 2012). Inside the order Carcharhiniformes, the most diverse among sharks, 59 species within 12 valid genera comprise the family Carcharhinidae, which are widely distributed around the planet except on the poles (Compagno 1988; Fricke, Eschmeyer \& Van der Laan 2020). The hypothesis of relationships in Carcharhinidae were mostly proposed based 
on molecular topologies (e.g., Vélez-Zuazo \& Agnarson 2011; Naylor et al. 2012). Those trees represent major advances in our understanding of the major patterns of relationships in the group. However, many evolutionary questions remain, mainly related to the evolution of phenotypic traits and relationships at the species-level.

Phylogenetic studies within Chondrichthyes are not scarce, although most are usually focused on more inclusive taxonomic ranks (e.g., Maisey 1984; 2012; Shirai 1992). de Carvalho (1996) and Shirai (1996) are amongst the most accepted and cited studies regarding a higherlevel of Elasmobranchii phylogenetic relationships through morphological data, once relevant anatomical characters were described by those authors. Within Carcharhiniformes, Compagno (1988) proposed a phylogenetic hypothesis, although based on a non-quantitative synapomorphy scheme. In recent years, novel hypothesis based on morphology (e.g., Klug \& Kriwet, 2010; Silva et al. 2015; Silva, Vaz \& Carvalho 2017; Moreira et al. 2019; Stone \& Shimada, 2019; Soares et al. 2020) were developed, focused on solving relationships above the species-level. Curiously, Kikuchi (2004) seems to be the only morphological phylogenetic hypothesis at species-level on any groups of Carcharhinidae.

\subsection{Phylogenetic position of Isogomphodon within Carcharhinidae.}

The first author to include I. oxyrhynchus in any hypothesis was Compagno (1988). Through an exhaustive survey of morphological data from living sharks and using autapomorphies and synapomorphies to infer the phylogenetic relationships among species groups (i.e., a non-quantitative analysis), Isogomphodon was positioned as sister group of Triaenodon. Kikuchi (2004) analysed the relationships of Rhizoprionodon and included Isogomphodon oxyrhynchus as an outgroup. Overall, the results from Kikuchi (2004) allocated I. oxyrhynchus as a sister taxon to Negaprion brevirostris. Naturally such outcome has to been seen in the context of an analysis focusing on Rhizoprionodon only, and thus not including dense representation of taxa relevant for resolving relationships of I. oxyrhynchus. Still, her hypothesis was one of the few, if not the only yet, to include I. oxyrhynchus in a quantitative analysis using solely morphological evidence.

Molecular phylogenetic studies have been more forthcoming in including Isogomphodon oxyrhynchus. Rodrigues-Filho et al. (2009), in a study focusing on elasmobranch identification and based on mitochondrial sequences (12S and $16 \mathrm{~S}$ ), placed $I$. oxyrhynchus as most similar to Carcharhinus porosus (Fig. 10). Those authors suggest that $I$. 
oxyrhynchus might belong to the genus Carcharhinus, an idea earlier raised by Compagno (1984). Neither authors actually implemented such nomenclatural changes.

Vélez-Zuazo \& Agnarsson (2011) proposed a new hypothesis on shark relationships based on COI, NADH2, Cytb, 16S and Rag-1, i.e., four mitochondrial genes and one nuclear gene, respectively. Using Bayesian analysis, those authors confirmed I. oxyrhynchus as sister group of Carcharhinus porosus. Naylor et al. (2012a) offered a broad molecular-based elasmobranch phylogeny through a Bayesian analysis using NADH2. Their results allocated Isogomphodon as sister group to a clade containing most species of Carcharhinus (Figs. 12 and 13). C. porosus, though not the sister group, was somewhat also closely related to Isogomphodon oxyrhynchus. Naylor et al. (2012b) also considered I. oxyrhynchus as a terminal, in which they put it as sister taxon to $C$. isodon with $C$. porosus as sister-group to the clade $I$. oxyrhynchus + C. isodon.

Sorenson et al. (2014), using one nuclear (Rag1) sequence and four mitochondrial genes (cox1, Cytb, 16S and NADH2) placed Isogomphodon oxyrhynchus as sister group of Carcharhinus macloti, although C. porosus also appears close to the clade I. oxyrhynchus $+C$. macloti. The paper infers the habitat preference of both C. porosus and I. oxyrhynchus as brackish, an expected result considering the sympatry of those two species. Cachera \& Le Loc'h (2017), employing three mitochondrial genes (Cyt-b, 12S and 16S) and one nuclear (RAG1), proposed I. oxyrhynchus as the sister taxon of a clade composed of multiple Carcharhinus species. Although they did not include $C$. porosus in their analysis, they did include $C$. isodon, a taxon which has been considered by Naylor et al. (2012b) as closely related to I. oxyrhynchus, was presented in the Carcharhinus clade (Figure 34).

One of most recent phylogenetic studies which included I. oxyrhynchus was Feitosa et al. (2018). That study was not primarily phylogenetic, but rather intended to use molecular markers for species identification in fish markets. Using NADH2 and COI, I. oxyrhynchus was placed as most similar to the Carcharhinus species (C. limbatus, C. leucas, C. acronotus, $C$. porosus and $C$. falciformis) with NADH 2 and closely allocated to C. acronotus when COI was used as the molecular marker. Finally, Naylor (2021) provided a database of current Elasmobranchii comparative information, both anatomical and molecular (available at: sharkrays.org) which places Isogomphodon oxyrhynchus within a large polytomy including most species of Carcharhinus.

Morphological and molecular data on Isogomphodon oxyrhynchus and its confamilials are here compared and synthesized as an aid to resolve its phylogenetic position inside 
Carcharhinidae. Although the morphological survey was not intended to be a quantitative phylogenetic analysis, it is hoped that a detailed comparative anatomy will provide a solid basis to illuminate previously published results. Additionally, a novel molecular analysis is conducted in order to test the position of I. oxyrhynchus in its family on a database broader than that available for previous studies. Our hypothesis also provides a framework against which comparative anatomical data can be mapped and understood in a phylogenetic context. Results not only yield a critical view on the phylogenetic position of Isogomphodon, but also allow an understanding about the evolution of its peculiar morphological features. 


\section{Material and Methods}

All available literature information on Isogomphodon oxyrhynchus was collected, compiled and reviewed, both from morphological (Compagno 1988; Kikuchi 2004) and molecular sources (Rodrigues-Filho et al. 2009; Vélez-Zuazo \& Agnarsson 2011; Naylor et al. 2012a; 2012b; Sorenson et al. 2014; Cachera \& Le Loc'h 2017; Feitosa et al. 2018). A compilation of their overall results is listed on Table 1.

Molecular analyses included all sequences and markers for Carcarhiniformes available on GenBank (NCBI: txid30483), until June $26^{\text {th }}$ of 2019. Sequence data were downloaded with the package PhyLoTA (Sanderson et al. 2008, available at: http://loco.biosci.arizona.edu/pb), aligned using Mafft (Katoh et al. 2002) and then gathered within the Zungaro server (FAPESP proc. 2014/26508-3). Models and partitions were selected using PartitionFinder2 (Lanfer et al. 2017) and phylogenetic inferences were generated with RAxML (Stamatakis 2014). Tree search methodology was based on Maximum Likelihood, in which each molecular marker has its own partition using an evolutionary GTR model. The final phylogenetic hypothesis was based on three topologies obtained under three different treatments based on different degrees tolerance to missing data: $0 \%, 10 \%$ and $25 \%$. The results on number of orthologous regions and number of paired bases are listed on Table 2. Molecular analyses were performed in collaboration with Dr. Victor A. Tagliacollo.

Morphological characters were organized according to Sereno (2007) and arranged in a binary-based morphological matrix in order to allow clear visualization of character-state distributions. CT-Scan images were obtained with the MZUSP CT-Scan (Model: Phoenix $v \mid$ tome $\mid x \quad m$ microfocus - General Electric) and from the database available in https://sharksrays.org/ (Naylor 2021). Dissected specimens are listed in the Material Examined section. Characters were described from four anatomical complexes: Neurocranium, mandibular arch, pectoral girdle and pelvic girdle. States assigned as (0) or (1) not necessarily imply in plesiomorphic versus apomorphic conditions. Similarities and differences were quantified through a permutational comparison of all taxa and then elaborate a similarity matrix. Most morphological discussion focused on C. porosus because of its recurrent placement as phylogenetically close to I. oxyrhynchus in previous studies. Remaining species here analysed were also compared and contrasted with I. oxyrhynchus. Their preparation technique used for analysis is listed on the Examined Material Section. 
A total of 12 taxa, including Isogomphodon oxyrhynchus, were comparatively examined. Then were described 46 morphological characters (20 on the neurocranium, 13 on the mandibular arch, seven on the pectoral girdle and five on the pelvic girdle), posteriorly codified for analysis purposes. The coding and similarity matrix are listed on tables $3-6$. From the 11 taxa compared with Isogomphodon oxyrhynchus, six were examined as CT-Scan images from Naylor (2021), two were directly dissected for this study and three were previously prepared. Some species were analysed on the basis of more than one kind of preparation (See Analysed Material). CT-Scan images may yield artifactual aberrations, and need to be carefully scrutinized, for example false foramina which are actually calcification failures (Fig. 7).

The database sharkrays.org provided information on six taxa of Carcharhinidae, whereas others were examined through dissections using forceps and bistouries in alcohol preserved specimens, previously cleared and stained individuals or CT-Scanned as in Chapter 1 of the present dissertation. Nomenclature terms for skeletal and muscular structures followed Compagno (1988). Some species (e.g., Carcharhinus perezii) were compared with I. oxyrhynchus though not included in the character coding due to lack on information of other anatomic regions (in this case, solely information of the neurocranium of $C$. perezii was accessed). Apart from the coded characters, brief and relevant considerations are drawn on the most evident variations between Isogomphodon oxyrhynchus and the other analysed species. The proposed morphological characters are listed on 3.2. 


\section{Results}

\subsection{Comparative anatomy of Isogomphodon oxyrhynchus}

This section starts with a one-to-one comparison between Isogomphodon oxyrhynchus and Carcharhinus porosus. The motivations for this are twofold. First, to provide a view for the kind of variation which is detected, coded and mapped in the subsequent section. Second, the two species have been repeatedly hypothesized as phylogenetically close (Vélez-Zuazo \& Agnarson, 2011; Naylor et al. 2012a; present study) and thus deserve a more direct comparison in features that go beyond the proposed characters. The neurocranium is the most modified region of I. oxyrhynchus, as illustrated on Fig. 1.

\subsubsection{Isogomphodon oxyrhynchus and Carcharhinus porosus}

Although the rostral node of the examined C. porosus (MZUSP 84440) was damaged, Compagno (1988) reports that this species has a rostral fenestra completely closed, with rostral cartilages nearly equal in mean diameter (see Compagno 1988, Fig. 19.10 - G). Both species have a minute rostral fenestra, although on I. oxyrhynchus this aperture is narrow-oval shaped whereas on $C$. porosus such structure is rather circular (See Compagno 1988 - Fig. 19.10 Illustration G). The thickness of the rostral cartilages is not significantly different among both taxa, although the posterior end of the medial rostral cartilage of $C$. porosus is thinner when compared to I. oxyrhynchus, which has an enlargement of such region. In other words, the transition between the medial rostral cartilage and the anterior portion of the cerebral cavity is abrupt on $C$. porosus whereas I. oxyrhynchus presents a gradual transition. Regarding the anterior fontanelle, C. porosus has a shallow groove on this region, with a discrete anterior calcification in the medial region that generates a minute foramen-like structure (See fig. 26), whereas in I. oxyrhynchus this region is strongly $\mathrm{V}$-shaped without a foramen. The preorbital process of $C$. porosus is similar to its congenerics: a 'spatula-like' structure that covers the anterior eyeball dorsally. In I. oxyrhynchus, on the other hand, this structure is minute and reduced, which is probably autapomorphic. Both species have thin, long, and slender postorbital processes, although on I. oxyrhynchus this structure apparently is directed more anteriorly than on $C$. porosus. The postorbital process of both species is slender and elongated, although on $I$. oxyrhynchus, this structure is anteriorly oriented whereas in C. porosus it is directed laterally. Regarding the parietal fossa, I. oxyrhynchus has an oval-shaped cavity while C. porosus has a 
clear circular shape on this region. The wing-shaped projection on the occipital condyle is possibly an autapomorphy of $I$. oxyrhynchus, therefore $C$. porosus has a different condition which is rather similar to the observed in its congeneric taxa.

In the mandibular arch, the main difference is on the ventral portion of the Meckel's cartilage, which in I. oxyrhynchus has a well delimited 'C-shaped' recess in the medial edge of the cartilage, whereas in $C$. porosus such condititon is absent. The general structure of the mandibular arch in I. oxyrhynchus is narrower than in C. porosus. Both C. porosus and I. oxyrhynchus have the ethmoid processes of the palatoquadrate wider than deep. Although $C$. porosus was dissected, information on the pectoral girdle and pelvic girdle was also gathered from Silva (2014), which also illustrated the appendicular skeleton of C. porosus. The scapulocoracoid of I. oxyrhynchus is slightly larger than that of $C$. porosus, especially on the ventromedial portion, and the radials are also proportionally longer in this species when compared to C. porosus.

The pelvic girdle shows few the differences between these two taxa. Nonetheless, the puboischiatic bar of C. porosus is slightly bulkier than that of I. oxyrhynchus, and it has lateral edges with mild concavities which contrasts with the smoother and slightly more angular posterior margin in the latter. The basipterygium of both taxa are similar in shape, with the first enlarged pelvic radial bifurcated solely in I. oxyrhynchus.

\subsection{List of morphological characters - Carcharhinidae}

The 46 proposed characters are original from this study and were elaborated based on the 12 examined taxa.

\subsubsection{Neurocranium}

1. Cn1 - Rostral cartilages, signs of hypercalcification

- (0) With hypercalcified zones (Rhizoprionodon porosus, R. terranovae, Loxodon macrorhinus)

- (1) Without hypercalcified zones (Isogomphodon oxyrhynchus, Carcharhinus porosus, C. plumbeus, C. galapagensis, Rhizoprionodon lalandii, R. taylori, Triaenodon obesus, Negaprion brevirostris, Scoliodon macrorhynchos) 
The rostral cartilages, within Carcharhiniformes, are rod-like tripodal structures, being two laterals dorsally and one medial ventrally (Compagno 1988; 1999). These regions are embedded in an Lorenzini Ampoule network within the rostral region, and the calcification degree is usually homogeneous, however some Carcharhinidae taxa (e.g., some Rhizoprionodon species and Loxodon macrorhinus) have hypercalcified zones that occur both within the rostral node and along the cartilage axis (Compagno 1988). Herein, the hyper calcification is easily identified by a thick, whitened pattern, and its occurrence both on the rostral node and along the axis of the rostral cartilages is here coded as state 0 .

2. Cn2 - Rostral node, rostral fenestra, occurrence

- (0) Absent (Loxodon macrorhinus, Triaenodon obesus, Negaprion brevirostris, Scoliodon macrorhynchos)

- (1) Present (Rhizoprionodon porosus, R. terranovae, Isogomphodon oxyrhynchus, Carcharhinus porosus, $C$. plumbeus, $C$. galapagensis, Rhizoprionodon lalandii, $R$. taylori).

The rostral fenestra is an opening located on the apical junction of the lateral rostral cartilages and the medial rostral cartilage (tip of the snout region). Although this structure is variable in shape, its occurrence is clearly visible even when restricted to a minute aperture or when the circumference is not completely closed (which is classified here as state 1).

3. Cn3 - Rostral node, anterior edge, shape

- (0) Narrow edged, with width of anterior tip shorter than the length of medial rostral cartilage (Rhizoprionodon terranovae, Loxodon macrorhinus, Isogomphodon oxyrhynchus, Carcharhinus porosus, C. plumbeus, C. galapagensis, Scoliodon macrorhynchos)

- (1) Wide edged, with width of anterior tip nearly equal to the length of medial rostral cartilage (Rhizoprionodon porosus, $R$. lalandii, R. taylori, Triaenodon obesus, Negaprion brevirostris)

On the rostral node, the junction of lateral rostral cartilages with medial rostral cartilage, the morphology of anterior edge can vary from a pointy, narrow-edged confluence to a node with a wide junction of rostral cartilages. This character is a potential reflex to the nostril shape in external anatomy, and for cases where the rostral node has an incomplete circumference 
around the rostral fenestra, the distance between the two apical portions of the lateral rostral cartilages was considered the width of the anterior edge.

4. Cn4-Confluence of medial rostral cartilage base with anterior ectethmoid region, conformation (Fig. 10)

- (0) Abrupt, with a narrow posterior edge of lateral rostral cartilage connected to the ectethmoid region (Rhizoprionodon porosus, $R$. lalandii, $R$. taylori, $R$. terranovae, Triaenodon obesus, Negaprion brevirostris, Loxodon macrorhinus, Carcharhinus porosus, C. plumbeus, $C$. galapagensis)

- (1) Gradual, with an enlarged posterior edge of lateral rostral cartilage connected to the ectethmoid region (Isogomphodon oxyrhynchus, Scoliodon macrorhynchos)

The confluence region between the ectethmoid region (i.e., anterior to the basal plate, between the nasal capsules) and the medial rostral cartilage is variable among the neurocrania of the Carcharhinidae taxa. The two states here proposed were differentiated based on how the medial rostral cartilages posterior edge is connected to the ectethmoid region: in a narrow with width nearly equal to the rostral cartilage width or with a wider connection.

5. Cn5 - Ridge connecting base of lateral rostral cartilage with edge of anterior fontanelle, curvature (Fig. 11)

- (0) Strongly curved, with angulation closer to $90^{\circ}$ than to $180^{\circ}$ (Carcharhinus porosus, $C$. plumbeus, C. galapagensis, Rhizoprionodon porosus, $\underline{\text { R. terranovae) }}$

- (1) Mildly curved, with angulation closer to $180^{\circ}$ than to $90^{\circ}$ (Isogomphodon oxyrhynchus, Rhizoprionodon lalandii, R. taylori, Triaenodon obesus, Negaprion brevirostris, Loxodon $\underline{\text { macrorhinus, }}, \underline{\text { Scoliodon macrorhynchos) }}$

The lateral rostral cartilages are connected to the lateral edges of anterior fontanelle in the dorsal portion of the neurocranium. Such confluence in Carcharhinidae species occurs inevitably with a certain degree of curvature, although in different angulations among the neurocrania morphologies. To perform the codification of this character, the analysis on lateral view is required, in which the angulation was measured using the lowest point of the curvature as the angle vertex. 
6. Cn6 - Basirostral fenestra, occurrence

- (0) Absent (Carcharhinus plumbeus, Rhizoprionodon lalandii, R. taylori, Triaenodon obesus, Negaprion brevirostris, Loxodon macrorhinus, Scoliodon macrorhynchos)

- (1) Present (Isogomphodon oxyrhynchus, Rhizoprionodon porosus, R. terranovae, Carcharhinus porosus, C. galapagensis)

Differently from the nasal fontanelle, the basirostral fenestra is a paired structure located ventrally on the confluence of medial rostral cartilage with the basal plate. It is usually covered in a thin layer of connective tissue, with no associated structure to these apertures.

7. Cn7 - Nasal fontanelle, conformation

- (0) Visible in dorsal view (Rhizoprionodon lalandii, R. taylori, R. porosus, $\underline{\text { Rhizoprionodon }}$ terranovae, Carcharhinus plumbeus, C. porosus, C. galapagensis, Scoliodon macrorhynchos)

- (1) Not visible in dorsal view (Isogomphodon oxyrhynchus, Triaenodon obesus, Loxodon macrorhinus, Negaprion brevirostris)

The aperture connecting the anterior proximal margin of the nasal capsule with the cerebral cavity is delimited as the nasal fontanelle (Compagno 1999). Its visualization requires the remotion of the connective tissue protecting the brain, although on the CT-Scan images such procedure is not necessary. Within Carcharhinidae, the conformation of such apertures is here coded based on their visibility in dorsal view.

8. $\mathrm{Cn} 8$ - Anterior fontanelle, extension related to pre-orbital process

- (0) Anteriorly narrow, with edge located anterior to the line of pre-orbital process (Rhizoprionodon lalandii, Rhizoprionodon taylori, Rhizoprionodon porosus, Rhizoprionodon terranovae, Carcharhinus plumbeus, Carcharhinus porosus, Carcharhinus galapagensis, Triaenodon obesus, Loxodon macrorhinus, Negaprion brevirostris)

- (1) Posteriorly extended, with edge located posterior to the line of pre-orbital process (Isogomphodon oxyrhynchus, Scoliodon macrorhynchos) 
Regarding this structure, the anterior fontanelle is a neurocranium region located between the posterior endings of the lateral rostral cartilages, in which a membrane is also located to physically protect the frontal facet of the brain, located beneath the posterior edge. In terms of shape, it varies in forms, but its posterior edge locations have two evident conditions, where they are either anterior or posterior to the line of pre-orbital process. In I. oxyrhynchus, the shape of the anterior fontanelle is likely a synapomorphy to the group, which is here coded as 1: a strong deepen 'V-shape'. To avoid the coding base on the pure morphology of this structure, which is highly variable, the pre-orbital process is pointed as the qualifier to provide more accuracy to the character.

\section{Cn9 - Pre-orbital process, morphology}

\section{- (0) Slender and cylindrical (Scoliodon macrorhynchos)}

- (1) Flattened, sometimes bifurcated (Rhizoprionodon lalandii, $R$. taylori, $R$. porosus, $R$. terranovae, Carcharhinus plumbeus, C. porosus, C. galapagensis, Triaenodon obesus, Loxodon macrorhinus, Negaprion brevirostris)

\section{- (2) Nearly truncated (Isogomphodon oxyrhynchus)}

Within Carcharhinidae, the preorbital process is a structure that precedes the orbit and anteriorly delimits the beginning of the region where the eye-associated musculature is located (Compagno 1999). In Carcharhinidae, this process is always present, although varying in shape and length, which is here coded in three states and considering the state 2 as an autapomorphy to I. oxyrhynchus. Scoliodon macrorhychos also presented a unique state, which is state (0), and most examined taxa were coded as state (1).

10. Cn10 - Anterior fontanelle, posterior profile, shape

- (0) Semicircular, U-shaped, truncate or broadly angled (Rhizoprionodon lalandii, Rhizoprionodon taylori, Rhizoprionodon porosus, Rhizoprionodon terranovae, Carcharhinus plumbeus, Carcharhinus porosus, Carcharhinus galapagensis, Triaenodon obesus, Loxodon macrorhinus, Negaprion brevirostris, Scoliodon macrorhynchos) 
Within Carcharhinidae, the posterior profile of the anterior fontanelle is highly variable in terms of shape ( $c f$. Compagno 1988). However, Isogomphodon oxyrhynchus has a condition which this region has an evident narrow 'V-shaped' profile that is not observed in any other taxa. Consequently, the state (1) is autapomorphic to Isogomphodon oxyrhynchus.

\section{Cn11 - Orbitonasal foramina, positioning in dorsal perspective}

- (0) Not visible in dorsal view (Rhizoprionodon lalandii, $R$. taylori, $R$. porosus, $R$. terranovae, Negaprion brevirostris)

- (1) Visible in dorsal view (Isogomphodon oxyrhynchus, Scoliodon macrorhynchos, Carcharhinus plumbeus, C. porosus, C. galapagensis, Triaenodon obesus, Loxodon macrorhinus)

The orbitonasal foramina are a pair of apertures located anteriorly on the orbital region and posterior to the nasal capsule. Although always present in Carcharhinidae, their conformation within the neurocranium is variable, being either closely positioned or more spaced between each other, which implies in their visibility in dorsal view.

\section{Cn12 - Medial keel on basal plate, occurrence}

- (0) Absent (Carcharhinus plumbeus, C. porosus, C. galapagensis, Rhizoprionodon taylori, Scoliodon macrorhynchos, Loxodon macrorhinus)

- (1) Present (Isogomphodon oxyrhynchus, R. lalandii, R. porosus, R. terranovae, Negaprion brevirostris, Triaenodon obesus)

The medial keel on basal plate is an elevation located between the ectethmoid condyles on the ventral surface of the neurocranium. In terms of associated structures, this region is connected with the anterior margin of the palatoquadrate cartilage, between the paired ethmoid processes of the palatoquadrate. Here, the medial keel is simply characterized as a parallel protuberance which is visible in ventral view.

\section{Cn13 - Post-orbital process, size related to pre-orbital process}

- (0) Equal or nearly equal in length (Carcharhinus plumbeus, C. porosus, C. galapagensis, $\underline{\text { Rhizoprionodon taylori, } R \text {. lalandii, } R \text {. porosus, } R \text {. terranovae, Triaenodon obesus, Negaprion }}$ brevirostris) 
- (1) Post-orbital process larger in length than pre-orbital process (Isogomphodon oxyrhynchus, Scoliodon macrorhynchos)

- (2) Post-orbital process smaller in length than pre-orbital (Loxodon macrorhinus)

The post-orbital process is an expanded region of the neurocranium which is located immediately posterior to the orbit and associated with the optic capsule (Compagno 1999). Here, the state 0 is considered when the difference in size between the pre-orbital and postorbital processes is not higher than $10 \%$ of the total length of the post-orbital process.

\section{Cn14 - Post-orbital process, connection with optic capsule}

- (0) Confluent with the optic capsule (Carcharhinus plumbeus, C. porosus, C. galapagensis, Rhizoprionodon taylori, $R$. lalandii, $R$. porosus, $R$. terranovae, Negaprion brevirostris, Scoliodon macrorhynchos, Loxodon macrorhinus)

\section{- (1) Separated of the optic capsule (Isogomphodon oxyrhynchus)}

\section{- (?) Undetermined (Triaenodon obesus)}

Within the neurocranium structure, the post-orbital process is often completely fused with the optic capsule (with both structures calcified to form a connection). Such condition is present in all examined genera of Carcharhinidae except in Isogomphodon, once the CT-Scan images and dissected specimen revealed that in Isogomphodon, this structure has a weaker calcified path with the optic capsule (1), which consequently classified it as an autapomorphy for state for Isogomphodon.

\section{Cn15 - Postorbital process, hyper calcification}

- (0) Absent (Isogomphodon oxyrhynchus, Triaenodon obesus, Carcharhinus plumbeus, C. porosus, C. galapagensis, Rhizoprionodon taylori, $R$. lalandii, Negaprion brevirostris, Scoliodon macrorhynchos, Loxodon macrorhinus)

- (1) Present (Rhizoprionodon porosus, $R$. terranovae) 
As well as the rostral node, a high degree of calcium deposition, a thicker mineralized layer, can be observed in the postorbital process of some taxa. Here, these characters seem to be present solely in Rhizoprionodon porosus, therefore the only terminal with state (1).

\section{Cn16 - Posterior wall of anterior fontanelle, conformation}

- (0) Smooth, with no notch or foramen (Isogomphodon oxyrhynchus, Triaenodon obesus, Carcharhinus plumbeus, C. galapagensis, Rhizoprionodon lalandii, R. terranovae, Negaprion brevirostris, Scoliodon macrorhynchos, Loxodon macrorhinus)

- (1) With an epiphyseal notch or foramen (Carcharhinus porosus, Rhizoprionodon taylori, $R$. porosus)

Within Carcharhiniformes, the posterior upper wall of anterior fontanelle significantly varies in morphology among the extant taxa, going from straight-lined on its edge to ' $\mathrm{V}$ shaped'. The epiphyseal nerve (which is related to light detection) runs through in this region (Compagno 1999), eventually associated with a foramen or notch (Kikuchi 2004). Although a foramen is easily detectable, the 'notch' requires a definition, which is considered present when the distance between the edges of this eventual notch is no longer than $20 \%$ of the length of between the edges of the anterior fontanelle.

The states of $\mathrm{Cn} 8$ postulated the extension of the anterior fontanelle related to the preorbital process, therefore not considering the presence of the notch on the anterior fontanelle. The 'V-shaped' observed on Isogomphodon oxyrhynchus might lead to the interpretation that this notch is somehow present in the posterior end of the ' $\mathrm{V}$ ', it is here however considered absent on this taxon.

17. Cn17 - Suborbital shelf, visibility in dorsal view (Fig. 12)

- (0) Reduced, not or slightly visible in dorsal view (Carcharhinus plumbeus, C. galapagensis, Rhizoprionodon taylori, R. terranovae, Loxodon macrorhinus)

- (1) Laterally expanded, clearly visible in dorsal view (Isogomphodon oxyrhynchus, Carcharhinus porosus, Triaenodon obesus, Rhizoprionodon lalandii, R. porosus, Negaprion brevirostris, Scoliodon macrorhynchos) 
As the name states, the suborbital shelf is a region of the orbit where the eyeballs and their associated musculature are rested. Although this character is rather conserved in terms of morphology, the main variation is simply the visibility of such structure on dorsal view (i.e., with cranial roof parallel to the substrate), which is potentially a reflex of the size of the eye musculature.

\section{Cn18 - Frontal Fontanelle, occurrence}

- (0) Absent (Isogomphodon oxyrhynchus, Triaenodon obesus, Carcharhinus plumbeus, C. porosus, C. galapagensis, Rhizoprionodon taylori, $R$. lalandii, $R$. porosus, $R$. terranovae, Negaprion brevirostris, Scoliodon macrorhynchos, Loxodon macrorhinus)

\section{- (1) Present ( - )}

The essential difference between the frontal fontanelle and the parietal fossa, apart from the positioning (frontal fontanelle is anterior to the parietal fossa), is the associated foramina that are only present in the parietal fossa. Although all the compared taxa are state 0 , some Carcharhinus species (e.g., C. obscurus and C. perezii) have such aperture present (Compagno 1988).

19. Cn19 - Medial sulcus posterior to the parietal fossa, occurrence (Fig. 13) - (0) Absent (Triaenodon obesus, Carcharhinus plumbeus, C. galapagensis, Rhizoprionodon taylori, Negaprion brevirostris, Scoliodon macrorhynchos, Loxodon macrorhinus)

- (1) Present (Isogomphodon oxyrhynchus, Carcharhinus porosus, Rhizoprionodon lalandii, $\underline{R}$. porosus, $R$. terranovae)

The parietal fossa describes a region located between the optic capsules and allocates the perilymphatic foramina, besides its association to the ducts that lead to the inner ears. Although it is usually circular to oval shaped cavity, it sometimes possesses a posterior sulcus that originates on the terminal edge, in which its occurrence is here coded as state (1).

20. Cn20 - Stapedial fenestra, conformation 
- (0) Completely separated from the internal carotid foramen (Isogomphodon oxyrhynchus, Triaenodon obesus, Carcharhinus plumbeus, C. porosus, Scoliodon macrorhynchos, $\underline{\text { Rhizoprionodon terranovae) }}$

- (1) Fused with the internal carotid foramen superficially in a single external circumference (Carcharhinus galapagensis, Rhizoprionodon lalandii, $R$. porosus, $R$. taylori, Loxodon macrorhinus, Negaprion brevirostris)

The stapedial fenestra is an aperture located on the posteroventral region of the neurocranium, being directed towards the internal margin of the basal plate. Within Carcharhinidae, there are two clear states of its conformation, which is either separated from the carotid foramen or superficially confluent with it, but with still two apertures present.

\section{Cn21-Occipital condyle, shape}

- (0) Expanded, with posterior projections that are nearly equal or equal in size with first vertebral centrum (Isogomphodon oxyrhynchus, Carcharhinus plumbeus, C. galapagensis, Rhizoprionodon taylori, $R$. lalandii, $R$. terranovae, Negaprion brevirostris)

- (1) Narrow, with projections not longer than half of the first vertebral centrum (Triaenodon obesus, Carcharhinus porosus, Scoliodon macrorhynchos, Loxodon macrorhinus, $\underline{\text { Rhizoprionodon porosus) }}$

The posterior-most structure of the neurocranium is the occipital condyle, a structure with not only holds the anterior insertion of epaxial musculature but enhances the connection with the first vertebral centrum.

\subsubsection{Mandibular Arch}

1. Cma1 - Meckel's cartilage, internal facet, morphology (Fig. 14)

- (0) Straight, with no well delimited recess (Triaenodon obesus, Carcharhinus plumbeus, $C$. porosus, C. galapagensis, Rhizoprionodon taylori, $R$. lalandii, $R$. porosus, $R$. terranovae, Negaprion brevirostris, Scoliodon macrorhynchos, Loxodon macrorhinus)

- (1) With a delimited 'C-shaped' recess in the medial-ventral facet of the palatoquadrate, occurrence (Isogomphodon oxyrhynchus) 
On the internal medial facet of Meckel's cartilage, a tendon is located which connects the posterior region of this structure to its anterior edge. Although this connection is often hidden or partially visible in the internal facet of the Meckel's cartilage in some carcharhinids, in Isogomphodon it is more visible due to a 'C-Shaped' gap which is present ventromedially. State (1) is an autapomorphy of I. oxyrhynchus.

2. Cma2 - Hyoid arch, ratio

- (0) Hyomandibular cartilage shorter than ceratohyal cartilage (Isogomphodon oxyrhynchus, Triaenodon obesus, Carcharhinus plumbeus, C. porosus, C. galapagensis, Rhizoprionodon taylori, $R$. lalandii, $R$. porosus, $R$. terranovae, Negaprion brevirostris, Loxodon macrorhinus)

- (1) Hyomandibular cartilage nearly equal in length to the ceratohyal cartilage (Scoliodon $\underline{\text { macrorhynchos) }}$

In elasmobranchs, immediately anterior to the mandibular arch (i.e., Palatoquadrate and Meckel's cartilage) is located the hyoid arch, which is composed by two paired pieces (the hyomandibula - dorsal - and ceratohyal - ventral) and a single fused Basihyal. Although their morphology is rather conserved in terms of minor differences among Carcharhinidae taxa, their length has differences. The state $(0)$ is considered present when the difference between both measurements is higher than $25 \%$ of the length of the ceratohyal.

3. Cma3 - Ethmoid process of the palatoquadrate, proportion of dimensions - (0) Height larger than length (Scoliodon macrorhynchos)

- (1) Length larger than height (Isogomphodon oxyrhynchus, Triaenodon obesus, Carcharhinus plumbeus, C. porosus, C. galapagensis, Rhizoprionodon taylori, R. terranovae, Negaprion brevirostris, Loxodon macrorhinus)

- (2) Length and height nearly equal in size (Rhizoprionodon terranovae, $R$. lalandii, $R$. porosus)

The ethmoid process of the palatoquadrate is a 'tubercle-like' projection located anteriorly on the dorsal palatoquadrate facet. Within Galeomorphii, such structure is positioned in rest anteriorly to the ethmoid region (which justifies the nomenclature) when the mouth is 
closed, and it is covered dorsally by a thick layer connective tissue. Compagno (1988) and Compagno (1999) identified such structure as 'orbital process of the palatoquadrate', nonetheless Shirai (1996) asserts that such nomenclature is limited solely to the superorder Squalomorphii. Although this coverage might mislead the coding of such character, the orbital process of the palatoquadrate is delimited based only to the calcified region. The state (2) is considered present when the difference between both measurements is not higher than $10 \%$ of the length of this structure.

\section{Cm4-Anterior articulation of Meckel`s cartilage (Fig. 15)}

- (0) Symphysis Narrow (Triaenodon obesus, Carcharhinus plumbeus, C. porosus, $C$. galapagensis, Rhizoprionodon taylori, R. lalandii, R. porosus, R. terranovae, Negaprion brevirostris, Scoliodon macrorhynchos, Loxodon macrorhinus)

\section{- (1) Symphysis Elongated (Isogomphodon oxyrhynchus)}

In the ventral anterior portion, the connection between the two pieces of the Meckel's cartilage occurs with no calcified zones, but rather by connective tissue. The extent of how this connection occurs is variable, i.e., the length of this region is determined by how large these separated pieces are within this facet. The state (1) is considered when the length of the connection facet nearly reaches the ventral medial portion of the Meckel's cartilage, although such code is, so far, an autapomorphy of Isogomphodon.

\section{Cma5 - Upper teeth, total counting of functional teeth of both sides}

- (0) Up to 30 (Carcharhinus plumbeus, C. porosus, C. galapagensis, Rhizoprionodon taylori, $\underline{R}$. lalandii, R. porosus, R. terranovae, Scoliodon macrorhynchos, Loxodon macrorhinus)

- (1) More than 30 (Isogomphodon oxyrhynchus, Triaenodon obesus, Negaprion brevirostris)

For this character, the total number of teeth in the upper rows was counted considering solely the first row of functional teeth within the palatoquadrate. Teeth formula information on Triaenodon obesus was collected from Compagno (1988).

6. Cma6 - Lower teeth, total counting of functional teeth of both sides 
- (0) Up to 30 (Carcharhinus plumbeus, C. porosus, C. galapagensis, Rhizoprionodon taylori, R. lalandii, $R$. porosus, $R$. terranovae, Negaprion brevirostris, Scoliodon macrorhynchos, Loxodon macrorhinus)

- (1) 30 or more (Isogomphodon oxyrhynchus, Triaenodon obesus)

For this character, the total number of teeth in the lower rows was counted considering solely the first row of functional teeth within the Meckel's cartilage. Teeth formula information on Triaenodon obesus was collected from Compagno (1988).

7. Cma7 - Ethmoid process of the palatoquadrate, origin position according to the teeth row (Fig. 16)

- (0) Above the $5^{\text {th }}$ teeth or before (Carcharhinus plumbeus, C. porosus, C. galapagensis, $\underline{\text { Rhizoprionodon taylori, } R \text {. lalandii, } R \text {. porosus, } R \text {. terranovae, Loxodon macrorhinus) }}$

- (1) After the $5^{\text {th }}$ teeth (Isogomphodon oxyrhynchus, Triaenodon obesus, Negaprion brevirostris, Scoliodon macrorhynchos)

Details on the ethmoid process of the palatoquadrate are elucidated on Cma3 statement. In order to code this character, an anterior vertical line (see Fig. 14) was drawn in the anterior margin of the ethmoid process of the palatoquadrate, and the teeth numbering, was detected based on the medial teeth, if present as the first, or based in the first teeth to appear in the row. Consequently, the first tooth this line contacts is identified and considered for the coding. The teeth region that the line first connects to does not impact the coding.

8. Cma8 - Palatoquadrate, posterior surface posterior to the articular facet, morphology (Fig. 17)

- (0) Straight (Carcharhinus plumbeus, Rhizoprionodon porosus, Negaprion brevirostris, Loxodon macrorhinus)

- (1) Curved/concave (Isogomphodon oxyrhynchus, Triaenodon obesus, Carcharhinus porosus, C. galapagensis, Rhizoprionodon taylori, R. lalandii, R. terranovae, Scoliodon macrorhynchos) 
In lateral view, the morphology of the posterior region of the palatoquadrate immediately posterior to the articular tubercle, is variable from a straight line to curved, concave shape. The state (1) is delimited by the condition seen on Negaprion brevirostris, which presents just a mild curvature present on such region and it is considered as such. Additionally, this state might be present as a concavity anteriorly and posteriorlu directed.

9. Cma9 - Meckel's cartilage, anterior region of adductor mandibulae insertion, length related to postdentary groove

- (0) Shorter or equal sized as the postdentary groove (Triaenodon obesus, Rhizoprionodon terranovae, Scoliodon macrorhynchos)

- (1) Longer than postdentary groove (Isogomphodon oxyrhynchus, Carcharhinus plumbeus, $C$. porosus, C. galapagensis, Rhizoprionodon taylori, $R$. lalandii, R. porosus, Negaprion brevirostris, Loxodon macrorhinus)

The adductor mandibulae region of insertion is a surface that extends from the region below the last teeth of the Meckel's cartilage to nearly the posterior ventral region of the palatoquadrate articulation facet, in a sulcus-like cavity. On the other hand, the postdentary groove is named to delimit the sulcus-like concavity located between the last posterior teeth and the articulation with the palatoquadrate.

10. Cma10 - Palatoquadrate, posterodorsal surface, morphology

- (0) Straight, with no elevation prior to the region close to the articulation tubercle (Isogomphodon oxyrhynchus, Carcharhinus porosus, Rhizoprionodon taylori, Scoliodon macrorhynchos)

- (1) Concave, with elevation prior to the region close to the articular tubercle (Triaenodon obesus, Carcharhinus plumbeus, C. galapagensis, Rhizoprionodon lalandii, $R$. porosus, $R$. terranovae, Negaprion brevirostris, Loxodon macrorhinus)

Within the palatoquadrate dorsal surface, posteriorly to the orbital process, an elevation might be present as a mild lateral crest. For this character, such elevation is described as an upward concavity located immediately before the articulation region, which also describes an anchoring point for the adductor mandibulae muscle. 
11. Cma11 - Meckel's cartilage, angulation of dentary row (Fig. 18)

- (0) Less than $30^{\circ}$ (Isogomphodon oxyrhynchus, Triaenodon obesus, Carcharhinus plumbeus, C. porosus, Rhizoprionodon taylori, $R$. lalandii, $R$. porosus, $R$. terranovae, Scoliodon macrorhynchos)

- (1) $30^{\circ}$ or more (Carcharhinus galapagensis, Loxodon macrohinus, Negaprion brevirostris)

For this character, a straight horizontal line has been drawn from the anterior most point of the Meckel's cartilage to its most posterior point, and the angulation generated between this line and the curvature of the teeth rows was measured (Fig. 16). Although the curvature of the dentary row is not a straight line, the angle was measured using as a landmark the fourth teeth after the row started to move upper from the line.

12. Cma12 - Meckel's cartilage, postdentary concavity, size in relation to palatoquadrate height (Fig. 18)

- (0) More than 50\% of Meckel's cartilage height (Rhizoprionodon lalandii, Scoliodon macrorhynchos)

- (1) Less than or equal to 50\% of Meckel's cartilage height (Isogomphodon oxyrhynchus, Triaenodon obesus, Carcharhinus plumbeus, C. porosus, C. galapagensis, Rhizoprionodon taylori, $R$. porosus, R. terranovae, Negaprion brevirostris, Scoliodon macrorhynchos)

In the rear end of the dentary rows of the Meckel's cartilage, a concavity is present between the last posterior teeth and the articulation with the palatoquadrate, which is here named as postdentary groove (pdg). The length of such region is variable, although to better detect and code it, a comparison with the height of the Meckel's cartilage is performed. The height of such structure was measured tracing a perpendicular line from the medial portion of the articulation tubercle to the most distant point on the ventral surface.

13. Cma13 - Meckel's cartilage, proportion between height and length (Fig. 18)

- (0) Height less than $40 \%$ of length (Isogomphodon oxyrhynchus, Triaenodon obesus, Rhizoprionodon lalandii, R. terranovae, Scoliodon macrorhynchos, Loxodon macrorhinus) 
- (1) Height equals $40 \%$ or more of length (Carcharhinus plumbeus, C. porosus, $C$. galapagensis, Rhizoprionodon taylori, R. porosus, Negaprion brevirostris)

To perform the coding in this character, the length was measured tracing a line from the anterior most region, immediately bellow the teeth positioned dorsal to where the calcified surface begins, to the posterior most point. On the other hand, the height considered the highest portion of the articulation tubercle with the palatoquadrate and the lowest point on this structure, as performed on Cma12. Once measured, the values were compared based on the length measurement.

\subsubsection{Pectoral Girdle}

1. Cpg1 - Posterior-triangular process of the scapula, occurrence (Fig. 19) - (0) Absent (Isogomphodon oxyrhynchus, Carcharhinus plumbeus, C. porosus, C. galapagensis, Rhizoprionodon lalandii, R. taylori, R. porosus, R. terranovae, Negaprion brevirostris, Loxodon macrorhinus)

\section{- (1) Present (Triaenodon obesus, Scoliodon macrorhynchos)}

The posterior-triangular process of the scapula is a posteromedial projection that has a highly variable shape occurs in a significant number of taxa (Silva, Vaz \& Carvalho 2017). This process describes an anchoring point for the epaxialis muscle, a deeply associated fiber with the pectoral fin. The occurrence of this character is merely classified here as present and absent once Triaenodon obesus is one of few species that does not have such structure (Silva et al. 2017, Fig. 6) and in Scoliodon macrorhynchos such structure is absent as well.

2. Cpg2 - Diazonal foramen aperture, morphology as viewed in lateral view - (0) Oval shaped (Isogomphodon oxyrhynchus, Carcharhinus galapagensis, Rhizoprionodon taylori, $R$. porosus, Negaprion brevirostris, Triaenodon obesus, Scoliodon macrorhynchos)

- (1) Circular shaped (Carcharhinus plumbeus, C. porosus, Rhizoprionodon lalandii, R. terranovae, Loxodon macrorhinus) 
The diazonal foramen is a widened aperture that is located on the ventral medial portion within the coracoid bar, which allows the passage of the artery that irrigates the pectoral fins. Such foramen is more visible in lateral and posterior views, although is shape in lateral view is variable. It usually describes an oval opening, although eventually it presents a circular-shape considering the dimensions between the extremities, in which both states are easily discernible.

3. Cpg3 - Mesopterygium, number of associated radials

- (0) 3 (Isogomphodon oxyrhynchus, Carcharhinus porosus, Rhizoprionodon lalandii, $R$. taylori, R. porosus, Loxodon macrorhinus)

- (1) 4 or more (Carcharhinus plumbeus, C. galapagensis, Rhizoprionodon terranovae Negaprion brevirostris, Triaenodon obesus, Scoliodon macrorhynchos)

Within the basal elements on the pectoral fin, the medial one is the mesopterygium (Compagno 1999). It is connected to the medial posterior portion of the coracoid bar and it has proximal radials connected to it. The number is usually not as high as seen in the metapterygium, usually restricted to 3 to 5 radials.

\section{Cpg4-Metapterygium, number of associated radials}

- (0) Up to 17 radials (Isogomphodon oxyrhynchus, Carcharhinus plumbeus, C. porosus, Rhizoprionodon taylori, $R$. porosus, $R$. terranovae, Negaprion brevirostris, Loxodon macrorhinus, Scoliodon macrorhynchos)

- (1) More than 17 radials (Carcharhinus galapagensis, Rhizoprionodon lalandii, Triaenodon obesus,)

The posterior most element in the pectoral elements, usually the most expanded and enlarged is the metapterygium (Compagno 1999). Its connection to the coracoid bar (i.e., the facet for mesopterygium) varies in terms of positioning and conformation, although for Carcharhinidae it has a condition which is separated from the facets of propterygium and mesopterygium (Silva et al. 2015). It also represents the connection piece in which most proximal radials of the pectoral girdle are connected to. Here, the total number of radials directly connected to the metapterygium is coded. The posterior portion of the metapterygium is usually a separated piece, in which here is considered part of the metapterygium for the radial 
counting. In other words, the radials connected to this separated piece are considered connected to the metapterygium and, therefore, included on the counting.

5. Cpg5 - Ventral posterior portion of the coracoid bar junction, morphology (Fig. 20)

- (0) Pointed (Isogomphodon oxyrhynchus, Carcharhinus galapagensis, Rhizoprionodon lalandii, R. taylori, $R$. porosus, $R$. terranovae, Scoliodon macrorhynchos)

- (1) Rounded (Carcharhinus plumbeus, C. porosus, Negaprion brevirostris, Loxodon macrorhinus, Triaenodon obesus)

Although the presented images (Fig. 18) might lead to conclude that the coracoid bar is composed by two separated pieces, it is actually a single structure that as a ventral junction which probably has a weaker calcification, therefore not detectable through CT-Scan. The pointed state (0) is considered when the angulation of the posterior extremities is more perpendicular (i.3., closer to $90^{\circ}$ ) than obtuse, which is coded as (1).

6. Cpg6 - Scapula, upper anterior edge, morphology (Fig. 21)

- (0) Straight lined (Isogomphodon oxyrhynchus, Carcharhinus porosus, Rhizoprionodon lalandii, $R$. taylori, $R$. porosus, $R$. terranovae, Negaprion brevirostris, Loxodon macrorhinus, $\underline{\text { Scoliodon macrorhynchos) }}$

- (1) With mild, tubercle-like projection (Triaenodon obesus, Carcharhinus plumbeus, $C$. galapagensis)

\section{- (?) Undetermined (Negaprion brevirostris)}

The coracoid bar upper pointy edge is cylindrical, although the morphology of the anterior surface of this region discretely varies, from a smooth regular surface to a concave region with a mild tubercle-like projection. Triaenodon obesus is a clear example of state 1, although calcification failures on Isogomphodon oxyrhynchus and on Negaprion brevirostris led to the code 'undetermined'. 
7. Cpg7 - Scapulocoracoid bar, ventral posterior surface between the articulation facets of meso- and metapterygium, curvature (Fig. 22)

- (0) Nearly perpendicular, with angle closer to $90^{\circ}$ than to $180^{\circ}$ (Carcharhinus plumbeus, Rhizoprionodon lalandii, $R$. terranovae, Negaprion brevirostris)

- (1) Mostly concave, with angulation closer to $180^{\circ}$ than to $90^{\circ}$ (Isogomphodon oxyrhynchus Carcharhinus porosus, C. galapagensis, Rhizoprionodon taylori, R. porosus, Triaenodon obesus, Scoliodon macrorhynchos, Loxodon macrorhinus)

To interpret such character, it is necessary to analyse the lateral view of the scapulocoracoid bar, although a schematic figure is presented on figure 20. Essentially, the transition between the posterior ventral junction of the two halves of the coracoid bar and the pectoral basal facets presents a curvature, however in different degrees of inclination. The clearest contrast is observed comparing Isogomphodon oxyrhynchus which is state 0 , and Carcharhinus plumbeus, state 1.

\subsubsection{Pelvic Girdle}

1. Cpv1 - Puboischiadic bar, proportion between height and length (Fig. 23) - (0) Height equal to or less than $40 \%$ of length (Isogomphodon oxyrhynchus, Carcharhinus plumbeus, C. porosus, C. galapagensis, Rhizoprionodon lalandii, R. taylori, R. porosus, Negaprion brevirostris, Triaenodon obesus, Scoliodon macrorhynchos)

\section{- (1) Height more than 40\% of length (Rhizoprionodon terranovae, Loxodon macrorhinus)}

The puboischiadic bar corresponds to the rectangular, boomerang-shaped structure that is the main base of the pectoral girdle. This piece is the anchoring point for the basipterygium and for the first enlarged pelvic radial. The 'equal' condition of state (0) is considered present when the difference between the two states is not higher than $10 \%$ of the puboischiadic total length.

2. Cpv2 - Puboischiadic bar, anterior facet, morphology (Fig. 24) 
- (0) Smooth edged (Isogomphodon oxyrhynchus, Carcharhinus plumbeus, C. porosus, C. galapagensis, Rhizoprionodon lalandii, $R$. taylori, $R$. porosus, $R$. terranovae, Triaenodon obesus, Loxodon macrorhinus)

- (1) With a mesial notch (Negaprion brevirostris, Scoliodon macrorhynchos)

The anterior facet on the puboischiadic bar is usually a straight, smooth surface that is shorter than the posterior facet, which is rather curved. The mesial notch present is this region (state 1) is evidenced in Scoliodon macrorhynchos (see fig. 24), whereas the smooth surface is illustrated on Isogomphodon oxyrhynchus.

\section{Cpv3-Basipterygium, number of associated proximal radials}

- (0) 15 or less (Isogomphodon oxyrhynchus, Carcharhinus plumbeus, C. porosus, $C$. galapagensis, Rhizoprionodon lalandii, $R$. taylori, $R$. porosus, $R$. terranovae, Loxodon macrorhinus, Scoliodon macrorhynchos)

\section{- (1) More than 15 (Triaenodon obesus, Negaprion brevirostris)}

Differently to the pectoral girdle, which has three basal elements and three types of radials, the pelvic girdle is simplified in one 'basal element, the basipterygium, and solely proximal and distal radials (Compagno 1999; Silva 2014). The basipterygium is the anchoring point to the pelvic proximal radials, whose number varies from species to species.

\section{Cpv4 - Puboischiadic bar, posterior facet, angulation (Fig. 23)}

- (0) Mildly curved or nearly straight (Carcharhinus galapagensis, Rhizoprionodon lalandii, Triaenodon obesus, Negaprion brevirostris, Loxodon macrorhinus)

- (1) Strongly curved (Isogomphodon oxyrhynchus, Carcharhinus plumbeus, C. porosus, Rhizoprionodon taylori, $R$. porosus, $R$. terranovae, Scoliodon macrorhynchos)

As stated on the Cpv01 character statement, the puboischiadic has a rectangular to boomerang-like shape, although the posterior facet is always curved in a certain degree. Here, this degree is measured based on the angulation, in which less than $160^{\circ}$ is considered evidently curved (state 1), whereas more than that is considered mildly curved to nearly straight (state 0 ). 
5. Cpv5 - Basipterygium, first enlarged radial, morphology

- (0) Simple (Carcharhinus plumbeus, C. porosus, C. galapagensis, Rhizoprionodon lalandii, R. taylori, $R$. porosus, $R$. terranovae, Negaprion brevirostris, Triaenodon obesus)

- (1) Bifurcated (Isogomphodon oxyrhynchus, Loxodon macrorhinus, Scoliodon macrorhynchos)

The first radial connected to the puboischiadic bar is usually expanded, which is named, as in Silva (2014), as first enlarged radial. Its morphology varies in terms of general shape, although the main feature here coded is its bifurcation, which illustrates that such structure is potentially a fusion of the first pelvic proximal radials.

\subsection{Novel molecular analysis}

The results from the novel molecular hypothesis here proposed were performed in collaboration with Victor A. Tagliacollo. In all three obtained topologies, Isogomphodon oxyrhynchus was positioned as sister-group of Carcharhinus porosus. The positioning of such clade varied in the three selected treatments of missing data, in which $0 \%$ it was allocated as sister group of a clade that includes Carcharhinus + Nasolamia + Prionace + Triaenodon, 10\% as sister-group to Triaenodon and 25\% in the same position to the obtained on $0 \%$. The final outcome of total of partitions, paired based and included terminals are listed on Table 2. 


\section{Discussion}

Compagno (1988) provided a vast database on information of Carcharhinidae, which is part of the most complete and detailed work on the anatomy of Carcharhiniformes. Classically relevant and highly detailed anatomical papers on elasmobranchs are not scarce (e.g., Allis 1923; Gilbert 1973; Compagno 1977; Lima et al. 1997; Goto 2001; Maisey 2004; Casas et al. 2005), although in the past decade several authors provided significant improvements on the knowledge of elasmobranch anatomy (e.g., Loboda 2013; 2016; Fontenelle 2013; Silva 2014; Silva et al. 2015; Moreira et al. 2018; Soares et al. 2020). Apart from the present comparisons with the examined specimens, these articles and other relevant literature provided important data to better compare Isogomphodon oxyrhynchus with other Carcharhinidae. In terms of limitations, most of the specimens were analysed through CT-Scan with low resolution and a short number of taxa were documented due to the lack of access to more material. It is possible that the non-analysed genera (e.g., Glyphis, Lamiopsis, Prionace) have similarities with Isogomphodon that have not been here reported. Nonetheless, relevant results were collected and here presented.

Among taxa examined for comparative anatomy, those most similar to I. oxyrhynchus are Triaenodon obesus, Carcharhinus porosus and Scoliodon macrorhynchos, whereas the most different were Loxodon macrorhinus, Negaprion brevirostris, and Carcharhinus plumbeus. T. obesus, C. porosus and N. brevirostris have all been previously positioned as sister-taxon or closely related to I. oxyrhynchus, although solely the first two were allocated as such based on explicit phylogenetic analysis (e.g., Naylor et al. 2012a), and support for the last is scant (e.g., Kikuchi 2004).

The cephalic region, especially the neurocranium, contains most of the informative variation in the skeleton of Isogomphodon oxyrhynchus. The entire neurocranium outline of $I$. oxyrhynchus is narrower than that of most other examined taxa, a particularity evident in both dorsal and lateral views. Despite that, it is unwarranted at this time to consider such trait as autapomorphic, a conclusion which would call for a detailed morphometric analysis. The most noticeable autapomorphies candidates for Isogomphodon are located in the neurocranium. The 'V-shaped' anterior fontanelle, narrow cranial roof and occipital condyle with 'wing-shaped' projection are particularly conspicuous. Again, all such traits, notwithstanding their apparent unusual nature, all involve quantitative shape and size differences which are difficult to objectively delimit. The range of variation in the anterior fontanelle shape among 
Carcharhinidae is extremely wide ( $c f$. Compagno 1988), and it is difficult to determine a nonarbitrary transformation series among various observed states. In any event, the shape in Isogomphodon is unique by a wide margin, and it is very likely autapomorphic. We propose this a working hypothesis to be tested against more data and analysis. Lastly, the truncated condition of the preorbital process was amongst the coded autapomorphies. Crest-like projections on the distal lateral facet of the orbital nasal foramen were present in the juvenile MZUSP 101214 and absent in the adult MPEG3455, which is potentially unique. Although the elongated rostrum is one of the most notable features on the neurocranium of I. oxyrhynchus, other taxa such as S. macrorhynchos may present similarly long rostral regions, not to mention some Carcharhinus as C. signatus also present externally elongated snouts (Compagno 1984). Considering that those species are in distant positions on the Carcharhinidae phylogenetic hypothesis, such elongation is likely a confluence. Apart from these subjections, the remaining listed features on the neurocranium are strong candidates of autapomorphies of Isogomphodon.

The connection of the postorbital process with the optic capsule is a controversial character. As observed in CT-Scan images, Isogomphodon oxyrhynchus has an incomplete calcified path between those structures. Within the CT-Scan images of Triaenodon obesus obtained from Naylor (2021), such feature is also visible in this taxon, although this described separation occurs more posteriorly. Nonetheless, considering the resolutions on these images were not in a high quality and that CT-Scan of sharks often result in regions with poorly calcified landmarks, it is possible that in T. obesus this is a result of misdetection, therefore coded here as undetermined. Considering this assumption, one might ask: How could this be any different for Isogomphodon oxyrhynchus once this observation was initially performed based on CT-Scan only? Firstly, the resolution in which the images from the juvenile (MZUSP 101214) and the adult (MPEG 3455) were higher than those from Naylor (2012), and both specimens presented the same feature. Second, the dissections on the juvenile suggest that the postorbital process has a different kind of connective tissue bonding these two regions. Lastly, Compagno (1988) illustrated both neurocrania of Triaenodon obesus, which also presented such character, and to I. oxyrhynchus, in which he did not illustrate such character in neither of them, could be indicative that both species are eligible to present such condition. Due to the lack of available specimens of $T$. obesus, no dissections were performed in this taxon to confirm such assumptions.

The general outline of the mandibular + hyoid arches in Isogomphodon is narrower than in other examined taxa and as a whole more pointed than in other Carcharhinidae species. The 
mandibular arches of other genera are not only broader and wider, but their palatoquadrates also have a more pronounced posterior elevation, something not observed in Isogomphodon. The mandibular arch characters, the teeth of Isogomphodon, both in the upper and lower jaws, are far more numerous than in other species examined, a condition likely unique to the genus Compagno (1988). Triaenodon obesus also has numerous jaw teeth (GVP-uncat: 42 upper tooth and 46 lower), though not as many as in I. oxyrhynchus (MZUSP 101214: 55 upper tooth and 50 lower tooth). An evident autapomorphy for Isogomphodon is the 'C-shaped' hollow area on the medial portion of Meckel's cartilage. This region has a tendon that connects not only the extremities of the ' $\mathrm{C}$ ' but also extends between the anterior edge of Meckel's cartilage and the posterior internal facet. The tendon itself is not unique to the genus, being also present in other taxa such as Carcharhinus porosus and Rhizoprionodon porosus. Another autapomorphy candidate is the symphysis between the two pieces of the Meckel's cartilage, in which the only examined species that has this region elongated is I. oxyrhynchus. Within the palatoquadrate, the elevation on the posterior facet of the palatoquadrate is a character present in most examined species and absent in I. oxyrhynchus. This region is an insertion site for the adductor mandibulae muscle, one of the components of the adductor mandible complex (Motta \& Wilga 1995; Wilga \& Motta 1998; Casas et al. 2005; Soares \& Carvalho 2013).

Although myology was not the focus of this study, some observations were made which deserve mention. For instance, in I. oxyrhynchus the levator palatoquadrati is inserted in a more posterior region of the supraorbital crest, whereas in C. porosus such region is located more anteriorly. Also, the eye muscles obliquo superior and obliquo inferior have crossed arrangements in both species, although I. oxyrhynchus has those muscles with tendinous origins embedded in connective tissue near the pre-orbital process, whereas in C. porosus they are not associated with tendons in their origin. The remaining examined species were not compared in those observations as they were not available for dissection.

The coracoid bar of I. oxyrhynchus seems slightly bulkier than those of other taxa, as observed in Figs. 6 and 7 although more evident in the lateral view. A clear informative character in the pectoral girdle of sharks that has been previously explored (Silva et al. 2017) is the triangular process of the scapula, which in Carcharhinidae is a simple protuberance, a condition considered as unique for the family although presumably reversed in some genera such as Triaenodon (Silva et al. 2017). Curiously, Scoliodon macrorhynchos lacks a triangular process on the scapula, while its congeneric, S. laticaudus, has one (Silva 2014). The conditions on the articulation facet of the basal elements with the coracoid bar has been explored in Silva 
et al. (2015). However, the visualization of such characters on the CT-Scan images was compromised by their inaccurate resolution on the CT-Scan imaging, therefore not applicable for eventual coding for the present study. In some taxa such as Carcharhinus galapagensis, the posterior proximal radials are modified into round structures that are separated from the metapterygium (Silva 2014; Silva \& Carvalho 2015). This condition is not present in Isogomphodon, as corroborated with the CT-Scan images.

The main candidate as sister-group to Isogomphodon oxyrhynchus is Carcharhinus porosus, as supported both by previous and the present phylogenetic hypotheses based on sequence data and on comparative anatomy. Alternative hypotheses exist (e.g., Rodrigues-Filho et al. (2009); Naylor et al. 2012a; Feitosa et al. (2018); Sorenson et al. 2014; Cachera \& Le Loc'h 2017; Vélez-Zuazo \& Agnarson 2011) and are discussed below.

Rodrigues-Filho et al. (2009) and Feitosa et al. (2018) used their topologies as a tool for identification, not phylogenetic relationships, but nonetheless provide useful clues for subsequent analyses. Similarly, in the results obtained by Naylor et al. (2012b), it is stated: "While there is undoubtedly some phylogenetic information in the clustering, it is almost entirely restricted to the tips of the tree." Unfortunately, Carcharhinus isodon, a proposed sister-group to Isogomphodon oxyrhynchus, was not available for the present study. Of course, phylogenetic considerations have a decisive bearing on nomenclature, namely the validity of Isogomphodon. In the cases which I. oxyrhynchus is internested within species currently included in Carcharhinus, then the recognition of a separate genus would result in the latter being paraphyletic.

In Vélez-Zuazo e Agnarson (2011), C. porosus was classified as sister group to $I$. oxyrhynchus, which was the first molecular-based hypothesis to propose such relationship based on traditionally employes sequences (COI, NADH2, Cytb, 16S and Rag-1). The results from Sorenson et al. 2014, the only one which was calibrated, group I. oxyrhynchus as sister group to $C$. macloti, a clade which is closely related to $C$. porosus. Those results, however accounted for habitat preference in which C. porosus was coded as brackish, as well as $I$. oxyrhynchus. The results obtained in Cachera \& Le Loc'h (2017) allocated I. oxyrhynchus within a Carcharhinus clade that includes $C$. porosus, again not in a sister-group conformation. This hypothesis only corroborates with previous results by solely allocating Isogomphodon as related to Carcharhinus, which has already been strongly supported.

The present chapter revealed that while a sister-group relationship between $I$. oxyrhynchus and Carcharhinus porosus is well supported by the novel molecular hypothesis 
here proposed, the position of the clade I. oxyrhynchus + C. porosus drifted among different topologies (Table 2). In the $0 \%$ and $25 \%$ treatments of missing, the clade in question was in a position in which, in case C. porosus is to be transferred to Isogomphodon, the genus Carcharhinus would not be resulted as paraphyletic. Therefore, an eventual nomenclatural change (in this case Carcharhinus porosus would be referred as Isogomphodon porosus), would be plausible to occur if such phylogenetic conformations were confirmed by further analyses. On the other hand, this change is questionable, once the comparative anatomy revealed no exclusive similarity between I. oxyrhynchus and C. porosus. Of 45 characters investigated herein, 26 were coded as the same state for those taxa (i.e., approximately $58 \%$ of similarity), although, as previously stated, none were exclusive.

Carcharhinus porosus (Ranzani, 1839) is a rather common shark species that, similar to the genus Rhizoprionodon, has the second dorsal fin origin located posteriorly to the anal fin origin being the only Carcharhinus with this character, although it agrees with its genus allocation by lacking a pair of labial sulcus and pre-anal ridge (Gomes et al. 2010). The distribution area of $C$. porosus extends from the southern coast of South America to the northern Atlantic on the United States, being restricted to coastal waters. Therefore, their distribution area overlaps in certain regions, for example on the Maranhão state coast in north-eastern Brazil (Lessa et al. 1999). Considering that similarities were detected between those species, a robust morphological analysis likely will corroborate with past results. In terms of autapomorphies of C. porosus and its unique similarities with I. oxyrhynchus, none of them were detected in the present analysis, which is actually curious considering the previously proposed relationships among those two species. It is possible that other anatomical complexes such as musculature and external features might elucidate features that would group those taxa in a morphological perspective, which has so far been performed by molecular-based characters.

Naylor et al. (2012b) allocated two species as closely related to Isogomphodon oxyrhynchus: Carcharhinus porosus and C. isodon. The external morphology of both species gives no hint as to conditions remindful of those in Isogomphodon oxyrhynchus. The cephalic region and pectoral fins of the latter are the most modified regions externally. Posterior to that point, there are few traits which deserve notice. For instance, in I. oxyrhyrnchus and C. isodon the origin of the second dorsal fin is aligned with the anal-fin origin, whereas in C. porosus, as in Rhizoprionodon, the origin of the second dorsal fin is located further posteriorly. Additionally, the pectoral fin in I. oxyrhynchus is broader and longer than in any representatives of Carcharhinus. Despite that, a proper evaluation of the significance of such similarities and 
differences requires a study focused on quantitative morphometrics assessments, something beyond the scope of the present work.

In terms of tooth morphology, C. isodon and C. porosus exhibit interesting parallels with I. oxyrhynchus. In C. porosus, the upper tooth rows resemble those of Rhizoprionodon, Loxodon and some Carcharhinus species such as C. signatus (Compagno 1984). Contrastingly, the lower tooth morphology is most similar to that of Isogomphodon, such as an elongated crown with narrow roots on the anterior rows. In $C$. isodon, teeth from both upper and lower rows resemble those of Isogomphodon: narrow roots with elongated crowns on anterior rows (decreasing in size towards posterior rows). However, the teeth of $C$. isodon are larger in total size and lack a deepened mesial notch (see Chapter 3). Those observations might provide substantial clues for future advances. 


\section{Concluding Remarks}

The Daggernose Shark, Isogomphodon oxyrhynchus, has anatomical modifications on its skeleton that might impact in its phylogenetic position within Carcharhinidae. The neurocranium and mandibular arch were the structures that allocated most of the modified characters, therefore could be interpreted as autapomorphies to the genus Isogomphodon. The truncated pre-orbital process and 'V-Shaped' anterior fontanelle were the most likely unique characters on the neurocranium. In the splanchnocranium, the ' $\mathrm{C}$-shaped' hollow area, expanded symphysis on the Meckel's cartilage and total teeth counting were the strongest characters to be considered autapomorphies. The appendicular skeleton of I. oxyrhynchus presented a rather similar anatomy when compared to other Carcharhinidae, with the coracoid bar being slightly bulkier as the most notable difference. To substantiate such results, not only the analysis of more taxa is required but also the performing of a phylogenetic analysis by means of a specialized software. With the organization of the comparative anatomy as a binary coded matrix and the character statements formulated as suggested by Sereno (2007), the present chapter is a relevant starting point for future research. Regarding the phylogenetic discussion, most previous topologies that included this species as a terminal were based on molecular data, with Carcharhinus porosus as a likely sister taxon to I. oxyrhynchus. Even with a low number of compared taxa, the 46 characters here elaborate not only provided a significant baseline for future morphological phylogenetic research, but also resulted in $C$. porosus as one of the most similar species to I. oxyrhynchus, which would potentially corroborate with previous hypothesis that considered these species as sister group. The sympatry between these species also argues in favour of such result, although further investigation is required in order to confirm whether or not this distribution overlap would impact in the final topology. Additionally, the novel molecular topologies here presented, in all three treatments of tolerated missing data, allocated I. oxyrhynchus as $C$. porosus was sister-taxa. This clade positioning, however, is different among the treatments, in which $0 \%$ and $25 \%$ positioned it in the base of the Carcharhinus clade and in $10 \%$ as sister-group of Triaenodon obesus. 


\section{References}

Andreev, P. S., Coates, M. I., Shelton, R. M., Cooper, P. R., Smith, M. P., \& Sansom, I. J. (2015). U pper O rdovician chondrichthyan-like scales from $\mathrm{N}$ orth $\mathrm{A}$ merica. Palaeontology, 58(4), 691-704.

Applegate, S. P. (1967). A survey of shark hard parts. Sharks, skates and rays, 37-67.

Cachera, M., \& Le Loc'h, F. (2017). Assessing the relationships between phylogenetic and functional singularities in sharks (Chondrichthyes). Ecology and evolution, 7(16), 62926303.

Casas, A. L. D. S., Intelizano, W., Fernandes de Souza Castro, M., \& Bonfim Mariana, A. N. (2005). Nervios de la musculatura mandibular del tiburon toro Carcharias taurus (Rafinesque, 1810) (Chondrichthyes: Odontaspididae). International journal of morphology, 23(4), 387-392.

Compagno, L. J. (1977). Phyletic relationships of living sharks and rays. American zoologist, 17(2), 303-322.

Compagno, L. J. (1988). Sharks of the order Carcharhiniformes (Vol. 486). Princeton, New Jersey: Princeton University Press.

Compagno, L. J. V. (1999). Endoskeleton. Sharks, skates, and rays: The biology of elasmobranch fishes, 69-92.

de Pinna, M. C. (1991). Concepts and tests of homology in the cladistic paradigm. Cladistics, 7(4), 367-394.

de Carvalho, M. R. (1996). Higher-level elasmobranch phylogeny, basal squaleans, and paraphyly. Interrelationships of fishes, 3, 35-62.

Feitosa, L. M., Martins, A. P. B., Giarrizzo, T., Macedo, W., Monteiro, I. L., Gemaque, R., Nunes, J. L. S., Gomes, F., Scheinder, H., Sampaio, I., Souza, R., Sales, J. B., RodriguesFilho, L. F., Tchaicka, L. \& Carvalho-Costa, L. F. (2018). DNA-based identification reveals illegal trade of threatened shark species in a global elasmobranch conservation hotspot. Scientific reports, 8(1), 3347.

Fricke, R., Eschmeyer, W. N. \& Van der Laan, R. (eds) 2020. Eschmeyer's Catalog of Fishes:

Genera, Species, Available at:

(http://researcharchive.calacademy.org/research/ichthyology/catalog/fishcatmain.asp)

Electronic version accessed: [22th March 2020]. 
Goloboff, P. A., Farris, J. S., \& Nixon, K. C. (2008). TNT, a free program for phylogenetic analysis. Cladistics, 24(5), 774-786.

Hennig, W. (1950). Grundzüge einer Theorie der phylogenetischen Systematik Deutscher Zentral-Verlag. Berlin, Germany.

Hennig, W. (1966). Phylogenetic systematics. University of Illinois Press.

Katoh, K., Misawa, K., Kuma, K. I., \& Miyata, T. (2002). MAFFT: a novel method for rapid multiple sequence alignment based on fast Fourier transform. Nucleic acids research, 30(14), 3059-3066.

Klug, S., \& Kriwet, J. (2010). Timing of deep-sea adaptation in dogfish sharks: insights from a supertree of extinct and extant taxa. Zoologica Scripta, 39(4), 331-342.

Kikuchi, C. N. T. (2004). Relações filogenéticas entre as espécies do gênero Rhizoprionodon Whitley, 1929 (Elasmobranchii, Carcharhinidae)

Lanfear, R., Frandsen, P. B., Wright, A. M., Senfeld, T., \& Calcott, B. (2017). PartitionFinder 2: new methods for selecting partitioned models of evolution for molecular and morphological phylogenetic analyses. Molecular biology and evolution, 34(3), 772-773.

Lessa, R. P. T. (1987). Aspectos da biologia do cação-quati, Isogomphodon oxyrhynchus (Mueller \& Henle, 1839) (Chondrichthyes: Carcharhinidae), das reentrâncias maranhenses. Bol. Cien. do Mar. Fortaleza-Ceará, 44-1.

Lessa, R., Batista, V., \& Almeida, Z. (1999). Occurence and biology of the daggernose shark Isogomphodon oxyrhynchus (Chondrichthyes: Carcharhinidae) off the Maranhão coast (Brazil). Bulletin of Marine Science, 64(1), 115-128.

Lessa, R., Batista, V. S., \& Santana, F. M. (2016). Close to extinction? The collapse of the endemic daggernose shark (Isogomphodon oxyrhynchus) off Brazil. Global Ecology and Conservation, 7, 70-81.

Loboda, T. S., \& Carvalho, M. R. D. (2013). Systematic revision of the Potamotrygon motoro (Müller \& Henle, 1841) species complex in the Paraná-Paraguay basin, with description of two new ocellated species (Chondrichthyes: Myliobatiformes: Potamotrygonidae). Neotropical Ichthyology, 11(4), 693-737.Maisey, J. G. (1984). Higher elasmobranch phylogeny and biostratigraphy. Zoological Journal of the Linnean Society, 82(1-2), 33-54. 
Loboda, T. S. (2016). Revisão taxonômica e morfológica do gênero Paratrygon Duméril (1865)(Chondrichthyes: Myliobatiformes: Potamotrygonidae)(Doctoral dissertation, Universidade de São Paulo).

Maisey, J. G. (2012). What is an 'elasmobranch'? The impact of palaeontology in understanding elasmobranch phylogeny and evolution. Journal of Fish Biology, 80(5), 918951.

Moreira, R. A., Gomes, U. L., \& de Carvalho, M. R. (2019). Systematic implications of the caudal fin skeletal anatomy in ground sharks, order Carcharhiniformes (Chondrichthyes: Elasmobranchii). Zoological Journal of the Linnean Society, 185(1), 193-211.

Motta, P. J., \& Wilga, C. A. (1995). Anatomy of the feeding apparatus of the lemon shark, Negaprion brevirostris. Journal of Morphology, 226(3), 309-329.

Naylor, G. J. (1992). The phylogenetic relationships among requiem and hammerhead sharks: inferring phylogeny when thousands of equally most parsimonious trees result. Cladistics, 8(4), 295-318.

Naylor, G. J., Caira, J. N., Jensen, K., Rosana, K. A., Straube, N., \& Lakner, C. (2012a). Elasmobranch phylogeny: a mitochondrial estimate based on 595 species. The biology of sharks and their relatives, 31-56.

Naylor, G. J., Caira, J. N., Jensen, K., Rosana, K. A. M., White, W. T., \& Last, P. R. (2012b). A DNA sequence-based approach to the identification of shark and ray species and its implications for global elasmobranch diversity and parasitology. Bulletin of the American Museum of Natural History, 2012(367), 1-262.

Naylor, G. J. (2021). Chondrichthyan Tree of Life (Available at: https://sharksrays.org/)

Patterson, C. (1982). Morphological characters and homology. Problems of phylogenetic reconstruction, 21-74.

Rodrigues-Filho, L. F. D. S., Rocha, T. C. D., Rêgo, P. S. D., Schneider, H., Sampaio, I., \& Vallinoto, M. (2009). Identification and phylogenetic inferences on stocks of sharks affected by the fishing industry off the Northern coast of Brazil. Genetics and molecular biology, $32(2), 405-413$.

Sanderson, M. J., Boss, D., Chen, D., Cranston, K. A., \& Wehe, A. (2008). The PhyLoTA Browser: processing GenBank for molecular phylogenetics research. Systematic Biology, 57(3), 335-346.

Sereno, P. C. (2007). Logical basis for morphological characters in phylogenetics. Cladistics, 23(6), 565-587. 
Silva, J. P. (2014). Filogenia dos principais grupos de Chondrichthyes baseada na anatomia comparada do esqueleto das nadadeiras pares e suas cinturas (Doctoral dissertation, Universidade de São Paulo).

Silva, J. P. C., \& De Carvalho, M. R. (2015). Morphology and phylogenetic significance of the pectoral articular region in elasmobranchs (Chondrichthyes). Zoological Journal of the Linnean Society, 175(3), 525-568.

Silva, J. P. C., Vaz, D. F., \& De Carvalho, M. R. (2017). Phylogenetic inferences on the systematics of squaliform sharks based on elasmobranch scapular morphology (Chondrichthyes: Elasmobranchii). Zoological Journal of the Linnean Society, 182(3), 614630.

Soares, K. D. A. (2020). Comparative anatomy of the clasper of catsharks and its phylogenetic implications (Chondrichthyes: Carcharhiniformes: Scyliorhinidae). Journal of Morphology, 281(6), 591-607.Sorenson, L., Santini, F., \& Alfaro, M. E. (2014). The effect of habitat on modern shark diversification. Journal of Evolutionary Biology, 27(8), 15361548.

Soares, M. C., \& de Carvalho, M. R. (2013). Mandibular and hyoid muscles of galeomorph sharks (Chondrichthyes: Elasmobranchii), with remarks on their phylogenetic intrarelationships. Journal of Morphology, 274(10), 1111-1123.

Sorenson, L., Santini, F., \& Alfaro, M. E. (2014). The effect of habitat on modern shark diversification. Journal of Evolutionary Biology, 27(8), 1536-1548.

Stamatakis, A. (2014). RAxML version 8: a tool for phylogenetic analysis and post-analysis of large phylogenies. Bioinformatics, 30(9), 1312-1313.

Stone, N. R., \& Shimada, K. (2019). Skeletal Anatomy of the Bigeye Sand Tiger Shark, Odontaspis noronhai (Lamniformes: Odontaspididae), and Its Implications for Lamniform Phylogeny, Taxonomy, and Conservation Biology. Copeia, 107(4), 632-652.

Vélez-Zuazo, X., \& Agnarsson, I. (2011). Shark tales: a molecular species-level phylogeny of sharks (Selachimorpha, Chondrichthyes). Molecular phylogenetics and evolution, 58(2), 207-217.

Wilga, C., \& Motta, P. (1998). Conservation and variation in the feeding mechanism of the spiny dogfish Squalus acanthias. Journal of Experimental Biology, 201(9), 1345-1358. 


\section{Appendix A - Analysed Material}

Institutional abbreviations:

AMNH - American Museum of Natural History; GMBL - Grice Marine Biological Laboratory; MZUSP - Museu de Zoologia da Universidade de São Paulo; Uncat Uncataloged; WAM - Western Australian Museum - Perth, Austrália;

\section{Carcharhinidae}

Isogomphodon oxyrhynchus - MZUSP 101214, 1 Newborn (alc, skins samples for SEM); MZUSP 101214, 1 Juvenile male (Alc, CT-Scan); MZUSP 37289, 1 Juvenile Male (dissected); MPEG 3455, 1 Adult male (alc).

Carcharhinus perezii - MZUSP53007, 1 Juvenile head (alc, CT-Scan)

Carcharhinus porosus - MZUSP84440, 1 Juvenile female (alc, dissected)

Carcharhinus galapagensis - GMBL Uncat, 1 Juvenile male (CT-Scan - obtained from sharksrays.org)

Carcharhinus plumbeus - GMBL 7960, 1 Juvenile male (CT-Scan - obtained from sharksrays.org)

Loxodon macrorhinus - WAM 26211.001, 1 Adult male (cleared and stained - donation) Negaprion brevirostris - GMBL Uncat, 1 Juvenile female (CT-Scan - obtained from sharksrays.org)

Rhizoprionodon lalandii - Uncat \#1, 1 Adult female (dissected); MZUSP79819, 1 Juvenile (alc, skeleton preparation)

Rhizoprionodon porosus - MZUSP 84432, 1 Juvenile (alc, skeleton preparation), MZUSP Uncat (cleared and stained)

Rhizoprionodon taylori - WAM Uncat, 1 Juvenile male (cleared and stained - donation)

Rhizoprionodon terranovae - GMBL Uncat, 1 Adult male (CT-Scan - obtained from sharksrays.org)

Scoliodon macrorhynchos - AMNH 258155, 1 Adult male (CT-Scan - obtained from sharksrays.org)

Triaenodon obesus - USNM 216792, 1 Juvenile male (CT-Scan - obtained from sharksrays.org) 


\section{Figures and Tables}

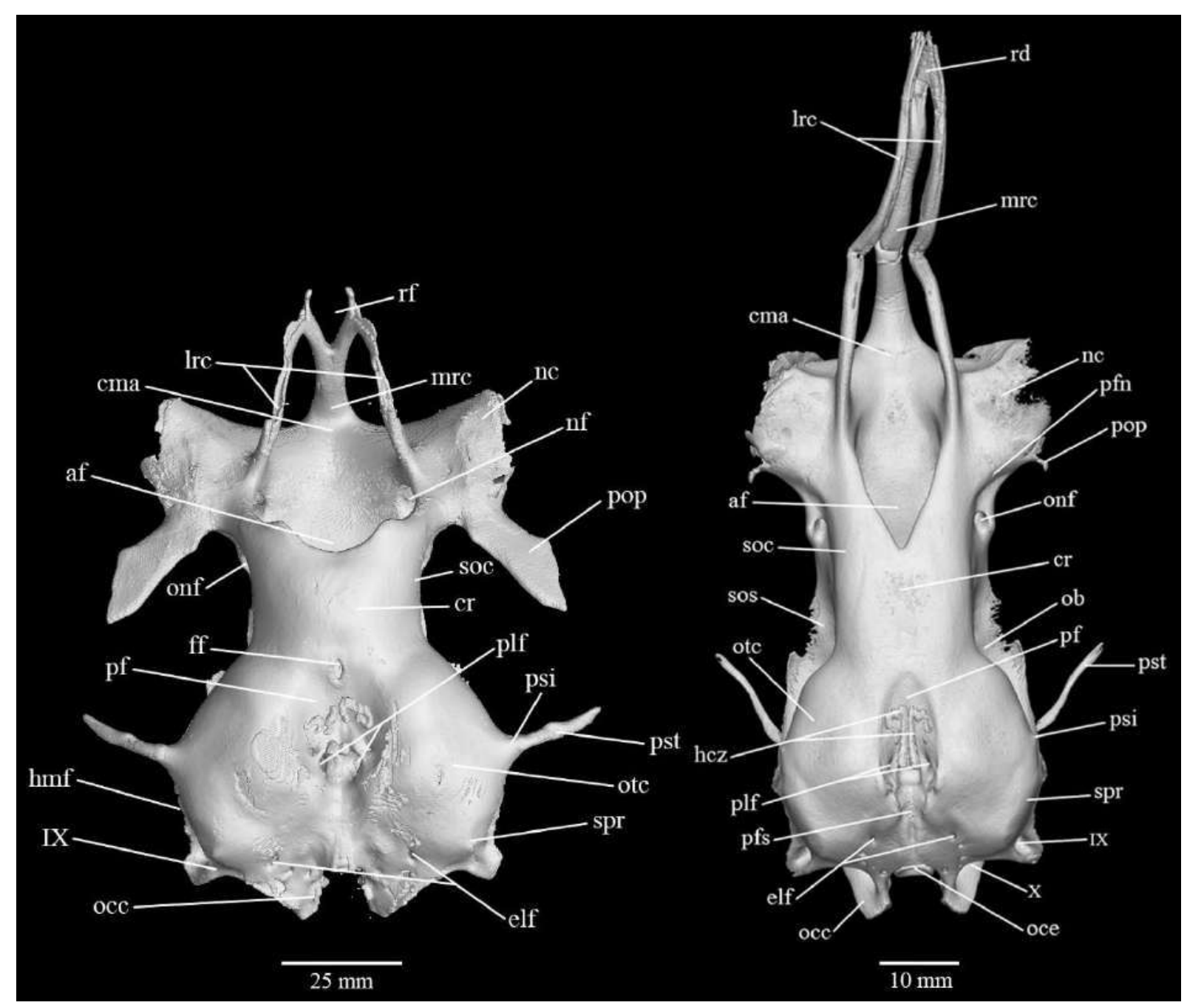

Figure 1. Comparative image between the neurocranium dorsal morphology of Carcharhinus species (Carcharhinus perezii - MZUSP 53007 - Left) and Isogomphodon oxyrhynchus (MZUSP 101214 Right) illustration the most modified region of the Daggernose shark in comparison to a generical and conserved morphology of a neurocranium from a Carcharhinus representant. Notice the elongated snout, reduced to nearly truncated pre-orbital process, narrow cranial roof, and V-Shaped anterior fontanelle. Anatomical abbreviations: af, anterior fontanelle; cma, confluence between the medial rostral cartilage and anterior fontanelle; cr, cranial roof; elf, endolymphatic foramen; ff, frontal fontanelle; IX, glossopharyngeal foramen; hcz, hypercalcified zones; lrc, lateral rostral cartilage; mrc, medial rostral cartilage; nc, nasal capsule; nf, nasal fontanelle; ob, orbit; occ, occipital condyle; oce, occipital centrum; onf, orbitonasal foramen; otc, otic capsule; pf, parietal fossa; pfn, profundus nerve foramen; pfs, parietal fossa sulcus; plf, perilymphatic fenestrae; pop, preorbital process; psi, postorbital process insertion; psl, posterior superior labial cartilage; pst, postorbital process; rd, rostral node; soc, supraorbital crest; sos, suborbital shelf; spr, sphenopterotic ridge; $\mathrm{X}$, vagus nerve foramen; 


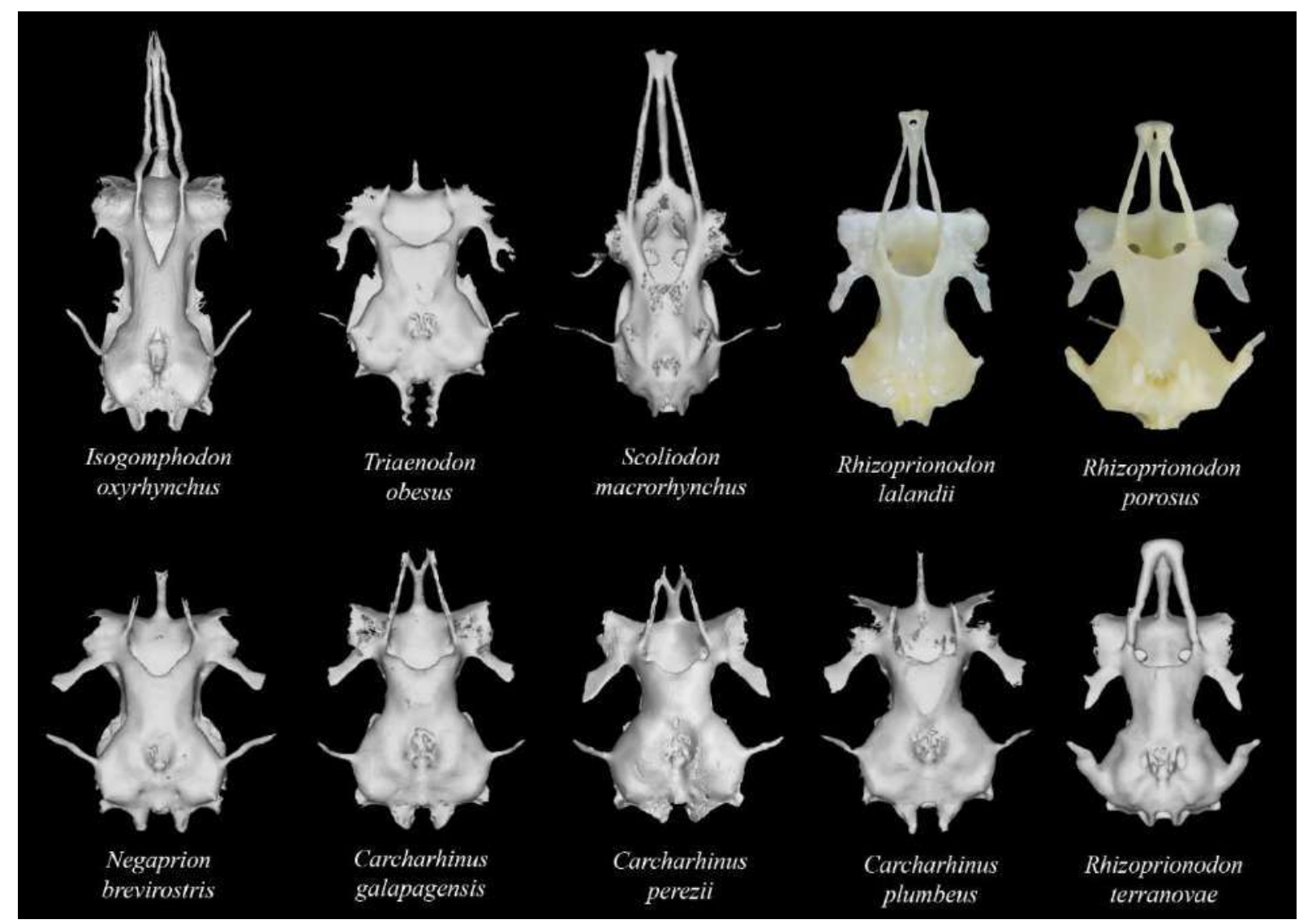

Figure 2. Comparative image between the neurocranium anatomy in dorsal view of exanimated Carcharhinidae sharks. Triaenodon obesus (USNM 216792), Scoliodon macrorhynchos (AMNH 258155), Negaprion brevirostris (GMBL Uncatalogued), Carcharhinus galapagensis (GMBL Uncatalogued), Carcharhinus plumbeus (GMBL 7960) and Rhizoprionodon terranovae (GMBL Uncatalogued) modified from Naylor (2021), available at: sharksrays.org. Specimen identification: Isogomphodon oxyrhynchus (MPEG 3455); Triaenodon obesus (USNM 216792); Scoliodon macrorhynchos (AMNH 258155); Rhizoprionodon lalandii (MZUSP 79817); Rhizoprionodon porosus (MZUSP 84432); Negaprion brevirostris (GMBL Uncatalogued); Carcharhinus galapagensis (GMBL Uncatalogued); Carcharhinus perezii (MZUSP 53007); Carcharhinus plumbeus (GMBL 7960); Rhizoprionodon terranovae (GMBL Uncatalogued); 


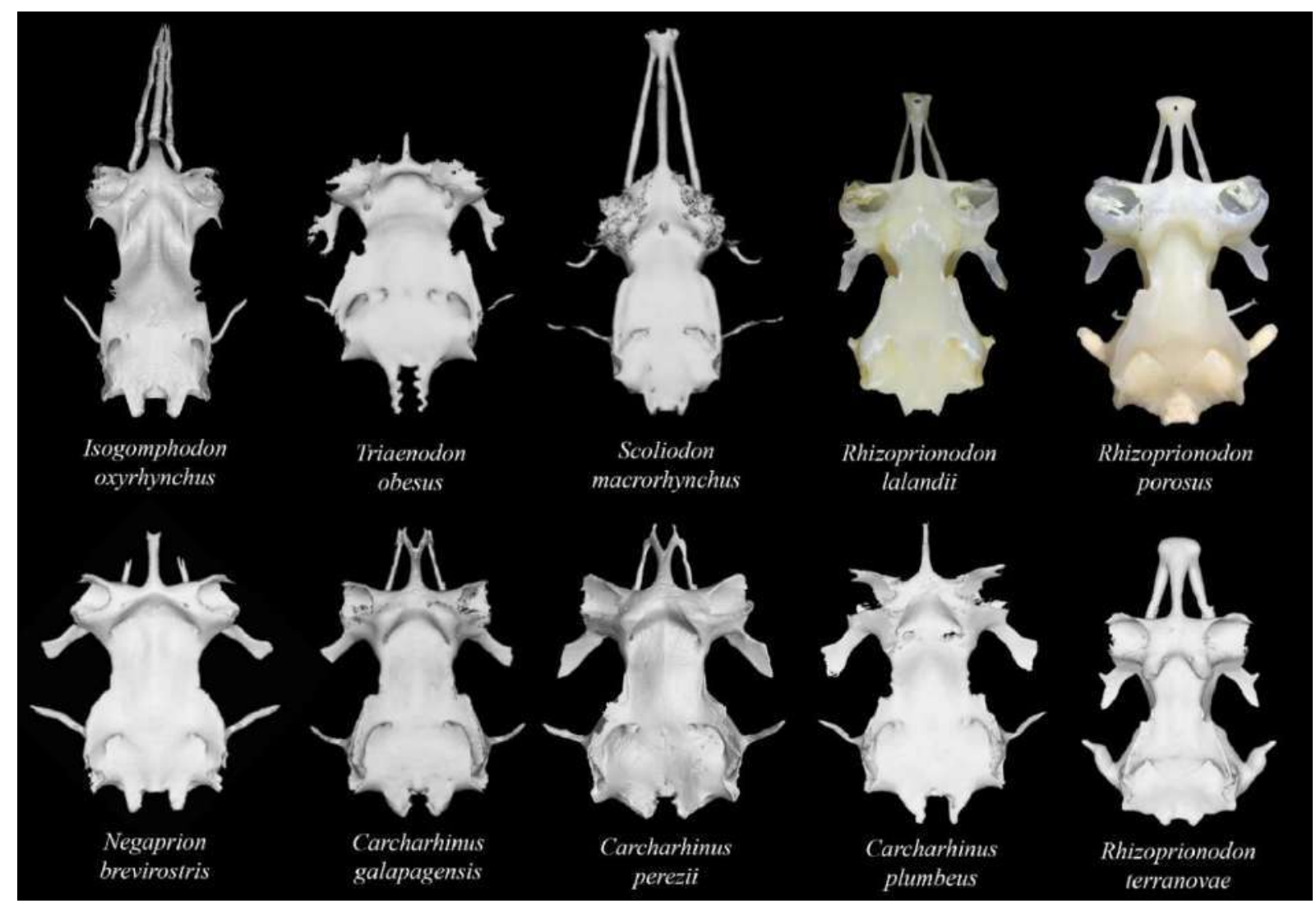

Figure 3. Comparative image between the neurocranium anatomy in ventral view of exanimated Carcharhinidae sharks. Triaenodon obesus (USNM 216792), Scoliodon macrorhynchos (AMNH 258155), Negaprion brevirostris (GMBL Uncatalogued), Carcharhinus galapagensis (GMBL Uncatalogued), Carcharhinus plumbeus (GMBL 7960) and Rhizoprionodon terranovae (GMBL Uncatalogued) modified from Naylor (2021), available at: sharksrays.org. Specimen identification: Isogomphodon oxyrhynchus (MPEG 3455); Triaenodon obesus (USNM 216792); Scoliodon macrorhynchos (AMNH 258155); Rhizoprionodon lalandii (MZUSP 79817); Rhizoprionodon porosus (MZUSP 84432); Negaprion brevirostris (GMBL Uncatalogued); Carcharhinus galapagensis (GMBL Uncatalogued); Carcharhinus perezii (MZUSP 53007); Carcharhinus plumbeus (GMBL 7960); Rhizoprionodon terranovae (GMBL Uncatalogued); 


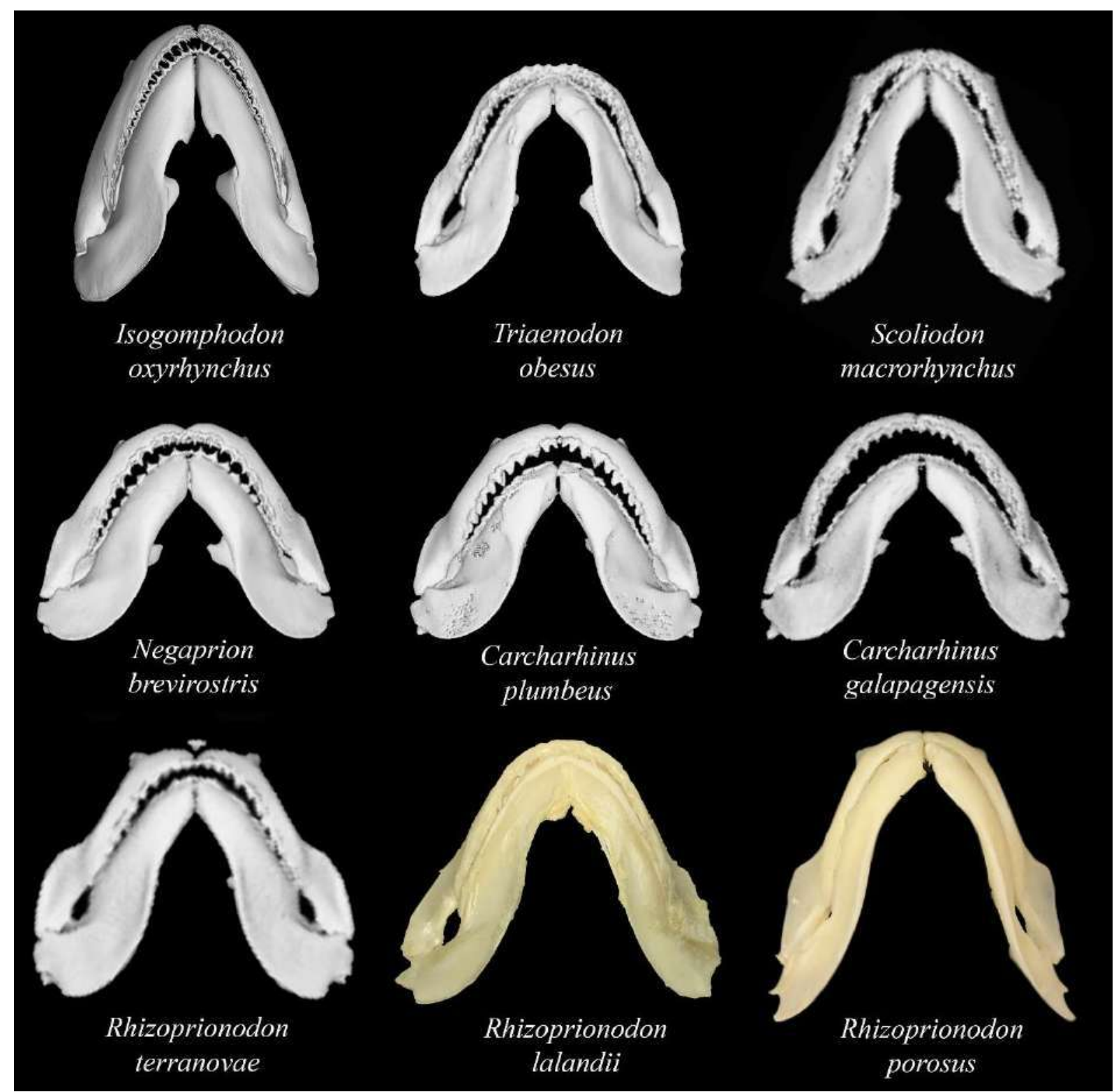

Figure 4. Comparative image between the mandibular arch anatomy in ventral view of exanimated Carcharhinidae sharks. Triaenodon obesus (USNM 216792), Scoliodon macrorhynchos (AMNH 258155), Negaprion brevirostris (GMBL Uncatalogued), Carcharhinus galapagensis (GMBL Uncatalogued), Carcharhinus plumbeus (GMBL 7960) and Rhizoprionodon terranovae (GMBL Uncatalogued) modified from Naylor (2021), available at: sharksrays.org. Specimen identification: Isogomphodon oxyrhynchus (MPEG 3455); Triaenodon obesus (USNM 216792); Scoliodon macrorhynchos (AMNH 258155); Rhizoprionodon lalandii (MZUSP 79817); Rhizoprionodon porosus (MZUSP 84432); Negaprion brevirostris (GMBL Uncatalogued); Carcharhinus galapagensis (GMBL Uncatalogued); Carcharhinus perezii (MZUSP 53007); Carcharhinus plumbeus (GMBL 7960); Rhizoprionodon terranovae (GMBL Uncatalogued); 


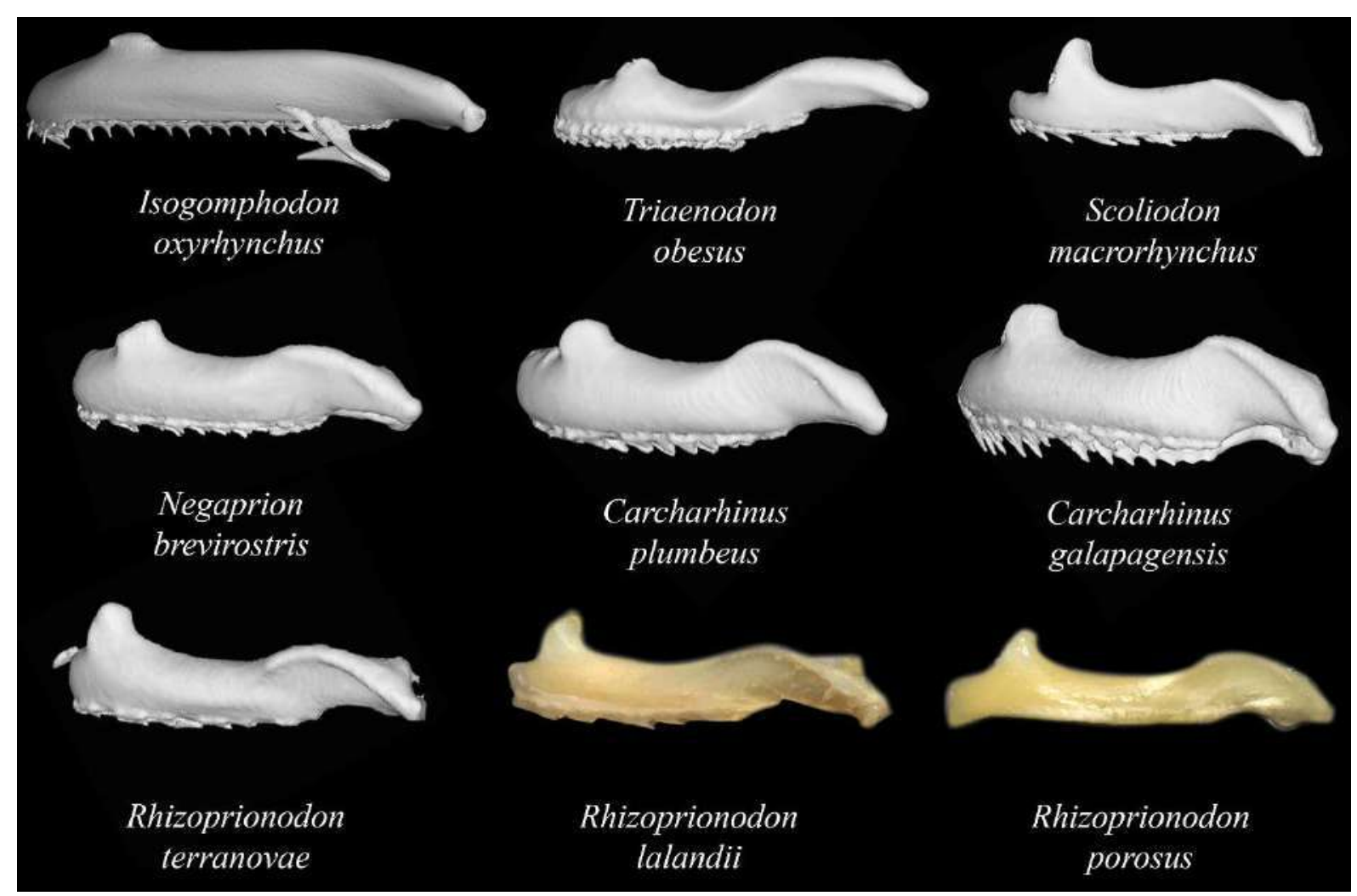

Figure 5. Comparative image between the palatoquadrate anatomy in lateral view of exanimated Carcharhinidae sharks. Triaenodon obesus (USNM 216792), Scoliodon macrorhynchos (AMNH 258155), Negaprion brevirostris (GMBL Uncatalogued), Carcharhinus galapagensis (GMBL Uncatalogued), Carcharhinus plumbeus (GMBL 7960) and Rhizoprionodon terranovae (GMBL Uncatalogued) modified from Naylor (2021), available at: sharksrays.org. Specimen identification: Isogomphodon oxyrhynchus (MPEG 3455); Triaenodon obesus (USNM 216792); Scoliodon macrorhynchos (AMNH 258155); Rhizoprionodon lalandii (MZUSP 79817); Rhizoprionodon porosus (MZUSP 84432); Negaprion brevirostris (GMBL Uncatalogued); Carcharhinus galapagensis (GMBL Uncatalogued); Carcharhinus perezii (MZUSP 53007); Carcharhinus plumbeus (GMBL 7960); Rhizoprionodon terranovae (GMBL Uncatalogued); 


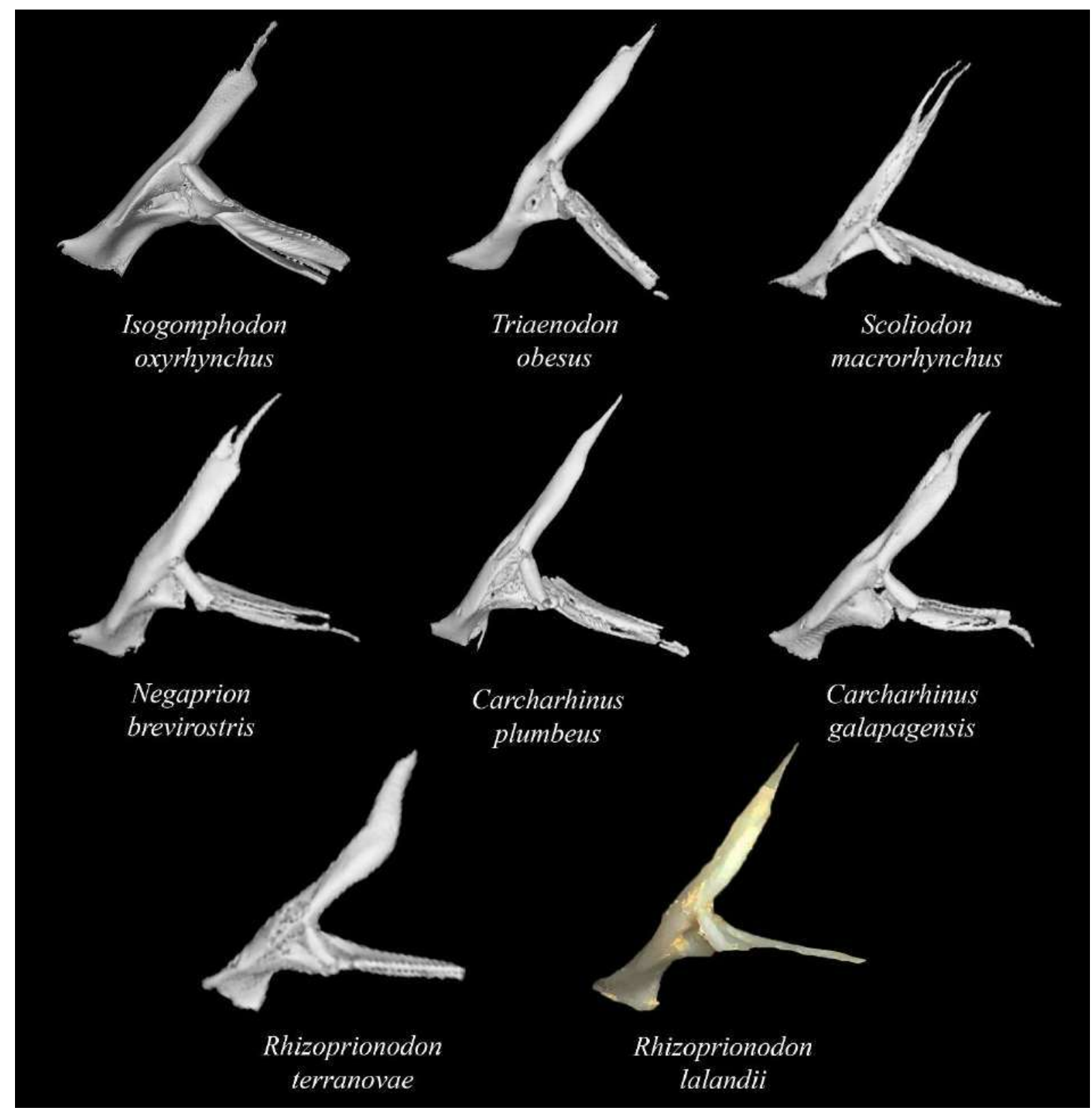

Figure 6. Comparative image between the pectoral girdle anatomy in lateral view of exanimated Carcharhinidae sharks. Triaenodon obesus (USNM 216792), Scoliodon macrorhynchos (AMNH 258155), Negaprion brevirostris (GMBL Uncatalogued), Carcharhinus galapagensis (GMBL Uncatalogued), Carcharhinus plumbeus (GMBL 7960) and Rhizoprionodon terranovae (GMBL Uncatalogued) modified from Naylor (2021), available at: sharksrays.org. Specimen identification: Isogomphodon oxyrhynchus (MPEG 3455); Triaenodon obesus (USNM 216792); Scoliodon macrorhynchos (AMNH 258155); Rhizoprionodon lalandii (MZUSP 79817); Rhizoprionodon porosus (MZUSP 84432); Negaprion brevirostris (GMBL Uncatalogued); Carcharhinus galapagensis (GMBL Uncatalogued); Carcharhinus perezii (MZUSP 53007); Carcharhinus plumbeus (GMBL 7960); Rhizoprionodon terranovae (GMBL Uncatalogued); 


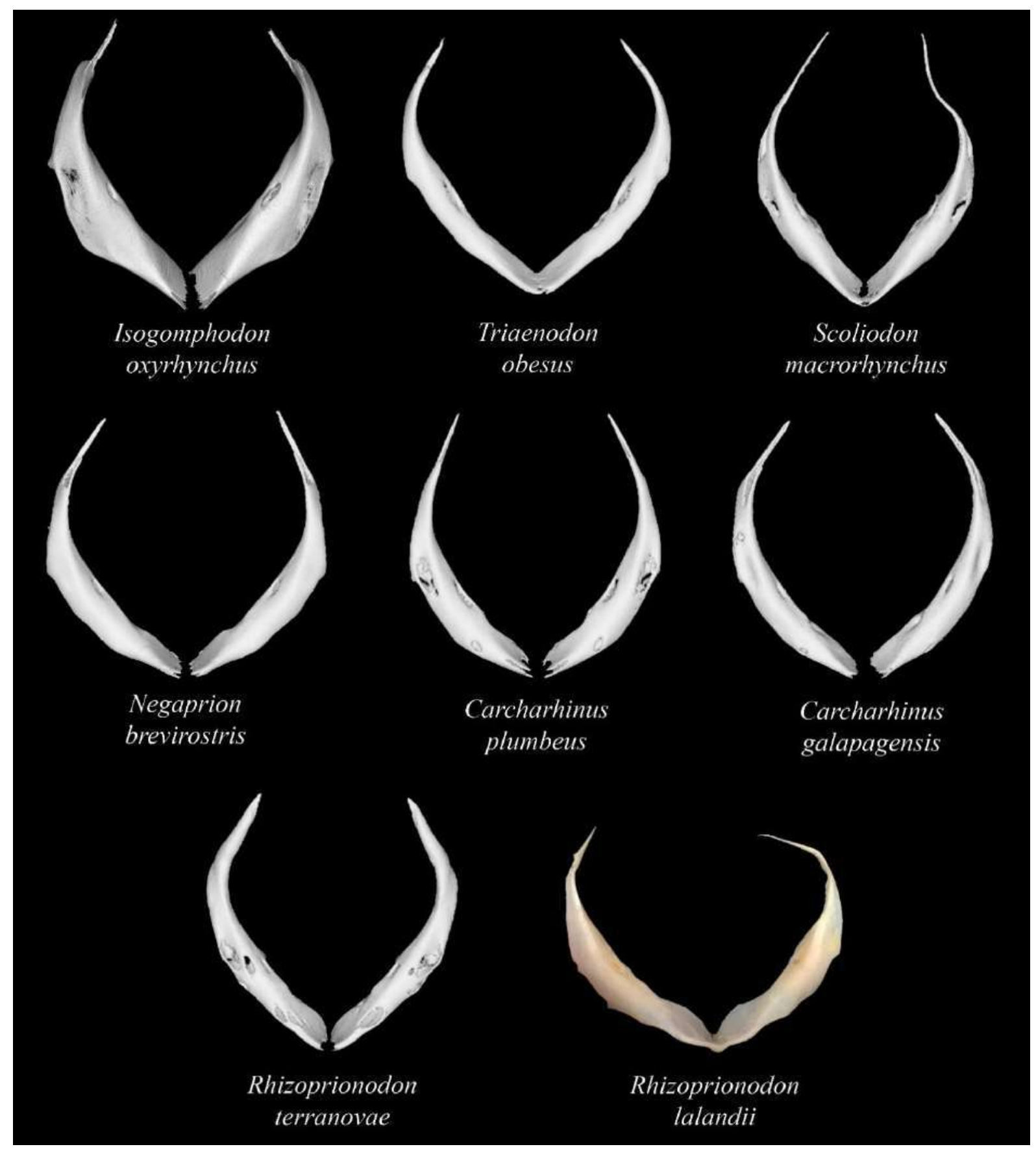

Figure 7. Comparative image between the coracoid bar anatomy in frontal view of exanimated Carcharhinidae sharks. Triaenodon obesus (USNM 216792), Scoliodon macrorhynchos (AMNH 258155), Negaprion brevirostris (GMBL Uncatalogued), Carcharhinus galapagensis (GMBL Uncatalogued), Carcharhinus plumbeus (GMBL 7960) and Rhizoprionodon terranovae (GMBL Uncatalogued) modified from Naylor (2021), available at: sharksrays.org. Specimen identification: Isogomphodon oxyrhynchus (MPEG 3455); Triaenodon obesus (USNM 216792); Scoliodon macrorhynchos (AMNH 258155); Rhizoprionodon lalandii (MZUSP 79817); Rhizoprionodon porosus (MZUSP 84432); Negaprion brevirostris (GMBL Uncatalogued); Carcharhinus galapagensis (GMBL 
Uncatalogued); Carcharhinus perezii (MZUSP 53007); Carcharhinus plumbeus (GMBL 7960); Rhizoprionodon terranovae (GMBL Uncatalogued);

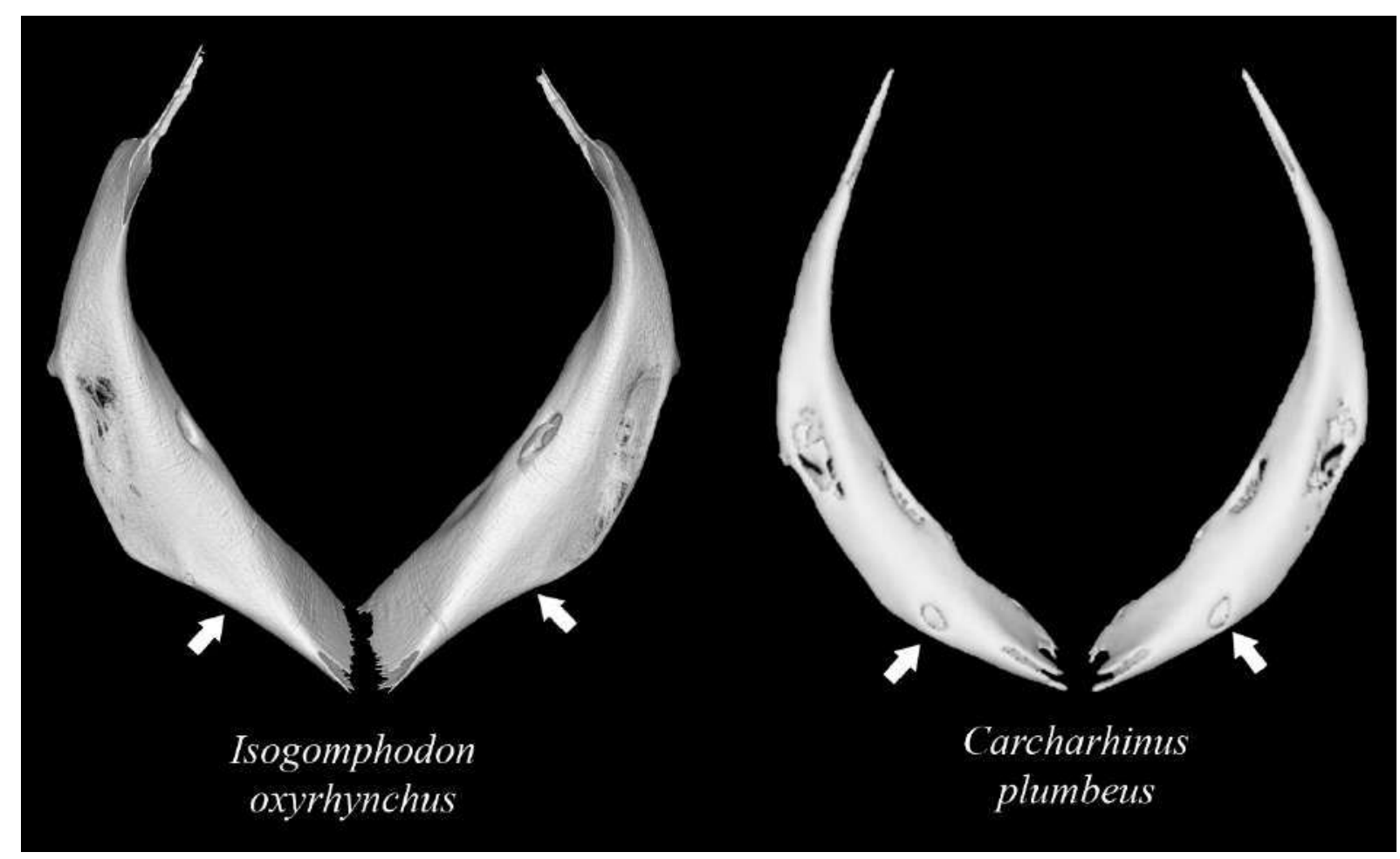

Figure 8. Comparative image between the scapulocoracoid morphology in frontal of Carcharhinidae sharks, focusing on two species. White arrow indicates a position where a possible foramen on the coracoid bar was located on Carcharhinus plumbeus and not on Isogomphodon oxyrhynchus. Carcharhinus plumbeus (GMBL 7960) modified from Naylor (2021), available at: sharksrays.org. Specimen identification: Isogomphodon oxyrhynchus (MPEG 3455); Carcharhinus plumbeus (GMBL 7960); 


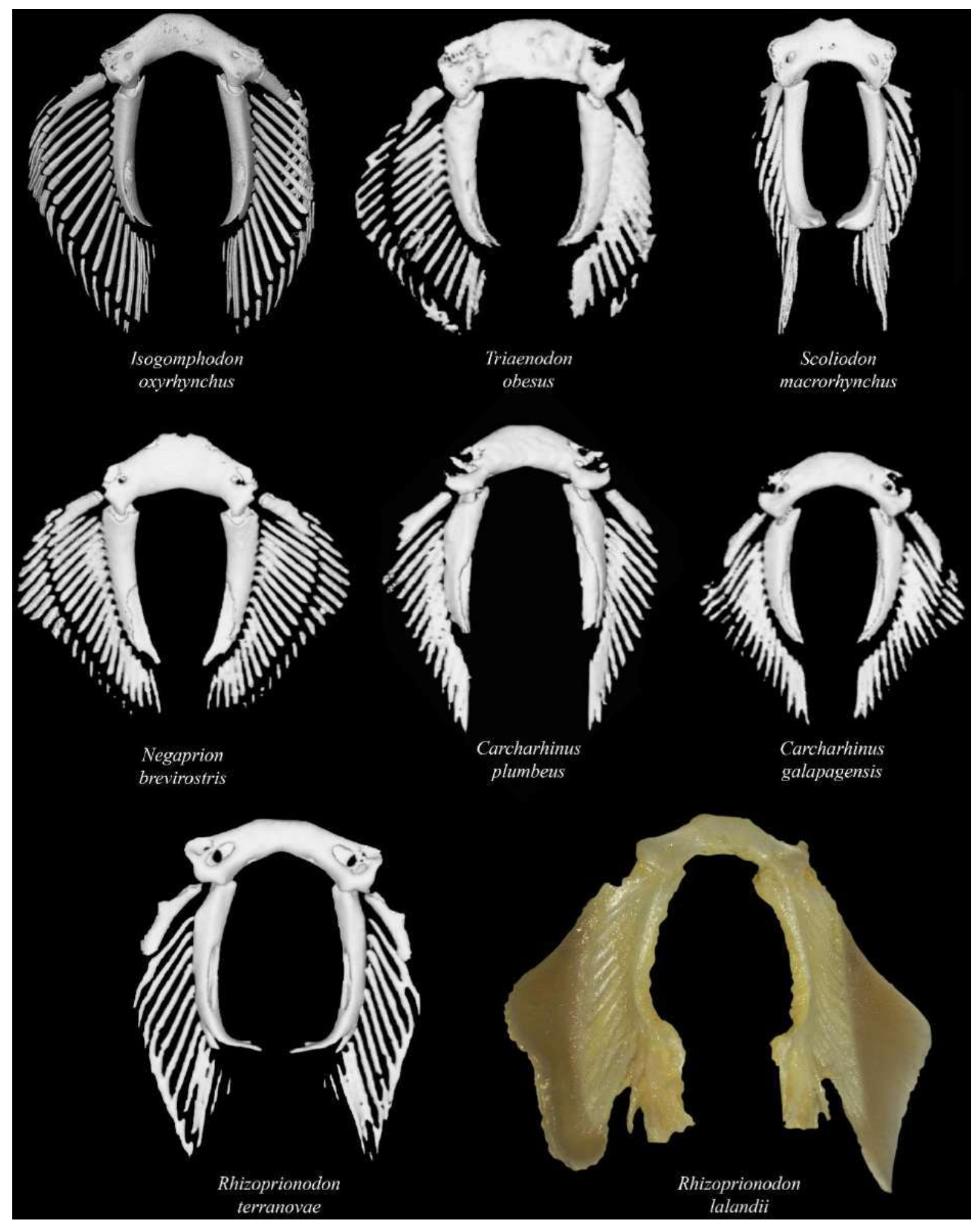

Figure 9. Comparative image between pelvic girdle anatomy in ventral view of exanimated Carcharhinidae sharks. Triaenodon obesus (USNM 216792), Scoliodon macrorhynchos (AMNH 258155), Negaprion brevirostris (GMBL Uncatalogued), Carcharhinus galapagensis (GMBL Uncatalogued), Carcharhinus plumbeus (GMBL 7960) and Rhizoprionodon terranovae (GMBL Uncatalogued) modified from Naylor (2021), available at: sharksrays.org. Specimen identification: Isogomphodon oxyrhynchus (MPEG 3455); Triaenodon obesus (USNM 216792); Scoliodon 
macrorhynchos (AMNH 258155); Rhizoprionodon lalandii (MZUSP 79817); Rhizoprionodon porosus (MZUSP 84432); Negaprion brevirostris (GMBL Uncatalogued); Carcharhinus galapagensis (GMBL Uncatalogued); Carcharhinus perezii (MZUSP 53007); Carcharhinus plumbeus (GMBL 7960); Rhizoprionodon terranovae (GMBL Uncatalogued);

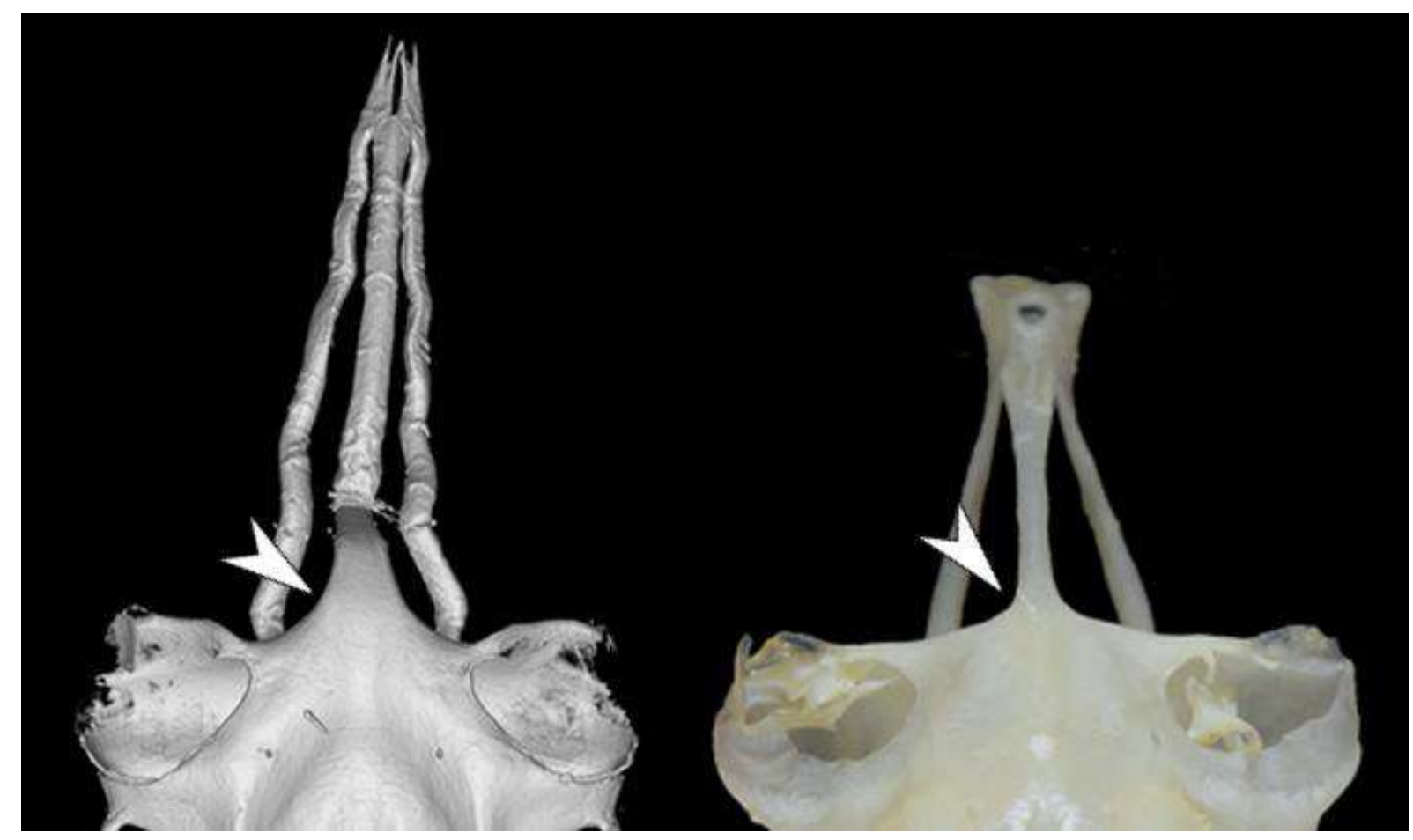

Figure 10. $\mathrm{Cn} 4$, in which the left is representing state 1 and the right, state 0 (Left - Isogomphodon oxyrhynchus MPEG 3455; Right - Rhizoprionodon lalandii MZUSP79817). 


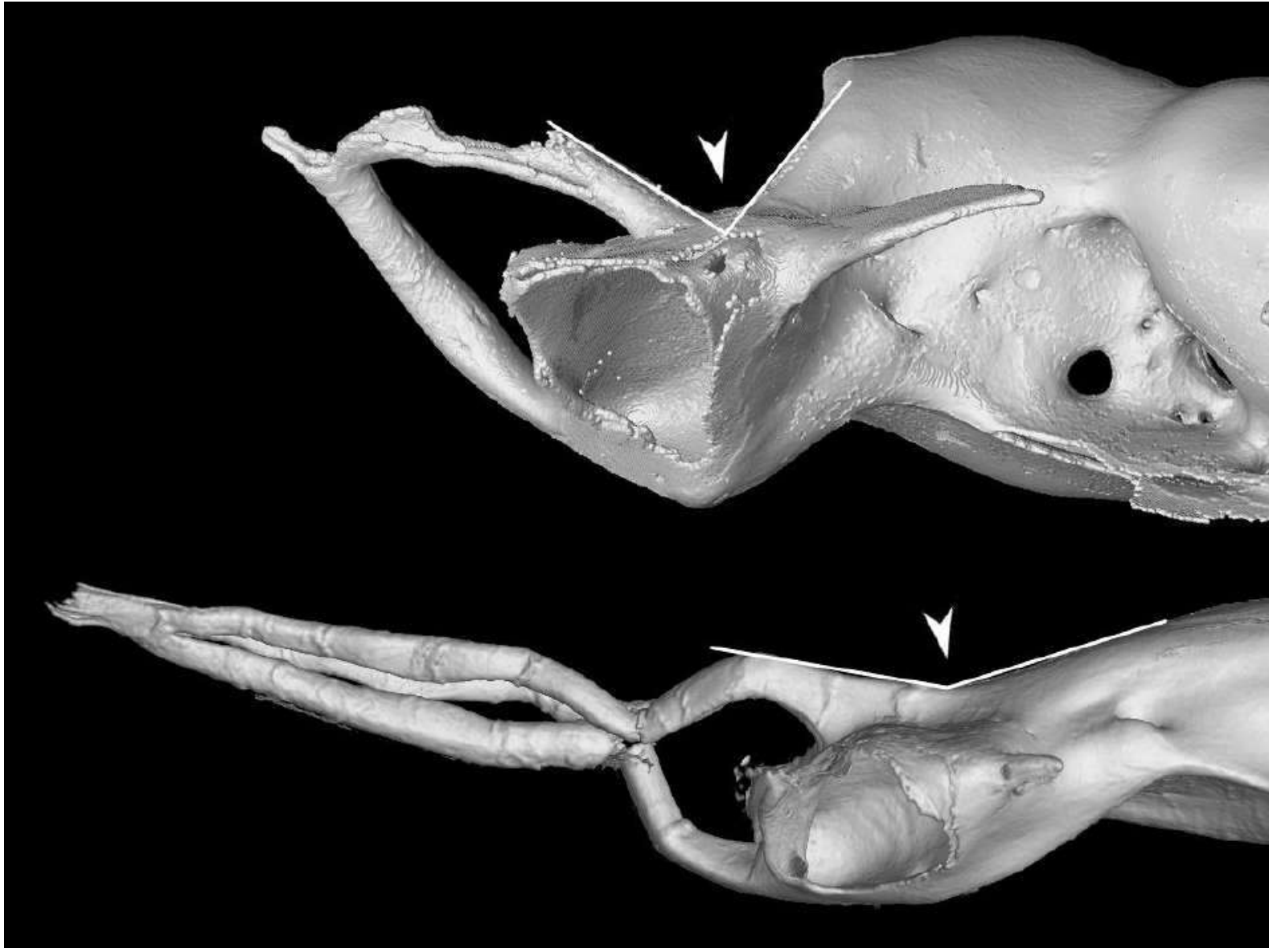

Figure 11. Cn5, in which the upper is representing state 0 and the lower, state 1 (Upper - Carcharhinus perezii MZUSP 53007; Lower - Isogomphodon oxyrhynchus MPEG 3455).

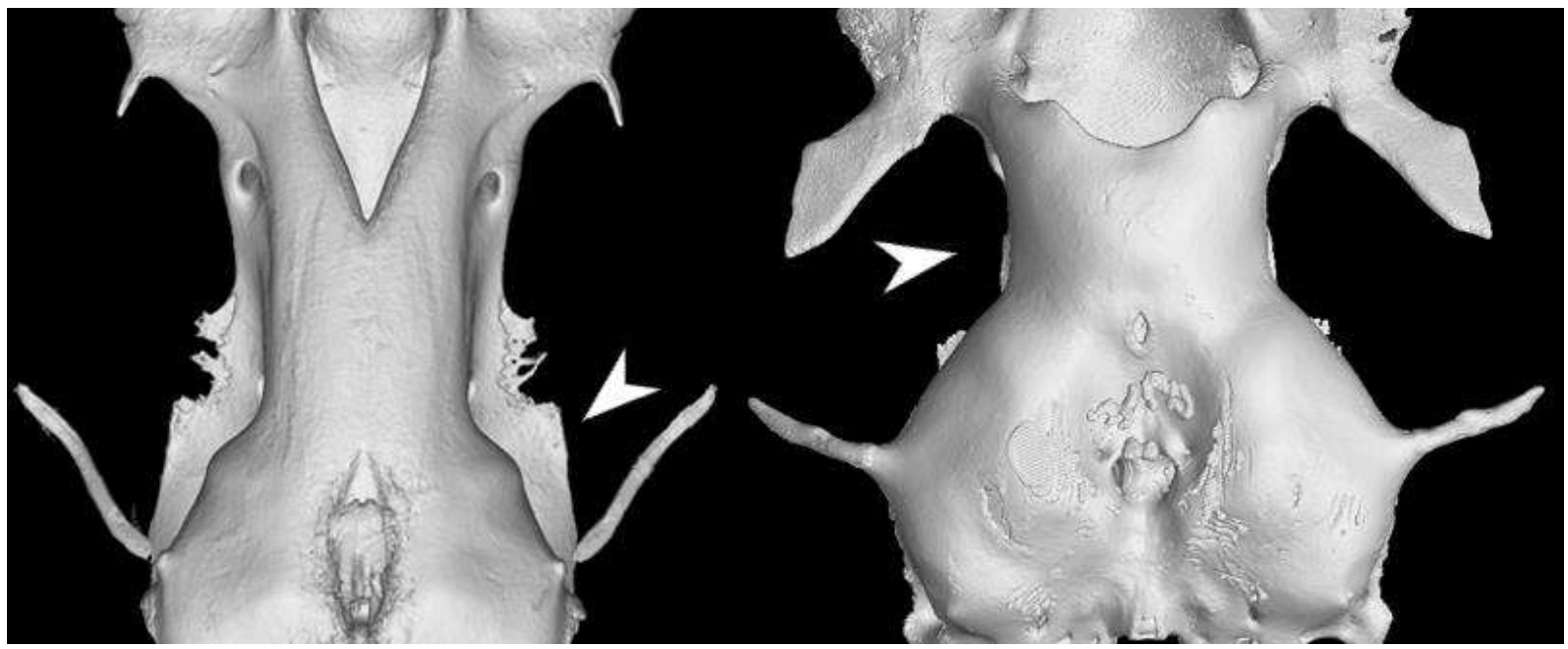

Figure 12. $\mathrm{Cn} 17$, in which the left is representing state 1 and the right, state 0 (Left - Isogomphodon oxyrhynchus MPEG 3455; Right - Carcharhinus perezii MZUSP 53007). 


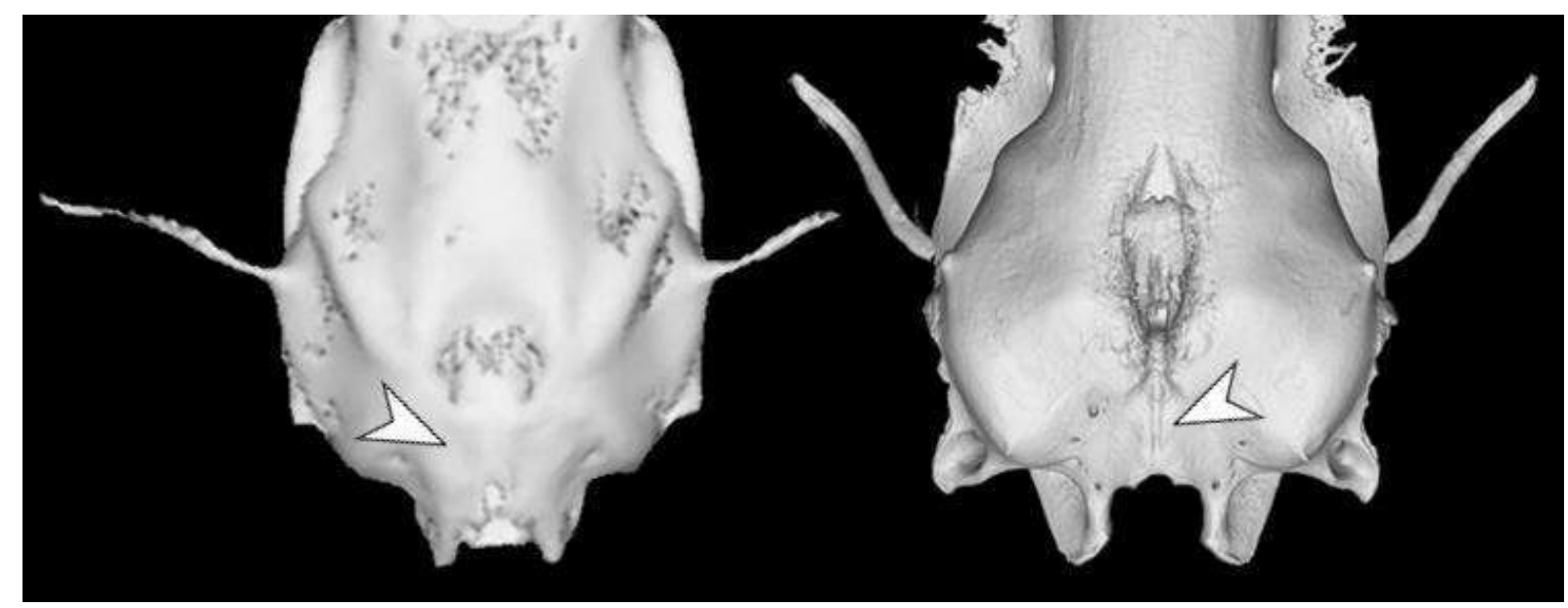

Figure 13. Posterior region of the neurocranium illustrating character Cn19. State 0 (left), State 1 (right). Left - Scoliodon macrorhynchus AMNH 258155; Right - Isogomphodon oxyrhynchus MPEG 3455. Images of Scoliodon macrorhynchos (AMNH 258155) modified from Naylor (2021), available at: sharksrays.org.

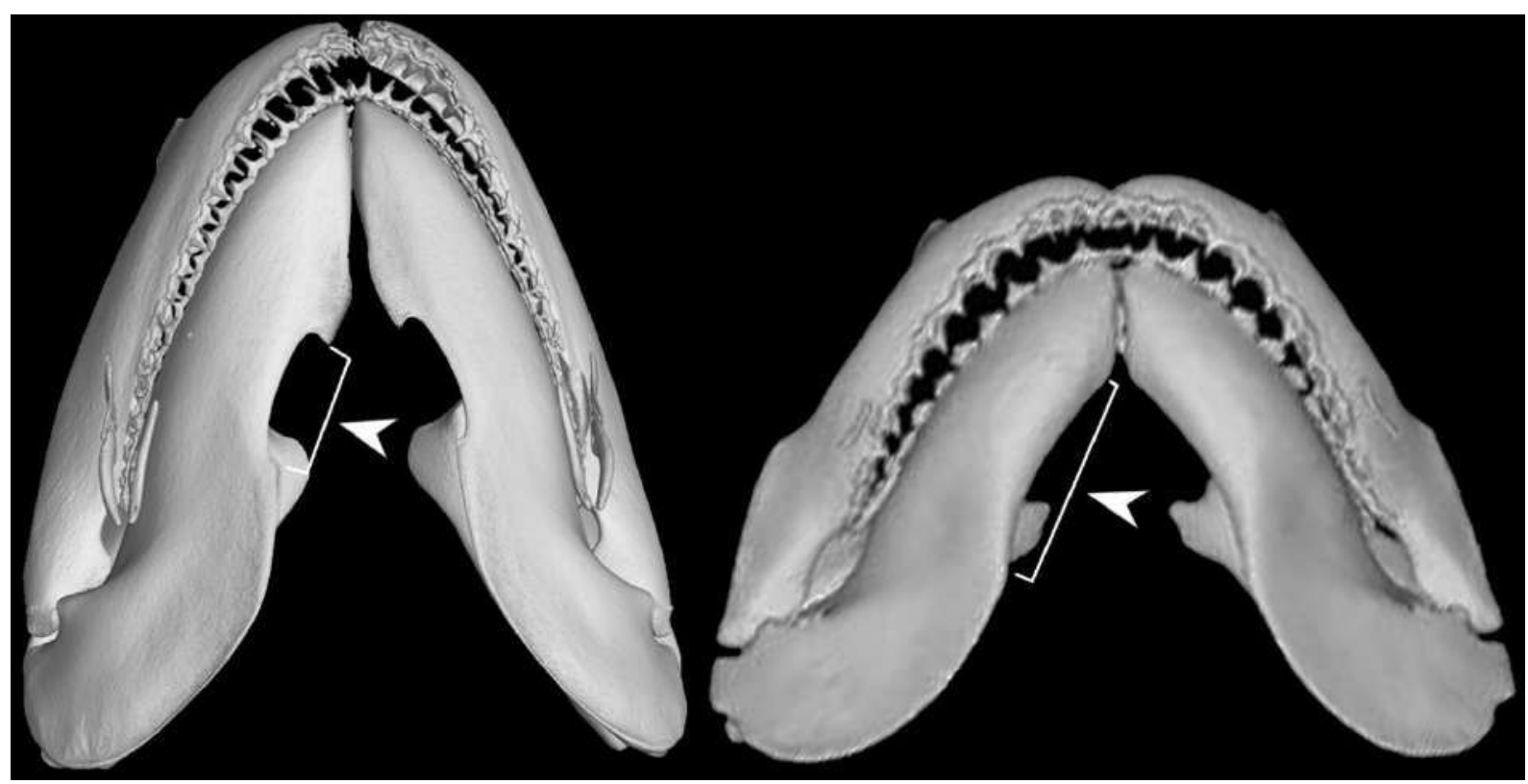

Figure 14. Mandibular arch in ventral view illustrating character Cma1. State 0 (right), State 1 (left). Left - Isogomphodon oxyrhynchus MZUSP 101214; Right - Carcharhinus galapagensis GMBL Uncatalogued. Carcharhinus galapagensis (GMBL Uncatalogued) modified from Naylor (2021), available at: sharksrays.org. 


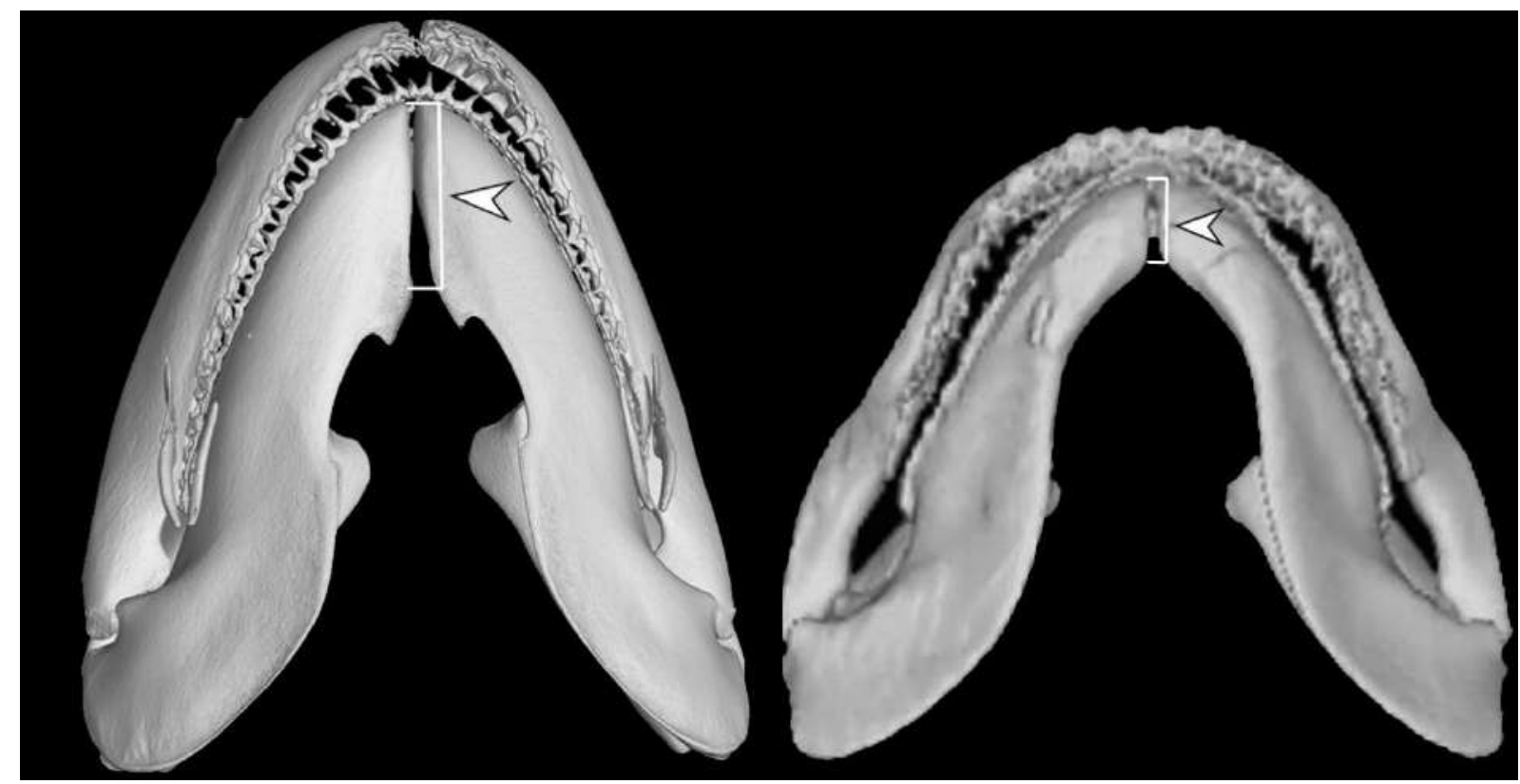

Figure 15. Mandibular arch in ventral view illustrating character Cma1. State 0 (right), State 1 (left). Left - Isogomphodon oxyrhynchus MZUSP 101214; Right - Triaenodon obesus USNM 216792. Triaenodon obesus (USNM 216792) modified from Naylor (2021), available at: sharksrays.org.

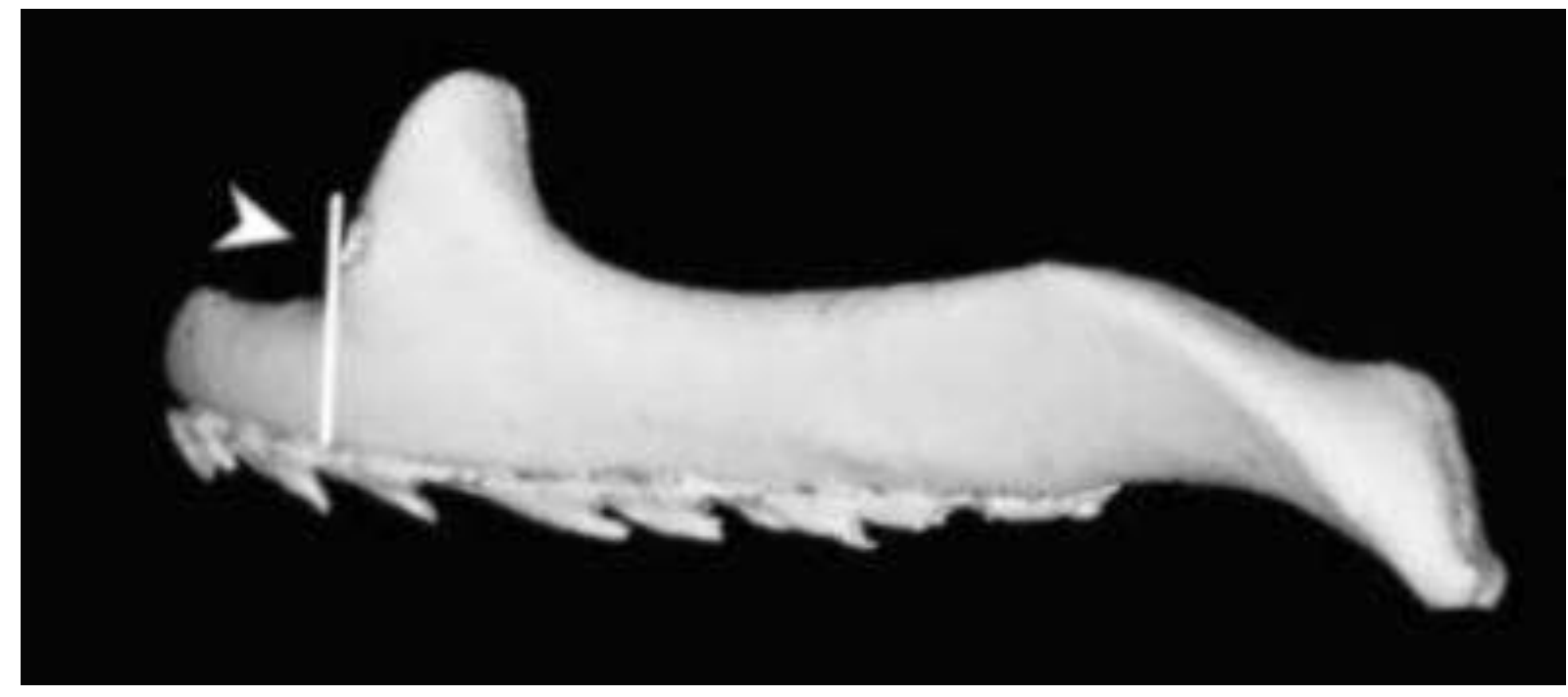

Figure 16. Schematic view of Cma7, illustrated on the palatoquadrate of Scoliodon macrorhynchus. Images of Scoliodon macrorhynchos (AMNH 258155) modified from Naylor (2021), available at: sharksrays.org. 


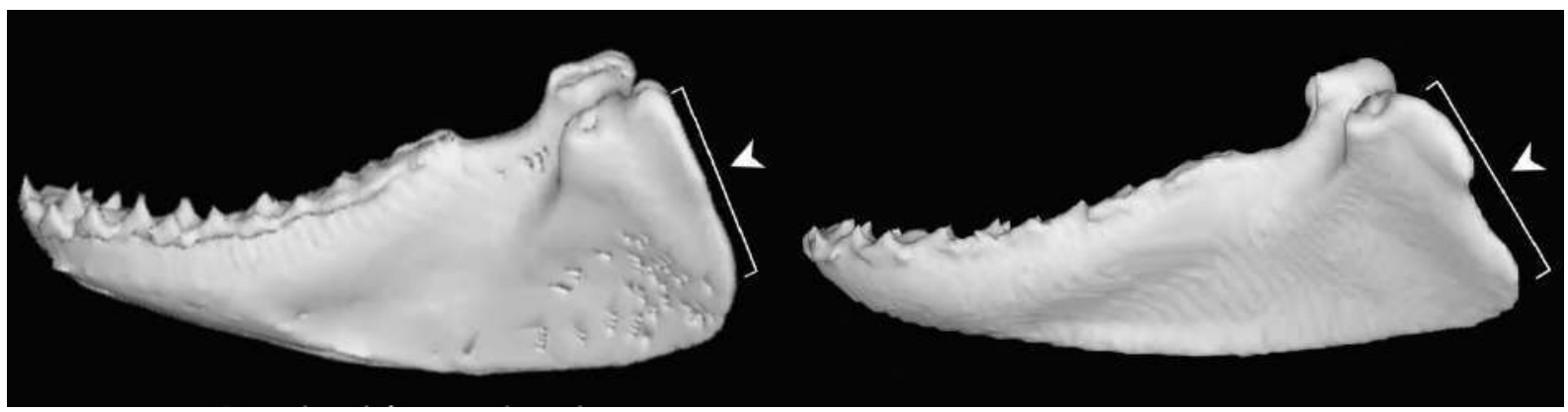

Figure 17. Meckel's cartilage in lateral view illustrating character Cma8. State 0 (left), State 1 (right). Left - Carcharhinus plumbeus GMBL 7960; Right - Rhizoprionodon terranovae GMBL Uncatalogued. Carcharhinus plumbeus (GMBL 7960) and Rhizoprionodon terranovae (GMBL Uncatalogued) modified from Naylor (2021), available at: sharksrays.org.

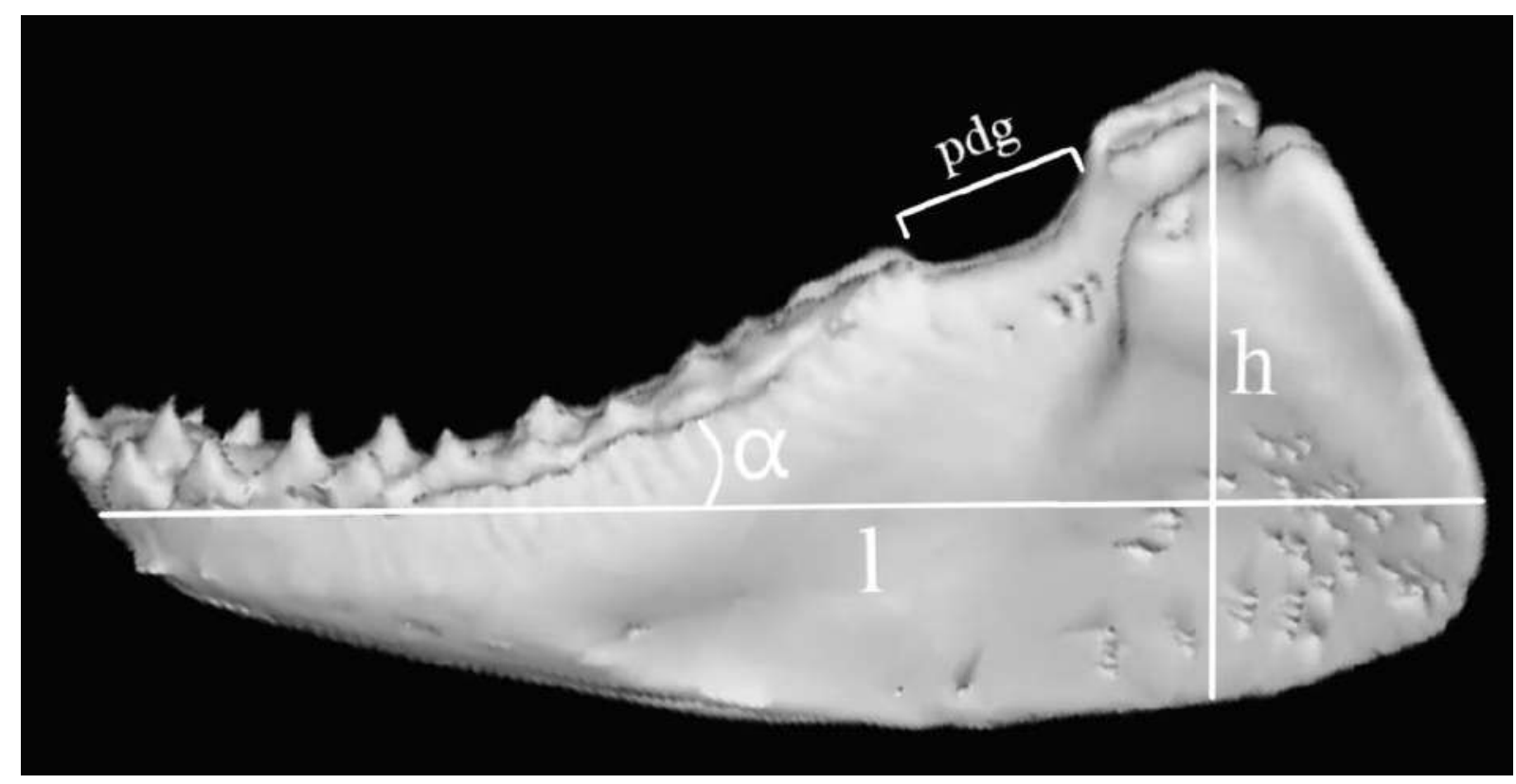

Figure 18. Schematic view of the measurements used on Cma11, Cma12 and Cma13, illustrated on the Meckel's cartilage of Carcharhinus plumbeus GMBL 7960 ( $\alpha$, angulation of dentary row; 1, length; h, height; pdg, postdentary groove;). Carcharhinus plumbeus (GMBL 7960) modified from Naylor (2021), available at: sharksrays.org. 


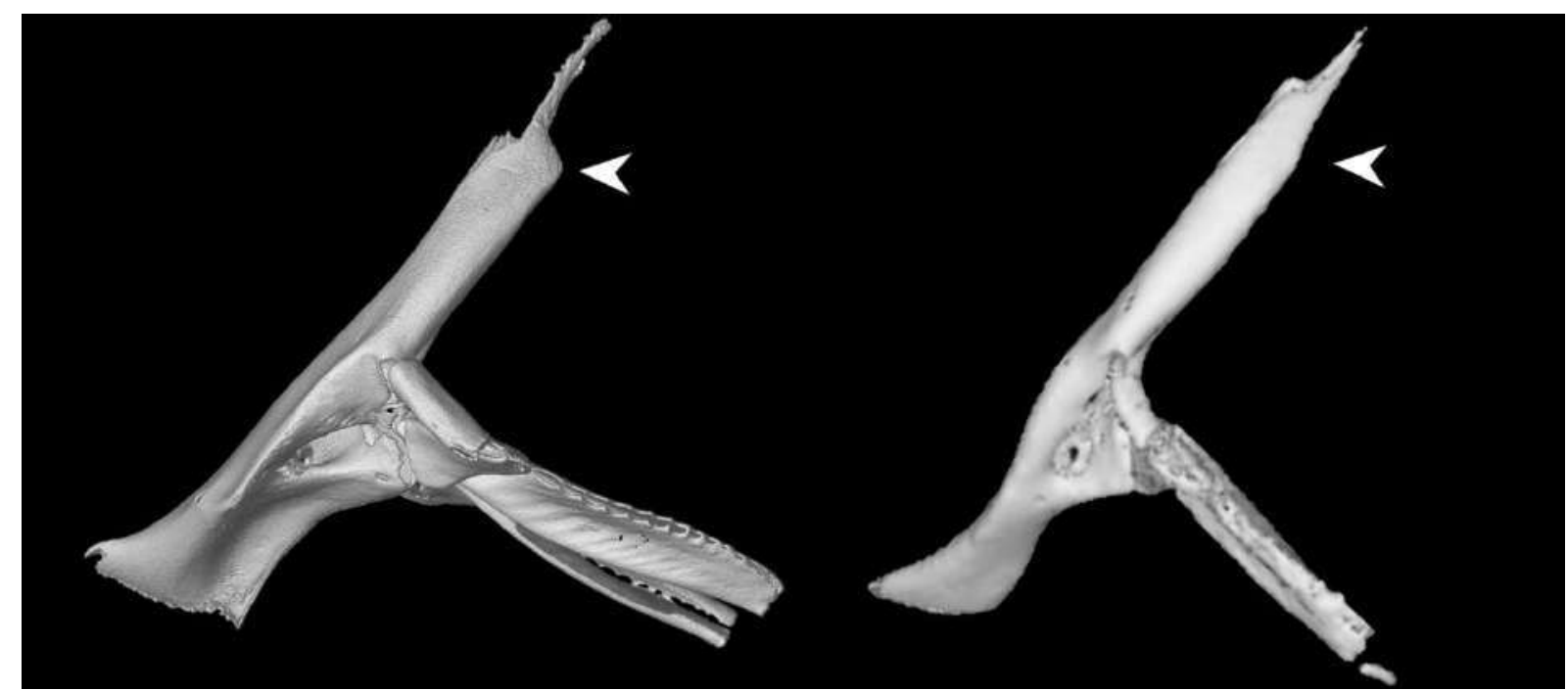

Figure 19. Scapula in lateral view illustrating character Cpg1. State 0 (right), State 1 (left). Left Isogomphodon oxyrhynchus MPEG 3455; Right - Triaenodon obesus USNM 216792. Triaenodon obesus (USNM 216792) modified from Naylor (2021), available at: sharksrays.org.

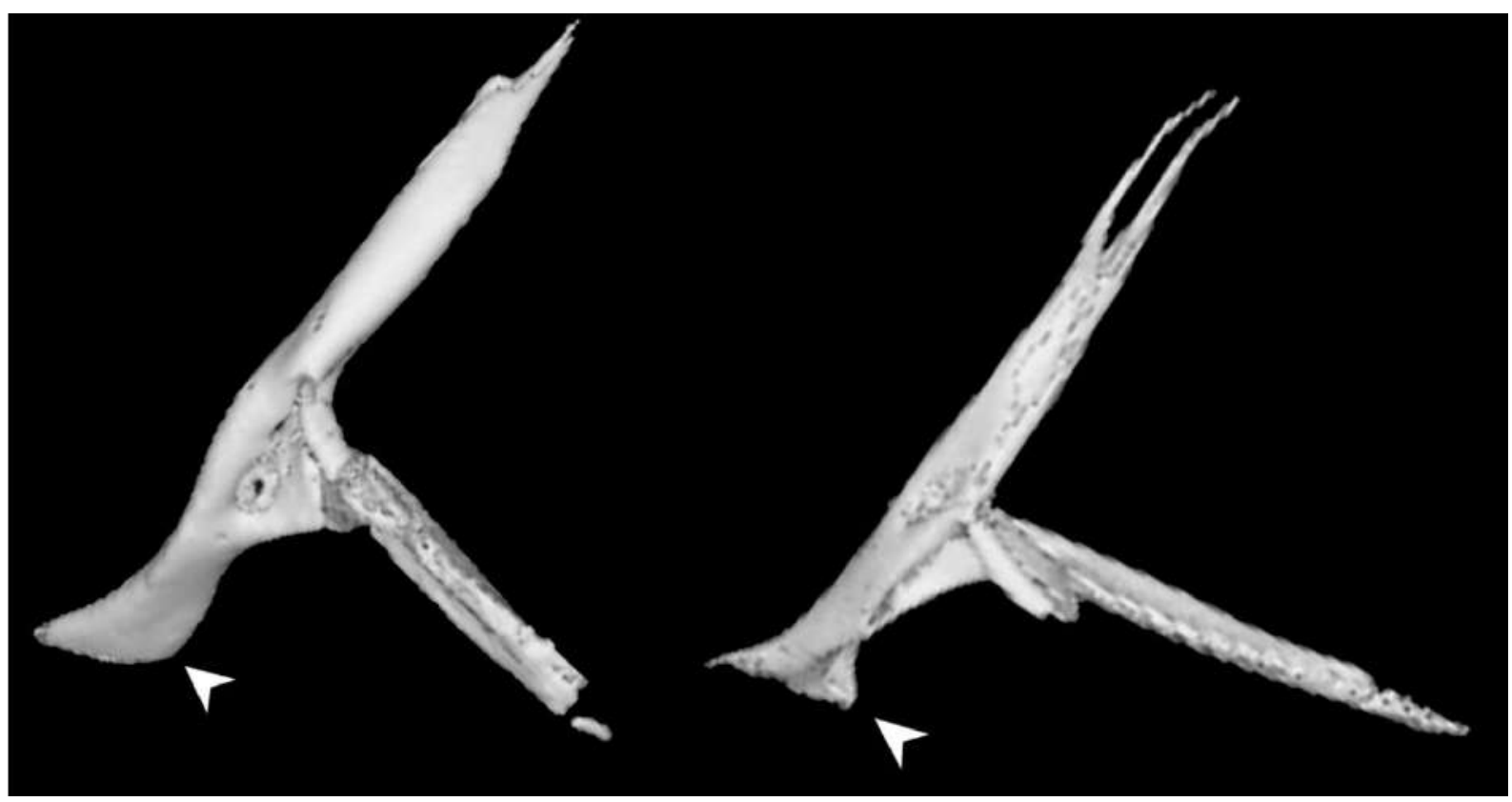

Figure 20. Scapula in lateral view illustrating character Cpg5. State 0 (right), State 1 (left). Left Triaenodon obesus USNM 216792; Right - Scoliodon macrorhynchus AMNH 258155. Triaenodon obesus (USNM 216792) and Scoliodon macrorhynchos (AMNH 258155) modified from Naylor (2021), available at: sharksrays.org. 


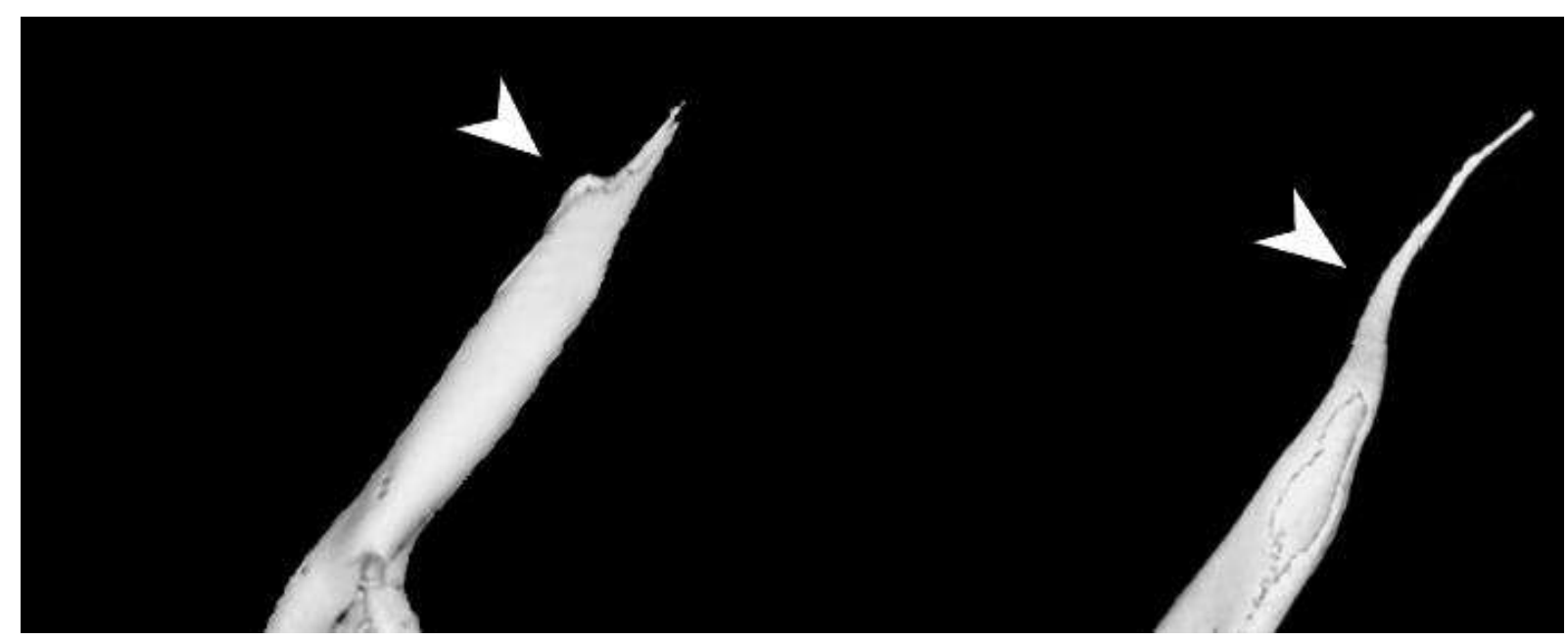

Figure 21. Upper portion of the scapula in lateral view illustrating character Cpg6. State 0 (right), State 1 (left). Left - Triaenodon obesus USNM 216792; Right - Scoliodon macrorhynchus AMNH 258155. Triaenodon obesus (USNM 216792) and Scoliodon macrorhynchos (AMNH 258155) modified from Naylor (2021), available at: sharksrays.org.

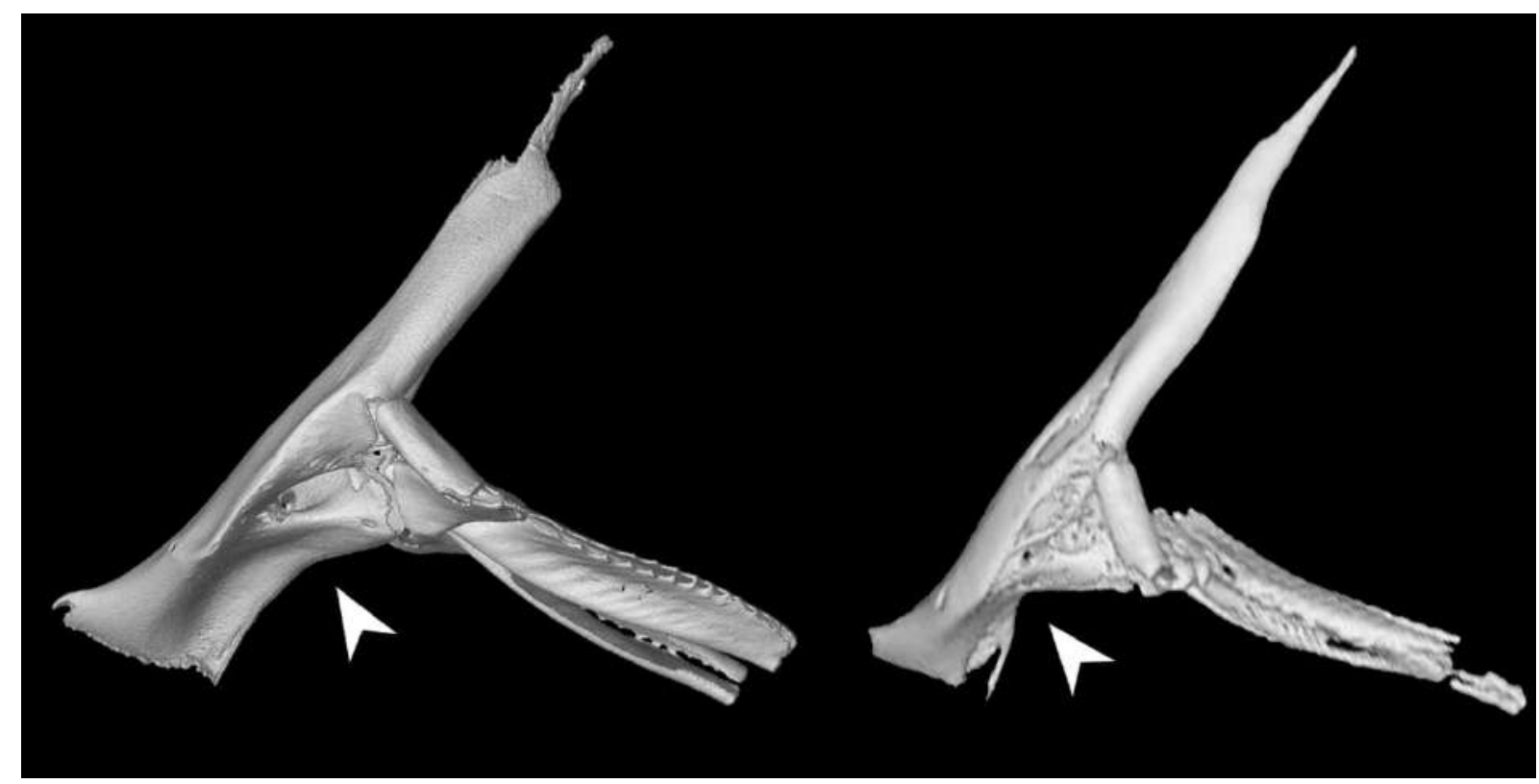

Figure 22. Scapula in lateral view illustrating character Cpg7. State 0 (right), State 1 (left). Left Isogomphodon oxyrhynchus MPEG 3455; Right - Carcharhinus plumbeus GMBL 7960. Carcharhinus plumbeus (GMBL 7960) modified from Naylor (2021), available at: sharksrays.org. 


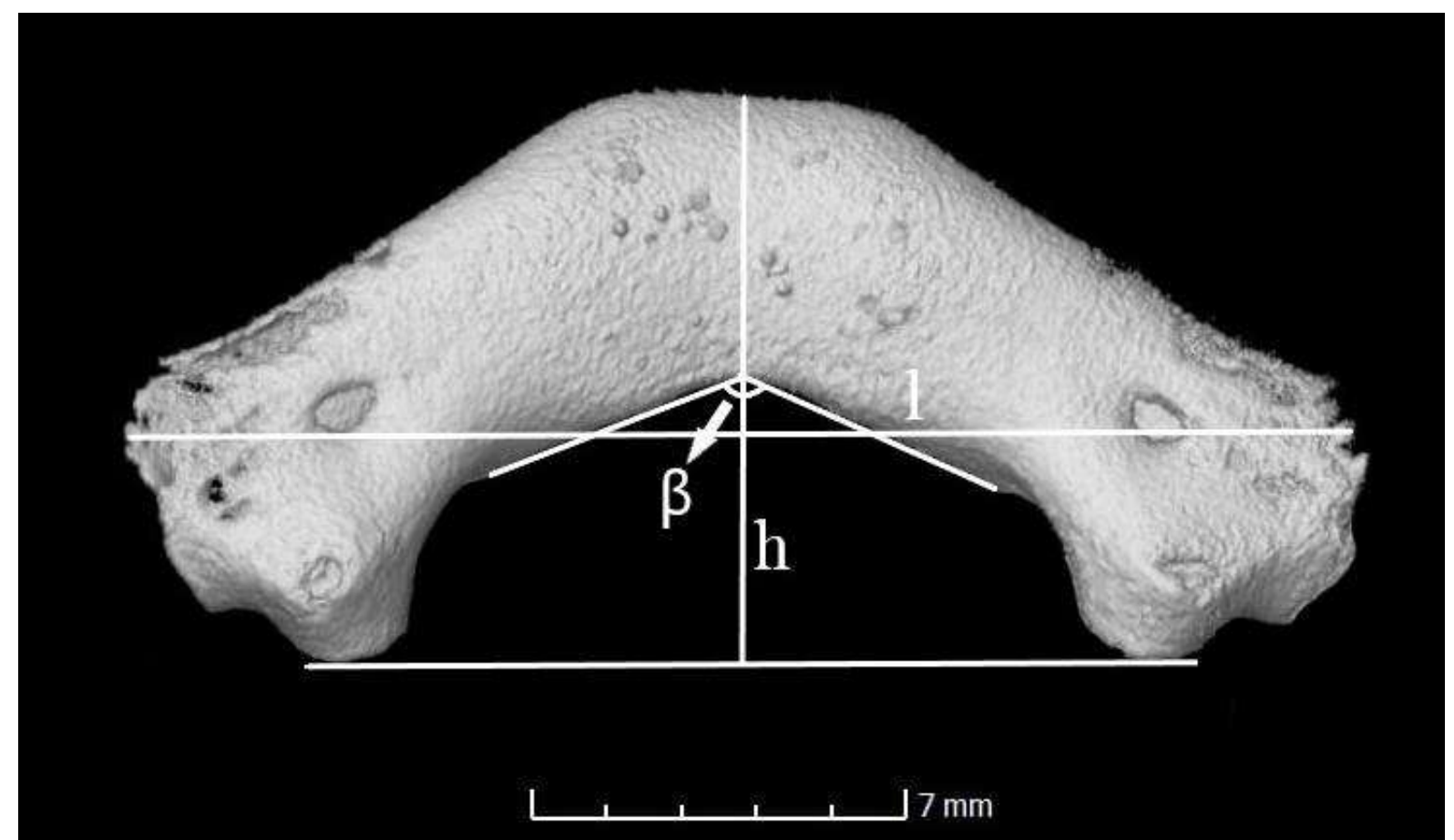

Figure 23. Schematic view of the measurements used on Cpv1 and Cpv4, illustrated on the puboischiadic bar of Isogomphodon oxyrhynchus MZUSP 101215. ( $\beta$, angulation of posterior facet of puboischiadic; l, length; h, height;)

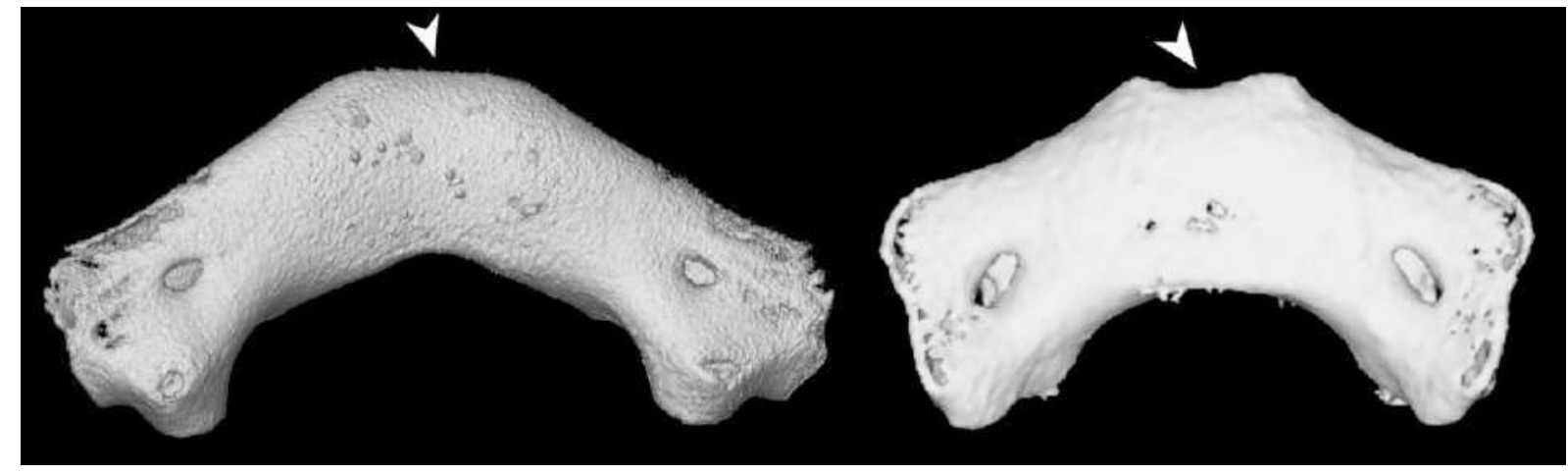

Figure 24. Puboischiatic bar in dorsal view illustrating character Cpv1. State 0 (left), State 1 (right). Left - Isogomphodon oxyrhynchus MZUSP 101214; Right - Scoliodon macrorhynchus AMNH 258155. Scoliodon macrorhynchos (AMNH 258155) modified from Naylor (2021), available at: sharksrays.org. 


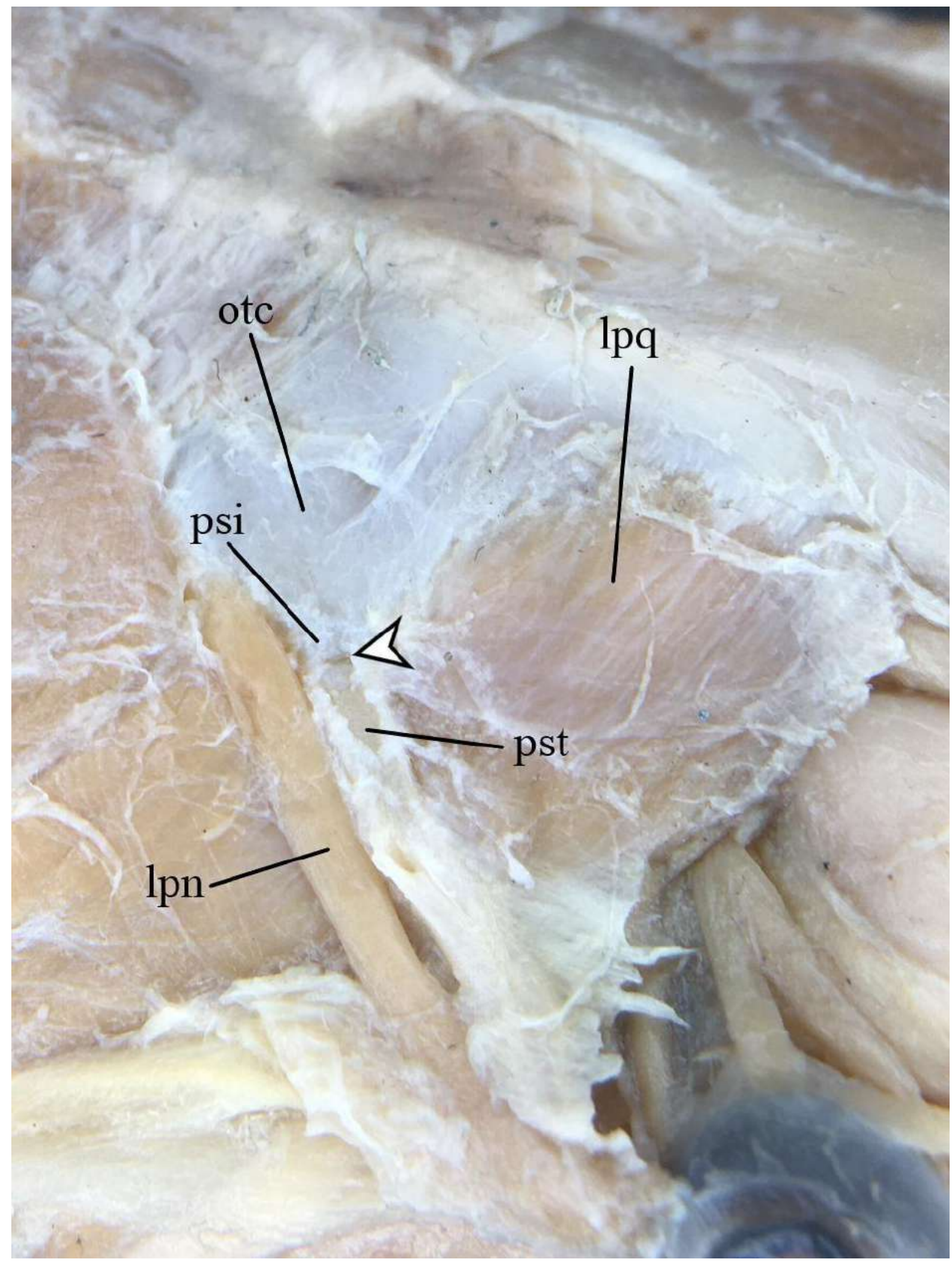

Figure 25. Dissection of MZUSP 37289 in the postorbital region, near the optic capsule. The white arrow indicates the weaker calcification surface between the postorbital process and its insertion on the optic capsule. Anatomical abbreviations: lpn, levator palpebrae nictitantis; lpq, levator palatoquadrati; otc, optic capsule; psi, postorbital process insertion; pst, postorbital process; 


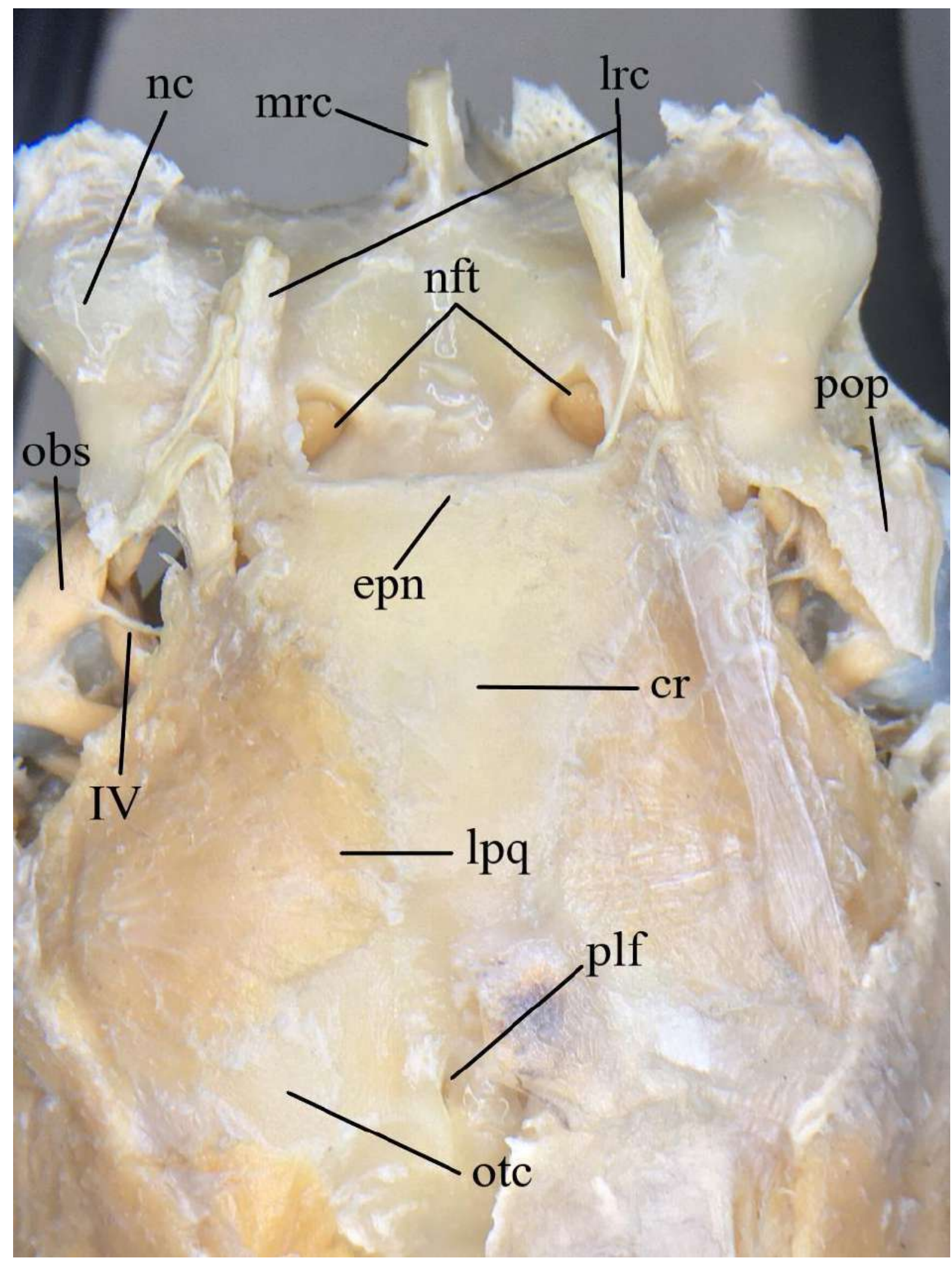

Figure 26. Dissection of MZUSP 84440 in the dorsal region. Anatomical abbreviations: epn, epiphyseal notch; lpq, levator palatoquadrati; lrc, lateral rostral cartilage; mrc, medial rostral cartilage; nc, nasal capsule; nft, nasal fontanelle; otc, optic capsule; plf, perilymphatic fenestrae, pop, preorbital process; obs, obliquo superior; IV, trochlear nerve; 
Table 1. Previous hypothesis that included Isogomphodon oxyrhynchus as a terminal

\begin{tabular}{|c|c|c|}
\hline Author & $\begin{array}{c}\text { Sister groups of } \\
\text { Isogomphodon oxyrhynchus }\end{array}$ & Character base \\
\hline Compagno (1988) & $\begin{array}{c}\text { Triaenodon and } \\
\text { Rhizoprionodon }\end{array}$ & Morphology \\
\hline Kikuchi (2004) & $\begin{array}{c}\text { Negaprion brevirostris } \\
\text { Rodrigues-Filho } \text { et al. (2009) }\end{array}$ & Molecular \\
\hline $\begin{array}{c}\text { Célez-Zuazo \& Agnarson } \\
(2011)\end{array}$ & Carcharhinus porosus & Molecular \\
\hline Naylor et al. (2012a) & Carcharhinus clade & Molecular \\
\hline Naylor et al. (2012b) & $\begin{array}{c}\text { Carcharhinus porosus } \\
\text { Carcharhinus macloti } \\
\text { Sorenson } \text { et al. } \text { (2014) }\end{array}$ & Molecular \\
\hline Cachera \& Le Loc'h (2017) & $\begin{array}{c}\text { Carcharhinus clade } \\
\text { (COI) and Carcharhinus } \\
\text { clade } \text { (NADH2) }\end{array}$ & Molecular \\
\hline Feitosa et al. 2018 & Carcharhinus porosus & Molecular \\
\hline Present study & Molecular \\
\hline
\end{tabular}

Table 2. Results on the present molecular phylogeny.

\begin{tabular}{|c|c|c|c|}
\hline Missing data & Number of partitions & $\begin{array}{c}\text { Number of paired } \\
\text { bases }\end{array}$ & $\begin{array}{c}\text { Number of terminals } \\
\text { (number of tips) }\end{array}$ \\
\hline $0 \%$ & 55 & $59176 \mathrm{z}$ & 512 \\
\hline $10 \%$ & 33 & 48963 & 497 \\
\hline $25 \%$ & 7 & 7397 & 433 \\
\hline
\end{tabular}


Table 3. Morphological matrix of the coded characters of the neurocranium

\begin{tabular}{|c|c|c|c|c|c|c|c|c|c|c|c|c|c|c|c|c|c|c|c|c|c|c|}
\hline \multirow{3}{*}{ Genus } & \multirow{3}{*}{ Terminal } & \multicolumn{21}{|c|}{ Neurocranium } \\
\hline & & 1 & 2 & 3 & 4 & 5 & 6 & 7 & 8 & 9 & 10 & 11 & 12 & 13 & 14 & 15 & 16 & 17 & 18 & 19 & 20 & 21 \\
\hline & & nc01 & nc02 & nc03 & nc04 & nc05 & nc06 & nc07 & nc08 & nc09 & nc10 & nc11 & nc12 & nc13 & nc14 & nc15 & nc16 & nc17 & nc18 & nc19 & Nc20 & nc21 \\
\hline \multirow[t]{2}{*}{ Isogomphodon } & $\begin{array}{l}\text { Isogomphodon } \\
\text { oxyrhynchus }\end{array}$ & 1 & 1 & 0 & 1 & 1 & 1 & 1 & 1 & 2 & 1 & 1 & 1 & 1 & 1 & 0 & 0 & 1 & 0 & 1 & 0 & 0 \\
\hline & $\begin{array}{l}\text { Carcharhinus } \\
\text { porosus }\end{array}$ & 1 & 1 & 0 & 0 & 0 & 1 & 0 & 0 & 1 & 0 & 1 & 0 & 0 & 0 & 0 & 1 & 1 & 0 & 1 & 0 & 1 \\
\hline \multirow[t]{4}{*}{ Carcharhinus } & $\begin{array}{l}\text { Carcharhinus } \\
\text { plumbeus }\end{array}$ & 1 & 1 & 0 & 0 & 0 & 0 & 0 & 0 & 1 & 0 & 1 & 0 & 0 & 0 & 0 & 0 & 0 & 0 & 0 & 0 & 0 \\
\hline & $\begin{array}{l}\text { Carcharhinus } \\
\text { galapagensis }\end{array}$ & 1 & 1 & 0 & 0 & 0 & 1 & 0 & 0 & 1 & 0 & 1 & 0 & 0 & 0 & 0 & 0 & 0 & 0 & 0 & 1 & 0 \\
\hline & $\begin{array}{l}\text { Rhizoprionodon } \\
\text { lalandii }\end{array}$ & 1 & 1 & 1 & 0 & 1 & 0 & 0 & 0 & 1 & 0 & 0 & 1 & 0 & 0 & 0 & 0 & 1 & 0 & 1 & 1 & 0 \\
\hline & $\begin{array}{l}\text { Rhizoprionodon } \\
\text { porosus }\end{array}$ & 0 & 1 & 1 & 0 & 0 & 1 & 0 & 0 & 1 & 0 & 0 & 1 & 0 & 0 & 1 & 1 & 1 & 0 & 1 & 1 & 1 \\
\hline \multirow{2}{*}{ Rhizoprionodon } & $\begin{array}{l}\text { Rhizoprionodon } \\
\text { taylori }\end{array}$ & 1 & 1 & 1 & 0 & 1 & 0 & 0 & 0 & 1 & 0 & 0 & 0 & 0 & 0 & 0 & 1 & 0 & 0 & 0 & 1 & 0 \\
\hline & $\begin{array}{l}\text { Rhizoprionodon } \\
\text { terranovae }\end{array}$ & 0 & 1 & 0 & 0 & 0 & 1 & 0 & 0 & 1 & 0 & 0 & 1 & 0 & 0 & 1 & 0 & 0 & 0 & 1 & 0 & 1 \\
\hline Loxodon & $\begin{array}{l}\text { Loxodon } \\
\text { macrorhinus }\end{array}$ & 0 & 0 & 0 & 0 & 1 & 0 & 1 & 0 & 1 & 0 & 0 & 0 & 2 & 0 & 0 & 0 & 0 & 0 & 0 & 1 & 1 \\
\hline Triaenodon & $\begin{array}{l}\text { Triaenodon } \\
\text { obesus }\end{array}$ & 1 & 0 & 1 & 0 & 1 & 0 & 1 & 0 & 1 & 0 & 1 & 1 & 0 & $?$ & 0 & 0 & 1 & 0 & 0 & 0 & 1 \\
\hline Scoliodon & $\begin{array}{l}\text { Scoliodon } \\
\text { macrorhynchus }\end{array}$ & 1 & 0 & 0 & 1 & 1 & 0 & 0 & 1 & 0 & 0 & 1 & 0 & 1 & 0 & 0 & 0 & 1 & 0 & 0 & 0 & 1 \\
\hline
\end{tabular}


Table 4. Morphological matrix of the coded characters of the mandibular + hyoid arches.

\begin{tabular}{|c|c|c|c|c|c|c|c|c|c|c|c|c|c|c|}
\hline \multirow{3}{*}{ Genus } & \multirow{3}{*}{ Terminal } & \multicolumn{13}{|c|}{ Mandibular + Hyoid Arches } \\
\hline & & 21 & 22 & 23 & 24 & 25 & 26 & 27 & 28 & 29 & 30 & 31 & 32 & 33 \\
\hline & & cma01 & cma02 & cma03 & cma04 & cma05 & cma06 & cma07 & cma08 & cma09 & cma10 & cma11 & cma12 & cma13 \\
\hline & Carcharhinus porosus & 0 & 0 & 1 & 0 & 0 & 0 & 0 & 1 & 1 & 0 & 0 & 1 & 1 \\
\hline Carcharhinus & Carcharhinus plumbeus & 0 & 0 & 1 & 0 & 0 & 0 & 0 & 0 & 1 & 1 & 0 & 1 & 1 \\
\hline & Carcharhinus galapagensis & 0 & 0 & 1 & 0 & 0 & 0 & 0 & 1 & 1 & 1 & 1 & 1 & 1 \\
\hline & Rhizoprionodon lalandii & 0 & 0 & 2 & 0 & 0 & 0 & 0 & 1 & 1 & 1 & 0 & 0 & 0 \\
\hline & Rhizoprionodon porosus & 0 & 0 & 2 & 0 & 0 & 0 & 0 & 0 & 1 & 1 & 0 & 1 & 1 \\
\hline & Rhizoprionodon taylori & 0 & 0 & 1 & 0 & 0 & 0 & 0 & 1 & 1 & 0 & 0 & 1 & 1 \\
\hline & Rhizoprionodon terranovae & 0 & 0 & 2 & 0 & 0 & 0 & 0 & 1 & 0 & 1 & 0 & 1 & 0 \\
\hline Triaenodon & Triaenodon obesus & 0 & 0 & 1 & 0 & 1 & 1 & 1 & 1 & 0 & 1 & 0 & 1 & 0 \\
\hline Negaprion & Negaprion brevirostris & 0 & 0 & 1 & 0 & 1 & 0 & 1 & 0 & 1 & 1 & 1 & 1 & 1 \\
\hline Scoliodon & Scoliodon macrorhynchus & 0 & 1 & 0 & 0 & 0 & 0 & 1 & 1 & 0 & 0 & 0 & 0 & 0 \\
\hline
\end{tabular}


Table 5. Morphological matrix of the coded characters of the appendicular skeleton.

\begin{tabular}{|c|c|c|c|c|c|c|c|c|c|c|c|c|c|}
\hline \multirow[b]{2}{*}{ Genus } & \multirow[b]{2}{*}{ Terminal } & \multicolumn{12}{|c|}{ Appendicular skeleton } \\
\hline & & 34 & 35 & 36 & 37 & 38 & 39 & 40 & 41 & 42 & 43 & 44 & 45 \\
\hline \multirow{3}{*}{ Isogomphodon } & & pcgt01 & $\operatorname{pcg02}$ & pcgt03 & pcgt04 & $\operatorname{pcg} 05$ & pcg06 & $\operatorname{pcg} 07$ & plv01 & plv02 & plv03 & plv04 & plv05 \\
\hline & Isogomphodon oxyrhynchus & 1 & 0 & 1 & 0 & 0 & 0 & 1 & 1 & 0 & 0 & 1 & 1 \\
\hline & Carcharhinus porosus & 1 & 1 & 1 & 0 & 1 & 0 & 1 & 1 & 0 & 0 & 1 & 0 \\
\hline \multirow[t]{2}{*}{ Carcharhinus } & Carcharhinus plumbeus & 1 & 1 & 0 & 0 & 1 & 1 & 0 & 1 & 0 & 0 & 1 & 0 \\
\hline & Carcharhinus galapagensis & 1 & 0 & 0 & 1 & 0 & 1 & 1 & 1 & 0 & 0 & 0 & 0 \\
\hline \multirow{4}{*}{ Rhizoprionodon } & Rhizoprionodon lalandii & 1 & 1 & 1 & 1 & 0 & 0 & 0 & 1 & 0 & 0 & 0 & 0 \\
\hline & Rhizoprionodon porosus & 1 & 0 & 1 & 0 & 0 & 0 & 1 & 1 & 0 & 0 & 1 & 0 \\
\hline & Rhizoprionodon taylori & 1 & 0 & 1 & 0 & 0 & 0 & 1 & 1 & 0 & 0 & 1 & 0 \\
\hline & Rhizoprionodon terranovae & 1 & 1 & 0 & 0 & 0 & 0 & 0 & 0 & 0 & 0 & 1 & 0 \\
\hline Loxodon & Loxodon macrorhinus & 1 & 1 & 1 & 0 & 1 & 0 & 1 & 0 & 0 & 0 & 0 & 1 \\
\hline Triaenodon & Triaenodon obesus & 0 & 0 & 0 & 1 & 1 & 1 & 1 & 1 & 0 & 1 & 0 & 0 \\
\hline Negaprion & Negaprion brevirostris & 1 & 0 & 0 & 0 & $?$ & 1 & 0 & 1 & 1 & 1 & 0 & 0 \\
\hline Scoliodon & Scoliodon macrorhynchus & 0 & 0 & 0 & 0 & 0 & 0 & 1 & 1 & 1 & 0 & 1 & 1 \\
\hline
\end{tabular}


Table 6. Similarity matrix of the morphological characters analysed on the compared taxa, summarizing the similarities in green and the differences in red.

\begin{tabular}{|c|c|c|c|c|c|c|c|c|c|c|c|}
\hline $\begin{array}{l}\text { I. } \\
\text { rhynchus }\end{array}$ & $\begin{array}{c}\text { C. } \\
\text { porosus }\end{array}$ & $\begin{array}{c}C . \\
\text { plumbeus }\end{array}$ & $\begin{array}{c}C . \\
\text { galapagensis }\end{array}$ & $\begin{array}{c}R . \\
\text { lalandii }\end{array}$ & $\begin{array}{c}R . \\
\text { porosus }\end{array}$ & $\begin{array}{c}R . \\
\text { taylori }\end{array}$ & $\begin{array}{c}R . \\
\text { terranovae }\end{array}$ & $\begin{array}{c}L . \\
\text { macrorhinus }\end{array}$ & $\begin{array}{c}T . \\
\text { obesus }\end{array}$ & $\begin{array}{c}N . \\
\text { brevirostris }\end{array}$ & $\begin{array}{c}S . \\
\text { macrorhynchus }\end{array}$ \\
\hline & 20 & 27 & 25 & 25 & 25 & 21 & 25 & 27 & 22 & 27 & 20 \\
\hline 26 & & 9 & 13 & 16 & 11 & 10 & 14 & 18 & 22 & 24 & 21 \\
\hline 19 & 37 & & 9 & 16 & 17 & 12 & 14 & 16 & 20 & 15 & 23 \\
\hline 21 & 33 & 37 & & 15 & 16 & 11 & 17 & 17 & 19 & 16 & 24 \\
\hline 21 & 30 & 30 & 31 & & 13 & 12 & 15 & 18 & 20 & 17 & 24 \\
\hline 21 & 35 & 29 & 30 & 33 & & 11 & 11 & 9 & 25 & 20 & 28 \\
\hline 25 & 36 & 34 & 35 & 34 & 35 & & 18 & 16 & 24 & 17 & 21 \\
\hline 21 & 32 & 32 & 29 & 31 & 35 & 28 & & 18 & 24 & 25 & 24 \\
\hline 19 & 28 & 30 & 29 & 28 & 37 & 30 & 28 & & 22 & 19 & 25 \\
\hline 24 & 24 & 26 & 27 & 26 & 21 & 22 & 22 & 24 & & 14 & 21 \\
\hline 19 & 22 & 31 & 30 & 29 & 26 & 29 & 21 & 27 & 32 & & 26 \\
\hline 26 & 25 & 23 & 22 & 22 & 18 & 25 & 22 & 21 & 25 & 20 & \\
\hline
\end{tabular}

I. oxyrhynchus

C. porosus

C. plumbeus

C. galapagensis

R. lalandii

$R$. porosus

R. taylori

$R$. terranovae

L. macrorhinus T. obesus

N. brevirostris S.

macrorhynchus

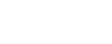

$$
\text { I. }
$$

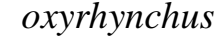

plumbeus

terranova 


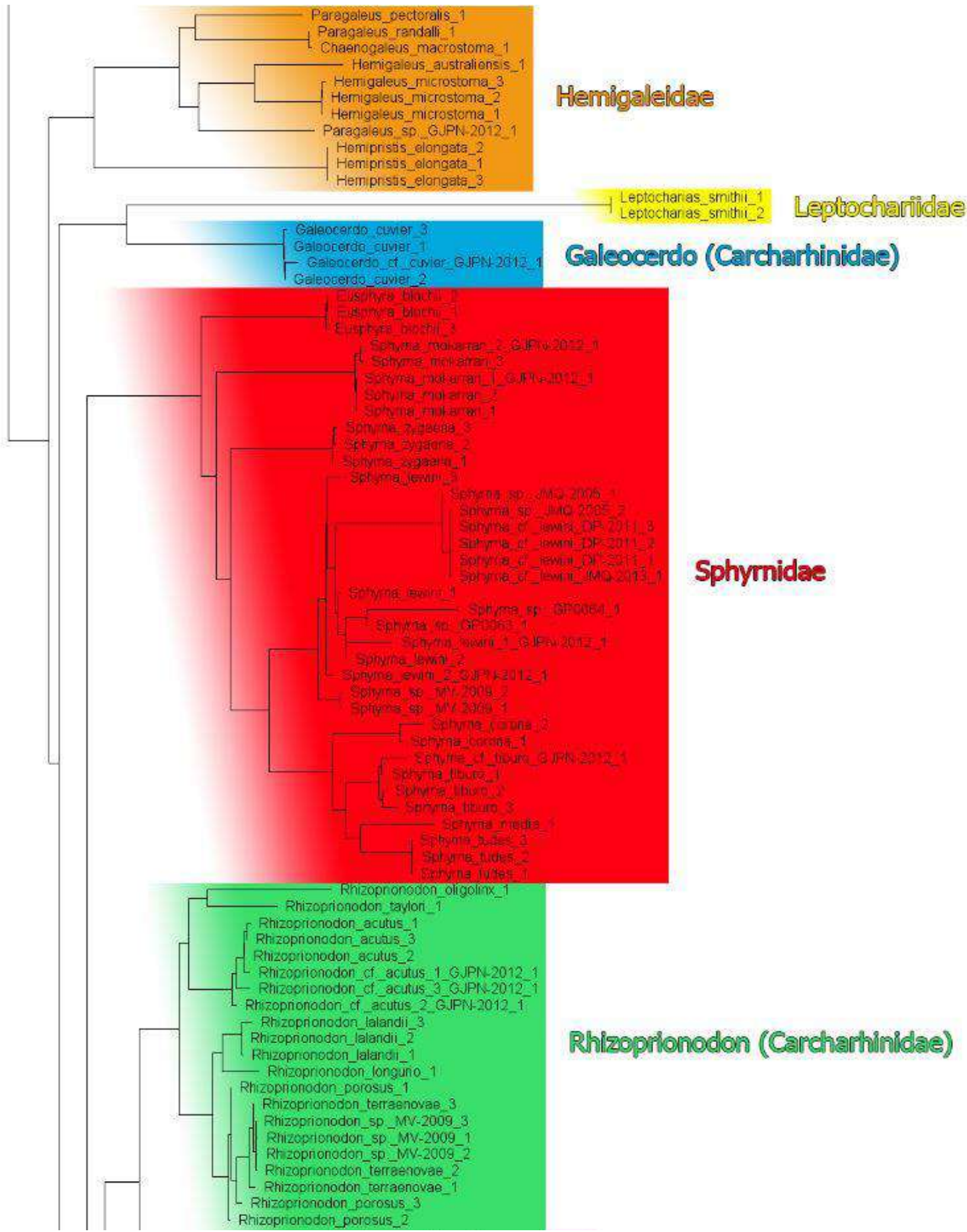

Figure 27. Consensus phylogenetic tree of Carcharhinidae rooted on Hemigaleidae, obtained through all available molecular sequences GenBank under the treatment of $\underline{\mathbf{0 \%}}$ of missing data. 


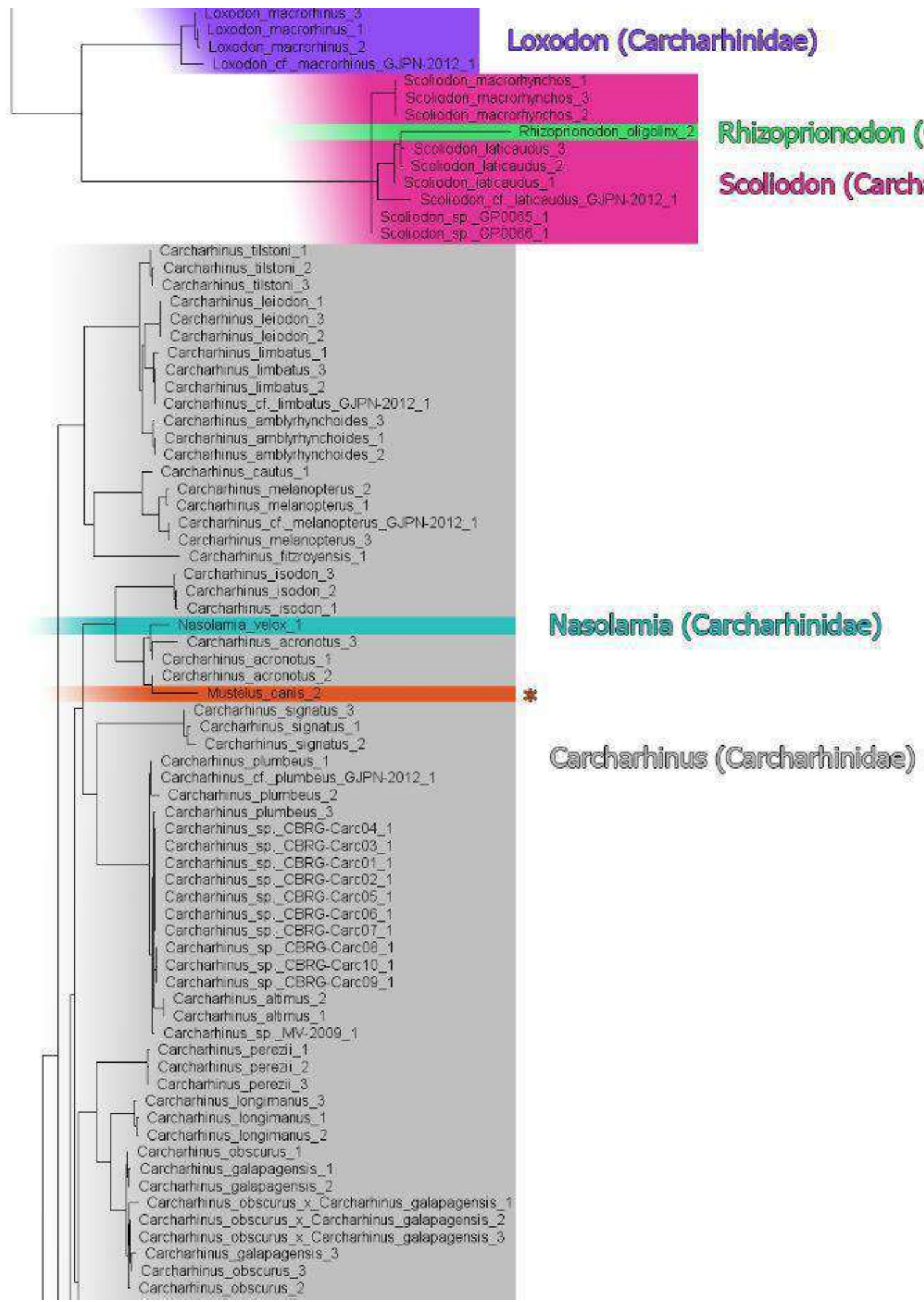

Figure 27. (Continued). *Terminal probably misallocated due to erroneous alignment. 


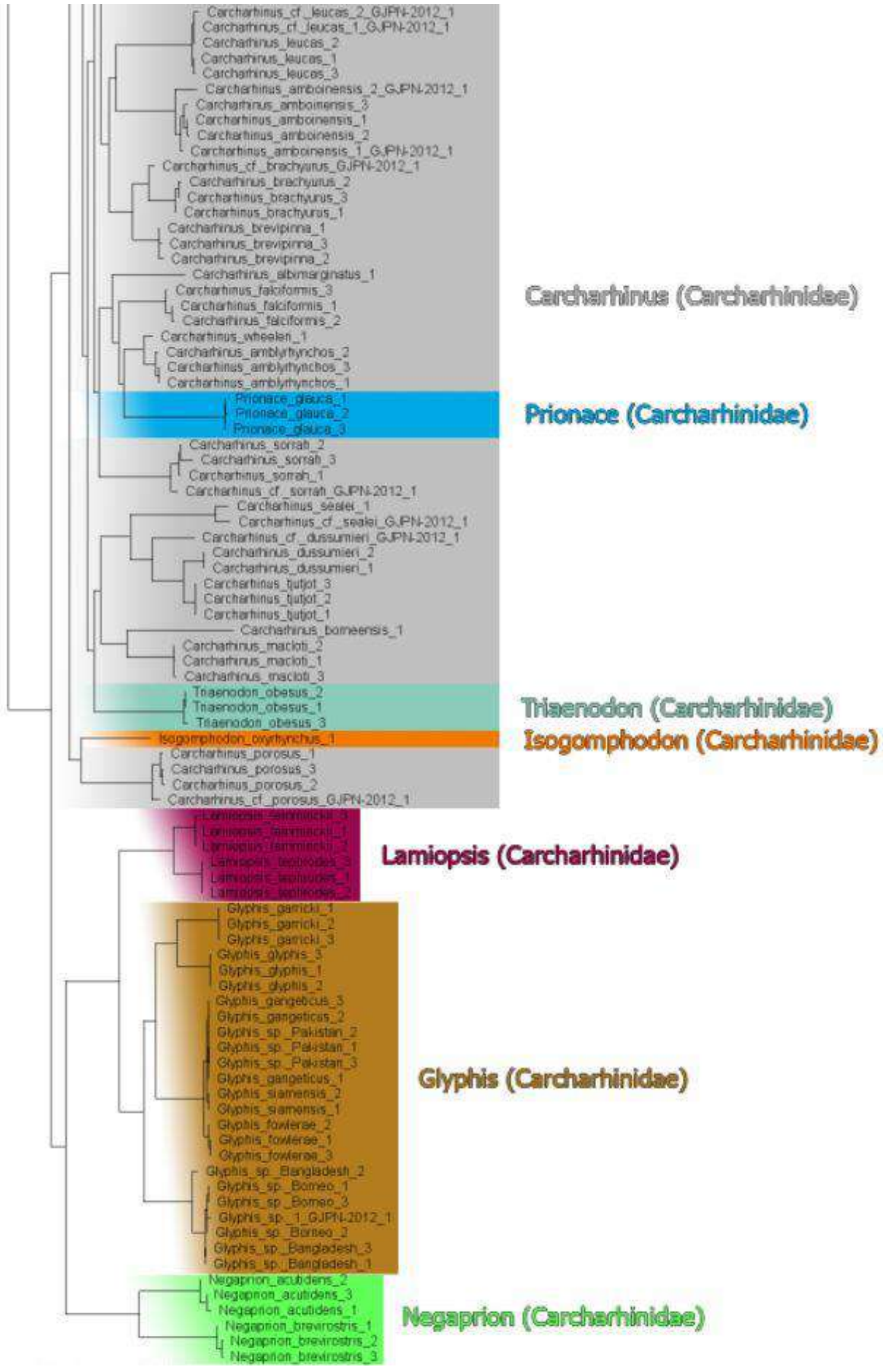

Figure 27. (Continued) 


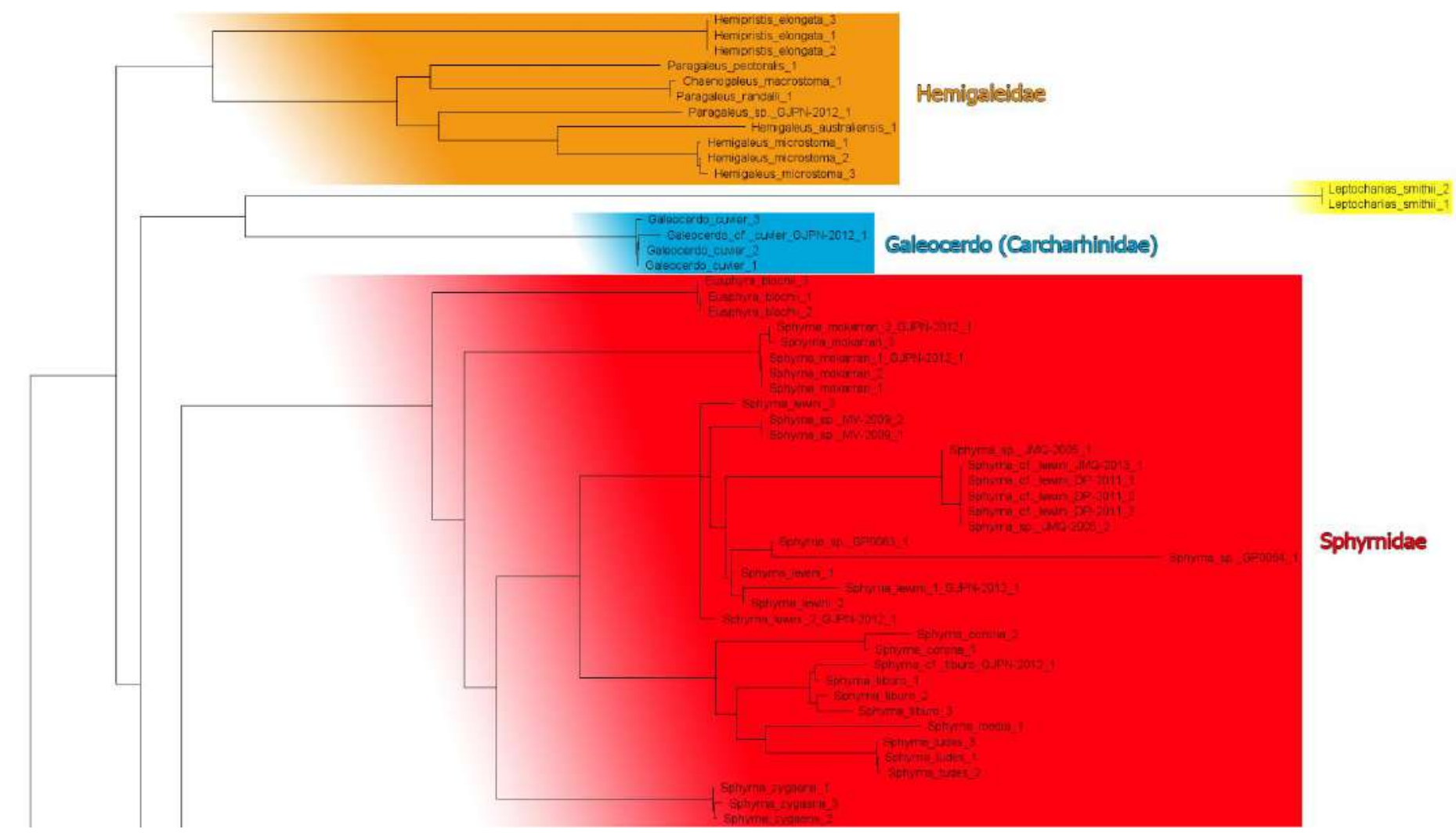

Figure 28. Consensus phylogenetic tree of Carcharhinidae rooted on Hemigaleidae, obtained through all available molecular sequences GenBank under the treatment of $\underline{\mathbf{1 0 \%}}$ of missing data. 


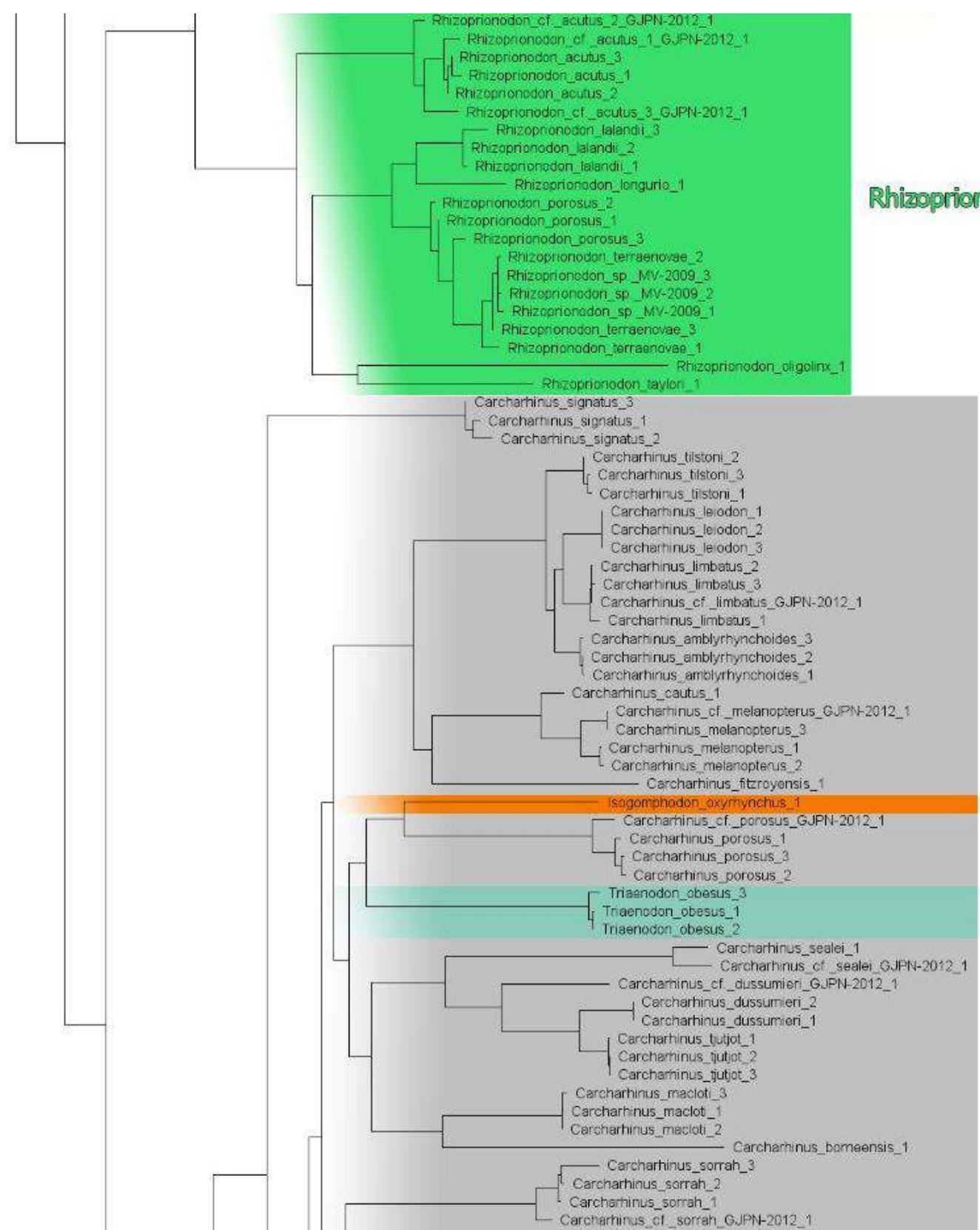

Cancharhinus (Carcharhinidas)

Isogomphodon (Carcharhinidae)

Triaenodon (Canchashinidae)

Figure 28. (Continued) 


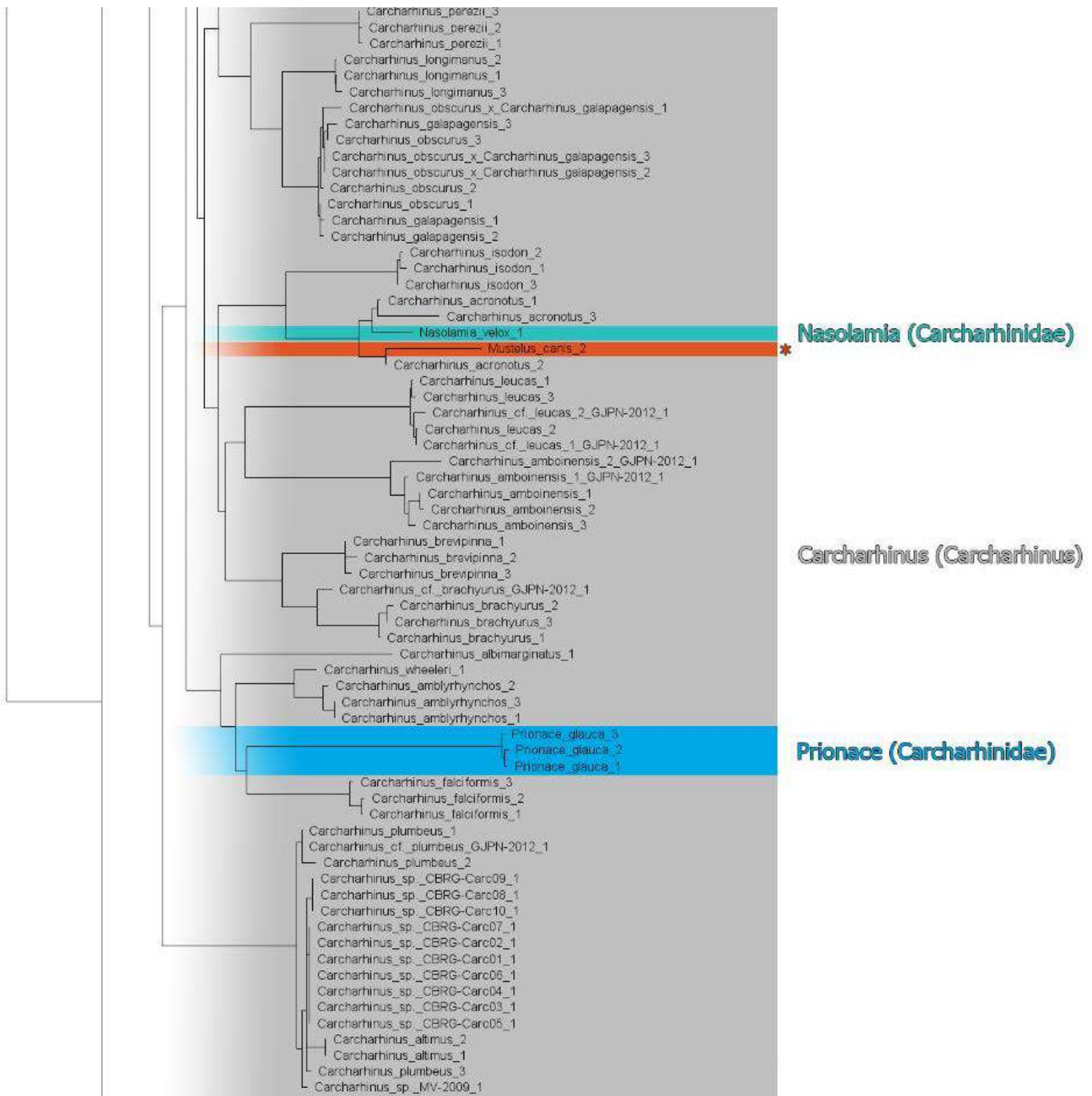

Figure 28. (Continued) *Terminal probably misallocated due to erroneous alignment. 


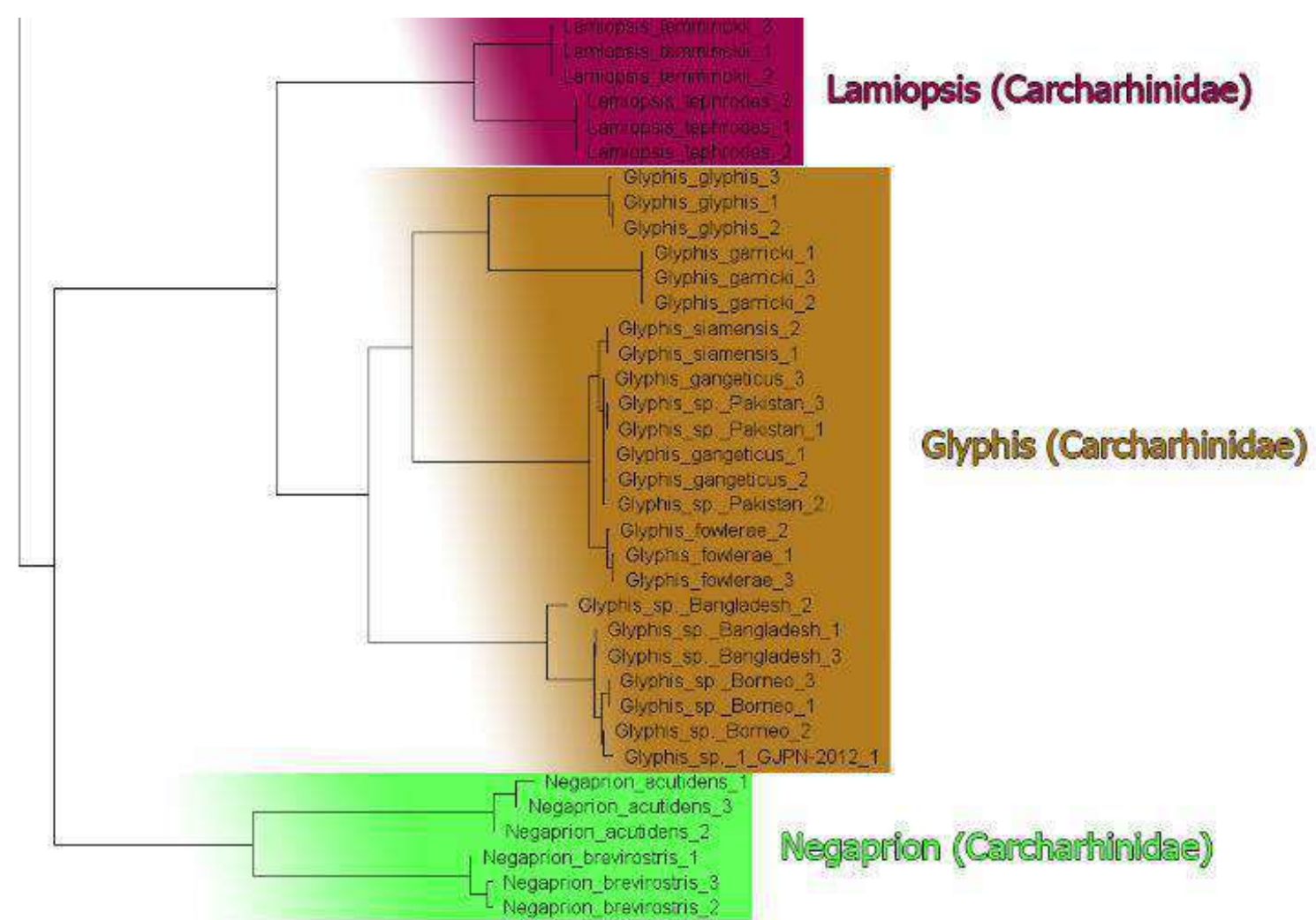

Figure 24. (Continued)

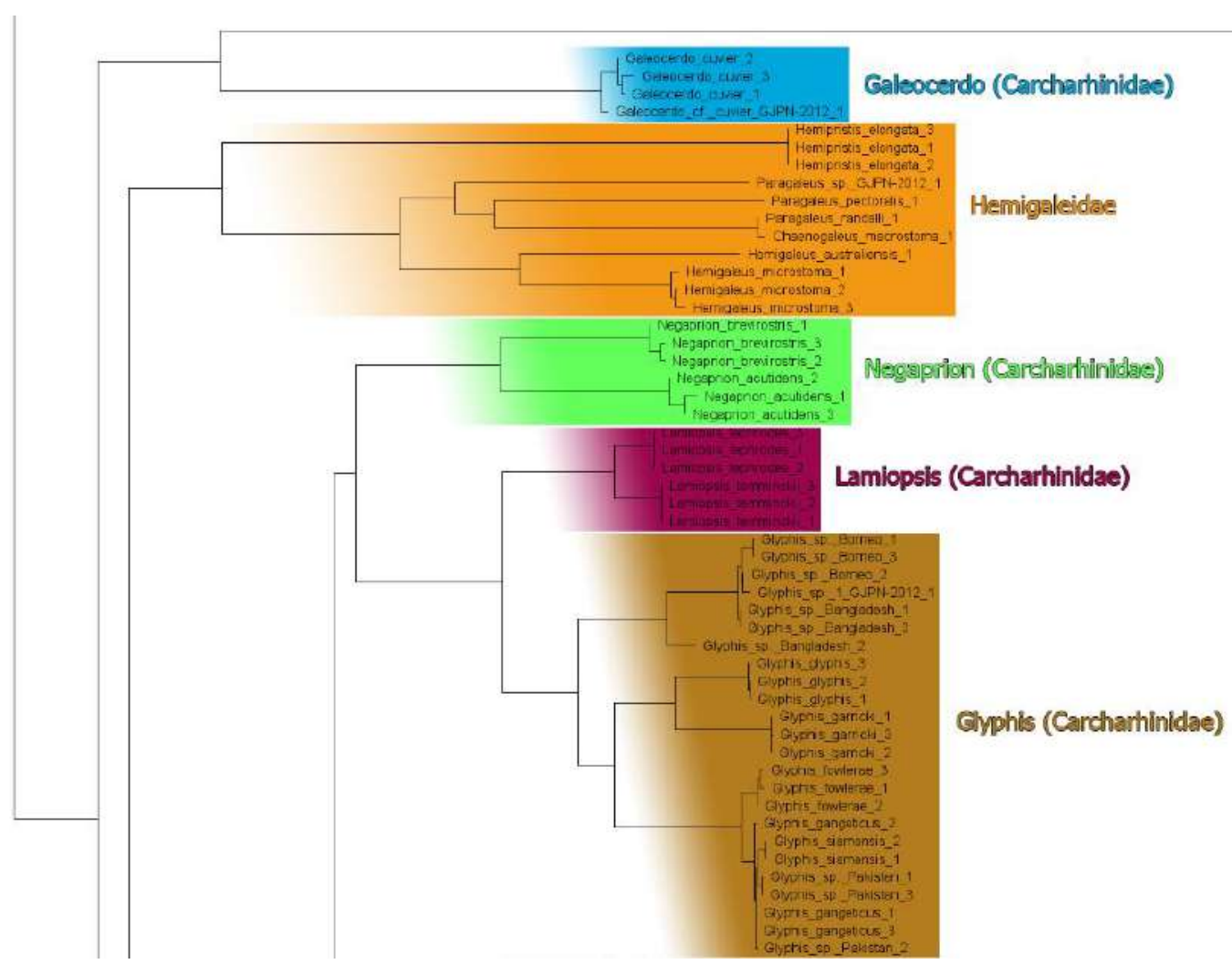

Figure 29. Consensus phylogenetic tree of Carcharhinidae rooted on Hemigaleidae, obtained through all available molecular sequences GenBank under the treatment of $\underline{\mathbf{2 5 \%}}$ of missing data. 


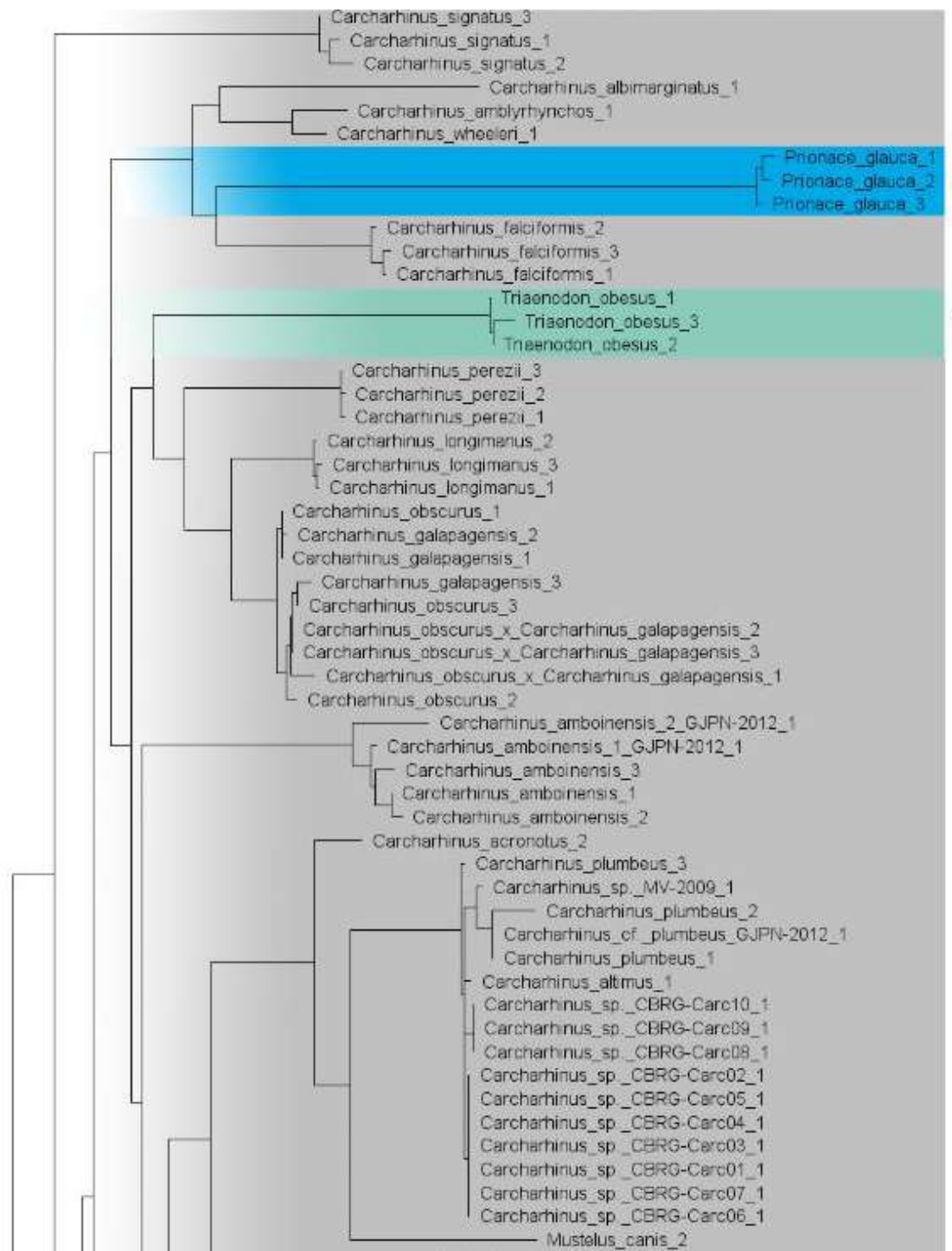

\section{Prionace (Carcharhinidae)}

Thiaenodon (Carcharhinidae)

Cancharhinus (Camcharhinidas)

Figure 29. (continued) 


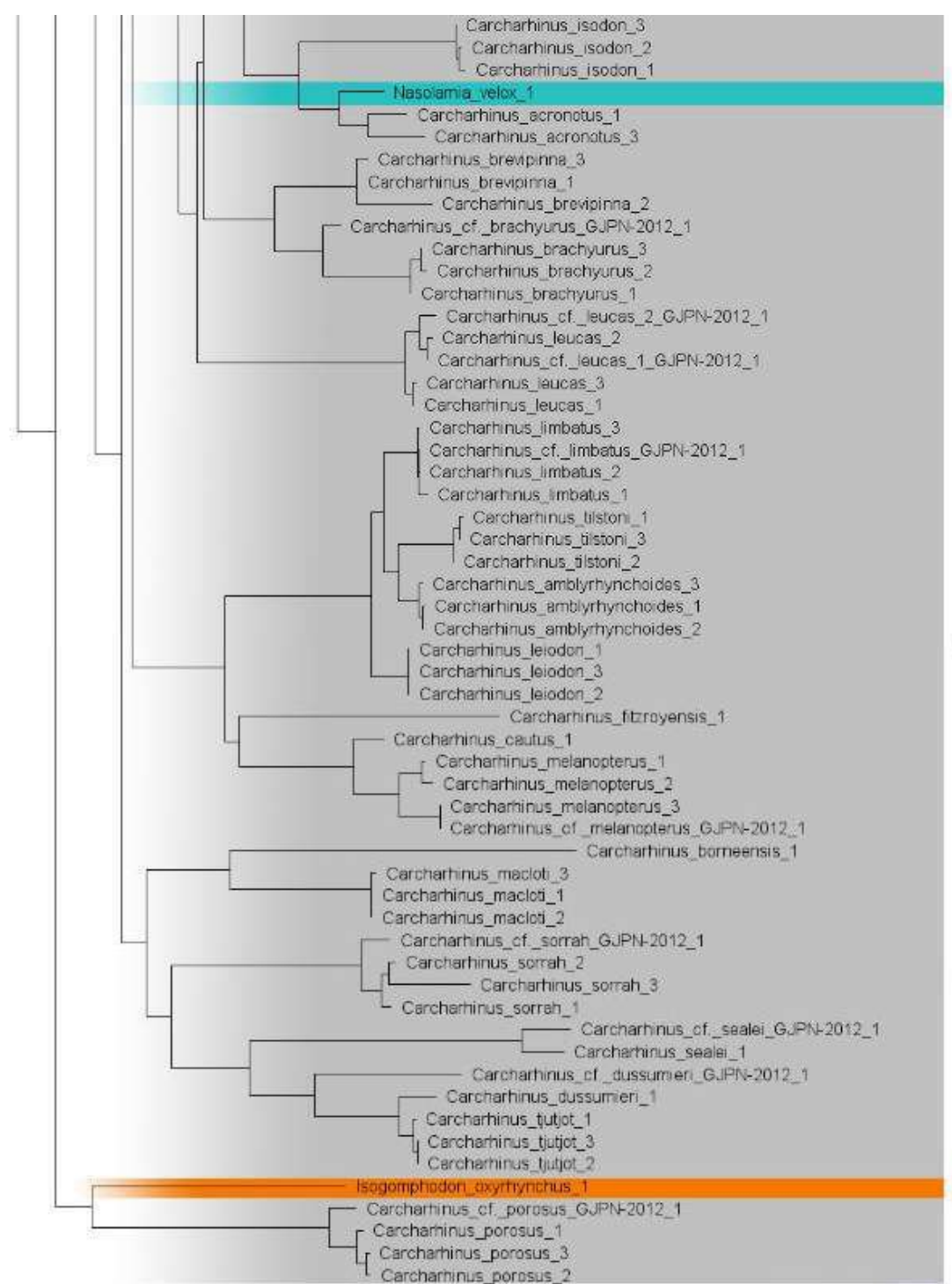

Nasolamis (Cancharhinidae)

Cancharhinus (Carchamhinidae)

Figure 29. (continued) 


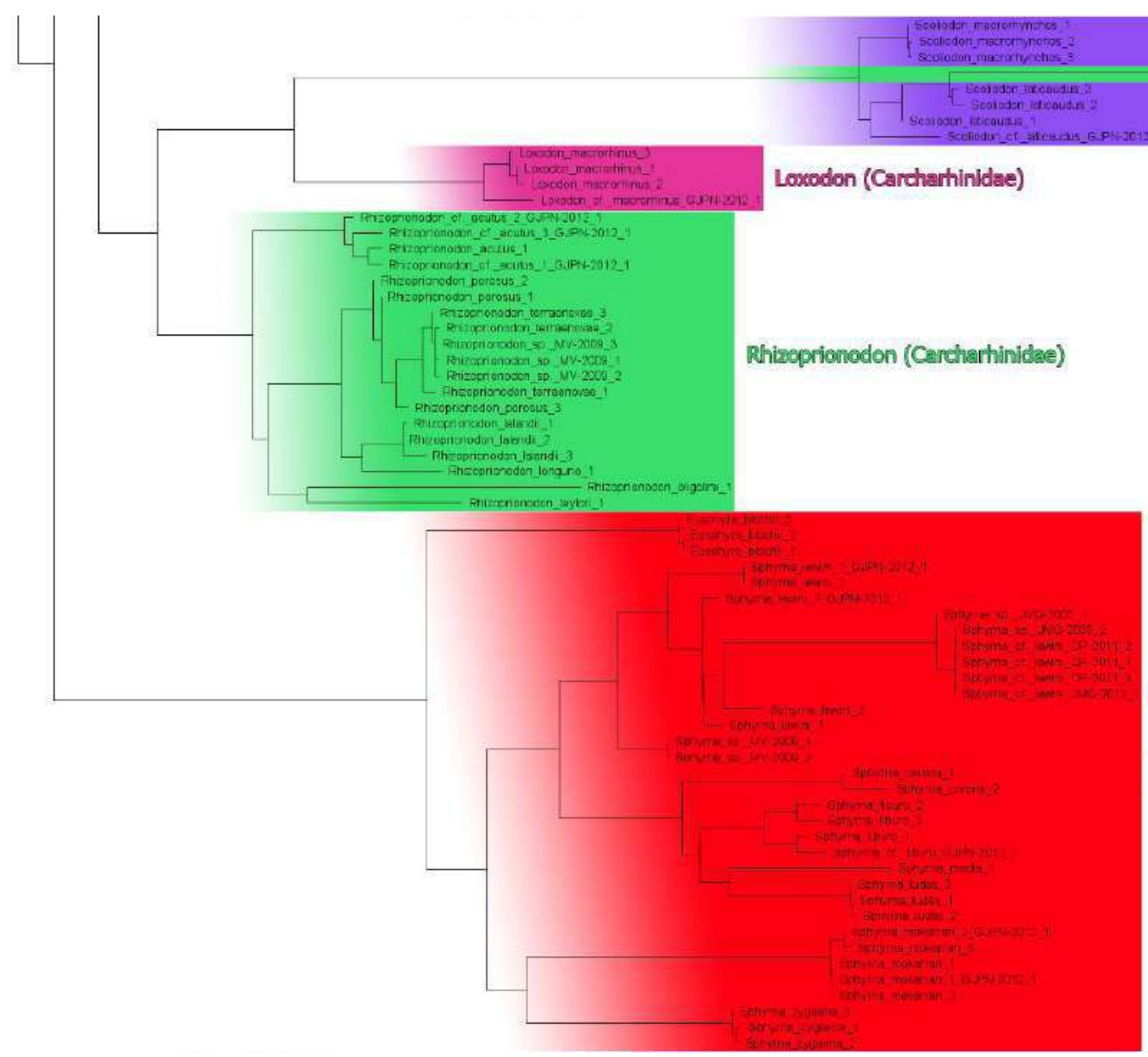

Rhlzoprionodon (Carcheathinidae) Sooliodon (Carcharhinidae)

Figure 29. (continued)

Sphymidae 

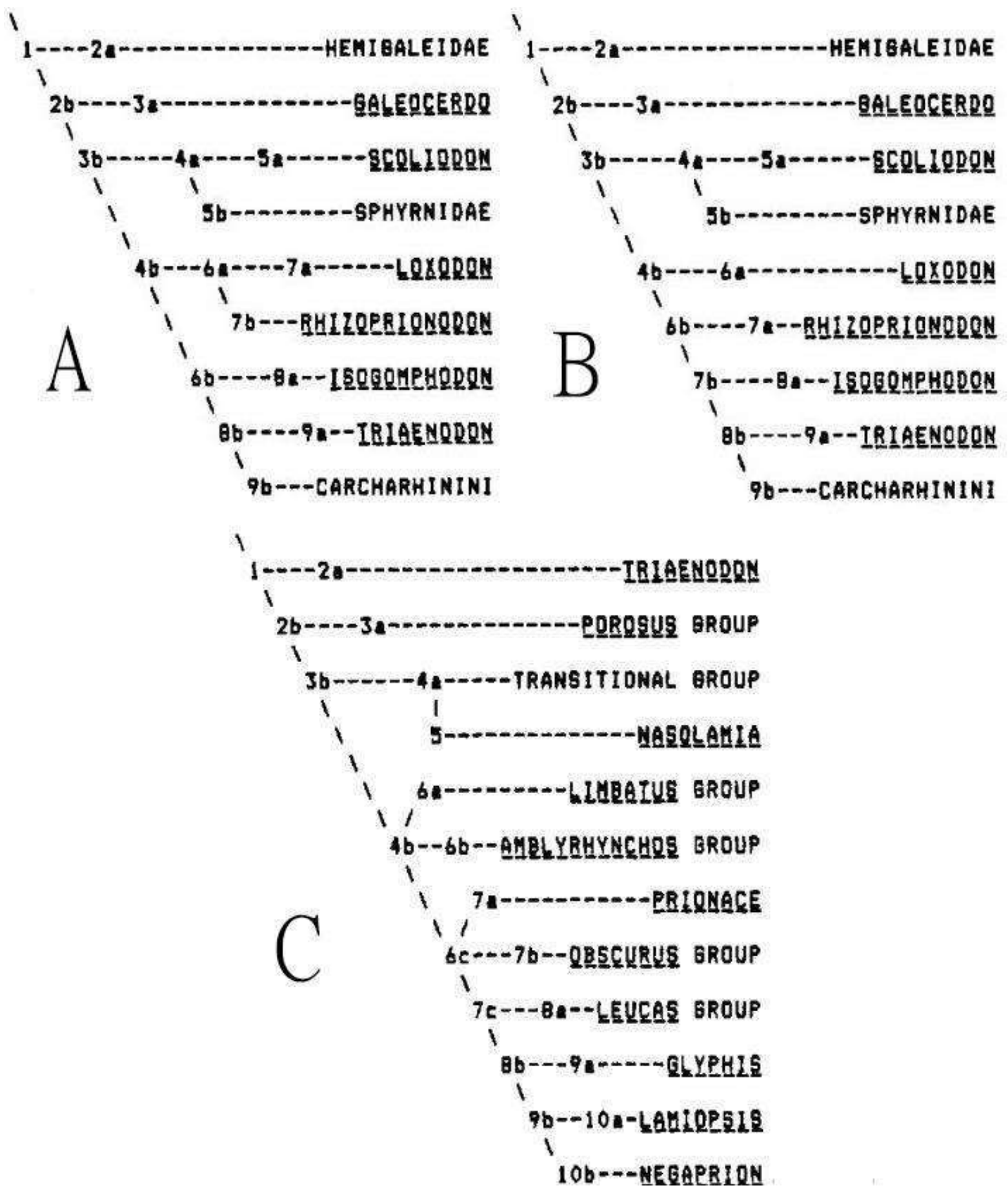

Figure 30. Topology proposed by Compagno (1988) in which Isogomphodon oxyrhynchus is allocated as a sister taxon of Triaenodon and Rhizoprionodon. 


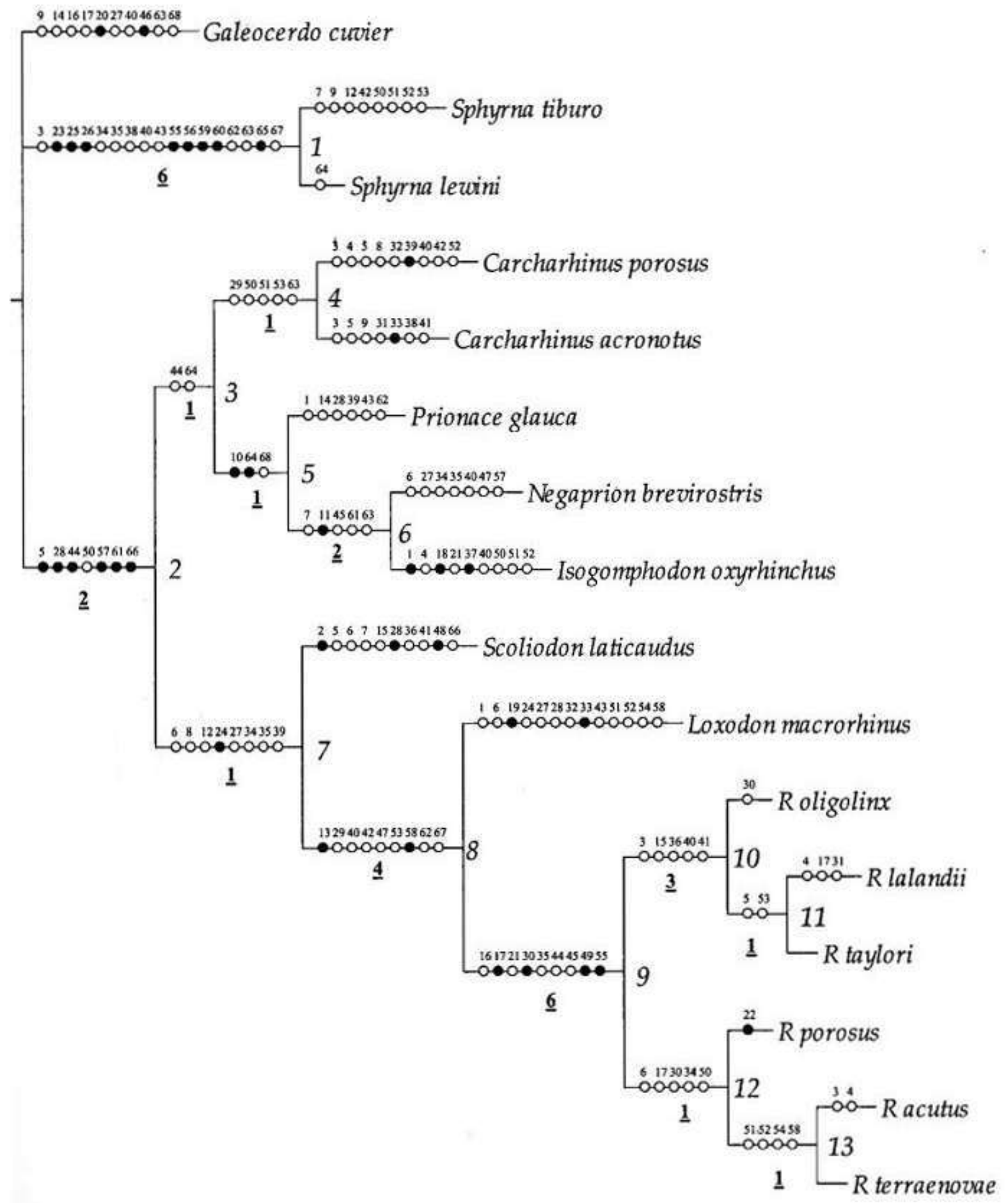

Figure 31. Topology proposed by Kikuchi (2004) in which Isogomphodon oxyrhynchus is allocated as a sister taxon of Negaprion. 


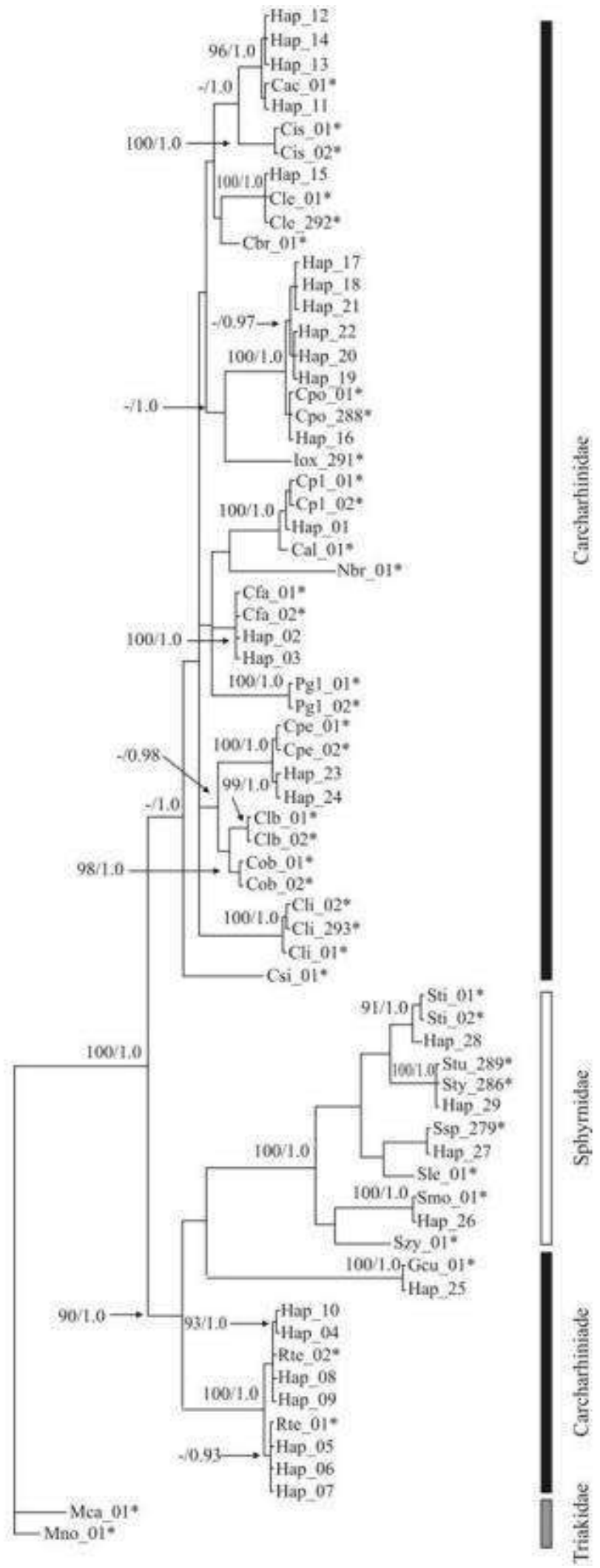

Figure 32. Neighbour-joining tree of Rodrigues-Filho et al. 2009, in which Iox_291 is the voucher that represents Isogomphodon oxyrhynchus. The abbreviations and acronyms are detailed in the original publication. 


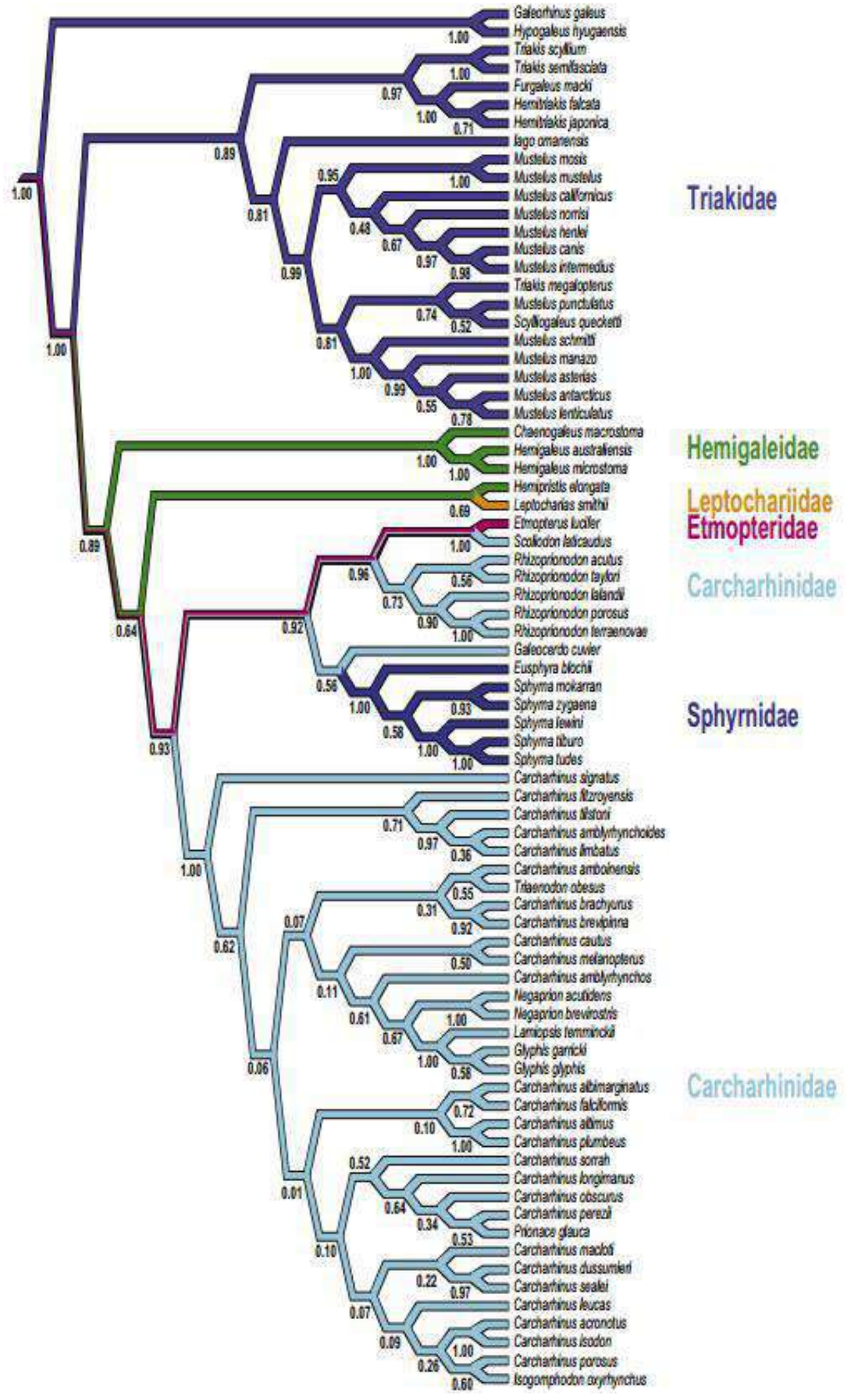

Figure 33. Consensus phylogenetic tree proposed by Vélez-Zuazo \& Agnarson 2011, positioning Isogomphodon oxyrhynchus as sister taxon of Carcharhinus porosus in the terminal portion of the topology. 


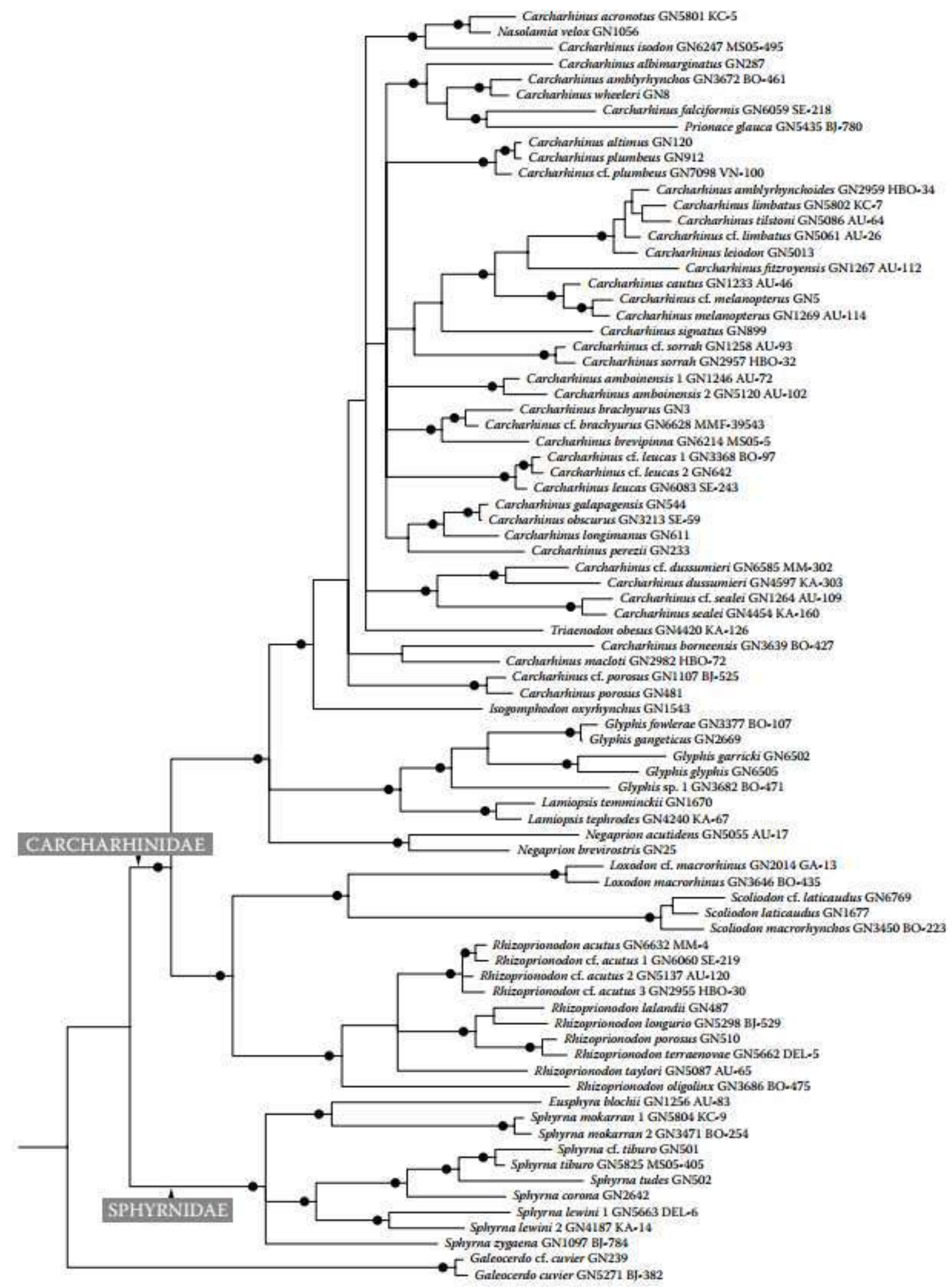

Figure 34. Topology proposed by Naylor et al. 2012a in which Isogomphodon oxyrhynchus is allocated as a sister taxon of Carcharhinus. 


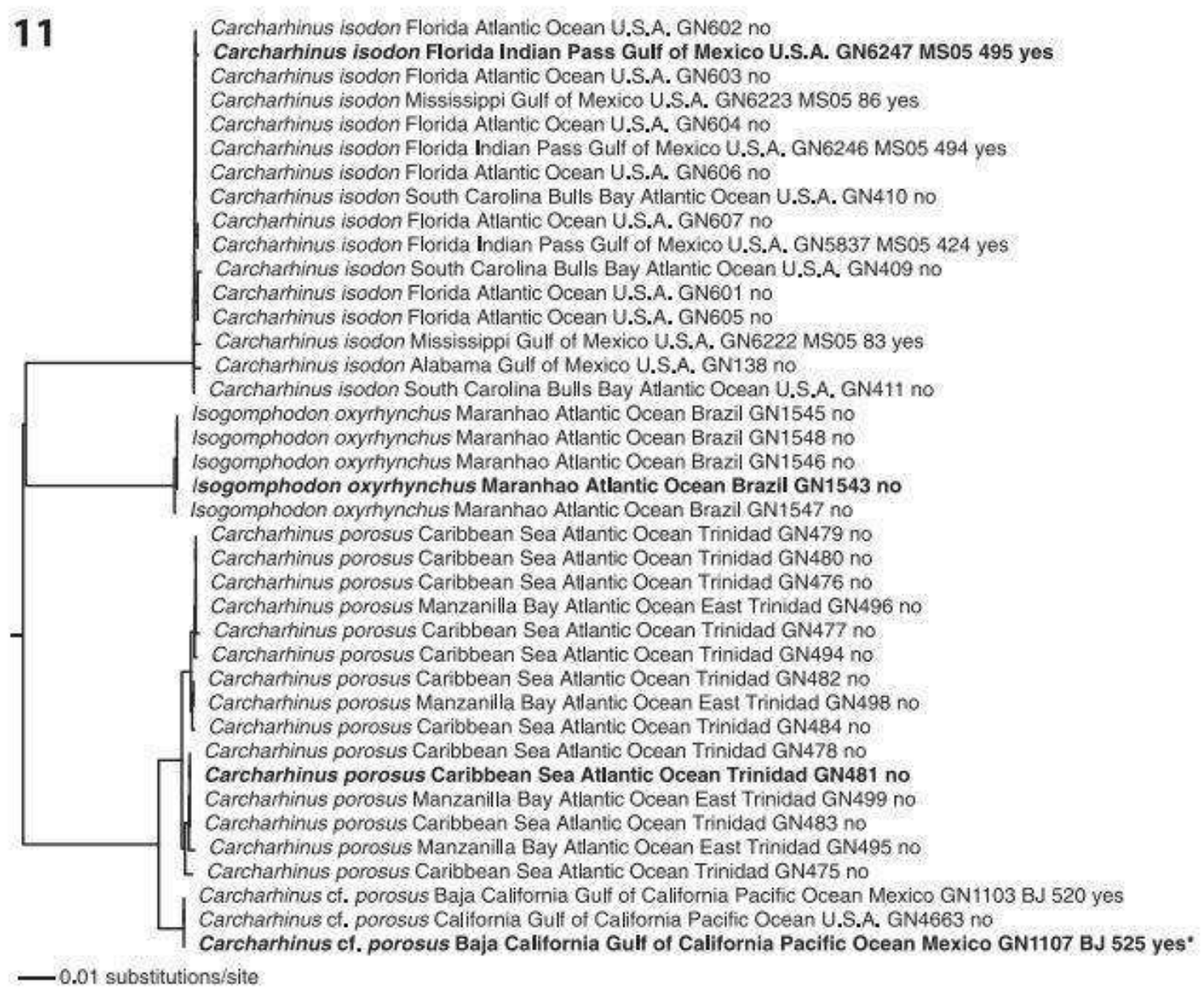

Figure 35. Partition of the topology proposed by Naylor et al. 2012b highlighting the sister taxa of Isogomphodon oxyrhynchus, which were essentially Carcharhinus porosus and Carcharhinus isodon. It is important to state that such conformation is under fair discussion once the employed methodology was focused on species identification rather than phylogenetic relationships. 


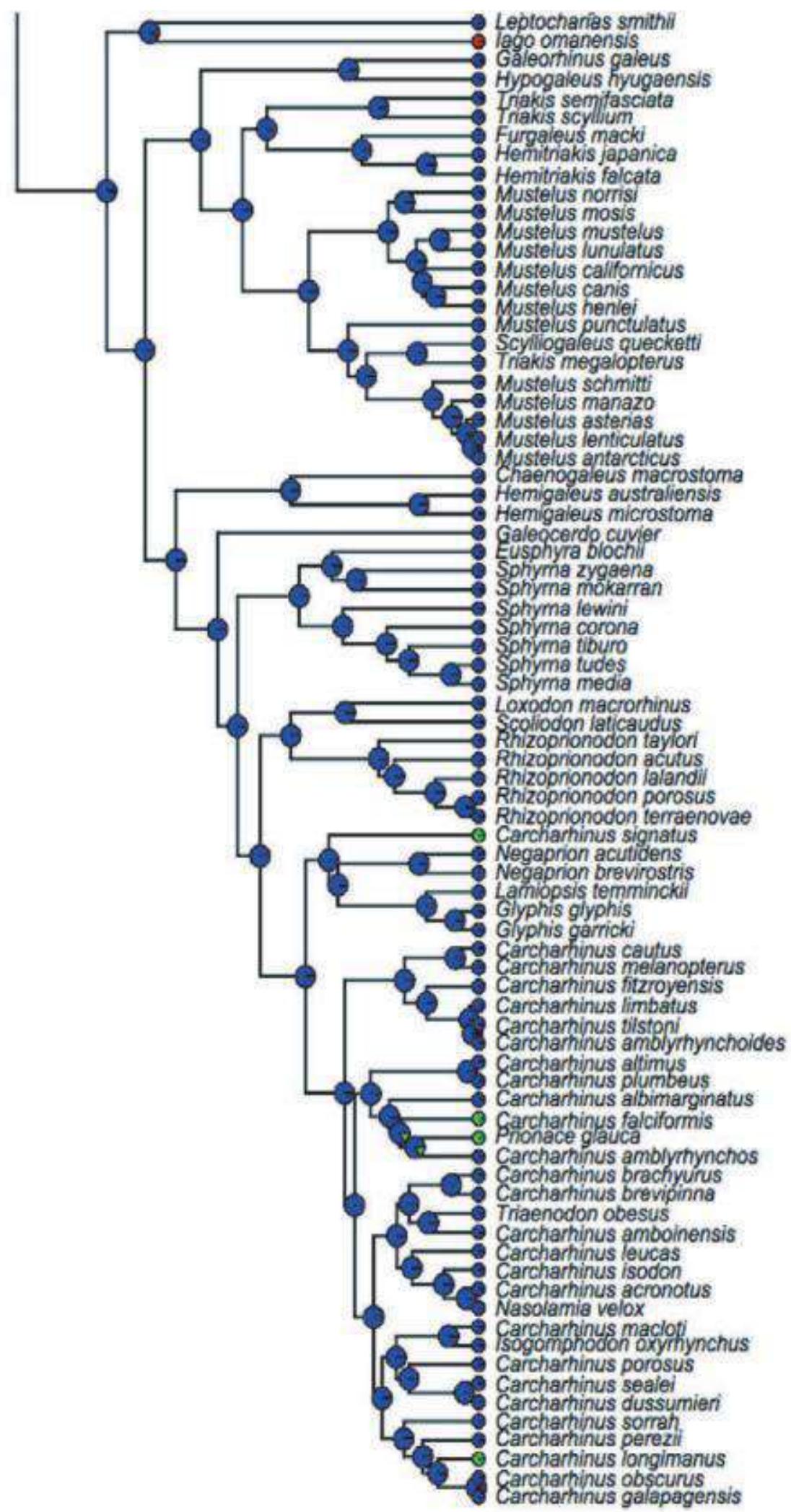

Figure 36. Phylogenetic hypothesis of Sorenson et al. 2014 using molecular markers but highlighting the habitat preference of each terminal through colour coding (see original publication for details). In this case, Isogomphodon oxyrhynchus is proposed as sister-taxon of Carcharhinus macloti. 


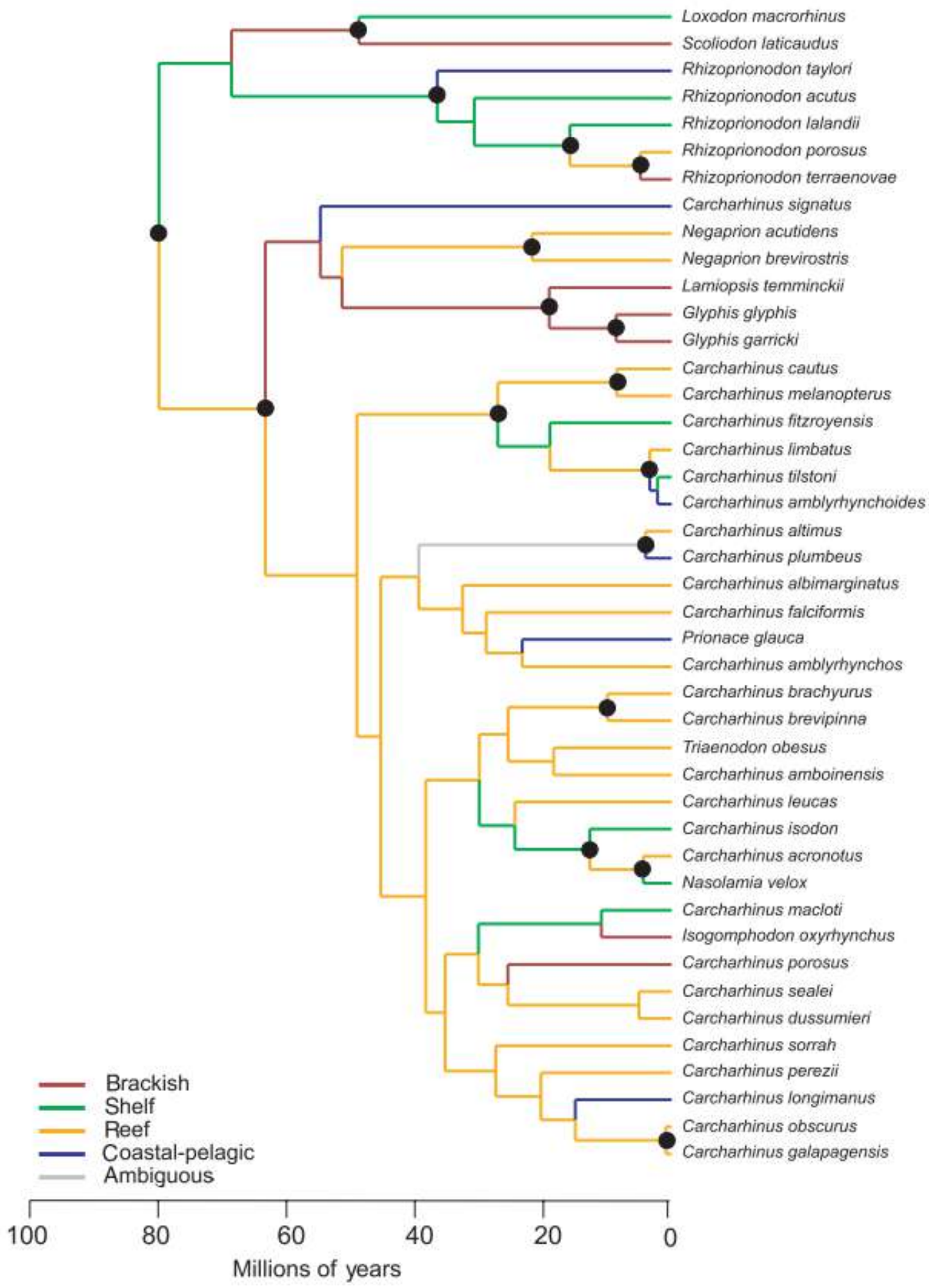

Figure 37. Calibrated phylogenetic hypothesis of Sorenson et al. 2014 using molecular markers but highlighting not only the habitat preference but also focusing on Carcharhinidae. As well as figure. 14 Isogomphodon oxyrhynchus is proposed as sister-taxon of Carcharhinus macloti. 


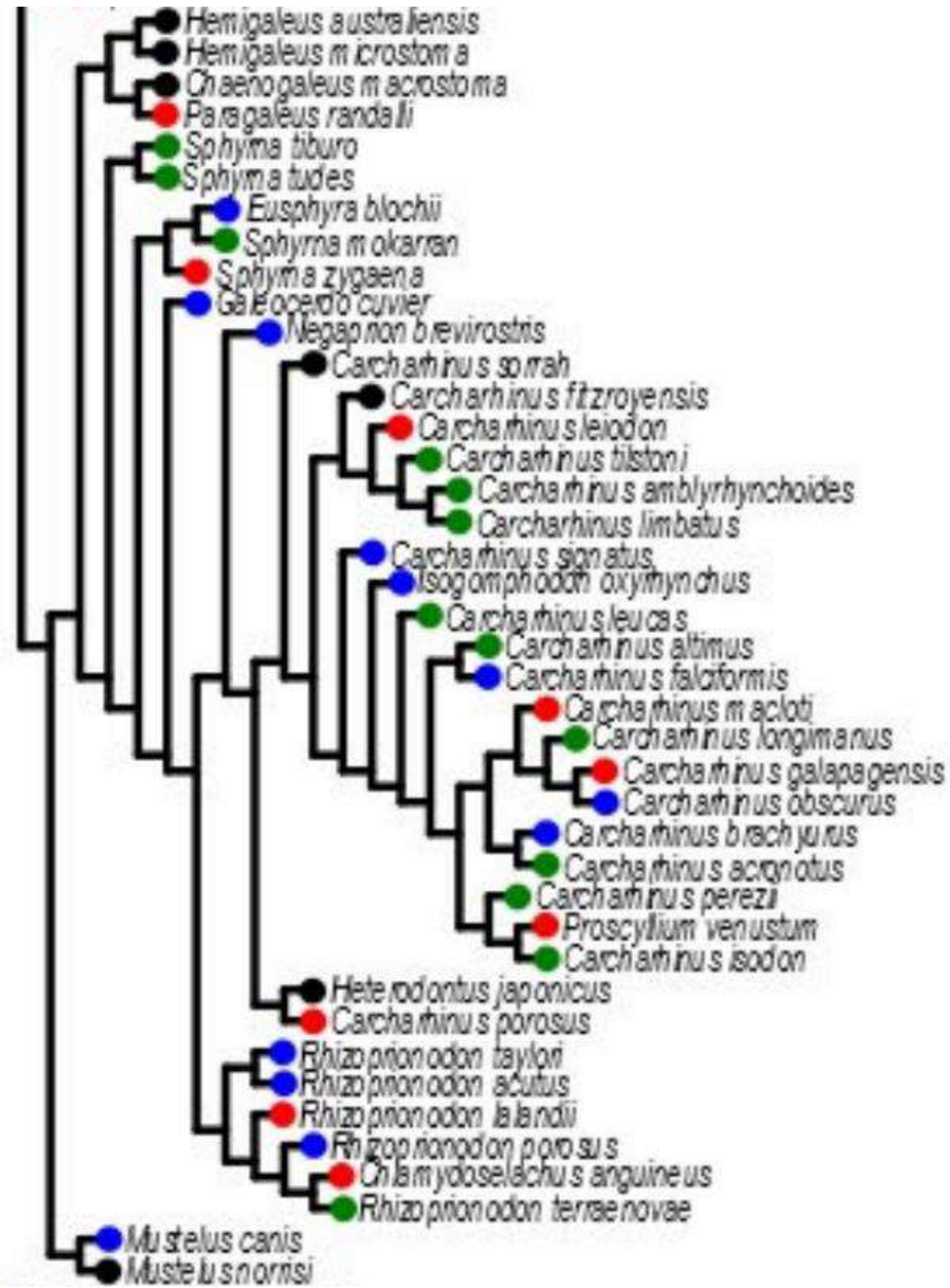

Figure 38. Partition of the topology proposed by Cachera \& Le Loc'h 2017 focusing on the Carcharhinidae + Hemigaleidae clade proposed on their original publication. Isogomphodon oxyrhynchus is here considered sister group a Carcharhinus clade. 


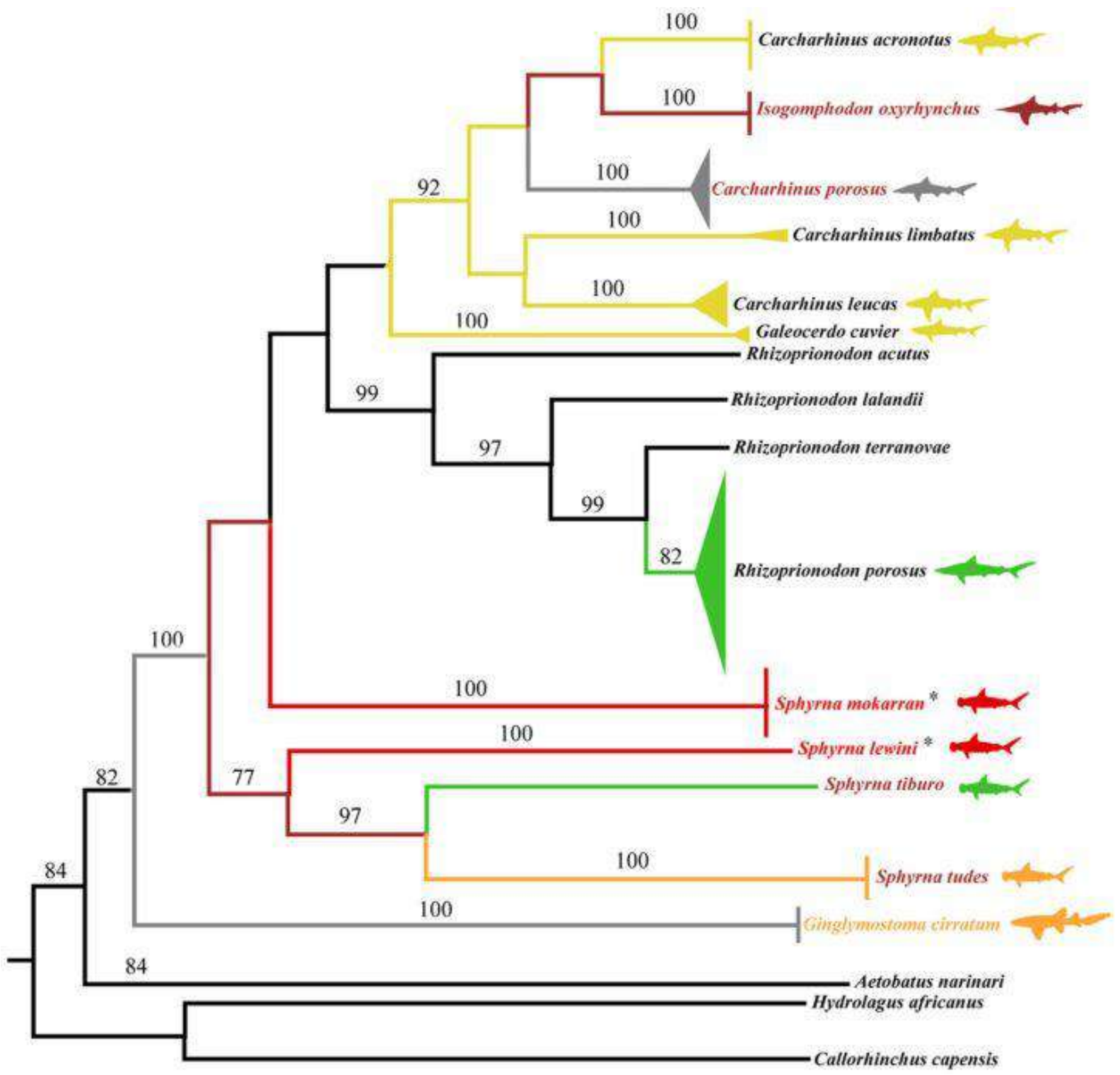

Figure 39. Neighbour-Joining tree proposed by Rodrigues-Filho et al. 2018 using COI as molecular marker. Although not represented by a high number of terminals, this analysis positions Isogomphodon oxyrhynchus as closely related to Carcharhinus acronotus. 


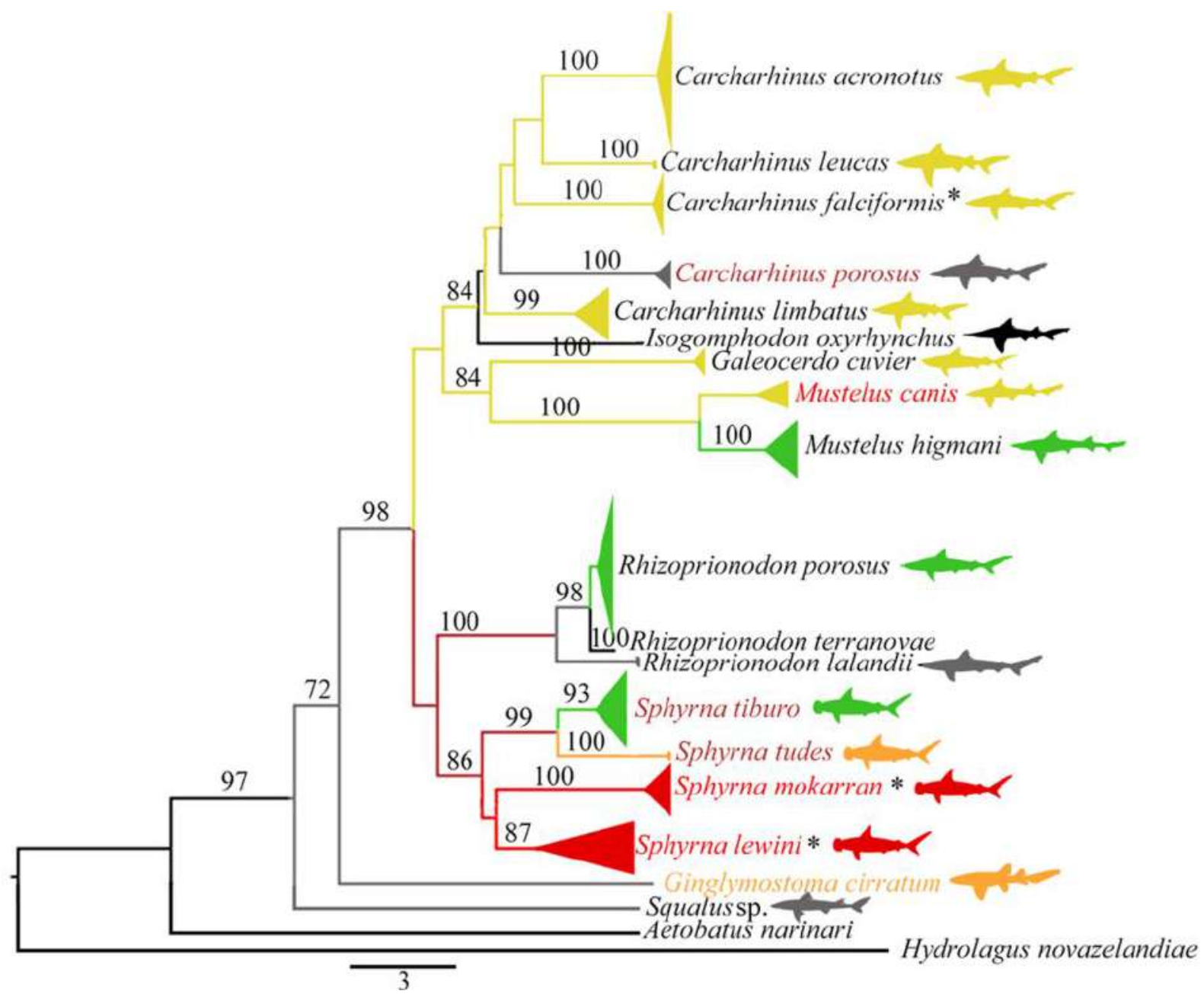

Figure 40. Neighbour-Joining tree proposed by Rodrigues-Filho et al. 2018 using NADH2 as molecular marker. Although not represented by a high number of terminals, this analysis positions Isogomphodon oxyrhynchus as closely related to a Carcharhinus clade that includes Carcharhinus falciformis, $C$. leucas, C. acronotus, C. porosus and C. limbatus. 
Chapter 3

A critical overview of fossil taxa attributed to Isogomphodon (Gill, 1862) based on tooth specimens 


\section{Introduction}

Paleontological records of chondrichthyans are abundant but mostly limited to strongly calcified structures, teeth, dermal denticles and occasionally vertebral centra. Complete skeletons and lithographic skeleton prints exist but are extremely rare (Lauritto 1999; Guinot et al. 2012; Frederickson et al. 2015). Identification of fossil elasmobranchs, like fossil taxa in general, provides potentially useful data for the molecular calibration and phylogenetic dating (Sorenson et al. 2014).

Fossil teeth attributed to the genus Isogomphodon comprise a surprisingly large array of taxa, including I. acuarius (Probst, 1879), I. lerichei (Datervelle \& Casier 1943), I. gracilis (Jonet 1966), I. caunellensis (Cappetta, 1970) and I. aikenensis (Cicimurri \& Knight, 2019). Isogomphodon lerichei and I. gracilis were described from the Southern Congo Basin and Costa de Caparica in Portugal, respectively. Isogomphodon acuarius and I. caunellensis have their type localities in Germany and France, respectively (Laurito 1999; Laurito \& Valerio 2008; Maisey 2012). The most recent described species, I. aikenensis, was found in North America, representing the first new species of Isogomphodon discovered in that region (Cicimurri \& Knight 2019). All those taxa are based exclusively on fossilized teeth and their allocation in the genus relies on dental comparisons with the sole extant species I. oxyrhynchus (Laurito 1999). Not all of them were initially allocated in Isogomphodon, with I. acuarius originally described in Alopecias, I. caunellensis in Aprionodon and I. lerichei and I. gracilis in Carcharhinus (Aprionodon). All of the aforementioned were later transferred to Isogomphodon, initially as synonyms of I. acuarius, by Laurito (1999) and, after multiple nomenclatural shifts, as valid species of Isogomphodon. (Cicimurri \& Knight, 2019).

Although Isogomphodon Gill (1862) was originally diagnosed on the basis of a number of unique or unusual characteristics, the only mention of tooth morphology by Gill (1862) was "Minute teeth or poorly teethed". Such scant information is actually informative to a limited degree since I. oxyrhynchus indeed has numerous minute teeth when compared to other Carcharhinidae. Of course, additional anatomical details are necessary to clearly identify, describe and assign fossil material to Isogomphodon, as done by subsequent authors (e.g., Lauritto 1999). Still, much work remains to be done on comparative dental anatomy of Isogomphodon and its closest relatives in order to test such assignments. The latest review of the species treated herein was Cicimurri \& Knight (2019), which provided brief considerations on each taxon, but without new morphological data. 
The identification of carcharhinids at genus level is useful for calibration of phylogenetic hypothesis, providing a necessary time frame for dating divergence events. Fossil records as a calibration tool for molecular-based trees has been employed by several authors to estimate the timing of node branching in topologies, once the occurrence of dated fossil taxa allows comparison with the employed molecular markers in the analysis (Adnet \& Cappetta, 2001; Klug \& Kriwet 2010). Additionally, fossil records or evidence from vicariance events are the best kind of data for calibration of molecular clocks (Straube et al. 2015). Although a number of phylogenetic studies have included the genus Isogomphodon as a terminal (Rodrigues-Filho et al. 2009; Vélez-Zuazo \&Agnarson 2011; Nayler et al. 2012a; 2012b; Sorenson et al. 2014; Cachera \& Le Loc'h 2017; Feitosa et al. 2018), the use of fossil teeth records as basis for calibration was only used by Sorenson et al. (2014), and the used tooth samples did not include those records of Isogomphodon. The most recent species of Isogomphodon to be described, I. aikenensis (Cicimurri \& Knight, 2019) provided accounts on the taxonomic history of the fossil species allocated in the genus, with a short discussion on the validity of their inclusion in Isogomphodon.

The present chapter offers a critical analysis of all fossil taxa so far allocated in Isogomphodon in order to assess in detail the evidence for their generic placement. The purpose of the present chapter is to provide more detailed descriptions of those fossil taxa, focusing on data relevant to test the evidence for their generic inclusion. 


\section{Material and Methods}

Tooth morphology data for fossil taxa were gathered from published sources, including original descriptions, illustrations, plus any additional relevant sources. Morphological information obtainable from isolated fossil teeth is limited by their very nature, and images of specimens are usually sufficient to gather most or all of the relevant data. Images from the literature (Table 1) were processed and organized using Photoshop, and the original descriptions are included in Appendix 1. Material of I. oxyrhynchus was directly examined both as preserved specimens and CT-Scan images. CT-Scan imaged were obtained with a Phoenix $v \mid$ tome $\mid x$ m microfocus - General Electric at Museu de Zoologia, Universidade de São Paulo. Tooth samples were removed using forceps and scalpels from four positions in the mouth: two anterior teeth (one from the upper and other from the lower jaw) and two lateral teeth (one from the upper and other from the lower jaw). Nomenclature and tooth numbering followed Compagno (1988 - see Figures 3.1 and 3.4). Literature sources for tooth morphology are listed in Table 1 and type localities are illustrated in Figure 1. Figures are edited to provide scale adjustments, pixel cleaning, improved resolutions, and schematic arrangements, as indicated in respective figure captions. Descriptions are organized according to upper and lower jaw positions as seen in whole specimens. For isolated teeth, i.e., most fossil taxa, such positional identification is often impossible, although some published sources claim to do so. 


\section{Results}

In this section, descriptions of tooth morphology are presented for all species currently assigned to Isogomphodon. The first one listed is I. oxyrhynchus, the only extant species in the genus and naturally the one with most complete data. The description provides the standard against which descriptions of remaining species, known from fossil teeth only, are organized. Translated original descriptions of each species are available in Appendix 1.

\section{Isogomphodon oxyrhynchus (Müller \& Henle, 1839)}

\section{Figs. $2-6$}

Carcharias (Aprionodon) oxyrhynchus Müller \& Henle, 1839 [Original description, Type Locality: Surinam];

Carcharhinus oxyrhynchus; - Bigelow \& Schroeder 1948 [synonymizing to Carharhinus];

Isogomphodon oxyrhynchus; - Gill, 1862 [transferred to Isogomphodon]; - Springer, 1950 [genus resurrection];

\section{Type locality: $\underline{\text { Surinam }}$}

\section{Period of Living: Extant}

General description: Isogomphodon oxyrhynchus has teeth with narrow, high and smooth cusps, associated with broad curved roots and transverse notches that are deeper on lower teeth. The anterolateral rows have higher crowns and roots than the posterior ones. Additionally, teeth are symmetric in most regions, with upper anterolateral teeth having narrow cusps and strong curves on the edges of their bases, while medial lower teeth, which Compagno (1988) named 'alternates', are significantly broader and with higher cusps than the medial ones from upper rows. The cusps on anterior teeth in both upper and lower rows, are higher and narrower than the posterolateral teeth ones. From anterior to posterior rows, the cusps gradually reduce in height, with most posterior ones without cusps, creating a visible morphological gradient along the jaws (Figs. $2-6$ ). The sigmoid profile of the crown in the upper teeth has a bases broader and cusps less pointed than those of the lower teeth. The mesial edges of most teeth (in the posterior rows a clear pattern was not visible) have a discrete ridge. In both jaws, the anterior 
teeth have slightly more curved lateral root edges than the adjacent ones. The posterior most rows lose their cusps beginning from the 22nd teeth onwards in the juvenile specimen (MZUSP 101214). No serrations were detected in any teeth with the CT-Scan, although they occur discretely in the cutting edge, especially on the upper ones, as seen on Figure 2.

Upper: The upper lateral teeth present broad roots and relatively short cusps when compared to the same position in the lower rows. Both anterior and lateral teeth are weakly serrated in the cutting edges (Fig. 2), in which the crown base is plain and has a deeper boundary on the labial facet than on the lingual facet. Within the lateral teeth, the crown base is broader and more angular in its curvature towards the lateral nodes. The cutting edges on the upper teeth reach the lateral shoulders, although the crown surface if thinner and less pronounced in this region. The medial sulcus does not reach the crown base on lateral teeth whereas at the anterior one reaches. The curvature on the anterior teeth is slightly less angular, with a crown surface dorsal to the distal lateral shoulder is straighter than the proximal. The concavity in the internode region is present and is mildly accentuated. The lateral teeth have the apical cusps pointer than those on the anterior teeth, which are usually slightly rounded.

Lower: The morphology of the lower tooth changes significantly from anterior to lateral position. The cusps are concave towards the lingual face, and the anterior teeth have narrow and thick roots, with an evident notch in the internode region, curved insertion bases and discretely rounded lateral nodes. Lateral teeth have roots broader and thinner, with a distal curvature less angulate than the proximal one. The lateral shoulders are nearly straight-lined, and the lateral nodes are sub-rectangular, which is more evident in the distal node. Both anterior and lateral teeth are smooth-cusped and have short crown base insertions in the root and pointy strongly sigmoid cusps, although on the anterior ones the cusp is inclined towards the left facet as viewed in lateral view. Lower teeth without serrations in the cutting edges.

Ontogenetic changes: Major ontogenetic drifts in teeth morphology are limited or nearly absent. Compagno (1998) states that adults have slightly bulkier cusps than juveniles, an observation corroborated here (Figs. 2 -6). 


\section{Isogomphodon acuarius (Probst, 1879)}

\section{Figs. $7-10$}

Alopecias acuarias Probst, 1879, p.140; lam. 2, figs. 76 and 77. [Original description; Type Locality: Southern Germany].

Isurus cf. novus (Winkler, 1874) - in Serralheiro, 1954, p. 49; lám.1, figs.15 and 16, no fig. 14. [Citation]

Vulpecula exígua (Probst, 1879) - in Serralheiro, 1954, p.54; lam.1, fig.26, no figs. 25 and 27. [Citation]

Carcharhinus cf. lerichei Dartivelle \& Casier (1943): - in Cappetta et al., 1967, p.292. [Citation] Aprionodon acuarias (Probst, 1879); - in Cappetta, 1970, p.57; la m.17, figs.1-20; - Case, 1980, p.90; lam.6, figs. 8 and 9 (A and B). [Citation]

Carcharhinus acuarius (Probst, 1879); - in Kruckow \& Thies, 1990, fig. 5 [Citation]

Isogomphodon acuarius (Probst, 1879); - Lauritto 1999 [transferred to Isogomphodon]; Lauritto \& Valerio, 2008 [new record]; Vialle, Adnet \& Cappetta, 2011 [new record]; CarilloBriceño et al. 2016 [new record]; Cicimurri \& Knight, 2019 [citation]; Carillo-Briceño et al. 2019 [new record];

\section{Time range: Lower Miocene to Lower Pliocene}

Holotype description: The holotype (Fig. 7, from Probst 1879 Lam.- 2, Figs. 76 and 77) has a deep curvature in the internode region, presenting a medial sulcus that reaches the crown base in lingual facet, and pointy, smooth and narrow cusp. The ventral root has rounded insertion bases, separated by a deepened internode facet. The lingual view shows the crown base restricted to the medial portion to the root, whereas on labial view it extends to above the shoulders, although not reaching the lateral edges of the root lobes.

General description: The anterior teeth have angular transitions crown-root, with a ventral internode region and a ventral curvature more accentuated in the anterior rows, which becomes shallower on laterals. The lateral shoulders have discrete projections. The upper teeth have asymmetrical cusps, with a curve on crown base being more angular towards one side. The crown base extends to above the lateral shoulders dorsal facet, with the boundary between these two regions being deeper on labial view than on lingual view. Regarding the roots, they are horizontally narrow, with circular lateral node edges, whereas the crowns drift from symmetrical and slender in the first lateral rows to asymmetrical and thicker in rows close to mouth corner (Fig: 9 - 15). The cutting edges are smooth, with no serrations. The lower teeth 
have narrow and symmetrical cusps, with curved root bases and medial sulcus not reaching crown base (except sample 4 fig 1.) The roots are thicker, with slender and strong cusps that do not become asymmetrical as moving towards the posterior lateral teeth rows (Fig 3: 16-20). The internodes are curved, cutting edges are smooth, without serrations in the cusps, and the crown base tends to reach the lateral nodes of the roots in the posterior lateral rows.

\section{Isogomphodon lerichei (Darteville \& Casier, 1943)}

\section{$\underline{\text { Fig. } 11}$}

Carcharhinus (Aprionodon) lerichei Dartivelle \& Casier, 1943 [Original description; Type Locality: Southern Congo Basin]: - Zbyszewski \& Moltinho d'Almeida, 1950, p. 340; Lâm. 6, fig. 104 [Citation];

Carcharhinus (Aprionodon) lerichei (Dartevelle \& Casier, 1943) var. Minuta nov.: - Jonet, 1966, p.69; lám.2, only figs. 7 to 13. [Var. minuta description; Type locality: Southern Congo Basin]

Isogomphodon lerichei (Darterville \& Casier, 1943); Lauritto 1999 [transferred to Isogomphodon]; - Cicimurri \& Knight, 2019 [Implicit revalidation]

\section{Time range: $\underline{\text { Upper Miocene }}$}

Type description: Overall, teeth of I. lerichei in Jonet (1966) present a mild curvature on the right side of the transition between the crown and the root (with few exceptions that are nearly symmetrical - specimens 10 to 12, in Fig. 11) and have broad, nearly symmetric roots. The angularity of the crown and the root on both sides appears asymmetrical on most samples, although some present this transition with equal angles. As most fossil species related to Isogomphodon, the labial facet of the crown in lateral view is plain and convex near the not, while the lingual surface is concave close to the root and curved.

General description: Some samples presented concavity close to the root in labial facet ( $2 b$, $7 b)$, nonetheless the most prominent concavity remains on the lingual side. The crown is rather shorter than the other species attributed to Isogomphodon, and the root insertion base has a weak and discrete concavity towards the medial sulcus (nearly plain on upper samples), which is slightly to nearly not visible on labial view. The medial sulcus is poorly visible in the imaging, although it seems to be rather shallow and to not reach the teeth crown. Regarding the cutting edges, the dentition of I. lerichei lacks serrations on these regions, although this assumption is taken based solely on the present images. Upper teeth (Fig. 11: 1, 2a, 2b, 3a, 3b, 4, 5a, 5b, 5c, 
6, 8a, 8b, 8c, 9, 13 of I. lerichei has different angles in the transition from the crown to the root, with the most pronounced angle being the right one on lingual view. In contrast to the lower teeth, the concavity on lingual facet is more evident. Although all teeth samples present a plain, convex root base, this feature seems more visible in the upper samples, although sample 13 is an exception. Lower teeth (Fig. 11: 7a, 7b, 10a, 10b, 11, 12a, 12b, 12c) has a concavity on the root base which is slightly more evident. Similar to teeth $2 b$, the sample $7 b$ presented a strong concavity in its internal facet, suggesting those samples are likely from parallel regions within the jaw. Nonetheless, $7 \mathrm{~b}$ has a stronger protuberance on the lingual facet, close to the root upper margin, and a more symmetric structure, while $2 \mathrm{~b}$ is slightly asymmetrical. Angles on the transition cusp-lateral shoulder in the lower teeth appear to be close to $90^{\circ}$ degrees, although samples $8 \mathrm{a}, 8 \mathrm{~b}$ and $8 \mathrm{c}$ also have shallower curves on the right side in lingual view. Additionally, the medial sulcus on the lower teeth do not reach neither the root base nor the crown surface. The smoothness on the teeth from the lower jaw is possibly a present feature, although such affirmation is still based on imaging.

\section{Isogomphodon gracilis (Jonet, 1966)}

\section{Fig. 12}

Carcharhinus (Aprionodon) gracilis Jonet, 1966, p.70; lam .2, figs.14 - 2 [Original description; Type Locality: Costa de Caparica - Portugal]

Isogomphodon gracilis (Jonet, 1966); - Cicimurri \& Knight, 2019 [transferred to Isogomphodon]

\section{Time Range: $\underline{\text { Upper Miocene }}$}

Type description: The cusps in I. gracilis are high and pronounced, with a strong and evident concavity in the medial portion of the root. Generally, the teeth of this species are slightly asymmetric towards the left side (as view from the labial facet) and the concavity is also more pronounces in the labial view.

General description: Laterally, the concavity in lingual facet is visible in both facets in some samples (Fig. 12 - 16b, 19b and 21), although others showed a convex labial view (15c). The curvature of the transition crown-root is wide (angle likely $>60^{\circ}$ ), with no serration within the cutting edges. The medial sulcus reaches the root base and does not touch the crown, and the root bases are more expanded towards one side, usually the right one as viewed from labial 
facet. The crown length varies among the specimens, being slightly thinner on 19a, 20a and $20 \mathrm{~b}$. The concavity on lateral view is evident in both facets, nonetheless teeth $15 \mathrm{c}$ has its labial facet plain and convex and lingual facet not as concave as the other samples. The upper teeth (Fig. 7: 14a, 14b) of I. gracilis have strong, point cusps and apparent smooth cutting edges. Regarding the medial sulcus, it is short and narrow, although visible in labial facet. From all the analysed specimens of this species, the only upper teeth specimen is nearly symmetrical, has its transition crown-root in the cutting-edge line wider in angle and a concave root. The lack of a lateral view limits the description on the convexity from both facets. The lower teeth (Fig. 7: 15a, 15b, 15c, 16a, 16b, 17a, 17b, 17c, 18, 19a, 19b, 19c, 20a, 20b, 21) vary in morphological features, although they generally have point, narrow and high crowns, shallow tubercles that allocate the medial sulcus and concave roots. On samples 19a, 19b, 19c, 20a, 20b, the cusps are higher and narrower, resembling features of some Lamniform sharks such as viewed in Mitsukurina owstoni. The medial sulcus is more visible in labial view and curvature on the cutting-edge transition is apparently close to $90^{\circ}$. The specimen of samples $19 \mathrm{a}, 19 \mathrm{~b}$ and $19 \mathrm{c}$, the root is thinner, and the medial sulcus originates at the very base. On specimen of samples $20 \mathrm{a}$ and $20 \mathrm{~b}$, the root base is curved on the edges and straight-lined in the medial portion, a unique feature only viewed in this species' specimen.

\section{Isogomphodon caunellensis (Cappetta, 1970)}

\section{Figs. $13-14$}

Aprionodon caunellensis Cappetta, 1970; p. 60; lâm. 16(23-27) [Original description; Type Locality: Caunelle (France)];

Isogomphodon caunellensis (Cappetta, 1970); - Cappetta, 1987; p. 121 [transferred to Isogomphodon]; Lauritto \& Valerio, 2008 [new record]; Cicimurri \& Knight, 2019 [Citation];

\section{Period of Living: Lower Miocene}

Holotype description: The cusps are pointed and strong, with crowns and their insertion on the root being narrow. The shoulders are angular, with the transition crown-root surface being weakly curvy to eventually straight (Sample 25, fig. 13). The medial sulcus is not visible in labial facet. The shoulders do not present projections and the root ventral facet is weakly Ushaped. 
General description: Generally, the root is thick and high, without serrations visible within the cutting edges. All samples present narrow based and tall crowns, apart from sample 24 in Fig. 13 and both samples of Fig 14., which have a broad crown insertion and shorter cusp. The medial sulcus is shallow, with its proximal origin not being visible in labial view (sample 27, Fig. 13). The shoulders are smooth, with no apparent protuberances on their surfaces. The boundary between crown and root is variable, being either straight or mildly curved. Additionally, as seen on sample 24 fig. 13, the crown base covers the entire shoulder dorsal surface. Most specimens are slightly asymmetrical, with exception of specimen 26 fig. 13. The upper teeth (Fig. 13 - 23 and 24) have the root lobes sub-rectangular to mildly circular, with the interlobes being mildly concave to nearly convex. On sample 23 fig. 13 the left transition (as viewed in the image perspective) between the shoulder and the crown is slightly less angular than the right side. Additionally, this specimen has lobes on the root with strong, mildly curved corners, although not circular. On figure 14, the specimens present curvy cusps, asymmetrical shoulders and deepen medial sulcus. The cusp presents a straight superior edge, although probably due to a damage during the fossilization process. The lower teeth (Fig. 13-25, 26 and 27) have pointy cusps and narrow root insertions, with obtuse transitions between crown and root. A mild internode concavity is also present. Most lower teeth samples have the boundary crown-root concave towards the upper direction in lingual facet. Nonetheless, specimen 27 Fig. 13 shows a curvature of such region directed to the ventral portion.

Isogomphodon aikenensis (Cicimurri \& Knight, 2019)

Isogomphodon aikenensis (Cicimurri \& Knight, 2019), p. 11, fig. 4 [Original description; Type Locality: South Aiken State, Aiken Country, South Caroline (United States of America)];

\section{Fig. $15(\mathrm{E}-\mathrm{P})$}

\section{Type locality: South Aiken State - United States}

\section{Period of Living: Eocene}

Holotype description: The holotype (SC2013.38.110 - Figure 15, specimen E) was described as upper anterior teeth, which has a narrow cusp, round-based lateral nodes and a crown base extending to the dorsal facet of lateral shoulders. Additionally, the interlobe area is deep in a discrete U-shape, the medial sulcus extends close to the crown base and the lateral nodes have round edges. 
General description: All illustrated specimens do not have serrations on the cutting edges, with generally asymmetrical shapes (except holotype, which is slightly symmetrical). The posterior teeth have broad and thick roots, with angular root-crown transitions (lingual view) and cutting edges visible and distinguishable on the lateral shoulders, with protuberances that resemble an expansion of the lateral nodes. The root width is generally similar in all samples, although some lower teeth have narrower roots. Cicimurri \& Knight 2019 employed the term 'T-Shaped' to describe the general morphology of theses specimens (Fig. 15, E - P). The curvature on the lingual view is apparently more pronounced on the medial-dorsal facet, close to the border between the crown and the root. The medial groove is shallow, reaching the crown base. The upper teeth (Fig. 15 - E, J, K, P) of I. aikenensis have narrower roots when compared to lower and posterior teeth (which jaw not specified by the authors), although the diameter of the cusps is similar among all samples. The holotype has a deeper interlobe area than the other upper sample. The curvature on the crown-root division is symmetrical on the holotype whereas the other upper teeth specimens have a less angular transition on the right side in lingual view. The lateral nodes are elongated and thin, with cutting edges on the lateral shoulders being from diagonal to horizontal. The lower teeth (Fig. 15 - F, G, H, I, L, M, N, O) are generally asymmetrical, with straight, narrow cusps and having the medial groove visible in labial view. The roots have oval edges on the lateral nodes, and the medial groove has hollow ventral extremities (i.e., the labial surface is absent in this region). Although most lower teeth specimens have perpendicular crowns, specimen figured in $\mathrm{H}$ and I on fig. 15 has a slightly curved cusps toward the distal lateral node. The internode area is shallow, although with a Ushape curvature. On specimen figured in H-I of fig. 15, the medial portion of its cusp is thicker. 


\section{Discussion}

Tooth morphology, as a basis, is a tool that has to be employed with caution in the identification of Elasmobranchii, both fossil and recent taxa, once the delimitation between informative and non-informative teeth are usually not objectively distinguished by most paleontological studies (e.g., lateral, symphisian and posterior teeth tend to have fewer diagnostic characters), thus putting in check the reliability of the fossil identifications (Naylor \& Marcus 1994). Dental characters are highly plastic, known to vary intraspecifically, ontogenetically, and positionally within the jaws (Raschi et al. 1982; Compagno 1988; Naylor \& Marcus 1994). Nonetheless, for Isogomphodon, the lateral nodes conformation and the crown height on anterior could potentially be used as a diagnostic character to differentiate the anterior to the lateral teeth. Still, taxonomic and phylogenetic conclusions based on teeth must carefully bracket all such variables as potential sources of error. In all cases, information available for extant species serves as the best template on how to interpret the significance of observed variation in fossil teeth. Depending on circumstances, information from isolated fossil teeth may range from fully conclusive to fully inconclusive. Of course, most cases fall between those two extremes. On the other hand, some specific genera such as Galeocerdo and Chlamydoselachus are sufficiently unique in their dental morphology to undoubtedly identify and allocate congeneric fossil specimens.

Firstly, the key-point that needs to be stated is the morphological delimitation of Isogomphodon teeth that would directly clarify the identification of the fossil taxa. Lauritto (1999) provides a genus-level dental description of Isogomphodon, comprising the following combination of characteristics: a high narrow slender cusp, concavity on lingual facet and convexity on labial surface on anterior teeth, a high root with lateral nodes closely positioned on anterior teeth and divergent on lateral teeth, deepen mesial groove, weak serration on upper anterior teeth (which is more evident on the cusp tip and nearly absent on the crown base) and smooth enamel. Results of the present study adds some new elements to a dental diagnosis of Isogomphodon, such as a morphological drift on the nodes from anterior to lateral teeth, especially on the lower jaw (closely positioned lateral nodes on the anterior rows - both upper and lower - that become more separated on the lateral positions), concave profile on lateral view of anterior lower teeth, narrow crown base with a strong sigmoid profile on the cusp, which gradually decreases in an anterior-posterior way, notched/curved internode facet and the presence of serrated cutting edges on the upper teeth. The gradual spacing between the lateral 
nodes along the teeth rows (especially on the lower teeth) occurs in many carcharhinids (see Naylor \& Marcus 1994), however it seems more pronounced in Isogomphodon. A clear delimiting character is teeth counting, in which Isogomphodon has numerous teeth varying from 49-60 in the upper rows and to 49-56 and in the lower rows (Compagno 1988), nonetheless for teeth specimens which were found isolated, such character has no use. Regardless, the anterior teeth of I. oxyrhynchus, both upper and lower, attain most of the characters that are potentially diagnostic, which are essentially the presence of serrations on the upper ones whereas the lower teeth present high, curvy crowns in lateral view with closely positioned lateral nodes and narrow internode ventral surface.

As far as it was observed by the present review, the genus Isogomphodon has not been categorized based on a single exclusive character in terms of morphology, but rather by a combination of features that would identify the teeth specimens as taxa from this group, starting with the general assumption that the sample has a 'Carcharhinidae-like' shape. The main concrete differences between the Carcharhinus teeth and the Isogomphodon teeth, as pointed out by previous authors, were essentially total size, teeth counting and the concave notched internode surface on lingual facet, in which comparatively Isogomphodon tends to have smaller, numerous teeth with a more pronounced concavity whereas Carcharhinus teeth is bigger, less numerous and essentially more flattened on the lingual facet. Additionally, the teeth morphology inside Carcharhinus has a component of variation in different individuals in a species level, for example sexual dimorphism and geographical distribution (Naylor \& Marcus 1994), therefore, considering the phylogenetic proximity of Isogomphodon to Carcharhinus species (e.g., Vélez-Zuazo \& Agnarson 2011; Naylor et al. 2012;), it seems rather possible to assume the same would be applicable to Isogomphodon. Considering the largely reported distribution of some fossil taxa such as Isogomphodon acuarius, a detailed taxonomic review for this species remains necessary.

Considering that total size has been used as a key feature in the cited descriptions of fossil Isogomphodon, which is usually smaller in adults compared to other carcharhinids (Compagno 1988), such character calls for a re-evaluation. Considering that determining the life stage and specimen total length based on teeth characters requires robust morphometric analysis and comparison of landmarks from the extant species (see Cooper et al. 2020), which was not performed by any description here considered, the total size of teeth is questionable character in the accurate identification of fossil species of Isogomphodon. Such feature is strongly explored by the original description of all taxa within the genus, often primarily 
regarded as diagnostic (e.g., I. caunellensis was described by Cappetta 1970 as bigger in size than its congeneric I. acuarius). Indeed, the teeth of Isogomphodon oxyrhynchus are smaller in total size (see Figs. 2 - 5 and Compagno 1988 - Plates 22 and 23), and although this might represent a valid character for the extant species, nonetheless it seems rather premature to consider that the same character would occur for taxa in which no other fossil record apart from teeth were collected and analysed. Additionally, the cusps height mildly increases with life stage in I. oxyrhynchus, which is asserted based on Compagno (1988) and on the observations of the juvenile (MZUSP 101214) and the adult (MPEG 3455). Within the fossil taxa, by the same time such increase is possible, none of the cited authors provided arguments to identify the life stage of the specimen from which their samples came from, not only raising the question whether they belong to an adult or a juvenile but also whether they general size is actually diagnostic. Moreover, total teeth counting, another highly informative character of Isogomphodon, is also a relative feature, once most fossil samples were often found isolated and therefore without evidence to argue they belong to the same specimen, even if found closely positioned.

Besides Lauritto (1999), Herman (1991) provides a dental characterization of the multiple taxa allocated within Carcharhiniformes, in which Isogomphodon was described as "Root neo-holaulacorhizid with a broad shallow median groove: root lobes expanded and generally obtusely angled - Principal cusp high and slender but medially and distally expanded, sometimes weakly serrated". Additionally, the principal cusp being longer than root length, mesial and distal cutting edges is another diagnostic feature proposed by Herman (1991). The present review endorses such features, although the 'medially expanded cusp 'description might not be applicable for I. oxyrhynchus, but perhaps for a genus level as I. acuarius on Fig. 3 (Specimen 3) fits to this description. It is important to notice, however, the contradiction of characters between Lauritto (1999) and Herman (1991), in which the first stated that the median groove is classified as deepen by the first author and shallow by the second.

It has also been noticed that a holotype is determined as a single teeth specimen, which is understandable once it is not always possible to collect teeth that came from the same dentary series. However, in some cases when it is possible to collect the dental series, more robust descriptions could be performed once it is known that teeth morphology in Isogomphodon varies not only among the upper and lower teeth, but also along the dentary rows of each jaw (Compagno 1988). Therefore, the method usually used to determine whether multiple isolated fossil teeth with different morphologies would actually belong to the same species or specimen 
but in different positions is subjected to flawed identifications. Cappetta (1970) is one of few examples that shows a dentary plus series of I. acuarius that likely came from an individual specimen.

The description of I. acuarius, I. lerichei and I. gracilis were rather simplistic and focused on the general morphological description rather than their taxonomical delimitation, once they were initially allocated in different genera (I. acuarius as Alopecias acuarius, I. lerichei and I. gracilis as Carcharias (Aprionodon) and I. caunellensis as Aprionodon). Posteriorly, based on the reviews and remarks provided by Lauritto (1999), Laurito \& Valerio (2006) and Cicimurri \& Knight (2019), all listed taxa were synonymized into and considered valid as Isogomphodon. Illustrations of teeth samples of Isogomphodon gracilis and Isogomphodon lerichei were obtained in Jonet (1966), which unfortunately not only lack scale bars within the images for size comparison but also images of labial, lingual and lateral views for all teeth specimens, which limits the discussion on those two taxa using measurement features and other metrics that depend on those views as argumentation. Curiously, he did not provide a clear difference that would separate I. gracilis from its congeneric species, although a brief description was published. The extant species in contrast has a unique feature when compared to its congenerics: the upper anterior and lateral teeth is weakly serrated. Therefore, inside the genus, I. oxyrhynchus is rather better delimitated if solely considering the teeth morphology.

\subsection{Isogomphodon acuarius (Probst, 1879)}

The original I. acuarius description was focused on generical features for the genus, with largest collected specimen size being potentially the most informative feature (Cicimurri \& Knight 2019). It was by far the most abundant and sampled species among its congenerics, not only with confirmed occurrence to South America and Europe (Probst, 1879; Lauritto, 1999; Laurito \& Valerio, 2008; Vialle, Adnet \& Cappetta, 2011; Carillo-Briceño et al. 2016; Carillo-Briceño et al.2019) but also with many specimens identified as I. acuarius. The analysed images from this taxon were here compiled from Probst (1879), Cappetta (1970), Lauritto (1999), Carrillo-Briceño et al. (2016), although remarks from Cicimurri \& Knight were herein considered. Each reference is analysed individually.

The specimen reported by Probst (1879), i.e., the holotype, has a morphology that fits to some of the characters for the genus (narrow elongated crown, enameloid profile and concave 
notched internode facet). Nonetheless, as this specimen was reported isolated, the clear diagnostic features (teeth counting, total size related to total size, gradated morphological drift along the rows) are not useful in this case. Cicimurri \& Knight (2019) stated that the teeth specimen illustrated by Probst 1879 has no significant differences from I. aikenensis and the description for the remaining collected specimens was limited to the genus itself, apart from largest collected tooth size $(7 \mathrm{~mm})$. Through the present comparisons with the extant taxon $I$. oxyrhynchus and with the characters that were proposed as potentially useful, the specimen in question might represent an Isogomphodon species based on the similarities with the proposed characters on this review. Regarding Cappetta (1970) I. acuarius, on the other hand, the samples could also represent an Isogomphodon species, once its lower teeth also have narrow roots, spaced lateral nodes on lateral teeth, high slender cusps, enameloid crowns, mild concavity on the base and narrow, slender cusps.

However, as observed in the specimens of Lauritto (1999), such key characters are absent, and still considered by the author as an Isogomphodon. The sample 3 on Fig. 10 has a cusp whose width is thick in most of the crown height, therefore putting into question whether it actually represent an Isogomphodon. Such specimens identified by Lauritto (1999) as I. acuarius specimens are more likely a Carcharhinus, considering characters such as a plain root medial ventral surface, thicker crowns and the angles between crown and lateral shoulders. Some teeth types of Carcharhinus isodon, C. brevipinna and C. limbatus have similar cusps morphology to Isogomphodon oxyrhynchus (narrow, smooth enamel and strongly sigmoid profile), although the root of C. isodon is plain and straight, whereas I. oxyrhynchus has a more pronounced medial scallop in the internode region of the root (see Compagno 1988 Plates 2223 and Naylor \& Marcus 1994 Fig. 2). The teeth identified as I. acuarius by Laurito (1999) has a similar morphology to $C$. isodon, although the teeth size in this last taxon is bigger than the genus Isogomphodon (see Compagno 1988 - Plates 22 and 23). To this moment, the present evidence sustains that I. acuarius is potentially an Isogomphodon species apart from Lauritto (1999) specimens. However, to better define whether or not I. acuarius is correctly allocated, the presential analysis of the specimens is mostly recommended. Based solely on the holotype, the genus classification is likely correct. 


\subsection{Isogomphodon lerichei (Datervelle \& Casier 1943)}

The only image source from Isogomphodon lerichei was compiled from Jonet (1966), as well as the description. Although Jonet (1966) actually describes a var. minuta to I. lerichei, still relevant morphological remarks on I. lerichei could be performed, however with caution to draw deep conclusions. Nonetheless, the author states that this variation differs from the type species based on its smaller size, narrow crown and thin root at the edges but slightly more pronounced at the centre.

Based on the present observations, some specimens illustrated by Jonet (1966) may be an Isogomphodon species while other can belong to another genera. Firstly, the designed holotype (Fig. $11-1,2 \mathrm{a}$ and $2 \mathrm{~b}$ ) is probably not an Isogomphodon, considering the crown curvature, plain root (with a mild concavity and very discrete notch) and weaker sigmoid profile. As well as the holotype, specimen 7 is also likely misallocated in its genus, considering especially its lateral shoulders (which are dorsally expanded in comparison to the other specimens). Lastly, the curvature (i.e., mild asymmetry in the specimen) seen in 3, 4, 5, 6 and 8 also puts in question the identification of these specimens. The remaining teeth are likely an Isogomphodon as they fit to the generic description here provided, however they need further review considering the author did not state whether they could belong to a single individual or multiple. However, considering they were all attributed to Isogomphodon and that the holotype presented significant differences to the genus description, the taxonomic nomenclature of $I$. lerichei is under discussion. In a case where these specimens all originate from the same mandibular arch, then they are not likely an Isogomphodon.

\subsection{Isogomphodon gracilis (Jonet, 1966)}

As well as Isogomphodon lerichei, images of I. gracilis were solely gathered from Jonet (1966), although it was from the original description and not a var. minuta as I. lerichei. Initially, Cappetta (1970) states that the teeth of I. gracilis are likely anterior teeth of I. acuarius, and as far as the analysis of the morphological features of both taxa, endorsing such statement would require a deeper analysis of more specimens once I. acuarius is the most common and abundant Isogomphodon taxon being reported on literature. Although the name I. lerichei is listed as a synonym of I. acuarius by Lauritto (1999), Cicimurri \& Knight (2019) considered such taxon as valid, but with no further morphological details. 
In terms of morphology, the teeth of I. gracilis are indeed rather similar to I. acuarius, nonetheless the root is slight thicker and the crown mildly wider on the Portuguese species (see specimens $14-16$ ). Also, the sample 14 of Fig. 12 is rather similar to sample 2 from Carcharhinus limbatus (Fig. 16), specially on the root length and curvature. On the other hand, specimens 19 to 21 (described as a type specimen) have features that might strongly argue in favour of previous hypothesis that they might actually belong to I. acuarius, which as once postulated by Lauritto (1999). The characteristics of these specimens agree to the genus description, however, to delimit whether it corresponds to a valid different taxon from its congenerics, further presential analysis of the specimens is strongly recommended once not only the images are in low quality but also due to the similarities with I. acuarius from CarriloBriceño et al. (2016).

\subsection{Isogomphodon caunellensis (Cappetta, 1970)}

Cappetta (1970) and Laurito \& Valerio (2008) were the cited literature from whence the illustrations were collected from. Generally, I. caunellensis was described and differentiated from I. acuarius and I. lerichei mainly based on total size, sigmoid profile and cutting edges, in which the Caunelle species has larger teeth, stronger sigmoidal shape in lateral view and absence of cutting edge on the cusp base from lower teeth (Cappetta 1970).

According to Cappetta (1970), few differences delimitate I. caunellensis from I. acuarius and I. lerichei. Essentially, he states that the cutting edges does not reach the crown base in both upper and lower teeth, as this last is described by having a more evident sigmoid profile, and mentions 'total size' as diagnostic, in which the author states that $I$. caunellensis has bigger teeth. Additionally, this taxon is described by Laurito \& Valerio (2008) with a high and widened neck in lingual facet towards the root margin. As observed here, one of the transitions from cutting edge to lateral shoulder is nearly straight lined (Fig. 8 and Fig. 9 specimen 25), which should also be considered for a diagnostic feature of the species. Specimen 25 , the designed holotype, has a morphology that resembles the lower anterior teeth of the extant species (see Fig. 2, samples 5 and 6), however it seems damaged in informative regions such as the lateral shoulders, which should be considered before concluding whether this taxon is valid for its genus. To confirm such observation, a presential, analysis is recommended.

The sample 24, based on its thicker crown base and curved lateral shoulders, does not fit as an Isogomphodon, whereas the remaining samples can potentially be already correctly 
allocated. Nonetheless, as fewer specimens of I. caunellensis have been reported on the literature, the morphological variation along the dentary rows was poorly described. It is important to say, however, that the images resolution might impact on the analysis of the morphological features, especially on sample 26 of Fig. 14. Compared to the congeneric species, the complete endorsement of I. caunellensis as a valid Isogomphodon needs further review to raise more morphological data and as far as here observed, was not strongly corroborated.

\subsection{Isogomphodon aikenensis (Cicimurri \& Knight, 2019)}

Recently, Knight \& Cicimurri (2019) describe a novel taxon from the North America region, the Isogomphodon aikenensis. As well as previous authors, one of the key features listed as diagnostic is the total size, however the provided delimitation of I. aikenensis is rather more complete, considering the authors listed the absence of serrations on all teeth and the root lobes morphology apart from other detailed characters that fit to Isogomphodon dental exclusivities. The recent description of I. aikenensis was more detailed and essentially focused, apart from other differences, on the absence of serrations, the convexity of lateral shoulders, the elongation of root lobes and total size to clearly delimitate this taxon from its congenerics, likely representing perhaps the most sustained valid Isogomphodon fossil taxon. Although the description of I. aikenensis is more robust and well discussed, its teeth morphology resembles more the living species than the other congeneric taxa, apart from the cutting edges which are not serrated in the north American species. Considering that both their type localities are less distant geographically, the possibility that I. aikenensis is a valid Isogomphodon is likely presumable.

\subsection{Fossil taxa allocation as Isogomphodon}

Teeth morphology as a tool to describe novel fossil taxa has been classically employed by some palaeontologists (e.g., Cappetta, 1970; Cicimurri \& Knight, 2019). For some Carcharhinidae genera that are known to have highly specific and diagnostic dental features, for instance Galeocerdo (Compagno 1988), dental morphology might be sufficient to correctly allocated the extinct taxon in question. In Isogomphodon, on the other hand, the extant species I. oxyrhynchus (which was the model used for comparisons to allocate the congeneric fossil 
species) has a vast number of characteristics apart from teeth morphology that justify its classification as a separate monospecific genus within Carcharhinidae, it seems rather premature to utilize such anatomical structure to describe novel taxa as Isogomphodon. Additionally, the diagnostic features here proposed for the dentary characters on this genus go beyond the analysis of isolated teeth.

Besides the high plasticity of dental characters that occur as deep as an intraspecific level of individuals from different populations (Naylor \& Marcus, 1994), the fossilization process might damage specimens in potentially informative regions (e.g., lateral nodes, serration on cutting edges, internode facet) as observed in Lauritto \& Valerio (2008) and Cappetta (1970), which might lead to erroneous conclusions.

On the other hand, the present review provided relevant delimitation on the tooth morphology of Isogomphodon, due to which some of the described taxa in this genus were corroborated (I. acuarius and I. aikenensis) in their allocation on the genus while others were refuted or put into question (I. lerichei, I. gracilis and I. caunellensis). The type localities of these species are geographically distant (see Figure 1), which might have impacts on their validity. Considering I. acuarius has been reported to occur in South America (Vialle, Adnet \& Cappetta, 2011; Carrillo-Briceño et al. 2016; Carrillo-Briceño et al. 2019), its validity has a favourable argument considering the extant I. oxyrhynchus is restricted to the Atlantic portion of this continent. Moreover, the narrow occurrence area I. oxyrhynchus, suggests that this species probably has evolved and developed unique morphological characters that go beyond dentary anatomy (e.g., reduced eyes, large pectoral fins, long snout), once the habitat of $I$. oxyrhynchus is restricted to turbid estuarine waters with low visibility. The paleoenvironment in which the fossil taxa occurred has not been described, which means that by the same they can be occurring in similar conditions to I. oxyrhynchus, they also can be adapted to a completely different environment. 


\section{Concluding Remarks}

The general morphology of the teeth in Isogomphodon involves features that require more completed data to better identify the fossil taxa that were attributed to this genus. Previous authors elected the main differences to delimit it from the other Carcharhinidae genera as total size and total teeth counting, Here, the morphological graduation along the rows, narrow cusps with angular transitions to the crown and evident concavity on internode ventral region, especially on the anterior tooth, are proposed in an attempt to delimitate informative and diagnostic features of isolated teeth, using I. oxyrhynchus as a model. At the moment, the only fossil taxon that can positively be included in Isogomphodon are I. aikenensis and I. acuarius. Other species, I. lerichei, I. gracilis and I. caunellensis have characteristics which are compatible with an inclusion in the genus but lack definite diagnostic features. Those taxa fit just as well within the range of variation seen in species of Carcharhinus (e.g., Laurito 1999). Total tooth size is a recurrent diagnostic character in much of the literature. In itself, such trait is obviously not decisive, being dependent on the age/size of specimens., which is evidenced in I. oxyrhynchus in which the adult has larger, bulkier teeth. Considering that there is a specific way of assessing body size or maturity stage of fossil teeth alone (e.g., Cooper et al. 2020), such method should have been applied before-hand in order to detect if 'total size' character is actually diagnostic. Otherwise, total tooth size is not in itself a decisive evidence. Another key point is the fossilization process, which potentially alters diagnostic features such as the cuttingedge surface, giving the false impression they are smooth when they could potentially be serrated. Nonetheless, the present review lists as a consequence, Except for I. aikenensis and I. acuarius. all other fossil taxa so far allocated in Isogomphodon should be considered as Carcharhinidae sp. or perhaps another genus. 


\section{References}

Adnet, S., \& Cappetta, H. (2001). A palaeontological and phylogenetical analysis of squaliform sharks (Chondrichthyes: Squaliformes) based on dental characters. Lethaia, 34(3), 234-248.

Bigelow, H. B. \& Schroeder, W. C. (1948). Fishes of the Western North Atlantic, Part I: Lancelets, Cyclostomes, Sharks. Memoirs of the Sears Foundation for Marine Research, 1 (1): 59-576.

Cappetta, H. (1970). Les Sélaciens du Miocène de la région de Montpellier. Palaeovertebrata, 3 (extraordinaire),1-139 p., 22 fig., 27 pl..

Carrillo-Briceño, J. D., Aguilera, O. A., De Gracia, C., Aguirre-Fernández, G., Kindlimann, R., \& Sánchez-Villagra, M. R. (2016). An early Neogene elasmobranch fauna from the southern Caribbean (western Venezuela). Palaeontologia Electronica, 1-31.

Carrillo-Briceño, J. D., Luz, Z., Hendy, A., Kocsis, L., Aguilera, O., \& Vennemann, T. (2019). Neogene Caribbean elasmobranchs: diversity, paleoecology and paleoenvironmental significance of the Cocinetas Basin assemblage (Guajira Peninsula, Colombia). Biogeosciences, 16(1), 33-56.

Cicimurri, D. J., \& Knight, J. L. (2019). Late Eocene (Priabonian) elasmobranchs from the Dry Branch Formation (Barnwell Group) of Aiken County, South Carolina, USA. Paleobios, 36, $1-31$.

Compagno, L. J. (1988). Sharks of the order Carcharhiniformes . Princeton, New Jersey: Princeton University Press.

Cooper, J. A., Pimiento, C., Ferrón, H. G., \& Benton, M. J. (2020). Body dimensions of the extinct giant shark Otodus megalodon: a 2D reconstruction. Scientific reports, 10(1), 1-9.

Dartevelle, E., \& Casier, E. (1943). Poissons fossiles du bas-Congo. Annales du Musée du Congo belge Ser. 3A. 2, 201-256. (1943-1959).

Frederickson, J. A., Schaefer, S. N., \& Doucette-Frederickson, J. A. (2015). A gigantic shark from the lower cretaceous duck creek formation of Texas. PLoS One, 10(6), e0127162.

Gill, T. (1862). Analytical synopsis of the Order of Squali and revision of the nomenclature of the genera. Annals of the Lyceum of Natural History of New York, 7, 367-413.

Guinot, G., Adnet, S., \& Cappetta, H. (2012). An analytical approach for estimating fossil record and diversification events in sharks, skates and rays. PLoS One, 7(9), e44632.

Herman, J., Hovestadt-Euler, M., \& Hovestadt, D. C. (1991). Contributions to the study of the comparative morphology of teeth and other relevant ichthyodorulites in living supraspecific 
taxa of chondrichthyan fishes. Part A: Selachii. No. 2c: Order: Carcharhiniformes, Families: Proscylliidae, Hemigaleidae, Pseudotriakidae, Leptochariidae and Carcharhinidae. Bulletin Institut Royal des Sciences Naturelle de Belgique. Biol, 61, 73-120.

Klug, S., \& Kriwet, J. (2010). Timing of deep-sea adaptation in dogfish sharks: insights from a supertree of extinct and extant taxa. Zoologica Scripta, 39(4), 331-342.

Jonet, S. (1966). Notes d'ichthyologie miocène. II-Les Carcharhinidae. Boletim do Museu e Laboratorio Mineralógico e Geológico da Faculdade de Ciências, 10(2), 65-88.

Laurito Mora, C. A. (1999). Los Seláceos fósiles de la localidad de Alto Guayacán (y otros ictiolitos asociados): Mioceno-Superior-Plioceno Inferior de la formación Uscari. San José, CR: CA Laurito.

Laurito, C. A., \& Valerio, A. L. (2008). Ictiofauna de la localidad de San Gerardo de Limoncito, Formación Curré, Mioceno Superior, cantón de Coto Brus, provincia de Puntarenas, Costa Rica. Revista Geológica de América Central, (39), 65-85.

Maisey, J. G. (2012). What is an 'elasmobranch'? The impact of palaeontology in understanding elasmobranch phylogeny and evolution. Journal of Fish Biology, 80(5), 918951.

Naylor, G. J., \& Marcus, L. F. (1994). Identifying isolated shark teeth of the genus Carcharhinus to species: relevance for tracking phyletic change through the fossil record. American Museum Novitates, 3109,1-53.

Naylor, G. J., Caira, J. N., Jensen, K., Rosana, K. A., Straube, N., \& Lakner, C. (2012a). Elasmobranch phylogeny: a mitochondrial estimate based on 595 species. In: Carrier, J.C.; Jeffrey, C.; Musick, J.A. \& Heithaus, M.R. The biology of sharks and their relatives. CRC Press. v.3, p. 31-56.

Patterson, C. (1981). Significance of fossils in determining evolutionary relationships. Annual Review of Ecology and Systematics, 12(1), 195-223.

Probst, J. (1879). Beiträge zur Kenntniss der fossilen Fische aus der Molasse von Baltringen. Hayfische. Jahreshefte des Vereins für vaterländische Naturkunde in Württemberg, 35:127191.

Raschi, W., Musick, J. A., \& Compagno, L. J. V. (1982). Hypoprion bigelowi, a synonym of Carcharhinus signatus (Pisces: Carcharhinidae), with a description of ontogenetic heterodonty in this species and notes on its natural history. Copeia, (1982), 102-109.

Springer, S. (1950). A revision of North American sharks allied to the genus Carcharhinus. American Museum novitates; no. 1451. 
Straube, N., Li, C., Claes, J. M., Corrigan, S., \& Naylor, G. J. (2015). Molecular phylogeny of Squaliformes and first occurrence of bioluminescence in sharks. BMC evolutionary biology, 15(162), 1-10.

Vélez-Zuazo, X., \& Agnarsson, I. (2011). Shark tales: a molecular species-level phylogeny of sharks (Selachimorpha, Chondrichthyes). Molecular phylogenetics and evolution, 58(2), 207-217.

Vialle, N., Adnet, S., \& Cappetta, H. (2011). A new shark and ray fauna from the Middle Miocene of Mazan, Vaucluse (southern France) and its importance in interpreting the paleoenvironment of marine deposits in the southern Rhodanian Basin. Swiss Journal of Palaeontology, 130(2), 241-258. 


\section{Analysed Material}

Institutional abbreviations:

MZUSP - Museu de Zoologia da Universidade de São Paulo;

\section{Carcharhinidae}

Isogomphodon oxyrhynchus - MPEG 3455, 1 Adult male (alc, teeth samples).

Carcharhinus brevinpinna - MZUSP84423, 1 Juvenile female (alc, teeth samples) 


\section{Annex A - Original description of the Isogomphodon fossil taxa}

\section{Isogomphodon acuarius (Probst, 1879)}

The teeth in Fig. 76 from the outside and Fig. 77 from the inside, of which our collection counts half a hundred, are 4 very evenly shaped among themselves; the size varies between - $0.01 \mathrm{~m}$ and $0.005 \mathrm{~m}$; the slender tip stands upright on the base; in some the base is more horizontally expanding; there are no developed secondary peaks, but on some teeth, there is an elevation of the enamel at the end of the base; they are massive. They differ from the teeth of the Aprion stellatus, which are roughly the same size and shape, in the lack of hollowing in two interior parts of the base; then the base is also shaped quite differently; not only that it remains crisper and thus the 'characteristic shape of the three-pointed star' is blurred, but it is also rounded at the base in an arc - while in the Aprion stellatus it runs in a straight line. Likewise, the teeth cannot be accommodated in the Seylleum family. The base of the latter is much more expressive and strongly protruding inward, even with the slimmer teeth. The entire type points our Zabne into the family of the Lamnids and here they can only belong to the Alopecias family because of their great uniformity. Judging by the size of the teeth, this species may have been of roughly the same size as the live fish (Al. Vulpes), from which MH emphasized that the ratio of the body to the tail was 3:5. Tertiary period must, however, according to the grandiose ratio of the Zibue: to conclude, a much larger way of peopling the sea.

\section{Isogomphodon caunellensis (Cappetta, 1970)}

Diagnosis: Rather large carcharhinid. Lower teeth with narrow crown, slender, in sigmoidal profile; the cutting edge does not reach the base of the cusp; the root is quite massive, little spread laterally. Teeth larger than triangular crown, fairly flat, inclined towards the commissure; sharp net; root fairly spreading transversely.

\section{Description:}

Upper teeth: A lateral tooth (fig. 23) shows a fairly high, triangular crown, tilted towards the commissure. The outer side is almost flat, slightly depressed in her mid-lower region. The outer limit of the crown is rectilinear. The internal face is not very convex. The branches of the root, which is moderately high, are a continuation of each other and each wear a heel which is not separated from the cusp by a notch. The mesial branch is longer than the distal. A more lateral 
tooth (Fig. 24) has a larger crown and more inclined towards the commissure; depression of the lower part of your outer face is larger.

Lower teeth: These teeth have a slender, straight crown with a fairly sigmoidal profile. accused. The faces, external, almost flat except at the base, and internal, rather strongly convex, are separated by a sharp edge which ends before reach the base of the cusp; therefore, the base of the crown has an almost circular section. The root, massive, has short branches and very wide apart. The basilar face, well developed, has a median groove net. The crown sends on each branch a short oblique heel.

Reports and differences: This shape, by its size and morphology, is easily distinguished from other genera of the family. She immediately separates from A. acuarius by its larger size; on the other hand, his upper teeth are clearly broader and sharper, on the lower teeth missing at the base of the cusp. By its dimensions, it is similar to A. lerichei of the Miocene of Bas-Congo; however, it is distinguished by its lower curvature teeth sigmoidal more pronounced, with a thicker crown whose cutting edge does not reach the base. Its upper teeth are wider than in the species African.

\section{Isogomphodon lerichei var. minuta (Datervelle \& Casier, 1943) - as in Jonet (1966)}

The teeth considered are small with a narrower and more pointed crown than in type specimen. Their height is between $3.5 \mathrm{~mm}$ and $8 \mathrm{~mm}$ length, their width between $5.5 \mathrm{~mm}$ and $9 \mathrm{~mm}$ and the $\mathrm{H} / \mathrm{L}$ ratios are 0.83 for the upper teeth and 0.856 for the lower teeth. The crown is triangular and has its very domed internal face, while its slightly convex external face is limited to two flats which form a sharp edge on its edges. The cusp is extended laterally by two very thin heels, low, sharp 'and completely smooth. They are often almost horizontal, and, in this case, they are mainly visible on the external face. The root is very prominent in the middle but flattens out at both ends. Its two branches form a very obtuse angle or else a very large radius arc of a circle. The median groove is very well marked. The teeth of the upper jaw have a crown with a narrow base and are inclined slightly towards the commissure and, sometimes, towards the interior of the mouth. The teeth of the lower jaw are very thin, and the crown is very sharp and curved towards the inside of the mouth. The inner face of it is very rounded and its outer face, almost flat, sometimes has a small triangular dimple at its base often there are small vertical folds. This variety differs from the type species by its smaller dimensions and the greater narrowness of 
the crown. The root is also thinner and with its flattened ends although it is prominent in its center. This variety has mostly been found in Tortonien VIIa although lower teeth have been found on Helvétien Vc and VIa.

\section{Isogomphodon gracilis (Jonet, 1966)}

The teeth of this species have a long, tapered crown. The total height $\mathrm{H}$ of the tooth is between $7 \mathrm{~mm}$ and $9.5 \mathrm{~mm}$ for a total width $\mathrm{L}$ of $5 \mathrm{~mm}$ and $7.5 \mathrm{~mm}$ which gives 1.29 for the average $\mathrm{H}$ / $\mathrm{L}$ ratio. The internal face of the crown is strongly domed, semi-circular, while the external face is only slightly convex $\mathrm{x}$ and often presents at its base a small triangular dimple with small vertical folds. The cusp is continued laterally by two very thin and almost horizontal low heels. They are visible on the external side while on the internal side, only their extremity is visible slightly exceeding the root. It also happens that the tails terminate vertically before the lateral end of the root. It may be curved inwardly of the mouth and in root has a slight sigmoid curvature. The root is stout and swollen in the center. Its branches are quite short and do not generally form an obtained angle, but their base is, in the center, rounded in an arc. A furrow bottom notches the middle of the root. The upper macho teeth are a bit wider and their heels continue to the tips of the root branches which are flatter and somewhat rounded. This variety exists from the Helvetian Vc (where the teeth are more often reduced to their crown), until the Helvetian VIa and Tortonian VIIa.

\section{Isogomphodon aikenensis (Cicimurri \&Knight, 2019)}

Diagnosis: Nearly 400 specimens are referred to the new species, which differs from Recent Isogomphodon oxyrhynchus (Müller and Henle 1841) in that all teeth have smooth cutting edges extending from the apex to the very base of the crown. In contrast, the upper teeth of the extant species are weakly serrated, and edges of lower anterior teeth are often limited to the upper half of the crown. In addition, the Eocene teeth have shorter cusps, many exhibit very convex lateral shoulders (especially more lateral positions), and the root lobes are more elongated and divergent (Herman et al. 1991, Compagno et al. 2005). The new species differs from the fossil species I. acuarius (Probst 1879), I. lerichei (Darteville and Casier 1943), I. caunellensis (Cappetta 1970), and I. gracilis (Jonet 1966) in being smaller in overall size. Additionally, the Dry Branch species has narrower upper anterior teeth and complete cutting edges on all teeth when compared to I. caunellensis, and the convexity of the lateral shoulders of anterior teeth 
appears to be more pronounced than on I. acuarius (Cappetta 1970, Case 1980, Müller 1999). The transition from main cusp to lateral shoulders appears to be slightly more angular on teeth of I. lerichei (Darteville and Casier 1943).

Description: These 393 distinctive teeth generally measure less than $5 \mathrm{~mm}$ in total height, although some anterior teeth are up to $7 \mathrm{~mm}$. All teeth have a tall and narrow cusp, and enameloid shoulders that extend nearly to the tips of the root lobes. The cutting edges of all teeth are smooth and extend to the tips of lateral shoulders. The holotype, SC2013.38.110, is an upper anterior tooth that is slightly asymmetrical (Fig. 4E). The crown consists of a tall, narrow and erect cusp, with cutting edges that diverge slightly towards the crown base. Elongate lateral shoulders extend obliquely onto the root lobes. The root is bilobate, with rather short and sub-rectangular lobes that are separated by a narrow but deep U-shaped interlobe area. The mesial lobe is slightly smaller than the distal lobe, and a deep but narrow nutritive groove bisects the flat lingual root face. Upper anterolateral teeth (Fig. 4P) are wider than those near the jaw symphysis. The cusp is narrow and flat as seen in more anterior positions, but slightly distally inclined. The lateral shoulders are more elongated and nearly horizontal. The subrectangular root lobes are lower, more elongated and divergent than on anterior teeth, and the interlobe area is wider but shallower. Lower anterior teeth (Fig. $4 \mathrm{H}, \mathrm{I})$ have a very tall and narrow cusp, with sub-parallel cutting edges. Lateral shoulders are short and nearly horizontal. The bilobate root is bisected by a deep, narrow nutritive groove, and the short lobes are widely separated by a U-shaped interlobe area. Lower lateral teeth (Fig. 4F, G) are similar to those in the upper jaw, but the cusp is narrower, lateral shoulders are shorter, the transition from cusp to shoulder is more angular, and the mesial root lobe is shorter than the distal one. The root is low and bilobate, with rounded and diverging lobes that are separated by a wide and shallow U-shaped interlobe area. A narrow but deep nutritive groove is located on the lingual face. Posterolateral and posterior teeth (Fig. 4L-O) are small with a T-shaped outline. The cusp is triangular but sharply tapering apically, rather low compared to teeth in more anterior positions. The cusp is vertical to slightly distally inclined, flat in profile. Lateral shoulders are short to elongate, perpendicular to the cusp, and the transition from cusp to shoulder is more angular than seen on more anterior jaw positions. The lateral shoulders in these positions are very convex at their distal extremities, resulting in a cusp-like appearance in lingual view. The root is bilobate with short, rounded, highly diverging lobes, which are separated by a broad, shallow 
to deep U-shaped interlobe area. The lingual face bears a centrally located narrow but deep nutritive groove. 


\section{Tables and Figures}

Table 1. Literature sources for fossil tooth images and/or remarks.

\begin{tabular}{|c|c|}
\hline Species & Literature \\
\hline Isogomphodon acuarius (Probst 1879) & $\begin{array}{c}\text { Probst (1879), Lauritto (1999), } \\
\text { Carrillo Briceño } \text { et al. (2016), } \\
\text { Cicimurri \& Knight (2019) }\end{array}$ \\
\hline Isogomphodon lerichei (Dartevelle \& Casier 1943) & $\begin{array}{c}\text { Jonet (1966), Cicimurri \& Knight } \\
\text { (2019) }\end{array}$ \\
\hline Isogomphodon gracilis (Jonet 1966) & $\begin{array}{c}\text { Jonet (1966), Cicimurri \& Knight } \\
\text { (2019) }\end{array}$ \\
\hline Isogomphodon caunellensis (Cappetta 1970) & $\begin{array}{c}\text { Cappetta (1970), Lauritto \& } \\
\text { Valerio (2008), Cicimurri \& Knight } \\
\text { (2019) }\end{array}$ \\
\hline Isogomphodon aikenensis (Cicimurri \& Knight 2019) & Cicimurri \& Knight (2019) \\
\hline Isogomphodon oxyrhynchus (Müller \& Henle 1839) & $\begin{array}{c}\text { Compagno (1988), Cicimurri \& } \\
\text { Knight (2019), present study }\end{array}$ \\
\hline
\end{tabular}




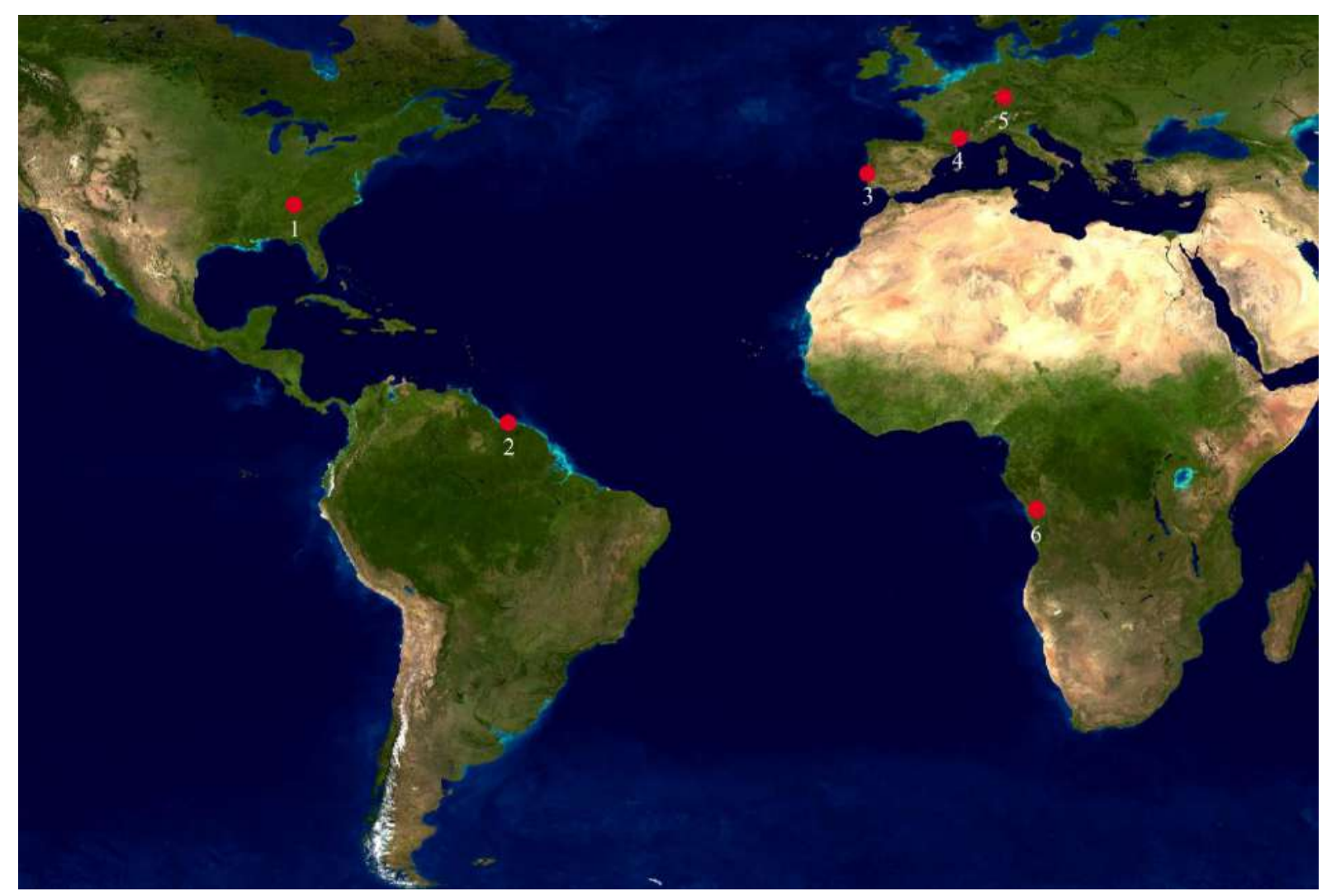

Figure 1. Type localities of the Isogomphodon fossil and extant species. 1. Isogomphodon aikenensis (Cicimurri \& Knight 2019) - Fossil; 2. Isogomphodon oxyrhynchus (Müller \& Henle 1839) - Extant; 2. Isogomphodon gracilis (Jonet 1966) - Fossil; 4. Isogomphodon caunellensis (Cappetta 1970) - Fossil; 5. Isogomphodon acuarius (Probst 1879) - Fossil; 6. Isogomphodon lerichei (Darteville \& Casier 1943) - Fossil; 


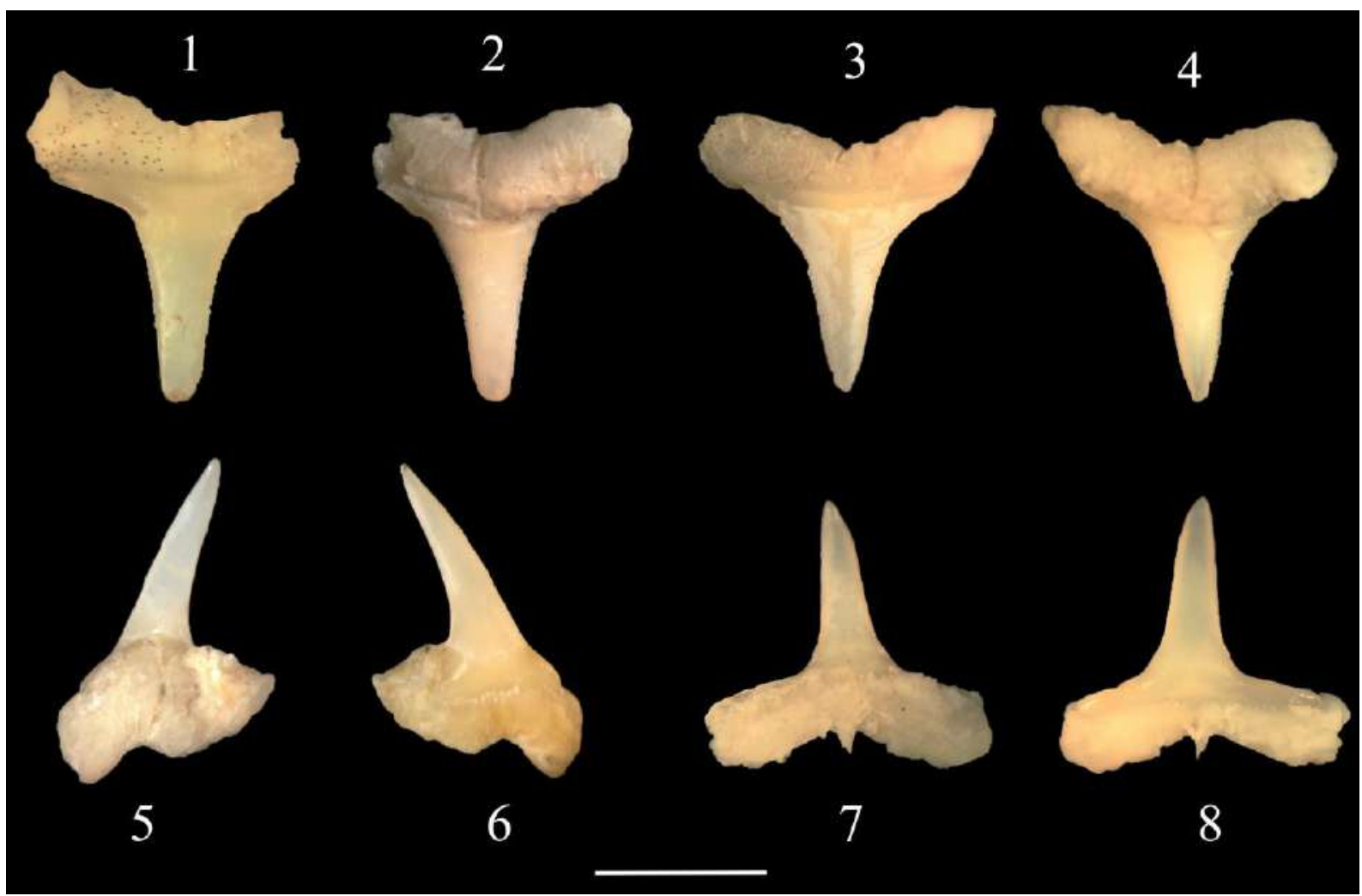

Figure 2. Teeth samples of Isogomphodon oxyrhynchus (MPEG 3455). Adult male. 1-2: Upper anterior teeth, in labial (1) and lingual (2) views; 3 - 4: Upper lateral teeth, in labial (3) and lingual (4) views; 5 - 6: Lower anterior teeth, in lingual (5) and labial (6) views; 7 - 8: Lower lateral teeth, in in lingual (7) and labial (8) views; Scale bar: $5 \mathrm{~mm}$.

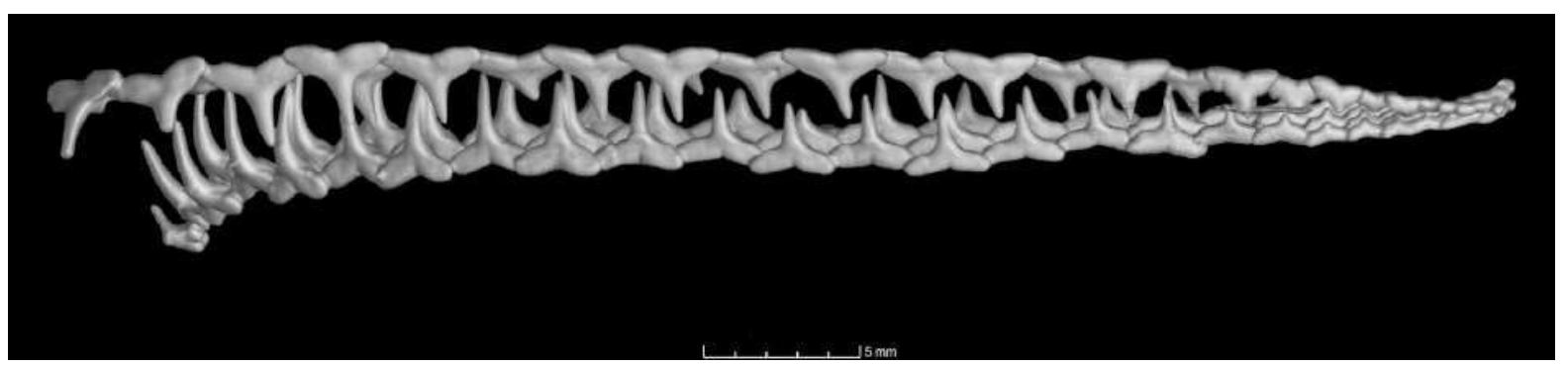

Figure 3. CT-Scan image of labial-lateral view of the dentition of Isogomphodon oxyrhynchus (MZUSP 101214), both upper and lower respectively positioned.

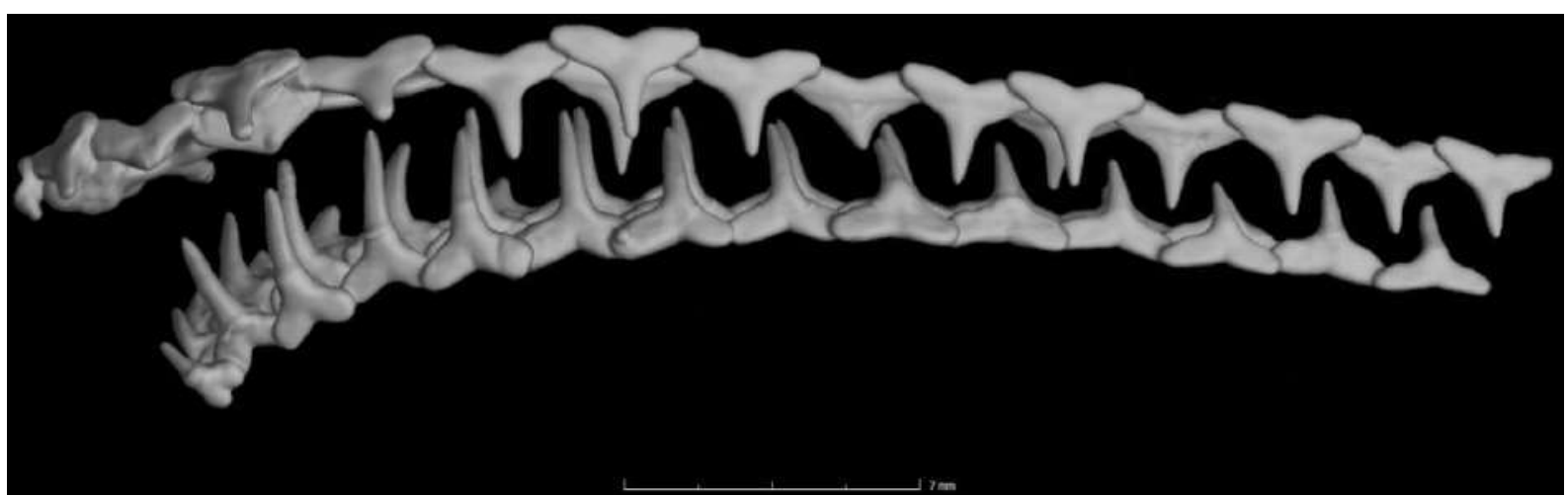

Figure 4. CT-Scan image of labial view of the dentition of Isogomphodon oxyrhynchus (MZUSP 101214), focusing on the anterior tooth rows, both upper and lower respectively positioned. 


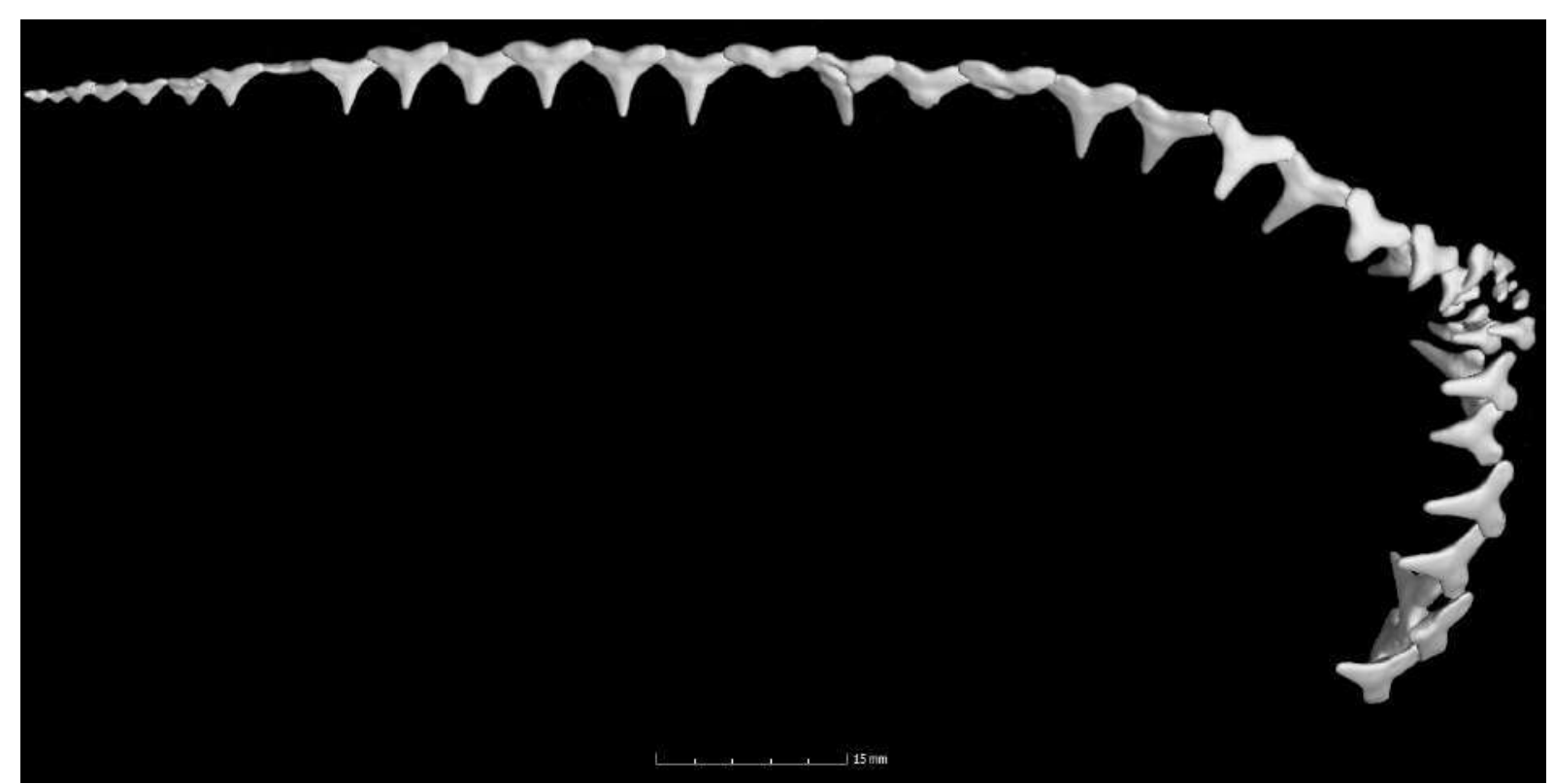

Figure 5. CT-Scan image of labial view of the upper dentition of Isogomphodon oxyrhynchus (MPEG3455).

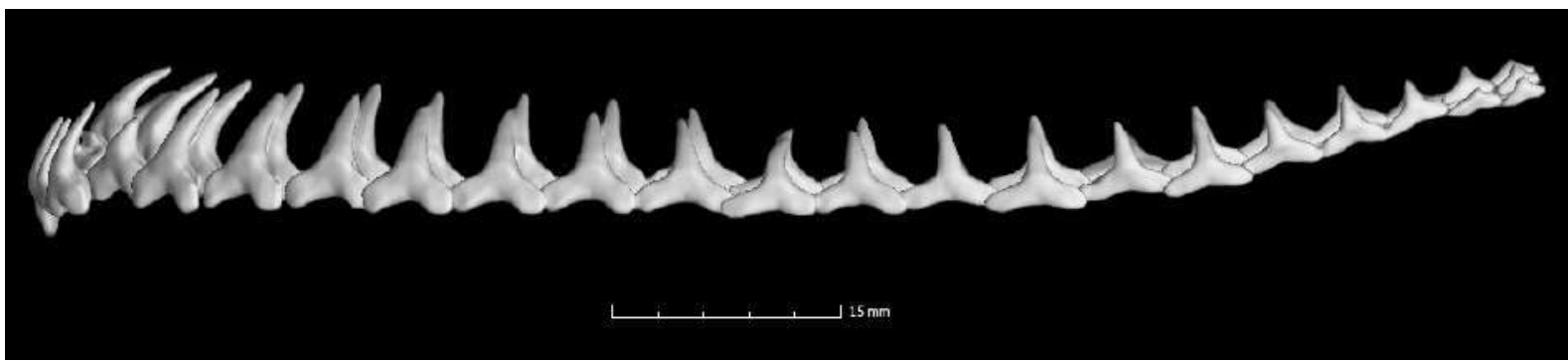

Figure 6. CT-Scan image of Labial view of the lower dentition of Isogomphodon oxyrhynchus (MPEG3455). 


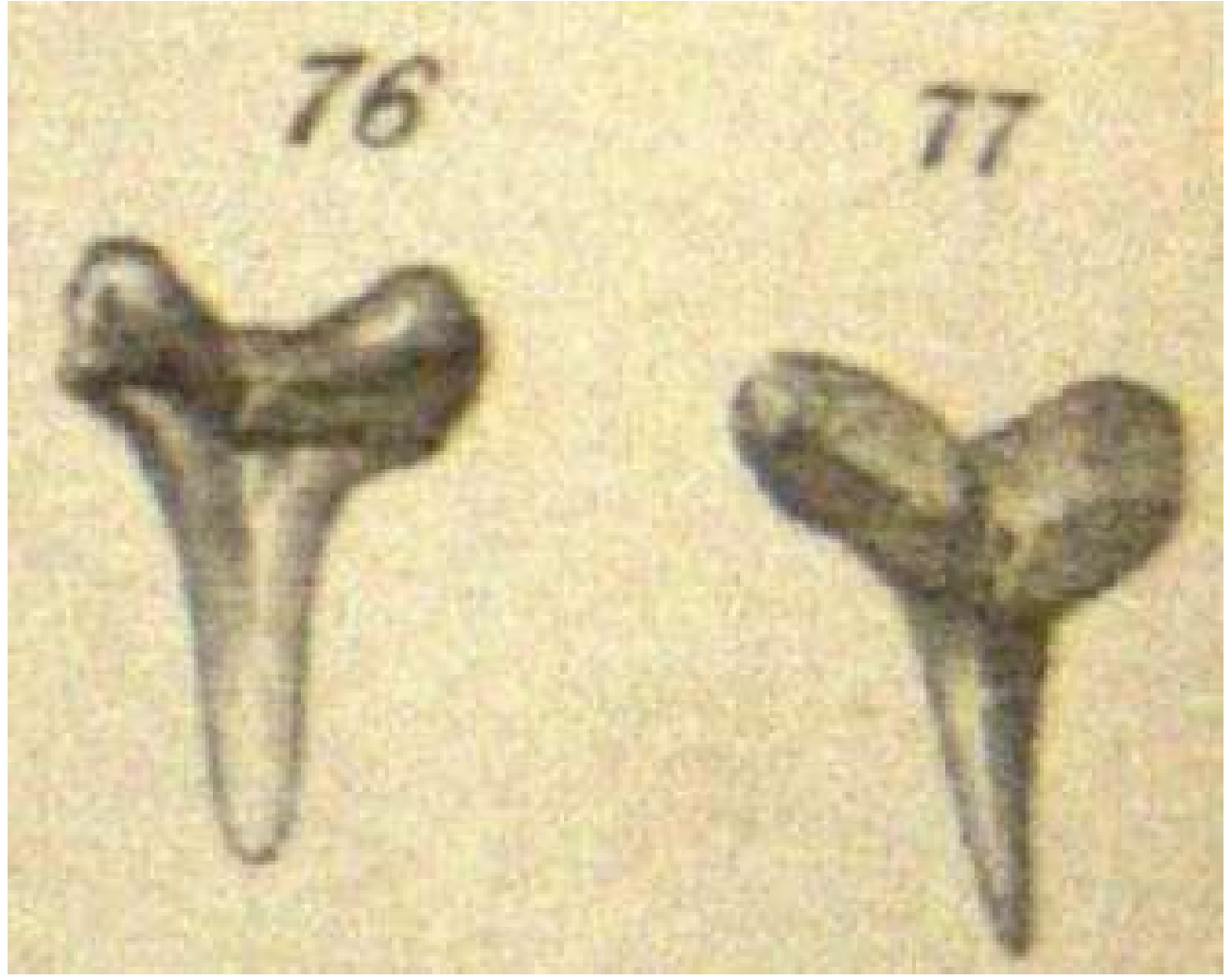

Figure 7. Holotype of Isogomphodon acuarius (Probst 1879). Original figure crop, as in Probst 1879. Teeth position not specified; 76 - labial facet; 77 - lingual facet. 


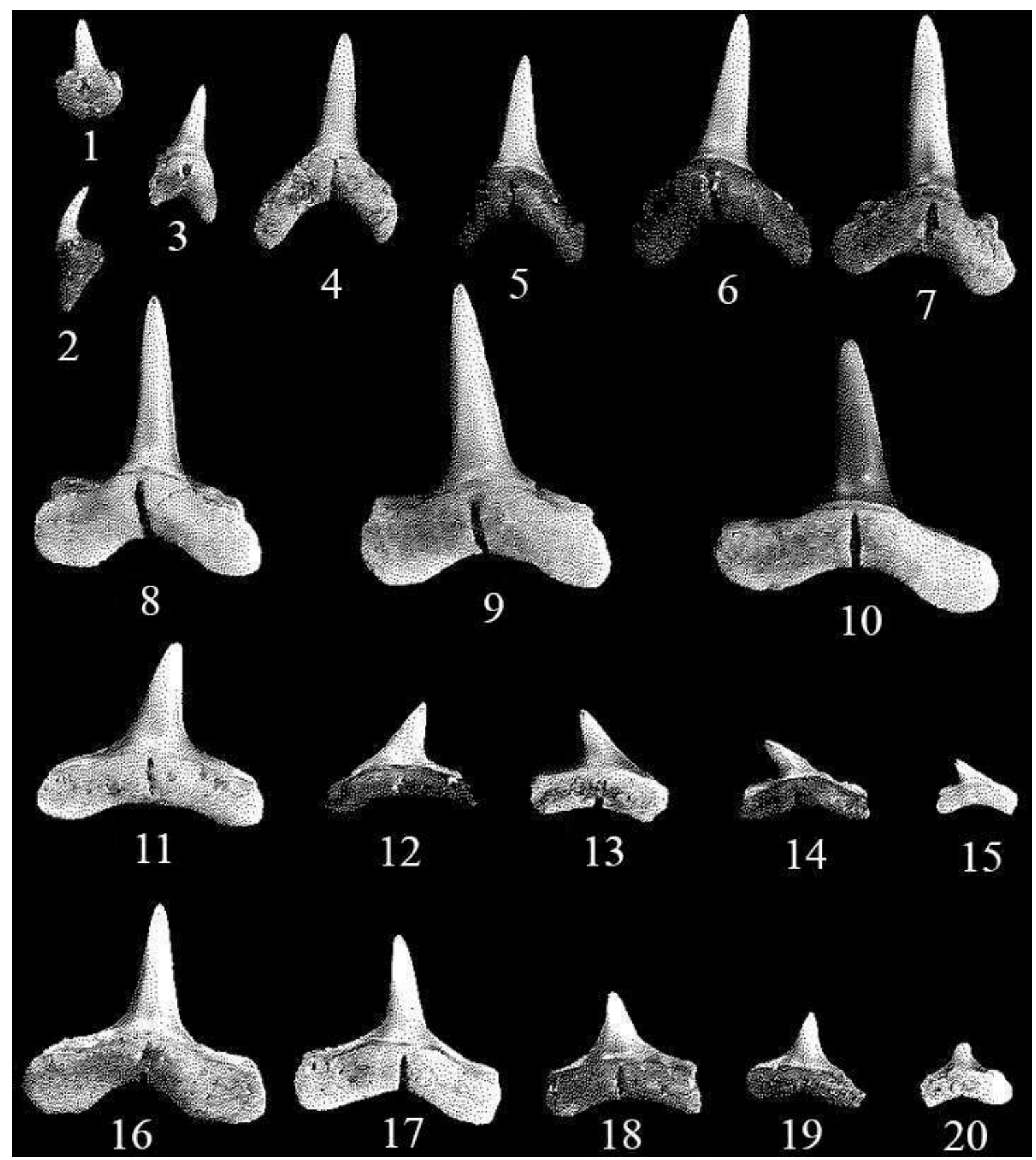

Figure 8. Tooth samples of Isogomphodon acuarius in Cappetta 1970. Image photoshop edited for schematic purposes. 1: (LPN 233) Parasymphysial teeth, lingual facet; 2: (LPN 234) Parasymphysial teeth, lateral facet; 3 : (LPN235) Parasymphysial teeth, lingual facet; 4 - 8: (LPN 236 - LPN 240) Anterior teeth, lingual facet; 9 - 15 : (LPN 214 - LPN 247) Upper lateral teeth in an anterior-posterior gradation, lingual facet; 16 - 20: (LPN 248 LPN 252). Lower lateral teeth in an anterior-posterior gradation, lingual facet, 


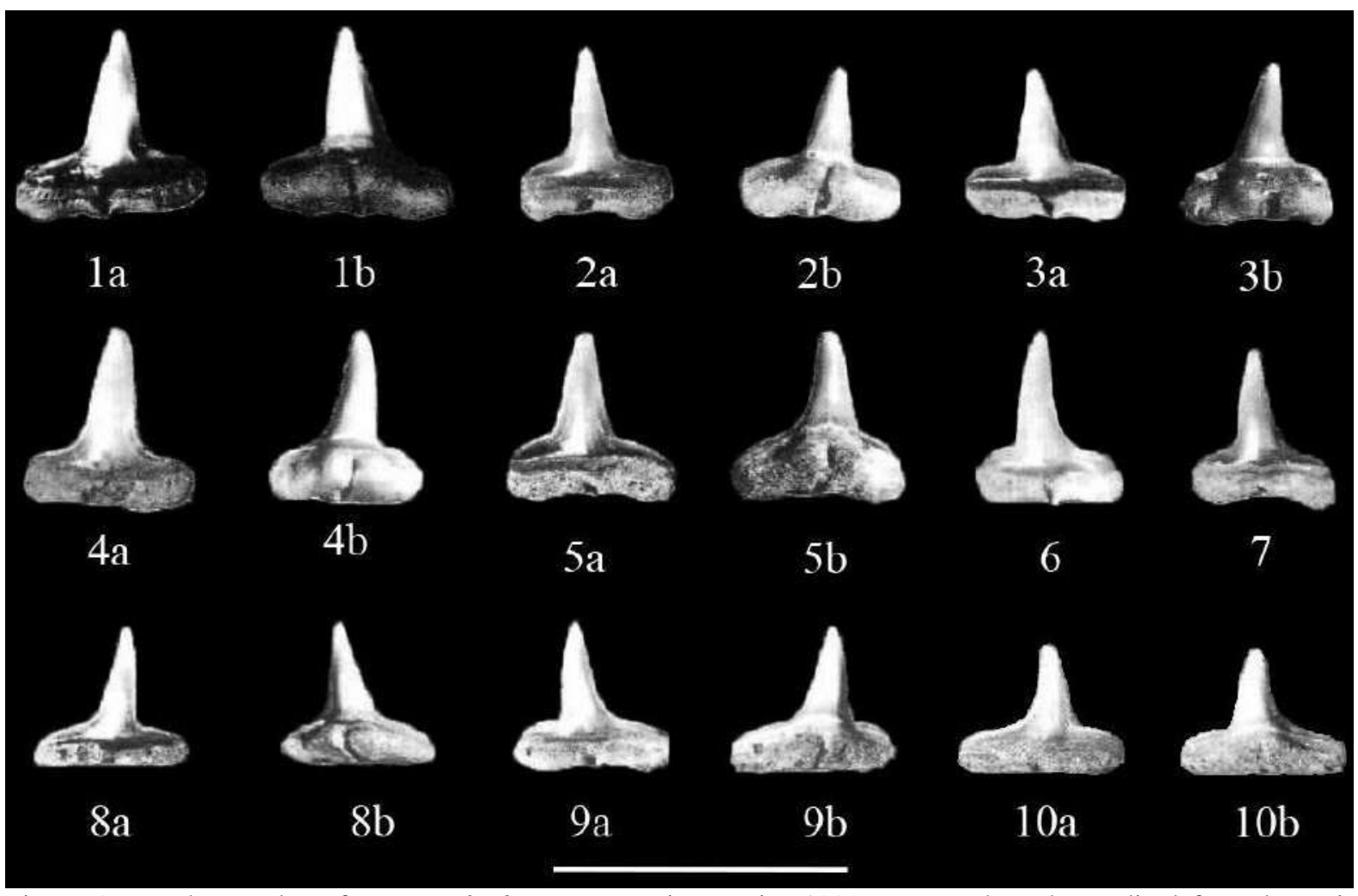

Figure 9. Tooth samples of Isogomphodon acuarius in Laurito 1999. Image photoshop edited for schematic purposes. Scale bar: 10mm; 1: (CLM-713) Anterior lateral teeth of uncertain position in labial (a) and lingual (b) facets; 2: (CLM-660) Lateral teeth of uncertain position in labial (a) and lingual (b) facets; 3: (CLM-643) Anterior lateral teeth of uncertain position in labial (a) and lingual (b) facets; 4: (CLM-650) Anterior lateral teeth of uncertain position in labial (a) and lingual (b) facets; 5: (CLM-665) Anterior lateral teeth of uncertain position in labial (a) and lingual (b) facets; 6: (CLM-649) Posterior lateral teeth of uncertain position in labial (a) and lingual (b) facets; 7: (CLM-642) Anterior lateral teeth of uncertain position in labial (a) and lingual (b) facets ; 8: (CLM663) Posterior lateral teeth of uncertain position in labial (a) and lingual (b) facets; 9: (CLM-670) Commissural teeth of uncertain position in labial (a) and lingual (b) facets; 10: (CLM-685) Commissural teeth of uncertain position in labial (a) and lingual (b) facets;

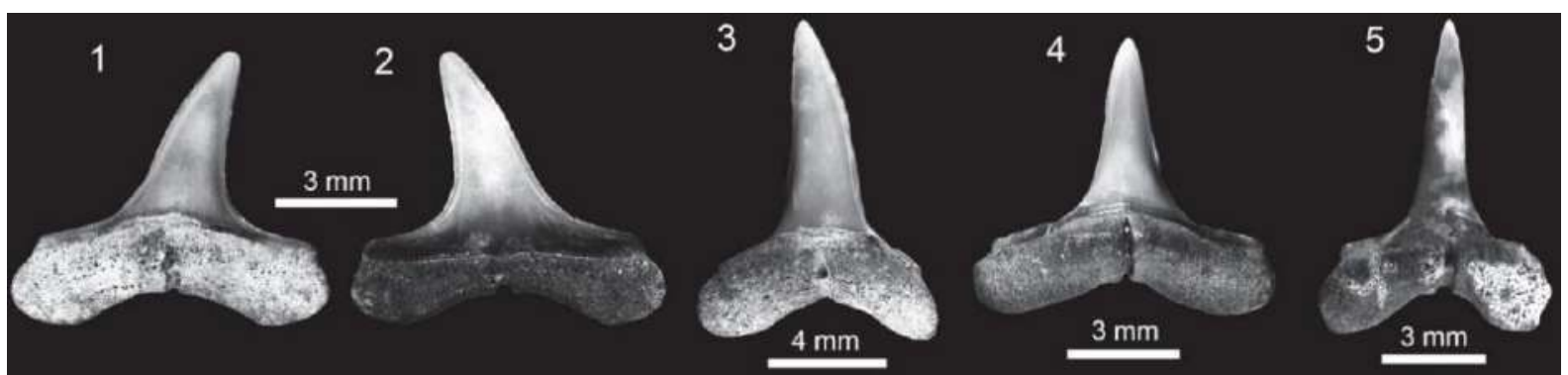

Figure 10. Isogomphodon acuarius (Probst 1879). Original figure, as in Carrilo-Briceño et al. 2016. 1 - 3 upper 4 -5 lowers. 


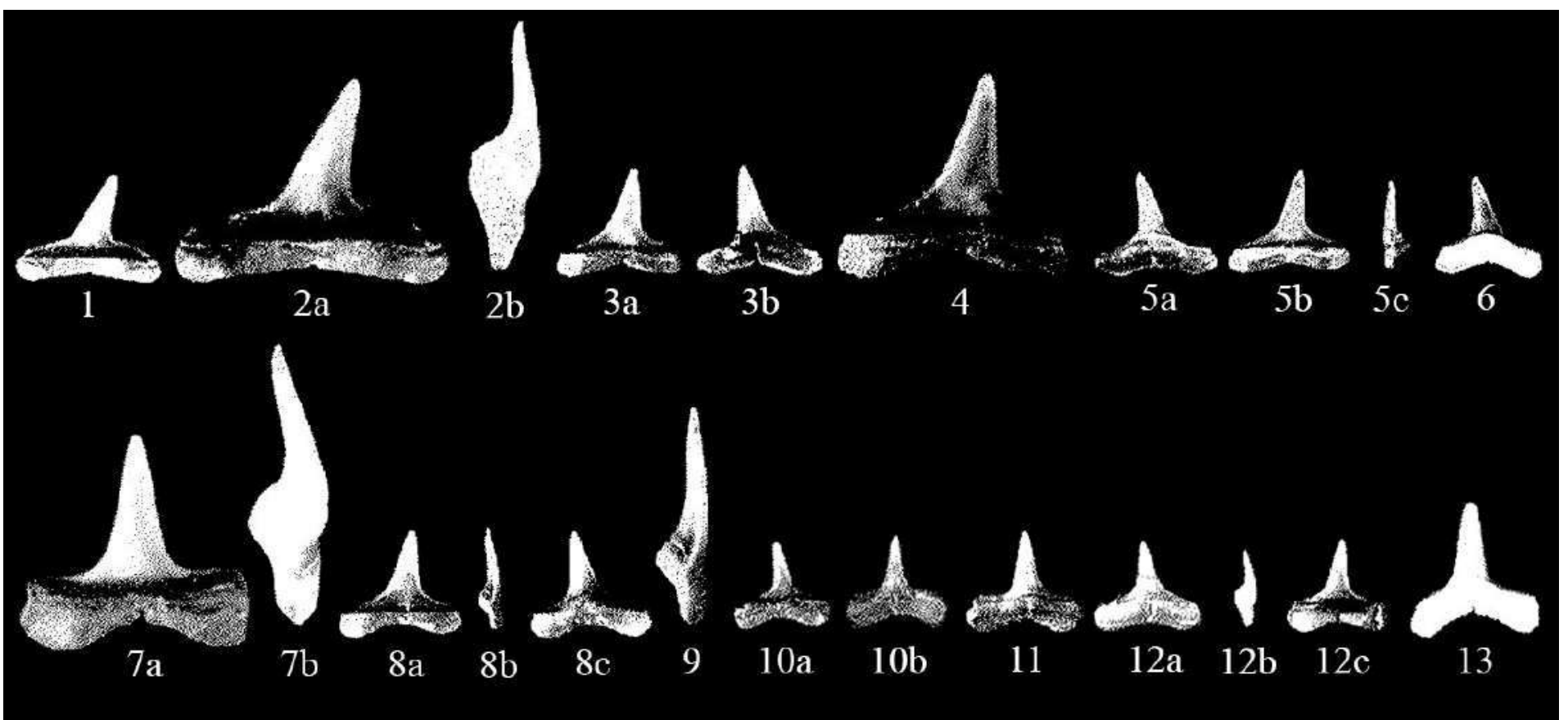

Figure 11. Tooth samples images of Isogomphodon lerichei (Daterville \& Casier, 1943), from Jonet 1966. Image was edited for schematic purposes, and teeth with the same number correspond to the same sample, as the letters describe different views. 1: TYPE - Labial facet of upper right teeth; $2 \mathrm{a}$ : Teeth sample 1 with $2 \mathrm{x}$ magnification labial facet; $2 \mathrm{~b}$ : Sample 1 with 2x magnification - lateral view; 3a: Upper right teeth - labial facet; 3b: Upper right teeth - lingual facet; 4: Teeth sample 3a with 2x magnification - labial facet; 5a: Upper right teeth - lingual facet; 5b: Upper right teeth - labial view; 5c: Upper right teeth - lateral view; 6: Upper right teeth - lingual facet; 7a: Lower right teeth with $2 \mathrm{x}$ magnification - labial facet; $7 \mathrm{~b}$ : Lower right teeth with $2 \mathrm{x}$ magnification - lateral view; 8a: Upper right teeth - lingual view; 8b: Upper right teeth - lateral view; 8c: Upper right teeth - labial view; 9: Teeth sample $8 \mathrm{~b}$, with 2x magnification - lateral view; 10a: Type - Lower left teeth - labial view; 10b: Type Lower left teeth - lingual view; 11: Teeth sample 7 in lingual facet - in 1:1 scale; 12a: Lower left teeth - lingual facet; 12b: Lower left teeth - lateral facet; 12c: Lower left teeth - labial facet; 13: Upper left teeth - lingual facet; 


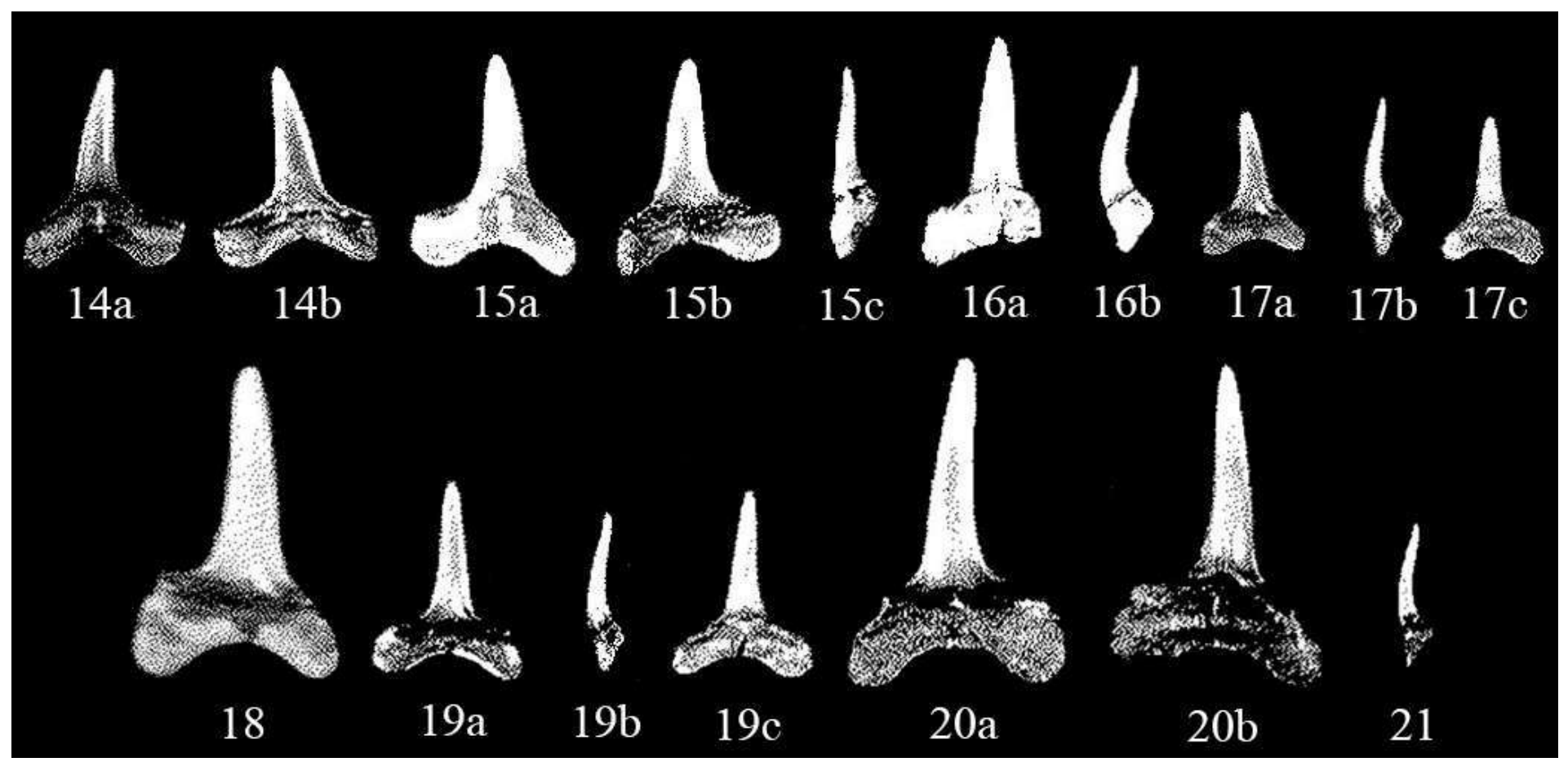

Figure 12. Tooth samples from Isogomphodon gracilis (Jonet 1966), from Jonet 1966. Image was edited for schematic purposes, and teeth with the same number correspond to the same sample, as the letters describe different views. 14a: Upper left teeth - lingual facet; 14b: Upper left teeth - lingual facet; 15a: TYPE - Lower teeth lingual facet; 15b: TYPE - Lower teeth - labial facet; 15c: TYPE - Lower teeth - lateral view; 16a: Lower teeth - lingual facet; 16b Inferior teeth - lateral view; 17a: Lower left teeth - lingual facet; 17b: Lower left teeth - lateral view; 17c: Lower left teeth - labial facet; 18: Teeth sample 17a, with 2x magnification; 19a: TYPE - Lower left teeth - labial facet; 19b: TYPE - Lower left teeth - lateral view; 19c: TYPE - Lower left teeth - lingual facet; 20a: Lower right teeth with 2x magnification - labial facet; 20b: Lower right teeth with 2x magnification - lingual facet; 21: Teeth sample 20 in 1:1 size - lateral view;
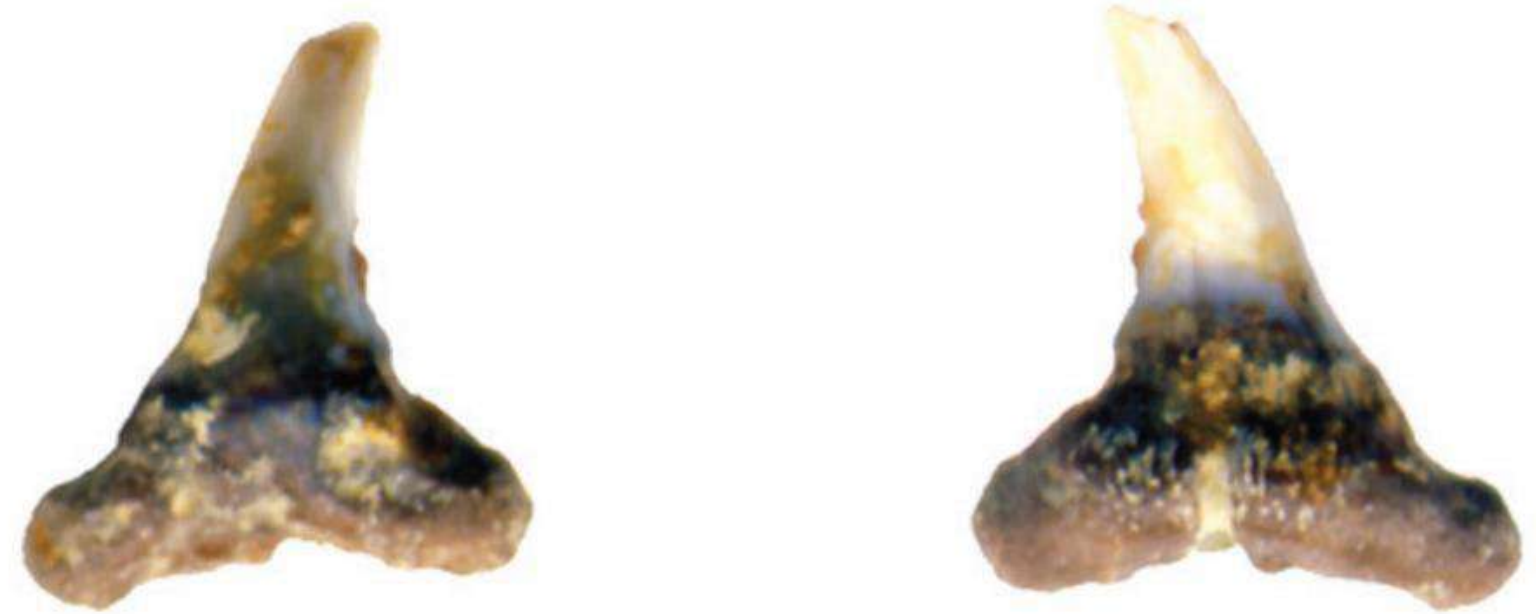

Figure 13. A possible upper tooth from Isogomphodon caunellensis (Cappetta 1970, original image from Lauritto e Valerio 2008). Labial view (right) and Lingual view (left). 


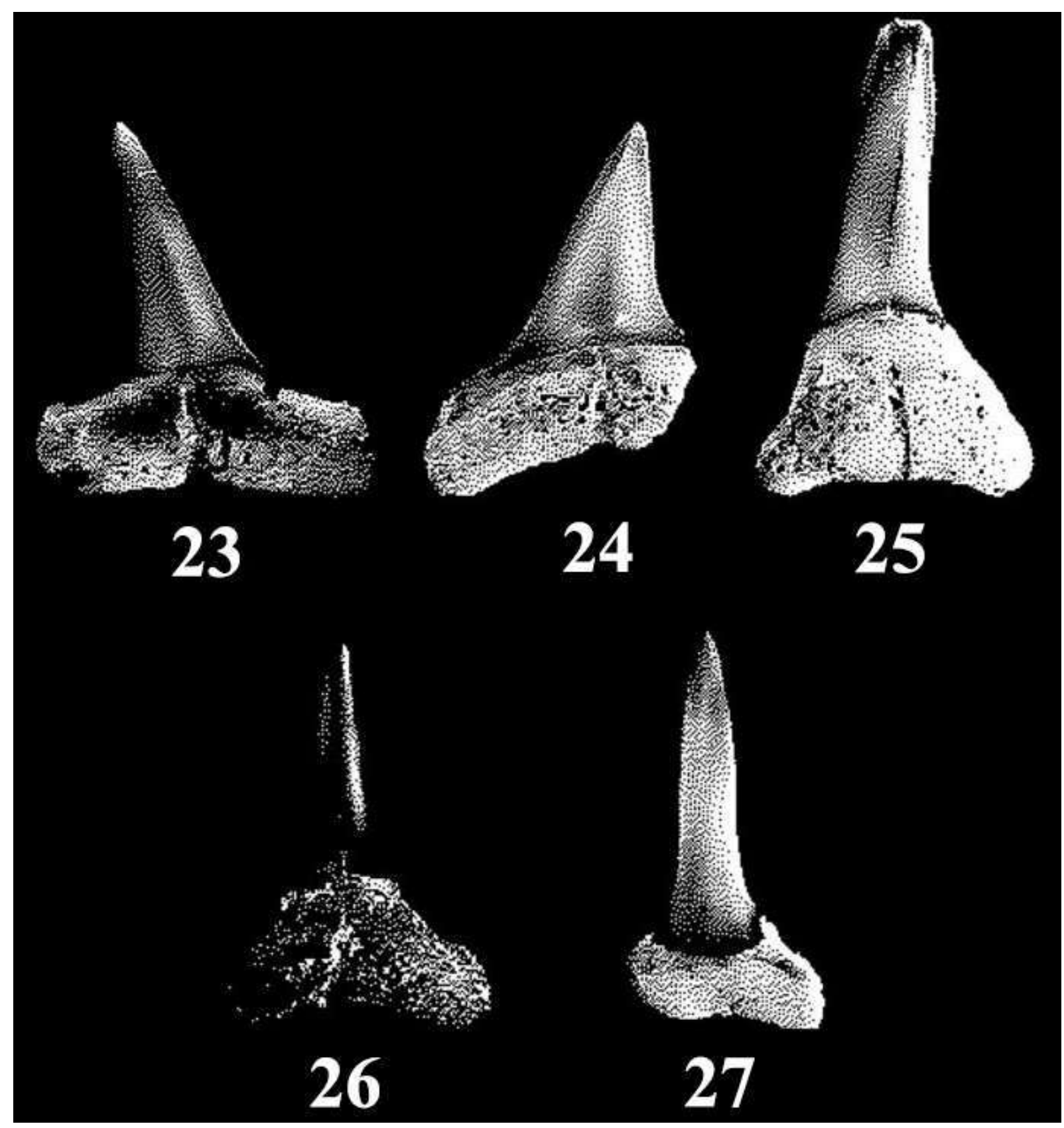

Figure 14. Tooth specimens of Isogomphodon caunellensis as in Cappetta 1970. Image photoshop edited for schematic purposes. Sample numbers: 23 - Superior teeth, lingual view; 24 - Superior teeth, lingual view; 25 TYPE, Inferior teeth, lingual view; 26 - Inferior teeth, lingual facet; 27 - Inferior teeth, labial facet. 


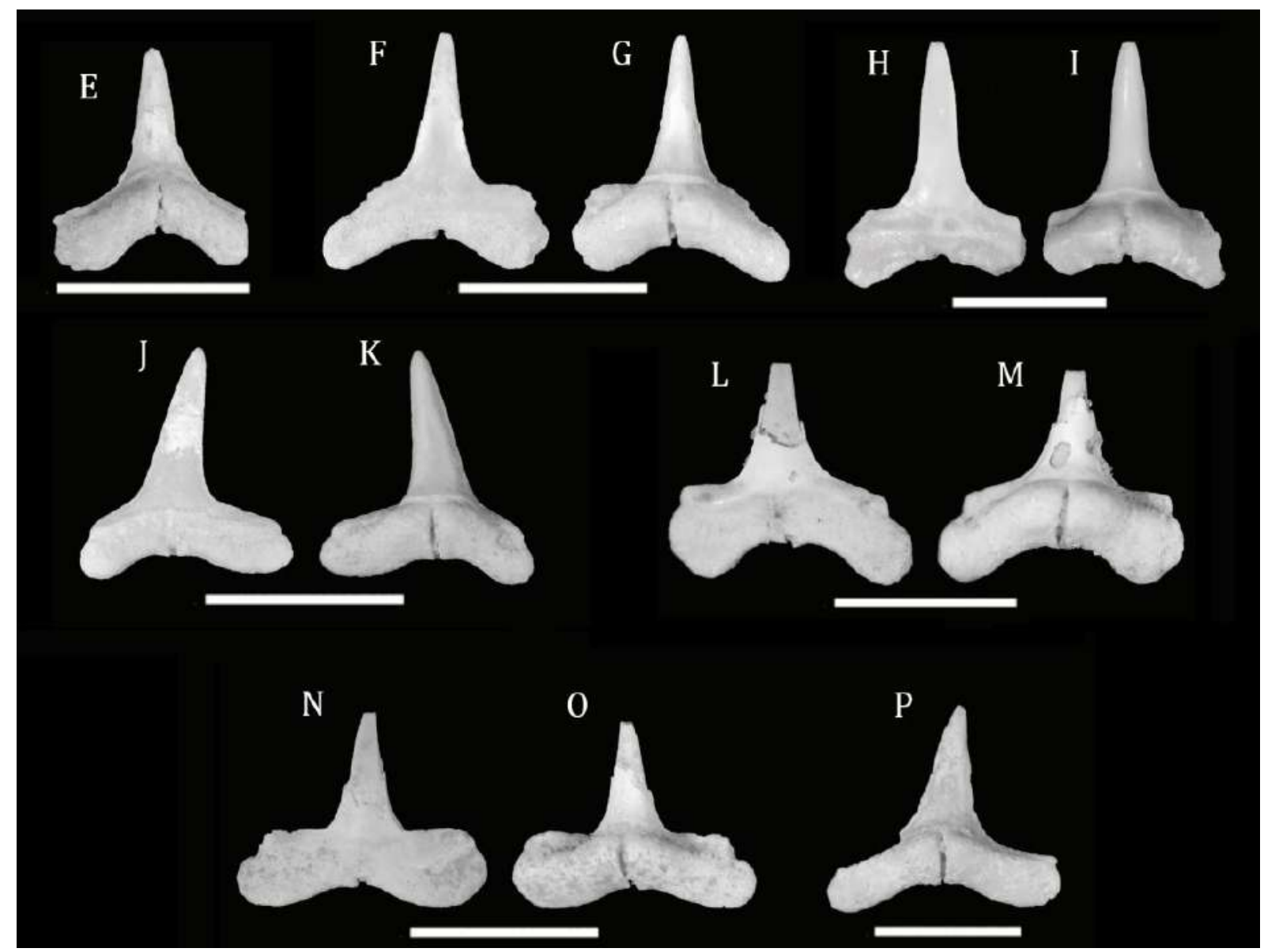

Figure 15. Tooth samples of Isogomphodon aikenensis $(\mathrm{E}-\mathrm{P})$ from Cicimurri \& Knight 2019. Image edited for schematic purposes. E: Holotype (SC2013.38.110) - Upper anterior tooth, lingual facet; F - G: Lower lateral tooth (SC2013.38.123) in labial $(\mathrm{F})$ and lingual $(\mathrm{G})$ facets; H - I: Lower anterior tooth (SC2013.38.119) in labial (H) and lingual (I) facets; J - K: Paratype (SC2013.38.112) - Upper lateral tooth in labial (J) and lingual (K) facets; L - M: Paratype (SC2013.38.114) - Posterolateral tooth in labial (L) and lingual (M) facets ; N - O: Posterior tooth (SC2013.38.115) in labial (N) and lingual (O) facets; P: Paratype (SC2013.38.111 - Upper anterolateral tooth in lingual facet; 


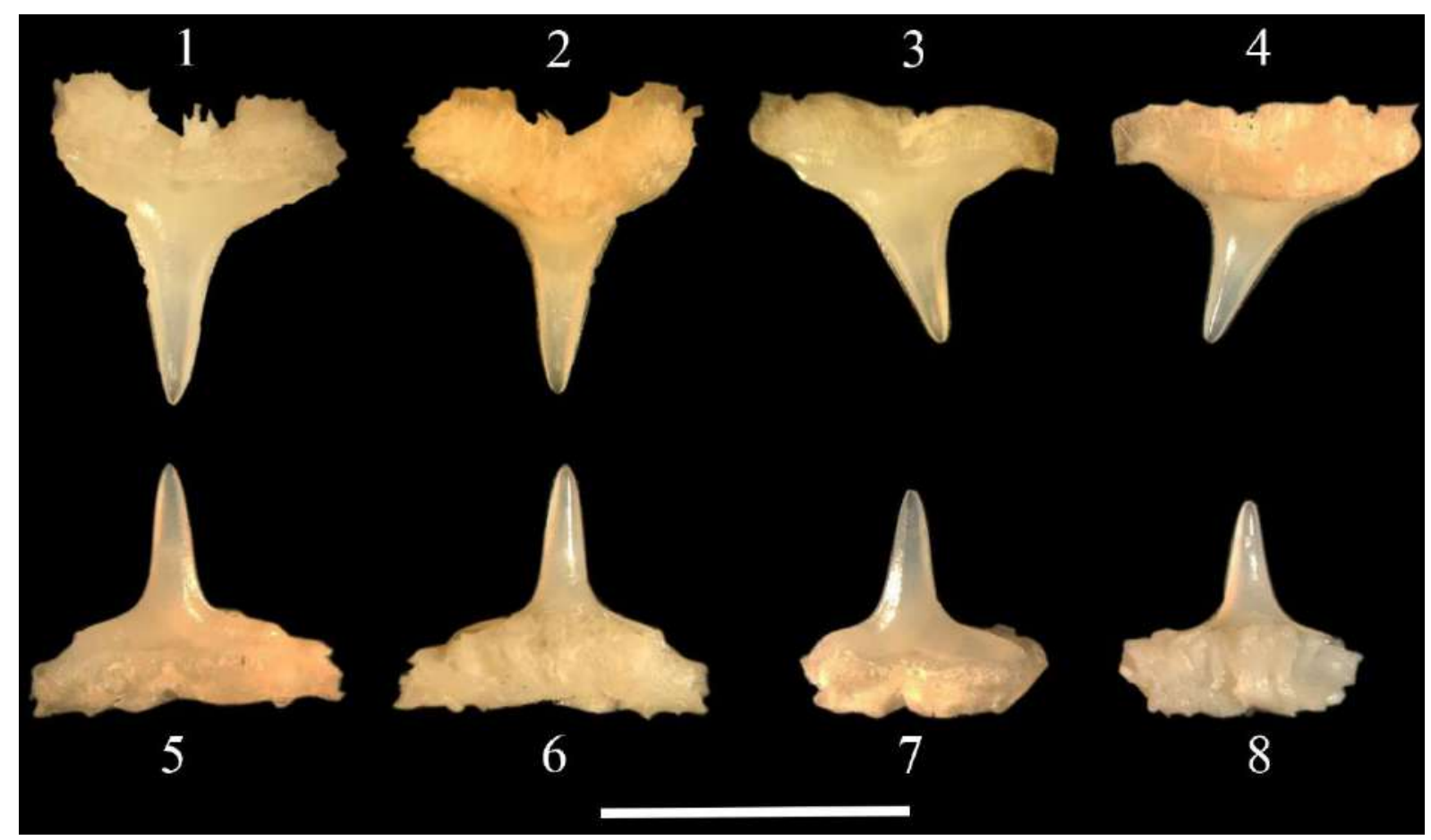

Figure 16. Teeth samples of a juvenile female Carcharhinus brevipinna (MZUSP 84423). Authorial image from the present study. 1 - 2: Upper anterior teeth, in labial (1) and lingual (2) views; 3 - 4: Upper lateral teeth, in labial (3) and lingual (4) views; 5 - 6: Lower anterior teeth, in labial (5) and lingual (6) views; 7 -8: Lower lateral teeth, in in labial (7) and lingual (8) views; Scale bar: $5 \mathrm{~mm}$. 